\title{
GEOPHYSICAL INSTITUTE
}

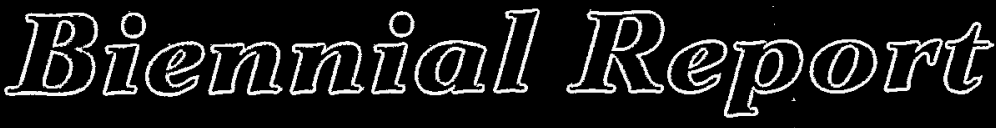

1993-1994

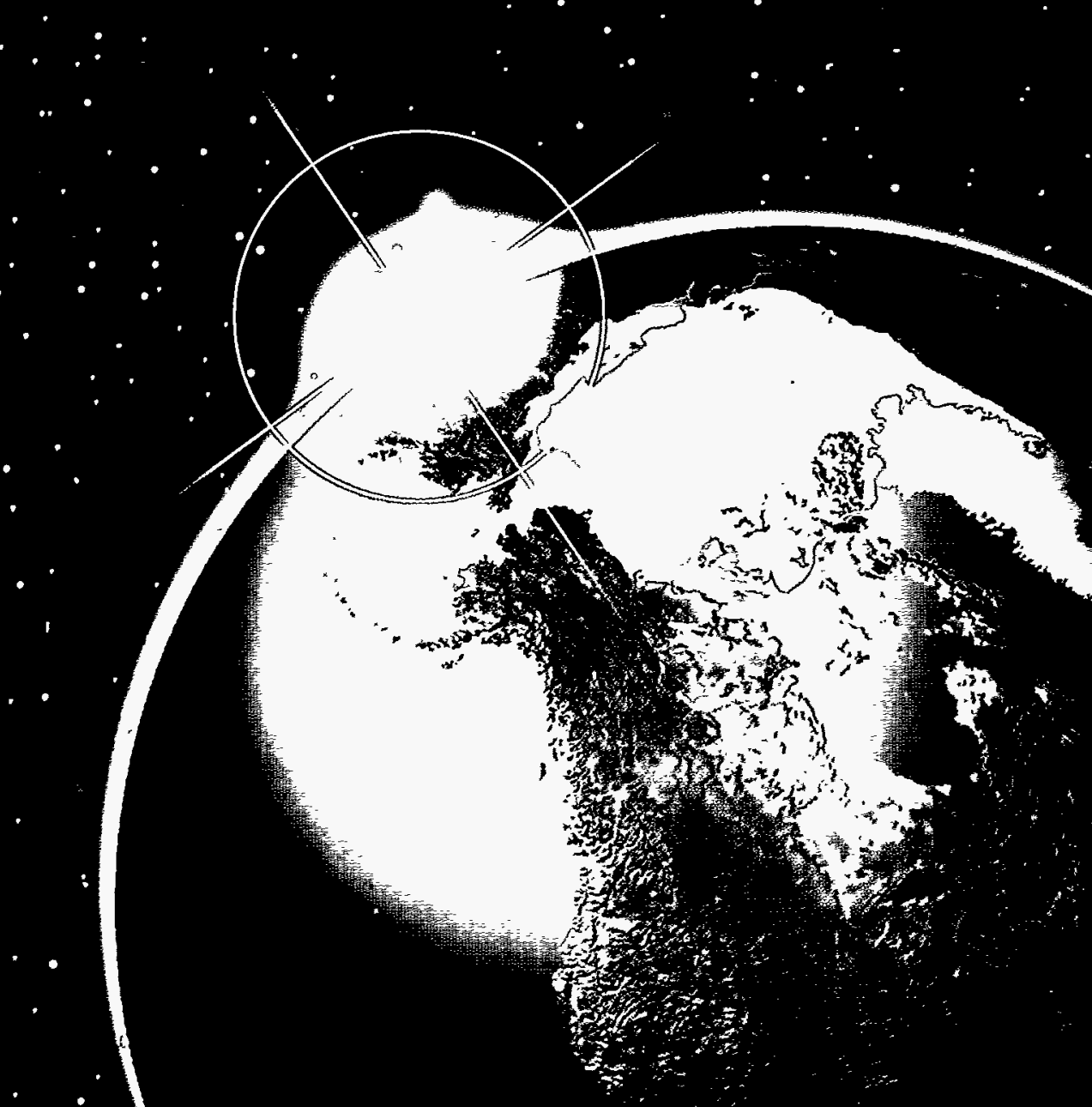




\section{Publisher's Note}

The 1993-94 Geophysical Institute Biennial Report was published November 1995 by the Geophysical Institute of the University of Alaska Fairbanks. Copies of this report are available free from the Geophysical Institute Information/Publications Office, 903 Koyukuk Drive, P.O. Box 757320, UAF, AK 99775-7320.

This report was produced by the Geophysical Institute Information/Publications Office and the Geophysical Institute Photo-Graphics Service Center.

\section{Cover Note}

An artistic impression of a solar eclipse decorates the front cover of the 1993-94 Geophysical Institute Biennial Report. The images on the front and back covers depict sea ice conditions on earth at a similar time of year (late August and early September). The front cover shows the minimum extent of the arctic ice pack in the northern hemisphere during the summer, while the back cover shows the maximum extent of the antarctic ice pack in the southern hemisphere during the winter.

Geophysical Institute Graphic Artist Deborah Coccia designed the cover.

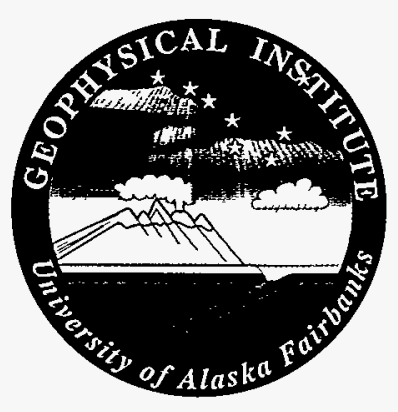

Universtr of A LASKA Fairbanks

The University of Alaska Fairbanks is an equal opportunity/affirmative action employer and educational institution. 
GJ-- 96004370

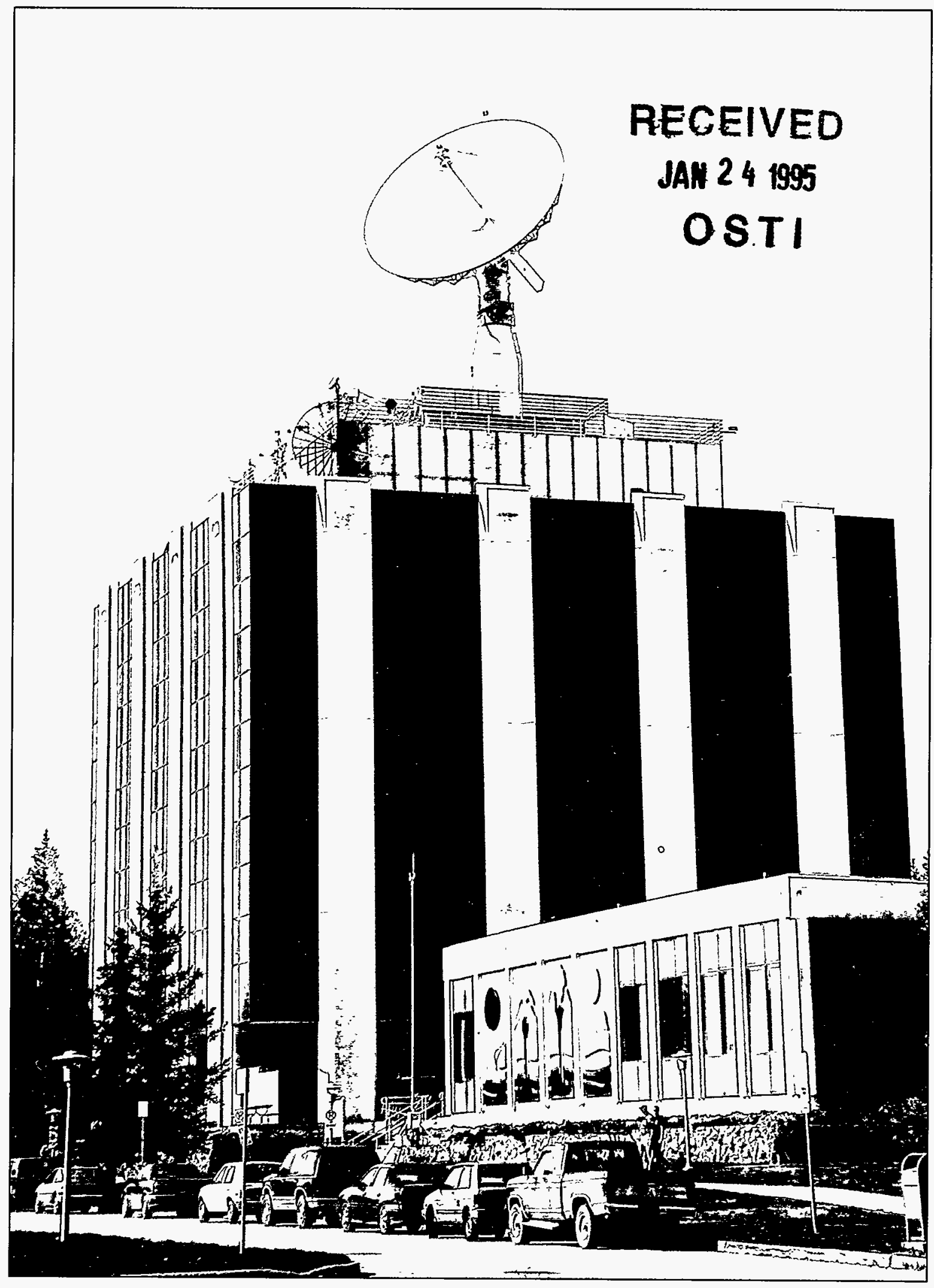

Biennial Report 1993-94 Geophysical Institute University of Alaska Fairbanks 


\section{Geophysical Institute*}

C.T. Elvey Building, 903 Koyukuk Drive, P.O. Box 757320, UAF, Fairbanks, AK 99775-7320

INTERNET E-MAIL: fygipub@aurora.alaska.edu TELEPHONE: 907-474-7558

FAX: 907-474-7290

TELEX: 354140 GEOPH INST FBK

TELEMAIL: GEOPH.INST.FBK

*Syun-Ichi Akasofu, Director

\section{University of Alaska Fairbanks Administration}

Signers' Hall, UAF, Fairbanks, AK 99775

Joan Wadlow, Chancellor

John Keating, Provost (2 February 1994-present)

Michael Rice, Vice Chancellor for Administrative Services

\section{University of Alaska Statewide System Administration}

Butrovich Building, UAF, Fairbanks, AK 99775

Jerome Komisar, President

Luis Proenza, Acting Vice President for Academic Affairs and Research (-1 July 1994)

'Nanne Myers, Assistant Vice President for Academic Affairs and Research

Brian Rogers, Vice President for Finance

Wendy Redman, Vice President for University Relations

William Kauffman, Vice President and General Counsel

Patty Kastelic, Executive Director of Human Resources

Abe Baggen, Director of Institutional Research

\section{University of Alaska Board of Regents **}

Sharon Gagnon President (1991-1999)

Virginia Breeze, Vice President (1989-1997)

Susan Stitham, Secretary (1987-1995)

Mark Helmericks, Treasurer (1987-1995)

Mary Jane Fate (1993-2001)

Eric Forrer (1989-1997)

Joseph Henri (1991-1999)

Michael Kelly (1991-1999)

R. Danforth Ogg (1993-2001)

Scott Otterbacher (1993-1995)

Lew Williams Jr. (1991-1999)

* Refer to contents for complete administrative listing.

** As of December 1994 


\title{
Geophysical Institute Advisors
}

The Geophysical Institute Advisory Consulting Board annually meets with the director to review current activities and to advise on management issues, particularly fiscal matters. Scientific review panels established for each of five research areas meet on a rotating basis, approximately once in three years, to consider recent progress and current research, and to plan for future development.

\section{Geophysical Institute Advisory Consulting Board (1994)}

\author{
Hugh Bradner, University of California, San Diego - Chair \\ Earl Beistline, Fairbanks \\ Gene Bierly, American Geophysical Union, Washington D.C. \\ Charles Kennel, (on leave) University of California, Los Angeles \\ Harry Persckek, Lexington, MA \\ Keith Runcorn, Imperial College of Science, Technology and Medicine, London \\ Philip Smith, Washington D.C. (effective August 1994)
}

\section{Geophysical Institute Scientific Review Panels (1994)}

\section{ATMOSPHERIC SCIENCES}

Robert Dickinson, University of Arizona

V. Ramanathan, Scripps Institute of Oceanography

Knut Stamnes, University of Alaska Fairbanks

John Winchester, Florida State University

\section{SNOW, ICE, AND PERMAFROST}

Roger Barry, University of Colorado

Keith Echelmeyer, University of Alaska Fairbanks

Arthur Lachenbruch, USDI Geological Survey

Charles F. Raymond, University of Washington

\section{VOLCANOLOGY}

Robert W. Decker, Double Decker Press

John Eichelberger, University of Alaska Fairbanks

Robert Kay, Cornell University

Peter Mouginis-Mark, University of Hawaii

Lionel Wilson, University of Lancaster

\section{SEISMOLOGY}

Hiroo Kanamori, California Institute of Technology

Carl Kisslinger, University of Colorado

Stewart Smith, University of Washington

Max Wyss, University of Alaska Fairbanks

\section{SPACE PHYSICS}

Syun-Ichi Akasofu, University of Alaska Fairbanks

Roger Arnoldy, University of New Hampshire

James L. Burch, Southwest Research Institute John Craven, University of Alaska Fairbanks

John Dawson, University of California-Los Angeles

Frank Djuth, Geospace Research, Inc.

Chester Gardner, University of Illinois

Ching-I. Meng, Johns Hopkins University

Raymond G. Roble, National Center for Atmospheric

Research

Roger Smith, University of Alaska Fairbanks

\section{DISCLAIMER}

This report was prepared as an account of work sponsored by an agency of the United States Government. Neither the United States Government nor any agency thereof, nor any of their employees, makes any warranty, express or implied, or assumes any legal liability or responsibility for the accuracy, completeness, or usefulness of any information, apparatus, product, or process disclosed, or represents that its use would not infringe privately owned rights. Reference herein to any specific commercial product, process, or service by trade name, trademark, manufacturer, or otherwise does not necessarily constitute or imply its endorsement, recommendation, or favoring by the United States Government or any agency thereof. The views and opinions of authors expressed herein do not necessarily state or reflect those of the United States Government or any agency thereof. 


\section{Contents}

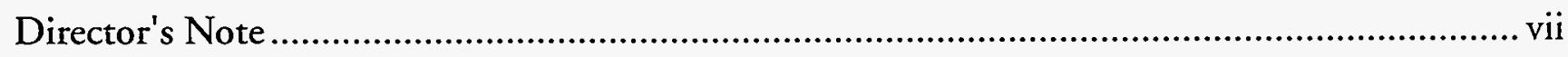

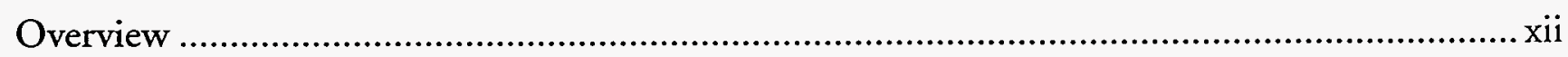

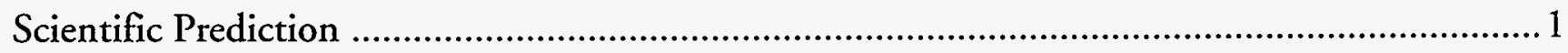

Can We Predict Geomagnetic Storms? …………………..................................................... 3

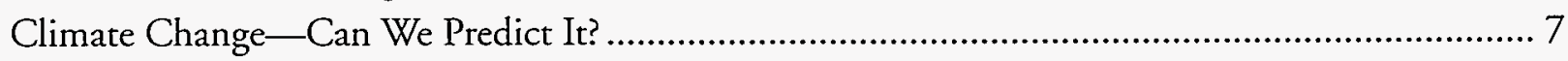

Predicting Glacier Surges ................................................................................................... 10

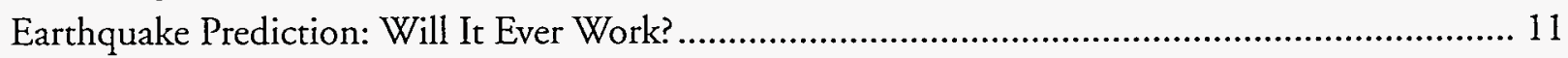

Can We Predict Volcanic Eruptions? ............................................................................... 14

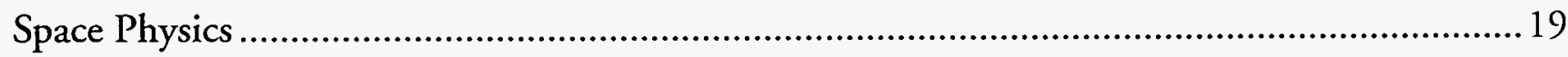

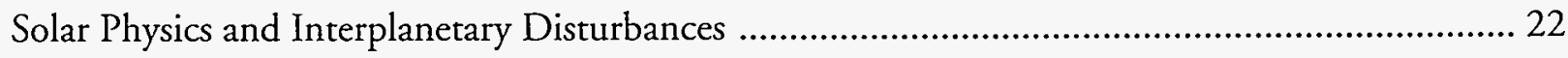

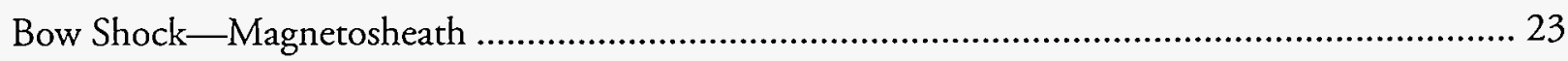

Magnetopause Boundary Layer: Magnetic Reconnection and Transport..................................... 25

Ionospheric Signatures of Dayside Boundary Layer Processes ..................................................... 30

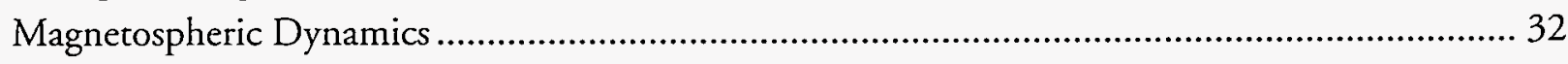

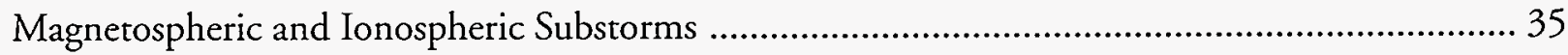

Aurora, Auroral Structures, and Current Systems .................................................................... 44

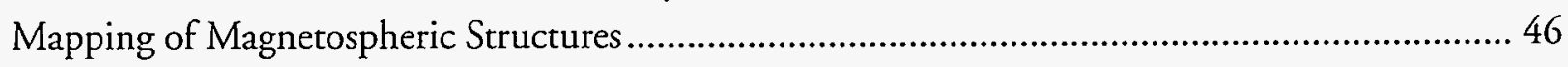

Magnetometer Studies: Auroral Currents, ULF Waves ........................................................... 50

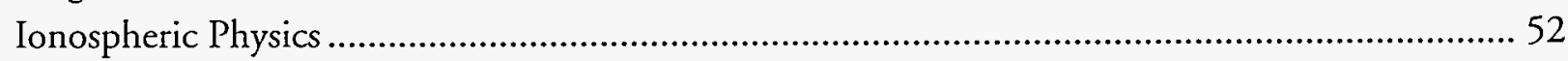

Modeling of Ionospheric Auroras ...................................................................................... 53

Thermospheric and Mesospheric Dynamics .......................................................................... 55

Upper and Middle Atmospheric Optical Flashes ................................................................... 59

Poker Flat Research Range ................................................................................................. 60

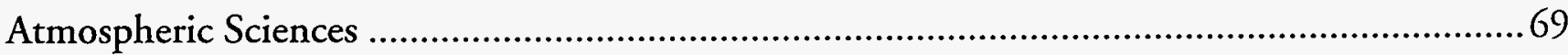

Regional Meteorology and Climatology …………………............................................... 72

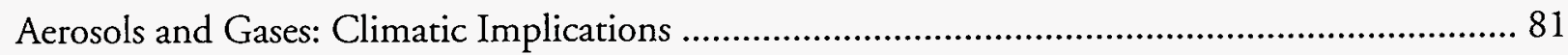

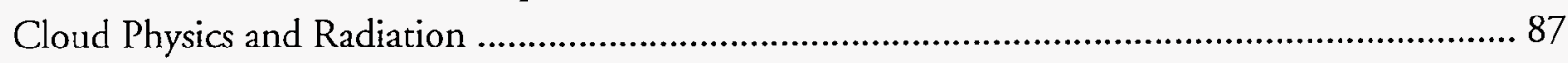

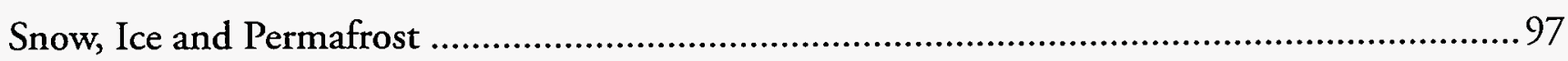

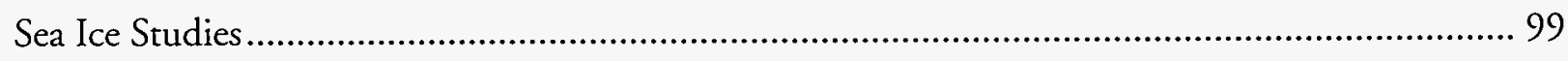

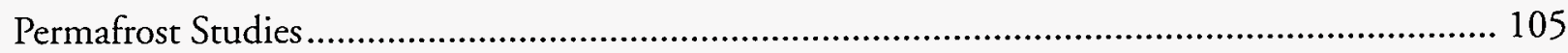

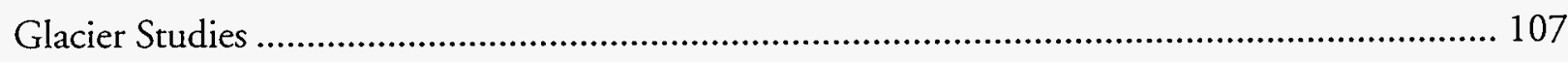

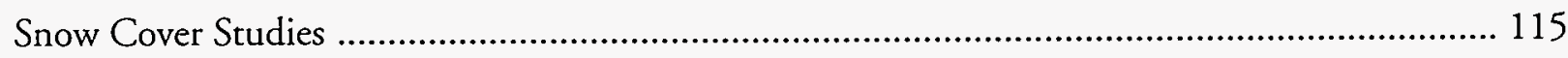

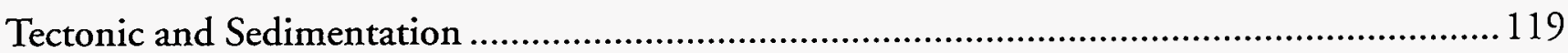

Stratigraphy, Sedimentology, and Structural Geology .......................................................... 121

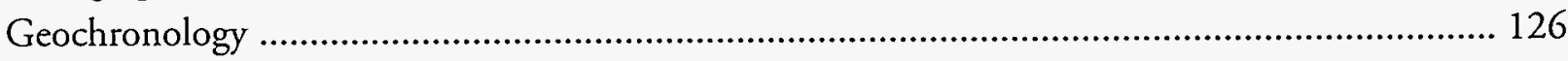

Paleomagnetism and Solid-Earth Geophysics .................................................................... 130 


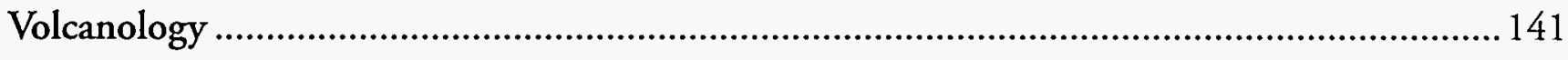

Monitoring and Hazard Mitigation .................................................................................. 143

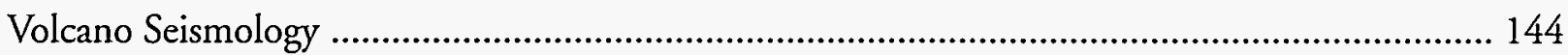

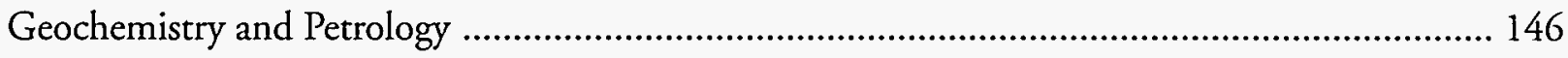

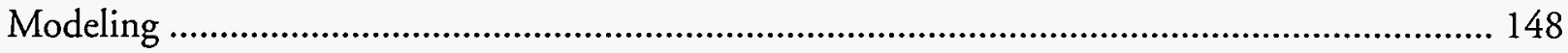

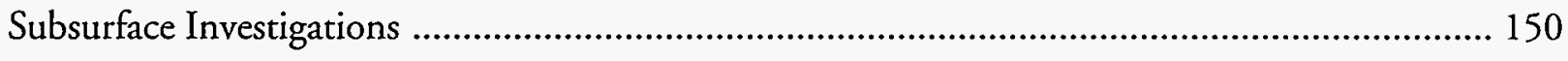

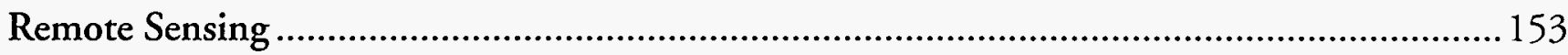

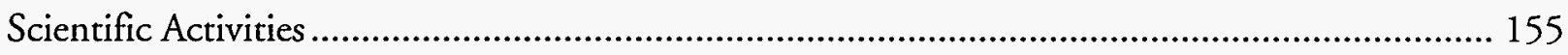

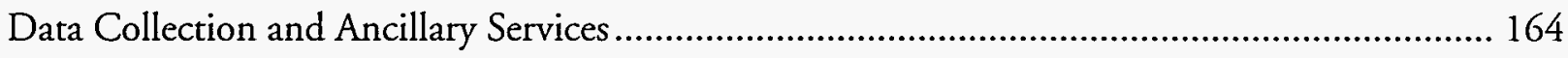

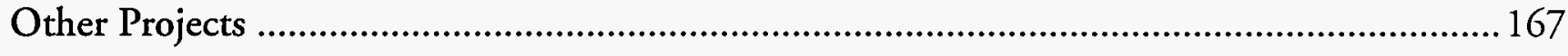

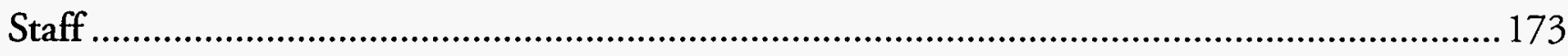

Faculty and Scientific Staff Listing by Group ...................................................................... 175

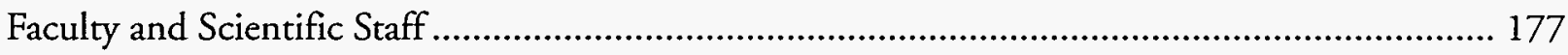

Administrative Staff by Department ................................................................................... 218

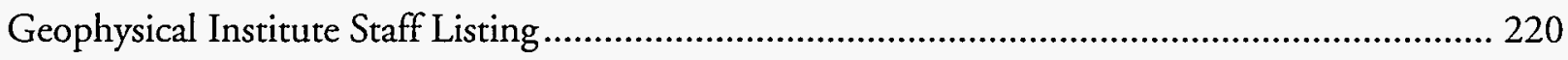

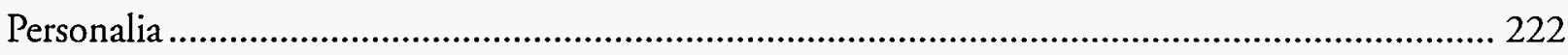

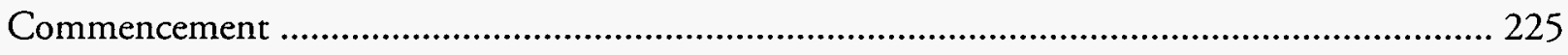

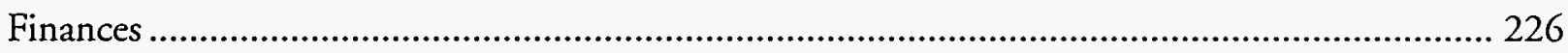

\section{Acronyms Used in Project Acknowledgments}

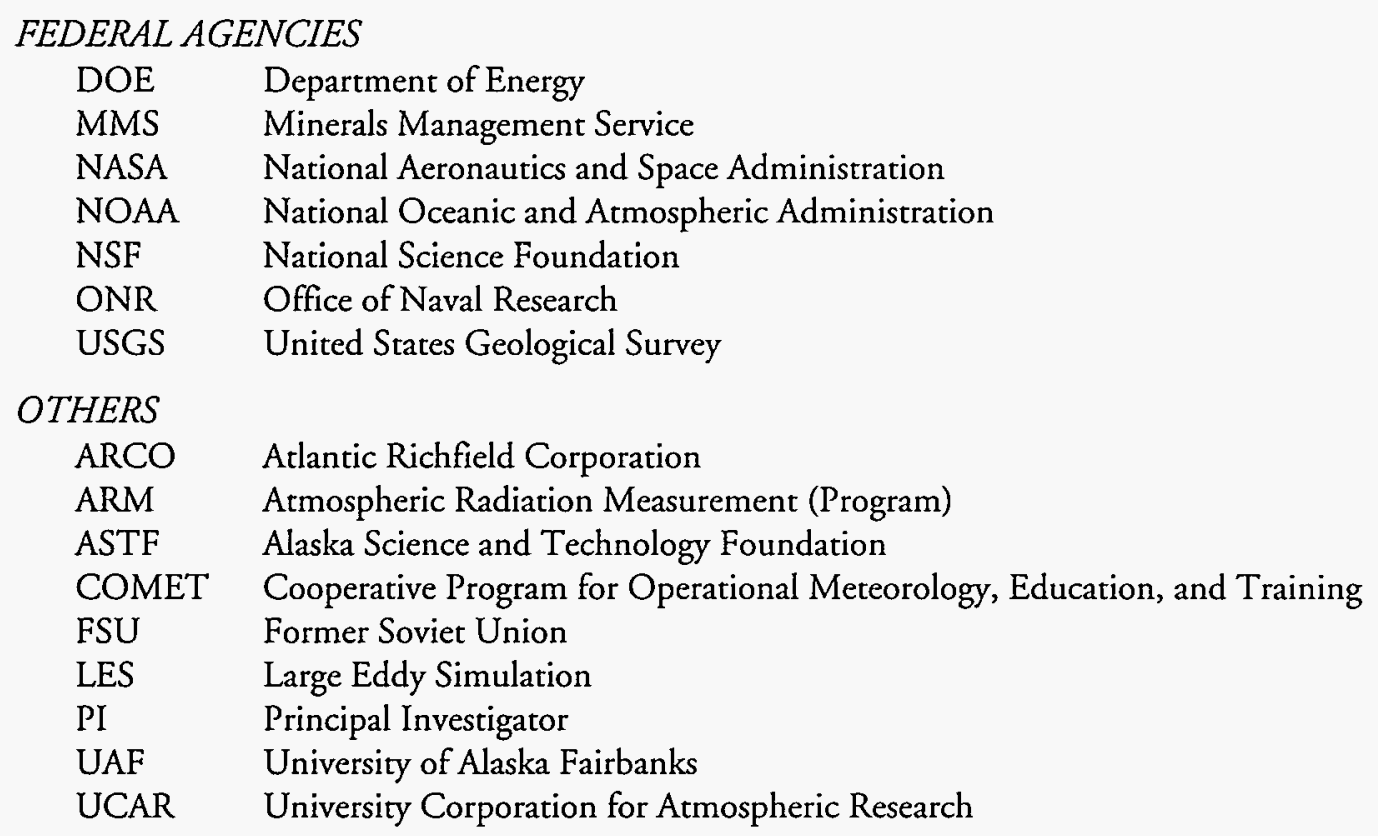




\section{Geophysical Institute Research}

\section{SPACE PHYSICS}

Solar and Interplanetary Physics

Magnetospheric Physics

Aeronomy and Auroral Physics
Ionospheric Physics and Radio Physics

Aeronomy and Middle Atmosphere

Computational Electrodynamics

ATMOSPHERIC SCIENCES

Climate Change

Atmospheric Dynamics

Atmospheric Chemistry and Physics

Radiation Budget

\section{SNOW, ICE, AND PERMAFROST}

Lake, River, and Sea Ice

Snow

Climate and Climate Change

Applied Science and Engineering
Permafrost and Seasonally Frozen Ground

Glaciers

Remote Sensing

Cooperative Projects on Climate Change

\section{TECTONICS AND SEDIMENTATION}

Stratigraphy and Sedimentology

Solid-Earth Geophysics

Tectonics

\section{VOLCANOLOGY}

Plate Margin Volcanism

Volcanism and Climate

Intraplate Volcanism

\section{SEISMOLOGY}

Earthquake Source

Tectonics

Velocity Structure

\section{SEISMIC HAZARD}

Volcanic Processes

Subduction Seismology

Paleoseismology

\section{SATELLITE REMOTE SENSING}

Air-Sea-Ice Interaction

Land Studies

Hazard Monitoring

K-12 Education Outreach Program
Structural Geology

Geochronology

Cooperative Projects in Atmospheric Sciences, Glaciology,

Tephrochronology, and Seismology
Earthquake Prediction

Plate Interiors

Tsunami
Cryospheric Studies

Climatology

Advanced Methodology 


\section{Director's Note}

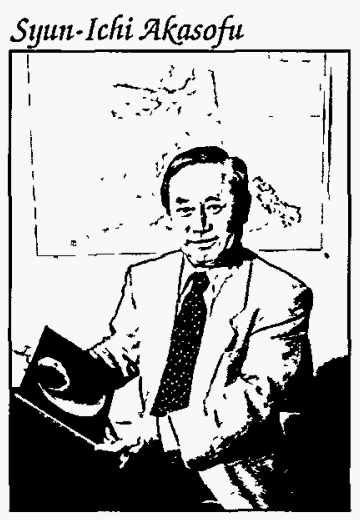

The Geophysical Institute is a unique research entity because it was established by an Act of Congress in 1946 to conduct arctic and polar research at the University of Alaska. Our staff has worked hard to fulfill its congressional mandate to play a central role in the nation's highlatitude research. In particular, we have experienced one of the most exciting periods of activity in the history of the institute during the last two years.

NASA's Space Physics Theory Program (SPTP) awarded a grant to the institute's space physics group to conduct theoretical study and computer simulations of various magnetospheric processes which include magnetic reconnection and magnetotail dynamics associated with magnetospheric substorms.

Sprites and Blue Jets, spectacular red and blue flashes that occur above thunderstorms and extend to the ionosphere, have been the focus of a new Geophysical Institute program. Institute researchers were the first to record hundreds of the flashes on color video. They captured the flashes above intense thunderstorms in the Midwest during a NASA-sponsored investigation.

Our 100th doctoral student graduated in 1994.

Poker Flat Research Range has ushered in a variety of improvements:

- The Communications Research Laboratory of the Japanese Ministry of Posts and Telecommunications has begun a 10-year program of middle atmosphere observations at PFRR in cooperation with the Geophysical Institute and the Environmental Technology Laboratory of the NOAA/Environmental Research Laboratories in Boulder, Colorado. The program will begin with the installation of a 256 -element imaging riometer at PFRR in the summer of 1995.

- The T. Neil Davis Science Operations Center, a 10,000-square-foot, two-story building with 18 observing domes and two launch-command areas, will be complete by the summer of 1995 .

- The Data Analysis Center and Geospace Environmental Data Display System (GEDDS) are in operation. This high-speed computer network shows real-time auroral, magnetic and ionospheric records from a large array of ground-based stations, together with satellite and rocket-orbital data.

- The Climate Change Monitoring Station will be in operation for a March 1995 campaign to measure background populations of tropospheric trace gases, aerosols, and the UV-radiation field.

- Launch facilities continue to improve. New telemetry and rocket assembly buildings, and a launcher enclosure for Pad 3 have been completed along with new cable trays, and two new 8-meter satellite telemetry antenna systems.

The Alaska Space Academy, a summer camp established in 1993 through the Alaska Space Grant Program, uses space exploration simulations in an outdoor setting to involve middle-school students in activities that demonstrate the relevance of math and science in their lives.

The High-frequency Active Auroral Research Program (HAARP) is being constructed at Gakona, near Glennallen. The Geophysical Institute is actively participating in scientific research associated with the project.

The Alaska Synthetic Aperture Radar Facility (ASF) continues to collect data from the European and Japanese Earth Resources Satellites (ERS-1 and JERS-1, respectively). The Science Division continues to work on advanced SAR analysis for studying polar processes, including glaciology, geology, sea ice, oceanography, forestry, volcanology and environmental hazards. In addition, ASF will receive data from ERS-2 and the Canadian RADARSAT Satellites in 1995. 
The Atmospheric Radiation Measurement (ARM) program, established by the U.S. Department of Energy, awarded scientific supervision of ARM research on the North Slope to the institute's atmospheric science group in 1991. Research on this project is well underway; construction of facilities is scheduled for 1995-96 and deployment of instrumentation is planned for 1996-97.

The Cooperative Institute for Arctic Research (CIFAR) was established by a Memorandum of Understanding between NOAA and the University of Alaska in 1994. Its goal is to conduct cooperative research in climate, atmospheric and ocean studies in the western Arctic.

The Center for Global Change and Arctic System Research (CGCASR) continues to establish interdisciplinary research connections with the Institute and UAF scientists in other departments, and to promote interdisciplinary global change research with funding from NSF, ONR, and NOAA.

The Wadati Chair in Climatology is held by Visiting Professor John Walsh from the University of Illinois. The chair, with additional grants from NASA and NSF, has enabled the Institute to establish a successful arctic climate-modeling group.

The Visualization Laboratory has been established in cooperation with the Arctic Region Supercomputing Center to give researchers access to sophisticated scientific visualization tools. These tools, along with high-quality printing and video production, allow researchers to explore and communicate their results.

The National Weather Service will lease space to collocate their new Fairbanks Weather Forecasting Office in the new addition Elvey Building addition. Collaboration between the National Weather Service and the institute's atmospheric sciences group will be enhanced through the sharing of equipment, data sets, work spaces and the interaction of researchers.

A New ${ }^{40} \mathrm{Ar} /{ }^{39} \mathrm{Ar}$ Mass Spectrometer and laser-dating system was installed in the spring of 1994 in the geochronology laboratory. Funding was provided by the National Science Foundation, the Alaska Science and Technology Foundation, and the University of Alaska. Recent results include precise dating of Miocene tephras, Quaternary basalts and single crystals of biotite and hornblende from Russia. Other projects include determining the uplift and thermal history of Mount McKinley and the timing of gold mineralization in the Fairbanks area.

The Alaska Volcano Observatory (AVO) successfully forecast the eruption of Redoubt and Spurr volcanoes, and predicted the movement of airborne volcanic ash clouds to the benefit of the state's airline industry.

The Remote Sensing and Seismology Groups have a pilot experiment in progress, to determine to what extent SAR interferometry can be used for monitoring volcanoes in Alaska.

The Alaska Earthquake Information Center (AEIC), a cooperative effort with the U.S. Geological Survey, monitors and catalogs earthquakes in Alaska and serves the state with information immediately following seismic events. Roger Hansen, AEIC coordinator, is also the new Alaska State Seismologist. Hansen brings valuable expertise in modern digital instrumentation and processing techniques to AEIC.

The Wadati Chair in Seismology is held by Max Wyss. He and his students have made progress in mapping stress directions in the Earth, in defining segmentation of plate boundaries, and in recognizing precursory seismicity patterns that might permit prediction of earthquakes similar to the M7.3 Landers 1992 shock.

The Seismic Microzonation Project was initiated for the Anchorage area, funded by the Alaska Science and Technology Foundation, the University of Alaska Fairbanks and the NEHRP program of the U.S. Geological Survey. Demand is increasing rapidly in Alaska - particularly for population centers - for relevant information for mitigating the damage porentials of destructive earthquakes to manmade structures. One of the main tasks of the microzonation project consists of site specific characterization of strong ground motion using a 15-station digital strong motion network. On supplementing users input, data from the network are processed by coinvestigators from the University of Alaska at 
Anchorage and Fairbanks, University of Southern California, Rensselaer Polytechnic Institute and Woodward-Clyde Consultants.

The U.S. Geological Survey has negotiated an agreement to transfer operation of the Earth Sciences Information Center and its map-distribution service to the Geophysical Institute's GeoData Center in 1995. The move enables the USGS to reduce its operating budget but preserves valuable service for the Fairbanks and university communities.

The Second International Conference on Substorms (ICS-2) was held on the UAF campus March 7-11, 1994, in the Salisbury Fine Arts Theater. Total registration for the Friday conference was 168, with representation from 11 countries: the U.S., Canada, Sweden, Norway, Finland, Germany, France, England, the Netherlands, Russia and Japan. The official proceedings of the conference comprise a total of 87 papers; 182 were presented in all. The chairman for local arrangements was J.D. Craven. In recognition of our contribution to the community through this conference, the Fairbanks Convention and Visitors Bureau acknowledged the ICS-2 as the "Meeting of the Month" for March 1994.

The Third Circumpolar Symposium on Remote Sensing of Arctic Environments was hosted in Fairbanks by the Geophysical Institute May 16-24, 1994. The conference, chaired by K. Dean and M. Jeffries, was a tremendous success; more than 100 scientists from the international community attended. The fourth conference is scheduled to be held in Copenhagen in 1996.

The Institure was involved with the following symposia:

- SAR Users Meeting, July 1993, University of Washington, Seattle (ASF was cosponsored). Organizers: W. Weeks and C. Wilson (Geophysical Institute), and D. Rothrock (University of Washington)

- Pacific Rim University Presidents Conference, September 1993, Anchorage. S.-I Akasofu assisted with organizing and securing support from the Japan Foundation

- Circumpolar Information Exchange: Shrinking the Circumpolar Community, 44th Annual Arctic Science Conference, September 15 - 18, 1993, Whitehorse (sponsored by the Arctic Division of AAAS; G. Weller, executive secretary)

- 1993 Magnetopause Workshop, September 20 - 23, 1993, Fairbanks. Organizers: L.-C Lee and A. Otto

- High-Latitude Ocean-Atmosphere-Ice Interactions-Measurements and Models: A Workshop, July 26 30, 1994, Fairbanks (co-hosted by Geophysical Institute and the Arctic Region Supercomputing Center). Organizers: G. Weller, A. Lynch and D. Corbett (ARSC)

- Bridges of Science between North America and the Russian Far East, 45th Annual Arcric Science Conference, August 25 - 27, 1994, Anchorage; August 29 - September 2, 1994, Vladivostok (cosponsored by Russian Academy of Sciences and the Arctic and Pacific Divisions of AAAS). U.S. Organizers: P. Anderson and G. Weller

- 1994 International Conference on Arctic Margins, September 5 - 9, 1994, Magadan (co-hosted by the Geophysical Institute). Financial chair: D. Stone; North American coordinator: D. Thurston (Anchorage Minerals Management Service)

The Institute has entered into the following agreements:

- Denver-FTRI, 1993: Letter of Intent to operate NDSC with the University of Denver

- DGGS, 1993: Memorandum of Understanding for collocation and cooperative research with the State of Alaska Division of Geophysical and Geological Surveys

- Japan-JNOC, 1993: Letter of Agreement for support of work at ANWR

- Japan-NASDA, 1993: Record of Discussion of Earth Observation Technology Cooperation

- Japan-Tokai University, 1993: Memorandum of Agreement for academic exchange with the Research Information Center of Tokai University

- Russia-NE Science Center (ICAM Magadan), 1993: Letter of Agreement for hosting the 1994 Conference on Arctic Margins 
The Institute has entered into the following agreements (continued):

- USGS-College Observatory, 1993: Memorandum of Agreement with the USGS establishing an Advocacy Council

- AADC, 1994: Agreement for the provision of administrative and coordination services by the Geophysical Institute in support of the Alaska Aerospace Development Corporation

- NOAA Environmental Research Laboratories, 1994: Memorandum of Understanding concerning the Cooperative Institute for Arctic Research (CIFAR)

- NOAA Environmental Research Laboratories, 1994: Memorandum of Agreement for use of buildings

- Russian Academy of Sciences (Magadan), 1994: Agreement with the Northeast Interdisciplinary Institute, Far East Division, for joint geophysical investigations in the basin of the Kolyma River, Russia

- USAF Phillips Laboratory (HLMS), 1994: Memorandum of Agreement to initiate a joint research and datacollecring capability benefiting both the U.S. Air Force and the Geophysical Institute

- USGS-Historical Alaska Geological Library, 1994: Memorandum of Agreement with the USGS for longterm lease and eventual ownership of the Historical Alaska Geological Library

- USGS-Map Office (ESIC), 1994: Cooperative Agreement establishing a state Earth Sciences Information Center within the Geophysical Institute

- USDI-Council of the National Seismic System, 1994: Charter to establish the Council of the National Seismic System 


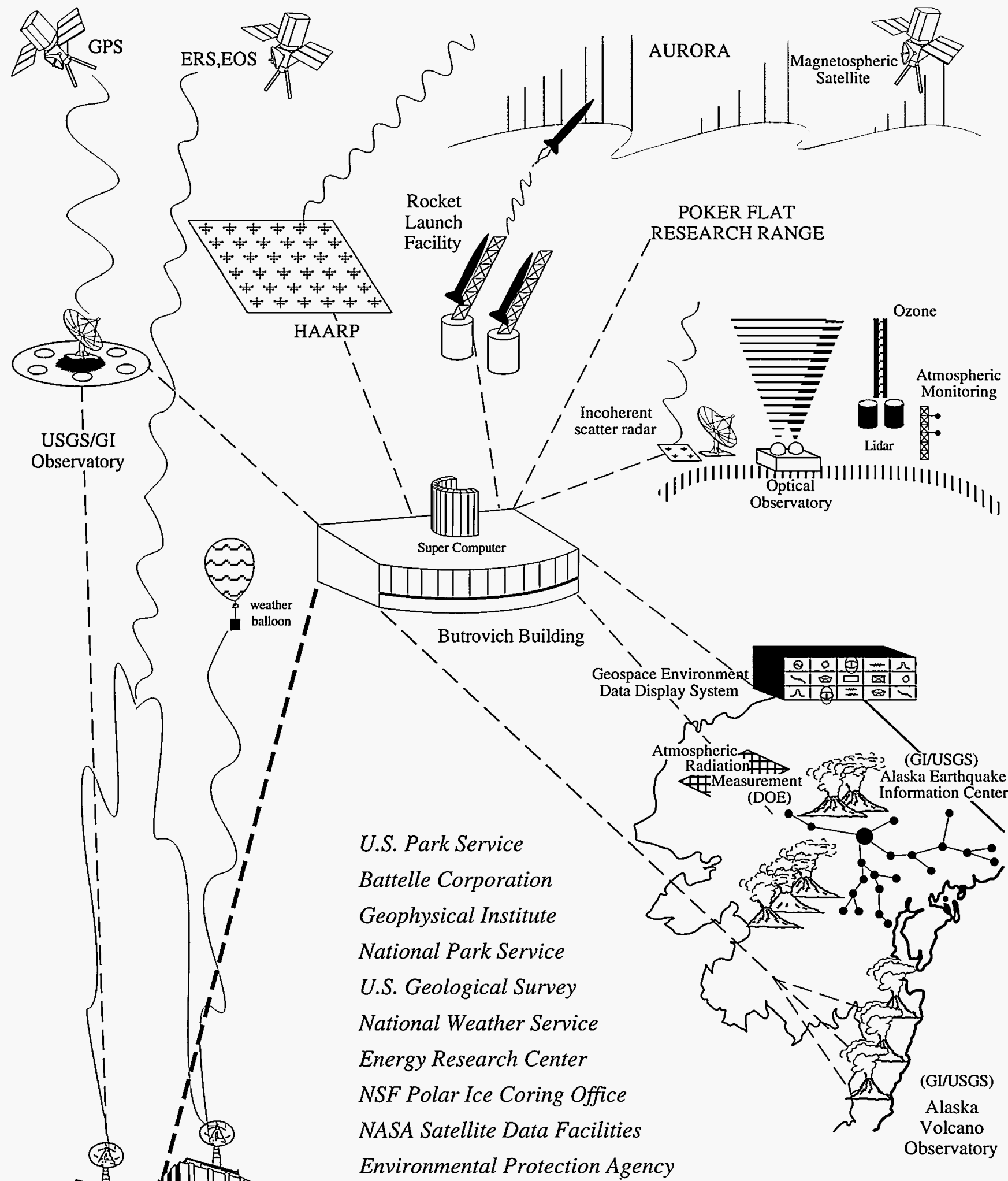

NASA Alaska Space Grant Program

Arctic Region Supercomputing Center

Alaska Division of Geological and Geophysical Surveys 


\section{Overview}

The Geophysical Institute is the only high-latitude research and academic center for geophysics in the United States. Specializing in research on arctic and subarctic phenomena, the institute offers graduate programs and opportunities for postdoctoral experience.

Faculty members at the Geophysical Institute teach borh graduate and undergraduate courses through the College of Natural Sciences, University of Alaska Fairbanks, and the Geophysical Institute participates with other universities and agencies in many cooperative research programs of state, national, and international significance.

During more than four decades of scientific work, the Geophysical Institute has earned international respect for research on the earth and its physical environment and for the training of graduate students in related disciplines.

Established by an Act of Congress in 1946, and passing to the University of Alaska in the 1950s, the scientific expertise of the Geophysical Institute now covers the spectrum of geophysical disciplines, ranging from outer space to the earth's inner core.

The Geophysical Institute is one of the few organizations in the country to closely associate scientists from these diverse disciplines.

Because of its strategic geographical, geological, and geomagnetic location, the institute regularly hosts visiting scientists from around the world, who use its facilities and interact with staff and students.

The special interests of Alaska, the university's existing scientific strength, and national priorities for arctic research and higher education demand continuing excellence from the institute.

Distinguished members of an advisory consulting board and several scientific discipline review panels regularly evaluate the scientific and instruction activities of the institute and advise the director and the university thereon.

Since its creation, the basic research and instruction activities of the institute have varied with changes in national priorities, enlargement of our discipline base, and changes to respond to State of Alaska needs.
In addition to carrying out the resulting mix of research and instruction programs, because of its knowledge of Alaska's conditions, the institute has responded to frequent requests over the years for assistance from other institutions in addressing national logistics and research support needs in Alaska.

As early as 1957-58, during the International Geophysical Year, the institute designed and supplied many of the hundreds of 16-mm "all-sky" aurora cameras created and placed throughout the world.

In 1959, the institute entered into contracts with the National Aeronautics and Space Administration for the construction of the first tracking station. The station was called Minitrack and it was located on the north campus at Ballaine Lake.

The Geophysical Institute later carried out the responsibility for the design, construction, and initial operation of the Gilmore Creek tracking facility, which was established in 1962 for the manned space flight program.

In 1968, the institute created Poker Flat Research Range, located about 30 miles northeast of Fairbanks. Operation of the range began the following year.

In 1987, the institute began the first stages of planning and negotiation for an upgrade and modernization program for Poker Flat Research Range to create a research facility of national significance.

Capital funding for a greatly improved scientific facility and observatory was provided through the U.S. Air Force and NASA.

When complete in 1996, Poker Flat will provide a high-latitude sounding rocket launch facility for studies of the aurora and other ionospheric phenomena. The range also will include a new observatory, which will make it well positioned to support intensive rocket and ground-based studies of climate and the arctic atmosphere.

In 1988, the institute responded to international needs by participating with NASA in the construction and operation of the Alaska Synthetic Aperture Radar Facility, the only tracking and receiving station in the United States for a series of international syntheric aper- 
ture radar satellites. This program resulted from agreements among the European Space Agency, Canada, Japan, and the United States. The State of Alaska provided funds for an addition to the C.T. Elvey Building to house the tracking, receiving and analysis equipment. The facility was dedicated in a ceremony in 1991.

Another significant program evolved from state financial problems in 1986 which resulted in the discontinuance of funding for the state seismologist in the Alaska Division of Geological and Geophysical Surveys.

Institute staff negotiated with the state survey for the transfer of both the departing seismologist and the activity budget in the program to the institute. The legislature also created by statute the new position of state seismologist which is located at the Geophysical Institute.

The establishment of the state seismologist position was instrumental as a basis for the subsequent creation and funding of the Alaska Volcano Observatory by Congress in 1988.

This new research organization is a cooperative program of the Geophysical Institute, the State of Alaska Division of Geological and Geophysical Surveys, and the U.S. Geological Survey. Created at the same time was an associated center of study at the institute called the Alaska Earthquake Information Center.

These changes occurred largely because Alaska's natural environment provides institute scientists with a geophysical laboratory of a kind almost unique on earth.

To investigate solar-terrestrial phenomena like the aurora, scientists capitalize on the high-latitude ionosphere, where these phenomena are accessible for observation from the ground, aircraft, rockets, and satellites.

Alaska's atmosphere is the birthplace of large-scale weather perturbations that often spread over the entire North American continent. It is also a sensitive indicator of conditions in the global atmospheric system.

Layers of snow, ice, and permafrost dominate Alaska's surface environment and the region's glaciers are the fourth-largest in the world.

Underneath Alaska, tectonic forces and plate motions shake and shape the state. Along with the dramatic events associated with volcanic and seismic activity, the state carries a potential of natural resources of largely undetermined scope, with the work of detailed mapping and quantifying yet to be accomplished.

The Geophysical Institute is housed in the eightstory C.T. Elvey Building on the West Ridge of the University of Alaska Fairbanks campus.

Instructional activities of the faculty are carried out through departments of the colleges of natural sciences and liberal arts. Nearly 150 faculty members and graduate students are engaged in the academic and research work of the institute, which is supported by a like number of professional engineers, technicians, supervisory personnel, and other specialists.

Accompanying increases in our major programs has been a rapid increase in institute basic research and research-support activities. In part this has been due to the added responsibilities of creating the Alaska Synthetic Aperture Radar Facility and supporting the European Space Agency and Japan Space Agency SAR satellites. The staff continues to grow and the present C.T. Elvey Building is increasingly unable to accommodate the needs of the several programs. Some of the institute's staff are now located off campus.

To meet the facility needs of this growing institution, the Geophysical Institute, with the assistance of the university administration, commissioned in 1991 a conceptual design study of an addition to the present C.T. Elvey Building.

The design was completed in the spring of 1992 and included an analysis of the space required and the cost of that space. Using the design as a basis for discussion and planning, the institute started a campaign to enlarge the building, and to complete design work.

Two state legislative appropriations were received and funding for the remainder is being sought from various sources. Construction of the new 79,000-squarefoot addition is scheduled to begin in 1995 .

Along with offices and laboratories, the present C.T. Elvey Building accommodates supporting services essential to the research program. These supporting services include business functions; machine, metal fabricating, carpentry, and electronics design and construction; 
instrument calibration; data processing and computer support; imaging services (computer graphics, photography, and drafting); publication services; library and archive facilities (including aircraft and satellite imagery); and word processing.

These services are available to institute personnel, other university units, and to a lesser extent, other agencies. For example: part of the maintenance of the University of Alaska statewide computer nerwork and the seismic data collection network are responsibilities of the electronic shop; the machine shop designs equipment and provides other support activities for the National Science Foundation's Polar Ice Coring Office; and the institute archives/GeoData Center serves a myriad of users for remote sensing data.

In addition, a growing segment of the general public avail themselves of the information and data services of the institute.

Much of the institute's observing equipment is located at field sites throughour Alaska, northwestern Canada, Svalbard, Norway, and Antarctica.

Most of the remote field sites are units of continuously operating networks of observing stations. A chain of auroral and magnetic stations extends from Talkeetna, northward through Alaska and the Canadian archipelago to Svalbard, Norway. The network of seismic stations spans central and southcentral Alaska, including the lower Cook Inlet and the Alaska Peninsula areas.

The Alaska Volcano Observatory is linked by a computer network and through cooperative field studies with laboratories in Alaska, Washington, and California, which all participate in the seismic monitoring of hazardous Cook Inlet volcanoes including Mount St. Augustine, Mount Redoubt, and Mount Spurr.

Poker Flat Research Range at Chatanika is the only university-operated sounding rocket research range in the world and the only high-latitude range on United States soil.

The Poker Oprical Observatory and the Fort Yukon Observatory, 200 miles to the north, are integral components of the range.

Initial improvements and code corrections as part of the Poker Flat upgrade have occurred. The Chena Valley Radio Facility, operated by the University of California at
Los Angeles and located 30 miles east of Fairbanks, has a powerful high-frequency transmitter for active ionospheric stimulation experiments.

The institute is admirably equipped to carry out large-scale data analysis, satellite-image processing, modeling of geophysical processes, and nonlinear plasma simulations that are otherwise not amenable to analytical solution.

In January 1993, the University of Alaska installed a CRAY-YMP supercomputer on the Fairbanks campus. This was followed in May 1994 with a T3D massive parallel processor and visualization equipment. The institute's needs for computational and visualization tools are well met with these new facilities.

ADVAL, the UAF Alaska Data Visualization and Analysis Laboratory, is housed at the institute. The laboratory provides spatial information analysis and image processing services for research and educational activities. It coordinates well with the Alaska Synthetic Aperture Radar Facility which receives, processes, and archives data from synthetic aperture radar (SAR) satellites.

ADVAL's major components are a receiving ground station, a SAR processor system, an archive and operations system, a derived products system for automated data extraction, and an interactive image analysis system for the nonroutine scientific analysis of SAR data.

The Geophysical Institute offers competitive graduate research assistantships that provide financial support through federal- or state-sponsored research projects. Assistantships may be awarded in geophysical topics or in association with other UAF research institutes for crossdisciplinary studies offering special training for careers in arctic science.

Graduate students are enrolled in M.S. and Ph.D. programs. Postdoctoral fellowships, tenable for one or two years with possible renewal for additional years, also are available. These are generally awarded competitively for research on specific topics.

Detailed information on graduate assistantships, postdoctoral fellowships, and technical support services can be obtained by writing to the Director, Geophysical Institute, University of Alaska Fairbanks, PO Box 757320 , Fairbanks, AK 99775-7320. 


\section{Scientific Prediction \\ $=$}

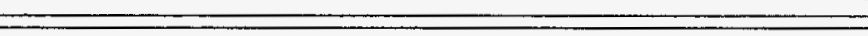

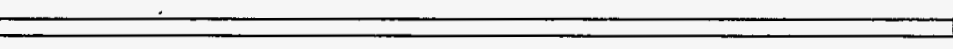

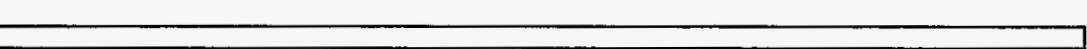

+2_

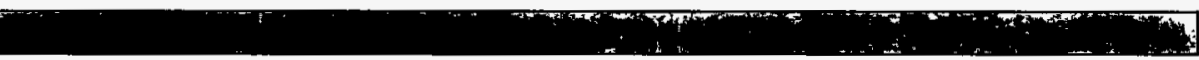
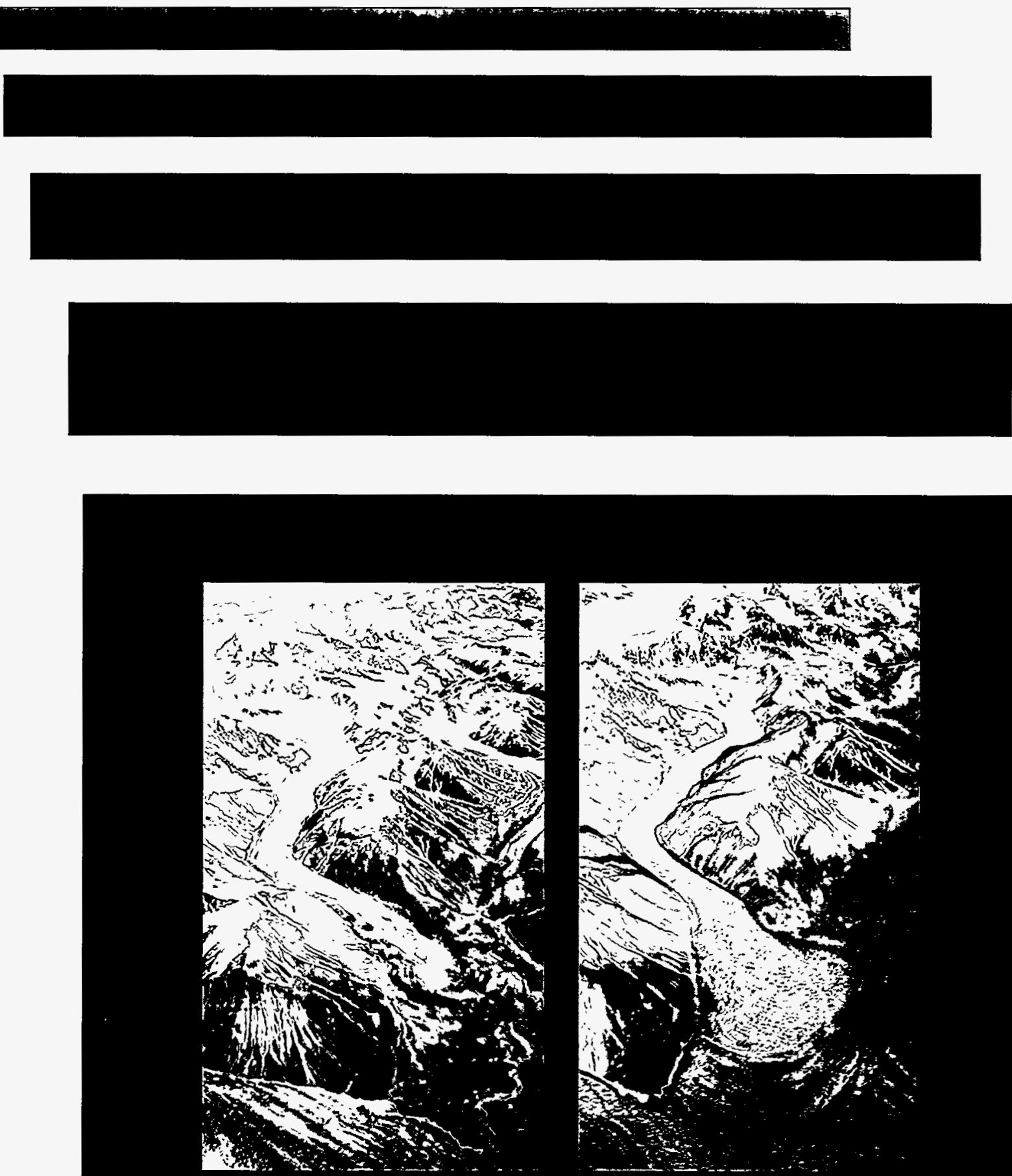


\section{SCIENTIFIC PREDICTION}

One of the ultimate tests of scientific results is predictability: we can trust our understanding when we say, accurately, "this event will be preceded by those clues," or "that manipulation will produce this result." Therefore, predictability also can be used as a measure of the progress in a particular discipline. Since we study a variety of natural phenomena at the Geophysical Institute, including geomagnetic storms, weather and climate, glaciers, earthquakes and volcanoes, researchers from these disciplines each have prepared a short article stating where the discipline stands in terms of predictability. It is hoped that each article is informative enough to illustrate the progress of its discipline.

\section{Can We Predict Geomagnetic Storms?}

\section{by Syun-Ichi Akasofu, Geophysical Institute Director}

One of the most important purposes of space weather research is to be able to predict geomagnetic disturbances for specific solar events. More specifically, modern storm forecasting should be able to predict at least two geomagnetic storm indices $\mathrm{AE}(\mathrm{t})$ and $\operatorname{Dst}(\mathrm{t})$ as a function of time after a specific solar event.

Space weather research is neither solar physics, solarwind physics, magnetospheric physics, nor thermo- spheric (ionospheric) physics. It is the discipline that integrates at least these four disciplines. Unfortunately, in spite of much progress in each of these disciplines in the past, there has been little effort to integrate them into a single discipline of geomagnetic storm prediction, so that there has been no significant progress in the storm prediction, namely prediccing $\mathrm{AE}(\mathrm{t})$ and Dst $(\mathrm{r})$ for a specific solar event. In other words, progress in each of the four disciplines is not the same as progress in the discipline of geomagnetic storm prediction, even if there is no doubt that progress in each of the four disciplines is important.

It is proposed that the modern forecasting scheme consist of two parts connected by a single equation that is a function of the solar wind speed $V$, the interplanetary magnetic field (IMF) magnitude $B$, and the polar angle $\theta$ of the IMF.

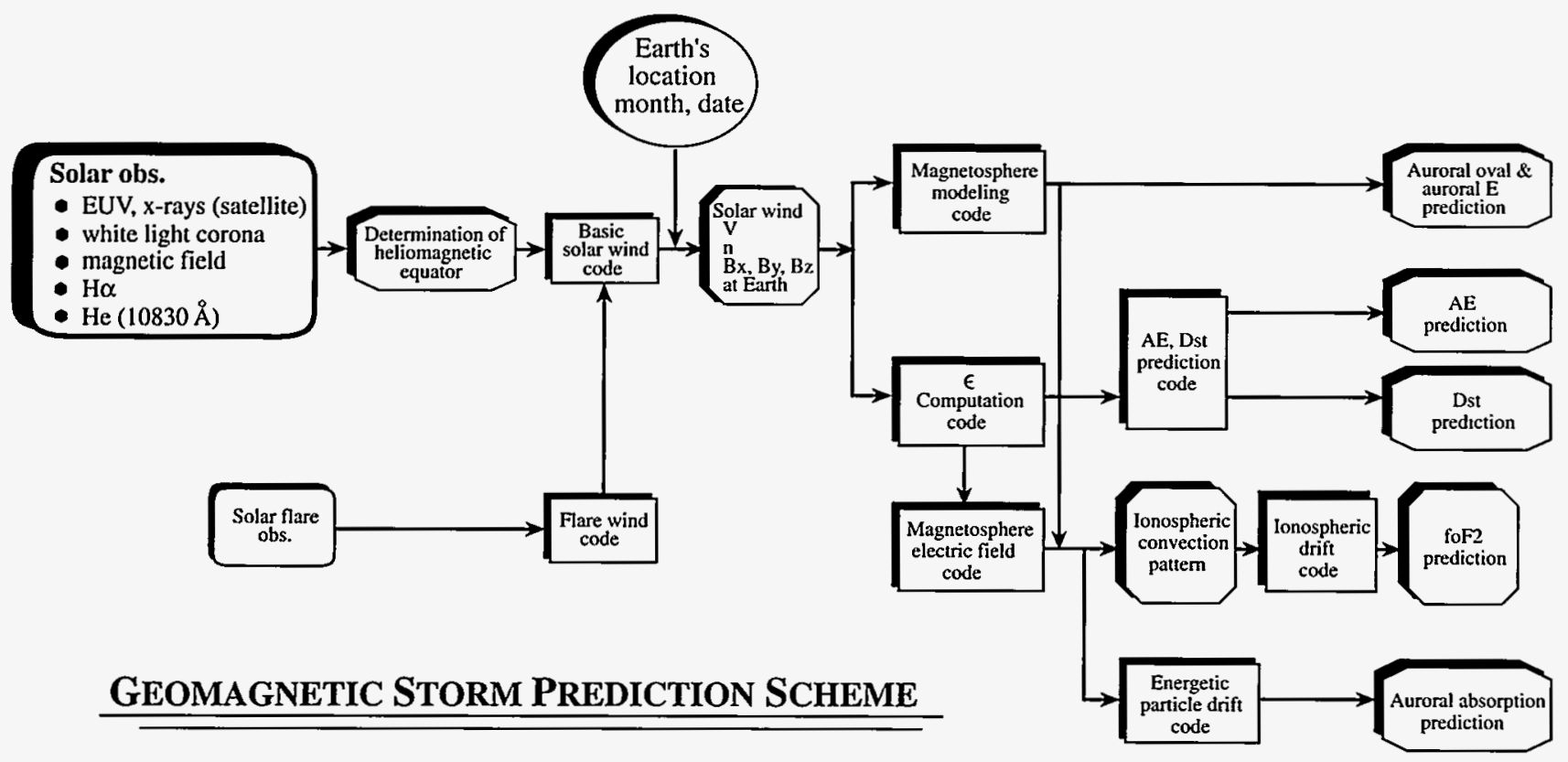


One of such functions is the socalled " $\varepsilon$ parameter," which represents the power $P$ of the solar windmagnetosphere generator: $\mathrm{P}$ (mega watts $)=20 \mathrm{~V}(\mathrm{~V} \mathrm{~km} / \mathrm{sec}) \mathrm{B}^{2}(\mathrm{nT}) \sin ^{4}$ $(\Theta / 2)$.

Having this particular function, the first part (step one) of the forecasting is to predict $V, B$, and $\theta$ at the location of the earth as a function of time for a specific solar event. The second part (step two) is to predict geomagnetic indices, such as the Dst and $\mathrm{AE}$ indices at the location of the earth as a function of time from the predicted $P(t)$. The whole scheme is illustrated in block diagram form in the figure on the previous page.

The figures on this page demonstrate such a possibility by "postdicting" Dst and $\mathrm{AE}$ on the basis of a past record and any deriva- tives of these indices. Knowing V, B, and $\Theta$ as a function of time, $P(t)$ is estimated first. Then, the Dst $(t)$ index is computed from the familiar equation:

$$
\frac{d D s t}{d t}=0.7 P(t)-\frac{D s t}{\tau}
$$

where $\tau$ is the lifetime of ring current protons; for simplicity, it is taken to be 8 hours in this case. A factor of 0.7 for $P$ is considered by noting that about 70 percent of $P$ is spent in producing the ring current belt. One can see that Dst thus computed resembles the observed Dst.

For the $\mathrm{AE}$ index, magnetospheric physics has nor advanced to the point of being able to predict theoretically the AE index from $P$. Thus, an empirical relationship between $\mathrm{P}$ and $A E$ is used for the time being, $\mathrm{AE}(\mathrm{nT})=600\left[\tanh \left(\log _{10} \mathrm{P}-\right.\right.$
18.9) $]^{2 / 3}+650$. The figure to the left below shows an example in which time variations of $P$ are quite different from those of the figure to the right. One can easily see that characteristics of the development of the storm depend on characteristics of variations of $\mathrm{P}$. Thus, it is possible to predict characteristic variations of $\operatorname{Dst}(t)$ and $A E(t)$, if $P(t)$ can be predicted at the location of the earth.

Step 1: (a) Specific Solar Event. A specific solar event is characterized by six parameters including: initial speed, $\mathrm{V}_{\mathrm{F}}$; area, $\sigma_{\mathrm{F}}$; duration, $\tau_{\mathrm{F}}$; start time, $T_{F}$; latitude, $\phi_{F}$; and longitude, $\chi_{F}$ of a solar flare.

An enhanced solar wind from a circular area is added to the background flow. The quantities $V_{F}$, $\sigma_{\mathrm{F}}$, and $\tau_{\mathrm{F}}$ depend on the intensity of individual events; the maximum
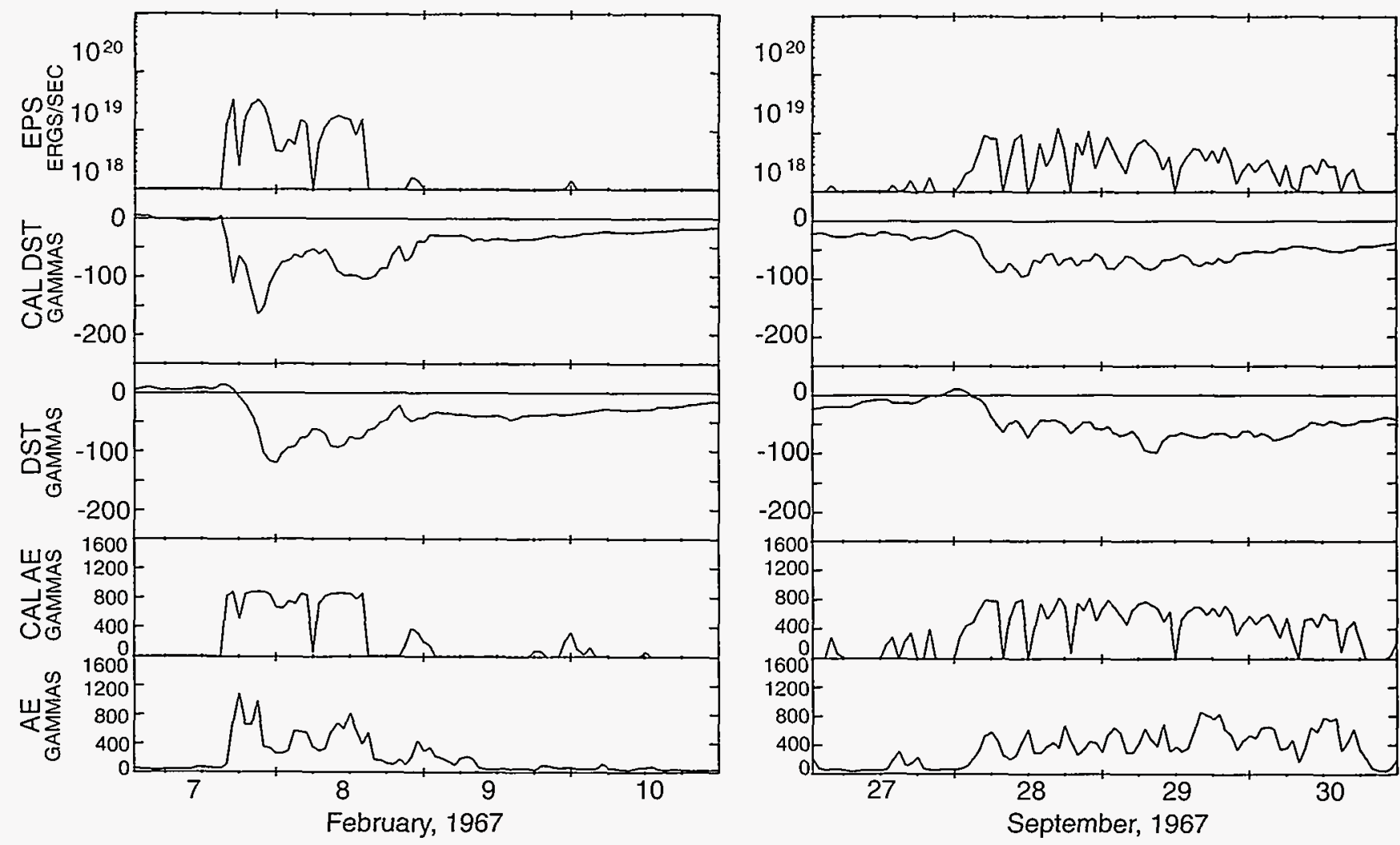

Shown in both figures above, from the top, are the $\varepsilon$-parameter, the predicted Dst as a function of time, the observed Dst variations, the predicted AE index and the observed AE index for the February 7 - 9, 1967 storm and for the September 27 - 30, 1967 storm. 


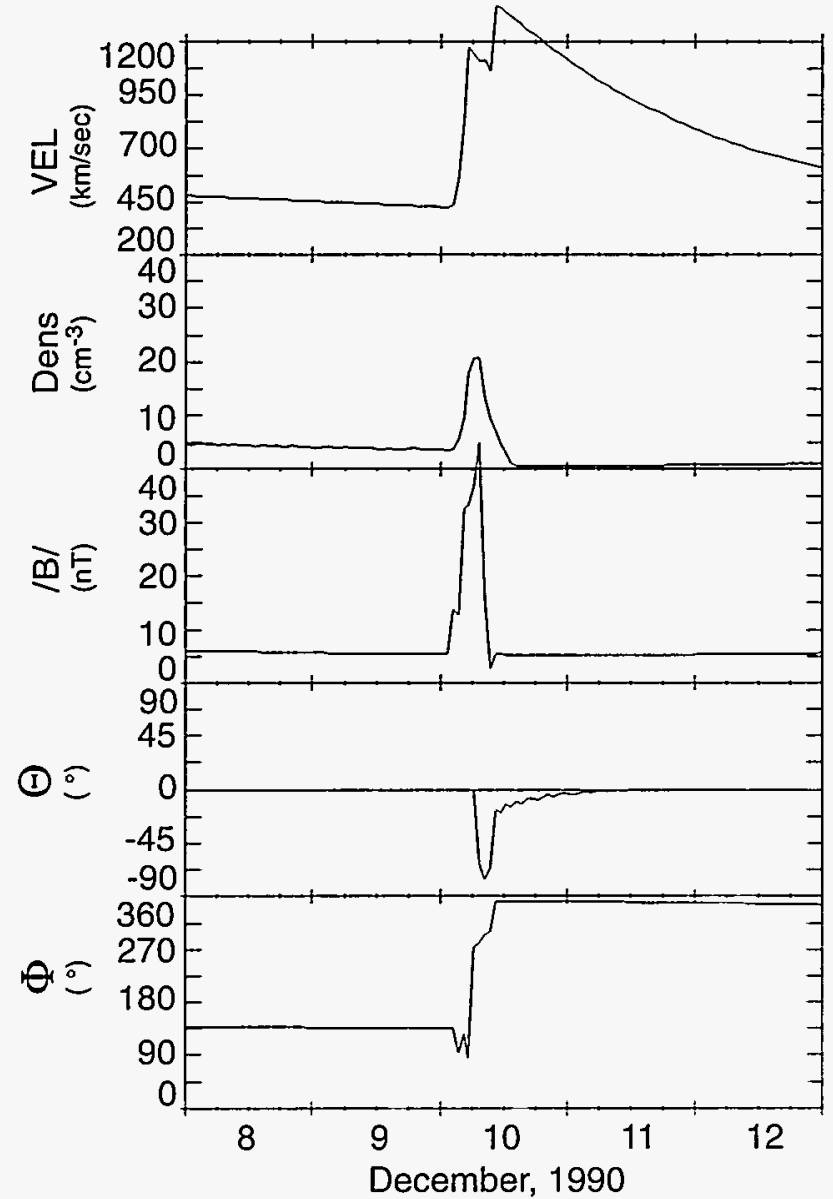

In the figure above, from the top, shown are variations of the solar wind speed, the density, the IMB magnitude, the $\Theta$ angle and the azimuth angle during the passage of the shock wave illustrated at the bottom of this page.

solar wind speed varies as a function of time (paramaterized by $\tau_{\mathrm{F}}$ ).

(b) Solar Wind Disturbances. An example of the resulting shock wave after a hypothetical solar event is shown in the figure to the right, using the distorted interplanetary magnetic field lines, superposed on the background spiral pattern. The location of the earth in this particular example is indicated by a star symbol. The figure at the top left of this page shows the computed variations of the solar wind quantities at the location of the earth, as the shock wave passes by the earth.

Step 2: The figure at the top of the next page shows, from the top, the estimated $\varepsilon$, the cross-polar cap potential $\Phi_{p c}, A E, D s t$ and the total energy dissipated in the polar ionosphere $\mathrm{U}_{\mathrm{I}}$ and the amount of temperature increase $\Delta \mathrm{T}$ in the thermosphere, estimated from the solar wind quantities shown above.

We have shown earlier that the expected Dst variations as a function of time can be obtained from $\mathrm{P}(\mathrm{t})$, using the familiar equation between $P(t)$ and $D s t(t)$. This is, unfortunately, not the case for the AE index, $\Phi_{p c}$ and $\Delta T$. However, there are a number of empirical relationships among $P(t)$ and $\Phi_{p c}, \Delta T$ and other quantities. Note that because $\theta$ has the largest variability $(300 \mathrm{~km} / \mathrm{sec}<\mathrm{V}<$ $1500 \mathrm{~km} / \mathrm{sec}, 3 \mathrm{nT}<\mathrm{B}<25 \mathrm{nT} ; 0^{\circ}<\theta<180^{\circ}$ ). At the present time, the polar angle $\Theta$ is the most difficult to predict among the solar wind quantities, but is the most crucial one in determining the $\varepsilon$ parameter. There are a large number of causes for changes of $\Theta$. It may well be that a magnetic cloud originates from the coronal mass ejection. However, even if this would be the case, it is not known at all whether $\Theta(t)$ can be predicted from a particular coronal mass ejection.

Calibration: It is important to be able to calibrate the results of the numerical prediction scheme during the transit of the solar disturbance to the earth. This is another aspect that the four individual disciplines do not consider. Fortunately, however, it is known that there occurs considerable interplanetary scintillation (IPS) after an intense solar event. The IPS after a major solar event in September 1978 was well documented. In order to compare the computed shock shape and the observed IPS, we construct a 3-D shape of the shock wave and
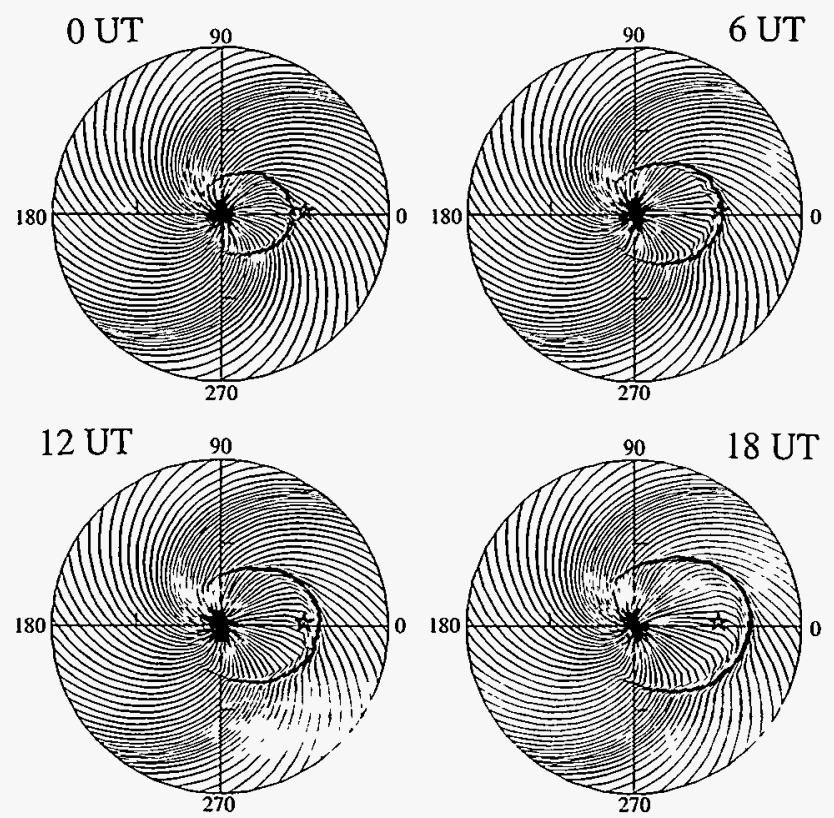

Shown above (Ecliptic Plane 2AU, 10 December 1990) are magnetic field lines from the same surface after an assumed solar event. Superposed in the familiar spiral pattern, the field lines are distorted by the propagating shock wave. The location of the earth is indicated by a star symbol. 


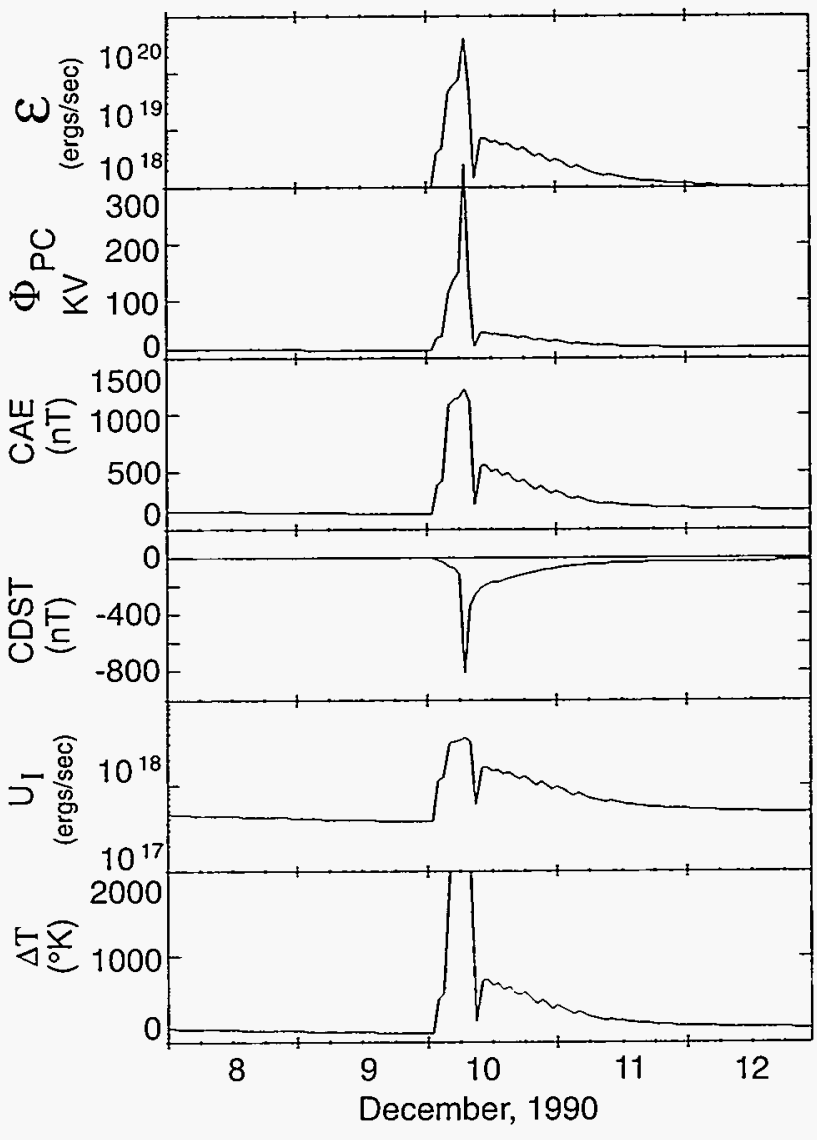

Sept 22, 1978

16 UT

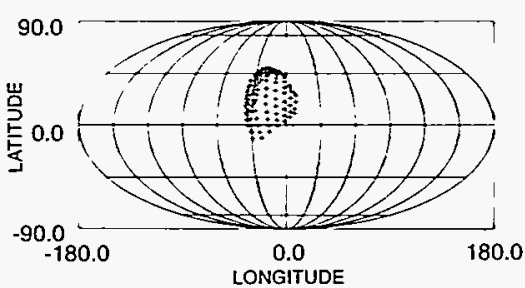

Sept 24, 1978

$16 \mathrm{UT}$

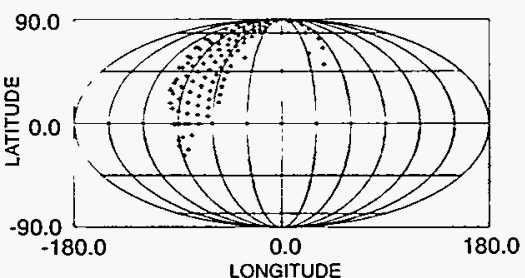

Sept 26, 1978

$16 \mathrm{UT}$

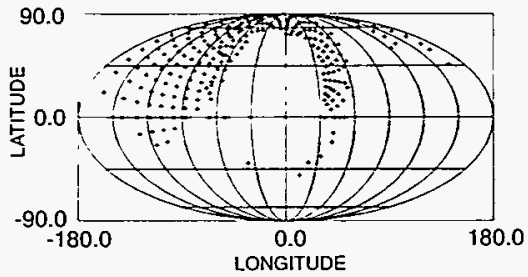

Sept 23, 1978 16 UT

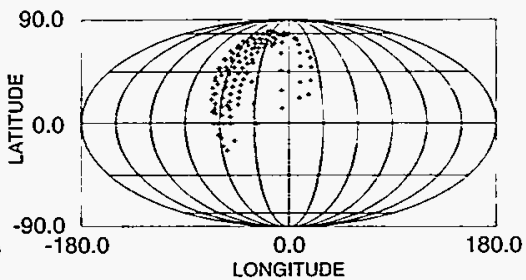

Sept 25,1978

$16 \mathrm{UT}$

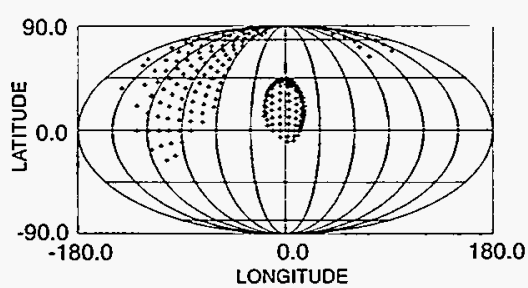

Sept 27, 1978

$16 \mathrm{UT}$

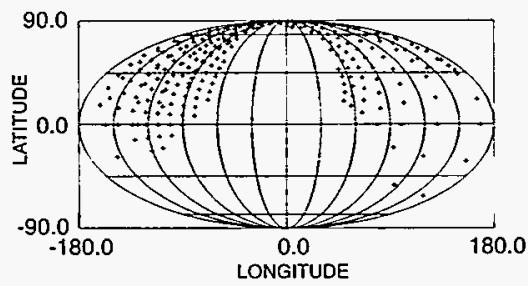

project it on the sky map (illustrated in the lower left). The computed strong IPS in the northwestern sky coincided with the location of the observed shock wave in the sky map in this particular case.

Applications: We demonstrated clearly that auroral activity induces currents in a power line system. Currents in the neutral line at the substation of the Healy-Fairbanks power line have been monitored, as well as the so-called earth current recorded at the Geophysical Institute, several miles from the substation. The figure on the next page illustrates that most of the fluctuations in the neutral line are identical to the earth current variations, which indicates the induced voltage between two electrodes, and shows that auroral activity induces currents in the power line system. This figure also shows currents in the circuit breaker system. Rapid changes in the neutral line cause pulses in the breaker system which would trip the breaker when the height of the pulses reach a certain critical level. If we could predict $A E$ as a function of time, power line companies could prepare for outages arising from auroral activity, and they would be able to distinguish between outages caused by auroral effects or by accidents.

Generally speaking, a medium auroral disturbance induces a voltage drop of about 1 volt/kilometer on the ground. The distance between Prudhoe Bay Oil Field at the North Slope and Valdez (the terminal of the pipeline) at the Pacific Coast is about 1000 $\mathrm{km}$, so medium auroral activity induces about 1000 volts across (north-south) Alaska. Since the total resistance of the pipeline is about $10 \mathrm{ohms}$, medium auroral activity induces about 100 amperes of current in the pipe. Our measurements show that current intensity of 1000 amperes is not uncommon. The pipeline is corroded where the current escapes into the ground.

Shown above at left, from the top, are the $\varepsilon$-parameter $(=P)$, the cross-polar cap potential $\Phi_{\mathrm{pc}}$, the AE and Dst indices, the total energy $\mathrm{U}_{1}$ deposited in the thermosphere, and the increase of the temperature $\Delta \mathrm{T}$ as a result of the increased para $\mathrm{P}$, which is estimated from the solar wind quantities shown at the top of the previous page. Shown at bottom left, the shock wave in front is projected onto the sky map. 
AURORAL EFFECTS ON POWER LINE

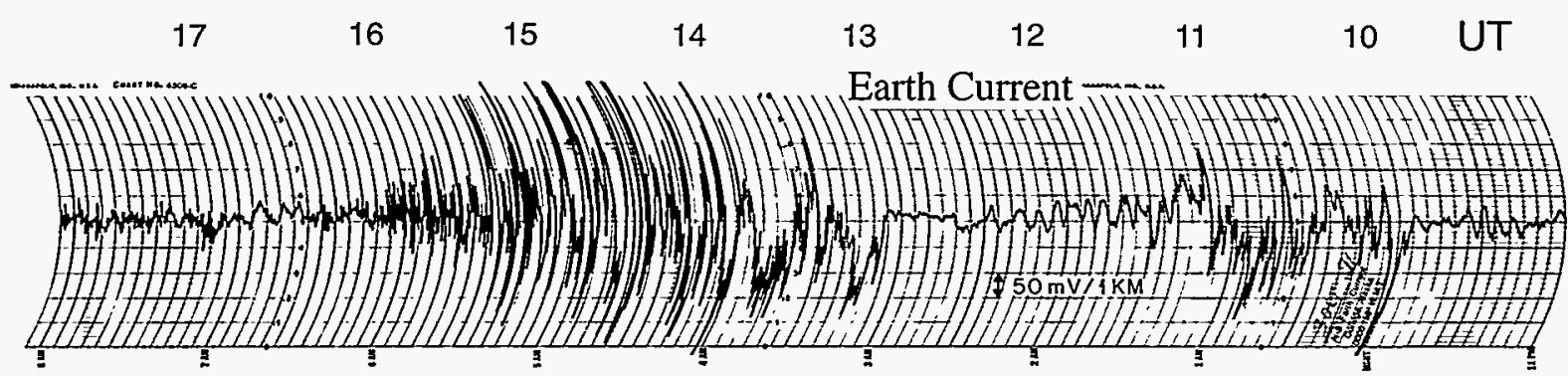

Gold Hill Autotransformer Neutral Current
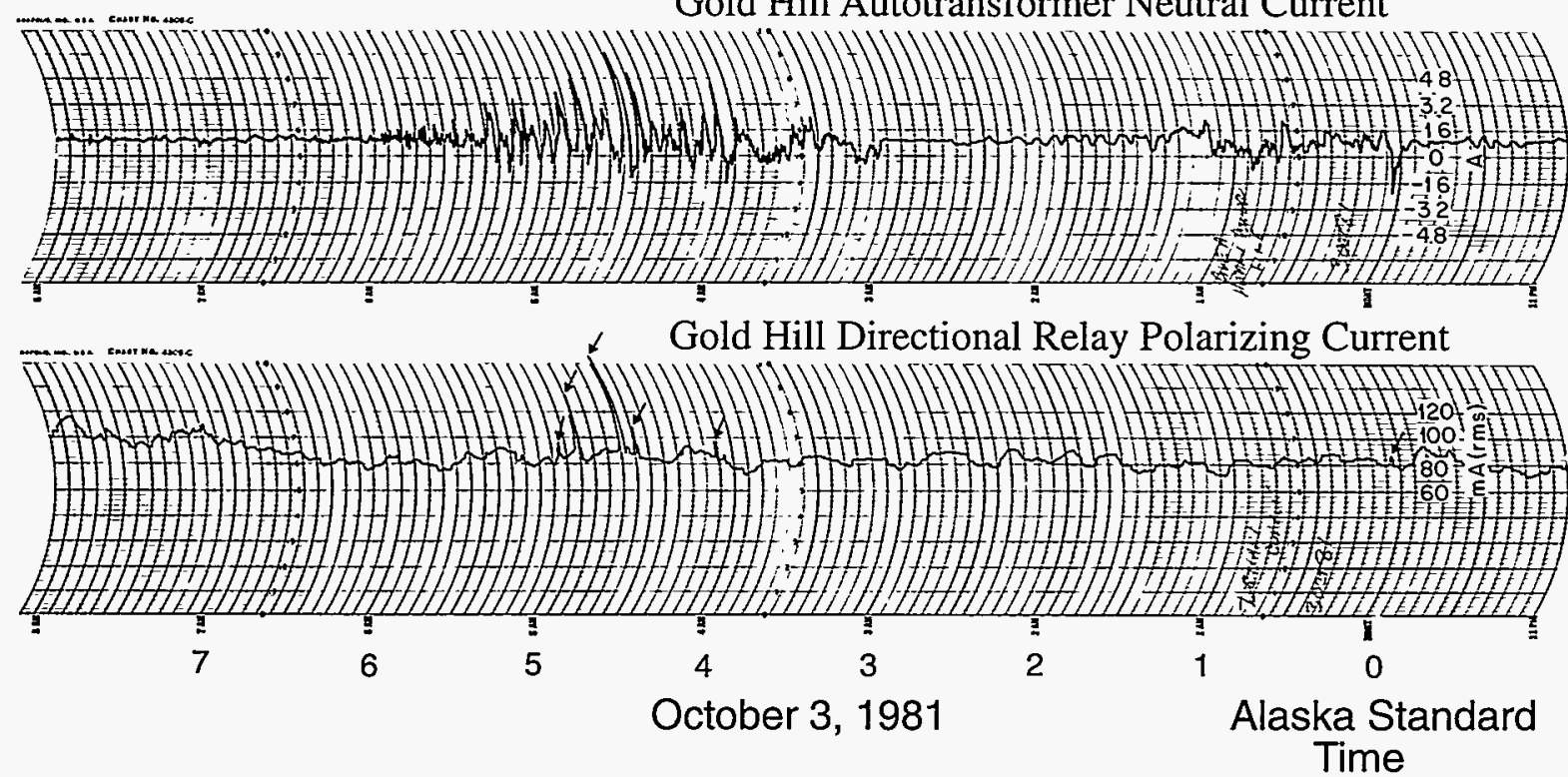

Shown above, from the top, are the "earth current" caused by auroral activity (observed at the Geophysical Institute); the current induced in the power transmission line measured at the substation (located several miles from the Geophysical Institute) and the relay current (the "circuit break" current) recorded at the substation.

\section{Climate Change-Can We Predict It?}

by Amanda Lynch, Assistant Professor of Atmospheric Sciences

The earth's climate always changes. The climate is vastly different now than it was during the last glacial maximum, 18,000 years ago, when ice covered much of North America and the global average temperature was $4^{\circ} \mathrm{C}$ cooler than it is today. It is different from the Cretaceous era, over 50 million years ago, when the climate was warm and tropical at high latitudes.

The climate is always evolving, and must be considered a living entity. In the past, climate change was triggered by natural fluctuations in the earth's orbit. (See the chart of global temperature estimates on the next pages.) Current and future climate change, by contrast, will be fueled in part from another source-human activity. Many activities associated with human economic development have altered the physical and chemical environment in ways that are known to modify the flows of energy and materials in the environment. It is natural to expect that these will lead to significant changes in the current climate equilibrium. The table (adapted from Schneider, 1992) on the following page sum-marizes a few of the human activities that can affect the local environment, and the expected effect on the climate.

Climate change, when it occurs, has a considerable impact on human and natural systems, which motivates a need for a quantitative evaluation of the mechanisms of climate 
change and equilibrium. The only quantitative tools we have for determining these mechanisms are models of various types, both theoretical and numerical. Models enable us to postulate a disturbance to one or more elements of the climate system, and determine the result. The complexity of the climate system means that a hierarchy of models is necessary. Theories describing the behaviour of small elements of the system, such as clouds or sea ice, are included along with simple energy balance models, and the four-dimensional coupled atmospheric-oceanicsoil-vegetation-cryosphere-chemistry models, known as climate system models.

Researchers at the Geophysical Institute are developing the Arctic Region Climate System Model
(ARCSyM), which is becoming recognized worldwide as an important tool in arctic climate research. Climate system models, which ideally include all known components of the climate system in a complex numerical representation, allow us to integrate the postulated changes to give us a picture of the changed climate as a whole.

Can we have faith in these predictions? Climate prediction, like most other forecasts of complex systems, must involve an extrapolation in which we attempt to determine future behavior from knowledge of past behavior and the present state. This is achieved by using equations believed to represent the physical, chemical and biological processes governing the climate system for the regions or phenomena of interest.
Thus, we must examine the quality of our knowledge of the present state and governing equations in order to judge the faith we have in furure predictions.

To determine the current state of the climate, we require observations. To determine the appropriate governing equations for the climate's behavior and mechanisms for change, we need to formulate theories, which are built from observations. All our knowledge of the climate, past and present, depends on the quality and quantity of these observations. Unfortunately, these observations are not always available for the particular part of the climate we wish to study. Sometimes the observations are available, but are of questionable quality. This is particularly true in the high latitudes, where extreme environ-

\section{Human Influences on Climate}

\section{Activity:}

Release of carbon dioxide by burning fossil fuels, and other greenhouse gases, e.g., methane, chlorofluorocarbons, etc.

Release of particles from industrial and agricultural practices. Soot and sulfur dioxide are of primary concern

Release of heat

Upward transport of chlorofluorocarbons and nitrous oxide

Land use changes: urbanization, agriculture, deforestation, etc.

Release of radioactive particles Effect on Climate:
Warms surface layers directly

Increases atmospheric absorption and emission of terrestrial infrared radiation

Sunlight-scattering and -absorbing particles influence albedo, atmospheric stability and cloud formation

Reduces stratospheric ozone, allows more solar radiation to reach surface

Affects surface albedo, subsurface hydrology, causes aerosol release

Increases conductivity of lower atmosphere, affects cloud formation
Importance of Effect:

Potentially a major influence on global climate and biological activity Net effect: Warming of lower atmosphere, cooling of stratosphere

Could have nonnegligible global effects, since estimate of residence time in the atmosphere is being reassessed upwards Net effect: Probably cooling

Locally significant now, could modify circulation

Net effect: Warming

Global but uncertain influence, due to biological effects and effect of increased ozone at lower altitudes Net effect: Probably warming

Global impact, but uncertain

Global impact, but uncertain 


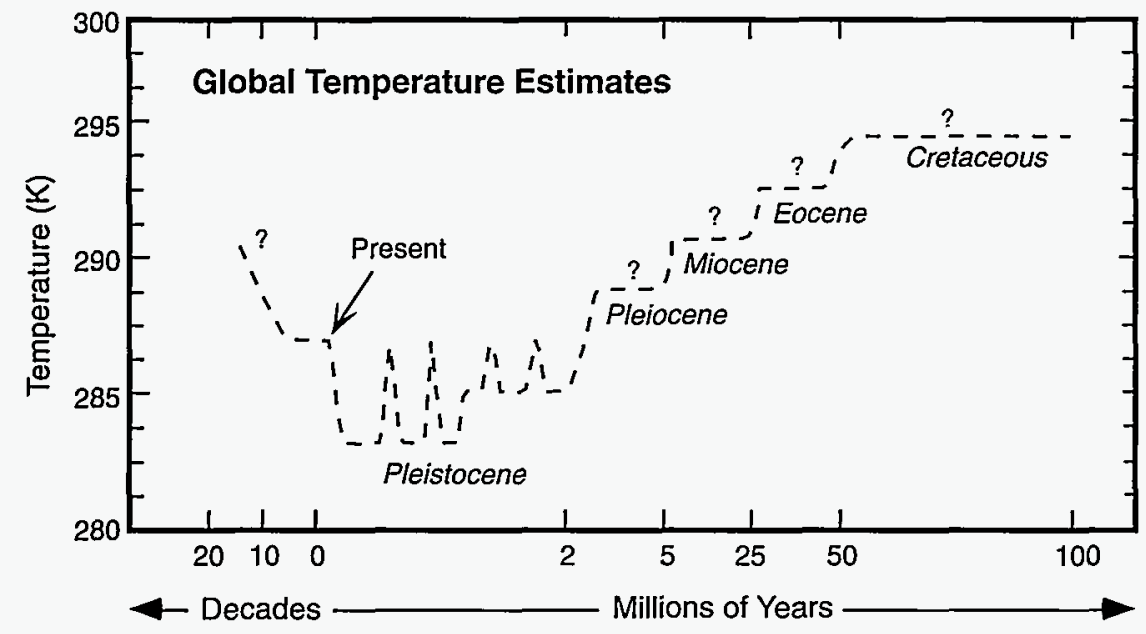

Climate change is illustrated above by global average temperature estimates. Estimates for the distant past are uncertain, although the differences between periods are fairly well known. The future increase in temperature is based on a possible scenario involving steadily increasing concentrations of carbon dioxide.

mental conditions make observations difficult and expensive to obtain.

Thus, it is important to continue to observe climatic phenomena to lead to a better understanding. Further, observations of particular phenomena must be examined in context with their role in the climate system as a whole. Many field studies are now planned, with experts in the numerical modeling of climate involved, to make sure that the time and expense of field programs yield useful information for the goal of climate prediction.

Examples of such field programs in the Arctic include SHEBA (Surface Heat Budget of the Arctic Ocean), the LAII (Land-AtmosphereIce Interactions) Flux Study, and ARM (Atmospheric Radiation Measurement), in which Geophysical Institute researchers, both observationalists and modelers, are heavily involved. In fact, the chief scientist for the Arctic ARM experiment is head of the atmospheric sciences group at the Institute. Further, in the case of numerical prediction, there are issues of spatial resolution, the integrator of knowledge of the climate system as a whole? Sub-gridscale phenomena such as clouds, atmospheric turbulence, and trees are represented collectively by their postulated impact on the grid-scale systems. The process of determining the appropriate representation is known as parameterization, which in itself involves many assumptions.

Climate system models have proven effective in analyzing the sensitivity of the climate to uncertain or even unpredictable variables. Uncertain climatic factors such as cloud parameters, could be varied over a plausible range, or alternative parameterizations could be used. The calculations would indicate which uncertain parameter or formula exerts the most leverage in climate processes. One could then concentrate research on narrowing the uncertainty surrounding these factors.

Uncertainty about parameterizations of key mechanisms, such as clouds, sea ice, and chemical cycles, is one reason the goal of climate prediction, the reliable, verified forecasting of key variables such as temperature and rainfall on a regional, time-evolving basis, is still out of our reach. These mechanisms are the subject of intense study at the Geophysical Institute, where researchers strive to understand both the processes themselves and their role in the climate system.

The problem is complex, but unfortunately the experiments which are underway are not merely of academic interest. Global change experiments are being performed right now within and on the laboratory that we and all other living things share-the earth. 


\section{Predicting Glacier Surges}

by Will Harrison, Professor of Physics

Glacier surges are recurring episodes of often spectacularly rapid motion, in some cases up to 100 meters per day or even more. Before-and-after photos of the surge of the Variegated

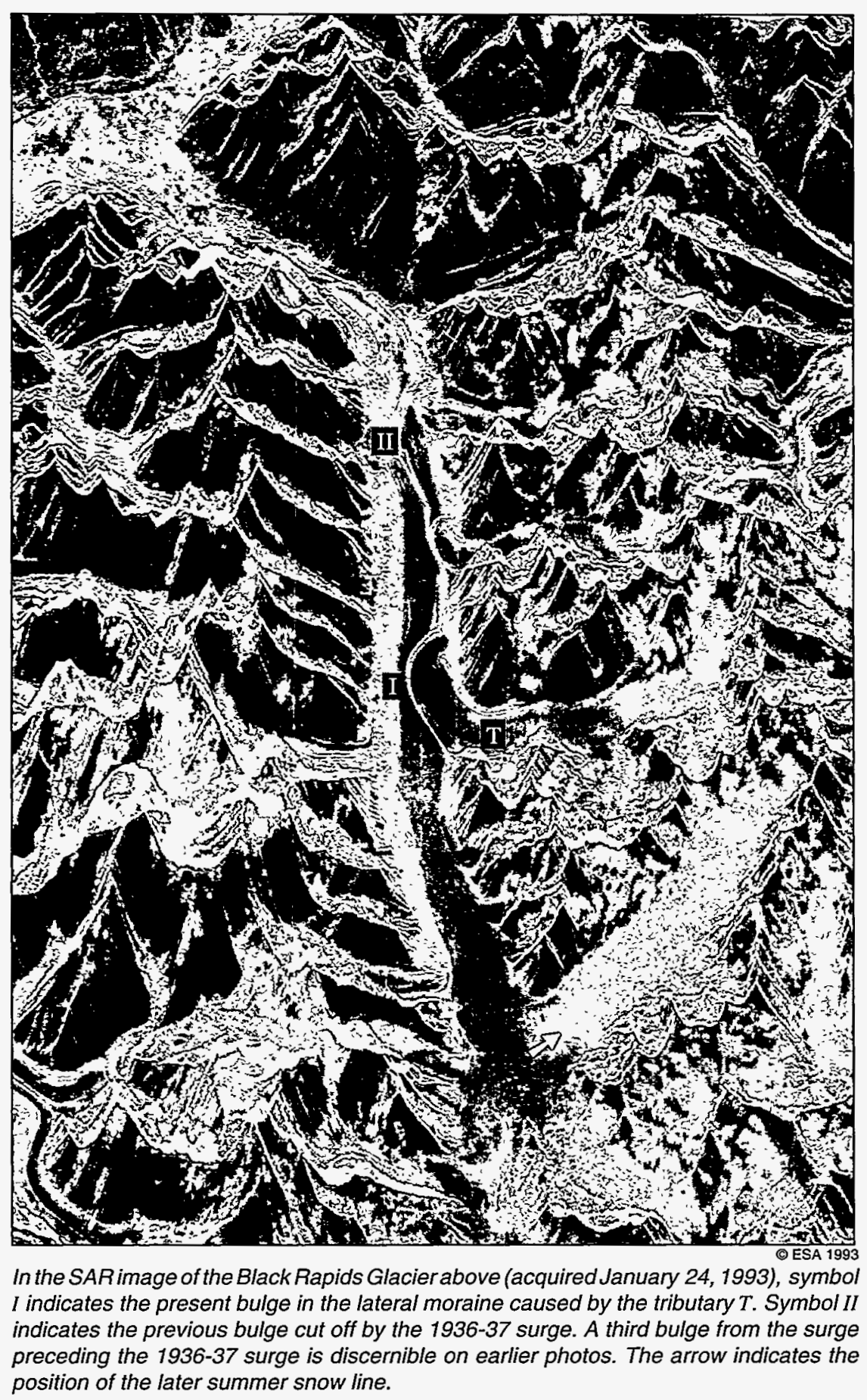

In the SAR image of the Black Rapids Glacierabove (acquired January 24, 1993), symbol $I$ indicates the present bulge in the lateral moraine caused by the tributary $T$. Symbol II indicates the previous bulge cut off by the 1936-37 surge. A third bulge from the surge preceding the 1936-37 surge is discernible on earlier photos. The arrow indicates the position of the later summer snow line.

Glacier, near Yakutat, Alaska, were taken by Austin Post in August 1964 and one year later (Figure 1). These photos show how much ice can be transferred down glacier in a surge.
Surges tend to be periodic, and the period between surges is fairly welldefined for a given glacier; a few decades is typical (although Variegated surges every 17 years or so). Surge-type glaciers are relatively rare, and where they occur they tend to do so in groups within a well-defined geographic distribution, which so far has defied explanation. The mechanism of surging is understood, however. Surges are due to the failure of a glacier's internal drainage system, leading to high water pressure at the bed of the glacier, and partial floating or failure of a sediment substrate.

Predicting the timing and strength of a surge are sometimes important. Perhaps the best example in Alaska is Black Rapids Glacier, which attracted national attention as the "Galloping Glacier" during its last surge in 193637. This surge stopped a kilometer or two short of the Richardson Highway, but past surges have dammed the Delta River and either overrun or flooded what are now the highway and the trans-Alaska Pipeline. Glacier-damned lakes tend to drain catastrophically; it is likely that some of the Black Rapids events were accompanied by major floods, which highlights the importance of trying to predict the timing and magnitude of the next surge.

One approach to prediction is to base it on past surge history. Some glaciers with short periods between surges have a recorded history of many surges; the best example in Alaska is Variegated Glacier. This approach has its problems, since surges are rarely strictly periodic, and 
because there is good evidence that some glaciers have changed in the past century from surge-type to normal; this seems to be particularly common in Spitzbergen, but it also probably has occurred in North America. Unfortunately, Black Rapids Glacier surged only once in historical times. Other surges are inferred from terminal moraines and their ages are uncertain. However, a periodic evolution of the pattern of a lateral moraine exists on the glacier. A major tributary pushes the moraine into a bulge where it enters the main branch of the glacier. The bulge is cut off by the next surge and is preserved down glacier. This pattern on Black Rapids Glacier is now äbout the same as it was before the previous bulge was cut off by the previous surge. This suggests that a surge of Black Rapids Glacier is imminent, a plausible situation if its surge period is similar to the known 50 - or 60 year pattern of the neighboring West Fork and Susitna glaciers.

The most reliable method of prediction is by field measurement of glacier speed and its evolution with time. Presurge accelerations may indicate that a surge is imminent, with a warning period of a couple of years. This method, and the historic one, both gave accurate predictions of the most recent surge of Variegated Glacier in 1982-83, but Black Rapids Glacier has proved to be more difficult. Its speed has waxed and waned over the last 20 years with no clear, sustained trend that would indicate the imminence of a surge. Possibly, under present climate conditions, Black Rapids Glacier can almost transport the ice that it receives without surging. If so, the timing of its next surge may depend sensitively upon what the climate does.

The strength of a Black Rapids surge, and whether it would reach the highway and pipeline, are different problems. The terminal moraines of previous surges suggest that recent surges have been relatively small, and that the possibility of the Glacier crossing the Delta River now is rather improbable. However, predicting the magnitude of a surge is more tenuous than predicting its timing. Surges in Alaska show a tendency to end

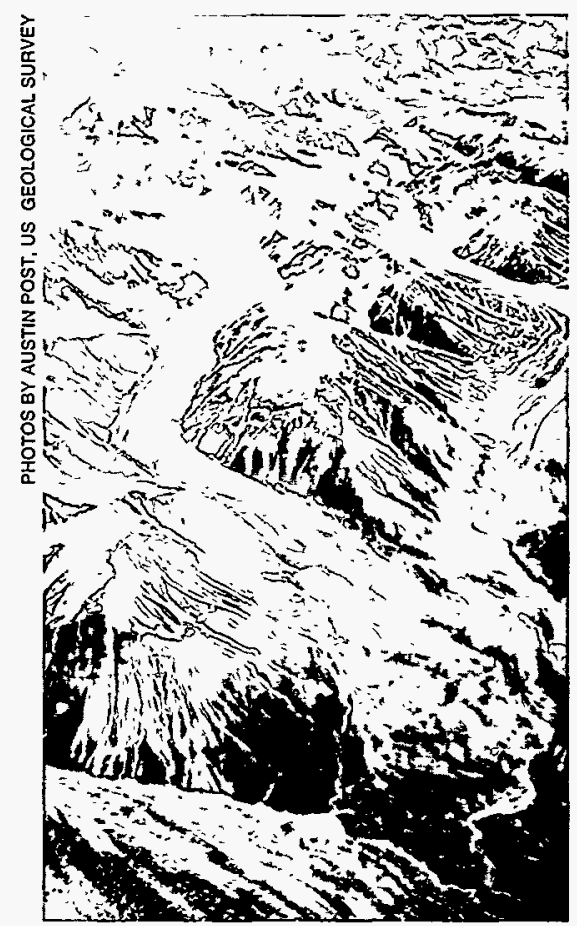

suddenly in July, apparently when abundant melt water is able to open the internal drainage systems. This means that a late spring or a cold summer might promote a large surge. In other conditions, the magnitude of a surge might depend on weather.

We still have a great deal to learn about the mechanics of glacier flow, particularly the flow of warm wet ones which slide on their beds and are so abundant in Alaska. Ar the moment, the simple, empirical methods of prediction are the best.

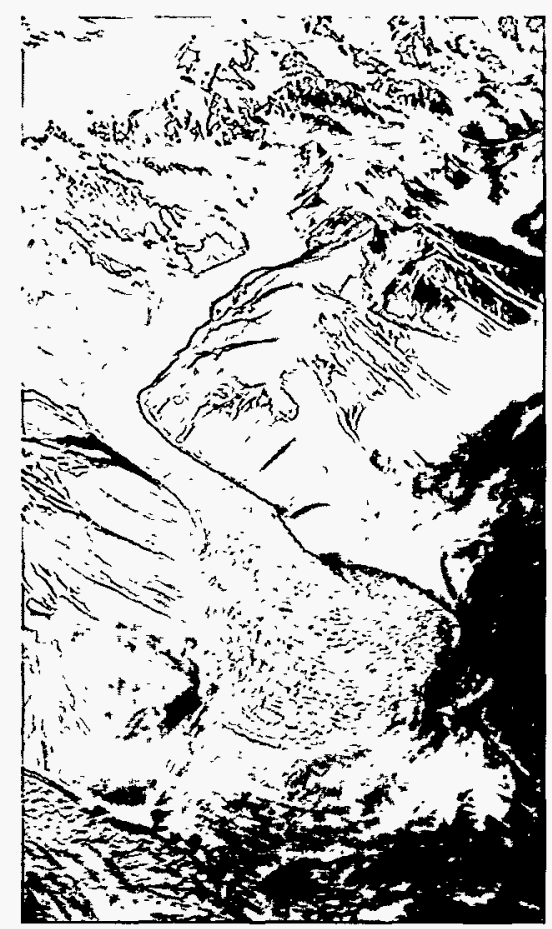

Shown is Variegated Glacier, near Yakutat, Alaska, in August 1964 (left) and one year later (right).

\section{Earthquake Prediction: Will It Ever Work?}

\section{by Max Wyss, Wadati Professor of Seismology}

Prediction is one of mankind's oldest and most elusive quests. Science is, in the view of rational people, the only means to develop information allowing predictions. If we understand the physics of a process, and if this process develops as a function of time, then we may be able to predict part of it. In the case of earthquakes, we are in a weak position, because we do not understand the details of the physical process. We do know that earthquakes are ruptures of faults (weak surfaces in the earth's crust) 
relieving stress that, in most cases, was built up over decades to thousands of years by movements of the lithospheric plates. However, we have only partial information about the state of stress in the earth's crust at any time or place, and we do not know the details of the rupture initiation.

The strain accumulated by plate motions can be measured geodetically. From the strain change we can estimate the associated stress change, under some assumptions. Since the crust can only stand so much stress (how much is poorly known and it must be variable), it would seem to follow that measurements of crustal deformations should play an important part in earthquake prediction

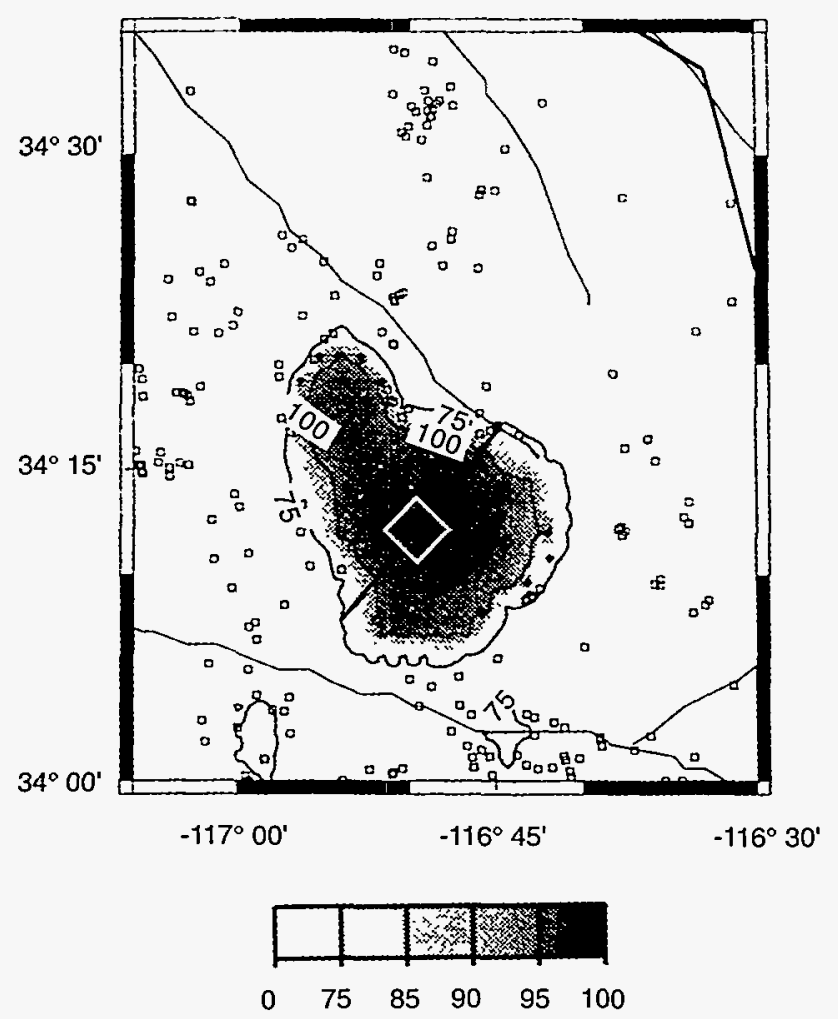

Seismicity Rate Decrease in \%

Shown is the epicenter map of the source volume of the Big Bear earthquake for December 1990 through 27 June 1992. The diamond marks the epicenter; the solid line through it marks the extent of the rupture of the mainshock. The percentage of the seismicity rate decrease (illustrated in the figure on the adjacent page) is labeled along the contours of the shading. The only other quiescence like this in southeastern California is the precursor anomaly before the Landers mainshock. The coincidence in space and time of these anomalies with the respective mainshocks strongly suggests that they are related. (From Wiemer and Wyss, Bull. Seism. Soc. Amer., 84, 900-916, 1994). efforts. However, up to the present, there exists not a single case history that is widely accepted in which an earthquake was, or could have been, predicted on the basis of crustal deformation measurements.

The chief reason why I believe that one day we will be able to predict some large earthquakes is that some of them have foreshocks and preshocks. Foreshocks and preshocks occur in the few days and up to about a year, respectively, before a large rupture. Neither can be distinguished from other earthquake swarm activity that is not followed by a mainshock. Today, we can say that when an earthquake occurs in California, there is a 5 percent chance that it will be followed during the next several days by a larger one in the immediate vicinity. Therefore, we are not in a position to use foreshocks or preshocks for actual predictions, but their very existence gives us hope. Clearly, they are part of the rupture process that begins before the main rupture. Since we know that local preparation processes exist, we should be able to figure out what parameters we can measure to detect them.

The parameters that have been proposed as useful precursors include seismicity patterns, crustal deformation, chemical changes of ground water and gases, level and temperature of ground water, changes of transmission properties for elastic waves, changes of the stress tensor, and electromagnetic disturbances, as well as several less credible ones. As chairman of the IASPEI (International Association of Seismology and Physics of the Earth's Interior) Subcommission on Earthquake Prediction, I have lead an evaluation of the claims of successful prediction methods, as part of LASPEI's contribution to the IDNDR (International Decade for Natural Disaster Reduction). This effort showed a vast schism between the believers and the nonbelievers. There are those who set the standards for acceptable work so high that it cannot be achieved for an experiment outside the laboratory, and there are those who think it is sufficient to send a twopage abstract to convince an international body of experts that they have a valid method of predicting earthquakes. The first group of these scientists resides mostly in the United States, the second mostly in China. Scientists in Japan, in general, occupy a middle position between these two extremes. It is surprising how much difference exists between the assessments of our capability in the problem of earthquake prediction by the seismological communities of different countries. In the U.S., the mention of 
research possibly useful for earthquake prediction is the death knell for a proposal to the agency charged with funding this type of work, while in China seismologists frequently claim to make correct predictions.

The occasional interference by people who derive their information from crystal balls or equivalent logic brings our research endeavor into the realm of suspicion. This suspicion is transformed into outrage in the seismological community, when scientists make insupportable claims. Unfortunately this happens not infrequently, because individuals are gripped by the messiah syndrome, or the lure of the limelight. Some investigators have managed to delude themselves into believing they have the solution to the problem, and they will not listen to any well-reasoned objections. Instead of meeting demands of higher standards of analysis and experiment design, they tout their so-called solution for the earthquake prediction problem to the mass media and unsuspecting officials. This has brought into question the credibility of prediction researchers in general. These phenomena are the chief reason for the evaluation by IASPEI of these claims.

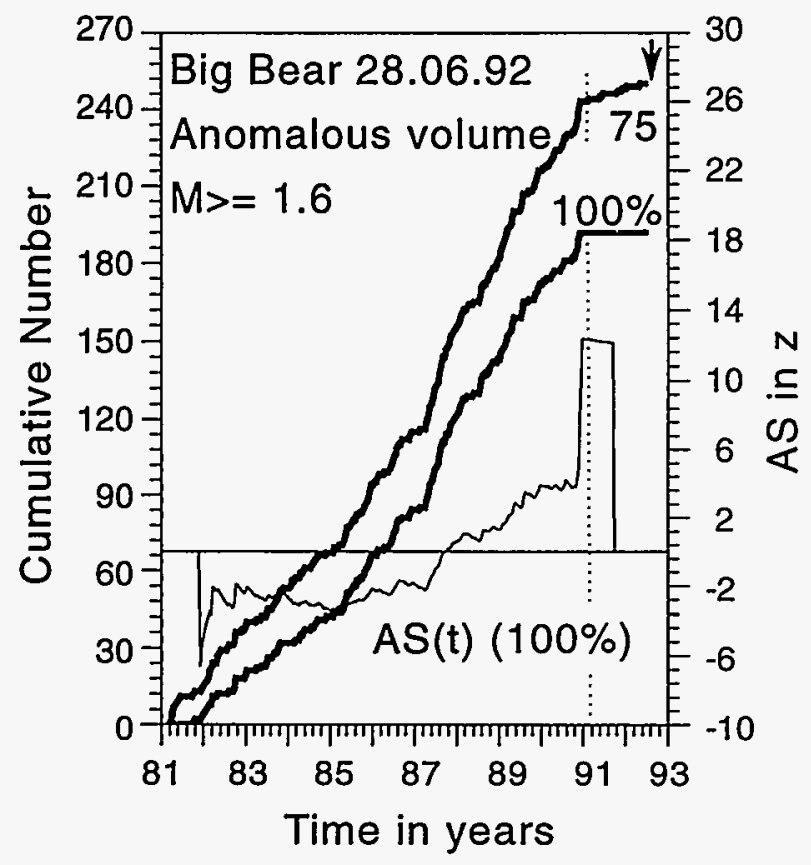

The cumulative number of earthquakes (bold lines) is shown as a function of time for the two areas defined by shading in the figure on the adjacent page. The low slope in these curves, occurring before and up to the mainshock (June 1992), is the quiescence anomaly. No other anomalies like this occurred anywhere else in southeastern California. An algorithm based on the standard deviate $Z$ estimates the significance of the rate of change as a function of time (thin line). (From Wiemer and Wyss, 1994).

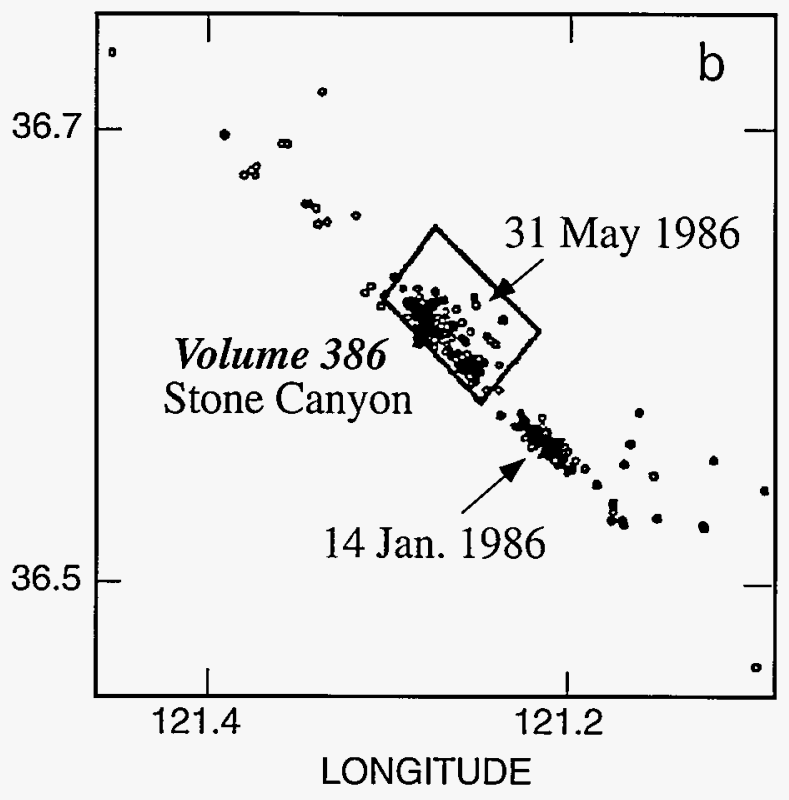

Shown above is the epicenter map of the aftershocks of the predicted earthquake of 31 May 1986, which occurred along the San Andreas fault (outlined by the seismicity trend), and of a small mainshock that was not predicted (14 January 1986). The polygon defined the location of the predicted event and was published by us one year in advance. (From Wyss and Burford, Nature, 329, 323-325, 1987)

In our evaluations, we found that it is not easy to judge a whole method, but it is relatively feasible to come to grips with a case history. Most of the 30 proposals of precursors submitted to IASPEI concerned case histories. Of these, 77.5 percent were rejected, 10 percent are in a category of undecided, and the remaining 12.5 percent have been placed on IASPEI's Preliminary List of Significant Precursors. They are: foreshocks; preshocks; quiescence before aftershocks; chemical and temperature change of ground water; and ground water level change. All of these have been accepted with several caveats. These include: (1) we do not know yet how to use these phenomena for actual prediction: (2) none of them are beyond the stage of case history (that is they have not been tested as a method); and (3) that there are unanswered questions about each of these experiments.

Why is it so difficult for us to make progress in the understanding of earthquake processes? The answer is because nature, not scientists, is in charge of the experiments.

Large earthquakes happen unannounced and infrequently, and most of them occur below parts of the earth's surface that are inaccessible for scientific measure- 
ments. Also, those source areas that are accessible still are separated from the investigators by at least $5 \mathrm{~km}$ of rock. Laboratory experiments are limited in their usefulness because there is a scaling problem, and we do not know exactly how to model the fault properties, nor the stiffness of the rock press.

Two mechanisms that can produce all of the precursors listed above were proposed about 20 years ago. The dilatancy-diffusion model relies on cracks opening under deviatoric stress to change the crustal properries, with varying pore pressure of ground fluids playing a key role. The slip-softening model proposes that slip on the fault surface is iniriated slowly at first, accelerating into catastrophic failure. Both models perform satisfactorily in the laboratory under the right conditions. Neither has been disproved, nor confirmed.

The precursor we are studying is seismic quiescence. This phenomenon is defined as a significant decrease in the rate of small earthquakes, which normally occur at a constant rate. The amount of decrease ranges from 100 percent at the center of the anomaly to about 40 percent at what we define as its periphery. The epi- center map of small earthquakes that occurred during the 1.6 years of precursor time before the Big Bear earthquake (M6.5, 28 June 1992, California) shows a clear absence of events in the source volume. The cumulative number of earthquakes that occurred in this volume as a function of time shows that it produced an average of 60 earthquakes per 1.6 year period, during the background time. This case history, and that of the Landers earthquake (M7.3) that occurred on the same day, are our best examples. This type of precursory seismic quiescence occurs in the source volume of about 50 percent of large earthquakes, and we find an approximately equal number of false and real alarms. For about a dozen case histories we have shown, a posteriori, that highly significant precursory quiescence existed. We correctly predicted one earthquake (M4.7, 31 May 1986, San Andreas fault) a year ahead of its occurrence. The probability that this earthquake happened at random is less than 5 percent. The aftershock zone filled exactly the segment of the San Andreas fault we predicted would fail before June 1986.

In an earthquake prediction, one has to specify the location, the time, and the magnitude of the expected event. For each of these parameters one has to give an error range. In addition, one has to specify the probability of the whole prediction to be fulfilled, and afterward, if one wishes to claim success, one also has to estimate what the probability was for the event to have correlated at random with the prediction. Significant progress has been made in recent years in sharpening the statistical tools by which the predictions are formulated and judged. Ultimately, a method will not be accepted unless it has passed a long series of real time tests that accumulated a statistical score defined as the passing score. The only proposed precursor for which this has been done is the swarm hypothesis, which has been abandoned because the test accumulated a negative score beyond that required for rejection.

This is the stage we are in with seismic quiescence: We must formulate an algorithm by which we can test in a statistical framework our proposed precursor in decades-long earthquake catalogs, to see if the accumulated score will allow either rejection or acceptance.

\section{Can We Predict Volcanic Eruptions?}

\section{by Stephen R. McNutt, Research Professor, Alaska Volcano Observatory}

Nearly every volcanic eruption is preceded by an increase in earthquake acrivity beneath or near the volcano, as shown in the figure on the next page. Evidence of heat and deformation of the ground are also common, but seismicity is most reliable. For this reason, seismology has become one of the most useful tools for eruption forecasting over the last two decades. At the present time, approximately 200 of the world's volcanoes are seismically monitored. This represents about one out of three of the 538 volcanoes that have erupted in historic times (Simkin,
1993). Over the last several decades, 55 to 70 volcanoes have erupted each year (Simkin, 1993). Alaska is home to 42 historically active volcanoes, eight of which are seismically monitored.

Types of Volcanic Earthquakes. Volcanic earthquakes may be defined as earthquakes that occur at or near 
volcanoes, generally within $10 \mathrm{~km}$, or are related to volcanic processes. Active volcanoes are the sources of a great variety of seismic signals, including high-frequency events, lowfrequency events, explosion quakes, and volcanic tremor.

Most high-frequency events are caused by shear failure or slip on faults, and differ from their tectonic counterparts only in their patterns of occurrence, which, at volcanoes, are swarms rather than mainshock-aftershocksequences. These events have clear $\mathrm{P}$ and $\mathrm{S}$ waves, and dominant frequencies are 5 to $15 \mathrm{~Hz}$. Most low-frequency events are caused by fluid pressurization processes, such as bubble formation and collapse, and also by shear failure, tensile failure, or nonlinear flow processes which occur at very shallow depths, for which attenuation and path effects play an important role. These events often have emergent $P$ waves, lack $S$ waves, and have dominant frequencies between 1 to $5 \mathrm{~Hz}$. Explosion quakes accompany explosive eruptions, and many are characterized by the presence of an air-shock phase on the seismograms. Volcanic tremor is a continuous signal of duration of minutes to days or longer. The dominant frequencies of tremor are 1 to $5 \mathrm{~Hz}$, similar to low-frequency events, and many investigators have concluded that tremor is a series of low-frequency events occurring at intervals of a few seconds.

Most seismometers used to monitor volcanoes are located on the flanks, at distances of 5 to $15 \mathrm{~km}$. Modern networks generally consist of eight or more stations, and data are telemetered to a common site and recorded on drum recorders, as well as digitally on computers. Event-triggered recording is common, yet to retain small events and emergent signals like tremor, continuous digital recording is performed at some observatories, including $\mathrm{AVO}$ at the Geophysical Institute. About a third of the monitored volcanoes have at least one station within $1 \mathrm{~km}$ of the active vent. A number of volcanoes are monitored indirectly because a nearby $(30-100 \mathrm{~km})$ seismic station is part of a larger regional seismic network.

Monitoring and Forecasting:The goals of volcano seismology include monitoring the present status of a volcano, forecasting eruptions, estimating size of eruptions in progress, and understanding the physical processes that are occurring. The primary focus of this article is forecasting eruptions. For this, goals are estimat-

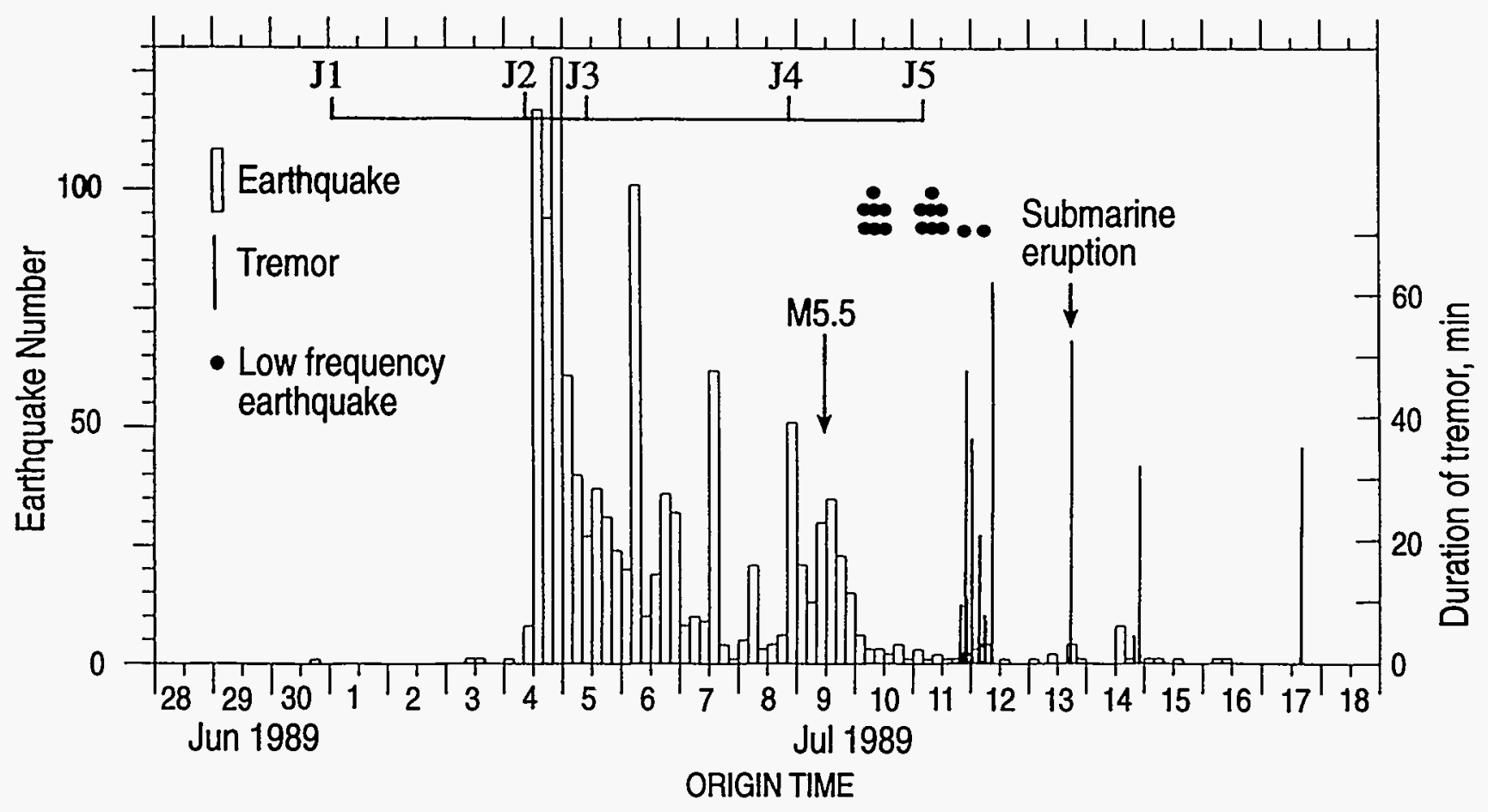

Shown is crustal seismicity associated with the 1989 Ito-oki submarine eruption. Number of earthquakes per 4 hours, duration of tremor events, periods of crustal movement (J1-J5), and occurrence of low-frequency earthquakes are indicated (after Ukawa, 1993). 


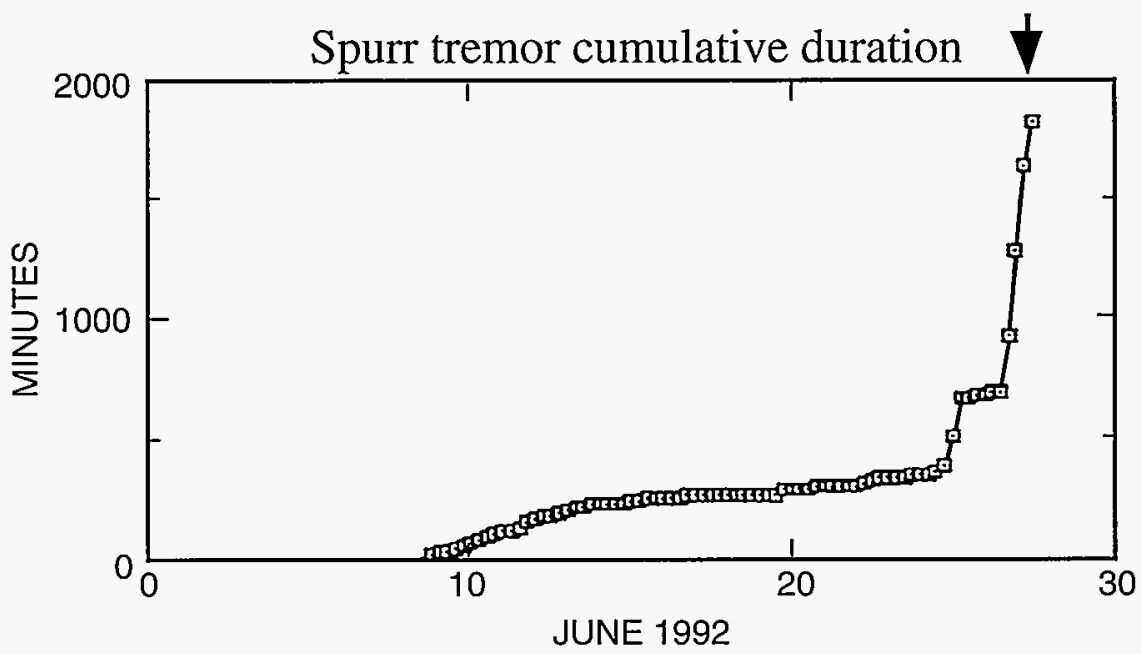

Mt. Spurr volcanic tremor cumulative duration versus time is shown above. Data are computed for 6-hour intervals. The eruption occurred (arrow) on June 27 (after McNutt et al., in press).
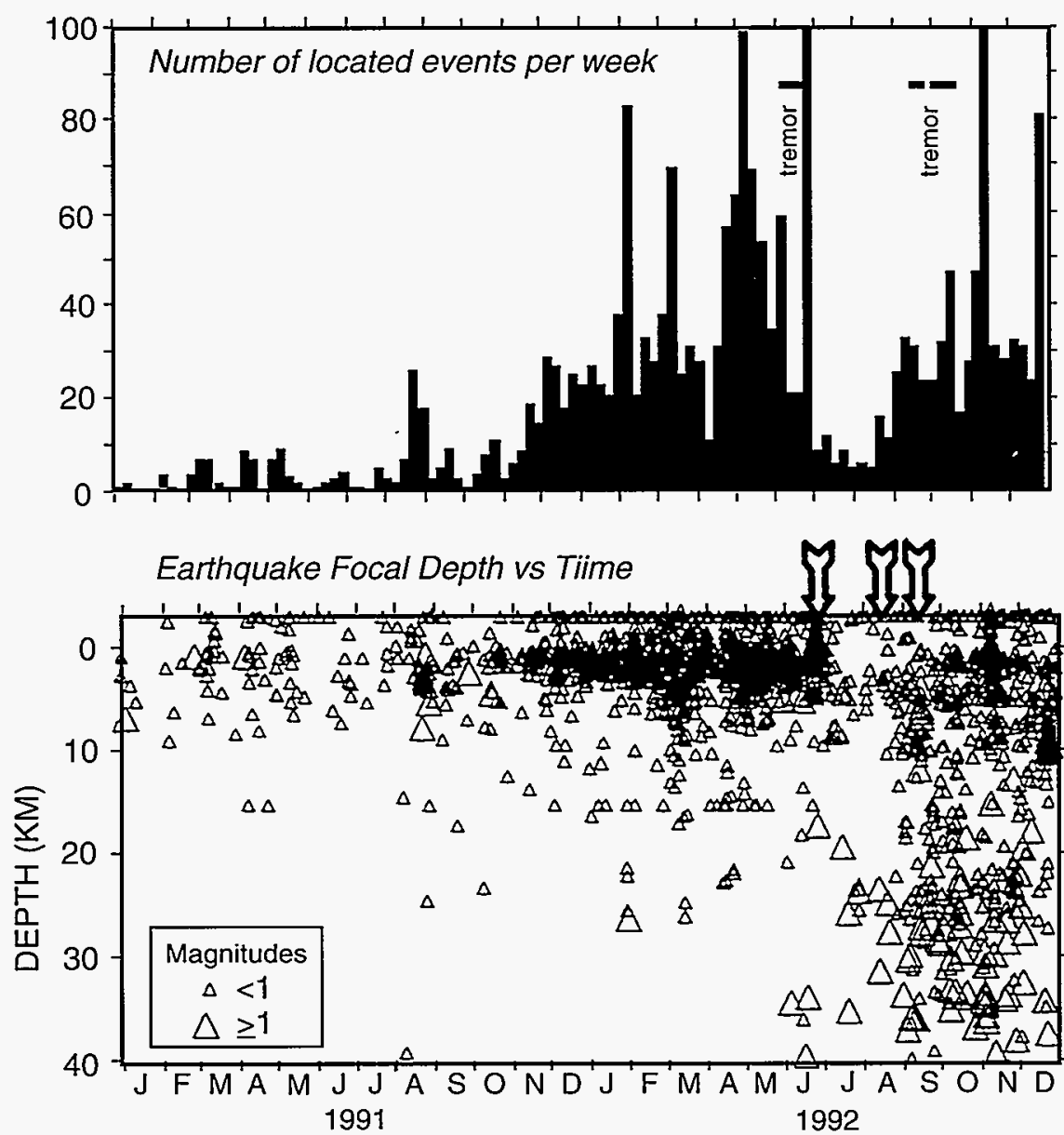

Seismicity in 1991 and 1992 associated with the eruptions of Mt. Spurr is shown. Top: Number of events per week. Episodes of volcanic tremor are shown as horizontal bars. Bottom: Earthquake depth versus time. The three eruptions are shown as arrows (after Alaska Volcano Observatory, 1993). ing the date and time, the place, the size and type, and the duration of eruptions. A variety of observations have a bearing on these problems.

Many successful eruption forecasts have been made because of an increase in seismicity above previously recorded background levels. This is based on the idea that the level of seismicity reflects the level of volcanic activity, and suggests that there is a constant long-term probability of eruption (usually assumed to be a Poisson process) and that the probability increases when earthquake swarms occur. The main physical processes monitored, however, are those associated with magma intrusion. Thus, there will always be a false alarm rate because some intrusions remain at depth whereas others reach the surface and erupt.

In general, large eruptions are relatively easier to forecast than smaller ones. The amount of magma involved is large, and earthquakes tend to be numerous as well as widely distributed spatially. Other precursors, such as deformation and steaming, are usually observed in conjunction with earthquake swarms. Small eruptions and many phreatic eruptions, by contrast, involve much smaller amounts of magma, and have subtle precursors or sometimes no precursors. They are, hence, generally harder to forecast.

Generally, volcanic earthquakes occur beneath the point of eruption. This helps reduce the monitoring problem to that of estimating the time of eruption, since the place is considered known. The problem of forecasting eruptions is thus simpler than that of forecasting earthquakes because there are fewer free param- 
eters. However, the initial earthquake swarms prior to the dacitic eruption of Mt. Pinatubo in the Philippines occurred about $5 \mathrm{~km} \mathrm{NW}$ of the volcano. Twelve days before the climactic eruption these earthquakes declined and vigorous swarms began beneath the eventual caldera (Wolfe, 1992; Harlow et al., in press).

It is expected that earthquake hypocentral locations would migrate from depth towards the earth's surface as magma rises from depths prior to eruption. There are, however, surprisingly few well-documented cases of such a trend. Large eruptions often follow long repose periods, and the earthquake swarms may include relatively large events.

Volcanic tremor is an especially common short-term precursor to eruptions. Barberi et al. (1992) have shown that tremor was the most common short-term precursor in a study of 132 historical cases of phreatic eruptions. This suggests a close link between tremor and hydrothermal processes.

Newhall and Dzurisin (1988) found that tremor was the most consistent short-term indicator of impending eruptions in a study of 200 calderas worldwide. McNutt (1992) showed that while most tremor episodes accompany eruptive activity, about 20 percent preceded eruptions on a time frame of ten days. Clearly, volcanic tremor is a common and important precursor to many eruptions.

Klein et al. (1987) showed that of 73 swarms studied, 31 percent accompanied eruptions, 42 percent accompanied intrusions, and 27 percent were associated with inflation episodes. Klein et al. noted that except for harmonic tremor gener- ated near an erupting vent, intrusions and eruptions were seismically indistinguishable. Thus, tremor is a key diagnostic to help distinguish eruptions from intrusions.

Generic Volcanic Earthquake Swarm Model: Common observations at many volcanoes suggest a general model of earthquake activity which may be used to forecast eruptions. The model, which we term a generic volcanic earthquake swarm model, is shown as a time series in the figure below. In the generic model, seismicity rates, types of events, and dominant processes are indicated in the commonly observed temporal sequence. Variations in the involvement of groundwater, as well as variations of other parameters such as composition, temperature, rate of flow, and volatile content of magma, can explain many of the observed differences in seismic activity at different volcanoes.

In the generic model, high-frequency earthquakes reflect shear fracture of the country rock in the vicinity

\section{Generic Volcanic Earthquakes Swarm Model}

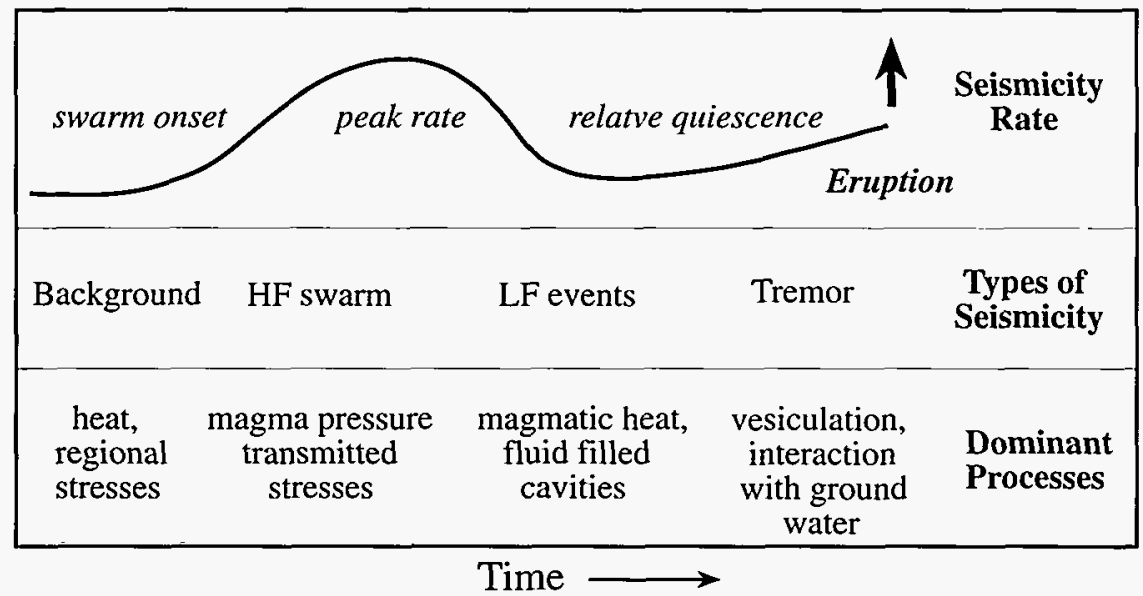

Shown is the schematic diagram of the time history of a generic volcanic earthquake swarm model. Seismicity rates are shown along with the main types of events observed at each stage. Some ideas about dominant processes are presented at the bottom of the figure. of volcanoes in response to increasing magmatic pressure. Since magma movement at depth may occur over large distances and long periods, the durations of high-frequency swarms are long. At shallow depths of one to three $\mathrm{km}$, several significant changes occur. First, volatiles begin to exsolve from the magma, changing its rheology, and, hence, its ability to transmit or reflect seismic waves. For example, exsolution of volatiles will increase the viscosity.

Second, the magma may encounter groundwater, modifying the shallow hydrothermal system and using some of the available magmatic heat. Most low-frequency events and tremor originate at shallow depths, and several models for their origin involve fluids: either water, magma, exsolved volatiles, or all three. Tremor caused by boiling of water is generally weaker than tremor involving magma movement.

Third, open cracks are found above three $\mathrm{km}$ depth in the earth, permitting venting of excess pressure. This 
may explain the relative quiescence after the peak rate of a swarm as well as the inverse relation between highfrequency events and tremor. Relative seismic quiescence occurred in up to 25 percent of a worldwide sample of 139 swarms (Newhall and Endo, 1987; Benoit and McNutt, 1994). Other factors may also cause quiescence, including: strain hardening; high temperatures near the brittleductile transition; increased water content (e.g., groundwater) lowering effective stress; or a reduction in strain rate. It is common for low-frequency events and tremor to occur after seismicity rate peaks, during times of relative quiescence. This may be explained by gradual shoaling of the source, particularly magma interacting with groundwater or exsolution of volatiles.

It is still true that each volcano is unique in its seismic behavior, but the observations suggest that variations in a few parameters, such as those discussed above, may account for the main differences. Minor variations in each of several parameters may greatly affect one observed parameter, such as swarm duration. The durations of earthquake swarms are characterized by a log-normal distribution with a mean of five days. No clear correlations exist between the duration of a swarm and the size or type of eruptive activity. This is not surprising, given the ranges of sizes, rates, compositions, and types of structures observed. Larger earthquakes occur at larger structures, but not necessarily in association with large eruptions, although there are a few examples of large events accompanying large eruptions.

Although seismology is a powerful tool in terms of eruption monitoring, it is no where near as powerful alone as it is when combined with other techniques. These include visual observations, heat, deformation, gas sampling, and others. Nevertheless, seismology is the backbone of most volcano observatories, and a strong seismic monitoring program generally results in the effective forecasting of eruptions and hazard mitigation. Future efforts will be undertaken to provide a more sophisticated physical basis for eruprion forecasting, and to search continually for new and existing high-quality data sets for better diagnostics. Such data, and welltrained and experienced scientists, will help reduce the potential for disasters at dangerous volcanoes.

\section{Some Successful Forecasts of Explosive} Volcanic Eruptions 1980-94

Mount St. Helens, Washington $1980-1986$

Galunggung, Indonesia .... 1982

Merapi, Indonesia 1984

Izu Oshima, Japan 1986

Banda Api, Indonesia 1988

Kie Besi, Indonesia 1988

Tokachi - Dake, Japan 1988

Galeras, Columbia. 1989

Izu-Tobu, Japan 1989

Redoubt Volcano. Alaska $1989-1990$

Kelut, Indonesia 1990

Unzen, Japan $1990-1991$

Mount Pinatubo, Philippines 1991

Colima, Mexico 1991

Lonkon, Indonesia 1991

Mount Spurr, Alaska 1992

Mayon, Philippines 1993

Mount Klyuchevskoi, Kamchatka 1994

Rabaul Caldera, Papua - New Guinea 1994

Popocatepetl, Mexico 1994 


\section{Space Physics

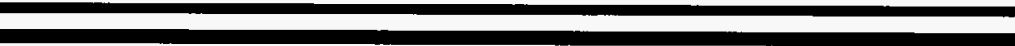 \\ (}

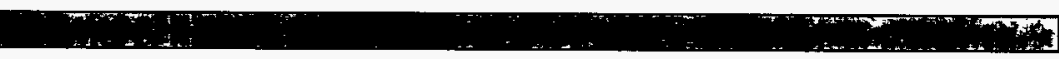

$\because 4$

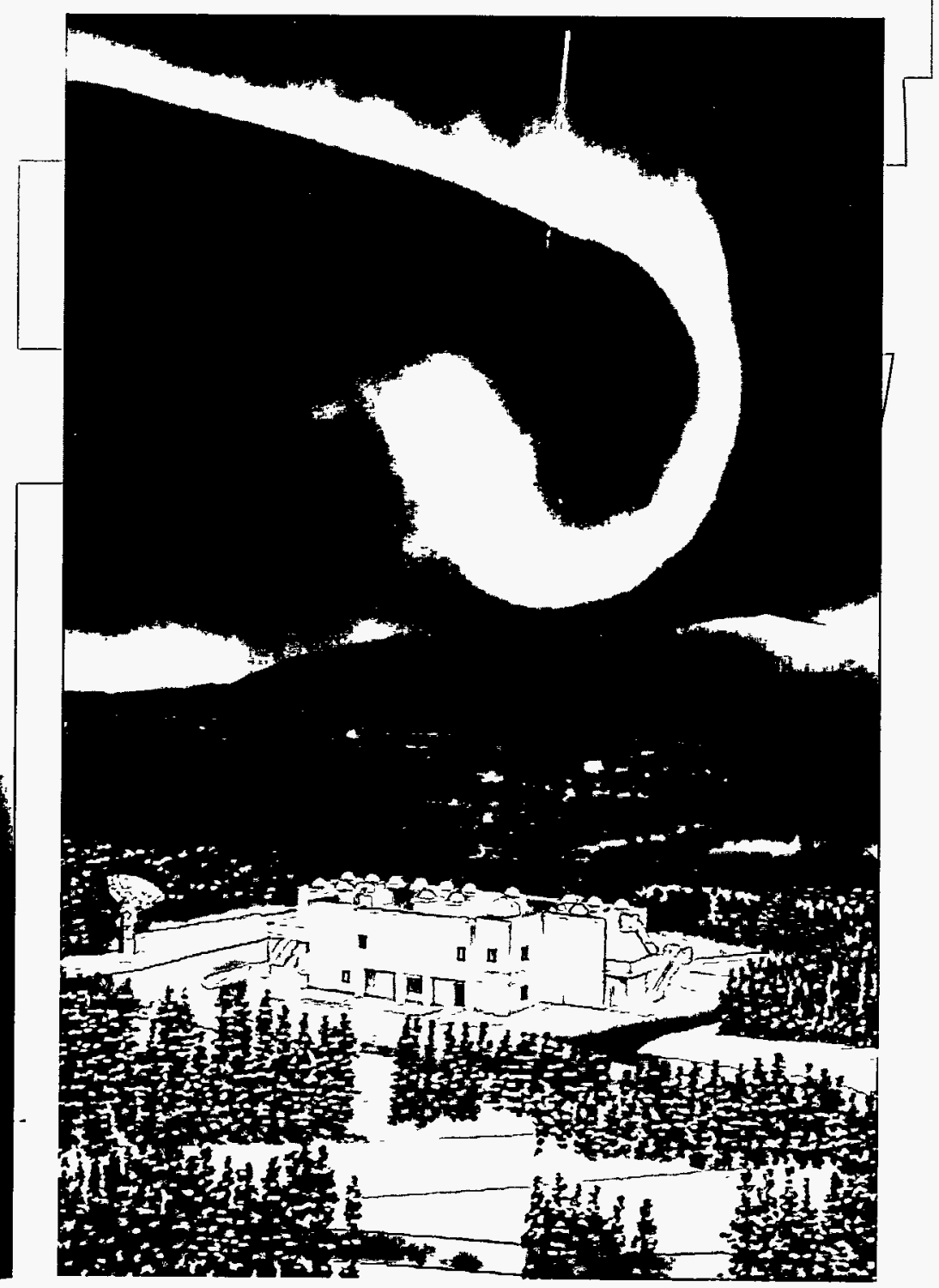




\section{SPACE PHYSICS}

Members of the Geophysical Institute's Space Physics Group study physical relationships within the earth's geospace environment, which extends from the sun to the earth's stratosphere. Major outstanding problems are associated with the coupling of energy, mass and momentum across boundaries between the various distinct regimes that exist in geospace.

Coupling processes within the plasma-dominated regimes from the sun to the upper ionosphere are challenging to describe both through measurements and computer simulations. Well-instrumented, multiplespacecraft missions are required in conjunction with well-conducted ground-based observations to understand the coupling processes, including mutual interactions and instabilities existing in and between local plasmas and plasma waves. For example, measurements of the large- and small-scale phenomena that occur at the boundary between the solar wind and Earth's magnetosphere are required on the basis of both satellite-based and ground-based observations to specify the important parameters of these regions.

Members of the group are active contributors to NASA's Global Geospace Science (GGS) mission within the overall International Solar Terrestrial Physics (ISTP) program. They also contribute to NASA's Space Physics Theory Program (SPTP) and to NSF's Geospace Environment Modeling (GEM) program. Theory, observations and computer simulations are combined in these programs to address the outstanding issues. Studies of the magnetosphere also have been extended to the Sun with successful simulations of the formation and eruptions of solar prominences.

Physical mechanisms of wave propagation and the creation of instabilities are important to descriptions of the ionosphere and neutral upper and middle atmosphere, as are convective transport, local chemistry, and solar contributions of electromagnetic energy and particles (after interactions with the magnetosphere).

Group members studying the upper and middle atmosphere have made important contributions to NASA's Dynamics Explorer (DE) and Upper Atmosphere Research Satellite (UARS) missions, and to the proposed Thermosphere-Ionosphere-Mesosphere Ener- getics and Dynamics (TIMED) mission. We also have contributed to NSF's Coupling Energetics and Dynamics of Atmospheric Regions (CEDAR) program. In addition, we have been major participants in active experiments for NASA's Combined Release and Radiation Effects Satellite (CRRES) and the suborbital program for investigations of plasma processes in the upper ionosphere.

Emphasizing the need for a coherent, comprehensive approach to ground-based studies of coupled regions in the atmosphere and ionosphere, the group is heavily involved in instrumentation and measurements made at UAF's Poker Flat Research Range and its downrange stations, at the Sondrestromfjord radar station in Greenland, and at optical observatories in Longyearbyen (Svalbard, Norway), Mt. John (New Zealand) and at the South Pole (Antarctica). These ground-based activities focus on rocket projects and global-scale programs, such as CEDAR, that can integrate data from diverse instruments and stations for studies of the coupled thermosphere-ionosphere-magnetosphere system.

The space physics theory group recently proposed a new mechanism for the substorm onset by successfully simulating the formation of a very thin current sheet in the near-earth plasma sheet. The group also provided a comprehensive study of the structure and transport at the magnetopause boundary layer. A mechanism for the generation of slow-mode structures and waves observed in the dayside magnetosheath also was proposed.

Recent measurements have pioneered a new phase in investigations of atmospheric electricity through the observations of high-altitude, upward-propagating flashes associated with tropospheric thunderstorm activity. The clear identification and improved observations of these energetic events further demonstrate the importance of the stratosphere and mesosphere to the global electric circuit, and illustrate a means of direct coupling between the tops of thunderclouds in the troposphere and the lower reaches of the ionosphere.

The T. Neil Davis Science Operations Center at Poker Flat Research Range is near completion. At the top of a low mountain overlooking the Poker Flat launch complex, the center will house a variety of optical instruments for studying auroras and mesospheric dynamics, 
and it will serve the needs of visiting scientists conducting rocket-borne experiments. The center also will serve as the focal point for the collecrion, display, and dissemination of other geophysical environmental data. Amenities include ample space for viewing the aurora and a conference room to encourage seminars and other scientific interchanges between staff and visitors.

A major new program of collaborative science has been initiated between the Geophysical Institute and the Communications Research Laboratory of Japan to investigate the middle and upper atmosphere. A set of new instruments built in Japan will be located at Poker Flat Research Range to measure energy deposition by waves and particles in the altitude range of 60 to $100 \mathrm{~km}$, and to measure winds and temperatures in the same region. An imaging riometer will be the first installation, followed by an imaging Fabry-Perot interferometer, a passive microwave sounder, a heterodyne spectrometer and a spaced-antenna wind radar.

HAARP, a program for High Frequency Active Auroral Research, has evolved from a DoD research program to a joint DoD-NSF research program. The Geophysical Institute is one of the participaring institutions in the HAARP program. Our scientists are interested in measuring the effects on the electron density, the electric field, the magnetic field, the VLF/ELF/ULF waves and the optical emissions in the auroral ionosphere produced by the HAARP high-power radar.

\section{Solar Physics and Interplanetary Disturbances}

Formation of Solar Prominences in Quadrupolar Magnetic Fields: A helmet-shaped magnetic arcade is often observed over a solar prominence and it sometimes surrounds more than one bipolar region. A survey by Tang (1987, Solar Phys. 107, 233) showed that more prominences are formed between bipolar regions than inside one bipolar region. Based on this observation, we have investigated the formation of prominences between two arcade systems.

In this study, two adjacent bipolar regions are mimicked by a quadrupolar field without $x$-point initially. On the footpoint level, we give

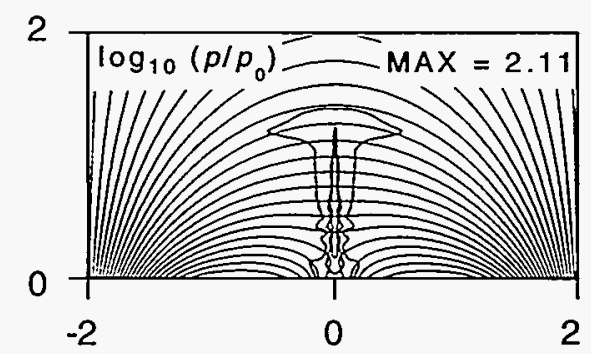

Shown is the formation of an inverse polarity prominence between two bipolar arcades. Plotted are contours of density variation $\left(\log _{10}\left(r / r_{\alpha}\right) 0\right)$, superimposed on field lines and contours of temperature variation $\left(\log _{10}\left(T / T_{\alpha} j 0\right)\right.$.

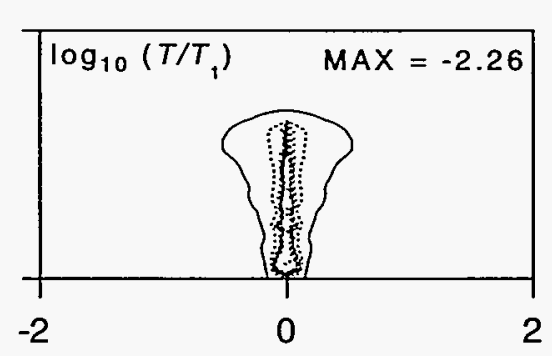

a shear and a slower converging motion. By the shear, both arcades expand separately and expel flux towards each other. A current layer is thus formed, starting from the neutral line, where the first magnetic reconnection takes place. The $\mathrm{x}$-point slowly rises in time with successive reconnection of field lines. The expansion of arcades induces the quasiadiabatic temperature drop, except near and below the $x$-point. The local density is decreased within the two expanding arcades, except near the $x$-point, but is increased in the overlying arcade. This density enhancement is mainly attributed to

the material outflow from the reconnection region. The sudden cooling to the chromospheric temperature first takes place in the overlying arcade. The successive condensation upward and downward results in a vertical sheet structure of the prominence. Since the plasma $\beta$ is very low ( 0.01$)$, the dips on field lines are not caused by the gravity of high mass, but by the intrinsic field geometry.

DOE Grant DE-FGO691ER13530: Scientific personnelL.C. Lee and G.S. Choe

Asymptotic Formation of Current Sheets in Sheared Magnetic Arcades: It is known that line-tied magnetic fields cannor fully open up with finite footpoint motions. It has thus been speculated that the open field is asymptorically approached with an indefinitely increasing shear. However, it was not certain whether a sequence of equilibria exists for all the footpoint displacements. In this study, we used a dynamic MHD code and a magnetofrictional code to 
investigate the behavior of magnetic fields with very large shears.

It was found that all the field lines keep ascending with increasing shear. The central current layer always bifurcates into two layers for a finite shear, and the distance between the footpoints of the current layer becomes smaller with increasing shear. After a certain amount of shear, most of the input energy is converted to the poloidal magnetic energy, while the toroidal magnetic energy is saturated. Our results show there is no nonequilibrium for any shear as far as a 2-D Cartesian geometry is concerned. It was also found that there is a critical shear beyond which magnetic reconnection is possible.

DOE Grant DE-FGO691ER13530 to University of Alaska: Scientific personnel-L.C. Lee and G.S. Choe

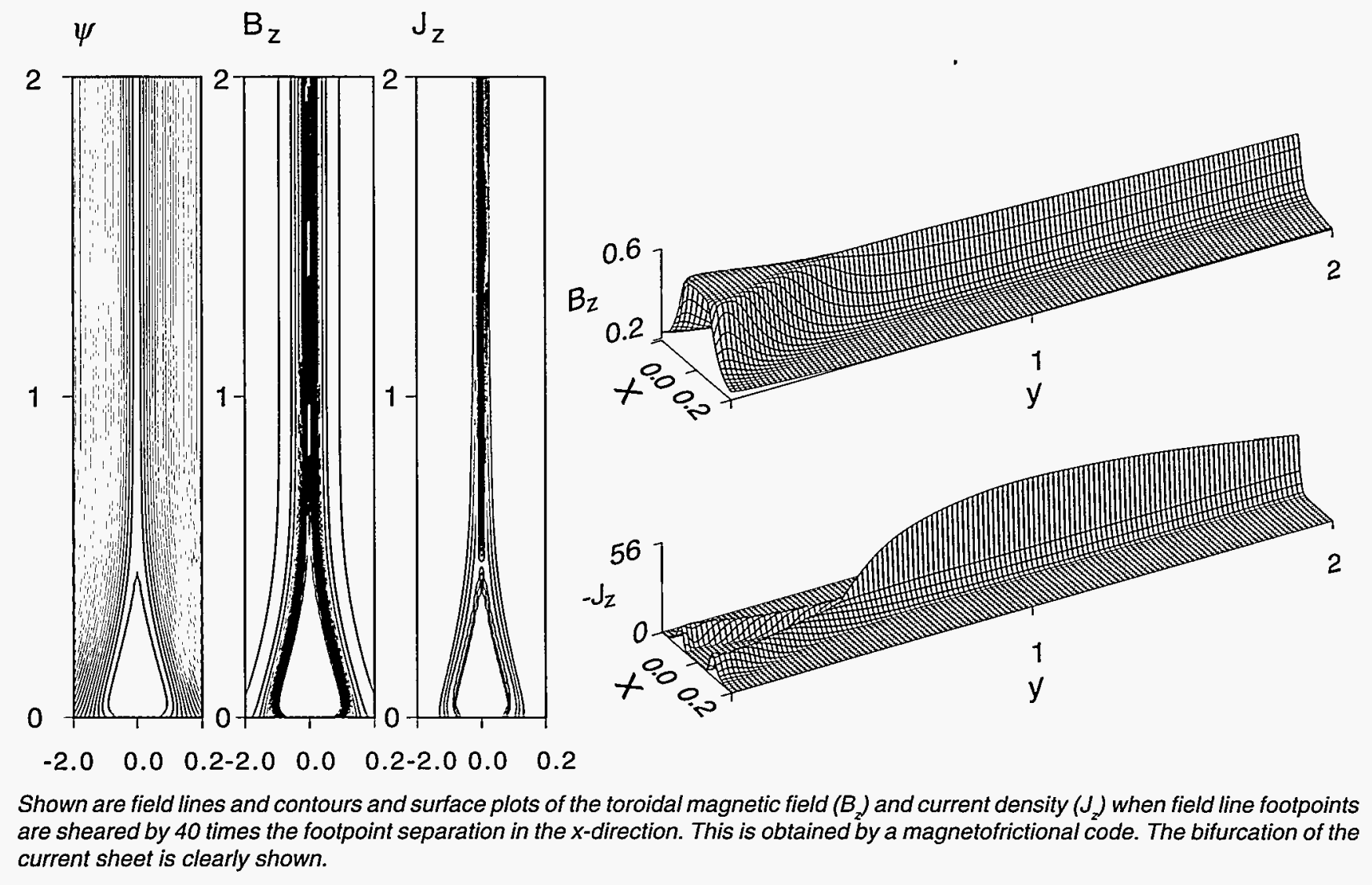

Shown are field lines and contours and surface plots of the toroidal magnetic field $\left(B_{z}\right)$ and current density $\left(J_{z}\right)$ when field line footpoints are sheared by 40 times the footpoint separation in the $x$-direction. This is obtained by a magnetofrictional code. The bifurcation of the current sheet is clearly shown.

\section{Bow Shock-Magnetosheath}

Observations of Slow-Mode Fluctuations in the Dayside Magnetosheath: The solar wind and interplanetary magnetic field drive geomagnetic activity, but it is the magnetic field and plasma in the magnetosheath that impinge directly onto the magnetosphere. Hence, an understanding of the solar wind control of the magnetosphere must include the processes associated with the bow shock and the magnetosheath. The magnetosheath, which is the region between the bow shock and the magnetopause, is highly turbulent. Previous studies of the magnetosheath waves concentrate on fluctuations of plasma density and magnetic field with periods smaller than two minutes. This research, however, studies fluctuations of the magnetosheath plasma and magnetic field with longer periods.

Plasma and magnetic field observations by ISEE 1 and ISEE 2 were examined to study large-scale fluctuations in the magnetosheath. We found that large-scale fluctuations with a period over two minutes are often present in the magnetosheath. The amplitudes of the fluctuating plasma density and magnetic field are comparable to the background values, and the thermal pressure and magnetic pressure were found to be anticorrelated. They appear nor only in the region close to the magnetopause, but also elsewhere in the magnetosheath. The corresponding plasma and magnetic observations from ISEE 3 and IMP 8 in the solar wind show that these fluctuations 
usually have their counterparts in the solar wind. But the signatures on the plasma density and magneric field are sometimes modified by the bow shock. Examples show that an Alfvénmode structure with a constant plasma density and magnetic field amplitude becomes a structure with strong plasma density variation in the magnetosheath. Statistics show that large-scale large-amplitude fluctuations are present in all 92 magne-

\section{Density Contours}
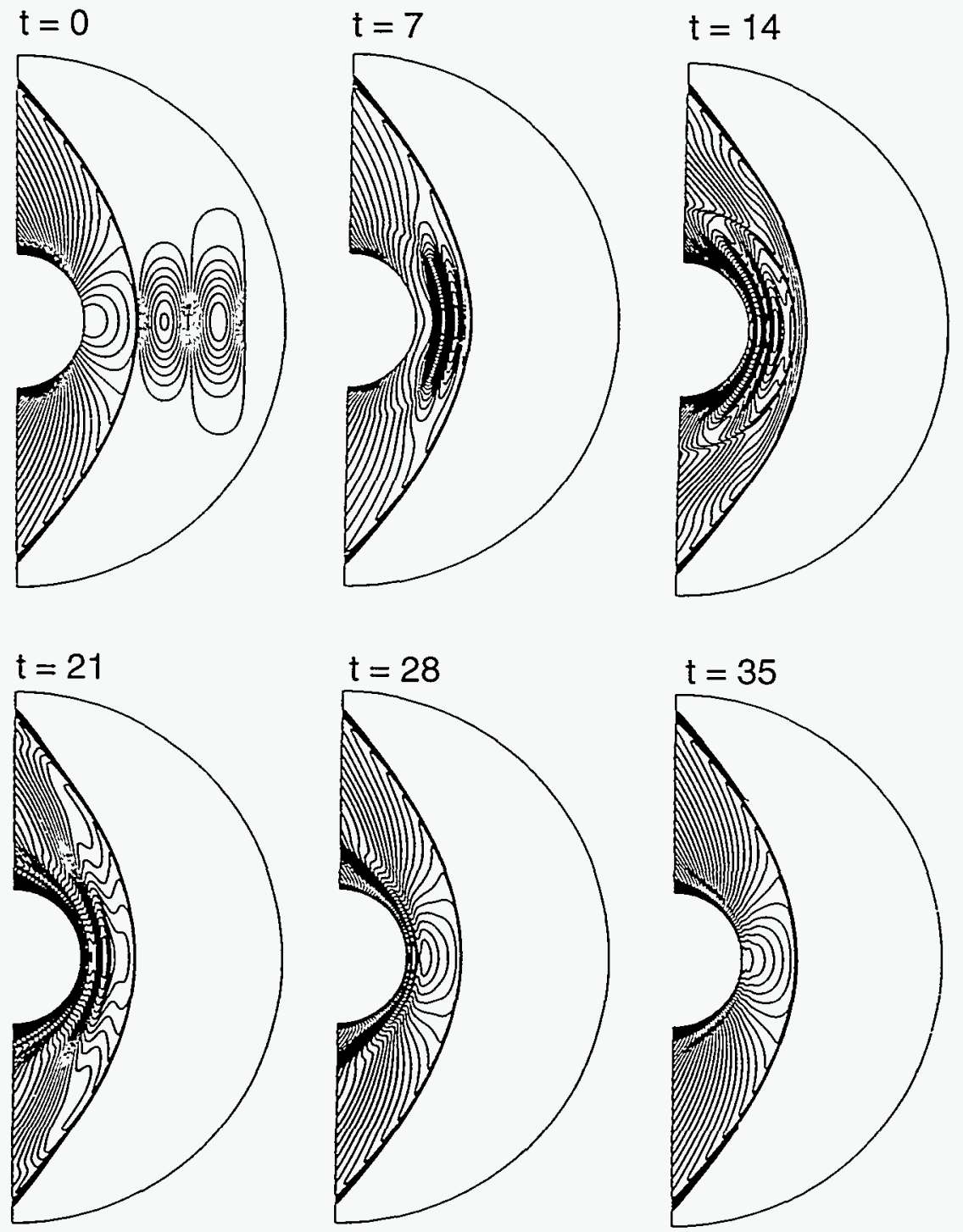

The interaction of interplanetary MHD waves with the bow shock leads to the generation of slow-mode waves observed in the inner magnetosheath. Plasma density contours at different times are shown for a simulation in which a fast-mode wave is initially imposed upstream of the bow shock. A fast-mode wave, two slow-mode waves, and an entropy wave are generated downstream of the bow shock in the magnetosheath after the incident wave interacts with the bow shock $\left(t=3.5 t_{A}\right)$. The fast-mode wave is reflected back at the magnetopause and steepens to form a fast shock $\left(t=7 t_{A}\right)$ before it merges into the bow shock. The propogation of the slow-mode waves in the direction normal to the magnetopause becomes very slow in the area close to the magnetopause. From $t=$ $7 t_{A}$ to $t=17.5 t_{A}$, the slow-mode waves stand in the inner magnetosheath for $\sim 15$ minutes.

tosheath crossings of ISEE 2 . About 90 percent of them are identified as slow-mode structure with anticorrelated plasma thermal pressure and magnetic pressure. More than half (62 percent) of the slow-mode structures are encountered in the inner half of the magnetosheath.

NASA Grant NSPTP-NAG5-1504 and NSF Grant ATM9111509: Scientific personnel-L.C. Lee, M. Yan, and P. Song

Generation of Slow-Mode Waves by the Interaction between the Bow Shock and Interplanetary MHD Waves: Slow-mode waves have been observed to appear frequently in front of the dayside magnetopause of the earth. The properties of these waves can be summarized as follows: (a) the variations in plasma density and magnetic field strength are anti-correlated; (b) the observed waves do nor convect with the magnetosheath plasma flow; they seem to stand against the magnetosheath flow; (c) the wavelength $\left(\lambda_{n}\right)$ in the direction normal to the magnetopause is typically 2000 to $5000 \mathrm{~km}$, while the wavelength $(\lambda)$ in the tangential direction is much larger than $\lambda_{n}$; and (d) a steepened plasma density profile with a shocklike structure also appears in the enhanced density region. Properties (a) and (b) indicate that the observed waves are slow-mode waves. Observations also indicate that these slowmode waves may come from the interaction of the bow shock and fluctuations in the solar wind.

Our simulations show that the interaction of interplanetary MHD waves with the bow shock leads to the observed slow-mode waves in the inner region of the dayside magneto- 
sheath. The transmission of one mode of MHD waves through the bow shock generates various modes of MHD waves, including fast-mode waves and slow-mode waves, in the magnetosheath. The fast-mode waves are reflected at the magnetopause, and propagate back towards the bow shock. These fast-mode waves can steepen during the propagation and form fast shocks before they finally merge into the bow shock. The propagation of slow-mode waves slows down as these waves approach the magnetopause. Both the plasma flow speed and the slow-mode phase speed normal to the magnetopause decrease to zero when approaching the magnetopause.

It was found, based on two-dimensional global MHD simulations (shown in the figure), that slow-mode waves are generated through the interaction between the bow shock and various MHD waves (fast-mode, Alfvén-mode, or slow-mode waves) in the upstream solar wind. The generated slow-mode waves stay in front of the magnetopause for a long time (over 15 minutes) before the wave energy is convecred away tailward. Since various waves are often present in the solar wind, this mechanism may lead to the frequent appearance of slow-mode waves in front of the magnetopause.

NASA GrantNSPTP-NAG5-1504 and NSF Grant ATM9111509: Scientific personnel-L.C. Lee and M. Yan

Interaction of the Magnetopause with Interplanetary Current Sheets: The interplanetary currentsheets may interace with the magnetopause current sheet as they propagate across the bow shock into the magnetosheath. When magnetic reconnection takes place in a current sheer, the strong magnetic tension force accelerates plasma to high-speed flow. On the other hand, satellite observations show that transient sunward plasma flows somerimes appear in the magnetoshearh, which are called anomalous plasma flow events. These events are found to be associated with interplanetary current sheets that come through the bow shock into the magnetosheath. We studied reconnection processes in a double current sheet system by two-dimensional MHD simulations and applied the results to the interaction between an interplanetary current sheet and the magnetopause current sheet.

Both the linear and nonlinear evolution of this process were analyzed for laminar perturbations. The results illustrate the existence of a linear regime with a symmetric and an antisymmetric mode and agree with previous analytic results. The nonlinear evolution shows a number of interesting new features and may explain some properties in corresponding studies of turbulent recon- nection. For wavelengths larger than twice the current sheet separation, the evolution of antisymmetric modes leads to an entire reconfiguration of the magnetic field and converts a major portion of the magnetic energy into kinetic energy.

Antisymmetric modes with smaller wavelengths and symmetric modes were found to saturate. The influence of the value of the resistivity on the reconnection rate decreases in the nonlinear evolution and the ratio of current sheet separation to wavelength seems to be of major importance. A comparison of the dynamics of periodic current sheets with the evolution of only two current sheets indicates that some of the results for the periodic system also apply to the evolution of only two interacting current sheets. These simulation results show that the explosive process of the magnetic reconnection in the double current sheet may generate the observed anomalous plasma flows and magnetic field reconfigurations in the magnetosheath.

DOE Grant DE-FGO691ER13530: Scientific personnelL.C. Lee, A. Otto, M. Yan, and D. Muzzell

\section{Magnetopause Boundary Layer: Magnetic Reconnection and Transport}

Structure of Reconnection Layers in the Magnetosphere: Magnetic reconnection can take place at a current sheet that separates two plasma regions with antiparallel magnetic field components. Through magnetic reconnection, magnetic energy can be efficiently converted into kinetic energy, leading to the ejection of high-speed plasma. A layered structure that contains several MHD discontinuities and expansion waves is formed in the high-speed outflow region in a quasi-steady reconnection. This layered structure is called the reconnection layer. In the earth's magnetosphere, magnetic reconnection can take place at the dayside 
magnetopause and at the flank of the magnetopause. Consequently, reconnecrion layers can be formed in these regions of the magnetopause.

We studied the structure of reconnection layers by solving the Riemann problem associated with magnetic reconnection at the magnetopause. The ideal MHD formulation, resistive MHD model, and hybrid model are used in the study. Petschek's symmetric reconnection model is generalized by including an

$$
\text { Dayside }(V \approx 0)
$$

asymmetry in the magnetic field and plasma density, a finite guide field $\left(B_{y}\right)$, the ion kinetic effect, and a shear plasma flow in the magnetosheath. At the dayside magnetopause, a large rotational discontinuity is found to bind the reconnection layer on the magnetosheath side; it can be identified as the magnetopause current sheet. Accelerated plasma flow is present in the boundary layer earthward of the magnetopause current sheet. Due to the mixing of plasmas

$$
\text { Flank }\left(V=V_{A}\right)
$$

\section{Observation}
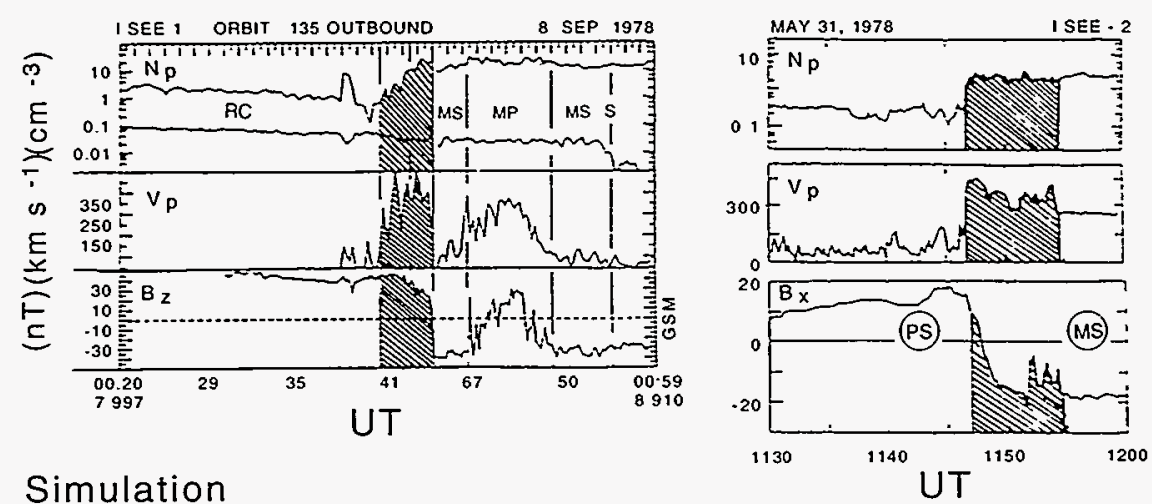

\section{Simulation}
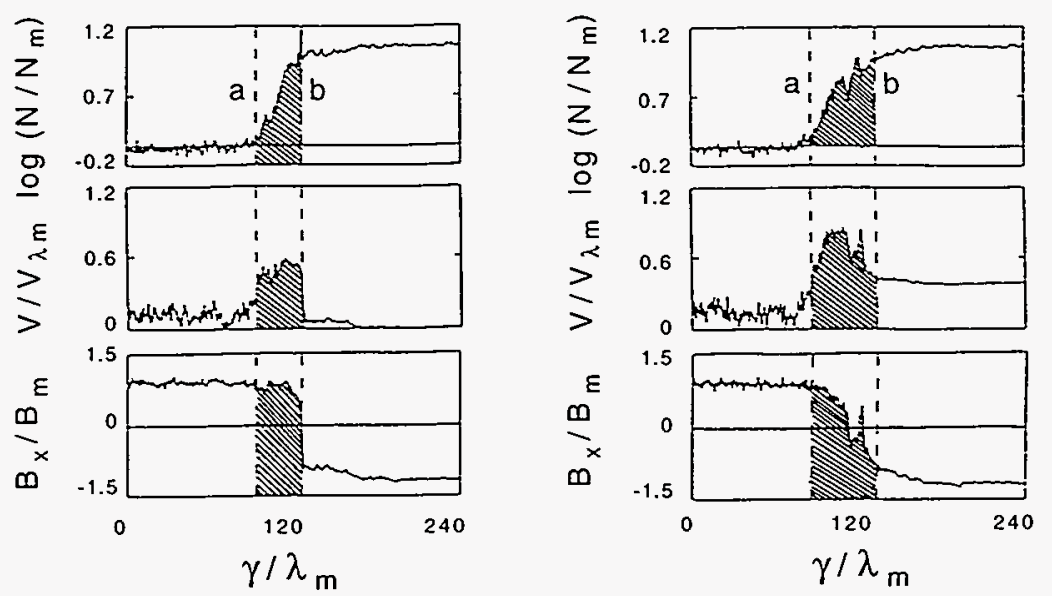

Hybrid simulations successfully reproduce the satellite observations of reconnection layers at (a) the dayside magnetopause and (b) the flank magnetopause. The magnetospheric side and magnetosheath side of the boundary layer are indicated by (a) and (b), respectively. At the dayside magnetopause, a large rotational discontinuity binds the reconnection layer on the magnetosheath side and can be identified as the magnetopause current sheet. Highspeed accelerated plasma flowis present in the boundarylayerearthward of the magnetopause current sheet. At the flank magnetopause, where a large plasma flow is present in the magnetosheath, the magnetic field transition region is very broad, and the accelerated flow is present in the entire field transition region. from the magnetosheath and magnetosphere, an increase in temperature ratio $T_{\|} / T_{\perp}$ occurs in the boundary layer, and the plasma population from the magnetosheath has a D-shaped ion velocity distribution.

In addition, we studied the structure of the reconnection layer at the flank magnetopause, where a large magnetosheath plasma flow is present. It was found that the field transition region in the flank reconnection layer is thick, and the highspeed accelerated flow is present in the entire field transition region. The results are consistent with satellite observations. The figure shows the structure of magnetopause boundary layer at the dayside magnetopause (small shear flow) and at the flank magnetopause (large shear flows) obtained from observations and simulations.

DOE Grant DE-FGO691ER13530: Scientific personnelL.C. Lee and Y. Lin

Linkage-Induced Magnetic Reconnection at the Dayside Magnetopause: One of the major unanswered questions of magnetospheric physics is the structure of the dayside magnetospheric boundary and the associated boundary layers, which map to the auroral ionosphere. This topic is important for the transport of solar wind plasma into the magnetosphere and the transport of magnetic flux from the dayside magnetosphere to the magnetotail. During periods of southward interplanetary magnetic field (IMF) orientation, magnetic reconnection can facilitate this transport as suggested in the first model by Dungey. During the past two decades, various models for recon- 
nection at the dayside magnetopause have been suggested, with increasing evidence and emphasis for three-dimensional processes. Three-dimensional reconnection at a localized spot generates two elbow-like flux tubes, which connect the IMF with the northern and southern polar ionosphere.

It is likely that three-dimensional reconnection occurs simultaneously at multiple patches on the magnetopause. A major problem with this picture is the interaction of magnetic flux tubes, which are generated at different reconnection sites. Multiple patches of reconnection can lead to the formation of interlinked magnetic flux tubes. Although the corresponding flux is connected to the IMF, the northward and southward connected branches are hooked into each other and cannot develop independently.

We have studied this problem in the framework of three-dimensional resistive magnetohydrodynamic simulations. The results illustrate the formation of a strong current sheet at the interface of two interlinked flux tubes if no dissipation is present at this interface. This current sheet is strongly tilted when compared to the original current sheet. In the presence of resistivity, the interaction of the two flux tubes forces a fast reconnection process which generates helically twisted closed magnetospheric flux. This linkage-induced reconnection (LIR) generates a boundary layer with layers of open and closed magnetospheric flux and may account for the brightening of auroral arcs poleward of the boundary between open and closed magnetic flux. Expected in situ signatures of flux rope linkage and LIR are: (a) a strongly perturbed magnetopause with large magnetic field components perpendicular to the original/model magnetopause; and (b) very strong flux rope signatures in the subsolar region. The results are particularly important for the upcoming CLUSTER mission which will be able to identify three-dimensional plasma structures.

NASA Grant NSPTP-NAG51504: Scientific personnel-A. Otto

Magnetic Reconnection in the Presence ofSheared Flow-Applications to the Earth's Magnetosphere: A major difference berween the observations of accelerated plasma at the dayside magnetopause and at the high/low latitude flanks of the mag-
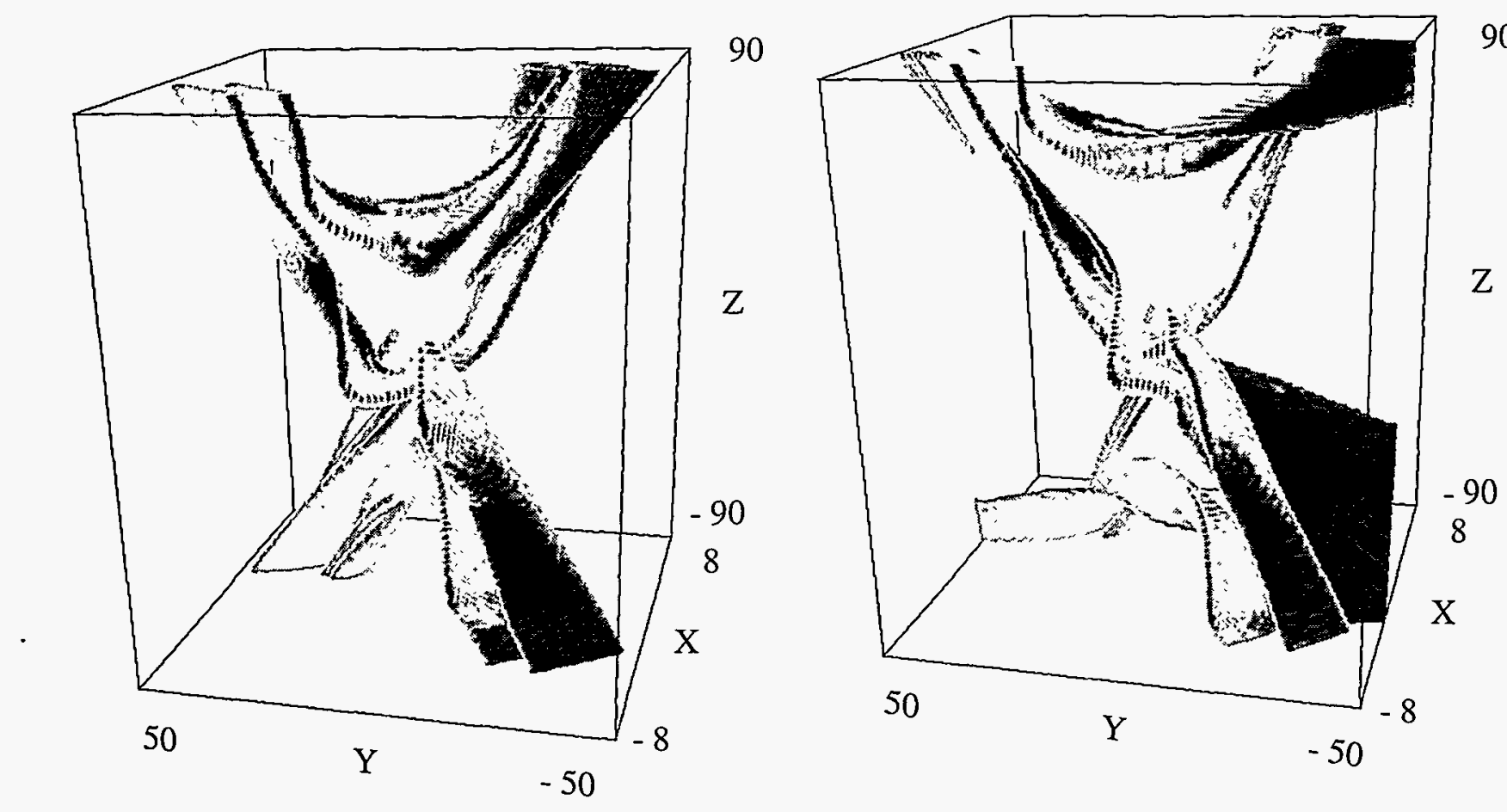

(A) Shown is a perspective view of the initial formation of interlinked magnetic flux tubes. The initial current layer is located in the plane $x=0$, and the $x$-axis points toward the sun. While the uppermost and lowermost flux tubes can move away from the center of the shown system, the motion of the interlinked flux tubes is restricted by their linkage. (B) Shown is the same view as (A), at a later time. An additional magnetic flux tube winds through the interlinked tubes. This flux tube is formed by strong magnetic reconnection at the interface of the two interlinked magnetic flux tubes. 
netosphere is the structure and the location of the accelerated flow relative to the major magnetic field transition region. At the dayside, the magnetic field reversal occurs sunward of the accelerated flow, and, at the flanks, the magnetic field transition region is equivalent to the region with the accelerated flow. Although flank observations of accelerated plasma have been interpreted in terms of magnetic reconnection, the lack of explanation for the different structure made this interpretation questionable.

In a series of resistive MHD simulations, we have demonstrated that the fast shear flow of the solar wind at the flanks of the magnetosphere modifies the reconnection structure and thus explains the observed differences. For symmerric current sheets without shear flow, plasma is accelerated by the $\mathrm{J} \times \mathrm{B}$ forces at two slow shocks that bound the outflow regions. In the case of asymmetric densities, the larger inertia on one side requires a larger $\mathrm{J} \times \mathrm{B}$ force and therefore a larger current on one side of the accelerated plasma. This explains the basic magnetopause and boundary layer structure at the dayside magnetosphere. A fast magnetosheath velocity in the direction of the accelerated plasma requires a smaller acceleration and thus smaller $\mathrm{J} \times \mathrm{B}$ force and current. Thus, the effect of a fast shear flow competes with the effect of the densiry asymmetry, and explains in terms of basic plasma physics the observed structure of the field reversal and the accelerated flow region flanking the magnetosphere.

Our results also predict that the two effects lead to a different structure of the boundary layer (acceler-
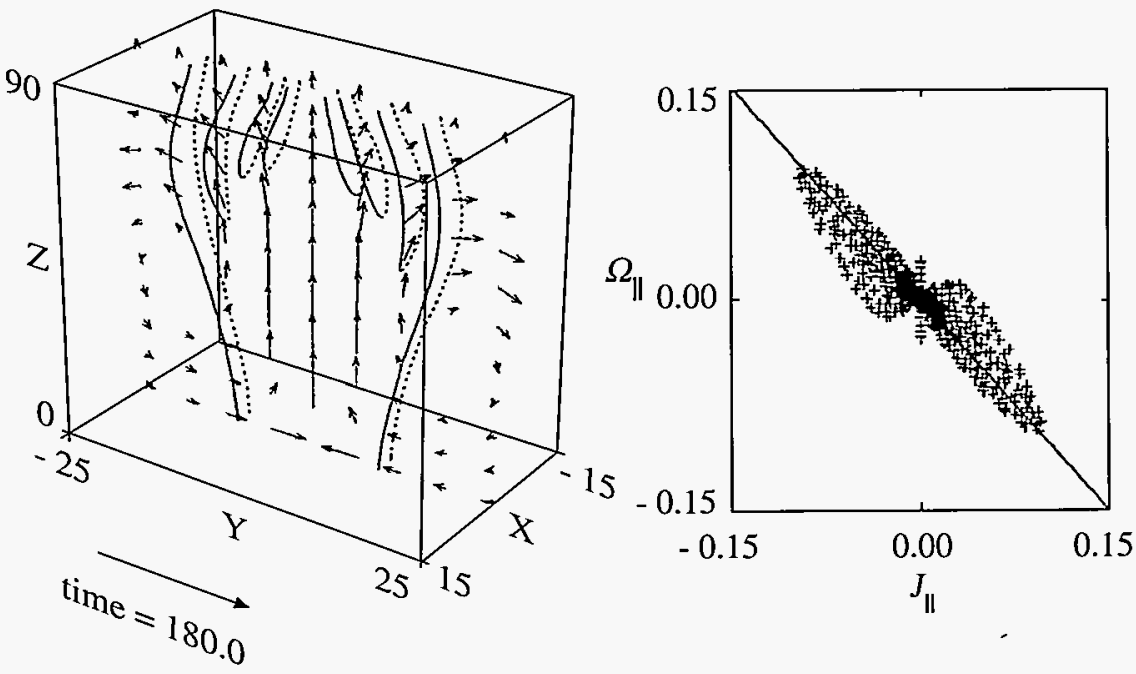

(A) A perspective view of magnetic field lines (solid and dashed) and velocity arrows for the three-dimensional magnetic reconnection process is shown. Reconnection occurs at the center of the lower boundary of the box. The $x$-direction points towards the sun. (B) $A$ test of the Walen relation for the field-aligned components of the current density and the vorticity is shown. The linear relation is consistent with field-aligned currents carried by Alfvén waves.

ated plasma) on the magnetospheric side for sunward acceleration (i.e. sunward of the reconnection site) at the flanks of the magnetosphere. The results apply not only to steady-state reconnection but also qualitatively to the bulge region at the leading edge of the reconnection geometry.

NASA Grant NSPTP-NAG51504: Scientific personnel-A. Otto, L. C. Lee, and A. La Belle-Hamer

Generation of Field-aligned Currents and Alfvén Waves by 3-D Magnetic Reconnection: It is known that strictly two-dimensional magnetic reconnection between antiparallel magnetic fields cannor generate fieldaligned currents (FACs). Thus, either a component of the magnetic field in the third dimension or threedimensional magnetic reconnection is required to generate FACs. Generation mechanisms of FACs are of central importance for the magnetosphere-ionosphere coupling.

We have carried out a three-di- mensional compressible MHD simulation to study the generation of fieldaligned currents (FACs) and Alfvén waves by magnetic reconnection for locally antiparallel magnetic fields across a current sheet. The initial state does not contain FACs. The results indicate that both FACs and Alfvén waves are generated by the 3$\mathrm{D}$ reconnection process. Two pairs of FACs are generated on each side of the current sheet. The polarities of the resulting FAC pair in the leading bulge region are opposite to those of a FAC pair in the trailing quasisteady region. It was further found that a large portion of the FACs $(\sim 40$ percent) are located in the closed field-line region. An examination of the Walén relation for the FACs and the parallel vorticity illustrate that Alfvén waves are generated and propagate away from the reconnection site.

Different from the 2-D process, three-dimensional reconnection generates a strong converging plasma 
flow along the third dimension into the reconnection region, and similarly an expanding flow in the reconnection bulge. The shearing motion of these flows generates the FACs that are not present in the corresponding 2-D process. These results provide an explanation for the observed Region 1 FACs at noon.

NASA Grant NSPTP-NAG51504: Scientific personnel-L.C. Lee, A. Otto, and Z.W. Ma

Magnetic Transients at the Dayside Magnetopause: Many models for the formation of transient events (magnetic flux transfer events) at the dayside magnetopause are based on magnetic reconnection. An alternative explanation of these events is a wavy magnetopause motion caused by solar wind pressure variations. To distinguish between the two mechanisms is of key importance for the global magnetospheric dynamics.

In comparing the two suggested processes, we have provided a detailed study of properties and the expected observational signatures of the two processes. The basic results are as follows: Based only on magnetic signatures, a unique identification of individual events is not possible, although pressure variations tend to generate weaker and more monopolar signatures of the magnetic field component $B_{N}$ perpendicular to the magnetopause. However, additional plasma data provides a multitude of properties that can be used to distinguish between a wavy magnetopause motion and magnetic reconnection.

All amplitudes, including magnetopause motion, magnetic field signatures, and total pressure of a wavy magnetopause motion, are proportional to the initial pressure variation, and therefore related. This motion can be generated by an increase or a decrease of the total pressure. Magnetic flux ropes that are generated by magnetic reconnection always imply an increase of the total pressure. Magnetic reconnection also leads to a strong plasma acceleration, while the plasma acceleration for boundary waves is usually small and proportional to the amplitude of the fast wave (e.g. determined from the total pressure variation). The $B_{N}$ signatures for magnetic reconnection are bipolar with a $+/$ - polarity for northward-moving and $\mathrm{a}-/$ - polarity for southward-moving structures. The dominant $B_{N}$ signature of boundary waves with a total pressure increase is positive (negative) for northward (southward) motion on the magnetospheric side. It is reversed for a decrease in total pressure. The magnetosheath signature of a boundary wave depends, in addition, on the IMF orientation.

We also have demonstrated that the compression and modulation of the magnetopause by a solar wind pressure increase can initiate magnetic reconnection, thus explaining several corresponding observations. The detailed summary of properties and signatures can guide observations of transient events to identify the underlying physical mechanism.

NASA Grant NSPTP-NAG51504: Scientific personnel-A. Otto, L.C. Lee, and Z.W. Ma

Kinetic Alfvén Waves as a Source of Plasma Transport at the Dayside Magnetopause: The nature of plasma transport across the earth's magnetopause is one of the most important problems in the study of the solar wind-magnetosphere coupling. As the shocked solar wind with variable plasma density and magnetic field impinges on the dayside magnetopause, it is likely to generate largescale Alfvén waves at the solar wind-magnetospheric interface. However, large gradients in the density and magnetic field at the magnetopause boundary effectively couple large-scale Alfvén waves with kinetic Alfvén waves.

In this study, we propose that the wave power converted into kinetic Alfvén waves may play an important role in plasma transport at the dayside magnetopause and in the electron acceleration along field lines. The transport can occur because, unlike the MHD shear Alfvén wave, the kineric Alfvén wave has an associated parallel electric field that breaks down the frozen-in condition and decouples the plasma from field lines. We calculated the deviation of the plasma from the field line, from which we estimate the diffusion coefficient associated with these bundles of decoupled plasma to be approximately $10^{9} \mathrm{~m}^{2} / \mathrm{s}$.

The plasma transport by kinetic Alfvén waves was found to be more efficient than that by waves with higher frequency, such as the ion cyclotron waves or lower-hybrid waves. The parallel electric field also may lead to acceleration of electrons along field lines in the magnetopause boundary, and may possibly provide an explanation for observed counterstreaming electron beams characterized by energies of 50 $200 \mathrm{eV}$.

NASA GrantNSPTP-NAG5-1504 and NSF Grant ATM9111509: Scientific personnel-L.C. Lee, J.R. Johnson, and Z.W. Ma 


\section{Ionospheric Signatures of Dayside Boundary Layer Processes =}

Ionospheric Signatures and Magnetospheric Cusp and Boundary Layer Processes in the Eastern Arctic: The project's goal is to relate the energetic particle observations made by the Defense Meteorological Satellite Program (DMSP) F6 and F7 satellites to ground-based optical observations of the aurora by using simultaneous DMSP photographs.

Dayside auroral forms near 10 hour Magnetic Local Time (MLT) in the northern hemisphere were observed simultaneously by the DMSP F6 and F7 satellites and by optical instruments at Longyearbyen, Svalbard $\left(78.108^{\circ} \mathrm{N}, 15.405^{\circ} \mathrm{E}\right)$. A number of passes in December 1983 were selecred from a larger suite of ground and space data to identify how the auroral forms observed in the optical data relate to the particle data and, hence, to the magnetospheric region of origin of the particle precipitation. The energetic particle boundaries detected by DMSP satellites were compared to auroral forms in the DMSP photographs.

The mean error between the DMSP particle data and the DMSP photographs, after correction for observing geometry, is approximately $17 \mathrm{~s}$ or $100 \mathrm{~km}$. Within this margin of error, the auroral forms identified in the DMSP photographs were all found to lie within the various magnetospheric boundaries identified using the particle data. The magnetospheric boundaries identified in the orbital plane were extrapolated along auroral forms in the photographs to the observatory on Svalbard. The majority of structured forms observed in the premagnetic noon region are associated with the magnetospheric low-latitude boundary layer precipitation. Other types of luminosity observed agree well with the relative energy deposition, according to the satellite.

December 9 and 10, 1983, were of particular interest because the $B_{z}$ component of the IMF $B_{z}<0$ was positive for a long period. The mantle aurora is seldom observed by the Longyearbyen meridian-scanning photometer (MSP). The long $B_{z}>0$ condition allowed discrete arcs to form and move northward from the mantle into the polar cap. An electron flux with a unique energy spectrum, which is of particular interest, is associated with discrete arcs poleward of the low-altitude boundary layer (LLBL) precipitation region. These arcs are found in the mantle region during IMF $B_{z}<0$ and expand into the polar cap region during periods of IMF $B_{z}>0$. Analysis of IMP8 (interplanetary monitoring platforms) magnetic field data suggests that al though polar cap arcs may be present at the time of a substorm onset, they appear as a result of the IMF $B_{z}>0$ conditions, rather than because they are a part of the auroral substorm process.

NSF Grant ATM92-24679: Scientific personnel-C.S. Deehr, R.W. Smith, J. Minow, M. Bierer, P. T. Newell, and C.-I. Meng

Ionospheric Signatures of the Patchy and Intermittent Reconnection at the Dayside Magnetopause: Reconnection at the dayside magnetopause, between the IMF (interplanetary magnetic field) and the geomagnetic field, is believed to be enhanced as the IMF turns southward. Supporting evidence for this belief is the equatorward motion of the cusp region. The cusp region is believed to be on newly reconnected open field lines. Indeed, particle flux is observed to be about four times higher in the cusp region around magnetic noon than in the pre- and postnoon regions, which map to the low-latitude boundary layer. This is similar to the ration of particle flux observed in ISEE crossings from the magnetosheath into the boundary layer.

The thickness of the low-latitude boundary layer (LLBL) increases away from the subsolar point. The thickness of the LLBL also increases as the IMF $\mathrm{B}_{\mathrm{z}}$ increases (i.e., thicker for northward IMF). When the IMF turns southward, the thickness of the LLBL should decrease because the antisunward convecting closed field lines are removed from LLBL enhanced dayside reconnection. Thus, the outer layers of the LLBL are steadily peeled away by enhanced dayside reconnection to result in thinning of the LLBL.

The support for enhanced dayside reconnection due to a southward turning of the IMF has been well documented in the open literature. However, the mode of enhanced dayside reconnection remains a controversial issue. The debate is centered on whether the dayside reconnection is spatially patchy and temporally uniform or spatially uniform and temporally bursty.

In this study, we outline the ionospheric signatures predicted by the patchy-intermittent (P-I) dayside re- 
connection model. Our model shows that the patchy reconnection generates spiky convection electric fields. The spiky convection electric field, in turn, drives the enhanced convection channel on closed field lines in the dayside ionosphere. The observed convection speed in the enhanced convection channel ranges from $\sim 0.5$ to $\sim 2 \mathrm{~km} / \mathrm{s}$. Our model predicts that the dayside auroral forms should move noonward and poleward along each enhanced convection channel in the low-latitude boundary layer. Multiple brightenings of dayside auroral forms should occur on a time scale of about two to four minutes, which is equal to twice the Alfvén bounce period between the ionosphere and the equatorial plane. Patchy reconnection is intrinsically intermittent due to the reconnection of elbow-shaped open flux tubes. The resulting reclosed flux tubes should contain a mixture of magnetosheathmagnetospheric plasmas as observed in the low-latitude boundary layer.

NSFGrantATM-8912359: Scientificpersonnel-J.R.Kan, C.S.Deehr, and L.H. Lyu (Institute of Space Science, National Central University, Chung-Li, Taiwan, ROC)

Studies of Dayside Auroral Dynamics: The Longyearbyen Optical Station $\left(78.108^{\circ} \mathrm{N}, 15.405^{\circ} \mathrm{E}\right)$ in the Svalbard Archipelago north of Norway has provided many years of observations of the dayside aurora using an all-sky-television, meridian-scanning photometers, and grating spectrographs. In recent years, we have made a comprehensive study of poleward-moving transient auroral forms that are typical of dayside aurora observed from very high lati- tudes but are not seen in the nightside aurora observable from Alaska. A search of available data has revealed more than 750 events occurring at different times and under different conditions. Most of these events are recorded on the all-sky television as well as by the meridianscanning photometer.

From early papers in 1975 to the present, it has been presumed that these events are associated with the influx into the magnetosphere of energy from the solar wind. The process of coupling of energy appeared to be discontinuous. It was imagined early on that events were occurring at the boundary of the magnetosphere, in which bursts of solar wind particles could gain access to geomagnetic field lines and precipitate into the atmosphere, causing aurora. Later identification of events like this by satellite-borne sensors gave rise to the term "flux-transfer event" for these phenomena.

The first publications on the dayside auroral transients were based on photographs taken at one-minute intervals in white light. With more modern techniques and better time resolution, it has been possible to show that most of these events occur with multiple brightenings, indicating that the source is able to re-energize the magnetic field line after the initiation of the event. Also, it appears that in the period of two hours, either side of geomagnetic noon, there is a predominance of emission in the $6300 \AA$ $O\left({ }^{1} D\right)$ line, indicating a soft electron flux. The importance of the study of multiple brightening events lies in the implications on the mechanism of their production. There are three types of events: one in which there is only one brightening; a second where there are two or more brightenings and continuous poleward movement; and a third where after a rebrightening, the auroral form remains bright and stationary. The average lifetime for the whole event is five minutes. For rebrightening events, the interval between first and second brightenings is about 2.2 minutes.

If further rebrightenings occur, they are spaced by shorter times. Eighty-two percent of all type-two events have two brightenings and about 18 percent have three. Typetwo events are more common (79 percent) than type-one events in the period of four hours surrounding geomagnetic noon. However, they are less common (41 percent) outside that period. Type-three events are relatively uncommon.

These observations suggest a mechanism that is able to recharge the magnetic flux tube several times. Possible contenders are the multiple $\mathrm{X}$-line reconnection mechanism (Lee et al., 1993) and the patchy reconnection mechanism of Kan (1988).

NSF Grant ATM93-02610: R.W. Smith, C.S. Deehr, L.C. Lee, K. Henriksen (University of Tromsö, Norway), G. Fasel, and J. Minow

Sounding of the Cleft Ion Fountain Energization Region: The goal of this project is to identify the ionospheric signature of the magnetospheric cusp in real time, so that a rocket payload can be flown over the region of the signature and measure the plasma processes associated with the upward acceleration of heavy ions that have been observed at higher altitudes from satellites. After we established that the greatest flux of 
energetic ions was associated with the presumed location of the magnetospheric cusp, and that the discrete arcs observed on the dayside are probably in the low-latitude boundary layer, we concluded that the best ionospheric indicator of the maximum ion outflow is the $6300 \AA$ / $5577 \AA$ emission ratio maximum associated with the signature of the magnetospheric cusp.
This event occurs over Svalbard for approximately an hour around 0830 UT, which is one hour behind local time there. A number of instruments will be employed to establish the ionospheric conditions, but the main strategy will be to look eastward for the arrival of the high $6300 \AA / 5577 \AA$ emission ratio region in the dayside aurora and time its approach to meet the rocket to be launched from Andoya Rocket Range in January 1995.

NASA Grant NAG5-693: Scientific personnel-C.S. Deebr, R.W. Smith, M. Conde, H.C. StenbaekNielsen, J.V. Olson, D. Lorenten, J. Minow, P. Kintner (PI-Cornell University), R. Arnoldy (University of New Hampshire), C. Pollack, T. Moore (MSFC), A. Egeland, and J. Holtet (University of Oslo)

\section{Magnetospheric Dynamics}

Particle-In-Cell Electromagnetic Codes in Complex Geometries: The project objective is to develop a series of codes to simulate the performance of high-power microwave and other plasma devices. The codes will be used in the design of such devices. A major design objective is to gain maximum efficiency in the conversion of beam electron energy to microwave energy extracted from the device. The device consists of an interaction chamber, resonant cavities and an extractor. The waves are generated by relativistic electrons gyrating in a strong magnetic field. Design is a trial and error process. A design is conceived, the cavities and chambers are machined, and then performance is painstakingly measured. The design cycle would be considerably shortened if it were possible to simulate performance of the various designs numerically. Changing the parameters in a code is much easier than machining the cavities. A numerical simulation is subject to infinite diagnostics, making performance evaluation much easier. Moreover, standard visualization packages can be used to display physical processes occurring in the device graphically, thus contributing to insight for improved design.

Particle-in-cell (PIC) electromagnetic codes are being developed for a generalized curvilinear coordinate system in which coordinate surfaces conform to boundary surfaces. The code employs an exactly conservative current algorithm so the code is entirely explicit. This lends the code to efficient implementation on massively parallel computer architectures. The boundary conditions will be modularized for flexibility. This also will facilitate the use of multiple coordinate patches. The use of multiple coordinate patches and modularized boundary conditions is necessary to simulate devices consisting of multiple chambers. These techniques will later be applied to the simulation of plasma torches using hybrid codes.

This project has relatively little to do with geophysics, but does use computer algorithms developed for global-scale modeling of the earth's magnetosphere.

AFOSR Grant F49620-94-1-0218: Scientific personnel-D.W. Swift
One-Dimensional Vlasov Structure and Disruption of Thin Current Sheet in the Magnetotail: The dynamics of current sheets was studied using one-dimensional (1-D) hybrid simulations. The particles are assumed to have initially a biMaxwellian distribution, which satisfies the marginal firehose stability criterion and a force balance condition in the direction perpendicular to the current sheet. Stable 1-D current sheets can be found in the anisotropic plasma provided that the ions are magnetized in a relatively thick current layer.

However, unstable thin current sheets evolve with a significant energy conversion from magnetic energy to plasma thermal and flow energy. In the unstable cases, the plasma is nonadiabatically heated in the direction perpendicular to the magnetic field. As a result, the particle distribution becomes more isotropic in the central region of the current sheet, leading to force imbalance and an earthward plasma flow. It also was found that a finite initial $B_{y}$ component can lead to magnetization of ions and stablize the 
current sheet. The nonadiabatic relaxation of the thin current sheet provides an effective magnetic energy dissipation in collisionless plasmas. The near-earth current sheet disruption and the earthward highspeed flow events observed in the mag-netotail also may be the result of thinning and the subsequent rapid evolution of the embedded current sheet without the presence of magnetic reconnection.

NSF GrantATM91-11509: Scientific personnel-L.C. Lee, H. Cai, and $Y$. Lin

Generation of New Magnetic Flux Near a Magnetic O-Line in Collisionless Reconnection: In a full-particle simulation of magnetic reconnection in a thin current sheet with electron gyroradius scale, we found the presence of a new dynamo process, in which a large amount of new magnetic flux near the magnetic O-line is generated associated with the compression of particles during magnetic reconnection. For the cases we have studied, the newly generated magnetic flux is about $\rho_{e} B_{0}$, where $B_{0}$ is the magnetic field far away from the current sheet, and $\rho_{e}$ is the electron gyroradius in terms of $B_{0}$ and the electron thermal velocity.

In the MHD model, Ohm's law is written as $E+v \times B=\eta J$, where $\eta$ is the resistivity. Near the magnetic O-line, B nearly vanishes and hence the electric field $\mathrm{E}$ and the current density $\mathrm{J}$ are in the same direction. No dynamo process, which requires $\mathrm{E} \cdot \mathrm{J}<0$, is allowed at the O-line in the MHD model. However, a dynamo process near the O-line can occur in collisionless plasmas. The momentum transport due to the off- diagonal elements of the plasma pressure tensor can lead to $\mathrm{E} \cdot \mathrm{J}<0$ near the magnetic $\mathrm{O}$-line.

If electrons can be supplied in the third dimension and hence the electrons play a role as a neutralized background, the reconnection process is mainly determined by ion dynamics. Therefore, the newly generated magnetic flux can be as large as $\rho_{i} B_{0}$, where $\rho_{i}$ is the ion gyroradius. The dynamo process near the O-line may lead to observable effects for the flux transfer events (FTEs) in the magnetopause and for the plasmoid formation in the magnetotail.

DOE Grant DE-FG06-91ER 13530: Scientificpersonnel-L.C. Lee and $\mathrm{H}$. Cai

Development of a Global-scale Hybrid Code of the Earth's Magnetosphere: This project is an ongoing effort to develop a numerical model of the coupled magnetosheath, magnetosphere and ionosphere. The ultimate objective of this project is a capability of predicting space weather based primarily on knowledge of the interplanetary magnetic field and plasma velocity and density in the upstream solar wind.

The project is built around a hybrid code. The hybrid code follows the exact motion of the ions and treats the electrons as a massless fluid in the self-consistent magnetic field. In a full particle code, considerations of computational stability require too many grid points, too many particles and too many time steps to be feasible for global-scale simulation. The hybrid code does not have these constraints, so time steps and distances between grid points more in keeping with the problem can be used. The advantage of the hybrid code over the magnetohydrodynamic code, more traditionally used for large-scale simulations, is that the hybrid code retains full ion kinetics.

Three innovative features have been incorporated into the code making it particularly suited for the simulation of the magnetosphere. One of them is the use of a generalized curvilinear coordinate system that can be specified as a table giving the absolute location of the grid points. This flexibility in gridding allows placement of a high density of grid points where high spatial resolution is needed, such as in the auroral ionosphere and magnetopause. Alow density of grid points can be used in the vast regions of the magnetotail lobes. Another innovation is the use of a fluid model for the cold, dense plasma inside the magnetopause. This plasma exhibits no kinetic behavior, so considerable computational savings are realized by using the fluid approximation in the near-earth regions. Another innovation is the subcycling of the magnetic field update to the particle push. Considerations of numerical stability require time steps short enough that a wave

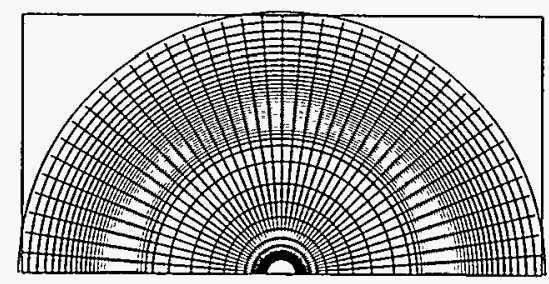

Shown is the coordinate system used in the simulation of the magnetic noon meridian plane. For clarity, only a third of the coordinate lines are displayed. The box extends to slightly more than 18RE from the center of the earth in the north, south and sunward directions. The region in the corners not covered by the grid lines is outside the simulation domain. 


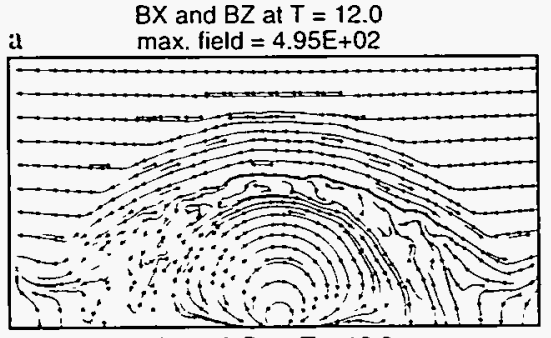

$\log$ of $B$ at $T=12.0$

b Contour from -3.359 to 2.291 by .403

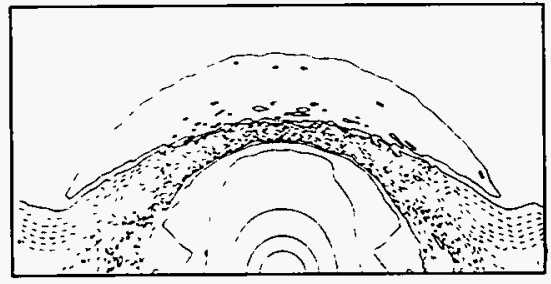

By contours at $\mathrm{T}=12.0$ c Contour from -2.401 to 2.154 by .455
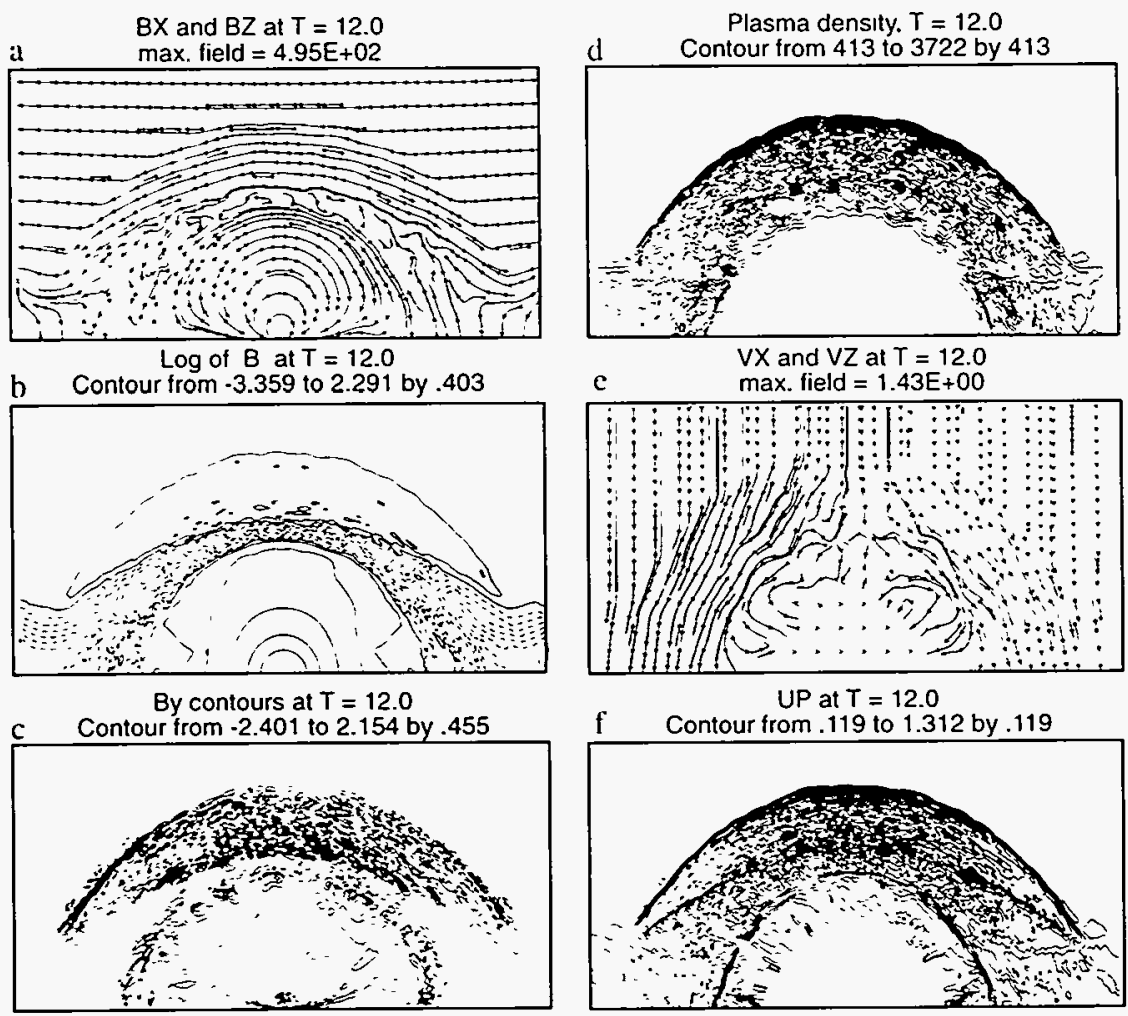

$\mathrm{UP}$ at $\mathrm{T}=12.0$

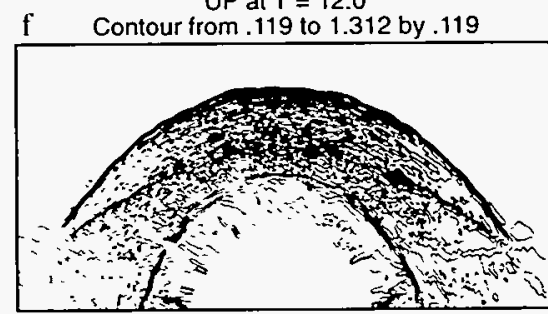

Magnetic field vectors (a), logarithm of the magnetic field intensity (b), the $y$-component of the magnetic field $(c)$, the plasma density $(d)$, the plasma flow vectors $(e)$, and the magnitude of the plasma flow speed $(f)$. Figure below is the same as the one above, except at the end of the run.

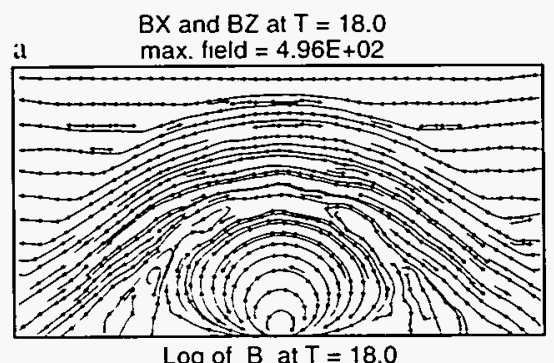

b Contour from -1.315 to 2.428 by .267

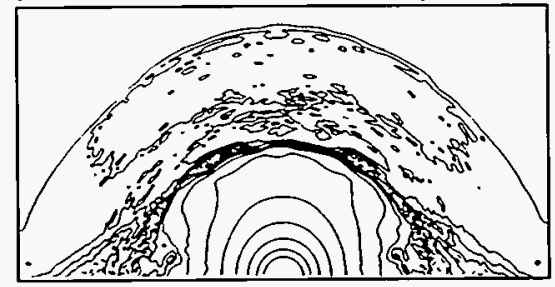

By contours at $T=18.0$

c Contour from -6.142 to 6.932 by 1.307

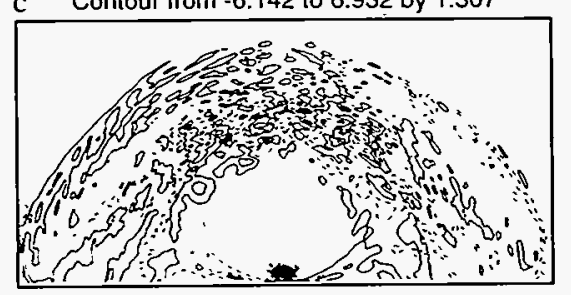

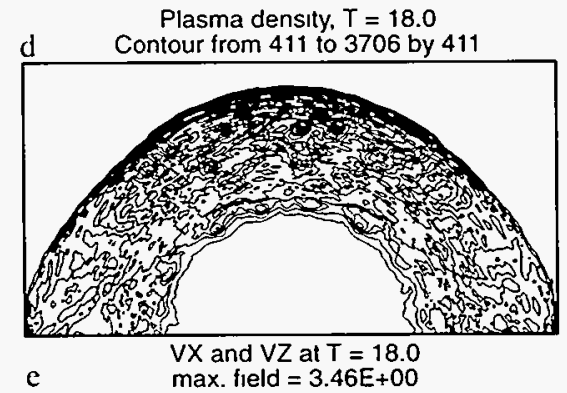

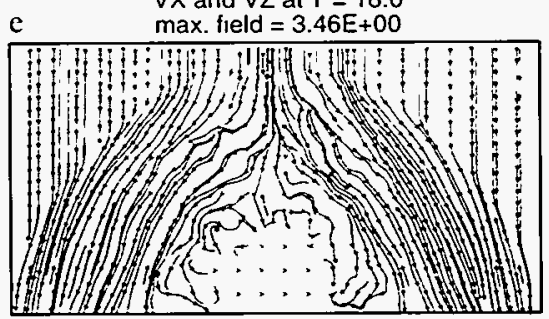

UP at $\mathrm{T}=18.0$

f Contour from .304 to 3.347 by .304

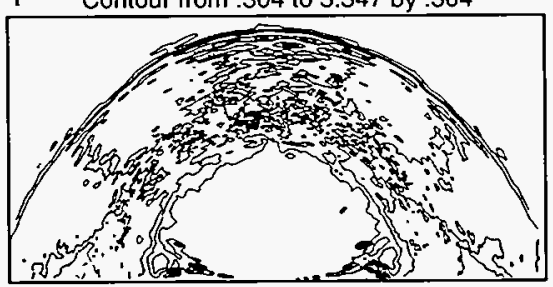

supported by the simulation cannot propagate across a grid cell in a time step. In the near-earth regions, wave phase velocities are high because of the strong magnetic field. The timesubcycling algorithm updates the magnetic field in short time steps, while the particle update, which is computationally more intensive, is updated with longer time steps.

A simulation incorporating these algorithms has been tested in a twodimensional model of the noon meridian plane of the earth's magnetosphere. The simulation follows the response of the magnetosphere to a southward turning of the interplanetary magnetic field (IMF). Figure 1 shows, for clarity of presentation, a small fraction of the $121 \times 123$ grid used in the simulation. The inner circle represents the ionospheric boundary, which is assumed rigid and non-conducting. Note the higher grid density at about 10 earth radii $\left(R_{e}\right)$ to capture the magnetopause and bow shock. Initially, the solar wind is assumed uniform, and there is no IMF inside 18 $R_{e}$. Beginning at $t=0$, a southward IMF is convected inward with the solar wind, ramping up to a constant value over a distance of $1 \mathrm{R}_{\mathrm{e}}$.

The top figure displays results of the simulation shortly after the southward IMF encountered the subsolar magnetopause. The sun is toward the top of the figures. Panel (a) shows the magnetic field vectors, which indicates tearing going on at the magnetopause. The bow shock also can be seen in the figure as the sharp deflection of the field lines. The magnitude of the field on a logarithmic scale is shown in panel (b). As can be seen in succeeding panels, the solid 
crescent-shaped contour in the upstream region shows the locations of both fast and slow shock fronts. Panel (c) shows the $y$-component of the magnetic field. The little islands near the magnetopause provide further evidence of tearing at the magnetopause. Note the contours extending from the cusp region to the highlatitude ionosphere. This is significant in that it indicates the presence of high-latitude field-aligned currents. These currents appear early in the simulation, so they do not depend on the presence of the IMF. They are likely due to differential motion between ions and electrons due to demagnetization of the ions in the cusp region. Panel (d) shows the density of the kinetic particles. Note the density pileup behind the fast shock, and the clumping and plasma blobs intruding into the magnetopause. Panel (e) shows the plasma flow vectors and the flow deflection at the location of the fast and slow shocks. Finally, panel ( $f$ ) shows contours of the plasma flow magnitude. Again, note the sharp velocity decrease at the bow shock and the plasma flow jump at the slow shock.

The lower figure shows the plasma and field configuration at the end of the run. The shock continues to expand because in two dimensions there is no way for magnetic field lines to pass around the magnetosphere, in the absence of greatly enhanced reconnection. Panel (a) shows a much thinner magnetopause and the appearance of a cusp in the polar regions, while panel (b) shows reconnected field lines. Panel (c) again shows field-aligned currents between the cusp and polar ionosphere. Panel (d) shows the discrete particle den- sity. Notice the plasma clumping at the low-latitude magnetopause, indicative of magnetic island formation from tearing. Panels (e) and ( $\mathrm{f}$ ) show the penetration and acceleration of ions into the cusp. The accelerated particles appear late in the simulation after reconnection has taken place, so it is likely they were accelerated as a result of reconnection. The slow shock has all but disappeared.

The simulation has exhibited, with considerable clarity, the fast and slow mode shocks and tearing at the magnetopause. In addition, it has exhibited two new effects not heretofore seen in a global-scale simulation, namely the currents linking the cusp and ionosphere and particle acceleration in the cusp. The next step is to expand the model to a full three dimensions to encompass the entire magnetosphere. In so doing, the code will have to be moved onto the $\mathrm{T} 3 \mathrm{D}$, the massively parallel supercomputer at the Arctic Region Supercomputing Center. The twodimensional simulation is being used as a basis for attracting support for the full three-dimensional project. Meanwhile, there are student efforts to expand the two-dimensional model to cover the entire noon-tomidnight meridian plane.

Scientific personnel-D.W. Swift

\section{Magnetospheric and Ionospheric Substorms}

Second International Conference on Substorms (ICS 2): The Second International Conference on Substorms (ICS 2) was held on the UAF campus from March 7-11, 1994, with all sessions of the five-day conference meeting in the Salisbury Fine Arts Theater. The Regents Great Hall was used throughout the week for poster sessions and all related activities. Total registration for the conference was 168, with representation from 11 countries: the United States, Canada, Sweden, Norway, Finland, Germany, France, England, the Netherlands, Russia and Japan. Attendance was comparable to that of ICS-1, which was held two years earlier in Kiruna, Sweden.

The number of papers presented was 182, and the official proceedings of the conference comprise a total of
87 papers. In addition to the highly successful scientific portion of the meeting, UAF also demonstrated to an international audience its ability to host such large conferences.

In recognition of our contribution to the community, the Fairbanks Convention and Visitors Bureau acknowledged the ICS-2 conference as the "Meeting of the Month" for March 1994. The meeting's conveners were J. R. Kan and S.-I Akasofu, and the local arrangements were organized by K. Berry, J. D. Craven (chair), J. Hume, D. W. Swift and D. R. Weimer.

NASA Grant NAGW 3663, NSF Grant ATM93-12667, and UA Natural Resource Funds: Scientific personnel-S.-I. Akasofu, J.R. Kan, J.D. Craven, D.R. Weimer, and D.W. Swift 
Development of the Substorm Current System in the Magnetosphere: The average pattern of the electric potential, current vectors and field-aligned currents in the ionosphere for a quiet time, the growth phase, the expansion phase, the peak period and the recovery phase of substorms were mapped on the equatorial plane of the magnetosphere by considering radial and azimuthal loop currents.

An improved Tsyganenko magnerospheric model was used for mapping. The radial component is in agreement with the statistical results from the AMPTE/CCE satellite. This result was applied in terms of the source of the ionospheric Pedersen currents and parts of the region-1 and region-2 field-aligned currents. The azimuthal component has an eastward component in the night sector, where the AMPTE/CCE satellite found a westward current. We show that their results can be explained as a combined effect of the cross-tail current, the ring current and a weak substorm wedge current.

Therefore, both our ground-based results and the AMPTE/CCE satellitebased results are in good agreement for the radial component and not inconsistent with each other for the azimuthal component, in spite of the fact that they are obtained by exrremely different methods.

These results prove also the presence of Bostrom's loop currents. The results suggest that an extensive ground-based network of magnetic observatories can monitor the loop currents in the equatorial plane, as well as the ionospheric currents and the field-aligned currents, on a continuous basis.

NSF Grant ATM93-11474:
Scientific personnel-W. Sun, S.-I. Akasofu, and Y. Kamide (Nagoya University Solar-Terrestrial Environment Laboratory)

Substorm Currents in the Equatorial Magnetotail: Characteristics of magnetospheric equatorial currents during substorms are determined from the vector magnetic field data acquired with the GOES 5 and GOES 6 satellites, separated by about 1.9 hours in MLT in geosynchronous orbit. These data have been used to determine the local time (azimuthal) and radial variation of the equatorial current. The divergence of the equatorial current was computed from these variations, and systems of field-aligned currents were deduced.

During the growth phase to the maximum phase of the tail-like reconfiguration of the near-earth magnetic field, a positive divergence (away from the equatorial plane) of the westward equatorial current occurs in the late evening to premidnight MLT sector, and a negative divergence (away from the equatorial plane) occurs in the premidnight to early morning MLT sector. The field-aligned current associated with these divergences flows into the ionosphere in the late evening to premidnight MLT sector and flows away from the ionosphere in the premidnight to early morning MLT sector. This flow direction pattern is the same as that of the region- 2 fieldaligned current system. During the expansion phase, a field-aligned current that is distinctive to the growthphase field-aligned current is generated in the same near-earth plasmasheet region. The field-aligned current flows away from the ionosphere in the late evening to premidnight MLT sector and flows into the ionosphere in the premidnight to morning MLT sector.

These field-aligned currents are due to a change in a sign of the divergence of the westward equatorial current. This flow direction pattern is the same as that of the region-1 fieldaligned current system and of the current-wedge model. This region-1 sense field-aligned current develops first near midnight at about five minutes after the expansion phase onset (as determined from the groundbased magnetometer data) and is delayed by 10 to 25 minutes farther away from midnight in the evening and morning MLTs, and continues until the end of geomagnetic dipolarization at the site of either GOES 5 or GOES 6, whichever is located closer to midnight.

We also have determined the presence of a radial current that flows toward the earth in the late evening to premidnight sector and flows away from the earth in the midnight to morning sector. The intensity of the radial currents increases before the expansion phase. Consequently, the patterns of field-aligned currents associated with various substorm phases are the superposition of currents driven by multiple sources with different temporal variations.

We have identified at least three different but related sources of fieldaligned currents during the growth and expansion phases. These sources are related to the divergence of the westward-flowing equatorial current and to distributions of pressure and magnetic field gradients that evolve in the magnetotail. These patterns 
include the current-wedge model during the expansion phase. When combined, these complicated systems support the basic region-1 to region2 field-aligned current flow pattern.

NSF Grant ATM93-11474: Scientific personnel-T. Iijima and M. Watanabe (University of Tokyo), T.A. Potemra and L.J. Zanetti (Johns Hopkins University), J.R. Kan and S.-I. Akasofu

Ground-based Observations of the Auroral Substorm: A combination of all-sky imagers (ASTV) and meridian-scanning photometers (MSP) was used to identify the optical signature of the growth phase, onset, expansion, and recovery of simultaneous events on the dayside from Longyearbyen, Svalbard. In the growth phase, segments of luminosity move at convection velocity $(1.5 \mathrm{~km} / \mathrm{s})$ westward along existing arcs and pulsate in the $\mathrm{MHz}$ frequency band superimposed on the westward movement. Ten minutes before the development of the small-scale arc structure associated with onset, the $6300 \AA$ emission begins to brighten, perhaps indicating a sudden increase in Fregion electron temperature. Atonset, the first low-energy, electron-induced arc poleward of the trapping boundary develops curls, brightens, and expands poleward a few minutes after the poleward expansion of the discrete electron arcs (westward traveling surge), but only after 21 hours MLT.

In the recovery phase, as in the expansion phase, the hydrogen emission generally avoids the discrete electron aurora and returns to the equatorward side after substorms before $01 \mathrm{hr}$ MLT as a part of the growth phase of the next substorm. After $01 \mathrm{hr}$ MLT, it remains on the poleward side of the main precipitation region, regardless of intensifications, for the rest of the night, but periodically invades the diffuse auroral region from the poleward side.

33 substorms in the

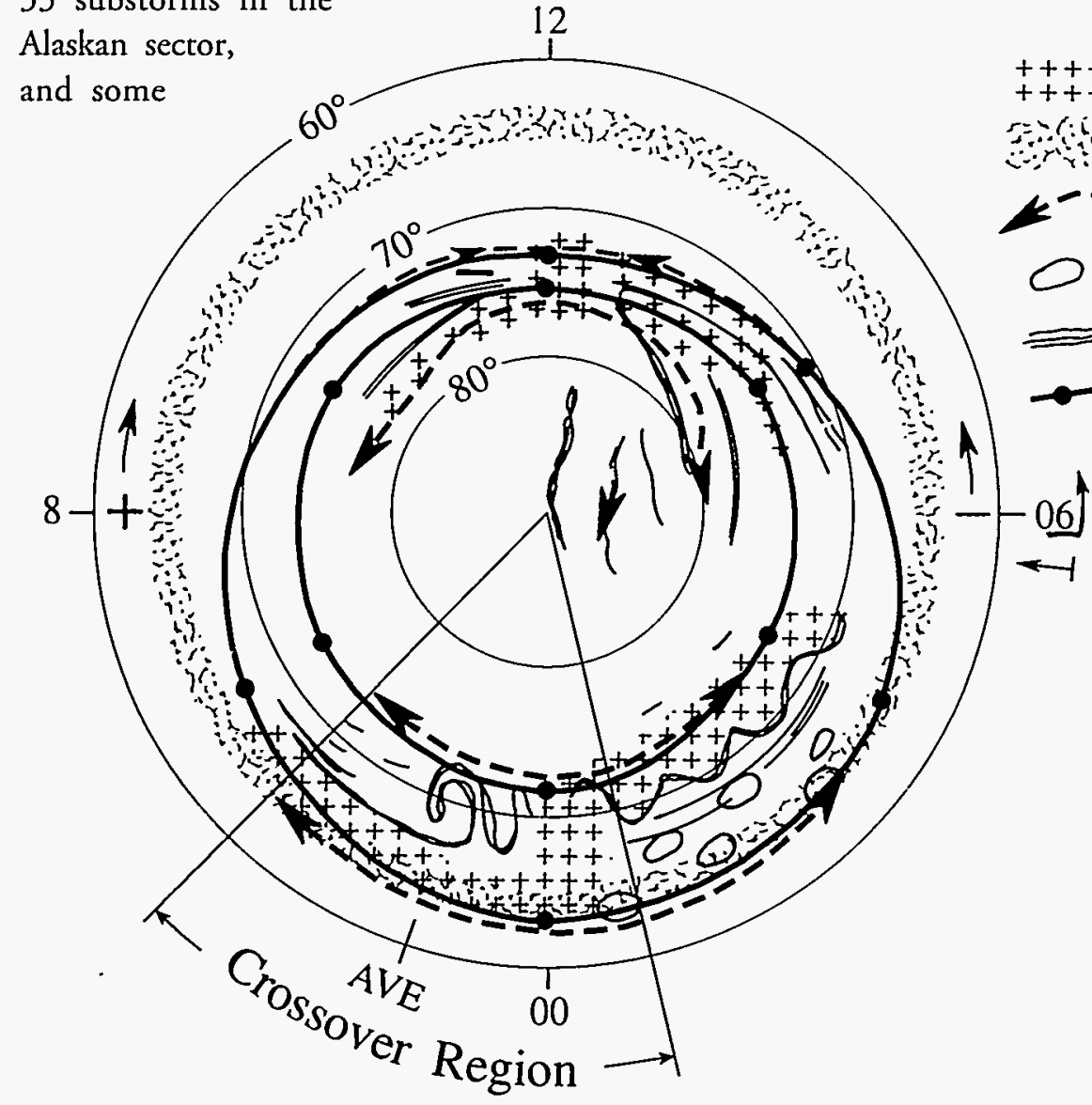

Shown above is a schematic view of the $\mathrm{H}$-and electron-induced aurora as a function of geomagnetic time and latitude.
Proton Aurora Diffuse Aurora Convection Motion Pulsating Aurora Discrete Arcs $Q=4$ Oval Geomagnetic Local Time Geomagnetic Latitude

For the 33 substorms observed from Poker Flat Research Range, the poleward crossover of precipitating protons occurs in a region between approximately $21 \mathrm{hr}$ and $01 \mathrm{hr}$ MLT, and is sharply peaked in occurrence frequency for an hour around $23 \mathrm{hr}$ and $01 \mathrm{hr}$ MLT. The average magnetic latitude at onset was 63.6 for protons and 64.6 for electrons. In general, the H-emissions and elec- 
tron-induced auroras are spatially and/or temporally separate, in a manner consistent with the hypothesis that they are indicators of downward and upward field-aligned currents, respectively.

NSF Grant ATM93-02610: Scientific personnel-C.S. Deehr, R.W. Smith, J.R. Kan, and B. Xu

Motion of the Auroral Bulge's Poleward Boundary in the Substorm Recovery Phase: There is general agreement that the large-scale features of the auroral substorm are readily observed with remote-imaging instruments on spacecraft. In particular, one can clearly observe the onset and expansion of the auroral bulge to some maximum size within the expansion phase, and one also can observe westward traveling surges along the oval in the evening sector, whether or not they actually travel.

Additional features later incorporated into descriptions of substorm phenomena are also observed, such as a growth phase following southward turnings of the interplanetary magnetic field (IMF), in which the diameter of the visible auroral oval expands prior to onser. Numerous additional, important details also are being provided by the auroral images on the Viking spacecraft. The recovery phase has not received the same level of attention as the seemingly more important earlier phases.

This work addresses the question of what happens in the recovery phase to the high-latitude poleward arcs of the auroral bulge. As originally defined, the recovery phase begins when auroral arcs along the poleward boundary of the auroral bulge end their poleward motion and begin an equatorward retreat. The beginning of this retreat is not necessarily immediate, for the poleward boundary may remain stationary at its most poleward location for up to 10 to 30 minutes before beginning its retreat toward lower latitudes.

Simultaneous observations used in this investigation are DE-1 auroral images of auroral substorms and ISEE measurements of the interplanetary magnetic field (IMF). Variations in orientation and magnitude of the IMF during optical observations are considered in order to remove or reduce confounding variations in the motion of the auroral features which could mask any dependence on the IMF's orientation.

It was found that in the recovery phase the poleward boundary of the expanded auroral bulge moves at a rate of $\sim 9.1 B_{z} \mathrm{~m} / \mathrm{s}$ in the evening sector near local midnight, where $B_{z}$ is the GSM $z$ component of the IMF, in nanoteslas. This motion is equatorward for $B_{z}<0$, and is generally consistent with the original qualitative description for such motions in the recovery phase, but the speeds measured here may be less than the speeds of individual arcs. The motion is poleward for $B_{z}>0$, where it becomes increasingly difficult to determine the motion during intervals of sustained magnetic quiescence.

Hence, the answer to the original question is in several parts. If $B_{z}<<$, the poleward boundary during recovery rapidly retreats equatorward toward latitudes of the primary auroral distribution. This is consistent with the early work. The rate of equator-ward motion decreases with increasing $B_{z}$. For $B_{z} \sim 0$, the poleward boundary remains at high latitudes and generally fades from detection. For $B_{z}>>0$, it appears that the highlatitude boundary becomes part of a developing contracted auroral oval.

NASA Grant NAGW 3441: Scientific personnel-J.D. Craven, L.A. Frank (University of lowa), and C.T. Russell(University of California, L.A.)

Unloading Instability Triggered by Alfvén Waves Disrupting the CrossTail Current in the Near-Earth Plasma Sheet: The concept of the substorm was first introduced by Akasofu [1964] in terms of a sequence of auroral morphological changes observed in the ionosphere. The auroral substorm morphology indicates that the substorm phenomenon is a global electrodynamic response of the coupled magnetosphere-ionosphere system to the dynamic forcing by the solar wind.

The substorm onset has been defined by the sudden brightening of the most equatorward aurora in the midnight sector. This was followed within a few minutes, by an explosive intensification and expansion of the auroral bulge. Occasionally, a substorm onset may not be followed by the expansion onset, which has been called the "pseudo" onset and has received some attention in recent years. The pseudo onset is initialed by brightening aurora at the substorm onset, but fails to develop fully into a typical substorm.

The purpose of the present study is to show that the explosive intensification of substorms can result from a system instability in the substorm wedge, called the unloading instability by Kan [1992]. The unloading instability is triggered by Alfvén waves 
reflected from the ionosphere and incident on the tail-like field region in the plasma sheet. This is different from the current instabilities which are triggered by wave-particle interactions, such as the cross-field current instability or the ballooning instability.

In this work, we first analyze the substorm phenomenology built upon extensive observational results in the literature. We then show how the unloading instability can lead the growth phase to the expansion phase of substorms. The unloading instability is characterized by amplifying Alfvén waves reflected from the taillike field region and by disrupting the intense cross-tail current at the same time. The energy for the unloading instability releases the stored energy by disrupting the cross-tail current which is -connected to the magnetopause current driven by the solar wind-magnetosphere dynamo located along the magnetotail.

The results of this study, suggest that the substorm expansion onset can be caused by the unloading instability in the substorm current wedge. The unloading instability is triggered by Alfvén waves from the ionosphere incident on the intense cross-tail current and dipolarizes the tail-like magnetic field. The induction electric field associated with the current disruption reinforces the dawn-to-dusk electric field to form a positive feedback loop in the current wedge. The expansion onset can result from the unloading instability if the magnetic field in the tail-like field region increases by more than 1.5 times its dipole-like field before undergoing tail-like reconfiguration. Energy for the unloading instability comes from the magnetic energy stored in the cross-tail current. Substorm onsets coincide or precede the expansion onset by less than 2 to 4 minutes, which is the round trip Alfvén transit time between the dipolarizing region and the ionosphere. Dipolarization in the nearearth plasma sheet should precede the expansion onset in the ionosphere be a one-way Alfvén transit time. An $\mathrm{X}$-line may form tailward of the dipolarization region as a consequence of the expansion onset.

NSF grant ATM-8912359: Scientific personnel-J.R. Kan, L.H. Lyu (Institute of Space Science, National Central University, Chung-Li, Taiwan, Republic of
China), and C.F. Kennel (University of California, Los Angeles)

Asymmetric Development of the Auroral Bulge During Substorm Expansion: The canonical model for the auroral substorm's expansion phase identifies in the midnight sector an expanding auroral bulge. This bulge is bounded at high latitudes by discrete arcs moving poleward and/or appearing at higher latitudes. The boundary's speed of poleward motion is measured at $100 \mathrm{~s}$ of $\mathrm{m} / \mathrm{s}$, with average speeds of $350-650 \mathrm{~m} / \mathrm{s}$. Slower equatorward motions for more irregular forms are observed at lower latitudes. This model provides only limited information about how

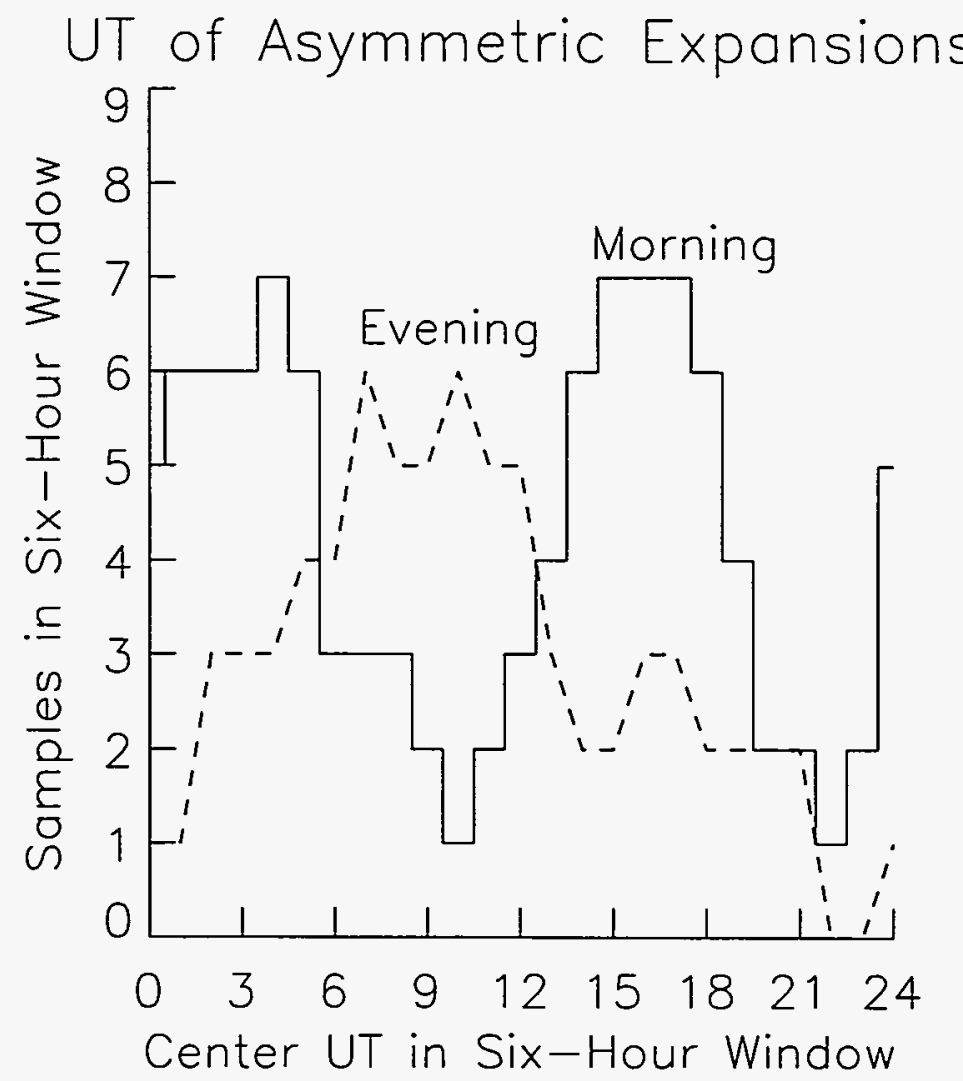

Asymmetric development of auroral substorms towards the morning or evening sector is influenced by the UT of substorm onset. The occurrence rate is shown in a six-hour sliding window as a function of the UT in the center of the window for 28 asymmetric expansions observed with DE 1 in 1981. 
the bulge evolves spatially during the expansion phase. Included are a westward traveling surge along the oval at the western end of the bulge and an expansion eastward into the morning sector that is more prominent in its latitudinal extent.

A well-defined auroral bulge is commonly observed in DE-1 auroral images from high-polar altitudes, and the poleward expansion in the midnight sector frequently evolves basically as sketched in the standard model. Equally outstanding examples exist for which the expansion phase develops differently. As an example, several authors have pointed out that the surge at the westward edge of the bulge is observed on occasions not to move any appreciable distance. In this case, the eastward expansion is dominant, and, but for the absence of a westward traveling surge, the evolution is similar to that presented in the standard model.

On other occasions, however, a westward expansion of the surge is dominant, which leads to a bulge asymmetrically distributed toward the west. Sorting observations of 28 asymmetric expansions according to the sign of the $y$-component of the interplanetary magnetic field yields a strong suggestion that the sign of $\mathrm{B}_{\mathrm{y}}$

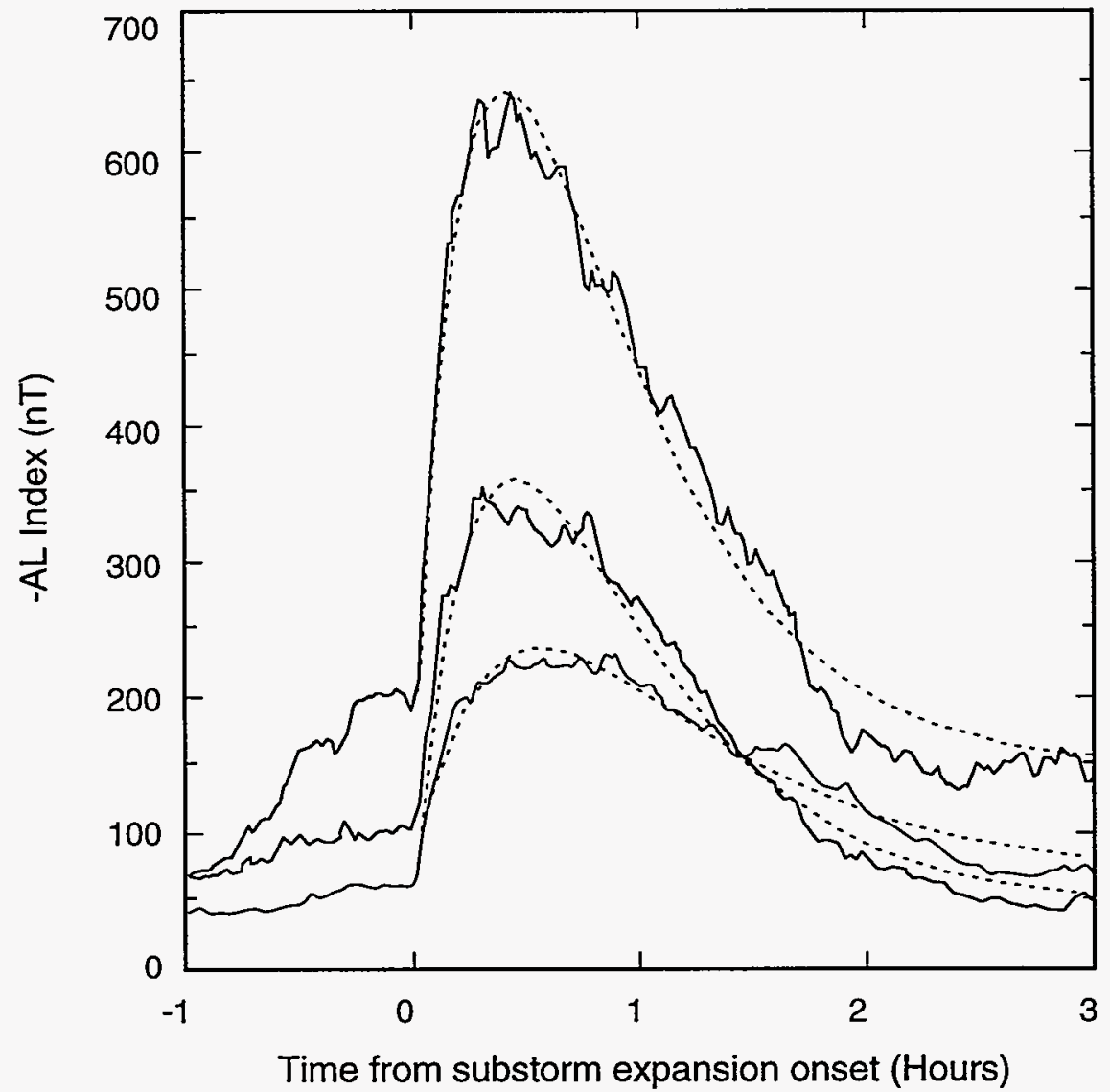

Average AL indices during substorms of increasing magnitude are shown. The three solid curves show the mean values of the AL indices from many substorms grouped according to peak magnitude and superimposed on a common scale from the start of the expansion phase. The dotted lines show the exponential equations which have been fit to these data. is an important parameter. For example, of the 12 asymmetric expansions into the evening sector, nine are associated with $\mathrm{B}_{\mathrm{y}}>0$.

Equally curious is the observation of an apparent UT dependence, as shown in the accompanying plot of the occurrence rate as a function of UT for sums over a sliding six-hour window. Clear relative maxima are seen at 0300 and 1600 UT for expansions into the morning sector, with an intervening maximum at 0900 UT for expansions favoring the evening sector.

Determination of the relative importance of the $B_{y}$ and UT contributions remains to be completed, as does a more complete analysis including expansions which do not suggest such asymmetries. However, the existence of such asymmetries in the distribution indicates that the longitude of a ground-based observer may be important in the determination of relative frequencies of occurrence for evening side versus morning side auroral phenomena in auroral substorms.

NASA Grant NAGW3441: Scientificpersonnel-J.D. Craven and L.A. Frank (University of Iowa)

Substorm Time Constants: It has been found that the auroral electrojet indices tend to follow a repeatable pattern during substorms. The AL index, which measures the westward electrojet current in the ionosphere, follows a characteristic curve that can be matched with an exponential equation.

This behavior indicates that the coupled magnetosphere-ionosphere system has inherent time constants as a result of the substorm process in 
which magnetic energy in the magnetotail is converted into kinetic energy in the plasma sheet, which is then dissipated by electrical currents through the ionosphere. These results show that the recovery phase of substorms, where the indices start to decline, occurs after a specific time interval due to the time constant of the energy dissipation, rather than due to a decrease in an external force in the solar wind.

NSF Grant ATM90-14148: Scientific personnelD.R. Weimer

Formation of a Very Thin Current Sheet in the Near-Earth Magnetotail and the Onset of Substorms: The onset of substorms is believed to be related to the formation and stability of thin current sheers in the near-earth $\left(-6 R_{e}>x>-20 R_{e}\right)$ magnetotail. The onser signatures are observed mostly in the near-earth region ( $x \sim-6$ to $-12 R_{e}$ ). The current sheet thinning starts before the onset of the expansion phase and the observed thickness of current sheets can be as small as 300 to $1000 \mathrm{~km}$.

Previous numerical studies showed that the tail can be stretched as convection proceeds and that a $B_{z}$-minimum can be formed in the plasma sheet. However, the obtained current sheet is much thicker than those observed prior to the substorm onset. A magnetofrictional method was developed to construct two-dimensional magnetohydrostatic equilibria of the earth's magnetosphere for a given distribution of entropy function $\left(p V^{\gamma}\right)$, where $p$ is the plasma pressure and $V$ is the tube volume per unit magnetic flux.

The accompanying figure shows an example in which a very thin current sheet with $B_{z}<0.5 n T$ and thickness $<1000 \mathrm{~km}$ is formed in the near-earth magnetotail $\left(x \sim-8\right.$ to $\left.-20 R_{e}\right)$ during the growth phase of a substorm. The tail-current sheets are found to become thinner as the entropy, the entropy gradient, or the tail-open magnetic flux increase.

Based on our study, the following scenario for the substorm growth phase and substorm onset, as illustrated in the frgure on page 24 , is proposed: (a) A localized non-adiabatic heating increases the entropy function $\left(S=p V^{\gamma}\right)$ and steepens the gradient of $S$ in $A$-space. Subsequently, the near-earth magnetotail is stretched tailward and forms a thin current sheet. (b) When the strict frozen-in condition is violated and particles are allowed to diffuse across field lines, inner field lines are dipolarized and outer field lines are more stretched tailward. (c) Continuing dipolarization and stretching make the current sheet thinner and thinner. (d) Magnetic reconnection may take place in the almost singular current sheer. (e) Reconnection generates a strong earthward flow and ejection of a plasmoid tailward.

DOE grant DE-FG06-91ER 13530: Scientific personnelL.C. Lee, L. Zhang, G. Choe, and H. Cai

(a) Magnetic Field Lines

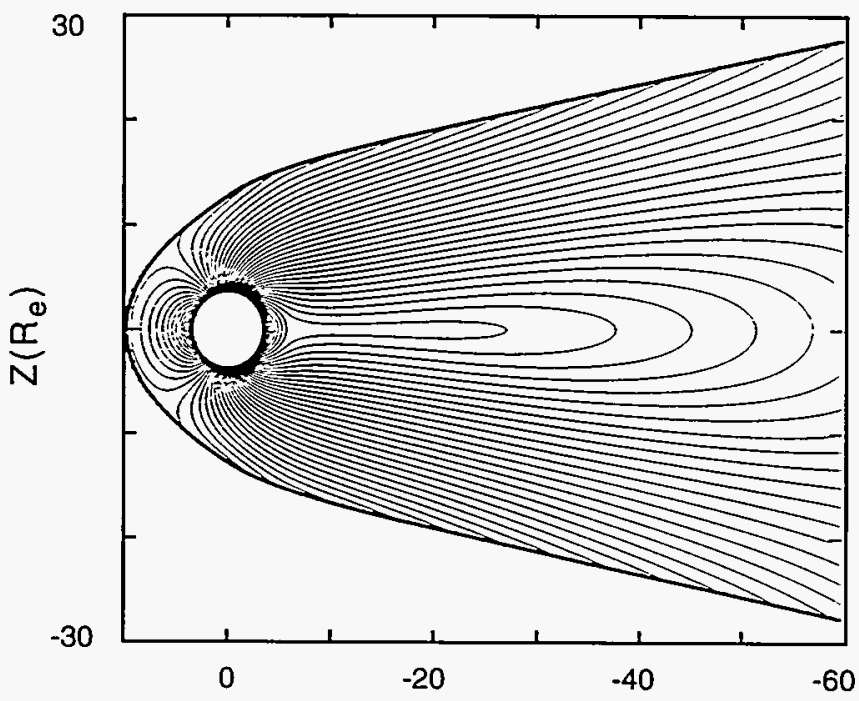

(b) Magnetic Field Lines

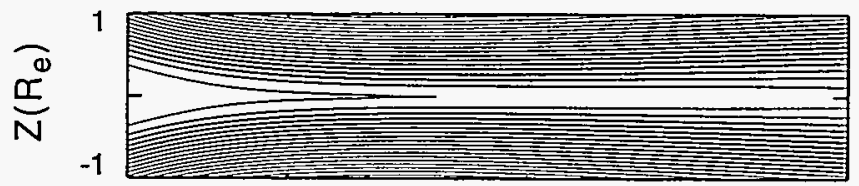

(c) Pressure Contours

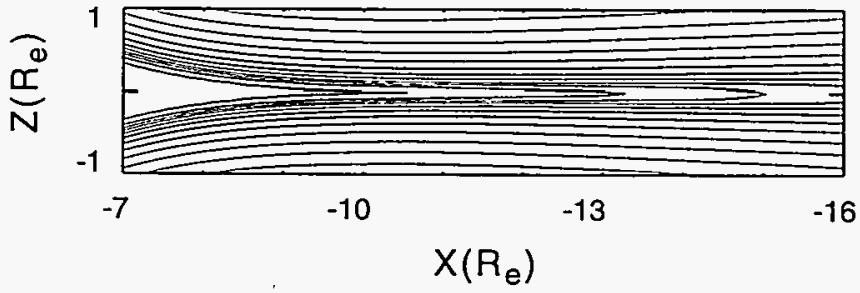

This two-dimensional global equilibrium configuration for the earth's magnetotail was obtained by the magnetofrictional method. The final equilibrium configuration depends on the boundary conditions imposed along the magnetopause and the distant tail boundary, as well as on the imposed specific entropy profile, where the specific entropy is defined as $S=P V^{g}$ and $V=U$ ds / $B$ is the volume in a unit flux tube. (a) Magnetic field configuration, in which a very thin $\left(d-0.05 R_{d}\right)$ current sheet is formed and the minimum $B_{z}$ is $\sim 0.24 n T$. (b) Magnetic field lines in the region between $-6 R_{\theta} \geq x \geq-17 R_{\theta}$ show a cusp-shape configuration. (c) Shown are the corresponding pressure contours. 


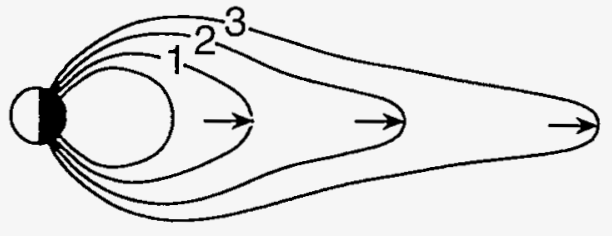

(a)
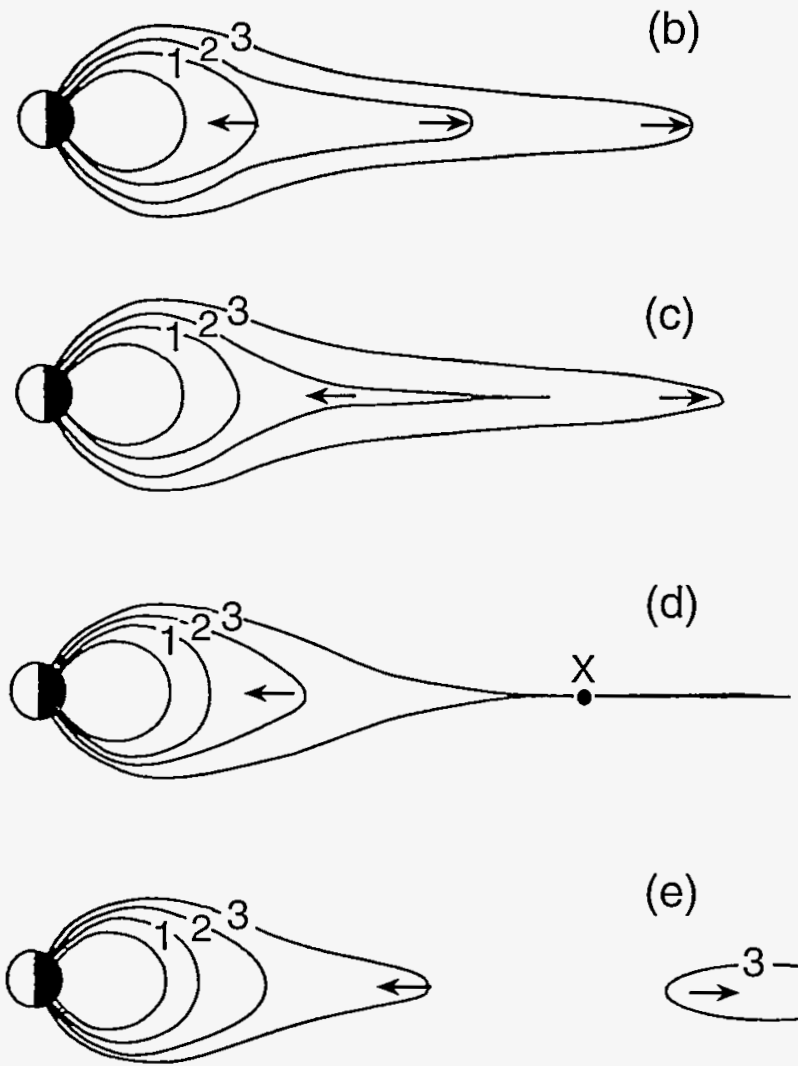

(e)

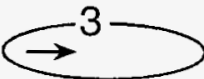

A new scenario for the formation of very thin current sheets and for the onset of magnetic field dipolarization and expansion phase (see the text for explanation) is shown.

The Average Ionospheric Electrodynamics for the Different Substorm Phases: The average parterns of the electrostatic potential, current vectors, and Joule heating in the polar ionosphere, as well as the associated field-aligned currents, were determined for a quiet time, for the growth phase preceding expansion onser, for the expansion phase, for the peak epoch, and for the recovery phase of substorms. For this purpose, the KRM magnetogram-inversion algorithm was applied to a data set from the six IMS meridian magnetometer chains, which were operated during the period of the International Magnetospheric Study (IMS).
This is the first attempt to obtain the average pattern of substorm parameters in the polar ionosphere, which is common to individual substorms for the different epochs. The global electric field current patterns are found.

On the basis of the current patterns over the entire polar region, it is possible to distinguish two components. The first one is related to the two-cell convection pattern (the directly driven component) and the other is the westward current in the dark sector, which is related to the wedge current (the unloading component). Time variations of the two components were determined for the four epochs and were shown to be considerably different. The dependence of these differences on the electric field and the conductivities (the Hall and Pedersen) was identified.

These and many other earlier results are consistent with the present ones, which are more comprehensive in our global study. There is some indication that both components are actually coupled. The facts that the two components exist and that their time variations are different indicate that we must be cautious in using a single electrojet index, such as $\mathrm{AL}$, in correlating with satellite data.

NSF Grant ATM93-11474: Scientific personnelS.-I. Akasofu, W. Sun, and Y. Kamide (Nagoya University Solar-Terrestrial Environment Laboratory)

Satellite Measurements through the Center of a Substorm Surge: Measurements have been obtained from the DE-2 satellite of electric and magnetic fields, plasma drifts and electron precipitation within a surge at the westward, leading edge of the auroral bulge at the peak of the substorm expansion phase.

The trajectory of the DE-2 satellite over the auroral emissions was determined from nearly simultaneous observations with the imager on the DE-1 satellite at a higher altitude. The electric field and plasma drift measurements have enabled us to deduce the basic configuration of the ionospheric electric potential, or plasma convection, around the surge.

The electric potential shows that the bulge is associated with a protrusion of the dawn convection cell into the dusk cell, poleward of the Harang discontinuity. This protrusion contains a westward electric field that strongly enhances the westward electrojet 

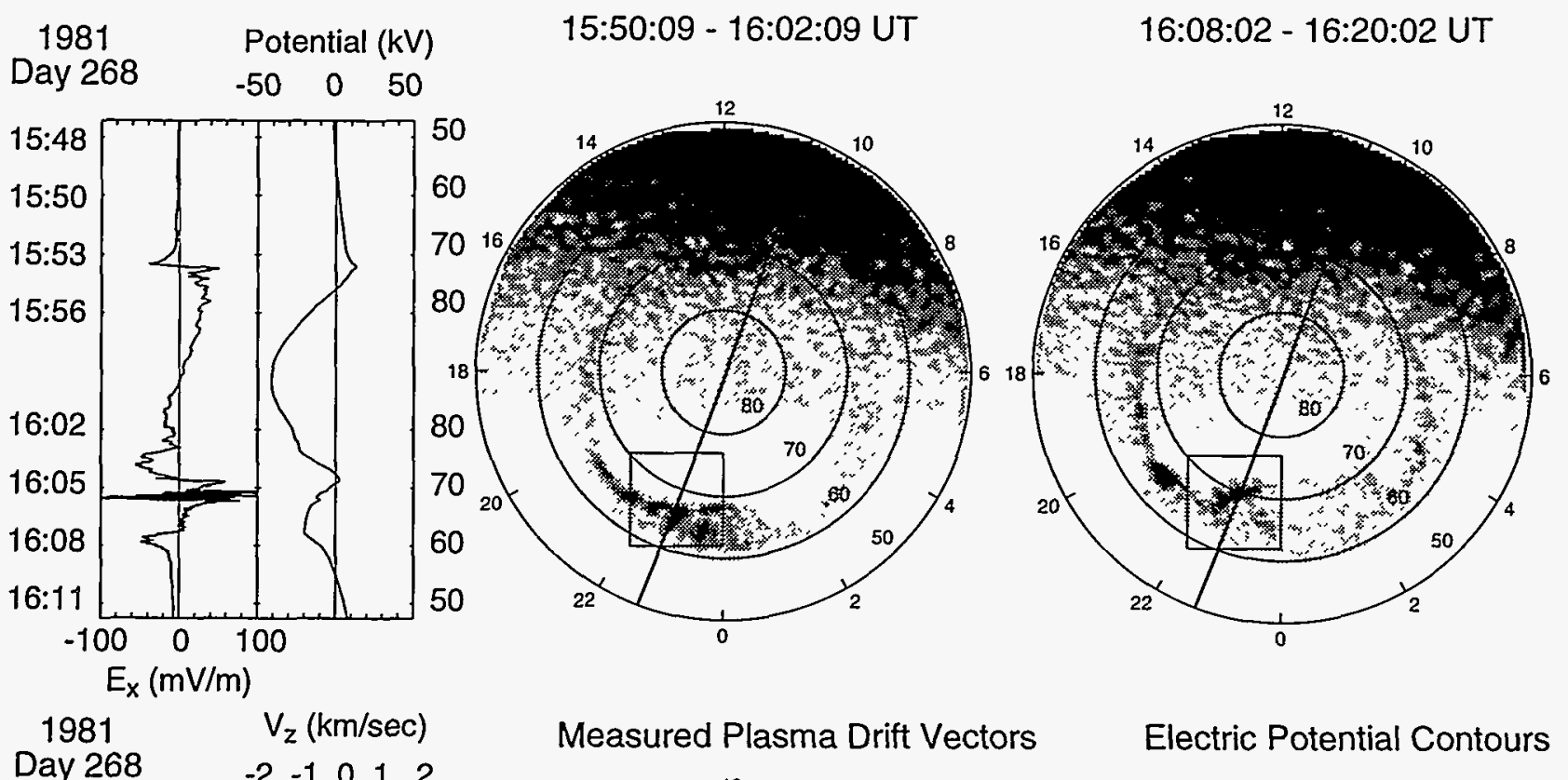

Day $268 \quad-2 \quad-1 \quad 0 \quad 1 \quad 2$
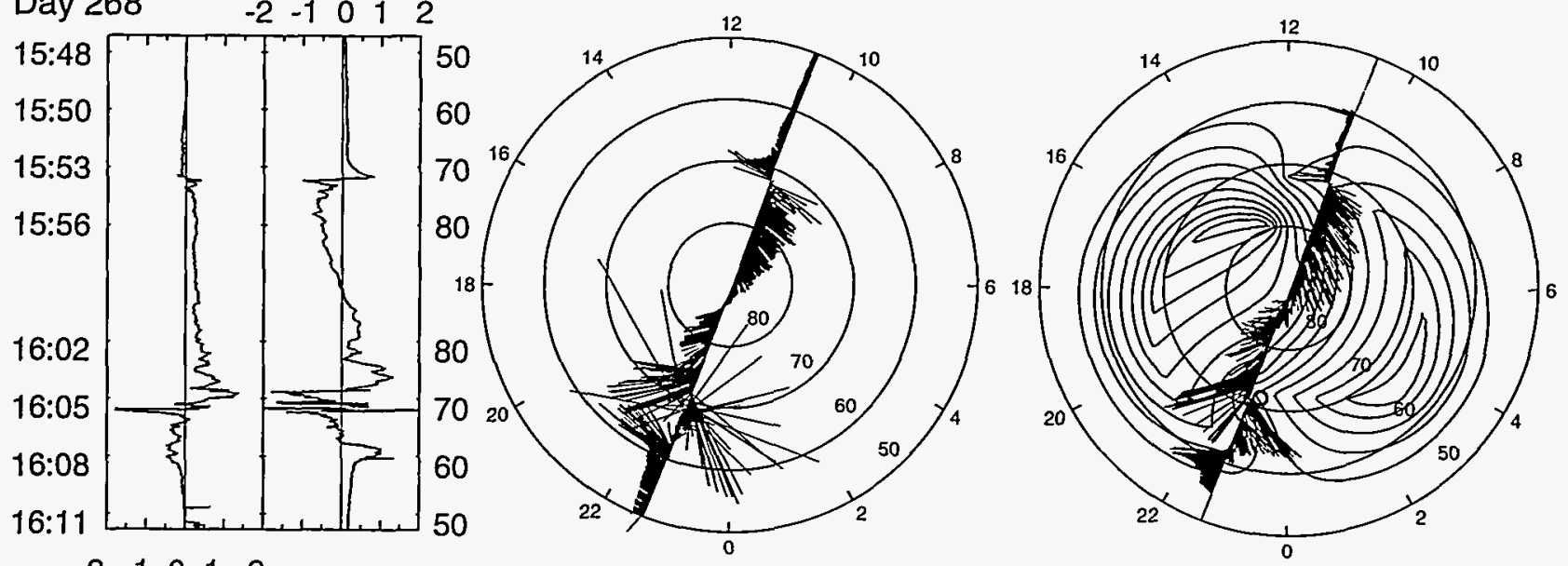

$$
\begin{array}{ccccc}
-2 & -1 & 0 & 1 & 2
\end{array}
$$

$\mathrm{V}_{\mathrm{x}}(\mathrm{km} / \mathrm{sec})$

Shown are satellite measurements through a substorm surge. The upper half of this figure shows two sequential images of the auroral oval, obtained from the DE-1 satellite on Julian Day 268 of 1981 , in which the locations of all image pixels have been translated into geomagnetic coordinates. These pictures show an expansion of the aurora during a large magnetospheric substorm. (The scale is reversed so that brighter areas appear dark.) The heavy black lines show the path of the lower-altitude DE-2 satellite through this auroral surge, while the graphs on the right show the electric fields and electric potential that were measured along the path of the satellite. The lower half of this figure shows plasma drift vectors that were measured on the DE-2 satellite, which are proportional to the electric fields. The bottom-right graph shows a contour map of a model of the polar cap electric potentials at this time. The distortion in the potentials around the surge have yielded important information about the electrodynamics of the substorm process.

current. This westward electric field, and the associated Cowling current, appear to terminate within the surge, which contains an intense, upward field-aligned current. The magnetic field measurements show that the region containing this field-aligned current is shaped like a cylinder, which contains a current that exceeds 500,000 Amperes.
NSF Grant ATM94-00516 and NASA Grant NAG51915: Scientific personnel-D.R. Weimer, J.D. Craven, L.A. Frank (University of Iowa), W.B. Hanson (University of Texas at Dallas), N.C. Maynard (Mission Research Corporation), R.A. Hoffman, and J.A. Slavin (NASA Goddard Space Flight Center) 


\section{Aurora, Auroral Structures, and Current Systems}

Auroral Radio Emission: The purpose of this project is to deploy two sensitive $\mathrm{LF} / \mathrm{HF}$ radio receivers in Alaska to monitor radio emissions from the aurora over the frequency range of $200 \mathrm{kHz}$ to $10 \mathrm{MHz}$.

The receivers were located at quiet radio sites at Two Rivers, Alaska ( 45 $\mathrm{km}$ east of Fairbanks) and at various other locations in the Alaska Bush. Eldon Thompson maintained and operated the Two Rivers site, and Don Hampton was responsible for the other sites.

During the course of the experiment, several instances of wide-band radiation (Auroral Radio EmissionARE) were observed associated with auroral activity corresponding to various plasma resonances and radiation from the interaction of electrons with the auroral plasma. Theoretical analysis and modeling efforts are currently in progress at Dartmouth to attempr to explain quantitatively the observations of ARE.

NSF Subcontract through Dartmouth College 5-34269: Scientific personnel-J. LaBelle (Dartmouth College) and R. Hunsucker

Automated Observatory at Eagle, Alaska: As part of a cooperative project with Los Alamos National Laboratory (LANL), wehaveinstalled a remote, fully automated observatory at Eagle, Alaska, near the AlaskaCanada border.

The observatory is close to the foot of the magnetic field line through a synchronous satellite instrumented by LANL. The intent is to compare visual auroral features with the energetic electron and ion fluxes at syn- chronous altitude. The site includes an all-sky television system, a Fabry-Perot interferometer for measuring high-altitude winds and temperatures, a riometer, and a magnetometer. All of the instruments operate automatically under computer control. A local caretaker checks the site daily, cleans snow off the domes, and changes tapes. The interferometer and the television camera can both be accessed by phone line from Poker Flat Research Range to check the operational status or to assist in evaluating auroral conditions for the launching of sounding rockets. Although we have operated all-sky cameras intermittently for a number of years, the Eagle station will provide the first comprehensive data set of this type from any Alaska site.

Los Alamos National Lab Grant PO4941Q0014-5E: Scientific personnel-T. Hallinan, R. Smith, M. Conde, H.C. Stenbaek-Nielsen, and J. Olson

Auroral Microphysics and Ion Conics: A sounding rocket to be launched from Poker Flat Research Range in February 1995 is intended to study the microstructure of auroral arcs and, in parricular, the nature of previously discovered lower hybrid solitary structures that have dimensions of only tens of meters and that may be responsible for acceleration of ions that stream upward from the ionosphere (ion conics).

The rocket will separate into two payloads that will allow simultaneous measurement at slightly separated locations. In order to relate the detailed rocket measurements to the aurora, we will operate low-lightlevel imaging systems at Poker Flat Research Range and at Barter Island on the Alaska Arctic Ocean Coast. Apogee of the rocket will be nearly overhead at Barter Island.

Our hope is to relate the detailed auroral morphology to the in situ measurements in order to form a better understanding of the causes of events such as flickering auroras, auroral rays, and fine arc structure. Work to date has included planning, and preparing instruments for the upcoming flight.

NASA Grant NAG5-5013: Scientific personnel-T. Hallinan, J. Kimball, and Roger Arnoldy (PIUniversity of New Hampshire)

Auroral Observations from the Space Shuttle: High-inclination orbits of the space shuttle regularly penetrate the auroral oval, usually encountering the nightside oval in a region south of New Zealand and Australia. This provides an opportunity to obtain, at low additional cost, stereo pairs of color images of the aurora as well as black-and-white video recordings. In a few minutes of elapsed time, it is possible to photograph auroras over many hours of local time.

The purpose of this project is to work with the Earth Observation Program at Johnson Space Center and with the Astronaut Training Office to encourage the astronauts to photograph the aurora on a timeavailable basis, and to provide guidelines and scientific information to the astronauts so that they can take the data in a way that is scientifically 
useful. Activities have included giving a seminar on the aurora at the Astronaut Training Office, writing a section on auroras for the new Earth Observation Program Astronaut Training Manual, assisting NASA officials in writing captions for press releases, and identifying the auroral data and obtaining copies.

The stereoscopic measurements of auroral rays have shown that rays in the polar cap are at much higher altitude than those in the auroral oval and that, within the oval, the variation in vertical extent is independent of the lower border altitude. From that, we concluded that the spread in electron energies is independent of the peak energy.

We also have observed a tall violet ray in one of the photographs. It has been determined that this is not sunlit, and an ongoing study of groundbased color TV data shows that occasional violet rays are observed in nonsunlit auroras. The nearly constant red/blue ratio within these rays suggests that the emissions are primarily from $\mathrm{N}_{2}$ and $\mathrm{N}_{2}{ }^{+}$. This is surprising in view of the high altitudes $(>300 \mathrm{~km})$ attained by some of the rays.

Although our interest is primarily in auroras, in the course of documenting the occurrence of thin-layered structures in auroras, we have observed a surprising variation in the airglow layers. The ratio of intensity of the green $(5577 \AA)$ to red $(\mathrm{OH})$ emissions is highly variable as is the separation in altitude between these two layers.

NASA Grant NAGW-2857: Scientific personnel-T. Hallinan, D. Lind (Utah State University), J. Kimball, and A. Roberts
The Shaped-Charge Injection of Field-Aligned $\mathrm{Ba}$ and $\mathrm{Ca}$ Plasma to Investigate the Physics of MassDependent Ion Acceleration: Ever since auroral physicists first found that the spectra and fluxes of electrons over discrete auroras peak in the energy range from 1 to $10 \mathrm{keV}$, there has been much controversy as to where and how the electrons are accelerated. The hypothesis with the longest standing in the community is that quasi-static parallel electric fields of 1 to $10 \mathrm{kV}$ potential drop at 1 to $2 \mathrm{R}_{\mathrm{e}}$ altitude play an important role in accelerating auroral electrons.

Recently, others have examined and discussed the evidence to support this hypothesis as well as the major qualifications and uncertainties that cast doubt on its validity. Auroral physicists do not agree on the relative contributions of quasielectrostatic fields and stochastic acceleration in turbulent electric fields in energizing auroral electrons and upstreaming ions. What does seem clear, is that some mass-dependent process is associated with the acceleration and/or dissipation of energy for the positive ion components. There is evidence from upstreaming ion conics and beams of mass dependency of energy, particularly between $\mathrm{H}^{+}$and $\mathrm{O}^{+}$.

It is not certain whether the mass dependence is a result of the process of forming conics at low altitudes, or whether it is a result of the ions being accelerated through a parallel electric field where ion-ion, two-stream interaction may transfer energy from the light ions to the heavier ions. The acceleration of ions upwards bears upon the acceleration of electrons downwards to form discrete auroras.
We proposed a definitive experiment to set boundary conditions to the models. The simultaneous injection on an auroral field line of $\mathrm{Ba}$ and $\mathrm{Ca}$ plasmas with similar initial velocities, and with pitch angles near $0^{\circ}$. The atomic weight of $\mathrm{Ba}$ is 137.34, and of $\mathrm{Ca} 40.08$, which is a ratio of 3.43 , close to the ratio of $\mathrm{O}$ to He. If we inject upward and parallel to $B$, two visible ion tracers of widely different atomic mass and essentially the same velocity, the ions will either experience the same gain in energy or they will not. In doing this, we eliminate one uncertainty about the origin of mass-dependent ion beams. The two feasible elements with a large ratio of atomic weights are $\mathrm{Ba}$ and $\mathrm{Ca}$, both of which have solar fluorescent ion lines in the visible spectrum.

In conjunction with a group at New Mexico Tech, we have built a Ca-lined shaped charge incorporated in a payload with a Ba-shaped charge to be launched under auroral conditions from Poker Flat Research Range in spring 1995.

We will observe the $\mathrm{Ca}^{+}$and $\mathrm{Ba}^{+}$ ions with low light level imagers from PFRR, Alaska, Ft. Smith, N.W.T, and from a Westwind-2 corporate jet aircraft flying our of Reno, Nevada. As we triangulate on the tips and determine the upwards motion of the two ion species, we will look very carefully for evidence of accelerations upwards (or downwards) that cannot be explained by gradient $-\mathrm{B}$, gravity, or $\mathrm{E} \times \mathrm{B} / \mathrm{B}^{2}$ drift imparting a parallel motion.

From the changes in velocity we expect to determine potential drops, and most importantly, wherher the energy changes are mass-dependent. In theory, there should be an ion-ion 
acoustic interaction between the $\mathrm{Ba}$ and the $\mathrm{Ca}$, which would transfer energy from the $\mathrm{Ca}^{+}$to the $\mathrm{Ba}^{+}$beams, and which should be evident in the velocities.

NASA Grant NAG5-5000: Scientific personnel-E.M. Wescott, H.C. Stenbaek-Nielsen, D. Hampton, $P$. Delamere, J. Desrochers, and D. Bostow

Fluorescent Plasma Tracer and Perturbation Experiments from Sounding Rockets: For a number of years we performed plasma injection experiments from sounding rockers for studies of auroral electrodynamics and field line tracing, perturbation experiments, and experiments to test the critical velocity effect proposed by Alfvén.

The work under the NASA grant involved completion of analysis of some of the experiments. In particular, we have completed a paper on rapid ray motions in barium ion clouds that offers compelling evidence for the existence of transient electric fields of order $100 / \mathrm{m}$ at elevations as low as $200 \mathrm{~km}$ during active aurora with rapid ray motions. The affected regions have dimensions on the order of a few kilometers and move across the magnetic field lines with velocities of 10 to $20 \mathrm{~km} / \mathrm{s}$. The barium ions show brightness enhancements of up to three times normal, which can be explained by a Doppler shift away from the solar Fraunhofer absorption lines.

NASA Grant NAG6-1: Scientific personnel-E.M. Wescott, H.C. Stenbaek-Nielsen, D. Hampton, and P. Delamere

Large-Amplitude Auroral Electric Fields Measured with DE-1: This study used measurements of electric fields on the DE-1 satellite in order to determine the probability distribution, as a function of altitude, of very large-amplitude electric field events.

These electric fields are detected for only a few seconds at a time when the satellite passes over the aurora. Their distribution has yielded clues to the nature of the magneric fieldaligned electric fields that accelerate auroral electrons.

NASA Grant NAG5-2249: Scientific personnel-D.R. Weimer and D.A. Gurnett (University of Iowa)

DE-1 and Viking Observations Associated with Electron Conical Distributions: Data from the electron detectors on the DE-1 and Viking satellites were examined for occurrences of events where the electrons had a conical energy distribution.

With a very careful analysis of the electric field measurements on DE1 , it was possible to find evidence for low-frequency (under $1 \mathrm{~Hz}$ ) electric field oscillations in the direction of the magnetic field coincident with some of these events.

These results indicate that auroral electrons may be accelerated by a resonance with an oscillating fieldaligned electric field.

NASA Grant NAG5-2249: Scientific personnel-J.D. Menietti (University of (owa), D.R. Weimer, $M$. Andre, and L. Eliasson (Swedish Institute of Space Physics)

\section{Mapping of Magnetospheric Structures}

Magnetic Mapping between the High-Latitude Boundaries of the Auroral Oval and the Plasma Sheet: It is currently popular to magnetically map auroral features and boundaries into the near-earth magnetotail using magnetic field models such as those of Tsyganenko. Equally ambitious empirical demonstrations are lacking in that the models created from averages of magnetic field observations are reasonable for quiet periods, but are much less reasonable for more active intervals when the field configuration is changing rapidly. The objective of this work is to use two observed physical boundaries assumed to be nearly identical, and to evaluate quantitatively the mapping between the two boundaries with increasing distance down the tail and with varying levels of magnetic activity.

The high-latitude boundaries of the plasma sheet (at high altitudes) and the auroral oval (at low altitudes) are used here. Position of the plasma sheet boundary is first determined using ISEE-1 or ISEE-2 measurements of the flux of 2-keV electrons, and the position is then mapped magnetically to auroral altitudes (ISEE). Simultaneous measurements with DE-1 are used to determine the poleward boundary of the aurora (DE) at the mapped local time of ISEE.

Fifty-six mappings are available in the southern hemisphere for the April-July period in 1983 under ei- 
ther quiet or active conditions. ISEE distances in the magnetotail range from $-\mathrm{X}_{\mathrm{gsm}}=8$ to $22 \mathrm{R}_{\mathrm{e}}$. Systematic differences are observed between the measured position of the plasma sheet-lobe boundary and the expected position based on ideal magnetic mappings, and these differences depend on the sign of the $\mathrm{z}$ component of the interplanetary magnetic field (IMF).

For example, using the 1982 magnetic freld model of Tsyganenko and Usmanov as a template for an investigation of the $B_{z}$ effect, it was found for $\left|Y_{g s m}\right|<5 R_{e}$ that the model underestimates the plasma sheet thickness for $B_{z}>0$ and overestimates it for $\mathrm{B}_{\mathrm{z}}<0$. In contrast, for ISEE at $\left|Y_{g s m}\right|>5 R_{e}$, the relation between the measured and modeled thickness and the sign of $\mathrm{B}_{\mathrm{z}}$ are reversed.

These observations further support efforts to replace the use of magnetic parameters such as $\mathrm{K}_{\mathrm{p}}$ with more responsive parameters such as $A E$, and encourage the eventual inclusion of instantaneous IMF values. A successful mapping from the auroral oval to the plasma sheet boundary will provide a reference frame to aid in the identification and mapping of structures at progressively lower latitudes throughout the region of discrete auroral features.

NASA Grant NAGW 2441: Scientific personnel-J.D. Craven, G.K. Parks (University of Washington), and L.A. Frank (University of Iowa)

Barium, Strontium and Calcium Experiments from the CRRES Satellite: Under the auspices of NASA, we participated in planning chemical releases, orbit selection, release rimes and aircraft and ground optical observations of chemical releases during four campaigns: The PEGSAT releases over northern Canada; CRRES releases G-13 and G-14 over the South Pacific based out of American Samoa; the highaltitude CRRES releases G-5, G-6, G-7, G-8 and G-10 from two aircraft based out of Sawyer AFB, Michigan; and the Caribbean perigee releases G-1, G-9, G-11A G-11B, and G-12 from a ground site on Aruba and in Argentina and from aircraft based out of Aruba and Argentina.

The results of the analysis of these four campaigns are partially complete. The South Pacific and spectral analysis of the Caribbean experiments have been published in two papers in the Journal of Geophysical Research and a third paper on the Caribbean conjugate experiments is in preparation. Analysis of the Alfvén's critical velocity experiments and the physics of the early time processes of the Caribbean releases are the subjects of two Ph.D. theses in progress.

The South Pacific critical velocity experiments: As part of the NASA CRRES chemical release program in September 1990, two Ba and one each of $\mathrm{Sr}$ and $\mathrm{Ca}$ canisters of a boron-titanium thermite mixture that vaporizes the element on ignition were released near perigee, after dusk in the South Pacific, to study the critical velocity effect proposed by Alfvén. The critical velocities of these three elements are 2.7,3.5, and $5.4 \mathrm{~km} / \mathrm{s}$ respectively, all well below the orbital velocity of $9.4 \mathrm{~km} / \mathrm{s}$. On 10 September 1990, a Sr and Ba pair (G-13, or CIV I) was released near Rarotonga at about $515 \mathrm{~km}$ altitude in a background electron density of $3.4 \times 10^{6} \mathrm{~cm}^{-3}$. On 14 September
$1990, \mathrm{G}-14$ or CIV II released a Ca and $\mathrm{Ba}$ pair west of $\mathrm{New}$ Caledonia near $595 \mathrm{~km}$ at an electron density of $1.5 \times 10^{6} \mathrm{~cm}^{-3}$. Ions of all three elements were observed with low-lightlevel imagers from two aircraft after they had transited up the magnetic field lines into the sunlight. Emissions from the spherically expanding neutral gas shells below the solar terminator, observed with cameras filtered for the $\mathrm{Ba}^{+}$ion line at $4554 \AA$ and also in unfiltered imagers for about 15 seconds after release, are probably due to excitation by hot electrons created in the CIV process. The ions created clearly lost much of their energy, which we now show can be explained by elastic collisions: $\mathrm{Ba}^{+}+\mathrm{O}$. Inventories of the observed ions indicate yields of 0.15 percent and 1.84 percent for $\mathrm{Ba}$ in the first and second experiments, 0.02 percent for $\mathrm{Sr}$ and 0.27 percent for $\mathrm{Ca}$.

Ionization from all the releases continued along the satellite trajectory much longer ( $>45 \mathrm{~s}$ ) than expected for a CIV process. The ion production along the satellite track versus time typically shows a rapid rise to a peak in a few seconds, followed by an exponential decrease to a level, essentially constant, rate. The characteristic distances for CIV I and II are 47 and $62 \mathrm{~km}$, respectively. We interpret the early time rise and exponential fall to be due to CIV ionization of 0.014 percent (CIV I) and 0.40 percent (CIV II) for the $\mathrm{Ba}$ releases. The later ions produced at a constant rate probably have origins from other processes such as stripping and associative ionization collisions with atmospheric constituents, primarily $O$, and charge exchange with $\mathrm{O}^{+}, \mathrm{He}^{+}$, and $\mathrm{H}^{+}$. We suggest 
that the much larger $\mathrm{Ba}$ ionization rate in CIV II over CIV I is due to the fact that the release occurred in the peak Ca density, where hot electrons were already present.

The bigh-altitude release campaign: During January and February 1991, we participated in the aircraft observation campaign for the CRRES high-altitude releases of $\mathrm{Ba}$ and $\mathrm{Li}$. The $100-\mathrm{km}$ ionospheric foot of the field lines connecting with the field lines of the release possibilities near apogee were located in a wide belt across northern Canada. Since the scientific goals of the releases involved the possibility of triggering pulsations in the aurora, or in the case of the large $B$ a releases, of triggering an auroral sub-storm, it was important to have observations of the auroras in the foor regions. Since the possible release points had conjugates scattered across the sparsely populated regions, it was nor feasible to site ground stations due to the high cost and the likelihood of cloud cover on any particular evening. Therefore, two Air Force C-135 aircraft were instrumented with optical and ionospheric sounding equipment to fly in the foot region.

We obtained an excellent data set of auroral observations for releases. No ionospheric effects due to the two Li releases were detected by any of the imagers. The barium release G-10 on 20 January was intended to initiate an auroral substorm. Indeed, shortly after the release there was an intensification of the auroral activity, which might be classified as a substorm. The radar at Millstone Hill also indicated anomalous activity somewhat to the east of the con- jugate point. Later, when careful analysis of the auroral activity of the evening of G-10 could be carried out, it was clear that the substorm had begun before the $\mathrm{Ba}$ release as a brightening well to the east near Greenland that propagated westward into the area where we observed it. Thus, there is probably no connecrion of the release of $B$ a near the equator at about $6 R_{e}$ and the substorm.

A second large barium release, G-8, was carried out during the next moon down window on 17 February. The auroras were active at the time of the release, and there is no evidence that the release was related to the activity.

The Caribbean campaign: At low magnetic latitudes, the equipotentiality of magnetic field lines has been considered to be a fact. If field-aligned current can flow from one ionosphere to the one in the conjugate hemisphere, differences in electric fields would be equalized although the magnetic variations would be different in conjugate areas. However, one $\mathrm{Ba}$-shaped charge experiment in 1971 provided evidence for significant nonequipotentiality on an $\mathrm{L}=1.22$ field line during very quiet magnetic conditions and low ionospheric electron densities. Two similar experiments under quiet conditions showed close equipotentiality. During July and August 1991, five releases of $B$ a vapor from the CRRES satellite over the Caribbean, at $\mathrm{L}$ values ranging from 1.22 to 1.40 , allowed further investigation of the drift rates at conjugate points. On one experiment, $\mathrm{G}-\mathrm{I}$, at $\mathrm{L}=1.38$, the data show significant nonequipotentiality during very active conditions and high ionospheric electron densities. On another experi- ment six days later, $\mathrm{G}-9$, at $\mathrm{L}=1.36$, also during active magnetic conditions, the data indicate field line equipotentiality. There was insufficient overlap of the northern and southern data to draw conclusions from the other three experiments.

Unfortunately, there are no useful ionospheric or magnetic data from the southern conjugate region to compare with various data from the Carribean region to form the basis of a physical theory of this phenomenon. However, it seems clear that the original explanation put forth to explain the 1971 experimental results-field-aligned current limitation due to insufficient electron density-must be wrong. The observations of the CRRES conjugate experiments provide some evidence for low $\mathrm{L}$ nonequipotentiality, but we have found no satisfactory explanation for the phenomenon.

NASA Grant NAS8-36321: Scientific personnel-E.M. Wescott, H.C. Stenbaek-Nielsen, D. Hampton, P. Delamere, D. Bostow, andJ. Desrochers

The Relationship between Ionospheric Convection and Magnetic Activity: This project used electric field data from the Dynamics Explorer-2 satellite, and solar wind data from IMP-8 and ISEE-3 in order to study the relationship among the ionospheric convection electric field and the solar wind, interplanetary magnetic field, and magnetospheric substorms. The auroral electrojet indices, derived from the worldwide chain of ground-based magnetometers, were also used. An innovative data analysis technique, which used data from a few thousand orbits, was used to show how the electric 
potentials, which drive the convection of plasma in the polar ionosphere, are influenced by the combined effects of the IMF orientation and substorm activity. Subtle, but very significant changes in the ionospheric electric potentials were detected during magnetospheric substorms. These changes were not detectable in prior examinations of individual satellite passes. This project showed for the first time the influence of magnetic reconnection in the magnetotail on the large-scale ionospheric electric fields.

NSF Grant ATM90-14148: Scientific personnel-D.R. Weimer and J.-H. Shue

Studies of Magnetospheric Imaging: Following up on previous work in simulation of glows from the plasmasphere, plasmasheet and polar cap outflow, new studies have been undertaken to determine actual glow distribution from measurements. Presently available data are fragmentary at best, hence approximate methods were considered in which the sparse data were supplemented by some assumptions about the variation of flux tube contents with longitude and time. For this work, it was assumed that the Field Line Interhemispheric Plasmaspheric (FLIP) model (Richards and Torr)

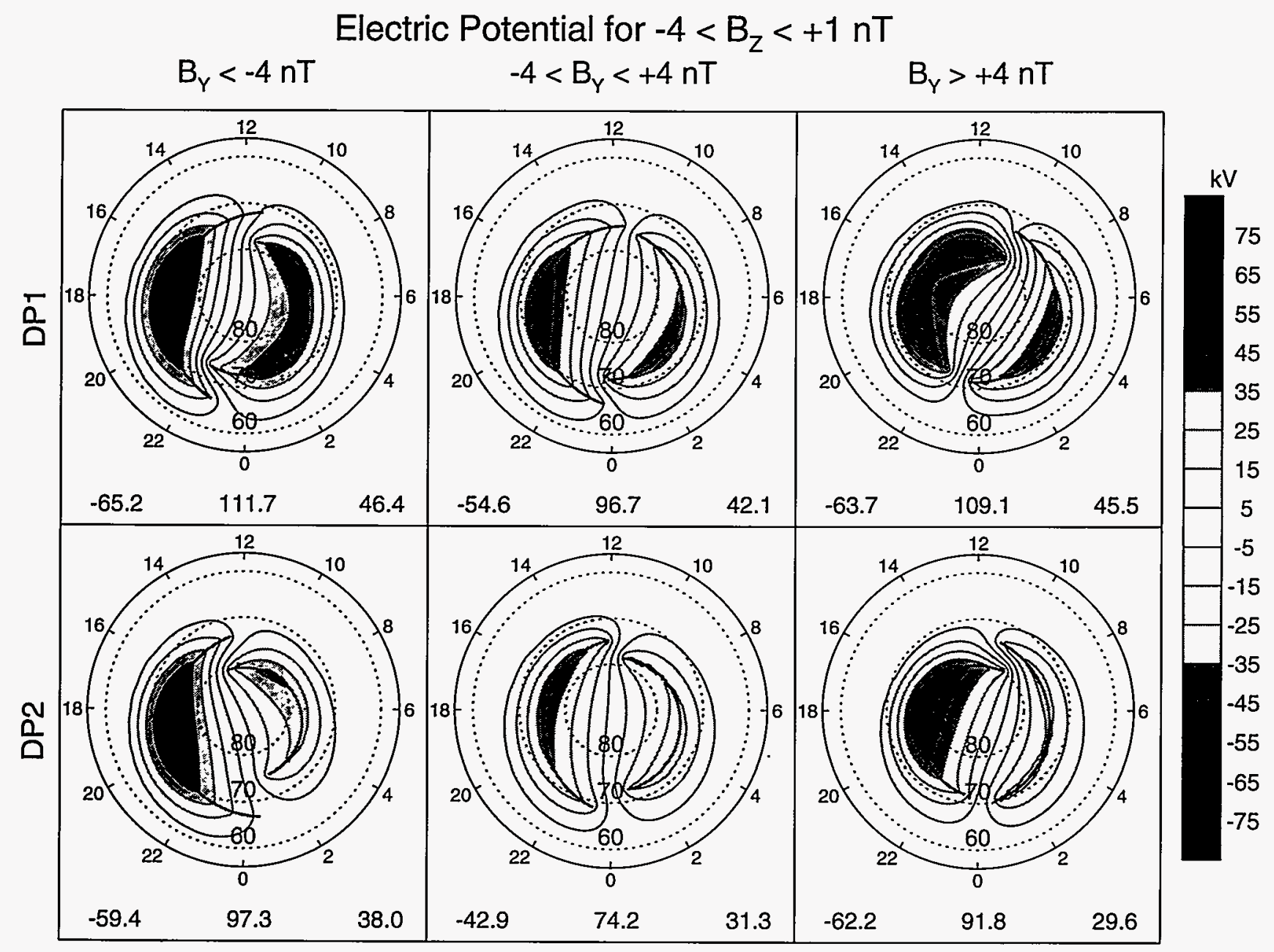

Shown are contour maps of electric potentials in the polar ionosphere. These maps were derived from an analysis of electric field measurements from over 2000 satellite passes over the polar caps. They show the influence of the dawn-dusk component of the interplanetary magnetic field (BY) for moderate values of the north-south component (BZ). These data were further divided into two categories according to the auroral electrojet indices. The bottom row shows the electric potentials for the cases where the $A U$ and $A L$ indices are of roughly equal magnitude, commonly designated as DP2, corresponding to the growth phase of magnetospheric substorms during which the polar cap expands due to magnetic merging on the dayside. The top row shows the potentials for the cases where the magnitude of the AL index greatly exceeds that of the AU index, designated as the DP1 configuration, which corresponds to the substorm expansion and recovery phase, during which magnetic flux is reconnection on the nightside. 
represented the essential physics of the redistribution of plasma along a flux tube so that the only unknown for each tube was the total tube content. Further, it was assumed that, up to a few earth radii, the high-latitude ion concentrations varied with their path along the field in the same way as the FLIP model at the boundary of the trough.

Using data from a rocket flight from Poker Flat Research Range (NASA 29.016), flown in quier geomagneric conditions in March 1982, a quasi-tomographic method was applied with a sparse matrix and the assumprions as described above. A trough was found at 65 degrees lati- tude in the northern hemisphere, as expected, but also a large flux tube ion population was found in the polar cap, contrary to previous studies and current theoretical work. The auroral data showed that a weak auroral form existed about $100 \mathrm{~km}$ north of the rocket.

One possibility for the high polar cap values is that the helium ions observed were connected to the aurora arc, rather than the polar cap. Although the measurements did contain information about the southern hemisphere, since lines of sight extended across the equator when looking south, the proportion of the total signal contributed was too small to permit reliable determination of flux tube contents. Using data from an orbiting satellite carrying a $304 \AA$ EUV photometer, it would be possible to extend the technique to provide more global distributions of helium ions.

Work is now in progress to test the global method, in readiness for data from satellite experiments to be flown in the next five years.

NASA Grant NGT 40022: R.W. Smith, D.E. Garrido, C. Marsh, D.W. Swift, A.B. Christensen (Aerospace Corporation), Y. Chiu and R.M. Robinson (Lockheed Space and Missiles Co.)

\section{Magnetometer Studies: Auroral Currents, ULF Waves}

Operation of the Alaska-Canada High-Latitude Magnetometer Chain: Magnetometers and riometers in the Alaska-Canada chain of stations follow a line of nearly constant geomagnetic longitude from Talkeerna, Alaska (62 degrees magnetic latitude), to Sachs Harbor, NWT (77.3 degrees magnetic latitude). Operation of the chain began in 1976 as part of the International Magnetospheric Study (IMS) program. Data from the magnetometers and riometers are transmitted automatically through the NOAA GOES satellites to the Space Environment Laboratory (SEL) in Boulder, Colorado, where they are reformatted and placed in the archives of the World Data Center. The data are available in real time through the SELDADS computer system.

Data from the meridian chain are an important resource to the magnetospheric research community. Real-time data is used as a diagnostic for rocket and radar studies of the polar ionosphere. Using the data from the meridian chain alone, or in conjunction with other magnetometer observations, one may reconstruct the pattern of ionospheric currents transecting the meridian chain and infer quantities such as energy deposition and the heating of the ionosphere. The data are an integral part of the Geo-Environmental Data Display (GEDDS) system which makes real-time ionospheric diagnostics available through the Poker Flat Research Range network.

NSF Grant ATM-8912155: Scientific personnel-J.V. Olson and S.-I. Akasofu

Magnetometer Observations at Gakona, Alaska: For several months during the early portion of 1994 , we made observations using a fluxgate magnetometer of the magnetic fields at Gakona, Alaska. The observing site is the future home of the HAARP ionospheric heater. In order to prepare for the analysis of data and to provide diagnostic support for experiments when the heater comes on line, we have begun to compile a catalog of data which will serve as a baseline.

The Gakona site, located at $55^{\circ}$ geomagnetic latitude, is a subauroral site. Consequently, we have found large variations are present during disturbed geomagnetic conditions, indicating the presence of significant overhead currents which are part of the auroral electrojet. Data from the site are currently archived at the Geophysical Institute and are available via network FTP access.

Funded by the Mitre Corporation: Scientific personnel-J.V. Olson

A Conjugate Study of ULF Waves at the Cusp-A GEM Project: During the first stage of the NSF/ GEM program we began a study of ULF waves associated with the mag- 
netospheric cusp in conjugate hemispheres. High-latitude stations at Longyearbyen, Svalbard, Norway, in the northern hemisphere and at Davis, Antarctica, provided data from fluxgate and induction magnetometers for this study. Data from Davis were provided by Dr. B. Fraser through support from the Australian ANARE program. We also have collaborated with experimenters at Longyearbyen who operated a meridian scanning photometer (msp).

Early in our study it became apparent that to make progress in understanding the spectrum of magnetic fluctuations at the cusp we would need to correlate with optical and satellite measurements, the purpose of which was to establish the location of the magnetometer with respect to the ionospheric footprint of the cusp/ boundary layer system. Correlations with the msp data allowed us to identify signals that were associated primarily with the central cusp from those associated with the surrounding boundary regions. We are continuing to analyze those signals using a variety of signal-processing techniques to unravel the data.

The spectrum of signals is quite complex, but can be separated into two elementary bands: a low-frequency band, $\mathrm{Pc} 5$, with frequencies from 1 to $10 \mathrm{MHz}$, and a somewhat higher frequency band ranging from $10 \mathrm{~m} \mathrm{~Hz}$ to above $50 \mathrm{MHz}(\mathrm{Pc} 3)$. The Pc5 band correlates well with optical signals and we find individual pulses occurring on the morning and afternoon sides of the central cusp associated with poleward moving forms in the msp data. We believe these to be the signatures of current filaments moving poleward associ- ated with boundary processes. In the central cusp in the Pc 5 band slightly more coherent wave packets are observed representing the driven oscillation of the nearby closed field lines. We anticipate the analysis of these signals will provide clues to the processes occurring at the dayside magnetopause.

Signals in the Pc3 band are comprised of two types. The first is a coherent oscillation which is wellcorrelated between sites. These are correlated with solar wind conditions and probably represent waves generated upstream of the magnetopause finding their way to the cusp ionosphere. The second class of signals in the Pc3 band is an incoherent, broadband of signals produced by lo$\mathrm{cal}$, quasi-periodicprecipitation of particles into the ionosphere. They are localized events with spatial coherence less than $100 \mathrm{~km}$. We believe these are associated with boundarylayer dynamics. Since we believe that the cusp region may be an important source of ULF energy in the magnetosphere, we have begun a set of studies of wave propagation in a realistic magnetospheric model.

Early results seem to indicate that significant wave energy can penetrate the inner magnetosphere where it can contribute to theULF background.

NSF Grant ATM-9213361: Scientific personnel-J.V. Olson, B.J Fraser, M.G. McHarg, C.A. Szuberla, and J. Williams

\section{A Conjugate Study of ULF Waves} at the Cusp-An Extension: As an extension of our work within the NSF GEM project, we have begun to extend our analysis of conjugate ULF waves associated with the high-lati- tude cusp/boundary layer regions to include inter-and intrastation correlations. In our previous work, we found a strong correlation between hemispheres in the long-period Pc5 band ( 1 to $10 \mathrm{MHz}$ ). Now, we are looking at the local correlations of events which are observed at conjugate sites.

In an initial survey of correlations between signals detected at various stations in one hemisphere, we have found a significant phase variation ordered by local time. There is a persistent and consistent sign change in the phase difference berween stations. We believe these phase changes reflect the nature of the oscillations driven on the last closed field lines by boundary processes.

AFOSR Grant F49620-95-1-0005: Scientific personnel-J.V. Olson, B.J. Fraser, C.A. Szuberla, H. Zhu, and J. Williams

Inversion of the Auroral Electrojets from Magnetometer Chain Data Based on the Flexible Tolerance Method: An improved method has been developed to invert a one-dimensional east-west electrojet from the $\mathrm{H}$ component of the geomagnetic disturbances measured along a meridian chain of magnetometer stations. This nonlinear inversion method is based on the flexible tolerance method. The main difference between this new method and the previous methods is that the height, the width and the location of the auroral electrojet current are determined together from the data by the flexible tolerance method, rather than assumed or calculated independently as in the previous methods. It should be noted also that the method de- 
vised in this study is fast enough to allow a real-time display of the inverted electrojet current distribution by a workstation.

NSF Grant ATM93-11474: Scientific personnel-W. Sun, $Y$. Kamide (Nagoya University Solar-Terrestrial Environment Laboratory), J.R. Kan, and S.-I. Akasofu

Correlations Between Auroral and Equatorial Electrojets: In a collaborative effort between the STE Laboratory of the University of Nagoya

\section{Ionospheric Physics}

Support and Communications for the High-Latitude Monitoring Service at Elmendorf AFB: The HighLatitude Monitoring Service (HLMS) is located at Elmendorf Air Force Base, Alaska, and has been in continuous operation since the early 1960s. HLMS was sponsored and supported by the NOAA Space Environment $\mathrm{Lab}$ (SEL) and the Air Weather Service of USAF. HLMS provided selected high-latitude and solar-terrestrial data in near real-time to numerous customers. In 1993, both of the sponsors made the decision to cease funding HLMS in spite of the use of the data by various organizations.

In order to keep this valuable source of high-latitude geophysical data available to the users, the Geophysical Institute of the University of Alaska Fairbanks organized a group of users to aid in the continuation of HLMS at least through May of 1995. One-time funding commitments were made by NOAA/SEL-Boulder, $\mathrm{CO}$; the Geophysical Institute; the High-Power Active Auroral Project and the Geophysical Institute, geomagnetic recordings have been initiated at Kotzebue, Alaska, in the auroral zone, and at Koror, Republic of Pelau, at the magnetic equator. The signals recorded at Kotzebue reflect the changes in the auroral electrojet that accompany global storm and substorm events. Koror, on the other hand, shows magnetic signals that are a reflection of the equatorial electrojet. We have found a direct correlation between the two data sets and found a direct corre-

(HAARP); and the Naval Security Group Command through the Applied Research Labs of Penn State University. At this time, we support a three-quarter time site technician and provide the necessary logistical support for HLMS. Data acquired at HLMS includes information form a three-axis magnetometer, $30 \mathrm{MHz}$ Riometer, $50 \mathrm{MHz}$ auroral radar and total electron content polarimeter. Some of these data are placed on the Internet, and all of the data are transmitted to the Geophysical Institure. It is hoped that additional support for HLMS will be obtained from the user community to keep HLMS operating.

Department of Commerce Grants PO 40RANR 402881 and PO 194639, and support funds from Penn State University: Scientific personnel-R.D. Hunsucker

D- and E-Region IncoherentScatter Radar Observations at Sondrestrøm, Greenland: The primary objective of this work is to make new observations of the very- spondence between the hour-long variations. In fact, it is easy to see correlations on a finer level when the data are carefully analyzed. We also have noted a correlation between auroral activity and the magnitude of the diurnal component in the equatorial electrojet, a new observation. We are continuing to gather and analyze the data in order to understand these observations.

Scientific personnel-S.-I. Akasofu, J.V. Olson, W. Sun, K. Yumoto, and $K$. Shiokawa

high-latitude ionosphere with high spatial resolution $(300$ and 600 meters) using the NSF incoherentscatter radar facility at Sondrestrøm, Greenland. Experiments have been conducted to measure thin metallicion structures in the lower ionosphere at heights from 80 to $120 \mathrm{~km}$. The radar has been operated in an elevation scan mode to map the latitudinal extent of these thin structures.

Under the action of the large-scale electric fields, which are frequently present at high latitudes, long-lived metallic ions $\left(\mathrm{Fe}^{+}, \mathrm{Mg}^{+}, \mathrm{Ca}^{+}, \mathrm{etc}\right)$, which originate from meteor ablation, may accumulate into very thin layers with about one-km thickness. We have begun an experimental program to map the characteristics of these structures. A numerical model also has been developed to predict the spatial occurrence of these layers, and, by comparison with observational data, we expect to verify the relative roles of electric fields and neutral winds in the formation mechanisms.

The model is capable of simulating the time-dependent behavior of differ- 
ent ion masses to demonstrate vertical motions, formation of multiple layers, etc. Another task accomplished is the development of a large-scale, time-dependent Eulerian model of the polar ionosphere. This model, in its final stages of development, is expected to be used for comparisons with radar and other experimental data.

NSF Grant ATM-9213969: Scientific personnel-B.J. Watkins, D.D. Rice, S. Bhattacharyya, S.A. Maurits, D.F. Bedey, and Q.-L. Min

Site Support for the Digital Ionospheric Sounder: The Geophysical Institute provides site support services to the Air Force for the operation of the digital ionospheric sounding system (DISS). Institute personnel are responsible for site upkeep, utilities, and routine monitoring. Data are forwarded to the Air Force Geophysical Laboratory at Hanscom AFB, MA.

U.S. Air Force Contract F6550393-M-5028 and F65503-94-M5002: Scientific personnel-B.S. Delana

\section{Modeling of Ionospheric Auroras}

Theoretical and Experimental Research on the Aurora Borealis: The optical aurora is the result of a variety of physical and chemical processes that occur in the upper atmosphere when it is bombarded with energetic particles. This project is a continuing effort to understand the details of the interaction between precipitat- ing particles and atmospheric gases. This includes not only the excitation of spectral emission features, but also the effect of the aurora on the composition, dynamics, energetics, and electric properties of the upper atmosphere.

Over the years, we have developed a sophisticated electron transport code that solves the linear Boltzmann equation. We evaluated this code and the uncertainties that arise from various input parameters and assumptions-electron-neutral collision cross sections, angular redistribution in various types of collisions, and energy degradation. The electron transport calculation is an important part of auroral models.

Other energy sources for aurora come from precipitating protons. Charge exchange reactions between protons and atmospheric molecules convert a precipitating proton flux into a stream of a mixture of energetic hydrogen and protons.

We developed a numerical procedure to describe the transport of a proton/hydrogen stream into the atmosphere to be combined with our auroral model.

Polar wind is a continuous outflow of light ions from the earth's ionosphere near the poles. Helium ions which are generated in the thermosphere are transported upwards from a collision-dominated region of the atmosphere into the collisionless plasma of the magnetosphere.

The transition region between these two domains is particularly difficult to treat. This problem was solved with a numerical treatment of the Fokker-Planck equation. The model gives a satisfactory descrip- tion of the helium ion escape flux in the polar caps.

NSF Grant ATM 90-22197: Scientificpersonnel-M. H. Rees, $\emptyset$. LieSvendsen, and D. Lummerzheim

Particle Environment Monitor (PEM) on the Upper Atmosphere Research Satellite (UARS): On 12 September 1991 the UARS observatory was launched into orbit by the NASA space shutrle and was turned on in the following weeks. The satellite carries a plethora of instruments for the remote sensing of the middle atmosphere as well as for in situ observations.

The instruments give information of composition, temperature, and wind in the altitude range from 15 $\mathrm{km}$ to $120 \mathrm{~km}$ over the entire globe between about 80 degrees north to 80 degrees south. Our involvement with UARS is primarily through the PEM instrument. The PEM instrument consists of three major parts: a down-looking $\mathrm{x}$-ray imager to observe the aurora; a magnetometer; and a particle sensor to detect electrons and protons in the energy range from thermal to relativistic.

The particle measurements above the atmosphere (UARS orbits at 585 $\mathrm{km}$ altitude) are crucial input parameters to models for the thermosphere and middle atmosphere. High-energy particles penetrate the middle armosphere, causing local ionization and producing $\mathrm{x}$-ray emissions. The $\mathrm{x}$-rays penetrate even deeper into the atmosphere and contribute to ionization beyond the range of the original particles. Both of these ionization sources contribute to the chemistry in the middle atmosphere, a region that has attracted much attention. 
Southwest Research Institute Subcontract 77820: ScientificpersonnelM.H. Rees, and D. Lummerzheim

Theoretical and Experimental Research on the Aurora Borealis: The aurora appears in a great variety of forms and shapes. Tall auroral rays extend to a very high altitude and have an almost constant luminosity over many scale heights. With this investigation we are combining an auroral model and a fluid simulation to provide a local acceleration mechanism for electrons, which should produce the appearance of tall rays. The thrust of this investigation is aimed ar finding and confirming mechanisms that are responsible for much of the fine structure that makes up the shape of the aurora.

NSF Grant ATM 94-09485: Scientific personnel-D. Lummerzheim, A. Otto, and M.H. Rees

Composition and Temperature of the Auroral Ionosphere and the Lower Thermosphere: Although the aurora is a global phenomenon, an individual auroral curtain or ray is a small-scale phenomenon. Just as global weather models cannot include individual clouds, global models of the ionosphere cannot include the local effects of the aurora. The largescale effects of precipitating particles that also cause the aurora, however, have to be taken into account in global models.

For example, the NCAR TIGCM (Thermosphere-Ionosphere General Circulation Model) is dependent on suitable parameterizations of the aurora for this purpose. We have developed a one-dimensional ionospheric model that is geared to auroral mod- eling. This small-scale model accepts precipitating electrons as input and transports them into a neutral atmosphere that is given by large-scale models. Other sources of energy and ionization include solar EUV, solar $\mathrm{x}$-ray, galacticx-ray, geocoronal EUV, and magnetospheric heat flux. Most of these sources show a temporal dependence with time scales varying from seconds to hours. We solve the continuity and energy equations for the ionospheric major and minor ions in a time-dependent manner. As the model runs, an ionosphere evolves and we can calculate the density and temperature of ions and electrons, as well as emissions and brightness of the aurora.

We have applied this model to the interpretation of auroral observations in a number of situations. We were able to combine particle observations from spacecraft with ionospheric probing by the Sondrestrøm incoherent scatter radar in a characterization of the ionosphere in the cusp.

This presents a case of very-lowenergy electron precipitation in a sunlit environment. The other extreme is intense nightside aurora. In cooperation with the University of Southampton, England, we analysed data from an EISCAT campaign. Here we had high-time resolution radar observations and ground-based TV images and photometer observations of a bright and short-lived brightening of a narrow auroral arc.

Using an adaptation of the ionospheric model capable of a time resolution of a few milliseconds, we found the obser-vations to be consistent with an elemental auroral structure caused by near monoenergetic electrons at about $8 \mathrm{keV}$ carrying an energy flux of about $400 \mathrm{erg} \mathrm{cm}^{-2} \mathrm{~s}^{-1}$. This intense electron precipitation lasted for about three seconds and was confined to an arc no more than $-1 \mathrm{~km}$ thick.

NASA Grant NAGW 3037: Scientificpersonnel-D. Lummerzheimand M.H. Rees

Modeling of the Atmosphere-Ionosphere-Magnetosphere System: NASA's Global Geospace Science (GGS) program is part of the International Solar Terrestrial Physics (ISTP) initiative that will focus on the exploration of the solar terrestrial environment during this decade. GGS includes ground-based observation, data analysis and interpretation, theory, modeling, and several satellites. GEOTAIL and WIND already have been launched and are operational; POLAR, SOHO, CLUSTER, and INTERBALL are additional upcoming launches.

An important element of the ISTP/ GGS program is the combination and distribution of data from all instruments to all participating scientists. To provide an overview of the available data, a set of key parameters has been defined, and is distributed via $C D-R O M$ at regular intervals. The key parameter distribution also includes data from other satellites (IMP, GOES, and LANL) and several ground-based observations, including incoherent scatter radar, high frequency radar at various sites, ionosonde, magnetometer, riometer, and optical observations.

In preparation for the launch and data acquisition phase of the mission, we are developing codes to ana- 
lyze global images of the aurora that will be acquired by instruments on the POLAR spacecraft, which is Ischeduled for launch in December 1995. The aurora will be imaged in several wavelengths characteristic of atmospheric species. A detailed study of spectral emissions may be used to characterize the energetic particles causing the aurora. As a result of this analysis, we will obtain ionospheric conductances and auroral precipitation patterns on a global scale. All available ionospheric data and derived parameters will be combined to give a consistent description of the ionosphere and thermosphere.

NASA Grant NAG5-1097: Scientific personnel-M.H. Rees, D. Lummerzheim, and R.G. Roble (National Center for Atmospheric Research)

Formation and Evolution of Thin Discrete Auroral Arcs: Properties of auroral discrete auroral arcs are: (a) the small width of these curtains of luminosity of $40 \mathrm{~m}$ to a few $100 \mathrm{~m}$; (b) deformation and splitting of arcs/ field-aligned current sheets; (c) recently observed fast shear flow close to and along discrete arcs; (d) the relation to field-aligned plasma transport; and (e) the relative motion between plasma and discrete arcs, i.e. the arc is not frozen into the plasma.

We have suggested a model of current striation (CS) for the formation of very thin auroral arcs, which is based on a three-dimensional magnetic reconnection process in the inner magnetosphere. The process requires a field-aligned current (FAC) layer that separates sheared magnetic fields. The reconnection regions are arranged along vertical $x$-lines due to the upward field-aligned current layers. In the suggested model, multiple arc systems imply a corresponding number of parallel vertical $x$-lines.

The CS model was investigated by 3-D numerical simulations, which demonstrate several characteristic features of discrete auroral arcs. The mapping of a localized parallel electric field in a sheared magnetic field generates narrow electric potential structures elongated in the west-east direction. The width of these structures is smaller than the width of the current layers, demonstrating that only part of the field-aligned current is carried by energetic particle precipitation. The individual process leads to the splitting of one FAC sheet into two current sheets. In a cascade from large to small geocentric distances, this can generate the observed multiple current sheets at ionospheric altitudes. The splitting of the FAC sheets is associated with upward and downward bulk plasma motion, which may contribute to the plasma transport from the ionosphere into the magnetosphere. The developing strong horizontal shearing motion generates convection electric fields, showing the same amplitude and altitude dependence as observed for spiky electric fields.

DOE GrantDEFG06-91ER 13530 and NASA Grant NSPTP-NAG5-1504: Scientific personnel-A. Otto and $G$. Birk (Ruhr-University Bochum)

\section{Thermospheric and Mesospheric Dynamics}

Studies of Mesospheric Dynamics:

These studies have been conducted using observations of wind, temperature and airglow emission rate from the Amundsen-Scott Station (South Pole), Antarctica. The observations were made optically using the $P_{1}(2)$ doublet of the (6-2) band of the hydroxyl Meinel system at $8400 \AA$. They were treated as a time series to find the periods of persistent oscillations. Since measurements were made at eight equally spaced azimuths, data were collected pertinent to an equal number of local times. Hence, it was possible to observe the passage of wave fronts of global-scale waves as they advanced in local time, and also to determine the azimuthal wave number of identified oscillations.

The observations permitted the investigation of waves with periods extending from 2 hours to 200 hours. In this range, two regions appeared in the winds that dominated the power spectrum: between six and 12 hours, and from 30 to 90 hours. It was found that the waves in the former were westward, while those in the latter were eastward. All persistent waves had azimuthal wave number one. Hence, there was no observable semidiurnal tide, even though periods close to 12 hours did appear. This result agrees with tidal theory, which predicts that tides diminish to zero as the pole is approached. It was suggested that the dominant wave in this range, at about 10 hours each period, was a Lamb wave.

The spectrum of waves in the temperature showed little activity, and did not show significant power at the same periods as for winds. Also, where there was sufficient power to identify 
the azimuthal wave mode, the azimuthal wave number was found to be zero. Stronger waves appeared in the emission rate spectra. Like the temperature waves, all of these were wave number zero also. The absence of waves number one in temperature and emission rate and the presence of those waves in the wind is understandable in terms of the damping of higher mode waves by viscosity.

The amplitudes of the wind waves observed at different azimuths were not equal, but varied over a factor of two. This is difficult to understand if waves are thought to circle the pole with a local source. It is preferable to consider a distant source with meridional propagation towards the pole. The wave amplitude variation can be accounted for by differences in the propagation characteristics as a function of local time or azimuth.

Time-dependent studies of these waves show that there are variations of wave amplitude occurring on timescales of days. In one case on record, it appears that an oscillation near three days period dies, locally generating another wave disturbance that has a longer period and that begins with a short burst of waves having much greater temperature amplitude than normal. Possibly, this is a case of a breaking planetary wave.

NSF Subcontract through University of Washington 600232: $R$.W. Smith, G. Hernandez (University of Washington), and J.F. Conner

Ion Drift and Ion Temperature Studies: From high-resolution observations of the $7320 \AA$ oxygen ion doublet, determinations of the ion temperature and line-of-sight ion drift were obtained. Since the neu- tral winds and temperatures were determined simultaneously at the same height, it was of interest to compare the values obtained on a statistical basis. Ion temperatures were found to be 100 to $200 \mathrm{~K}$ higher than neutral temperatures, and lineof-sight ion drifts normally exceeded the wind. This finding was consistent with the expectation that the neutral atmosphere is driven by the drag of ion-neutral collisions. The heating effect of ion drag raises the ion temperature more than neutral temperature because of the much lower concentrations of ions.

NSF Subcontract through University of Washington 600232: R.W. Smith, G. Hernandez (University of Washington), G.F. Fraser (University of Canterbury, New Zealand)

Study of Upper Thermospheric Temperatures at the South Pole: Using the six years of observations of upper thermospheric temperatures made at South Pole from 1989 to the present, a study was made of the behavior of daily averages and diurnal averages. Comparison was made with the MSIS86 thermospheric model as a benchmark.

Daily averaged temperatures were found to be in reasonable agreement with the MSIS86 prediction. At solar maximum, low geomagnetic activity resulted in temperatures lower than predicted by $100 \mathrm{~K}$. At high activities, observed temperatures were higher than predicted, again by about $100 \mathrm{~K}$. Away from solar maximum, the amplitude of these departures decreased and there was better agreement between measurement and model.

Observed diurnal variations corresponded to the model in amplitude and phase. However, there were notable temperature gradient effects in the data, which consistently showed a higher temperature towards higher latitude on the nightside and much weaker gradients on the dayside. Comparing the diurnal variation with the average wind pattern observed, it appears that the low temperature occurs when air is advected into the polar cap from lower latitudes on the dayside and the high temperature occurs where air is expelled to lower latitudes on the nightside. In an averaged sense, the temperature measurements reflect the heating imparted to a thermospheric air parcel crossing the polar cap.

NSF Subcontract through University of Washington 600232: R.W. Smith, G. Hernandez (University of Washington)

Vertical Winds: Measurement of the Doppler shift of optical emissions from the aurora and airglow can be used to infer vertical winds in the atmosphere at the height of emission. Such winds must cause major raising or lowering of the atmosphere, and are expected to be of small scale and to be short-lived when they exist. Several studies have been done to investigate the occurrence and strength of vertical winds in the auroral zone and at higher latitudes.

Using the high-resolution FabryPerot Interferometer at Poker Flat Research Range, vertical winds were measured at lower and upper thermospheric altitudes. In the 14 days of measurement, the major event consisted of a vertical upward wind of about $150 \mathrm{~m} / \mathrm{s}$ in the upper thermosphere which lasted for approximately 20 minutes. A corresponding up- 
ward wind of $40 \mathrm{~m} / \mathrm{s}$ was observed in the lower thermosphere. The magnitude of the upper thermosphere wind was consistent with scattered reports from other observers at other places and times. Data from the meridianscanning photometer and all-sky television camera showed that an important factor concerning the cause of this upward wind was its occurrence just on the poleward side of arcs in the expansion phase of a geomagnetic substorm. Indeed, the breakup started within a few minutes of the observed large vertical wind. Subsequent measurements of lower thermospheric upward winds made at Fort Nelson, British Columbia, confirmed that the association with a prebreakup arc system is quite common.

Observations of vertical winds from South Pole (Amundsen-Scott Station) show a different behavior which is related to geomagnetic time and apparently not to aurora arc position. It was found that a daily cycle of vertical winds occurs in the upper thermosphere in which there is a peak of downward wind near geomagnetic noon and upward wind neargeomagnetic midnight. The amplitude of the variation depends upon geomagnetic activity, reaching a peak of $150 \mathrm{~m} / \mathrm{s}$ for $\mathrm{Kp}=9$. At very low activity, the amplitude was $40 \mathrm{~m} / \mathrm{s}$. Also, the downward wind at noon was usually stronger than the upward wind at midnight, and it was more dependent on geomagnetic activity. In the lower thermosphere, the daily variation was strong at high geomagnetic activity, with a $40 \mathrm{~m} / \mathrm{s}$ amplitude.

The cause of upward winds associated with auroral arcs is believed to be Joule heating in the E-region of the ionosphere. The large downward winds at the South Pole are thought to be due to a strong divergence in the horizontal component of wind brought about by a divergence in the ion drift pattern.

NSF Subcontract through University of Washington 600232: R.W. Smith, G.D. Price, and G. Hernandez (University of Washington)

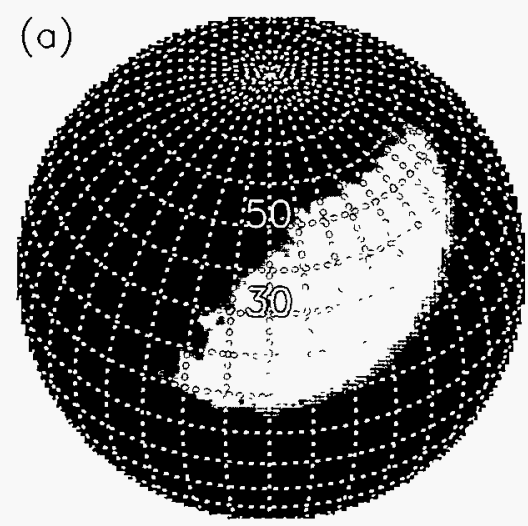

(b)
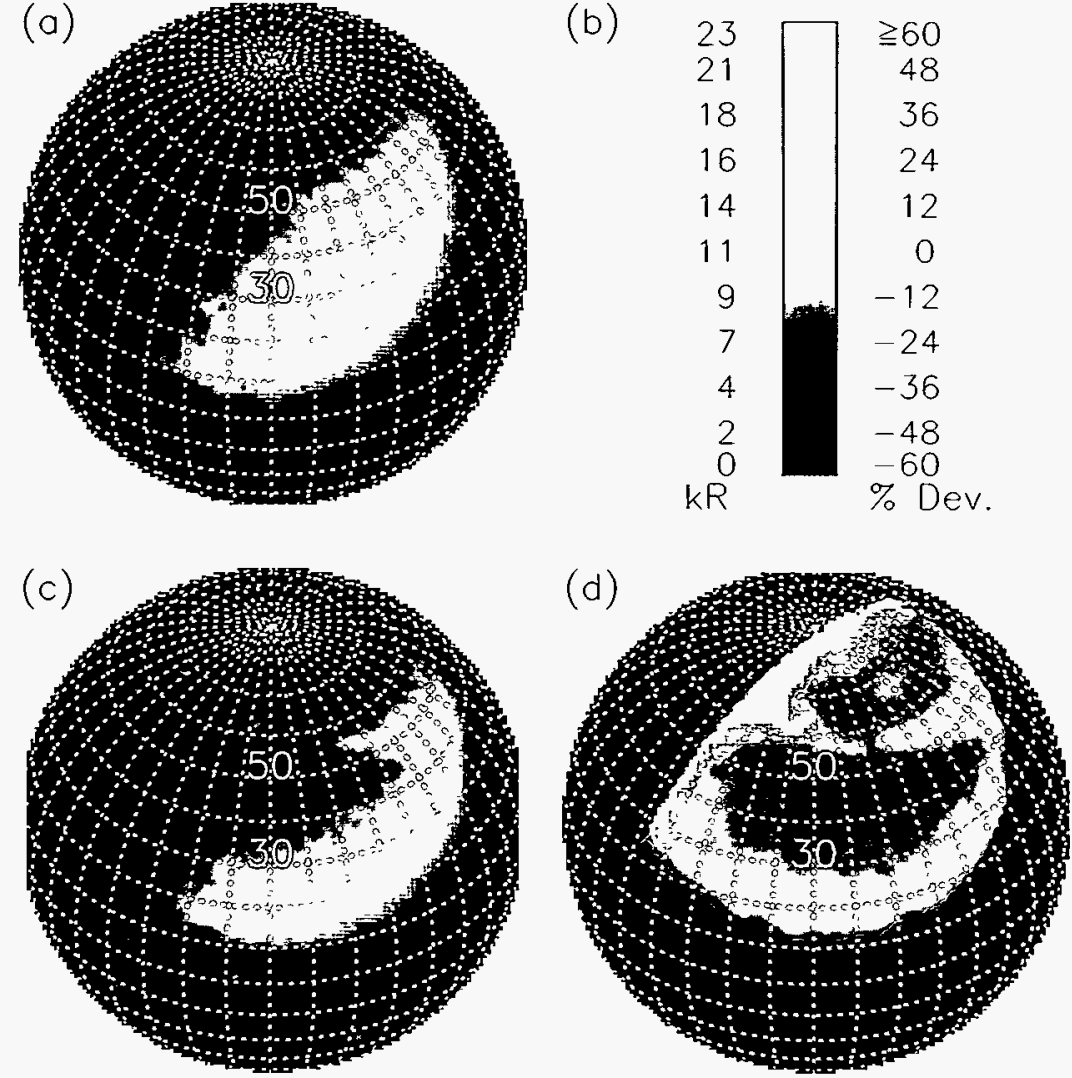

Variations in the FUV terrestrial dayglow at $130.4 \mathrm{~nm}$ denote changes in thermospheric composition. The FUV dayglow at 123-160 nm shown in panel (a) was obtained on a magnetically quiet day with an auroral imager on the DE-1 spacecraft. Dominant contribution is by atomic oxygen at $130.4 \mathrm{~nm}$. The steady increase in brightness with decreasing solar zenith angle is characteristic of the quiet-time images. The sun is to the right in this remapping of the original image, which uses an orthographic projection with geographic coordinates. The center of the image is 40 degrees $N, 30$ degrees $W$, at $\sim 0830$ local sun time. The North Pole is toward the top. Absolute brightness is coded in panel (b) in units of kilorayleighs (left column). In contrast, the dayglow in a highly active day (22 October 1981) is shown in panel (c). Note the aurora at high latitudes and the decrease in FUV brightness at midlatitudes near the morning terminator. The percent change in FUV brightness during the active period relative to reference values computed from a large collection of observations in magnetically quiet periods is shown in panel (d). The area equatorward of 55 degrees $N$ and bounded by the black contour is $\sim 10^{7} \mathrm{~km}^{2}$, and the maximum depression in brightness is $\sim 55$ percent. This represents greater than a factor of two decrease in the thermospheric column density of atomic oxygen. The percent change is coded using the right column in panel (b). Auroral emissions at high latitudes artificially increase the percentage deviation. 
later at subauroral latitudes in the morning sector of local time.

For example, in-situ observations with the ESRO-4 spacecraft show that the densities of $\mathrm{Ar}$ and $\mathrm{N}_{2}$ can increase at the $280-\mathrm{km}$ altitude by factors as great as -100 and -10 , respectively, while $\mathrm{He}$ decreases by $\sim 10$. The variations in $\mathrm{O}$ are smaller in magnitude and can increase or decrease. The spatial extent and magnitude of the variations both increase with increasing magnetic activity. The $\mathrm{O} / \mathrm{N}_{2}$ ratio at the $280-\mathrm{km}$ altitude can decrease by more than a factor of 10 , and the variations in composition can extend to geomagnetic latitudes below 30 degrees during periods of intense magnetic activity.

Variations in composition also are observed through spacecraft-based optical observations, where, for example, S3-4 observations in the nadir direction at altitudes $<270 \mathrm{~km}$ and near 60 degrees latitude showed $>30$ percent decreases in brightness during the active period for O-related emissions at $130.4,135.6$ and $164.1 \mathrm{~nm}$.

In each of these studies, the instantaneous spatial extent of O-density variations was only determined as a function of latitude, and temporal resolution was limited by the orbital periods of the spacecraft.

In this new work, the analysis extends these earlier observations by others so that variations in $\mathrm{O}$ can be determined simultaneously in both latitude and longitude, and monitored wirh improved temporal resolution. This is possible with available broadband FUV observations on the DE-1 spacecraft, for which the dominant response is at $-130 \mathrm{~nm}$ and in the presence of two dominant sources: photoelectron excitation by solar
EUV radiation and resonant scattering of the solar component at 130.4 $\mathrm{nm}$. The observations show that localized decreases in the FUV brightness are generally observed in the morning sector following intense auroral activity in which the $\mathrm{AE}$ index exceeds $\sim 1000-1500 \mathrm{nT}$ for several hours.

These decreases are interpreted as being the consequence of the auroralassociated heating, which reduces the thermospheric column density of $\mathrm{O}$ relative to that of $\mathrm{N}_{2}$. An outstanding example of such a decrease is summarized in the accompanying figure, where the FUV dayglow has decreased by more than 25 percent over an area in excess of $10^{7} \mathrm{~km}^{2}$, and the maximum localized depression in brightness is $\sim 55$ percent.

We conclude that an $\mathrm{O}$ density depression in excess of a factor of two has occurred within this region. The maximum depth of the decrease lessened during more than two hours of observations. These remote observations provide the first instantaneous, two-dimensional measurement of the large-scale spatial extent of such a change in thermospheric composition.

NASA Grant NAGW 3436: Scientific personnel-J.D. Craven, T.J. Immel, L.A. Frank (University of (owa), A.C. Nicholas, and D.J. Strickland (ComputationalPhysics, Inc.)

Studies of Upper Thermospheric Dynamics: The June 1991 Storm: Utilizing data gathered from our instruments at Amundsen-Scott Station (South Pole), Antarctica, and Mr. John, New Zealand (44 S, 171 E), a study has been made of the geomagnetically active period extending from 5 - 13 June 1991. Optical measurements of upper thermospheric winds and temperatures were made from both sites. Clear weather continued from 1 - 15 June at South Pole, and 11 - 13 June at Mt. John, enabling a study covering a wide range of latitudes.

The geomagnetic $\mathrm{Kp}$ index varied from one to nine in the period of observation. Thermospheric temperatures at South Pole were observed to peak near $2200 \mathrm{~K}$ at the height of the disturbance, falling to $1200 \mathrm{~K}$ after it had subsided. The winds in excess of $600 \mathrm{~m} / \mathrm{s}$ were measured at South Pole. At Mt. John, which is normally well equatorward of the auroral zone, there were large gradients in wind and temperature, indicating that the storm effects did reach to middle latitudes. In addition, there was visible aurora towards the pole.

Comparison was made with standard wind and temperature models showing that there were distinct disagreements in wind direction and magnitude near Mt. John. Meridional winds were in reasonable agreement at South Pole, but, again, there were disagreements in the zonal components. On the other hand, the temperatures recorded were similar to those predicted by the MSIS86 model.

In the wind model, no account had been taken of changes in the ionospheric convection pattern, which would be expected for the changes of the By component of the interplanetary geomagnetic field. It was concluded that the omission of this factor in determining the winds could well account for the differences between observation and model, particularly at high latitudes. It also appeared that the major discrepancy between data and model at 
midlatitudes was more obvious for one sign of By than the other. However, there are too little data to justify a firm conclusion in this case.

Funded by NSF Subcontracts through University of Washington 600232 and 385298: Scientific personnel-R.W. Smith, G. Hernandez (University of Washington), G.F. Fraser, S. Smith, and M.Clark, (University of Canterbury, New Zealand)

Model Auroral Electron Precipitation Patterns for Coupled Thermosphere-Ionosphere Simulations: This work is creating new globalscale models of auroral electron precipitation at improved temporal resolution for use with the general circulation models of the coupled thermosphere and ionosphere.

Basis for the new models is the existence of an extensive collection of DE-1 auroral images obtained simultaneously at ultraviolet and visible wavelengths, and from which the energy flux, $Q_{e}$, and characteristic energy, $\mathrm{E}$, of the precipitating auroral electrons can be estimated using existing numerical codes for the luminous output as functions of model input electron spectra. The unknown albedo background at visible wavelengths must be estimated using an iterative technique that compares $\mathrm{Q}_{\mathrm{e}}$ and $\mathrm{E}$ obtained with each of the two-color pairs of images.

One model is being prepared as a function of AE for all levels of activity, quiet to active, and the second is for isolated, simple substorms as functions of time, peak $A E$ in the substorm, and the initial diameter of the auroral oval. Global-scale maps of conductance due to ionization produced by the incident electrons then can be inferred. The models are intended for investigators in need of realistic model energy inputs from auroral electron precipitation on a truly global scale, but who do not require for a particular investigation the highly detailed information contained in the original spacecraft images.

NASA Grant NAGW 3436: Scientific personnel-J.D. Craven, D.W. Hembroff, and T.J. Immel

\section{Upper and Middle Atmospheric Optical Flashes}

Red Sprites and Blue Jets: Lightning from thunderstorms recently has been determined to produce several types of luminous electrical phenomena in the middle atmosphere above the storms, in the space between the tops of the storm systems and the ionosphere. "Red Sprites" and "Blue Jets" are two types of oprical discharges associated with thunderstorms that have been studied in a series of jet aircraft flights over the American Midwest conducted by the Geophysical Institute during June and July 1994. Only recently have Red Sprites and Blue Jets been studied as interestinggeophysical phenomena. Anecdotal accounts of these events have appeared sporadically in the literature for more than 100 years, but it has only been within the past few years that they have been documented on video tape. We have been engaged in one of the first systematic efforts to document and study them as electrodynamic links in the global electrical circuit. We have also studied their possible impacts on the chemistry of the middle armosphere. Red Sprites are diffuse red luminous events above thunderstorms that appear to be associated with simulta-

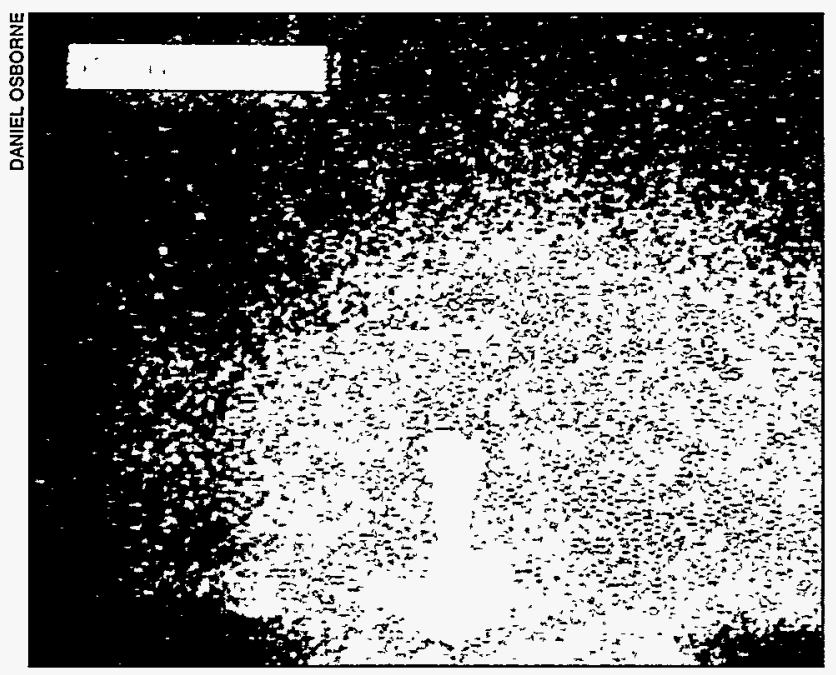

This large Blue Jet was filmed from a plane over eastern Arkansas on June 30,1994, using a very wide angle low-lightlevel, black-and-white television camera. The existence of these Jets has been reported in anecdotal accounts during the last century. neously occurring lightning flashes in the underlying storm system. Sprite characteristics differ from those of classical cloud-to-ground discharges or intracloud discharges. They are of longer duration than return strokes, typically persisting for less than $10 \mathrm{~ms}$, and they extend from $35 \mathrm{~km}$ to more than $90 \mathrm{~km}$ altitude and range from $10 \mathrm{~km}$ to $50 \mathrm{~km}$ across, with volumes of several thousand cubic $\mathrm{km}$. They are thus mesospheric D-region phenomena. The upper regions are typically 


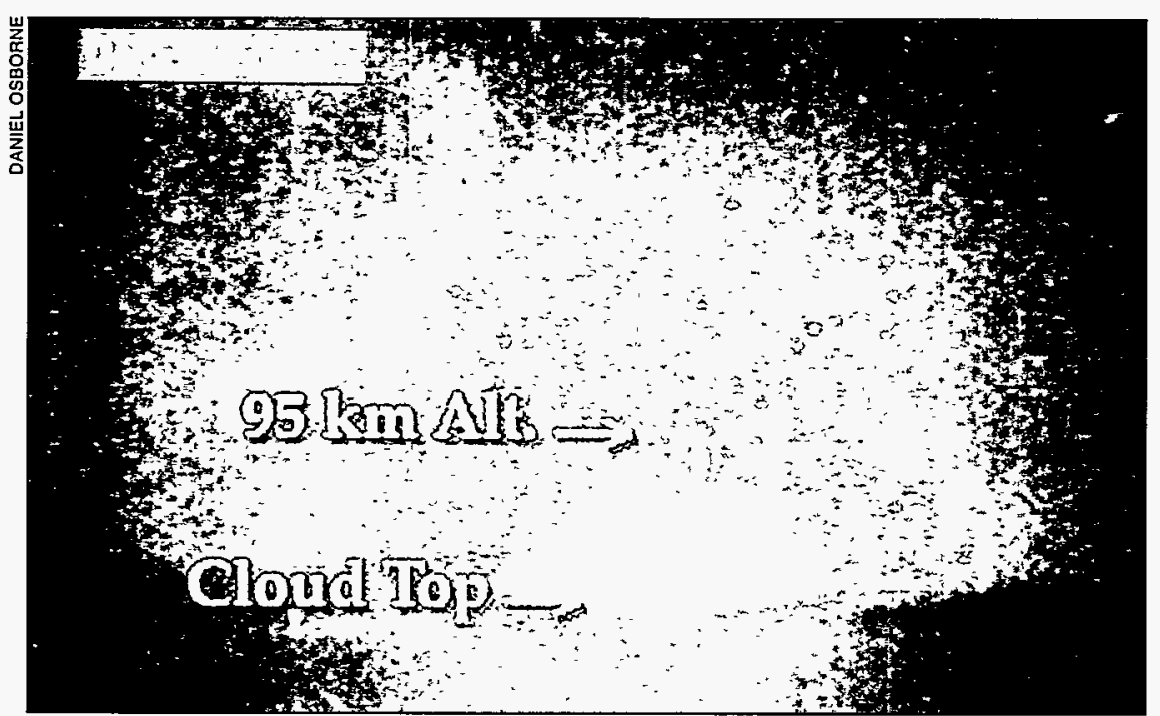

This family of Red Sprites was captured on video over the Midwest on July 6, 1994. A determination of their height and size was made by using triangulation of images of the same Sprites taken from two different aircraft.

red within the 50 to $90 \mathrm{~km}$ region, while the lower extremities at altitudes of 35 to $50 \mathrm{~km}$ are predominantly blue.

The lower blue region and the red tops of Sprites exhibit tendrils or dendritic structure. Red Sprites are not produced by every cloud-toground or intracloud stroke, but instead appear to be strongly associated with very large positive cloud-toground stokes occurring in the late stages of thunderstorm evolution.

Optical brightness measurements of Red Sprites show that the optical strength is at least equal to that of a strong aurora. However, it is believed that the red color arises from radiating molecular nitrogen and oxygen, with possibly some contribution from hydrogen Balmer alpha. Based on these assumptions, the associated electron density in Red Sprites is estimated on the order of $10^{5}-10^{6}$ $\mathrm{cm}^{-3}$ with mean energy of $2-4 \mathrm{eV}$. These data support the idea that the electrodynamic energy in Red Sprites is sufficient to induce significant chemical perturbations over several thousand cubic kilometers in the space above mesoscale storm systems. Present data are inadequate to assess if the resultant perturbations are longlived or important on a global scale, but ongoing research is directed toward addressing this question.

Blue Jets, as the name implies, are a distinctly different phenomenon, imaged by accident during several of our aircraft flights to study Red Sprites. They are narrowly collimated beams of blue light that spurt up from the tops of some active thunderstorm systems, propagating upward at speeds of approximately 100 $\mathrm{km} / \mathrm{hr}$ and reaching to altitudes of about $40 \mathrm{~km}$. Occasionally, an upward hemispherical shock front appears to be launched from the terminus of the Blue Jet, propagating upward another $10 \mathrm{~km}$ before dispersing at about $50 \mathrm{~km}$. It is currently believed that the blue color indicates emission from neutral or ionized molecular nitrogen. If this is true, then the corresponding elec- tron density is comparable to that of Red Sprites. An especially puzzling aspect of Blue Jets is the relatively long lifetime of the event $(-200 \mathrm{~ms})$, which is much longer than the lifetime of free electrons at altitudes of 20 to $40 \mathrm{~km}$.

Research on Red Sprites and Blue Jets is in its infancy. In current activities we are concentrating on two different aspects of Sprite and Jet phenomenology. The first is to obtain measurements of their optical spectra, which should provide crucial information on the production mechanisms. The second is to undertake a survey mission to ascertain the occurrence of these events in equatorial thunderstorm regions.

An aircraft mission is planned to the Amazon basin during February and March in 1995 to address these questions.

NASA Grant NAG5-5019 and NSF Grant ATM-9217161: Scientific personnel-D. Sentman, E. Wescott, D. Hampton, and $M$. Heavner; Engineering personnelD. Osborne

\section{Poker Flat Research Range}

Poker Flat Research Range (PFRR) is operated by the UAF Geophysical Instituteand is theonly university-owned rocket range in the world. It is primarily used for scientific sounding rocket launches, and the mission objectives usually involveauroral and polar middle -upper atmosphere research, but the relationship of the range to UAF provides additional support for groundbased, orbital, and engineering research, as well. 
The site is located on the Steese Highway, approximately 30 miles northeast of Fairbanks, making it the highest latitude launch site in the western hemisphere. The flight zones extend generally northward over northern Alaska and the Arctic Ocean.

The National Aeronautics and Space Administration (NASA), the Department of Defense (DoD), the National Science Foundation (NSF), and the National Oceanic and Atmospheric Administration (NOAA) all provide funding for various aspects of operations and research, but NASA/Wallops Flight Facility is responsible for the flight operations and contract oversight.

About 260 major sounding rocket experiments have been launched from PFRR, for an average of 10 launches annually (plus more than 1700 meteorological rockets), since construction began in 1968. All of the major sounding rocket types have been launched at least once from PFRR, but more than 60 of the early launches involved NikeTomahawk motors. The development of the larger 3-and 4-stage Black Brant X, XI, and XII vehicles has led to fewer, larger and more complicated missions in recent years.

A number of federal, state and private agencies, including the Bureau of Land Management and the State of Alaska, are involved in authorizing the impact of sounding rockets and payloads on the $100,000 \mathrm{~km}^{2}$ of downrange land. This large flight zone over land makes payload recovery practical and economical for a wide range of mission payload and vehicle configurations. Recovery of payloads on the arctic ice pack has not yet been deemed practical. All traffic in the flight zones is approved, monitored and coordinated by the Federal Aviation Administration (FAA). Countdown and flight information is also maintained in real time with the North American Air Defense Command.

The range head is a $21-\mathrm{km}^{2}$ area owned by UAF. Approximately half of this is for booster impact and longterm environmental studies. The other half is divided into three parts, designated as launch, telemetry, and science operations areas.

In the launch area are four principal launch pads: Pads 1 and 2 have 3,400-kg MRL rail launchers; Pads 3 and 4 each have a 9,000-kg rail launcher, but the Pad 4 launcher is scheduled for removal and replacement with a $15,000-\mathrm{kg}$ Athena rail launcher and enclosure. Pad 5 has a $2,000-\mathrm{kg}$ twin-boom rail launcher, which is currently dedicated to the Student Rocket Program as a part of Alaska Space Grant Program activities at UAF. Launch operations are directed from a $250-\mathrm{m}^{2}$ blockhouse. Windweighting data are acquired with an 80-m meteorological tower and balloon tracking by radar. Vehicle tracking is accomplished with a Tradat system in telemetry and a C-band radar, operated by NASA/WFF. NASA plans to upgrade this radar in 1995 . There is a $180-\mathrm{m}^{2}$ facility for rocket motor storage, and two (180- and $\left.250-\mathrm{m}^{2}\right)$ rocket motor assembly buildings. The $600-\mathrm{m}^{2}$ payload assembly building and Class-100 clean room are equipped to handle payloads up to 1 meter in diameter and 8 meters long, and weighing up to $4,500 \mathrm{~kg}$.

Other launch area facilities include two temporary office buildings, a balloon inflation building, maintenance shops, fuel depot, and an extensive inventory of specialized vehicles and trailers.

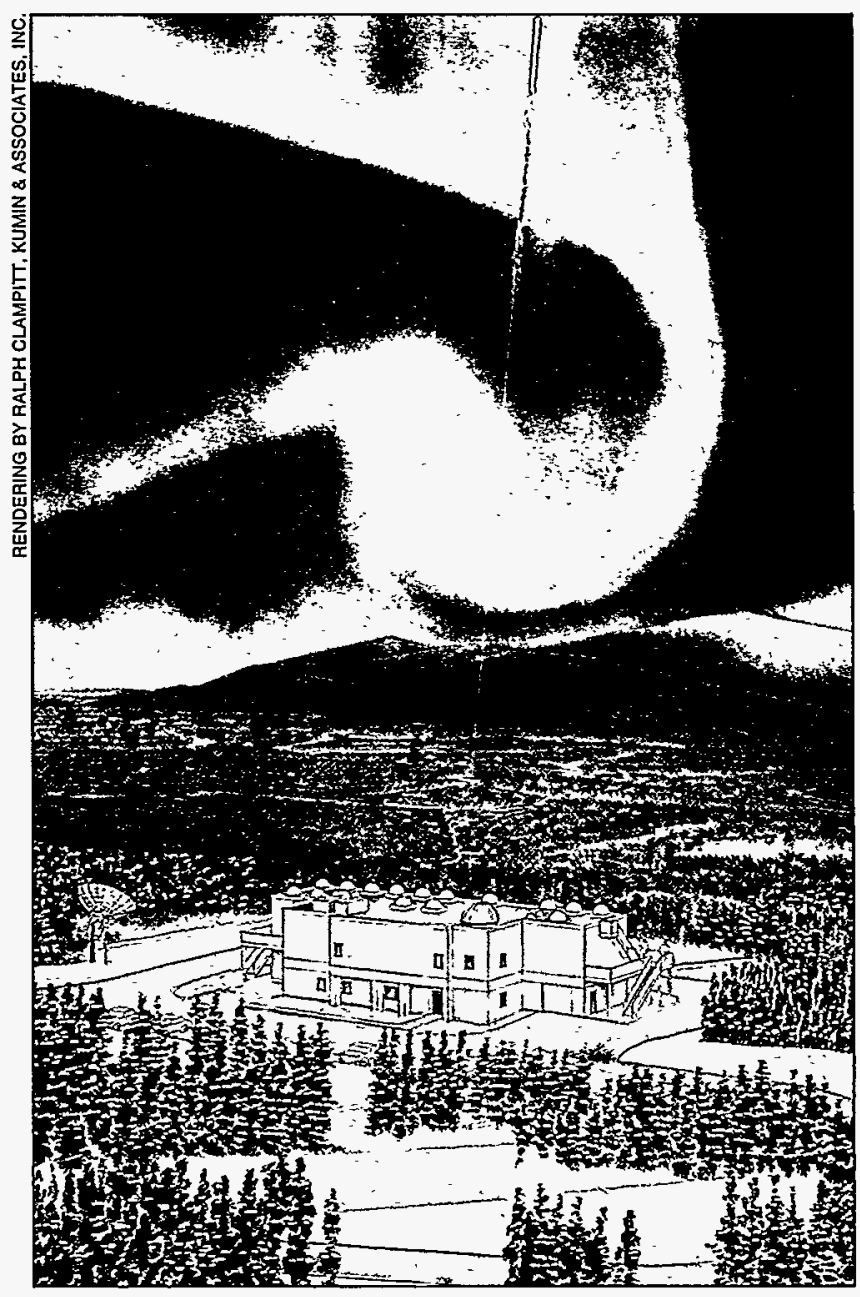

This architect's rendering pictures the T. Neil Davis Science Operations Center, scheduled for completion at the Poker Flat Research Range in 1995. 


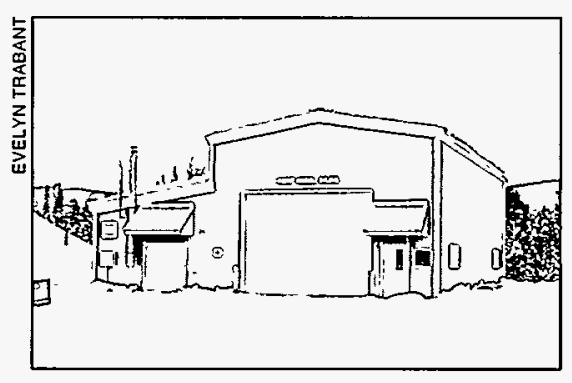

Shown is the rocket motor assembly building at Poker Flat Research Range.

The telemetry area is on a hill overlooking the launch complex. In addition to the $390-\mathrm{m}^{2}$ telemetry building in the base of the $15-\mathrm{m}$ diameter radome, an adjacent $390-\mathrm{m}^{2}$ facility was recently completed to allow for expanding orbital tracking responsibilities. NASA/ WFF owns and operates both orbital and suborbital S-band and L-band tracking systems supported by one 2.5-m, one 5-m, and two 8-m diameter tracking antennas. An 8-m Redstone tracking antenna will be installed in 1995.

The communications system has recently been upgraded to include an optical fiber ethernet on the range and a $45 \mathrm{Mbps}$ (DS3) digital microwave link to UAF, where various links to the Internet are available.

Launch criteria are usually based on a scientific assessment of ionospheric conditions over a large area of the western Arctic. Data from an array of observatories in Alaska and western Canada and from the NOAA Space Environment Lab in Boulder, Colorado, are transmitted over a broad-band communications network and will be displayed at the T. Neil Davis Science Operations Center. This 900- $\mathrm{m}^{2}$ observatory will be completed in April 1995. It will provide space for local optical observations (imagers, spectrographs, and interferometers), two launch-team decision areas, and the Geospace Environment Data Display System (GEDDS).

Additional observations of the lower and middle atmosphere are beginning to come on-line with the establishment of the Climate Change Monitoring lab, a GI Na-resonance LIDAR installation, and a cooperative program between GI and the Communications Research Lab of
(40.003 UE), for Roger Arnoldy of the University of New Hampshire, misfired on ignition at 10:43:41 on January 27 , and the vehicle came apart soon after leaving the rail. This was the thirtieth failure at PFRR out of 249 missions, for an overall success rate of 88 percent. Post mission analysis of the accident by the NASA/ Wallops Flight Facility engineering section showed that the Talos motor probably dropped below tempera-

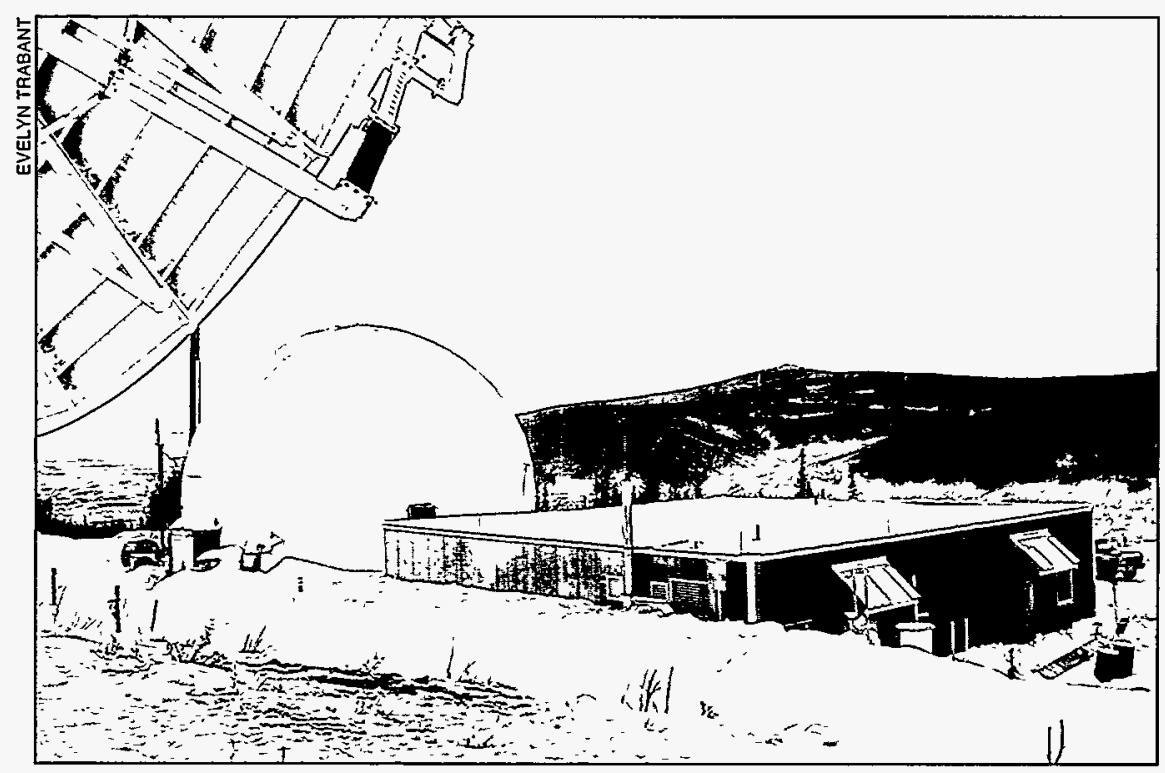

The new telemetry facility stands in the shadow of the new Transportable Orbital Telemetry Station (TOTS) $8 \mathrm{~m}$ antenna and the dome housing $2.5 \mathrm{~m}$ and $5 \mathrm{~m}$ antennas.

the Ministry of Posts and Telecommunications of Japan and the NOAA Environmental Technology Lab.

Operation and Maintenance of Poker Flat Research Range: Eleven sounding rockets were launched from Poker Flat during 1993 and 1994, bringing the total number of rockets launched to 257 since the inception of the range in 1968. All eleven missions were sponsored by NASA. The first of the rockets launched in 1993 was not a success. The first stage (Talos motor) of a Black Brant XII ture tolerance after 10 days on the rail at ambient temperatures of less than $-40^{\circ} \mathrm{C}$. This analysis led to a change in the design of the insulating

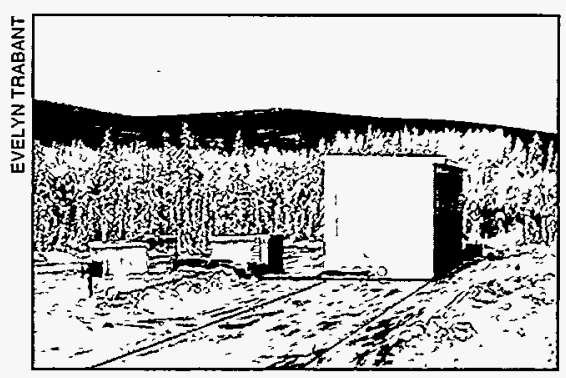

$A$ new launcher enclosure houses the $9000 \mathrm{~kg}$ rail launcher on Pad 3 at Poker Flat Research Range. 
box to extend it past the previously exposed rear engine fairing.

The second mission of the 1993 season was another Black Brant XII (40.004 UE) launched on April 2 at 10:09:00 UT for Charles Carlson of the University of California at Berkeley. Carlson's objective of measuring electric fields and waves at $1500-\mathrm{km}$ altitude above an auroral arc was accomplished and should lead to new knowledge of auroral electron acceleration processes.

The last mission of 1993 was a Nike/Black Brant VC (27.135 UE) launched on May 6 at 15:38:00 UT for George Parks of the University of Washington. Parks released a highaltitude balloon prior to the rocket launch in order to confirm that the dayside aurora was of the $x$-ray microburst type. The experiment successfully measured relativistic electrons associated with the microbursts in an attempt to determine their origin. The payload was recovered successfully, but a second launch (27.136 UE) was postponed.

The first launch of 1994 (27.137 UE) was scheduled at solar noon (22:00 UT) on January 30, 1994, for William Sharp of the University of Michigan. The data were compared with data from a similar mission launched from PFRR at noon on July 24,1992 . The objective was to determine seasonal changes in the concentration of naturally occurring, chemically active atoms and molecules near the $100-\mathrm{km}$ altitude region. Because the launch was scheduled for a certain time, it was witnessed by a large number of onlookers, mainly from Chatanika Lodge, located about one mile south of the range entrance.

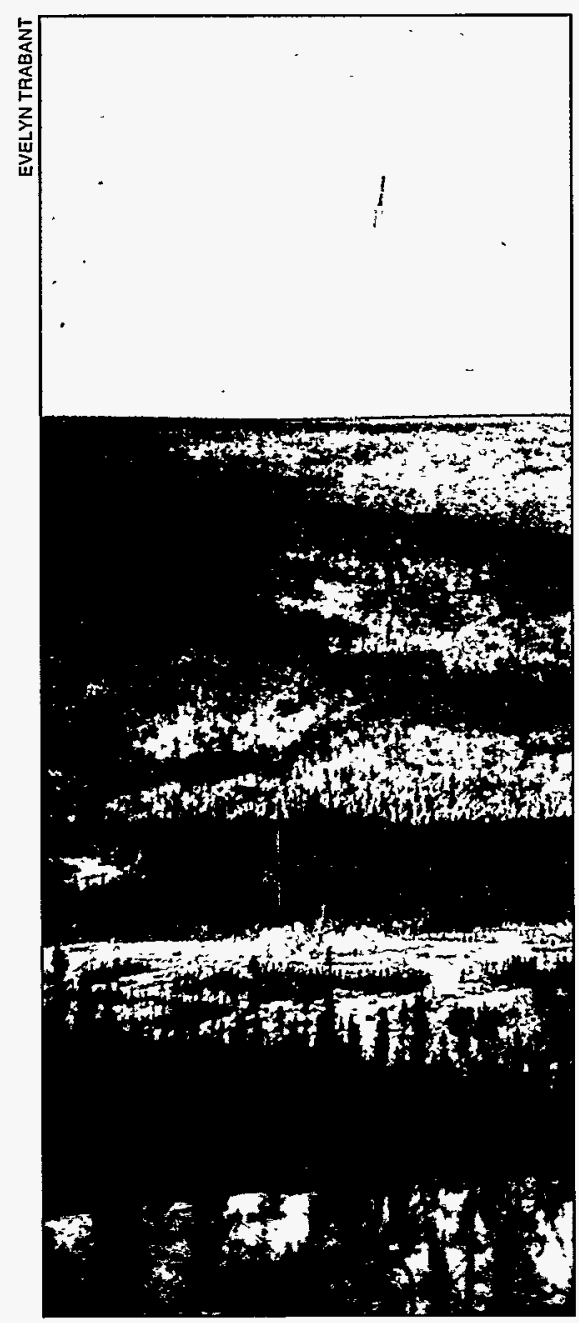

Launch number 250 at Poker Flat was a Nike-Black Brant $V$ for William Sharp of the University of Michigan.

TheARIA (Atmospheric Response in Aurora) campaign consisted of three rockets. An instrumented, Nike-Black Brant VC (27.131 CE, Andrew Christensen, Aerospace Corp.) was launched to measure atmospheric composition and incoming particle fluxes at 13:16 UT on February 12, 1994. It was supported by ground-based magnetometer and optical measurements of the input heating and momentum. The resulting complex effects on the atmosphere were measured also in the neutral and ionized winds using ground-based interferometers and
TMA trails from two Nike-Tomahawk vehicles (18.232 UE and 18.233 UE, Miguel Larsen, Clemson University).

At $73 \mathrm{ft}$ long, the Black Brant XII (40.005) for Roy Torbert of the University of New Hampshire was thelongest vehicle ever launched from PFRR. The complex payload was launched over auroral arcs above the north coast of Alaska at $08: 21$ on March 3, 1994, but the daughter and baby subpayloads, designed to remove spatial-temporal ambiguities from fields and plasma measurements during the flight, unfortunately did not deploy. However, the main payload, which functioned well throughout the flight, included a new instrument for measuring auroral electric currents.

The sixth rocket of the season was a Nike-Orion (31.071 UE) launched at 09:11:47 UT on March 7, 1994 for Edgar Bering of Rice University. The purpose of this flight was to test an $\mathrm{x}$-ray pinhole camera as a means of recording the position and motion of $x$-ray aurora from space. The payload underflew the nominal apogee of $85 \mathrm{~km}$ due to the early ignition of the Orion, so the parachute portion of the flight was not high enough to photograph the aurora at $\mathrm{x}$-ray wavelengths. The payload was recovered intact.

A Terrier-Black Brant VC (36.114 DE) was launched at 06:50:42 UT on March 11, 1994, for R. McCoy of the Naval Research Lab. The mission was to acquire extreme ultraviolet (EUV) and far ultraviolet emission spectra of the diffuse aurora. The mission was successful and the payload was recovered. The final launch of the 1994 season was a Taurus-Orion (33.063 UE) for Charles Barth of the 
University of Colorado. The flight occurred at solar noon (22:05 UT) on June 27, 1994, during a period of high auroral activity, to evaluate the role of the aurora in the production of nitric oxide, which is an active constituent in the lower thermosphere. Recovery was successful; the instrument will be launched again next year during a period of lower auroral activity.

NASA Grant NAS5-31637: Scientific personnel-C.S. Deebr, S.-I. Akasofu, R. Collins, T. Hallinan, R. Hunsucker, J. Olson, G. Shaw, R. Smith, H.C. Stenbaek-Nielsen, J. Strandberg, and D. Weimer

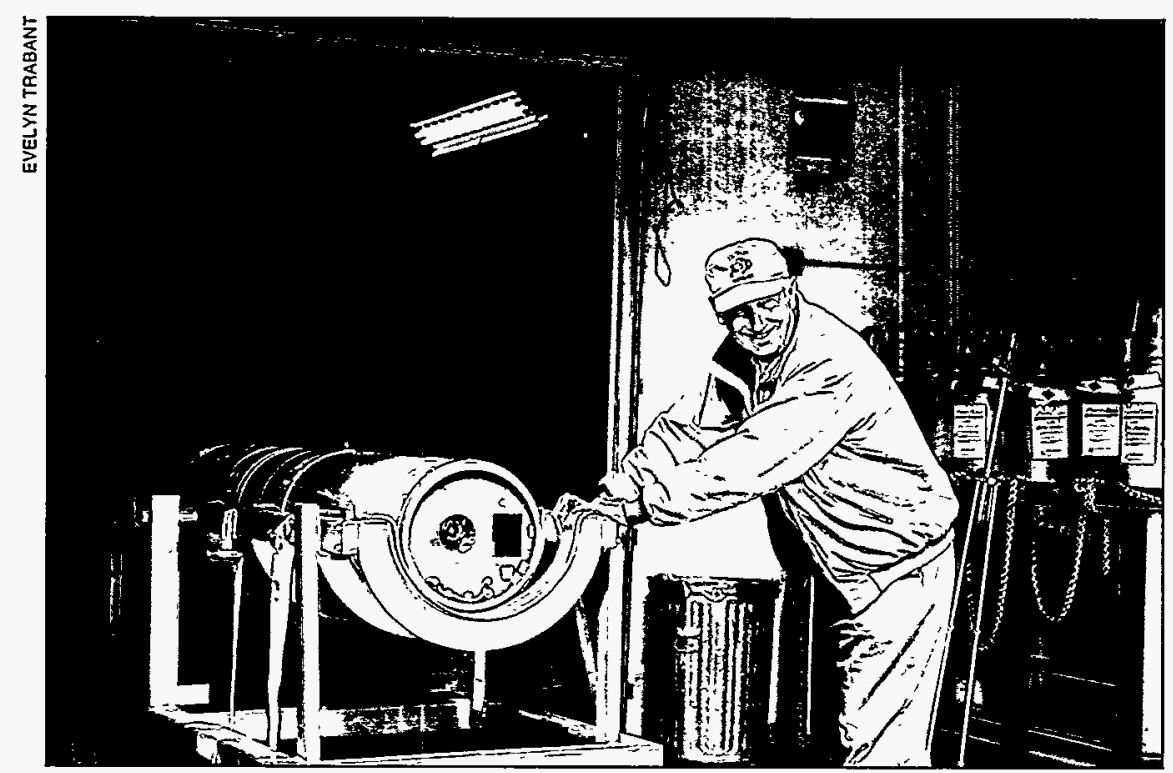

Charles Barth, of the University of Colorado at Boulder, wheels his payload out of payload assembly prior to his successful launch on June 25, 1994.

Improvement and Modernization of PFRR: A major upgrade to the PFRR facilities funded by Congressional appropriation was begun in 1990 and is continuing. Activity in 1993 and 1994 consisted of two major parts: launch facilities and research facilities.

Launch facilities - Funding for a preliminary engineering report on all of the construction to be com- pleted under the Congressional appropriation began in October 1992. The report was produced in 1993, based on the recommendations in the Implementation Plan.

An existing balloon launch facility at Barter Island on the north coast of Alaska was secured from the US Air Force to use as a downrange observatory. This facility was renovated into an observatory with provision for cameras, magnetometers and other critical instruments to be operated in support of rocket launches requiring ground support $600 \mathrm{~km}$ downrange. The instrumentation was installed and operational by the spring of

1993.Funding for the major facilities upgrade $(\$ 3.6 \mathrm{M})$ was received in August 1993; engineering and design work for all facilities then began. A new fuel storage and dispensing facility was constructed, along with foundations for a second Transportable Orbital Telemetry Station (TOTS) antenna for satellite and rocket telemetry to be installed by
NASA. The final phase of electrical code corrections was completed, along with a cable tray system between the block-house and launch pads 1, 2, 3, and 4. New building construction included C-Rocket Assembly Building (C-RAB), a new telemetry building, and a launcher enclosure for Pad 3.

Research facilities - Funding for the improvement and modernization of PFRR research facilities (\$6.95M) arrived in April of 1993. The PFRR Science Working Group (SWG) under Chairman C. Deehr, had established eleven projects in the implementation plan for the upgrade of Poker Flat Research Range.

By the end of 1994, some projects were complete, and most were planned for completion by September, 1995.

NASA Grant NAG5-699: Scientific personnel-R. Collins, $T$. Hallinan, R. Hunsucker, J. Olson, G. Shaw, R. Smith, H.C. StenbaekNielsen, J. Strandberg, and D. Weimer; Engineeringpersonnel-J. Desrochers, S. Estes, D. Osborne, and D. Rice

The Geospace Environment Data Display System (GEDDS): Today, information is becoming available in real time; the presentation of this data in a timely fashion and in a format that is easily comprehensible is a major task. This is particularly important when the data are to be used as the basis for a rocket launch. At Poker Flat, there are many auroral experiments where the success of the experiment relies on the ability of the investigator to send a rocket into an aurora. This, in essence, requires that the investigator be able to predict the aurora 10 to 20 minutes in advance, 


\begin{tabular}{|llll|}
\hline Project & Designator & Project Leader & PURPose To DeSigN AND BuILD \\
\hline Observatory & SOC & T. Hallinan & Science Operations Center \\
Optical Instrumentation & OPT & R. Smith & Optical instrumentation \\
Command Decision Center & CDC & H. S-Nielsen & Network data display \\
Launch Facilities & FAC & J. Strandberg & *not a science project* \\
Data Analysis Center & DAC & D Weimer & Data archive, process, display \\
Lidar & LDR & R. Collins & Lidars \\
Downrange Observations & DRO & J. Olson & Instruments and observatories \\
Communication & COM & S. Estes & Communications network \\
Climate Change & CCM & G. Shaw & Atmospheric trace gas, aerosol, \\
& & & and UV monitor station \\
VHF Radar & VHF & R. Hunsucker & Modernize VHF radar \\
Program Office & ADM & J. Strandberg & *not a science project* \\
\hline
\end{tabular}

so the launched rocket can reach the altitude at which the desired measurements are to be made. Thus, the task is to present the available data in such a fashion that a 15 - to 30 minute auroral forecast can be made.

Traditionally, launch support instrumentation has consisted of magnetometers, riometers, all-sky cameras, and meridian scanning photometers at Poker Flat and Fort Yukon, $200 \mathrm{~km}$ to the north. To accomodate today's large rockets, which require information from sites further downrange, we have instrumented a facility at Barter Island on the north coast of Alaska as well. Data from both Fort Yukon and Barter Island are telemetered over phone lines to Poker Flat and displayed there.

An integral facet of the upgrade to the facilities at Poker Flat is the Geospace Environment Data DisplaySystem (GEDDS). Efforts have been made to acquire as much geophysical data as possible in real or near-real time. The system has a permanent data line to NOAA/SEL, and the database there is also available at Poker Flat. The auroral radar in Anchorage is connected to the GEDDS, and a phone line to Elmendorf Air Force Base provides access to the most current DMSP passes.

On a campaign basis, Poker Flat has real-time access to the Canadian CANOPUS data, and a number of experiments have telemetered data to the range. An example of the latter are all-sky data from Fort Smith, Northwest Territories, in support of a Geophysical Institute barium shaped charge experiment conducted from Poker Flat. The data are acquired and displayed by the GEDDS as soon as they are available. We have emphasized this real-time requirement because of the intended rocket support; data made available with significant delays are of little help when aurora predictions are needed 15 minutes in advance.

A very important role fulfilled by the display system is the integrated display of quantities derived from the basic data. In support of the USAF SPIRIT II experiment, a mag- netometer data display program was developed to show maps with magnetic disturbance vectors at all sites, with data reporting to Poker Flat. The magnetic data can be inverted to display ionospheric currents and Joule heating. More recently, a special display has been designed showing the currents in the Poker Flat magnetic meridian which would be close to the path taken by rockets launched at the range.

Another integrated display is the geographical distribution of the aurora. This display takes all-sky images from all of the stations reporting, and, assuming an altitude of the auroral emissions, the all-sky format is converted to a geographical map. Data from up to six stations can be displayed simultaneously.

The assumed altitude can be changed by the user, so when an aurora is visible from more than one station, it is possible to perform a triangulation on the aurora to obtain the altitude of the lower border, which is indicative of the auroral electron energy. 
The data displayed are also archived for later use. Efforts are currently underway to make the data available to the larger scientific community. The goal is not only to make the archived dara available, but also to provide it real-time. It should be possible for a researcher elsewhere to connect to Poker Flat and to receive the current all-sky image or the current auroral map.

The GEDDS offers research opportunities beyond rocket experiments. The efforts underway provide remarkable and exciting possibilities for a global understanding of the solarterrestrial system, of which the aurora is just one part. Satellite measurements yield, at best, only a few scattered data points in space; to understand these data fully, they must be integrated with the much denser net of groundbased observations.

With a system like GEDDS this can be done in a timely fashion. Thus, the GEDDS can constitute a classroom for space scientists to learn how real magnetospheric processes develop and manifest themselves in ground-based measurements, in contrast to the textbook processes which in most cases are idealized and far removed from the actual phenomenon.

NASA Grant NAG5-699: Scientific personnel-H.C. Stenbaek-Nielsen; Engineering personnel-E. Hoch, D. Rice, and D. Risse

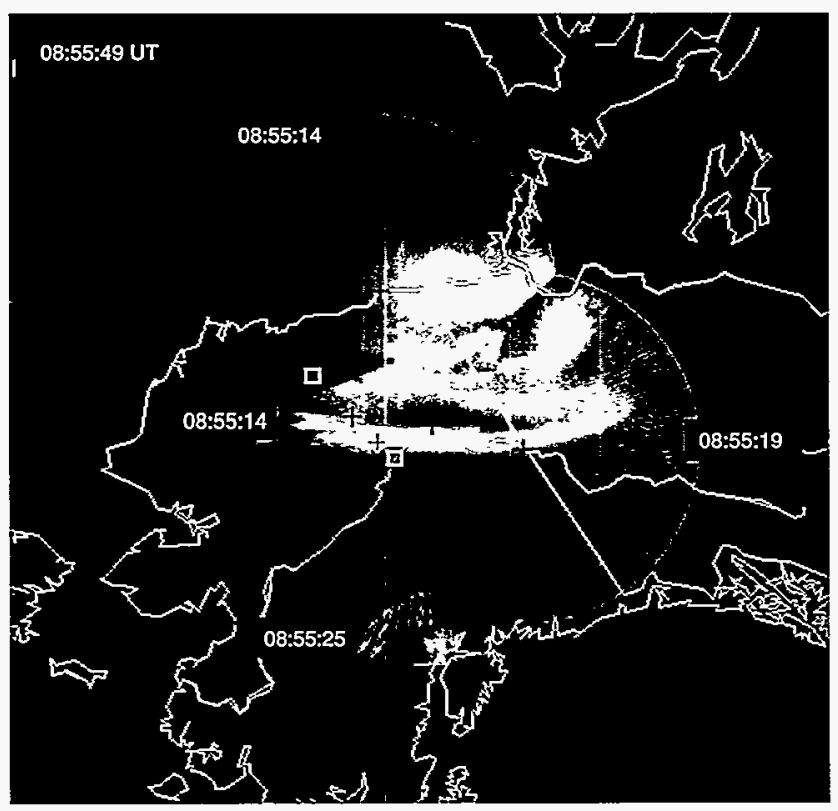

The composite above is an image from the auroral mapper showing the distribution of the aurora based on all-sky images taken from cameras at Poker Flat, and near the communities of Fort Yukon, Eagle, and Kaktovik, Alaska.
Development of a New Generation of Auroral Imagers as Part of the Poker Flat Improvement Plan: For nearly thirty years, the mainstay of our imaging program, both for auroral studies and for barium release studies, has been the image orthicon television system. Because of their low noise, high gain, and large photocathodes, these cameras consistently outperformed the newer miniature cameras developed commercially. However, their operation was labor-intensive and it was becoming increasingly difficult to obtain replacement parts. Accordingly, we have designed and constructed a new generation of lowlight-level imagers for auroral research. The new cameras use large input optics, $25 \mathrm{~mm}$ Gen II intensifiers, nonvignetting relay optics, CCD imagers, and 3 -stage Peltier Coolers to allow integration. The exposure time can be varied from standard television rates ( 30 frames per second) to exposures of up to four minutes. The controllers for the cameras allow remotely controlled operation via phone lines or local computer-controlled operation. Using the same basic building blocks, but with appropriate modifications, we have constructed new all-sky cameras, narrow-field cameras, and a refurbished television spectrograph to study the rapidly varying spectra of active auroral arcs.

NASA Grant NAG5-699: Scientific personnelT. Hallinan; Engineering personnel-J. Desrochers and D. Osborne

VHF Auroral Radar: Under this project, the Geophysical Institute supervised the final stage of the upgrade of the $50-\mathrm{MHz}$ auroral radar located at Elmendorf AFB, Alaska. The radar and antenna upgrade were performed by personnel of the NOAA Aeronomy Laboratory of Boulder, Colorado. Dr. Robert Hunsucker oversaw the upgrade and performed the final operational inspection. The data communication and monitor systems were also designed, installed and tested, and a user's handbook was prepared. The auroral radar is sited so that the antenna, which is only 2 degrees wide in azimuth, intersects the Eregion at near-normal incidence over Poker Flat Research Range (PFRR). Thus, the monitor displays the latitudinal location of field-aligned irregularities (FAR) in the auroral E-layer as a function of time. This information is then made available to scientists to assist in rocket launches. The data are also archived for later analysis.

NASA Grant NAG5-699: Scientific personnel-R.D. Hunsucker 
Poker Flat Upgrade-Lidar: A resonance lidar was brought on line at Poker Flat in November of 1994. The project was financed with upgrade funds and with some equipment donated from the University of Illinois.

The lidar system is capable of measuring aerosol, temperature and metal density profiles in the 15 to $100 \mathrm{~km}$ altitude region. Of particular interest at Poker Flat are mesosphere and lower thermosphere (70 to $110 \mathrm{~km}$ ) studies which are being carried out in collaboration with the other optical instruments on site.

Observations are initially focused on the structure of the meteoric metal layers to study winds and waves in the upper mesosphere and the effects of auroral activity on the upper mesosphere. A daytime capability is being developed to allow summertime observations and support of noctilucent cloud experiments in 1995.

NASA Grant NAG5-699: Scientific personnel-R. L. Collins and $M$. P. Krynicki

The Alaska Space Grant Program: The Alaska Space Grant Program (ASGP) was created in March 1991. Since that time, we have initiated a broad spectrum of new program activities aimed at enhancing aero- space-related research and education throughout Alaska. Our largest program is the Alaska Space Grant Fellowship Program, which provides stipends to university students working on aerospace research projects.

Many of the current Space Grant Fellows also are involved with another of our major programs-the Alaska Student Rocket Project. In this project, UAF students design, build and launch payloads from the nearby Poker Flat Research Range.

Our first launch occurred on 17 July 1992. Plans are under way to launch a second payload in 1995 which will include an advanced tracking system using the GPS navigation satellites. We plan to expand this program to allow the participation of students and teachers in public schools throughout Alaska.

We have launched a multifaceted educational outreach program under the direction of Assistant Professor Neal Brown.

The intent of this program is to bring exciting aerospace topics into classrooms throughout Alaska, thereby encouraging students to study math and science and to consider careers in science and engineering. A series of model rocket workshops was conducted at Poker Flat Research Range during the sum- mer of 1992. In the UAF Journalism and Broadcasting Department, Mark Linsalata's magazine design class produced a new magazine, Alaska Space Ventures, which highlighted exciting aerospace activities under way in Alaska; it was distributed to middle school students throughout the state to stimulate interest in science and technology.

Wealso sponsor a number of other programs, including small grants for initiating new research projects and for developing new aerospace curricula. A request for proposals for grants and fellowships is distributed in February of each year. The Alaska Space Grant Program continues to evolve rapidly, and we would welcome participation in helping to chart our future. Persons interested in receiving the ASGP Newsletter, or who have ideas for new program activities that would enhance capabilities in Alaska for aerospace research or education, are requested to contact the Alaska Space Grant Program Office at (907) 474-6833.

NASA Grant NGT 40022: Scientific personnel-J.G. Hawkins, N.B. Brown, K. Dean, C.S. Deehr, R. Pierce, J. Zarling (UAF School of Engineering), and $D$. Goering (UAF Mechanical Engineering Department) 


\section{Atmospheric Sciences}

a
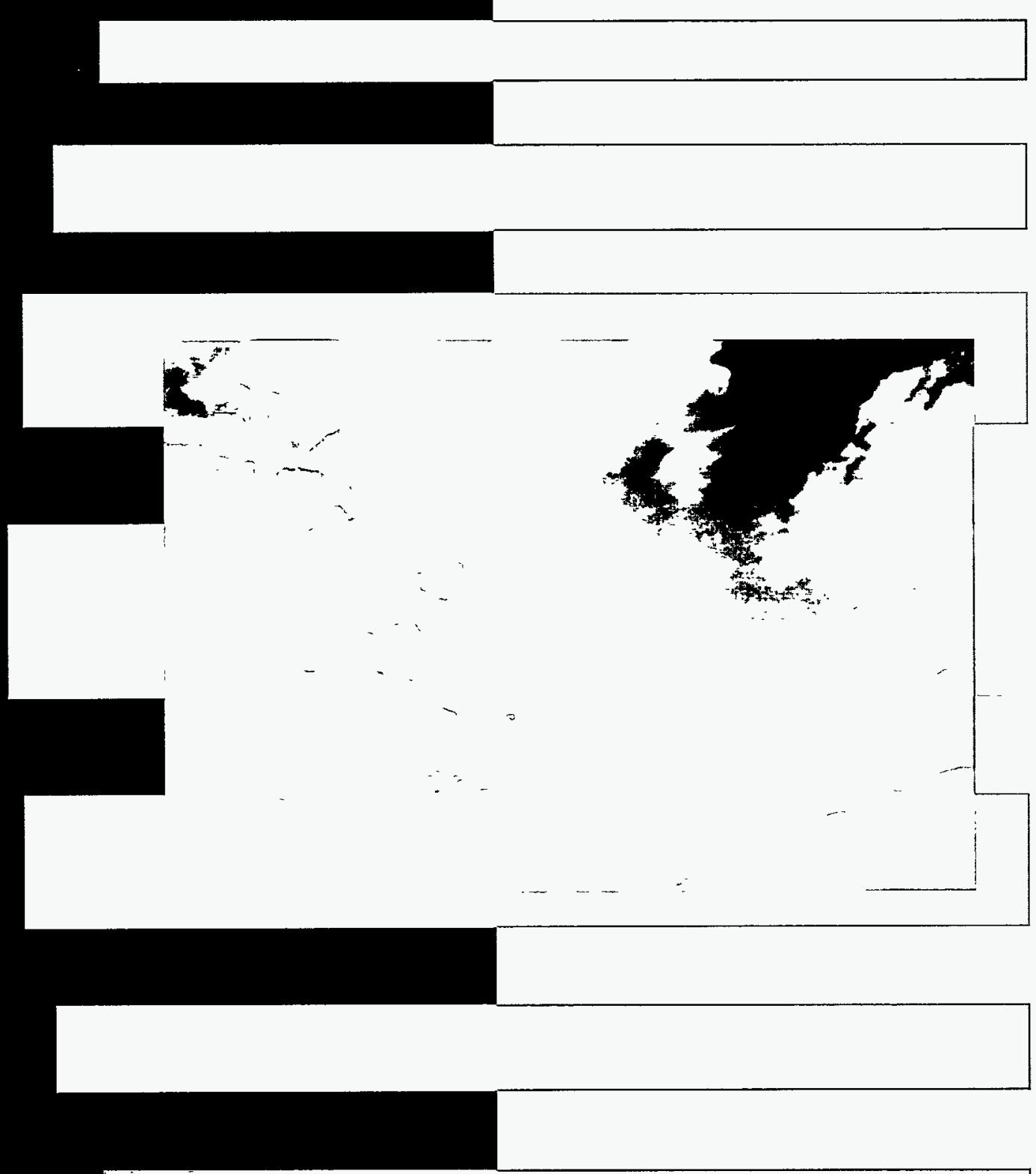


\section{ATMOSPHERIC SCIENCES}

Scholarly work conducted by the Atmospheric Sciences Group comprises a variety of related projects that are united under the theme of polar climate and global climate change research. The various projects are organized into three groups: Regional Meteorology and Climatology; Aerosols and Gases-Climatic Implications; and Cloud Physics and Radiation.

It is now well recognized that clouds constitute a major obstacle to reliable climate modeling and prediction. To address this issue, the Department of Energy (DOE) established the Atmospheric Radiation Measurements (ARM) program, a long-term field project designed to help unravel the role of clouds in the climate system by improving the treatment of clouds in climate models. Under this program, three major research sites will be established worldwide to study cloud-radiationclimate processes, feedbacks and interactions. One of these sites will be established in Alaska. Scientific supervision of ARM research in Alaska was awarded to the institute's Atmospheric Science Group by DOE in 1991. This project is well underway; construction of facilities is scheduled for 1995-96 and deployment of instrumentation is planned in 1996-97.

The Geophysical Institute has made considerable progress on the development of the Arctic Region Climate System Model, which is becoming an important tool in arctic climate research and is being used by several other universities. This mesoscale model allows for a detailed, high-resolution, computer simulation of the Arctic as a system. A major goal of this research is to investigate how the cloudy, and, at times, polluted arctic atmosphere interacts with the snow- and ice-covered land and ocean, and how the arctic climate system responds to natural and/or anthropogenic influences. The model focuses on two distinct high-latitude phenomena the presence of ice clouds during the polar night, and ice and snow surface conditions during large portions of the year.

To improve the performance of climate models at high latitudes, the institute has developed radiation models that properly treat the interaction with snow and ice surfaces. Thus, a comprehensive radiative model for the coupled atmosphere-sea ice-ocean system has emerged.
This model, the first one of its kind, allows for scattering and absorption by molecules, cloud and aerosol particles in the atmosphere, pure ice, air bubbles and brine pockets in the sea ice, and particulate matter in the ocean. The plan is to further develop this model to make it suitable for inclusion into large-scale and mesoscale climate models, such as the institute's Arctic Region Climate System Model, and to apply it to analyze data obtained in the ARM experiments.

Progress in atmospheric chemistry includes observations of UV radiation fluxes and theoretical studies of the impact of ozone depletions, aerosols and clouds on atmospheric photolysis rates, and on the penetration of UV radiation to the surface and into the ocean. The effects of growing pollution in the arctic atmosphere, and around the globe, are being studied, along with the oxidative capacity of the troposphere. The group participates in several missions under the umbrella of the International Global Atmospheric Program, including the Marine Aerosol and Gas Exchange Initiative, and the Pacific Exploratory Mission, with measurements from the Aleutian Islands. In addition, airborne and ground-based investigations of atmospheric trace constituents were conducted in the former Soviet Union, at Barrow, Alaska, at Poker Flat Research Range in Fairbanks, Alaska, over the Gulf of Alaska, and in Hawaii.

Regional meteorology and climatology studies in the Arctic and Antarctic continue. The coupling of katabatic (downslope) winds on the Antarctic Ice Sheet with geostropic wind fields is under investigation in a collaborative effort involving the institure and groups from France and Australia. In this study, climatological measurements are transmitted from the ice sheet by satellite to the home institutions. Trajectory predictions continue to be developed to track plumes from Alaska's eruptive volcanoes, which constitute potential hazards for local and international aircraft operations. Investigation of long-term trends include a joint project with the National Weather Service to evaluate trends in Alaska's climate.

The Atmospheric Science Group is increasing its breadth by interactions with other departments on campus, frequently under the auspices of the campus-wide 
Center for Global Change and Arctic System Research. We are working with other groups to assess the global electric circuit and its possible relation to global climate change, and to research the evolution of atmospheric gases and clouds. We also work closely with the Geo- physical Institute's Snow, Ice, and Permafrost Group to study the role of snow and ice in the climate system, including the interpretation of satellite Synthetic Aperture Radar (SAR) images received by the Alaska SAR Facility in the institute.

\section{Regional Meteorology and Climatology}

\section{The Meteorology of the Arctic}

Front over Alaska: A joint research project between the Geophysical Institute and the Forecast Office of the National Weather Service in Fairbanks has studied the arctic front over Alaska. Although there is still a lot we do not understand about the arctic front and the arctic air mass, this project has clarified a number of points.

First, the arctic front is not the major precipiration producer it was previously suggested to be. Classic arctic frontal passages produce anywhere from 0 to 56 percent of any given winter's snowfall in Fairbanks, and are responsible for rare snow events as far south as Anchorage. Even in Barrow, heavy snowfalls are likely to come from occluded systems originating in the North Pacific.

Second, the classic arctic front (the boundary between $\mathrm{CA}$ and $\mathrm{mP}$ air) is not the only way snowfall can be produced when arctic air is near the surface. Fronts berween two arctic air masses can produce small amounts of precipitation, as can overrunning events which appear only as weak troughs at the surface.

The figure to the right shows several minor precipitation events at Fairbanks along the top; the lower portion is a time-height cross section of wet bulb potential temperature overlaid by relative humidiry deficit. Air with wet bulb potential tempera- ture below $0^{\circ} \mathrm{C}$ was treated as arctic air, since it must have at least started the process of radiative cooling from maritime air. (This air on the figure is that below the boundary with gray above and white below.) Note that some precipitation events, notably the one near 204 hours, show much of the moisture within the arctic air.

Third, the density of freshly fallen snow is much less well correlated with temperature during snowfall than had been assumed previously.

Fourth, the arctic front may play a major role in the production of $\mathrm{cA}$ air by removing excess water very early in the cooling process. Paradoxically, this could actually slow the production of cA air, as the drier air should have a lower radiative cooling rate.

Fifth, we have identified several possible ways of looking at the arctic front, involving thicknesses and pseudoadiabats. At the present time, the usefulness of all but the simplest, the $1000-850 \mathrm{mb}$ thickness, is limited by data and model output.

Probably the major problems remaining are those having to do with the availability of data. Because of this, we chose for operational purposes to use the analyzed and predicted fields from the various atmospheric models. This approach has considerable advantages in that data are available at much closer spacing, and the prognostic fields are useful for forecasting. It also suffers from two major problems.

The first is the accuracy of the interpolated and prognostic fields, which are based on limited sounding and satellite data and models which are somewhat questionable in their ability to duplicate the observed strong surface inversions.

The second is the availability of the fields needed. Unfortunately, those models believed to give the best low-level results do not provide water vapor content as a three-dimensional field on ARO-NET. As a result, the equivalent potential temperature (EPT) and wet bulb potential temperature (WBPT) fields can not be calculated from these models.

In summary, we have a much better understanding of the arctic front and the arctic air mass than was the case at the beginning of this study. But we cannot yet say that we understand it nearly as well as we understand the polar front.

This project has involved very close and useful cooperation between the University of Alaska Fairbanks and the National Weather Service Forecasting Office in Fairbanks. Techniques developed jointly have been applied to real forecasting problems.

These contacts between UAF and NWS also have helped in the establishment of a new NOAA-University of Alaska Cooperative Institute, the Cooperative Institute for Arctic 
Research (CIFAR). We gratefully acknowledge the help of COMET in having helped to bring about this cooperation.

COMET Award UCAR S 9445462: Scientific Personnel-S.A. Bowlingand G.E. Weller; R. Thoman and T. Fathauer (NWS)

Meteorological Factors Controlling the Initiation of Snow Melt in Arctic and Interior Alaska: Snow melt on Alaska's North Slope is often complete within a week or so once melting starts. The date on which melting starts, however, may vary from year to year by as much as a month. Since the solar input at

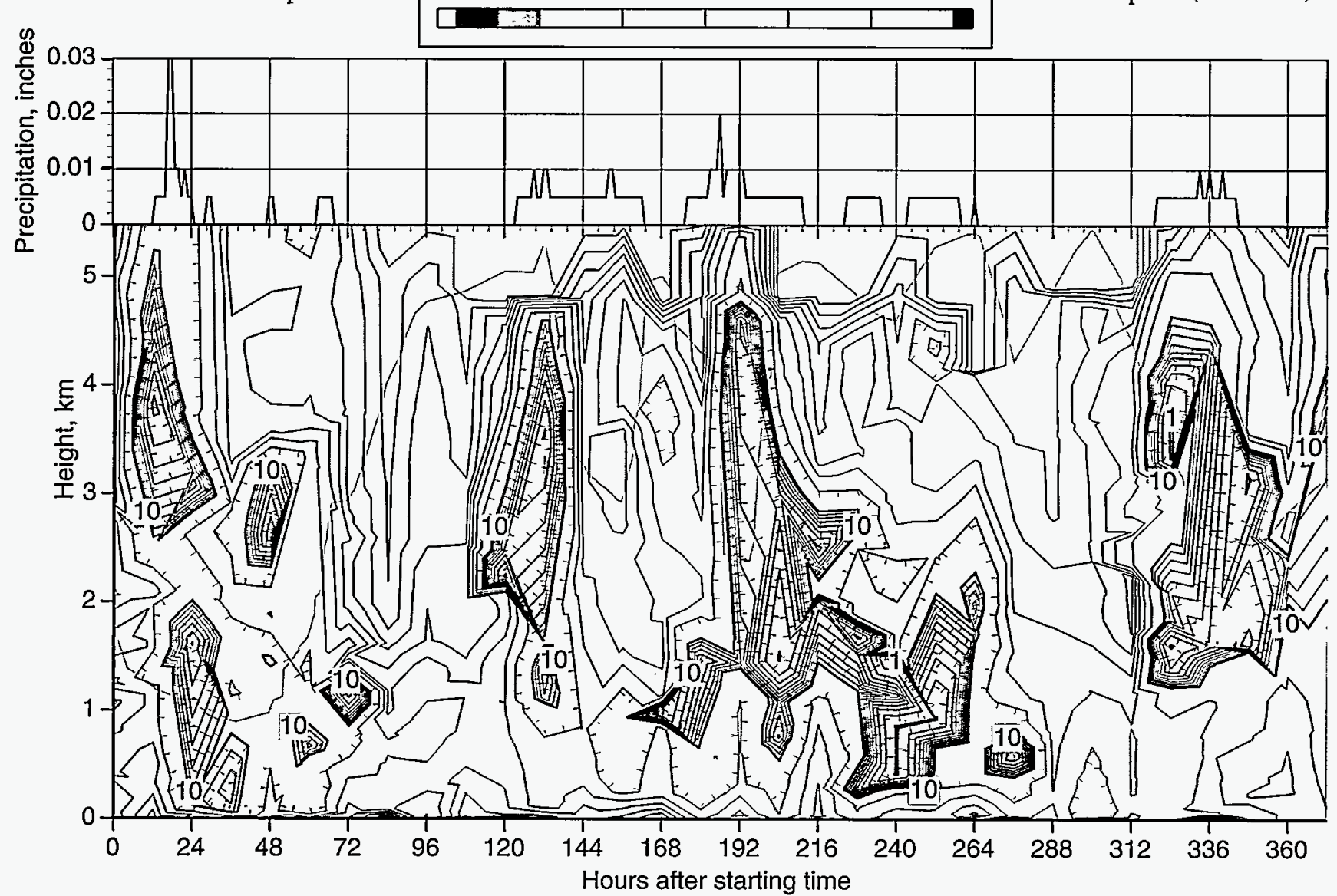

the top of the atmosphere is constant from year to year, the variation must be due to the effects of the atmosphere. We have been investigating the effect of the full atmospheric column on the surface energy balance over a snow surface, with emphasis on the net radiation budget over a surface held at $0^{\circ} \mathrm{C}$.

Sensitivity analysis has indicated that the single most important factor determining the date of snow melt is the water content of the atmosphere, both as clouds and as vapor. Temperature is also important, both di- rectly and through its influence on absolute humidity. Because of the high albedo, relatively low solar elevation angle, and constant outgoing thermal radiation during snow melt, most of the year-to-year variability in the radiation balance comes from the incoming thermal radiation. In the absence of clouds, over half of the thermal radiation comes from the lowest $500 \mathrm{~m}$ of the atmosphere. Thus, advection of warm, moist air at low levels is critical for snow melt. The effect of clouds on the daily radiation balance during snow melt depends critically on the liquid water path (the LWP).

Shown is a time-height cross section of wet bulb potential temperature (WBPT) at Fairbanks, covering the period from 2 pm February 7,1974 , through 2 am February 23, 1974. The gray scale breaks at $0^{\circ} \mathrm{C}$, which we have defined as the boundary between arctic air and maritime polar air; the WBPT increases monotonically with height in these soundings. The overprinted line contour chart is the relative humidity deficit, with the contour density sharply increased at 90 percent relative humidity (labeled 10). The precipitation chart at the top, based on hourly precipitation records, has traces coded as .005 inches $h^{-1}$. 

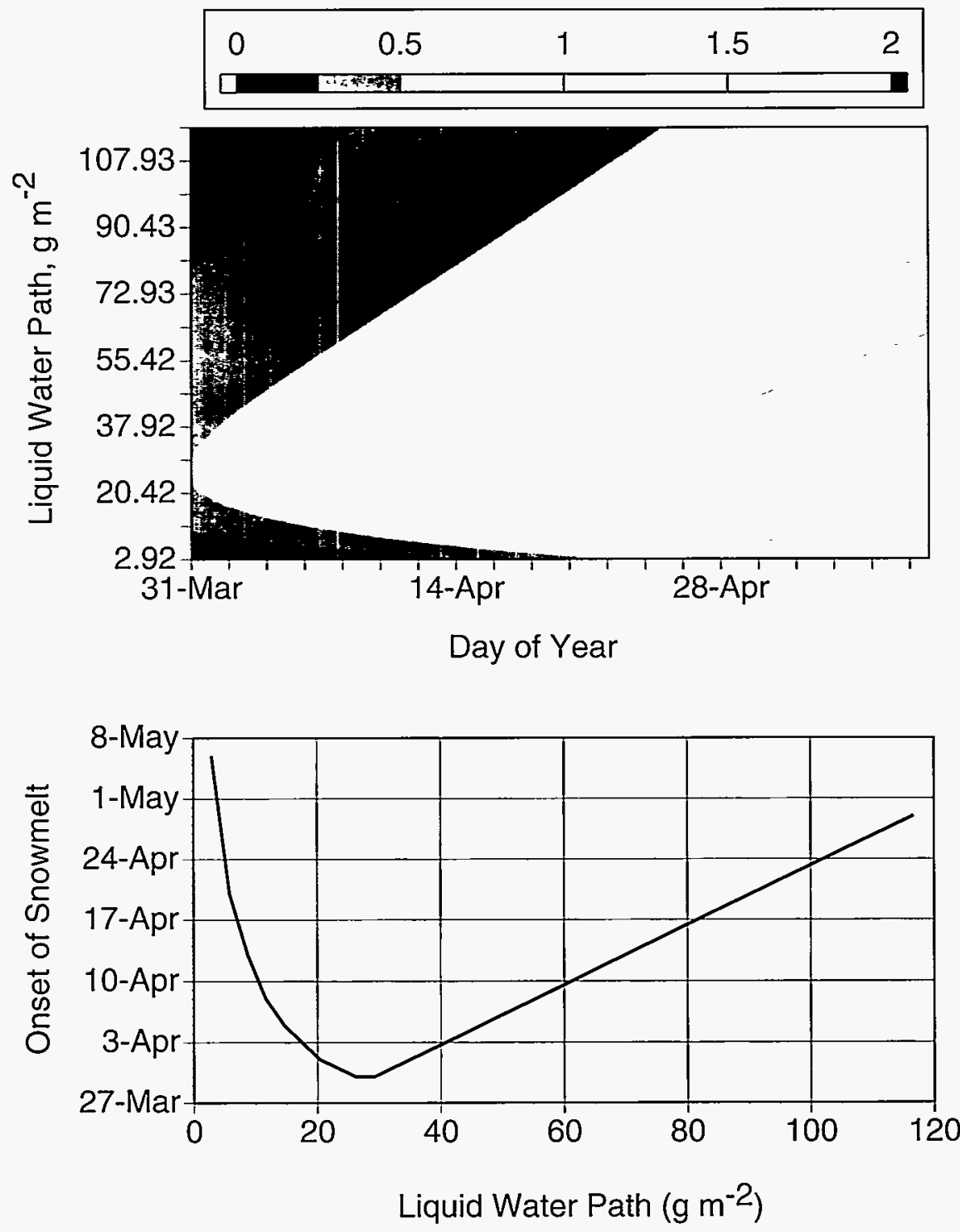

Top: Contours of calculated net radiation balance in $\mathrm{MJ} \mathrm{m}^{-2}$ day-1 are shown overa snow surface at $0^{\circ} \mathrm{C}$ as a function of date and liquid water path. All calculations were carried out with an atmospheric lapse rate of $65^{\circ} \mathrm{C}(100 \mathrm{~m})^{-1}$ and a surface temperature of $0^{\circ} \mathrm{C}$. Relative humidities were averaged from 12 years of Barrow soundings for the observed snow melt period.

Bottom: The date is shown on which the modeled surface energy balance is sufficiently positive to melt $1 \mathrm{~mm}$ water equivalent per day. Again, atmospheric properties are assumed constant over the time period shown. A more realistic seasonal warming of advected air would reduce the horizontal time range, but would not change the fact that snow melt would begin earlier with a cloud liquid content of up to $120 \mathrm{~g} \mathrm{~m}^{-2}$ than with a clear sky.

Downward thermal radiation increases very rapidly as the LWP increases, leveling out as the cloud becomes effectively opaque in the thermal infrared. At the same time, the ner solar radiation at the ground decreases, but more gradually. The result is that with an equivalent drop radius of $7.5 \mu \mathrm{m}$, the net all wave radiation peaks at a LWP around $25 \mathrm{~g} \mathrm{~m}^{-2}$ (geometrical optical depth roughly 5) and then falls off with increasing LWP. Even at a LWP of $120 \mathrm{~g} \mathrm{~m}^{-2}$, however, which is about the maximum for arctic stratus, the effect of clouds on the daily net radiation total is positive, in agreement with many observations.

In our theoretical model, the presence of clouds at the critical LWP could advance snow melt by over a month. Thus, the cloud-climate effect at high latitudes over snow is positive even in the presence of long arctic days.

Application of the model to real data for Barrow and McGrath is ongoing, but hampered by several problems with the data.

Because of drifting, Barrow snow depth data have a high degree of uncertainty. Water content, and particularly clouds, are poorly measured by atmospheric soundings, and our sensitivity modeling indicates that these data are critical. (It is not uncommon to have $10 / 10$ cloud cover recorded when no part of the sounding even reaches ice saturation, for instance.)

Finally, transfer of sensible and latent heat to or from the surface under strong inversion conditions is very poorly understood, though its effect seems to be small. A small field program will probably be needed to address at least some of these problems.

The relationship of the thickness between the $1000 \mathrm{hPa}$ and $500 \mathrm{hPa}$ pressure surfaces to the initiation of snow melt is holding up at least to the accuracy of the data on snow depth. As expected, the critical thickness at McGrath, where forest cover reduces regional albedo, is less than that at Barrow. The air temperature at McGrath during snow melt is also persistently above freezing, while the temperature at Barrow often remains below freezing through most of snow 
melt. This is tentatively attributed to the heating of the atmosphere at thermometer height by radiatively warmed, low-albedo trees at McGrath.

NSF Grant OPP92-14953: Scientific personnel-S.A. Bowling, K.H. Stamnes, T. Zhang, and $H$. Laurusevage

Mesoscale Climate Modeling: The strong sensitivity of polar climate to the simulated surface fluxes of heat, moisture and momentum is undoubtedly responsible for many of the deficiencies in the simulations of the Arctic by global climate models. However, rigorous explanations of these deficiencies have been lacking because of the complexity of the interactions between the atmosphere (including clouds), ocean, land, snow and sea ice.

The approach we have taken to reach an understanding of the role of the Arctic in climate is a high-resolution limited area model system approach. This approach, while expensive and difficult, is physically based and has yielded promising preliminary results, hence offering a wide range of applications.

The Arctic Region Climate System Model (ARCSyM) has been under development since 1992, and is now recognized as a leading regional model of the Arctic. ARCSyM has been developed to simulate coupled interactions among the atmosphere, sea ice, ocean and land surface of the western Arctic.

The atmospheric formulation is based upon the NCAR regional climate model RegCM2, and includes the CCM2 radiation scheme and the Biosphere-Atmosphere Transfer Scheme (BATS). The dynamic-ther- modynamic sea-ice model includes the Hibler-Flato cavitating fluid formulation and the Parkinson-Washington thermodynamic scheme linked to a mixed-layer ocean.

Simulations have been performed at a range of horizontal resolutions, from $7 \mathrm{~km}$ to $63 \mathrm{~km}$, in order to assess the performance of the model and to guide the development of new, high-latitude specific physical parameterizations.

Experiments at the coarser resolutions have addressed the model sensitivity to sea ice dynamics (rarely done in global climate models), the subgrid-scale moisture treatment, to ice phase physics in the explicit moisture parameterization, to changes in the relative humidity threshold for the autoconversion of cloud water to rainwater, and to changes in cloud parameters affecting cloud-radiative interactions.
In particular, it has been found that the parameterization of ice dynamics (rarely done in global climate models) and the ice phase in atmospheric moist processes are crucial in working towards an accurate arctic simulation. Further work in these areas is already underway.

In addition, it is clear that even with simple representations of these processes included, there are deficiencies in the resulting model simulations, which point to the need for similar systematic examination of other parameterizations, including radiative processes, soil hydrology and ocean circulation.

Since the implicit physical schemes outperform the more physically representative explicit schemes in the Arctic, more work needs to be done on these physically based schemes before their inclusion into GCMs is warranted.

SAR imagery (below left) from 25 February 1992 shows the large opening of the polynya in response to the southward drift of ice. The open water state in the polynya is short-lived in the low temperatures as new ice is reformed almost as soon as older ice is blown southward away from the coast. The corresponding ice concentration as simulated by ARCSYM is also shown (below right). The white area is the western half of St. Lawrence

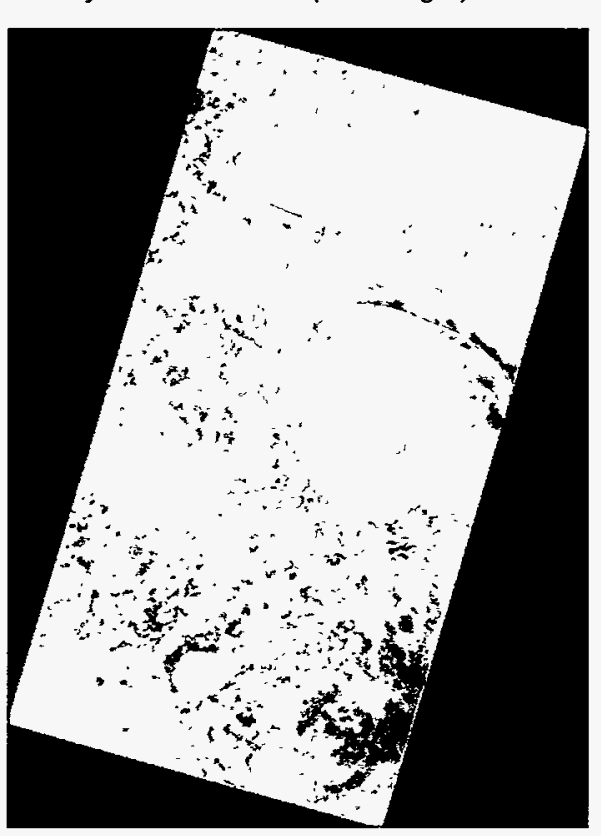
Island, and darker areas represent lower ice concentrations. Each square represents $49 \mathrm{~km}^{2}$. While limitations of resolution are apparent, a similar dramatic polynya opening is identified, with low ice concentrations extending over $70 \mathrm{~km}$ south of the island.

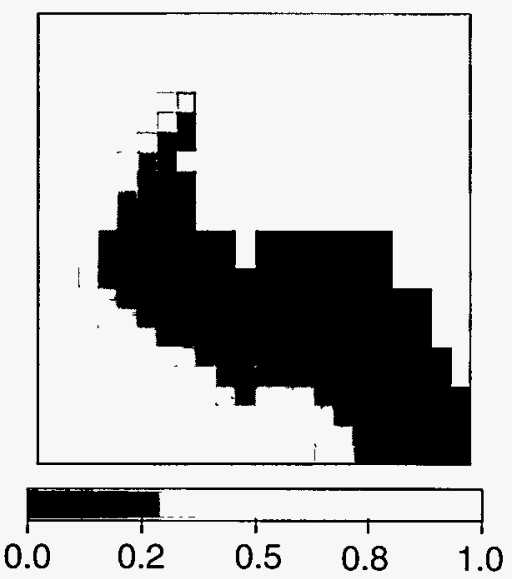


Simulations at finer resolutions have addressed the performance of the model in simulating specific events, such as the formation of the St. Lawrence Island polynya (SLIP). Satellite observarions of the polynya have previously been limited to largescale, low-resolution satellite imagery or costly field expeditions that are highly dependent on season and cloud conditions.

The recently launched European Space Agency Earth Remore Sensing Satellite 1 (ERS-1) which carries a syntheric aperture radar (SAR) has made a new form of high spatial and temporal resolution imagery and SMM/I) to produce a comprehensive picture of the ice circulation and the process of polynya formation.

Modeling a SLIP event (the opening and closing of the polynya) has been a difficult task due to the relatively small size of the polynya, leading to the necessity of a regionalscale coupled atmosphere/sea-ice model.

However, with the development of ARCSyM, simulation of the SLIP is now possible. The use of the newly acquired remote sensing products can now be extended further to model validation in addition to observation of the polynya event.

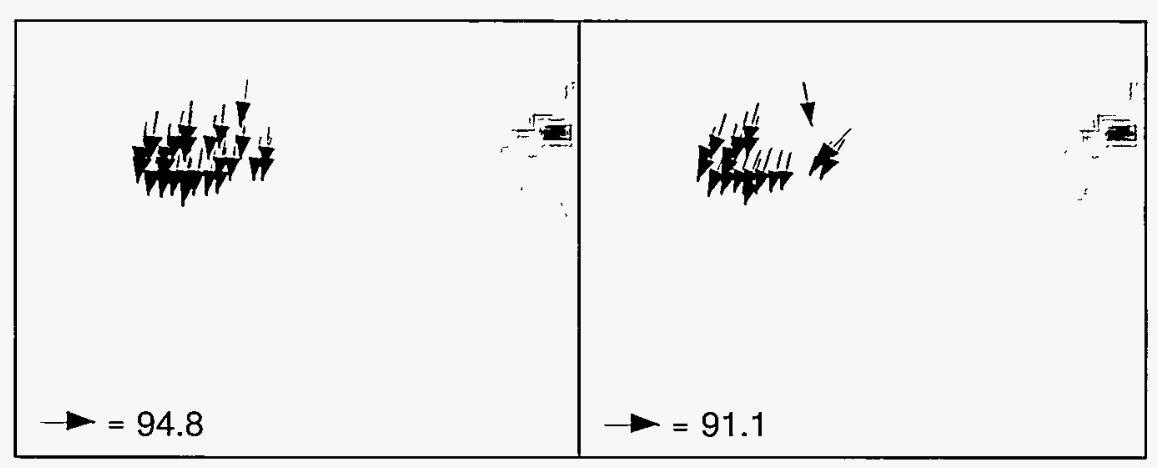

The St. Lawrence Island polynya in the Bering Sea is formed by prevailing northerly winds several times each winter season and is a haven for sea mammals. The ARCSYM model allows simulation of wind-driven ice drift vectors (right) which can be compared with ice drift derived from SAR imagery (left). Vectors shown are ice displacement in kilometers over a three-day period in February 1992. The model simulates the observed ice drift quite accurately, although the larger apparent size of the island due to a 7-km horizontal resolution causes more obvious flow around the eastern side of the island.

available for use. Because the SAR is an active $\mathrm{C}$-band microwave instrument, it is virtually unaffected by darkness, or by the dense cloud cover which tends to be prevalent during Bering Sea winters. The resulting SAR imagery also can be processed to create ice-motion products.

In addition, SAR imagery can be combined with more traditional remote sensing products (i.e., AVHRR
Work is proceeding in three phases. The first phase is concerned with assessment of the land surface-vegetation models. An appraisal of the date vegetation data is currently underway, with a year-long experiment being run to compare with the 1992 field data from the LAII-Flux Study Alaska North Slope Data Sampler. The Canadian Land Surface Scheme existing BATS package using up-to-
(CLASS) has been successfully coded, and will be linked to the ARCSyM for appraisal following the BATS assessment. Further, colleagues at NCAR are developing a simplified version of the Land Surface Exchange (LSX) package which also will be assessed as soon as it is available.

The second phase is the examination of the role of the cloud-radiation interactions. Sensitivity studies involving mixed phase moist processes, shallow convection, cloudradiative parameters and more efficient radiative transfer schemes are underway.

The third phase involves the coupling of a regional ocean circulation model and a high-resolution, mixedlayer model, to complete the basic suite of component models in the climate system model. This work is currently being undertaken in conjunction with researchers at Rutgers University.

The ultimate goals of the research are as follows:

- to provide a prioritization of the problems and issues confronting modelers of the Arctic system;

- to create a climate system model that is appropriate for long-term simulations of high-latitude climate; and

- to creare a coupled model for studying individual phenomena such as polynya formation and land surface run-off, which require such an approach.

NASA Grant NAGW 2829, and NSF Grant DPP 9214810: Scientific personnel-G.E. Weller, A. Lynch, and D. Bailey; J. Walsh and W. Chapman (University of Illinois); D. Haidvogel (Rutgers University); J. Tilley (University of Colorado) 
An Investigation of AtmosphereLand Interactions under Arctic Conditions: This project began in the fall of 1994. An atmosphereland surface-permafrost model is being developed to improve our physical understanding of the transfer of momentum, heat, and water between the atmosphere and the land in arctic environments. We will couple an existing atmospheric radiative transfer model to a snowactive layer-permafrost model through the surface energy balance.

The coupled model will include resolution of vertical profiles of temperature and moisture content from the armosphere, through snow cover, vegetation and the active layer, to the deep permafrost. The coupled model will be tested and calibrated using the currently available field data. Then, the calibrated coupled model will be used to investigate the effects of variations of lapse rate, moisture content of the atmosphere, aerosols and clouds on the surface energy balance, snow cover, the active layer and permafrost.

DOE National Institute for Global Environmental Change (NIGEC) Western Regional Center at the University of California, Davis, Grant94023: Scientific personnel-K.H. Stamnes, T.E. Osterkamp, and T. Zhang

A Numerical Study of the Diurnal Variation of the Adélie Land Katabatic Wind Regime: Katabatic winds occur with great frequency over the Adélie Land sector of Antarctica. Data collected by automatic weather stations (AWS) along a string of stations from Adélie Land to the high interior plateau show a marked diurnal cycle of the wind and temperature regimes of the lower atmosphere during the short austral summer period.

Numerical simulations of the katabatic wind regime have been conducted for the variety of solar forcing and synoptic conditions assuming a clear sky environment.

Results suggest that the katabatic wind is a robust feature of the antarctic boundary layer. Significant disruption of the low-level drainage features occurs only during the midsummer months because of solar heating of the ice slopes and synoptic activity along the Adélie Land coastline.

During midsummer, modeled ground temperatures undergo a diurnal cycle with an amplitude of $15^{\circ} \mathrm{C}$ near the coast, which is in agreement with the AWS observations.

The katabatic wind decreases considerably during the middle of the day, as wind speeds at coastal sites become reduced by more that one half the early morning values.

Numerical simulations depict a rapid summer-to-winter transition of katabatic wind conditions over the one-month period from midFebruary to mid-March. The surface energy budget becomes negative for the entire 24-hour period after mid-February, corresponding to the rapid onset of wintertime katabatic wind regime.

NSF Grant DPP90-70969: Scientific personnel-T. R. Parish (Department of Atmospheric Science, University of Wyoming, Laramie); G.D. Wendler; and P. Pettré (MeteoFrance, Centre Nationalde Recherches Météorologiques)
Interactions Between Katabatic Winds and Polynyas at the Adélie Coast, Eastern Antarctica: Infrared satellite images of the coastal area off Adélie Land were examined together with two wind data sets. One data set was from the manned French station, Dumont d'Urville, and the other data set was derived from an Automatic Weather Station (AWS), during the 1986 austral winter.

A correlation between the development of open water areas (polynyas) and the appearance of extremely strong offshore winds can be drawn.

The wind direction tended to be more perpendicular to the coastline during these extreme events, showing the katabatic origin of the increase in wind strength.

In the study area, the influence of the katabatic wind on the sea ice extends 20 to $100 \mathrm{~km}$ offshore. Sea ice motion farther off the coast seems to be more dominated by synoptic scale weather systems.

Broader scale atmospheric influences may create large polynya structures that influence the development of coastal winds, as the temperature contrast between open water and the cold continent generates its own circulation.

NSF Grant DPP 9070969: Scientific personnel-G.D. Wendler and U. Adolphs

Schumann Resonances: The combination of the electrically conducting earth and the ionosphere separated by the insulating armosphere forms a spherically symmetric, planetary sized resonant cavity. The electromagnetic normal modes of this cavity, the Schumann resonances, are excited by the impulsive currents of light- 


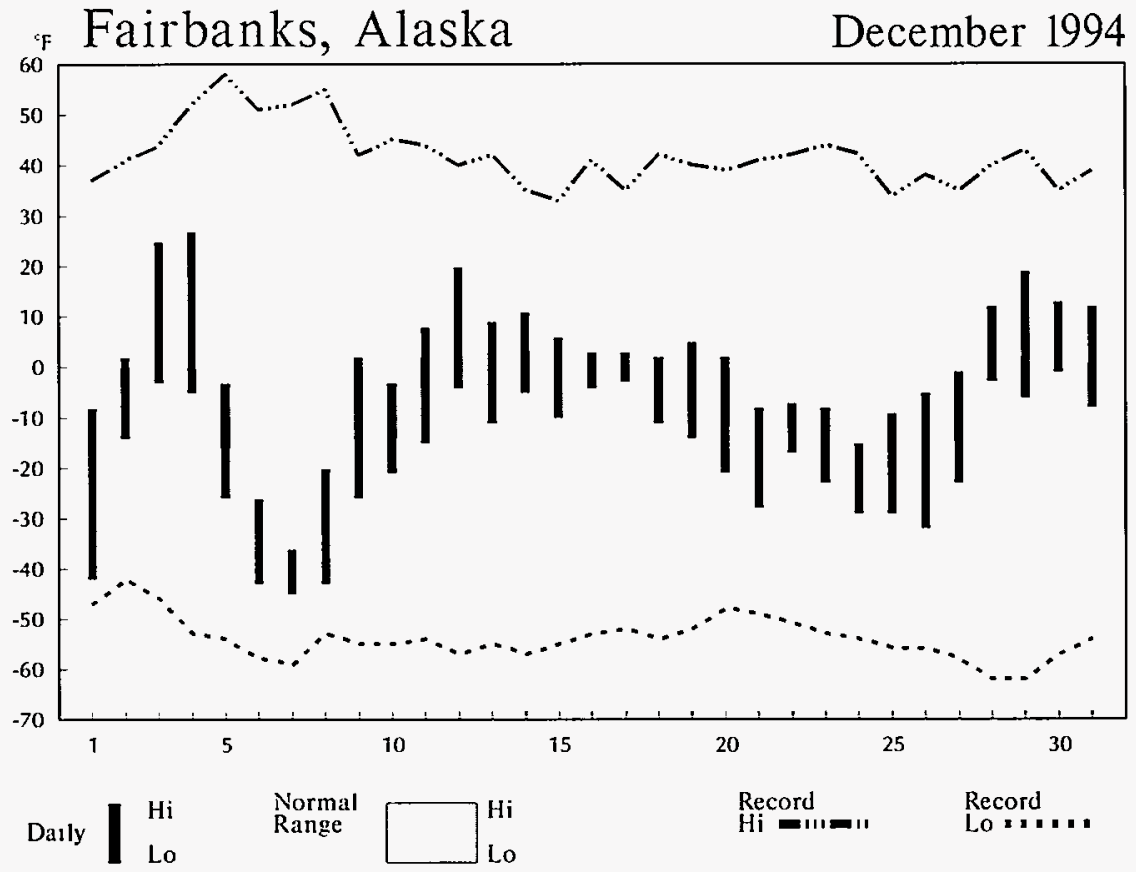

Shown above is the December 1994 climate summary for Fairbanks, Alaska, produced by the Alaska Climate Research Center at the UAF Geophysical Institute. The summary shows that temperatures rose and fell above the normal range several times in December.

ning to produce a line spectrum at the normal mode frequencies of the cavity, $8,14,20,26,32,38 \ldots \mathrm{Hz}$.

The Schumann resonances are the dominant natural electromagnetic background in the frequency range 3-60 Hz. Since the scale length of the modes is of global proportions, the resonances may be detected virtually anywhere on the planet.

The amplitude of the resonances is a direct measure of the incoherent superposition of the global totality of lightning. These properties of the Schumann resonances therefore permit monitoring the global totality of lightning using only a few ground stations, a measurement that is otherwise difficult to make.

Since 1993, we have been engaged in a joint project with Earle R. Williams of MIT to test the hypothesis that Schumann resonance intensities may provide a sensirive measure of changes in tropical continental surface temperatures. The link between global temperature and Schumann resonances is that a small change in surface temperature has been observed to produce a large change in convection and thunderstorm formation in the tropics.

This increase in thunderstorm intensity, if it occurs on a global scale, may be detected as a corresponding intensification in the Schumann resonances, effectively permitting one to construct a "global thermometer" based on Schumann resonance intensities.

Testing this hypothesis requires accurately modeling the propagation effects of the resonances within the earth-ionosphere system, and accumulating continuous resonance data over timescales spanning hours, days, seasons, and years.

To this end, we have been engaged in performing synoptic measurements of the Schumann resonances from a small network of globally distributed stations located in Alaska, California, Western Australia, and Brazil.

Data accumulation through the end of 1994 was sufficient to begin making long base line comparisons among stations. We anticipate that by the end of 1995 we will have the necessary accumulation of data to account for the principal departures from symmetry in the caviry, and be able to make direct comparisons with surface temperature in a direct test of the global thermometer.

NSF Grant ATM92-17161: Scientific personnel-D.D. Sentman and $M$. Heavner

Alaska Climate Research Center: The Alaska Climate Research Center at the Geophysical Institute is the twin of a similar center at the University of Alaska Anchorage. The main mission of these centers is to respond to inquiries on the meteorology and climatology of Alaska from public, private and government agencies, as well as from researchers around the country.

Most of the climatological data available for Alaska have been accumulated at these two centers; Anchorage has much of the old data as hard copy, and most of the Fairbanks center's data are in digital form. Our center issues monthly summaries and weather reviews which are published in several newspapers around the state (see above figure). We also conduct research on a number of meteorological/climatological topics at high latitudes.

State of Alaska funds: Scientific personnel-G.D. Wendler, S.A. Bowling, and G.E. Weller 
Long Climate Records in Alaska: The question of whether the climate is warming (or otherwise changing) is by no means settled. While instrumental records exist from the late nineteenth and early twentieth century in many parts of the world, they are rarely continuous and uniform over time.

This lack on continuity in information leads to the question of whether observed changes are real or due to changes in instrument location and shielding, changes in the surroundings (e.g., the growth of cities or clearing of forest), or changes in operational procedures (e.g., the time of day at which observations are made).

All of these possible sources of error must be examined in order to produce a time series of temperatures which may be used to assess climate change.

In Alaska, there are a number of stations with relatively long records, in some cases reaching back into the late nineteenth century. However, none of these stations has a continuous record at a single site, and several of the better records in terms of continuity have problems with possible heat island effects.

We have examined these stations in hopes of correcting for three types of error: biases due to changes in the time of observation, discontinuities in station history, and progressive changes in station surroundings, particularly those due to urbanization.

1. Biases due to changes in the time of observation have been examined at more southerly stations, but the extremely rapid changes in sunrise and sunset times at higher latitudes in Alaska, together with the complete loss of a daily cycle of temperature in midwinter north of the Arctic Circle, will result in a different pattern of errors from this source in Alaska than is seen in middle latitudes.

Observations suggest that this type of change may introduce an error of more than $1^{\circ} \mathrm{F}$ in spring and summer monthly mean temperatures.

The study also indicates that individual years would vary considerably in monthly mean temperature depending on the time of observation, even though the sun remains below the horizon.

This result underlines the importance of using a number of years of hourly temperature observations to calibrate the necessary corrections.

2. Correcting discontinuities in station history normally involves attempting to estimate missing data from neighboring stations as well as accounting for changes in station location or instrumentation. Changes of this type involve comparing station records with those of neighboring stations (rare in many parts of Alaska) or, in a few cases, with different types of record (such as atmospheric soundings during the last 40 years).

This error currently appears to be the most intractable type in Alaska records.

Unfortunately, discontinuities of this kind are currently being introduced into the record as a result of modernization of the weather observation network.

Changes in siting or instrumentation should, if at all possible, have an overlap of at least a year, and preferably five years or more, with the old location. This is not financially feasible for all changes, but a limited subset of climate stations should be

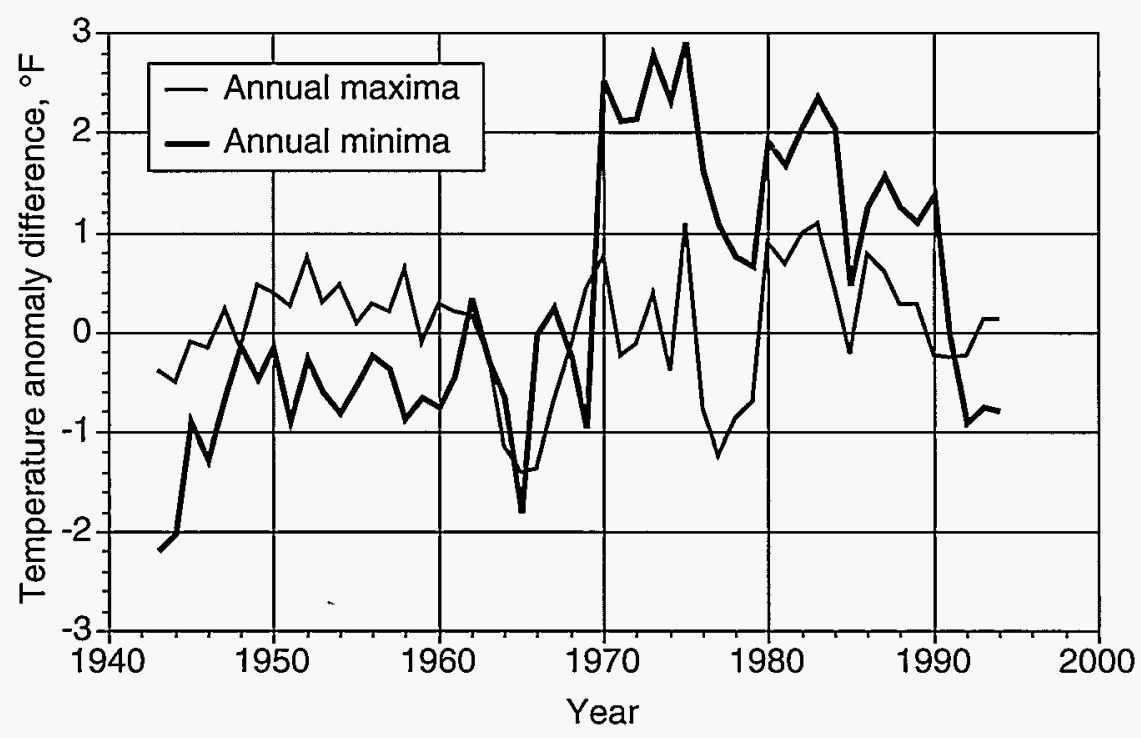

Shown is the difference between Fairbanks mean annual maximum (thin line) and minimum temperature anomalies and the average of those for Big Delta, Fairbanks, McGrath, McKinley, Northway, Tanana and University. Anomalies were calculated as deviations from averages including only the years between 1943 and 1990 for which annual means were available for all seven stations. Significant sensor moves of Fairbanks were recorded in 1963 and 1991. There are no recorded changes at any of the seven stations in 1969 or 1970 . The change may be due to airport construction. 
designated as baseline stations, with special consideration given to keeping their records continuous.

3. Some of the stations with the most complete records (e.g., the university at Fairbanks) are in areas known to have relatively strong urban influences on recorded temperatures.

We are investigating corrections for this effect by using paired urban and rural stations and by comparing surface temperature trends (which are affected by urbanization) with temperatures higher in the atmosphere at sounding stations.

Since Alaska heat islands are very shallow because of the steep ground inversions, temperatures as low in the atmosphere as $950 \mathrm{mb}$ should not be affected by heat island effects.

The technique can only be used back into the 1940s and is limited to a small number of sites, but these sites include McGrath, Nome, Barrow, and Kotzebue, most of which are prime candidates for long-term records.

Unfortunately, comparisons of Fairbanks with surrounding stations have shown what appears to be a step change in Fairbanks rather than the kind of gradual warming that would be expected from a heat island effect (please see figure on the previous page).

At the present time it appears unlikely that it will be possible to correct any Alaska stations well enough to obtain reliable temperature trends back into the first half of the century. There are, however, several stations with potentially usable records dating back to the 1950s.

NOAA Grant NA16RC 0518: Scientific personnel-S.A. Bowling
Participation in Arctic Monitoring and Assessment Program (AMAP): The Geophysical Institute, collaborating with scientists from other nations, is involved in the Arcric Monitoring and Assessment Program, an international endeavor of the circumpolar arctic nations to assess the growing problem of contamination in the different components (land, sea, air and biological tissue) of the arctic system.

During the academic year 199394, UAF Associate Professor Dan Jaffe was on sabbatical at the University of Oslo.

Funded by the U.S. Department of State to participate in AMAP, Jaffe worked with the AMAP secretariat in Oslo as a member of the official U.S. delegation on a number of AMAP task force and experts working meetings. With earlier funding from the State of Alaska, Jaffe has participated in the AMAP process since its inception in 1991.

The Geophysical Institute is preparing a chapter for an AMAP report that will review and assess heavy metals in the Arctic.

In this phase of the project, a group of approximately 40 experts have been identified to request up-to-date databases for use in the assessment.

Karopalov, in Russia, and J. Pacyna, in Russia, are working with Professor Glenn Shaw from the Geophysical Institute to prepare the report. They attended the AMAP meeting in Washington D.C. in October 1994.

Shaw has been appointed the official U.S. representative to AMAP's Assessment Steering Group.

ONR Grant N00014-94-11028:Scientificpersonnel-G.E. Shaw and D. Jaffe; J. Pacyna (Norwegian Institute for Air Research); Karopalov (Institute of the Environment, Moscow, FSU); Project leader-E. Myers (NOAA)

Early Environmental Warning System: Two automatic weather and radiation monitors from Los Alamos National Laboratory in New Mexico were installed at the Geophysical Institute as part of a long-range early environmental warning system being tested in the Arctic.

One of the monitors is on top of UAF's Geophysical Institute Elvey Building; the other is in front of the building, gathering meteorological data and assessing levels of gamma radiation in the atmosphere.

Under this collaboration among Los Alamos, UAF and ADEC, the monitors are being tested in subarctic conditions for at least a year to see if the automated stations can be dispatched in high latitudes as part of an extensive global monitoring system dubbed NEW-NET, or the Neighborhood Environmental Watch Network.

The monitors are similar to an air sampling station which was placed here two years ago under an agreement between UAF, ADEC and the U.S. Environmental Protection Agency to monitor possible radioactive contamination of arctic air resulting from past Soviet activities. It was one of several monitors installed around Alaska following reports which began to surface in 1992 about the FSU's dumping of nuclear and other military and industrial waste.

Participants: G.E. Shaw; T. DeLaca (UAFArctic Research Office); L. Sanders (Los Alamos National Laboratory) 


\section{Aerosols and Gases: Climatic Implications}

The Physical and Chemical Characteristics of Aerosols: Increasingly, researchers are beginning to recognize the importance of atmospheric aerosols on climate and possible climate change. Even in remote regions, the air contains aerosols that can affect the radiation budget of the earth in two ways.

First, sunlight can be reflected off or absorbed by the aerosol, resulting in a change in radiation and energy balances in the atmosphere. The aerosol can also act as cloud condensation nuclei (CCN). The radiative properties of clouds are determined, to some extent, by the number of $\mathrm{CCN}$ in the air at the time a cloud forms. Thus, by affecting the radiative properties of clouds, CCN can indirectly influence the planetary albedo and climate.

Natural aerosols (i.e., not caused by anthropogenic activity) consist primarily of sulfate compounds. Dimethylsulfide (or DMS) is released from the oceans of the world as a biological byproduct from phytoplankton. The sulfate is produced from a complex set of chemical reactions which transform the gaseous DMS to sulfate aerosol.

In other words, the climate of the earth may be controlled to some extent by microscopic oceanic creatures.

Our goals in this study are to measure the chemical composition of natural aerosols and their physical characteristics. The chemical information will help us to understand the origin and chemical processes which lead to the formation and subsequent changes in the aerosols in the atmosphere.

The physical measurements include size distribution, growth as a function of relative humidity, and the ability of the aerosols to act as $\mathrm{CCN}$. These measurements will help us to ascertain the importance of aerosols on the earth's radiation bal- ance by both the direct and indirect mechanisms discussed previously.

NASA Grant NAGW-3728 Scientific personnel-G.E. Shaw, R.L. Benner, D. Veazey, C. Jodwalis, and W. Cantrell

Gaseous Sulfur Intercomparison Experiment: The NSF sponsored an international intercomparison of techniques used for measurement of sulfur dioxide in the atmosphere. Invitations were extended to only seven research groups from around the world for the study, which was hosted by the University of Delaware.

The UAF group was invited because they have been at the forefront of developing a sensitive sulfur detection system-known as the sulfur chemiluminescence detector, or SCD. This instrument can measure as little as $10^{-14} \mathrm{~g}$ of sulfur, which makes it useful for analysis of trace levels typical in the atmosphere.
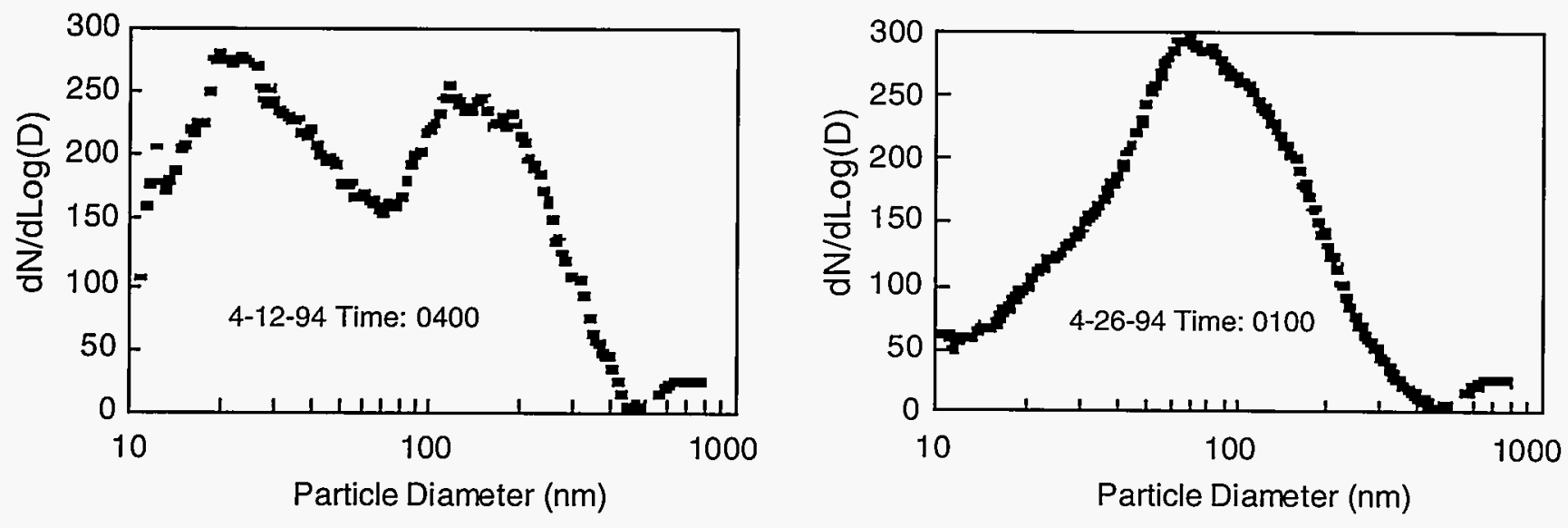

A vivid example of the differences between aerosols that have a different life history can be found in the two figures above. Data on the graph on the right were measured from the free troposphere near the top of Mauna Loa in Hawaii, and are characteristic of aerosols that have not been influenced by clouds. Data for the graph to the left were obtained from the marine boundary layer over the shore of the island of Hawaii as the trade winds carried clean air onshore. The bimodal distribution is characteristic of aerosols that have been influenced by clouds. A simple model of the cloud/aerosol interaction confirms this interpretation of the size distributions. 
Sulfur dioxide levels ranging from 0 to 1 part per billion were introduced to a sampling manifold in this double-blind experiment.

Each research group was then given 1.5 hours to measure the gas in the manifold. In addition to sulfur dioxide, other gases that could potenrially interfere with different measurement techniques were added to the sample manifold.

Results from each group have been submitted to an advisory group, but the results are yet not known.

University Faculty Small Grants Program WO5710: Scientific personnel-R.L. Benner and J. Wu

The Long-Range Over-the-Water Dispersion Experiment: The earth's surface is nearly 80 percent ocean and the planetary albedo is dominated by clouds over the ocean surface.

The dynamics of mixing water vapor, sulfur gases, aerosols and other chemicals is important to understand how these clouds form and how they

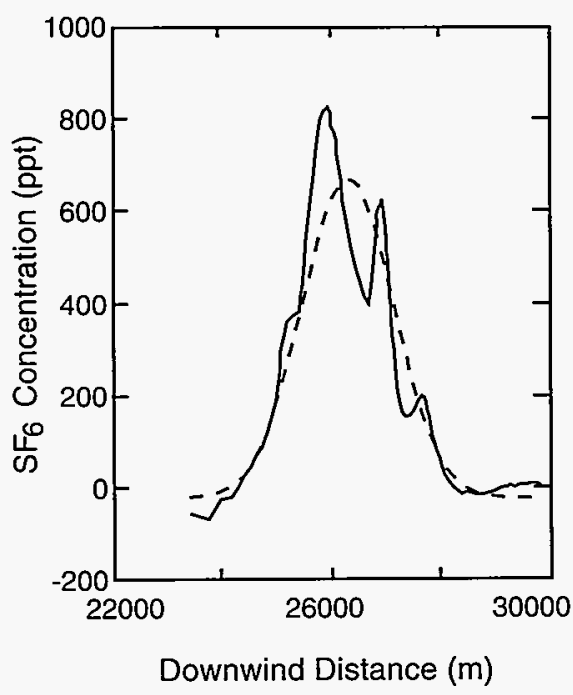

Pictured in the graph above is an $\mathrm{SF}_{6}$ plume observed from aircraft. The dotted line is the best-fit Gaussian representation. might be affected by global-scale change to the environment. There is, therefore, a considerable interest in the dynamics of mixing in the marine boundary layer (MBL).

An experiment was conducted off the coast of Hawaii to develop a better understanding of these processes.

Sulfur hexafluoride $\left(\mathrm{SF}_{6}\right)$, which is an inert gas often used to study motions in the atmosphere, was dispersed in a line by a C-130 aircraft flying at about 30 meters above the ocean surface.

Five boats and a Cessna 302 measured the tracer as it moved downwind with real-time continuous $\mathrm{SF}_{6}$ analyzers. The tracer was still observable with the analyzers $120 \mathrm{~km}$ from the release at levels as low as 50 parts per trillion.

The $\mathrm{SF}_{6}$ instruments were developed by Assistant Professor ofChemistry Richard Benner of the Geophysical Institute. Data from this study are still being analyzed.

NOAA Grant 40RANR304617: Scientificpersonnel-R.L. Bennerand C. Jodwalis

Real-time Sulfur Gas Concentration Measurements in the Marine Boundary Layer: A real-time sulfur chemiluminescence detector, which was custom-made at the Geophysical Institute, was used onboard the Woods Hole Oceanographic Institute research vessel Oceanus during the summer of 1992.

This unique instrument produced the first-ever measurement of concentration fluctuations of dimethylsulfide in the remote atmosphere.

The measurements have allowed us to gain new insights into the be- havior of sulfur gases in the marine environment. We found that temporal and spatial integration is an important consideration for performing accurate concentration measurements, even in a marine boundary layer of neutral stability.

We also were able to estimate the surface flux of dimethyl-sulfide into the atmosphere through variance and inertial-dissipation methods.

Comparison of our results shows a discrepancy between other flux estimate methods by nearly a factor of two. Current estimates suggest that natural sources of sulfur in the atmosphere are approximately equal in magnitude to anthropogenic sources.

The implications of our results, if correct, are that global average natural emissions of DMS dominate anthropogenic sources. The results also imply that current estimates of DMS oxidation rates and atmospheric lifetimes may need to be revised.

ONR Grant N00014-92-J-1296: Scientificpersonnel-R.L. Bennerand C. Jodwalis

Monitoring Arctic Haze and Sulfur Aerosol in Alaska: The Geophysical Institute is monitoring the concentration of sulfur aerosols and heavy metal contaminants in the atmosphere in Alaska.

It has been known for a long time that pollution products, mostly from industrial sources in Eurasia, are contaminating the Arctic. The contamination, called arctic haze, is widely spread and reaches arctic and subarctic regions in Alaska.

We have installed air sampling equipment at Barrow (on the north tip of Alaska), at a hilltop location at Poker Flat Research Range in central 
interior Alaska and at Homer, on the shore of the Gulf of Alaska. The time record of the concentrations of trace atmospheric constituents varies methodically throughout the year.

At the arctic and subarctic stations at Barrow and Poker Flat, the concentration of a number of heavymetal contaminants including arsenic, selenium, antimony, cadmium, nickel, copper, and zinc increased in the winter months to concentrations comparable to those observed in polluted industrialized regions.

However, in the summer and autumn months, the air in Alaska was very clean, comparable to locations in the central Pacific Ocean.

At the Homer site on the Gulf of Alaska, there is no strong evidence for imported pollution. The gulf has clean air as far as industrial markers are concerned.

However, very interesting elevated concentrations of the sulfur-containing aerosols, methane sulfonic acid and sulfate, occurred in the summer months,apparently being released from phytoplankton in the biologically rich and productive gulf.

Researchers are interested in such biologically produced sulfur aerosols; they serve as nuclei on which water condenses to make cloud droplets.

ONR Grant WO 5264: Scientific personnel-G.E. Shaw and R.L. Benner; W. Maenhaut (Institute for Nuclear Technology, Ghent, Belgium)
A Study of the High-Latitude Nitrogen Oxide Reservoir: The goal of this project is to identify the forcing mechanisms, both natural and human-caused, for tropospheric nitrogen oxides and ozone in the verify global, three-dimensional pol- lutant transport models.
This work involved comparing the computer-calculated ozone and ni- trogen oxide concentrations against observed data.

high latitudes. Tropospheric ozone is an important species due to its influence on the cleansing mechanisms of the atmosphere. It is also a greenhouse gas, and is toxic to humans and vegetation.

Tropospheric ozone is known to form from reactions of nitrogen oxides in the troposphere (e.g., smog chemistry).

In contrast to stratospheric ozone, which is decreasing, tropospheric ozone is increasing in the northern hemisphere due to anthropogenic emissions of nitrogen oxides.

During the spring of 1993 , measurements of $\mathrm{NO}_{\mathrm{x}}, \mathrm{PAN}$, ozone, hydrocarbons and alkyl nitrates were made at Poker Flat Research Range. Most of these species show a clear spring maximum which is consistent with an accumulation of these compounds throughout the northern hemispheric winter.

In the spring of 1994, an even more extensive group of compounds were measured at the Norwegian research station at $\mathrm{Ny}$ Ålesund in the high-arctic island group of Svalbard.

This campaign was conducted jointly with the Norwegian Institute of Air Research (NILU) and scientists from the Universities of Bergen, Oslo, and Stockholm. Simultaneously, we are developing detailed chemical-radiative transfer models to interpret our observations.

During a sabbatical at the University of Oslo, Associate Professor Dan Jaffe worked with other scientists to
NSF Grant ATM92-15127: Scientific personnel-D.A. Jaffe, H. Beine, J. Kelley, E. Pullman, and J. Herring

Impact of East Asian Emissions on $\mathrm{CO}$ and $\mathrm{O}_{3}$ Concentrations in the North Pacific Atmosphere: The goal of this project is to quantify the transport of pollutants to the North Pacific region from emissions in the Asian region.

In 1994, a new observational station was established at Shemya, Alaska, at the end of the Aleutian chain.

This station was established because we believe that pollutants from East Asia (e.g., China, Japan, Korea) are transported to Alaska. The Shemya site provides an excellent opportunity to evaluate this hypothesis. Instrumentation for carbon monoxide and ozone were installed in Shemya during June 1994, and the data is accessed remotely from the Geophysical Institute.

We intend to install similar instruments at a second site in the Pacific on the island of Guam. In January 1995, Associate Professor Dan Jaffe is scheduled to visit Guam and identify an optimal location for the instruments. We expect that carbon monoxide and ozone instruments will be installed later in the year.

We will combine our data from Shemya and Guam with data collected by NOAA from their atmospheric station on Hawaii to provide a Pacific-wide understanding of the transport of pollutants from Asia. Our preliminary analysis of this data indicates that all three of these sites (Shemya, Guam, and Hawaii) are recipients of Asian air pollution during certain periods of the year. 
NOAA Grant NA36GP0253: Scientific personnel-D.A. Jaffe, E. Pullman, and L. Zhang

$\mathrm{NO}_{2}$ Column Abundance Measurements and Trace Gas Chemistry Regulating Arctic Stratospheric Ozone Abundance: The objective of this project is to unravel the role of $\mathrm{NO}_{2}$ in the arctic ozone budget. Morning and evening slant column abundances are measured and used to study diurnal, seasonal, and dynamical cycles and the possible conversion of $\mathrm{N}_{2} \mathrm{O}_{5}$ into $\mathrm{HNO}_{3}$ on sulfate aerosols.

Radiative transfer computations are used to convert the slant path column abundances to vertical column abundances appearing in photochemical models.
A second instrument was installed in Svalbard, Norway in August 1994. By making similar measurements in Svalbard (78 degrees North) and Fairbanks (64 degrees North) we will be able to compare $\mathrm{NO}_{2}$ levels at a location that is expected to be frequently within the polar vortex (Svalbard) and one that is usually outside the vortex (Fairbanks).

Results from the 1993-1994 Fairbanks campaign: The $\mathrm{NO}_{2}$ vertical column abundance was shown to have a strong diurnal dependence. This is caused by the conversion of $\mathrm{NO}_{2}$ to $\mathrm{N}_{2} \mathrm{O}_{5}$ which in turn converts to $\mathrm{HNO}_{3}$ during the night.

In the arctic winter, when the days are short and the solar elevation is low, the photodissociation of $\mathrm{N}_{2} \mathrm{O}_{5}$ and $\mathrm{HNO}_{3}$ becomes very slow, so
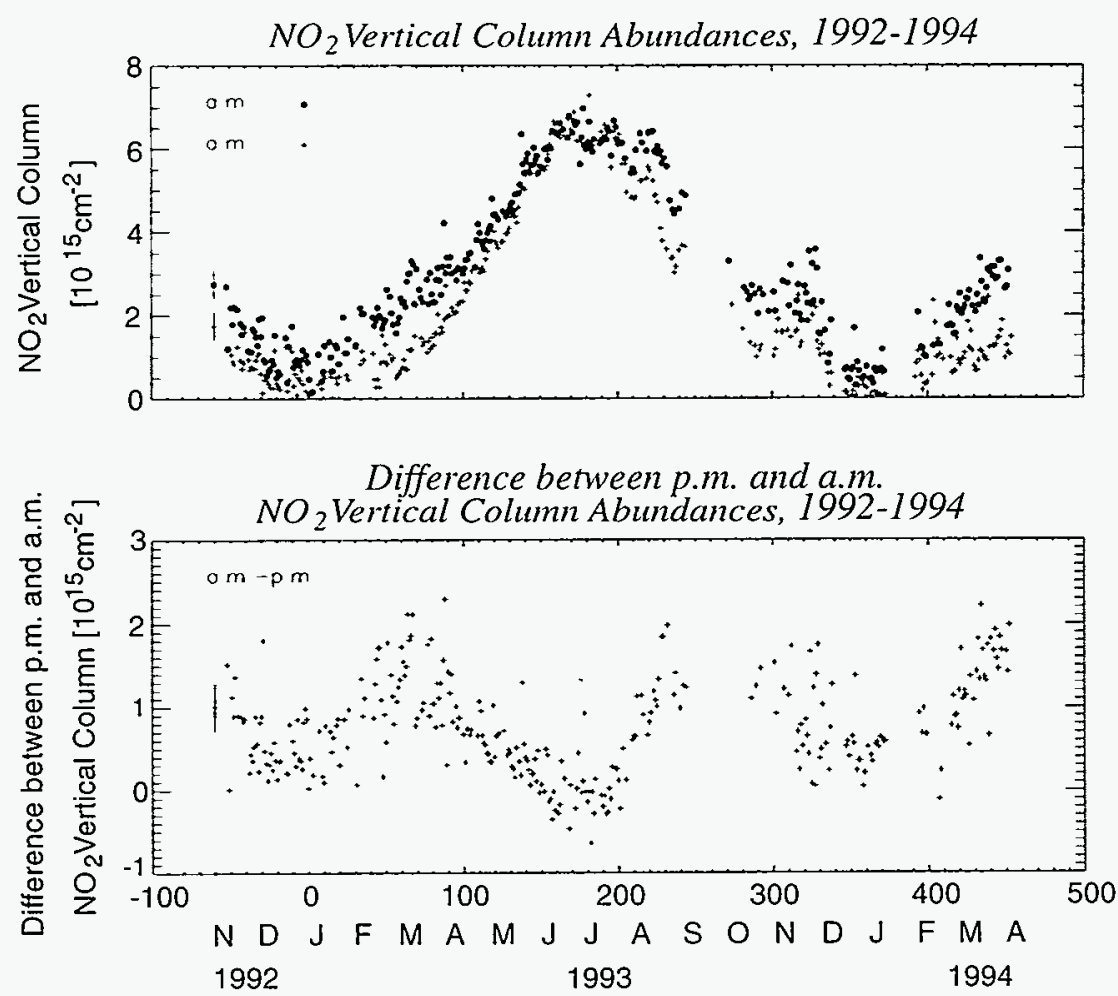

During the arctic summer, the night becomes short and most of the $\mathrm{N}_{2} \mathrm{O}_{5}$ and $\mathrm{HNO}_{3}$ will be photodissosiated to $\mathrm{NO}_{2}$ and seen in the top panel above. The bottom panel shows that in the spring and the fall when the night and day are approximately of equal length we have the biggest difference between morning (a.m.) and evening (p.m.) values of $\mathrm{NO}_{2}$.

the amount of free $\mathrm{NO}_{2}$ radicals decreases. During the arctic summer, the night becomes short and most of the $\mathrm{N}_{2} \mathrm{O}_{5}$ and $\mathrm{HNO}_{3}$ is photodissociated to $\mathrm{NO}_{2}$, as seen in the top panel of the figure on the previous page.

The bottom panel of the figure shows that in the spring and in the fall, when the night and day are approximately of equal length, we have the biggest difference between morning (a.m.) and evening (p.m.) values of $\mathrm{NO}_{2}$.

NSF Grant ATM-9302348: Scientific personnel-K.H. Stamnes, R.W. Smith, G.E. Shaw, J. Slusser, and $R$. Storvold

Lidar Measurements of Arctic Aerosols: An aerosol lidar is being operated at Sheep Creek Research Station in Fairbanks, Alaska, in collaboration with Nagoya University.

The data from this lidar is being used to study both tropospheric and stratospheric aerosols as part of ongoing research into the arctic haze phenomenon and the heterogeneous chemistry of the Arctic stratosphere.

Scientific personnel-R.L. Collins, and G.E. Shaw; T. Shabata (Nagoya University, Japan)

UV Radiation in the Southern Seas in Early Spring 1993: Radiation measurements were carried out during a research cruise of the Nathaniel B. Palmer in August and September 1993, from the tip of South America to Antarctica.

The validity of the measurements of hourly data had to be confirmed first because the measurements were carried out on a moving platform. Some of the results follow. 
- The radiative levels changed substantially during the trip, as both latitude and season changed. Daily values of global, UV-A and UV-B radiation were measured continuously during the trip.

- A mean value of the clearness index $\mathrm{K}_{\mathrm{r}}$ (total transmissivity) was found to be 0.44 . This is a low value, but the cloudiness was high (a mean value of 78 percent). The clearness index increased with decreasing cloudiness from 0.41 for overcast to 0.62 for clear-sky conditions. Individual daily values varied even more, but were always between 0.2 and 0.8 .

- The UV-A and UV-B radiation levels were related to the global radiation. However, correlation coefficients improved substantially, when the analysis was carried out for different cloudiness classes. Clouds reduced the global radiation the most, less the UV-A; the UV-B was the least affected.

- The relative intensity of the UV radiation increased with cloudiness. This was more pronounced for UV$B$ than for UV-A.

- The ozone concentration during the trip varied between 160 to 290 DU (Dobson Units). We entered and left the "antarctic ozone hole" several times.

- The UV-B radiation displayed a dependency on both ozone levels and cloudiness. A three-dimensional presentation is presented for UV-B versus ozone and cloudiness.

- The UV-B radiation was, on average, more than doubled when we were in the "antarctic ozone hole," while the UV-A radiation was hardly affected.

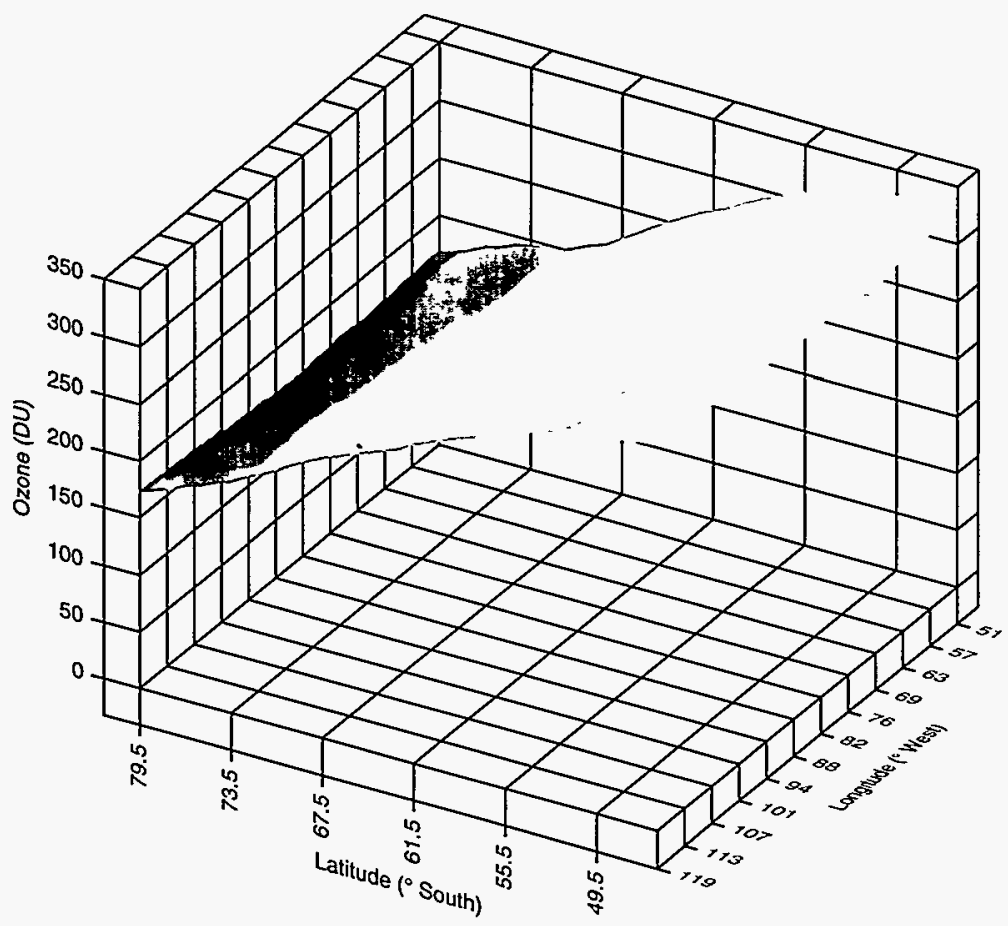

\section{Average Ozone Concentration}

The graph above shows ozone concentration in Dobson units (TOMS data) from Meteor 7. The values represent the mean conditions for the time of the 1994 cruise mentioned above. Note the substantial decrease in ozone concentration as the cruise continued southward. Daily values reached as low as $160 \mathrm{DU}$.

NSF Grant DPP 9070969: Scientific personnel-G.D. Wendler and T. Quakenbush

\section{Measurements of Ultraviolet Solar} Radiation with a Sunphotometer: A UV-visible multichannel instrument was acquired by the Geophysical Insitute and installed on the roof in August 1993 to study the relationships among ultraviolet radiation, ozone abundance and cloud effects. The plan is to deploy this device on the North Slope as part of the educational component of the ARM project.

This GUV-511 instrument, built by Biospherical Instruments, measures irradiance at $308,320,380$, and $450 \mathrm{~nm}$, with a passband of about $10 \mathrm{~nm}$. In addition, it measures the total photosynthetically active radiation between 400 and $700 \mathrm{~nm}$.

The instrument is designed to require a minimum of maintenance; it is temperature stabilized at $40^{\circ} \mathrm{C}$, and, therefore, operative during the cold winter.

By combining irradiance measurements with radiative transfer computations, we can determine total ozone abundance, UV-dose rates, cloud optical depths and UV-spectra.

The total ozone abundance is obtained by measuring the $320 \mathrm{~nm} /$ $308 \mathrm{~nm}$ irradiance ratio. We have used a radiative transfer model to compute the corresponding ratio for 
a variety of ozone abundances and solar zenith angles and we created look-up tables from which the total ozone abundance can be determined by comparing measured ratios with the computed ones.

Further, the cloud optical depth may be obtained by using either the measured $340 \mathrm{~nm}$ or the $380 \mathrm{~nm}$ irradiance. By using these two parameters in the radiative transfer model, a solar spectrum may be computed.

The time resolution of the GUV measurements is one minute, and after 15 months with nonstop logging, a large set of data has been collected.

These data have now been systematized and analyzed. Ozone values, UV doses and optical cloud depths for the whole period (August 1993 to

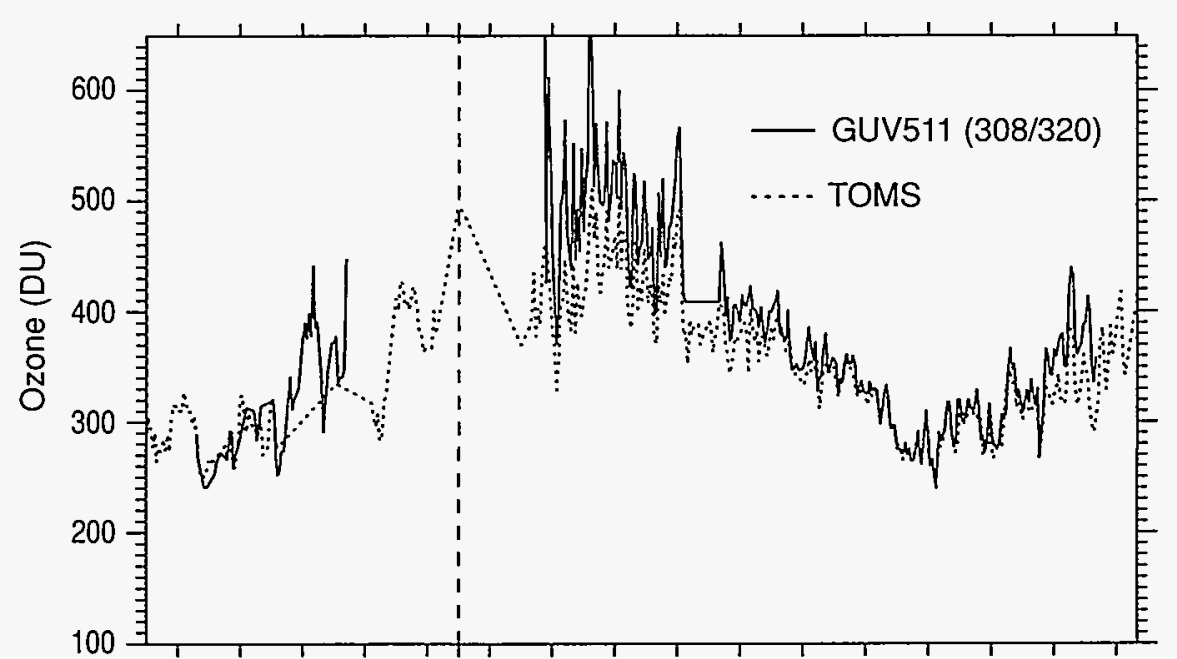

Aug Sep Oct Nov Dec Jan Feb Mar Apr May Jun Jul Aug Sep Oct Nov 1993

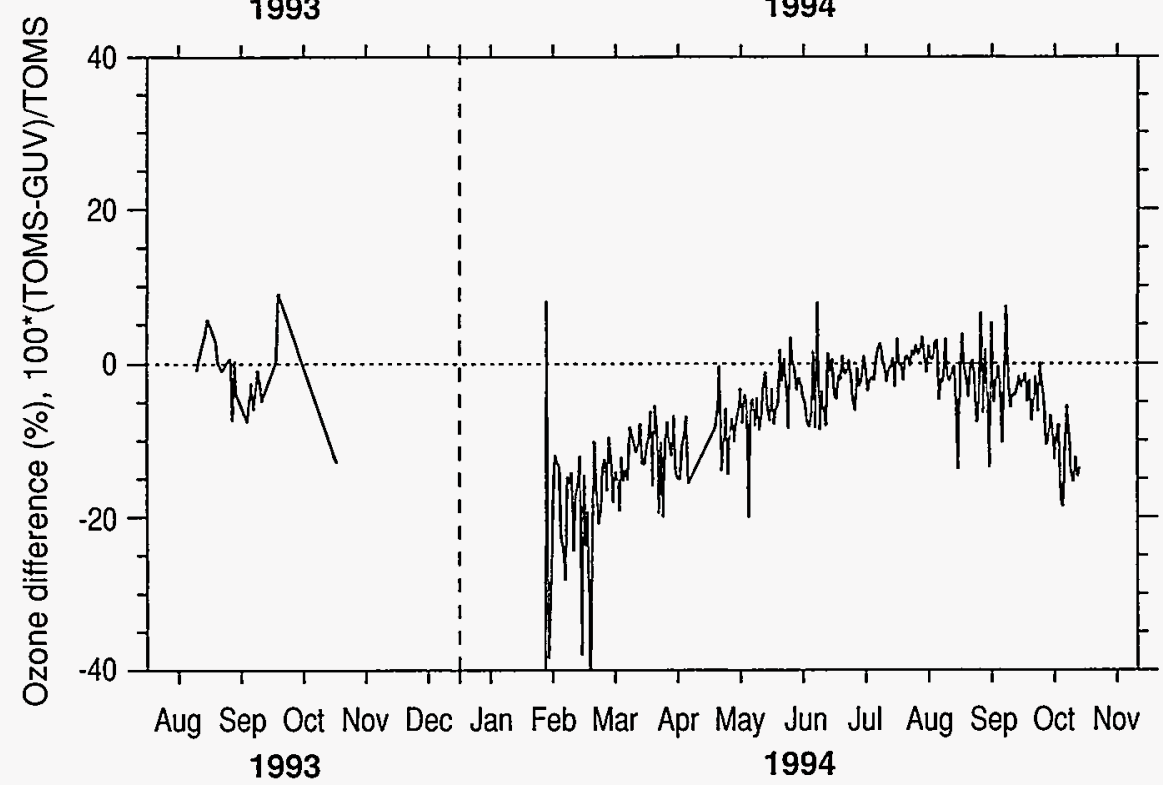

The two graphs above show a comparison of ozone values from GUV-511 and TOMS. In winter, the 308 channel from GUV-511 give signals that are too weak, and no ozone values can be calculated. Instead, the 320/380 irradiance ration can be used in the winter.
December 1994) have been calculated.

The data have been compared with ozone measurements from other instruments, preferentially with ozone values from a nearby Dobson instrument (a ground-based spectrometer) and with satellite data from TOMS (onboard the Meteor 3 satellite).

The agreement between GUV and TOMS is good during the summer period, but the deviation is somewhat larger in the winter. This discrepancy might be due to the different vertical ozone distributions that normally occur during winter and summer seasons.

Use of "wrong" winter ozone profile might cause errors in the computed look-up tables, but probably can be corrected by introducing a more appropriate winter ozone profile in the radiative transfer model.

Analysis of GUV data (comparisons between the four channels) has revealed some drift in the GUV instrument.

The drift is especially evident for the 340 channel and results in an overestimation of the calculated cloud cover and an underestimation of the ozone value. However, this drift can be corrected by determining new response functions for the instrument.

In February 1995, the GUV instrument will be taken to San Diego, where the necessary calibration will be performed. All the data can then be corrected.

So far, the data from GUV seem very promising. They will probably be both reliable and accurate when the instrument is properly calibrated.

NSF Grant DPP92-00747: Scientific personnel-K.H. Stamnes and T. Svendby 
Polar Ozone Depletion and Biologically Relevant Ultraviolet Radiation: This project makes use of the NSF UV-visible Monitoring Network in Antarctica, and at Barrow, Alaska, to quantify the relationship among ultraviolet radiation exposure, ozone abundance and cloud cover in the polar regions.

A comprehensive radiative transfer model is used to compare measured spectral irradiances with computed results in order to validate the model.

A radiative transfer model for the coupled atmosphere-ocean system has been developed and used to study the penetration of ultraviolet radiation into the ocean.

NSF-DPP; Polar Biology andMedicine Program, Grant DPP92-00747: Scientific personnel-K.H. Stamnes and J. Zeng
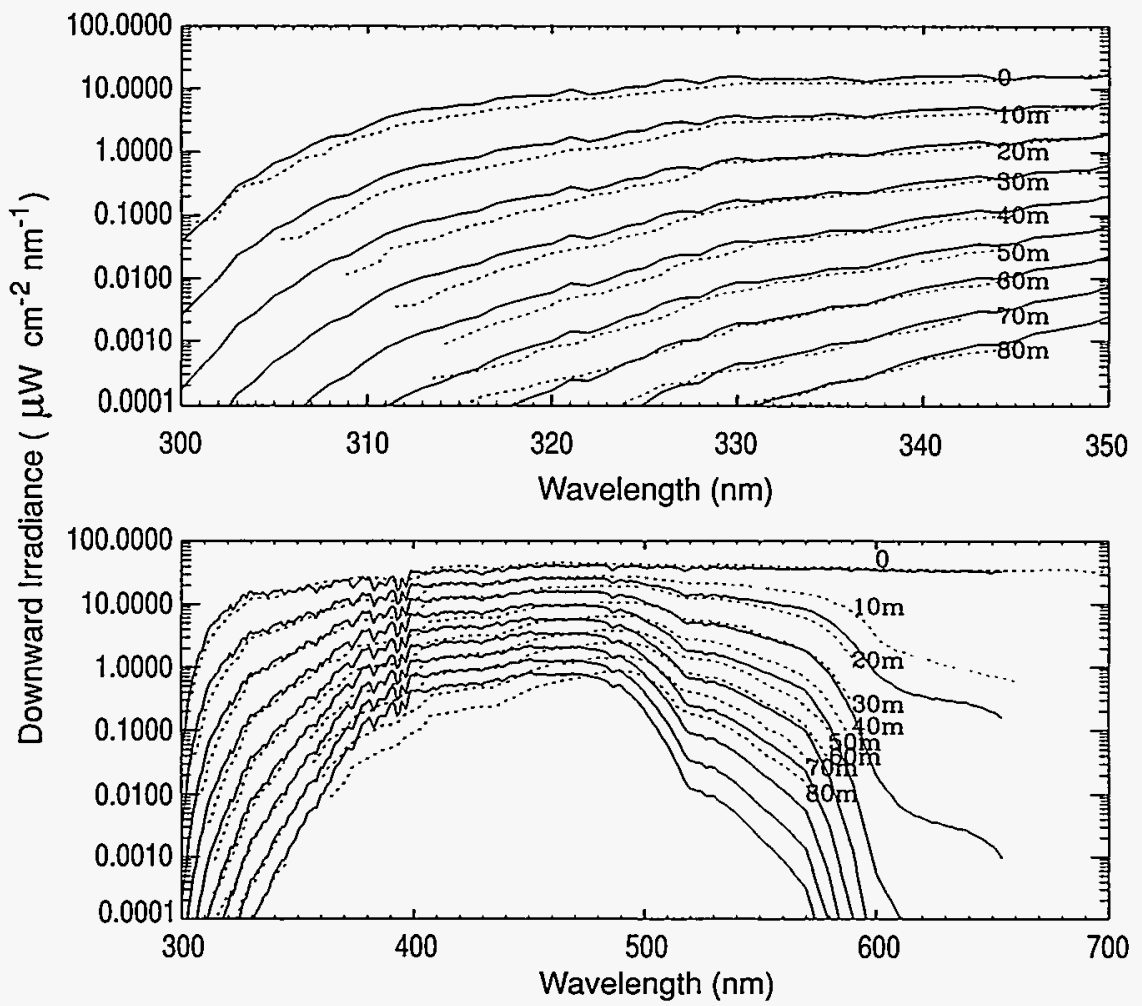

Shown above is a comparison study of undenwarer ultraviolet radiation between model computation and in situ measurements made in Southern Ocean, Antarctica. Solid lines are modeled and dotted lines are measured.

\section{Cloud Physics and Radiation}

The North Slope of Alaska (NSA) Atmospheric Radiation Measurement (ARM) Program: Uncertainties associated with cloud behavior are recognized as the major obstacle to progress in climate modeling and prediction.

ARM is a ten-year climate research program aimed at unraveling the role of clouds in the climate system. As such, it constitutes a major portion of the Department of Energy's contribution to global change studies.

The goal of ARM is to improve our understanding of the role clouds play in the climate system by studying the life cycle of clouds and their effect on the radiative energy bal- ance. The experimental framework is the cloud and radiation test-bed. The program's focus is on cloud behavior and on how clouds affect the radiative energy balance and, thereby, the climate. The ultimate aim is to use this information to improve the performance of global climate models.

The North Slope of Alaska site is the only high-latitude ARM site. High latitudes are important because they constitute the major heat sink for the earth.

Northern Alaska experiences great seasonal variation in solar illumination and surface properties. Also, the exchange of water between the atmo- sphere and the arctic land and ocean varies grearly between summer and winter.

Global climate models have demonstrated that the Arctic is a region of high climate sensitivity. Model experiments, in which the abundance of carbon dioxide is doubled, indicate the largest warming over the Arctic occurs in the winter.

These experiments provide compelling reasons for taking phenomena occurring in the polar regions more seriously.

Arctic processes have been treated so primitively in most models that we have no way of judging if the predictions are realistic, and we can 
not determine global ramifications. Should we expect the predicted warming to lead to an ice-free Arctic Ocean? If so, how soon will that occur? What is the impact of an icefree ocean on regional (Arctic) and on global climate?

The NSA ARM site is ARM's window on the Arctic. It will be used to provide much of the data required to answer the preceding questions, to improve global climate models so they can become more viable tools
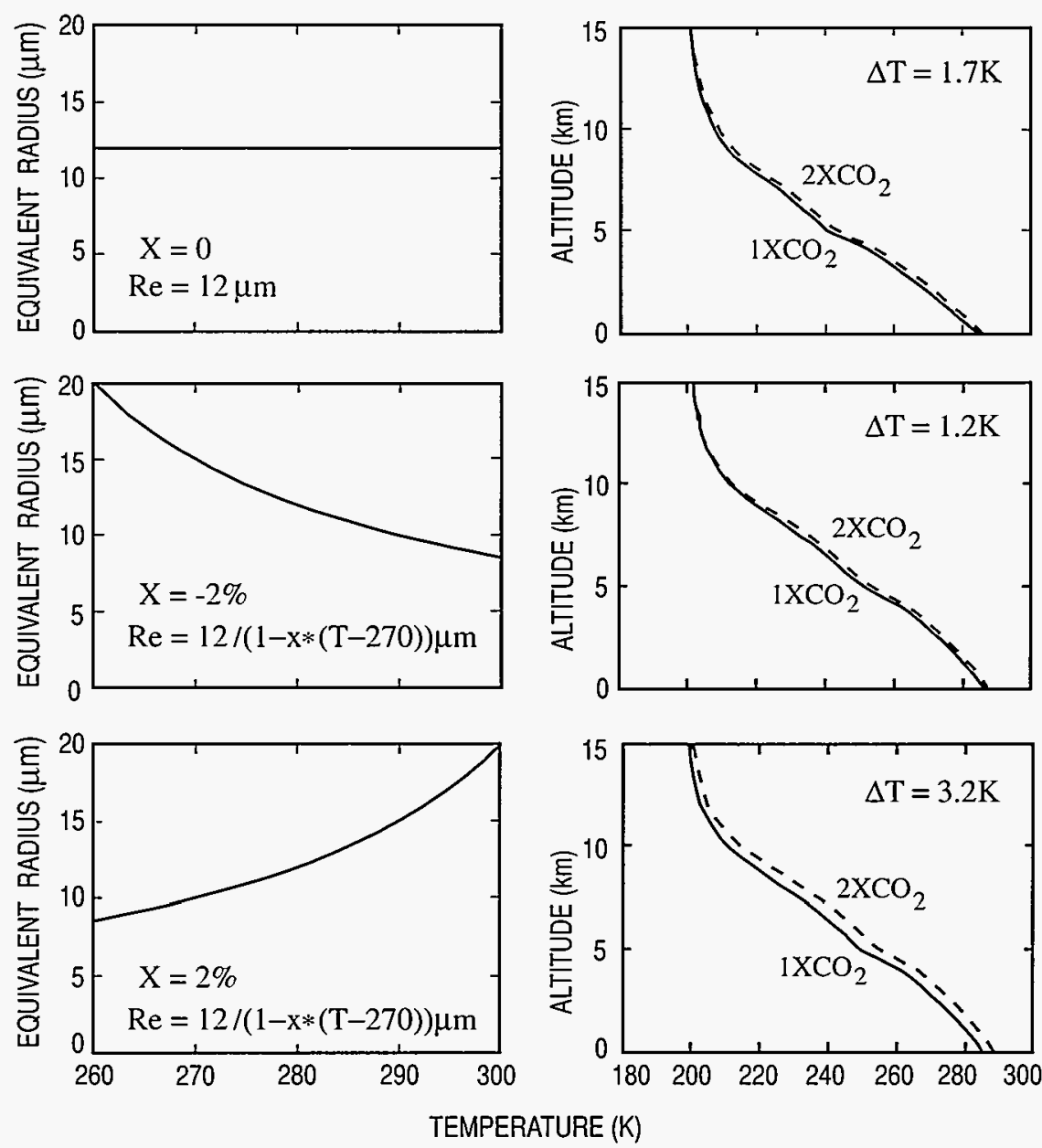

Cloud microphysical processes have a strong influence on the magnitude of the greenhouse effect as the three panels above illustrate. Top Panel: For fixed cloud drop size in the climate model, the equilibrium surface temperature increases $1.7^{\circ} \mathrm{C}$, for a doubling of the $\mathrm{CO}_{2}$ concentration. Middle Panel: If the cloud drop size increases 2 percent when temperature increases $1^{\circ} \mathrm{C}$, the equilibrium surface temperature increases $1.2^{\circ} \mathrm{C}$, for a doubling of the $\mathrm{CO}_{2}$ concentration. Bottom Panel: If the cloud drop size increases 2 percent when temperature increases $1^{\circ} \mathrm{C}$, the equilibrium surface temperature increases $3.2^{\circ} \mathrm{C}$, for a doubling of the $\mathrm{CO}_{2}$ concentration. arctic conditions at a very basic level. With this model, we expect to be able to analyze and extract the parameters that play a significant role in arctic cloud formation and in the arctic radiation budget.

The model will be used to provide insights into cloud behavior, as well as guidance for which cloud properties should be measured.

The Arctic is a key element in determining the radiation budget of the earth. Within the polar regions, the net radiation (incoming solar radiation minus outgoing infrared radiation) is negative.

To paint a complete picture of the Arctic, one must examine the prevalent atmospheric features. One such feature is a persistent layer of lowaltitude stratiform clouds found over the Arctic, predominantly from April to September.

These Arctic Stratus Clouds (ASC) modulate the earth's radiation budget by contributing to the vertical transport of heat. It is therefore crucial to understand the radiative properties of Arctic Stratus Clouds.

We believe that the radiative properties of ASC are strongly coupled to the microphysics of the cloud. To offer insight into the detailed interaction of radiation and cloud microphysics, we must construct a model that combines a detailed microphysics scheme and solid radiative transfer code.

Our aim is to determine whether this coupling is sufficient to describe the observed characteristic properties, such as the lifetime and multilayered structure, of stratus clouds. In addition, we hope to provide useful input to measurement programs at the NSA ARM site by isolating 
the measurable parameters that influence the overall radiative properties of these clouds:

Battelle Pacific Northwest Laboratory DOE Contract 091574-A-Q1: Scientific personnel-K.H. Stamnes, O. Lie-Svendsen, and J. Simmons

Cloud Microphysics and Climate Sensitivity: A Radiative-Convective Model (RCM) has been developed and used to study the link between cloud microphysics and climate.

The RCM includes a comprehensive radiation scheme in which cloud microphysical properties have been parameterized in terms of effective droplet radius and liquid water content.

Results from the RCM model indicate that potential changes in cloud droplet size in response to global warming (expected from increased emissions of greenhouse gases) may have substantial feedbacks on climate (see illustration above).

In collaboration with the group of Professor R. Somerville of the Scripps Institution of Oceanography, our radiation scheme, including the parameterization of cloud optical properties, is currently being implemented into a GCM to allow for a more comprehensive study of cloud feedback mechanisms.

DOE, Quantitative Links, Grant DE-FG06-90ER6120: Scientific personnel-K.H. Stamnes and Y.-X. Hu

Measurements of Cloud Condensation Nuclei: A cloud chamber is used to measure the supersaturation spectrum of cloud condensation nuclei.

The aim of the study is to investigate the role of nuclei in cloud for-

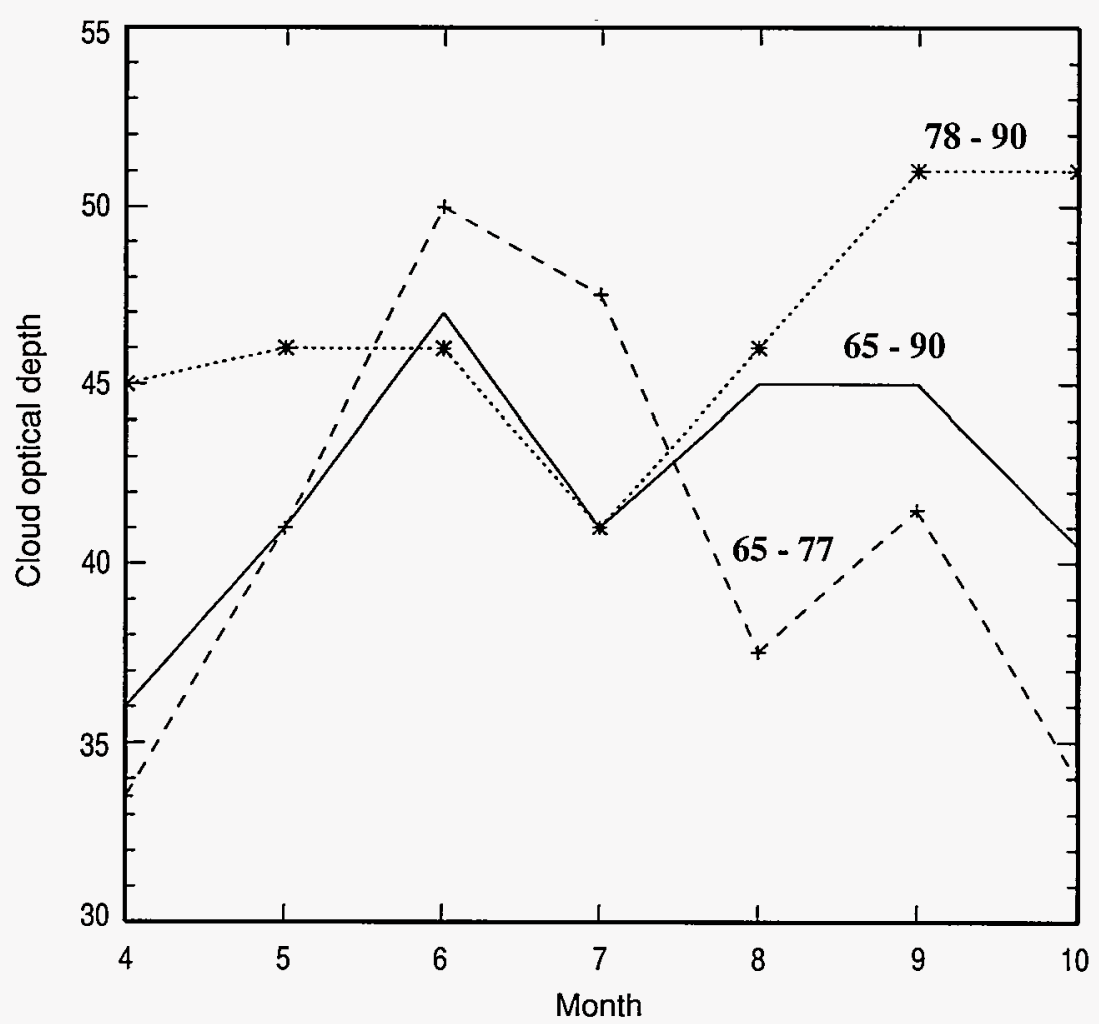

Shown above is the seasonal cycle of the cloud optical depth (median value at noon) at Bergen, 1965-1990.

mation, and to investigate how alteration in these nuclei (caused by industrial pollution reaching the Arctic) may affect cloud microphysics and radiative transfer.

We have found that cloud condensation nuclei are removed in air masses that have originated in the northern Pacific region and passed over the Alaska Range.

During such chinook flow, the condensation nuclei apparently are removed in precipitating clouds on the windward side of the mountain.

Our preliminary results indicate that the number of cloud condensation nuclei in arctic haze episodes is quite small. We will continue to work to obtain additional information on the CCN spectrum of the type that can be employed in cloud models.

Battelle Pacific Northwest Laboratory DOE Contract 091574-A-Q1:
Scientificpersonnel-G.E. Shaw, K.H. Stamnes, and Q. Ji

Local Cloud Climatology Based on the Analysis of Long-term Solar Irradiance Records: A unique cloudradiation data set obtained at Bergen (Norway) covering a 26-year period (1965-1990) was used in conjunction with a comprehensive radiative transfer model to infer cloud properties and to study their evolution over this period.

An examination of the optical depth-temperature relation suggests that the clouds over Bergen have a maritime character in the autumn, but a more continental (cold) character in the spring.

This study clearly indicates the effects of local orography on cloudiness in this region. We also found a slight trend toward increasing cloud 


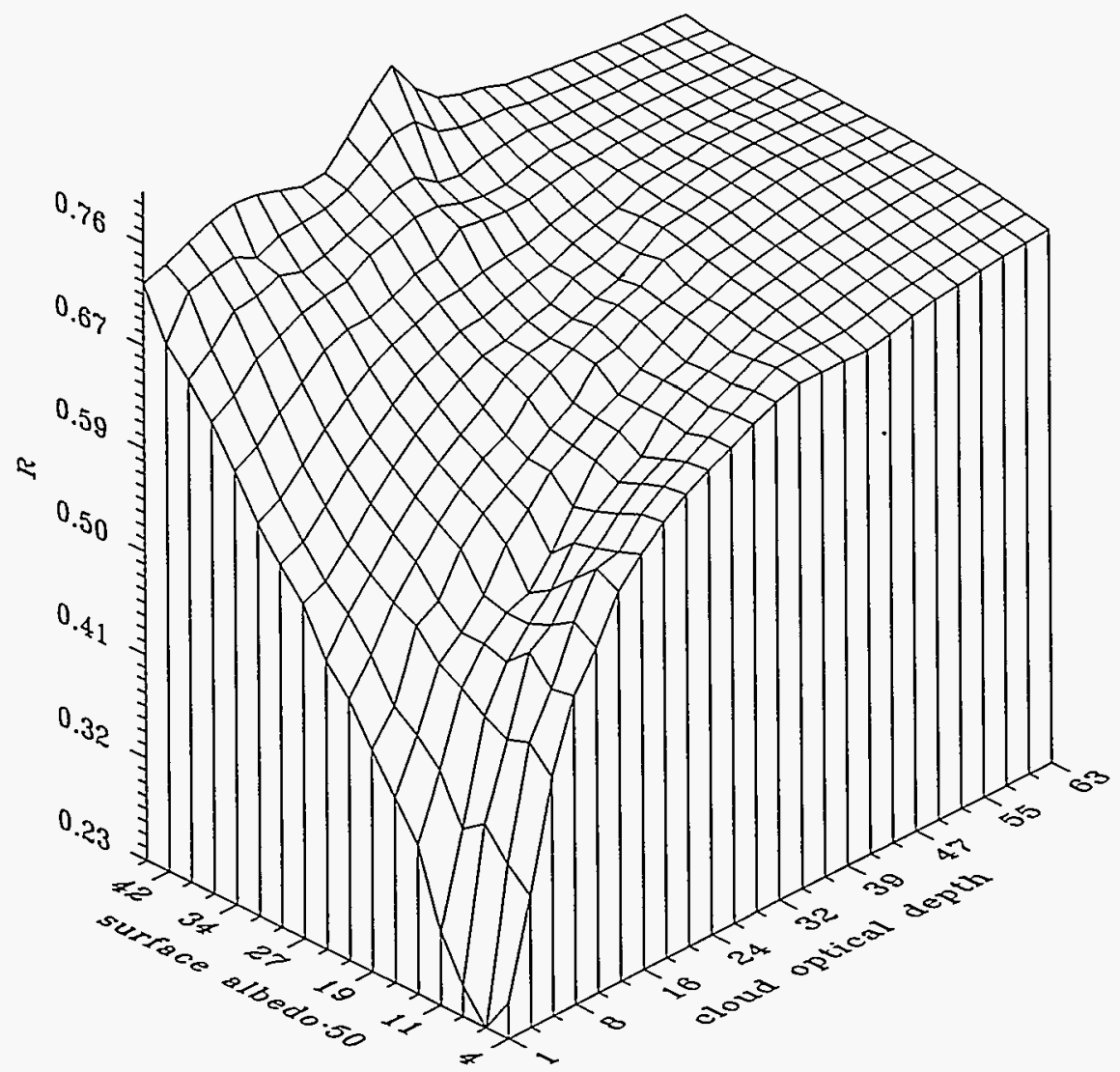

Shown in the graph above is the system albedo (R) for cloudy atmospheric conditions.

optical depth at noon (April through October) for this period.

Battelle Pacific Northwest Laboratory DOE Contract 091574-A-Q1: Scientific personnel-K.H. Stamnes and E. Leontieva

Cloud/Albedo Effects on the Radiation Budget in the Arctic: Broadband solar irradiance data from the NOAA Climate Monitoring and Diagnostics Laboratory facility in Barrow have been used in conjunction with radiative transfer computations to infer cloud optical depth over Barrow with emphasis on the joint role of surface albedo and cloud optical depth.

We plan to develop similar techniques for analysis of data from the multichannel MFRSR instrument.
A-Q1: Scientific personnelK.H. Stamnes and E. Leontieva

Arctic Qualification of Radiation Instruments: A Multi-Filter Rotating Shadowband Radiometer (MFRSR) was modified for use at high latitudes by Pacific Northwest Laboratory, and subsequently installed on the roof of the Geophysical Institure in September 1993.

The MFRSR provides spectrallyresolved total-horizontal, diffusehorizontal, and direct-normal irradiances at wavelengths 415,500 , $610,665,862$, and $940 \mathrm{~nm}$ with a nominal bandwith of $10 \mathrm{~nm}$ within the passband of a silicon photodiode.

The instrument is designed to perform routine measurements of atmospheric (aerosol) optical depth for monitoring anthropogenic climate modification and has been deployed by the Department of Energy's Quantitative Links and ARM measurement field programs, as well as by the U.S. Department of Agriculture.

The spectral channels were selected on the following basis: the $415 \mathrm{~nm}$ channel indicates contribution from molecular (Rayleigh) scattering; the 500,610 , and $665 \mathrm{~nm}$ channels indicate contributions from ozone (the Chappuis band); the $862 \mathrm{~nm}$ channel characterizes the transparent window region; the $940 \mathrm{~nm}$ channel is within a strong water vapor absorption.

The three irradiance components are derived from a single optical sensor, thus reducing intercalibration concerns about both absolute sensitivity and spectral bandpass, and guaranteeing synchronized measurements. A standard Eppley Precision Infrared Radiometer (PIR), on loan 
from the ARM program, has been equipped with a ventilator and muffin fan to alleviate problems with moisture and frost build-up on the PIR's dome.

In September 1994, the PIR was deployed on the roof of the Geophysical Institute along with a revised data-logging system that allows implementation of the same data processing program for a set of radiation and meteorological instruments.

A UV-visible multifilter instrument was acquired by the Geophysical Institute for investigating the feasibility of studying the relationship among ultraviolet radiation, ozone abundance and cloud effects with such a simple device.

This instrument, built by Biospherical Instruments, has channels at $308,320,380$, and $450 \mathrm{~nm}$, with a passband of about $10 \mathrm{~nm}$. In addition, it measures the total photosynthetically active radiation between 400 and $700 \mathrm{~nm}$.

The plan is to deploy this instrument on the North Slope as part of the educational component of the ARM project. In addition, the University of Denver (D. Murcray and F. Murcray) has deployed a BOMEM FTIR with about one wave number resolution at the Geophysical Institute.

Our current plans are to monitor the performance of all these instruments under a variety of weather conditions, including severely cold periods, to assess their potential for contributing to the North Slope of Alaska ARM site objectives in the near future.

Battelle Pacific Northwest Laboratory DOE Contract 091574-A-Q1: Scientific personnel-K.H. Stamnes,
E. Leontieva, T. Svendby; F. Murcray and D. Murcray (University of Denver)

Radiative Transfer in the Atmosphere-Sea Ice-Ocean System: A comprehensive radiative transfer model for the coupled atmospheresea ice-ocean system has been constructed and is being used to study the radiative interactions between the atmosphere, sea ice, and ocean. The consistent solution of the radiative transfer equation in this coupled system automatically takes into account the refraction and reflection at the air-ice interface.

The input parameters required by the model are observable physical properties (for example, the profiles of temperature, pressure and gas concentrations in the atmosphere; the
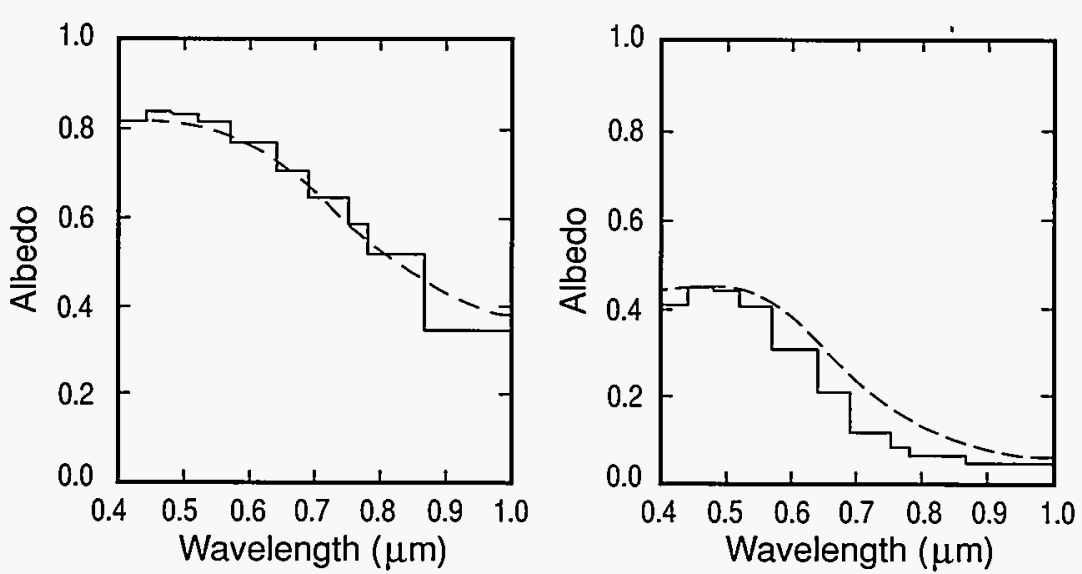

A comparison of observed spectral albedo (broken line) with model calculations (solid line) for melting multiyear white ice (left panel) and for melting first-year blue ice (right panel) is shown above.

water or ice content of clouds; the mean size of cloud particles; and the profiles of temperature, density and salinity in the ice).

This coupled model computes albedo self-consistently (see figure for comparison with observations) and also allows for a detailed study of the disposition of solar energy within each of the subsystems, as well as within the entire system.

The model also shows how the partitioning of solar energy within the system depends on atmospheric structure, including cloud cover and ice properties, such as temperature, salinity and density.

The modeling results show that as the ice density increases, the radiative absorption increases in the entire coupled system, as well as in the sea ice and in the ocean, while the absorprion in the atmosphere exhibits a slight decrease. As the ice salinity increases, the radiative absorption in the entire coupled system, in the ice, and in the ocean, decreases.

Most of the radiative energy absorption by sea ice occurs in a very thin top layer of the ice. This is 
In sea ice, the scattering by inclusions, especially the air bubbles in a few centimeters of the uppermost layer, plays a vital role to the solar energy absorption and partitioning in the whole system.

Enhanced scattering in this top layer will not only increase backscattering to the atmosphere, but also increase the absorprion in this top ice layer itself and decrease the radiation penetrating to the deeper layers of the ice and into the ocean. Ice thick- ness also exerts a significant influence on the radiative energy balance in the atmosphere-sea ice-ocean system, especially when the ice is thin.

An increase of ice thickness between 0 and $70 \mathrm{~cm}$ results in an increase in the radiative absorption in the ice and a decrease in the ocean absorbtion, as well as a decrease in the entire system. However, the total absorption in the entire system remains almost constant once ice thickness exceeds about $70 \mathrm{~cm}$. Never-
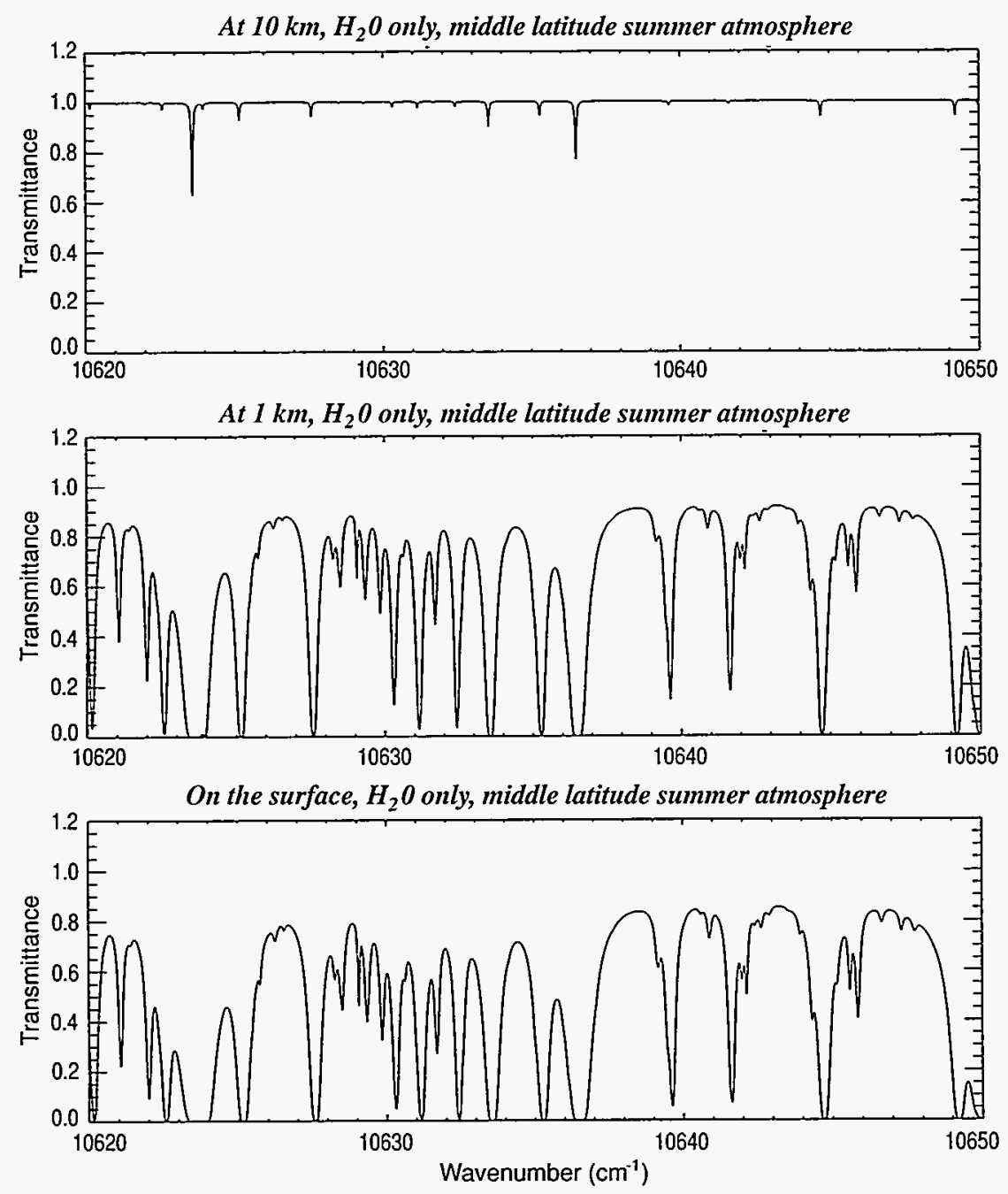

The three panels above show transmittance calculated from a merged FASCODE/ DISORT code at $10 \mathrm{~km}$, at $1 \mathrm{~km}$, and at the surface respectively. The resolution is 0.005 $\mathrm{cm}^{-1}$. Water vapor, the main absorber in this spectral region, is located below $10 \mathrm{~km}$, (see figure). The transmittance at $10 \mathrm{~km}$ (top panel) is 1 , except for some strong absorption lines. The bottom panel is the transmittance of the whole atmosphere.

theless, the absorption within the atmosphere is not sensitive to the ice thickness change.

The main role of clouds is to moderate any variation in the radiative energy budget caused by changes in ice properties and ice thickness. In other words, clouds reduce the sensitivity of the absorbed solar energy in every layer to changes in ice properties and thickness.

The couplings that we have considered thus far are purely radiative. The model will be refined to improve the treatment of the ice vertical properties for different ice types.

The plan is to combine the coupled radiative transfer model with models treating the evolution of ice, snow and clouds to investigate interactions and feedbacks in the polar climate system.

Battelle Pacific Northwest Laboratory DOE Contract 091574-A-Q1: Scientific personnel-K.H. Stamnes and $Z$. Jin

Work on Radiative Transfer Algorithms: A narrow-band model has been developed by Phillips Laboratory for computing thermal and solar radiance and transmittance with about one wave number resolution (MODTRAN).

The gaseous absorption coefficients are derived from the comprehensive HITRAN spectroscopic data base. This model includes accurate treatment of single scattering radiative transfer, but approximate (twostream) treatment of multiple scattering.

To enhance the validity and usefulness of MODTRAN, it has been merged with an accurate multiple scattering algorithm (DISORT). 
Since the resolution of MODTRAN is similar to that of FTIRs to be used in the ARM program, the merged MODTRAN/DISORT code is expected to be a useful tool for the analysis of FTIR data from the ARM sites including the North Slope of Alaska/Adjacent Arcric Ocean site.

Similar work on line-by-line codes is in progress with emphasis on including solar capability and rigorous multiple scattering into line-by-line algorithms, such as FASCODE and GENLZ.

Battelle Pacific Northwest Laboratory DOE Contract 091574-A-Q1: Scientific personnel-K. H. Stamnes, $X$. Fan, and N. Larsen

Radiative and Chemical Effects of Clouds and Aerosols: This project focuses on the atmospheric radiative processes and their effects on atmospheric composition. Clouds and aerosols scatter and absorb solar and terrestrial radiation. Such particulate matter may profoundly affect both the actinic flux and the radiative warming and cooling rates and, thereby, the atmospheric temperature structure.

This has important implications for atmospheric chemical composition because: 1) photolysis rates are affected, 2) many chemical reactions occur at temperature-dependent rates; and 3) the dynamic behavior of the atmosphere will change.

In this project we use our state-ofthe-art radiation model, valid throughout the troposphere and stratosphere, to undertake a systematic study of the influence of clouds and aerosols on atmospheric radiation and composition. This study is accomplished by incorporating our radiation model into one- and twodimensional armospheric chemistry transport radiation models.

To carry out this endeavor successfully, we collaborate with the atmospheric chemistry modeling groups headed by $\mathrm{G}$. Brasseur at the $\mathrm{Na}$ tional Center for Atmospheric Research, Boulder, Colorado, and Professor I. Isaksen at the University of Oslo, Norway.

Finally, we also use our radiation model to investigate the effects of clouds and aerosols on the feasibility of retrieving trace gas information by the remote sensing of ultravioletvisible radiation from the ground and from space.

NASA Grant NAGW 2165: Scientific personnel-K. H. Stamnes and A. Kylling

Study of the Arctic Radiative Energy Budget Using Ground-Based and Satellite Data: To study the radiative energy budget in the Arctic, this project makes use of NOAA polar orbiter data, Synthetic Aperture Radar (SAR) data, plus data from the NOAA Climate Monitoring and Diagnostics Laboratory and the NSF UV-visible monitor in Barrow, Alaska. These data are used in conjunction with radiative transfer models to infer the role of clouds and surface characteristics on the radiation environment.

Emphasis is placed on spatial and seasonal variability in the radiation budget and on the joint role of surface albedo and cloud cover in regulating the solar and terrestrial radiation. Planetary albedo and brightness temperature is provided by AVHRR, and SAR imagery provides surface type identification which may be used to assign albedo. With both SAR and AVHRR data, the radiative transfer modeling will be constrained from both sides of the atmosphere.

Existing radiation measurement programs in Barrow are used to test the methodology. These programs include a scanning spectroradiometer for the ultraviolet and visible range and broadband measurements of downwelling and upwelling solar and thermal infrared radiation. The Scripps Antarctic Research Center is providing image processing facilities for the entire project.

NSF-ARCSS (ARCtic System Science) Program, Grant DPP91-14010: Scientific personnel:-K.H. Stamnes and W. Han; D. Lubin (California Space Institute)

Study of Climate Change Mechanisms in the Cloud-Capped Planetary Boundary Layer over the Arctic Ocean Using a Hierarchy of Models Resolving Cloud Formation Processes: This project is focused on modeling and case studies of radiative, dynamical, and microphysical properties of low stratiform clouds (with emphasis on aerosol/cloud interaction) covering most of the Arctic Ocean during the summer.

A recently developed three-dimensional large eddy simulation model with explicit formulation of cloud microphysics, as well as its one-dimensional and two-dimensional reductions, will be used to simulate and parameterize radiative cloud forcing and transport of momentum, heat, and moisture.

The long-term goals of the project are to provide theoretical/modeling 
support of observational studies at selected sites in the Arcric, to understand cloud formation mechanisms and feedbacks, and to develop parameterization schemes for use in General Circulation Models, and contribute to other climate-related programs of in situ and remote measurements of cloud parameters.

NSF DPP-Polar Ocean and Climate Systems Grant OPP94-15350: Scientific personnel-V.V. Filyushkin and K.H. Stamnes; D. Lilly (University of Oklahoma)

Use of Data from the ADEOS Satellite, the Alaska ARM and SAR Facilities and the SHEBA Ice Camp Experiment to Study Cloud/ Radiation/Climate Interactions and the Ultraviolet Radiation Environment in the Arctic: Access to data from the sensors onboard the Advanced Earth Observing Satellite (ADEOS) will enable us to conduct comprehensive studies of the polar environment.

The primary aim of the proposed work is to study the annual cycle of cloud/radiation interactions in a variety of meteorological conditions using $A D E O S$ data in conjunction with ARM and SHEBA observations to develop parameterizations of cloud processes and their impact on the radiation budget that are suitable for inclusion in climate GCMs in the Arctic.

Instruments such as the Polarizarion and Directionality of Earth Reflectances (POLDER), Advanced Visible and Near IR Radiometer (AVNIR), and the Ocean Color and Temperature Scanner (OCTS) will provide data on the reflectance of clouds and the surface at various spatial resolutions. The POLDER, considered to be the main cloud instrument, will provide horizontal resolution at a footprint of $6 \mathrm{~km}$ by $7 \mathrm{~km}$.

Measurements will be taken for solar zenith angles of less than 75 degrees during spring, summer and fall in the Arctic.

The OCTS, designed for studies of low-reflecting scenes, can, however, provide valuable informarion on cloud climatology in the Arctic at low sun elevations during winter.

The thermal IR channels of the OCTS can be used to characterize the IR component of the radiative budget in the Arctic during the entire year.

The AVNIR provides very high spatial resolution of $8 \mathrm{~m}$ and $16 \mathrm{~m}$ over the footprint of $80 \mathrm{~km}$ by 80 $\mathrm{km}$. The dynamical range of AVNIR permits the measuring of cloud reflectances.

Our main goal is to use the data from the AVNIR to study the spatial characteristics of low clouds in the Arctic in conjunction with the highresolution LES modeling of the cloud-capped boundary layer.

Ground-based and aircraft indirect and in situ cloud data will be utilized to facilitate extraction of information about the cloud parameters from the satellite observations.

Another important goal is to use ADEOS data to study stratospheric chemistry and the impact of ozone changes, cloud cover and aerosol loading on biologically relevant ultraviolet radiation.

ADEOS instruments, such as the Improved Limb Atmospheric Spectrometer (ILAS), the Interferometric Monitor of Greenhouse Gases
(IMG), and the Total Ozone Mapping Spectrometer (TOMS) will provide data on spatial distribution of ozone and a number of important ozone chemistry-related species such as $\mathrm{NO}_{2}, \mathrm{HNO}_{3}, \mathrm{~N}_{2} \mathrm{O}, \mathrm{CH}_{4}$, etc.

The instruments will provide data series of various spatial/temporal coverage. The assimilation and analysis of the measurements will, thus, require the use of comprehensive photochemical/transport modeling of the arctic stratosphere chemistry.

The time series of ozone and parameters of cloud cover will be used to study the influence of clouds on the levels of biologically relevant solar UV radiation at the surface.

The project can be viewed as an interdisciplinary study glued together by the common denominator of radiative processes, theoretical and observational means and the set of instruments onboard the ADEOS.

Modeling activities in preparation for the launch of the satellite (scheduled for 1996) are underway.

Endorsed by NASA and selected by the National Space Development Agency of Japan, Project No. 1063: Scientific personnel-K.H. Stamnes and V.V. Filyushkin; F. Murcray (University of Denver); S.-C. Tsay (NASA Goddard Space Flight Center)

Arctic Programs Related to, and Coordinated with, the NSA ARM Program: An extension of the Alaska ARM effort to the Adjacent Arctic Ocean (AAO) will occur in conjunction with SHEBA (Surface Heat Budget of the Arctic Ocean), an eighteen-month field experiment based at a manned drifting sea ice camp in the perennial pack ice of the Arctic Ocean. 
This $\$ 20 \mathrm{M}+$ experiment is led by the National Science Foundation as part of its Arctic System Science (ARCSS) program and by the Office of Naval Research.

The SHEBA field program is scheduled to begin in April 1997. The SHEBA observational effort will emphasize the relationship between radiative fluxes (especially as affected by surface- and cloud-radiative interactions), the mass balance of sea ice, and the storage and retrieval of energy and salt in the mixed layer of the ocean.

The SHEBA and ARM efforts are sufficiently complementary that the scheduling and siting have been designed to maximize the synergism, so that SHEBA permits the extension of the Alaska ARM project to the icecovered waters of the Arctic Ocean.

Another ARCSS effort that is of direct relevance to NSA ARM is the arctic trace gas Flux Study of the Land-Atmosphere-Ice-Interactions (LAII) component of ARCSS.

The Flux Study consists of (1) measurements of fluxes of trace gases $\left(\mathrm{CO}_{2}\right.$, methane) to the atmosphere and of water-transported materials to the ocean, (2) determination of the primary controls of the fluxes, and (3) scaling and synthesis to the regional scale (Alaska North Slope and beyond). The primary field sites are in the Kuparuk drainage basin of the Alaska North Slope.
The ultimate goal of this study is to assess the feedbacks between climatic change and the release of greenhouse gases from arctic terrestrial regions.

The LAII Flux Study interfaces with the NSA ARM effort both geographically (through field measurements in adjacent regions of the North Slope) and scientifically (through the link between surface radiative fluxes, soil/vegetation temperature and wetness, and rates of trace gas flux from/to terrestrial ecosystems).

The scaling and synthesis component of the LAII Flux Study utilizes the Arctic Regional Climate System Model (ARCSyM), which is now being run over a domain that encompasses both the LAII Flux Study area and the proposed NSA/ARM/AAO CART site.

The Arctic Climate System Study (ACSYS) is a new initiative of the World Climate Research Program. ACSYS is expected to span a period of approximately ten years.

ACSYS emphasizes the climate component of the arctic system through its focus on the Arctic Ocean, its sea ice cover, and its energy and water budgets. A topic of particular emphasis in ACSYS is the cloudradiative interaction that is crucial to a quantitative description and understanding of the surface energy budget in the Arctic. Both NSA
ARM/AAO and SHEBA are potential U.S. contributions to ACSYS.

It is expected that the findings of ARM and SHEBA will directly affect the ACSYS-coordinated iceocean modeling, which will likely be the key to an assessment of the stability of arctic sea ice in a changing climate.

Phase III of the First ISCCP (International Satellite Cloud Climatology Project) Regional Experiment (FIRE III) is a NASA-led effort that is planned to take place in the Arctic in conjunction with SHEBA and NSA/AAO.

The emphasis of FIRE is to provide in situ data that are currently lacking on cloud radiative properties to validate and improve satellite retrievals and GCM performance in the Arctic.

The use of instrumented aircraft is expected to play a major role in FIRE. Coordination between the FIRE III, ARM and SHEBA programs in the Arctic will be facilitated by the presence of several members of the FIRE III Science Team on the SHEBA Science Working Group, the ARM Science Team Executive Committee, and the ARM NSA/AAO Advisory Panel.

These projects and others related to, and coordinated with, the NSA ARM Program, will be discussed in more detail in future biennial reports. 

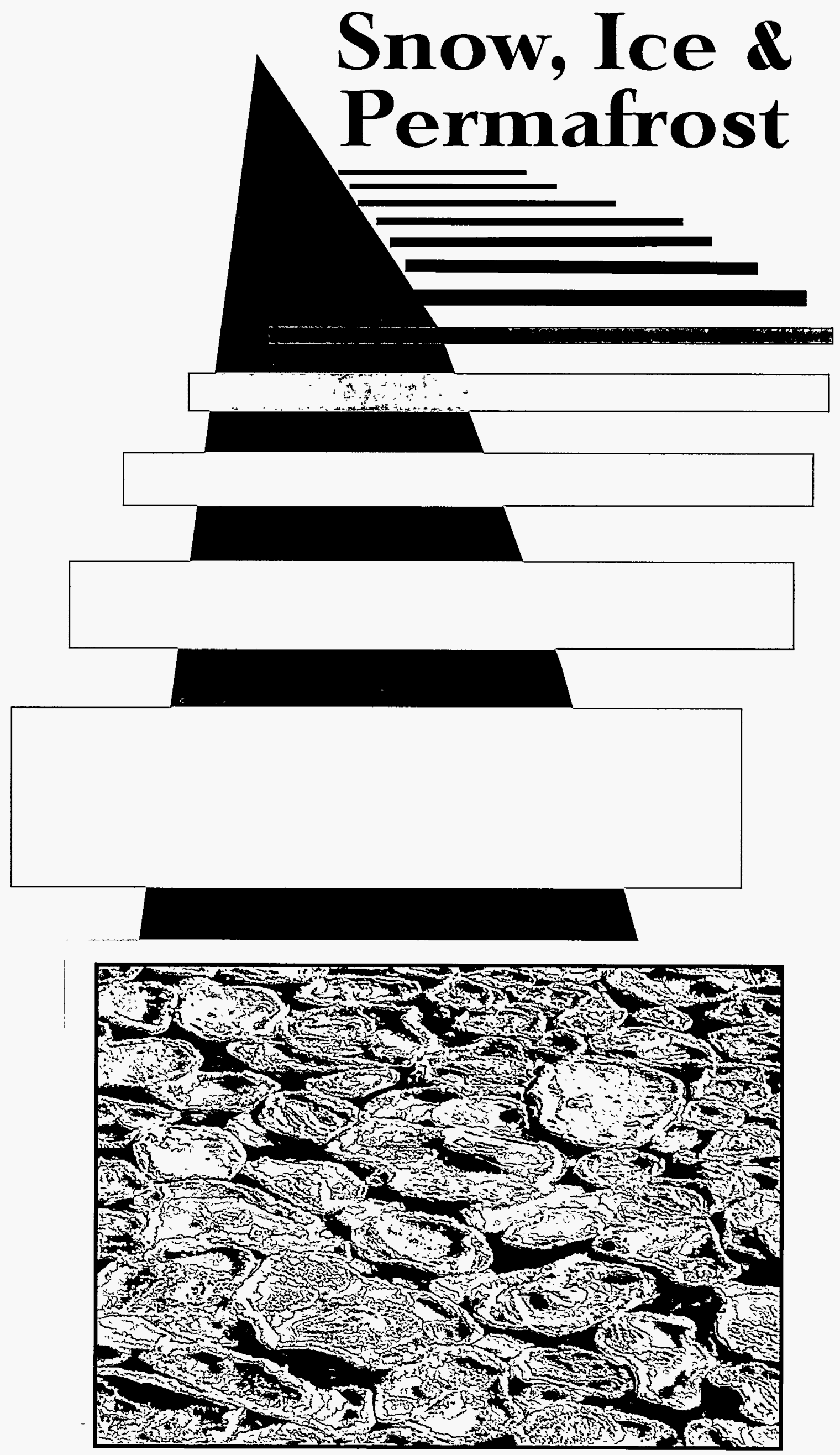


\section{SNOW, ICE and PERMAFROST}

Snow, ice, and permafrost research at the Geophysical Institute is directed toward developing a better understanding of the properties of these materials, of the processes occurring within them, and of the role they play in the polar and subpolar environments. Much of the research involves field investigations in Alaska, with some work carried out in Greenland and Antarctica. Some of the research is performed with the Alaska Synthetic Aperture Radar Facility (ASF) in the Geophysical Institute.

Sea ice studies cover a range of topics. One of these is how ice properties are related to meteorological conditions and growth histories. Others deal with the physical characteristics and development of ice in the Ross, Amundsen, and Bellingshausen seas, Antarctica, and the Beaufort-Chukchi Sea. The relationship between ice properties and radar backscatter is under study in order to improve the interpretation of SAR images. Some of the problems have an engineering focus. Permafrost research has been directed toward problems in heat and mass transport, including those involving salt, the response of permafrost to climate change (and the inverse problem of extracting information about past climate from present permafrost conditions), and the application of geophysical methods for detecting permafrost and related materials.
Glacier studies have recently focused on the dynamics of the fast flow of glaciers in Alaska and on the ice streams in Greenland and Antarctica. Studies have been broadened to include the use of satellite radar altimetry in ice sheet dynamics, and problems related to climate. In Alaska, the latter include the airborne measurement of glacier surface elevation changes, the identification of snow line positions using SAR data, and the determination of the accumulation rate on $\mathrm{Mt}$. Wrangell. In Greenland, the accumulation over the ice sheet has been reexamined, and SAR data have been applied to the refinement of the glacier facies concept. In Antarctica, modeling studies have examined greenhouse effects on the ice sheet and processes involved in ice stream flow.

Particularly in northern Alaska, snow studies have focused on seasonal snow cover-its quantity, the direction and magnitude of wind transport, its physical properties and structure, and its melting in relation to meteorological conditions.

The results of our snow, ice, and permafrost research are useful in the state of Alaska both in developing an understanding of scientific problems (including global change) and in the solution of applied problems (including the development of resources).

\section{Sea Ice Studies}

Relationships between the History and Structure of Sea Ice and its Physical and Mechanical Properties: This project involves two aspects of sea ice mechanics that are important building blocks leading to an improved understanding of the natural behavior of this common, naturally occurring arctic material.

The first aspect is the influence that structure, properties and growth history have on the strength and mechanical properties of sea ice at different scales. Currently, we are investigating the interrelations between 1) the degree of c-axis align- ment in the horizontal plane, the degree of substructure development, the vertical variation in grain size, the development of brine drainage features and 2) the local small-scale flexural and compressive strengths as determined on plates and beams. In particular, we are emphasizing the development of experimental and theoretical bases for estimating the properties of first-year sea ice during the spring and early summer when brine drainage channels are well-developed within the ice. These features, which can be centimeters in diameter and may extend vertically throughout most of the ice sheet, must strongly influence the bulk properties of sea ice at these times of year. Yet, to date, the presence of these ubiquitous features have been completely ignored in treatments of ice properties.

The second objective of the project is to study the refreezing rate of firstyear pressure ridges. Recent ridges are ubiquitous features of pack ice and must be considered in analyses of sea ice on any scale larger than that of individual floes. Yet data are lacking still concerning the speed with which these features reheal and thus 


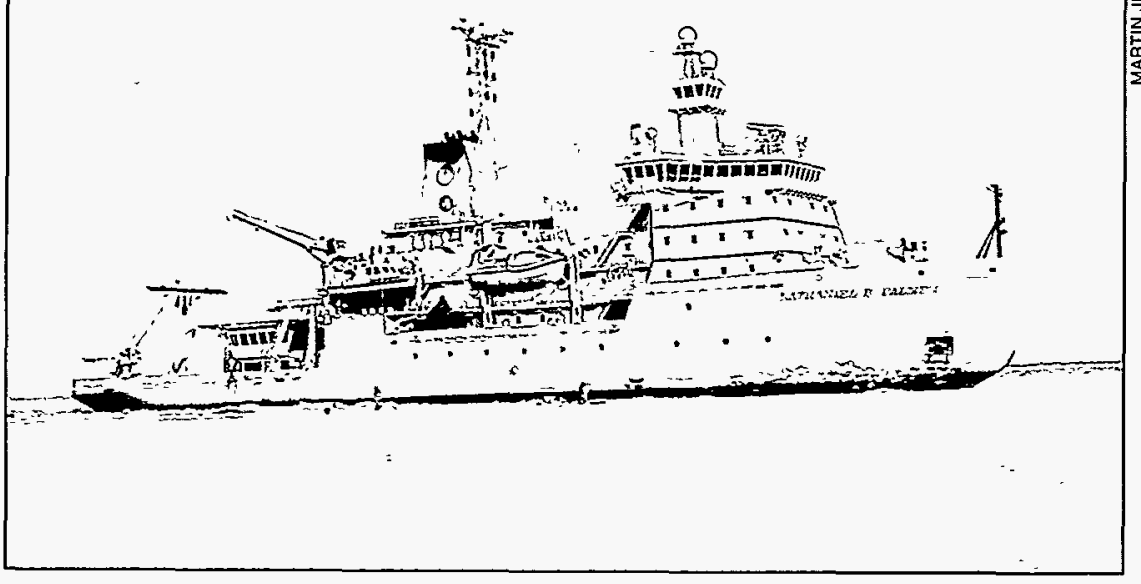

The R.V. Nathaniel B. Palmer is a 308-foot-long, 13,000 horsepower research vessel capable of continuously breaking through up to 3 feet of sea ice at a speed of 3 knots. The ship supports the U.S. Antarctic Marine Science Program and has been used extensively by Research Associate ProfessorMartin Jeffries of the Geophysical Institute.

reestablish continuity between the floe fragments. We are performing observational and modeling studies of this process. With these studies, we are collecting data on interrelations between ice temperatures, ice growth rates and ice salinity profiles. We plan to use this information to improve current numerical models that predict changes in the profile properties of sea ice during the complete ice growth season.

ONR Grant N00014-92-J-1429: Scientific Personnel-W.D. Harrison, L.H. Shapiro, and W.F. Weeks

Sea Ice: Physical-Structural Characteristics, Development, and SAR Signatures in the Pacific Sector of the Southern Ocean: The objectives of this research in the latewinter pack ice close to the time of maximum ice extent and thickness are: 1) to document the salinity and temperature characteristics of floes; 2) to document the structural variability and to identify the processes responsible for ice growth; 3) to operation and ice observations and measurements were concurrent with ERS-1 data acquisition at O'Higgins Base on the Antarctic Peninsula.

NSF Grant OPP91-17721: Scientific personnel-M.O. Jeffries, W.F. Weeks, S. Li, K. Morris, S. Cushing; R. Jaña (Instituto Antartico Chileno); Chuab Teong Sek (University of Kansas); H.R. Krouse (University of Calgary); A.P. Worby, R. Massom (Australian Antarctic CRC)

The Role of Snow in Antarctic Sea Ice Development and OceanAtmosphere Energy Exchange: The objective of this research in the winter antarctic pack ice is to understand the role of seawater flooding and snow ice formation in antarctic sea ice growth and their effects on the heat and mass balance of the ice. During a cruise of the R.V. Nathaniel B. Palmerin the Amundsen and Ross seas in September and October 1994, activities included: 1) documentation of the physical and strucrural characteristics of the snow cover; 2) determination of snow and ice

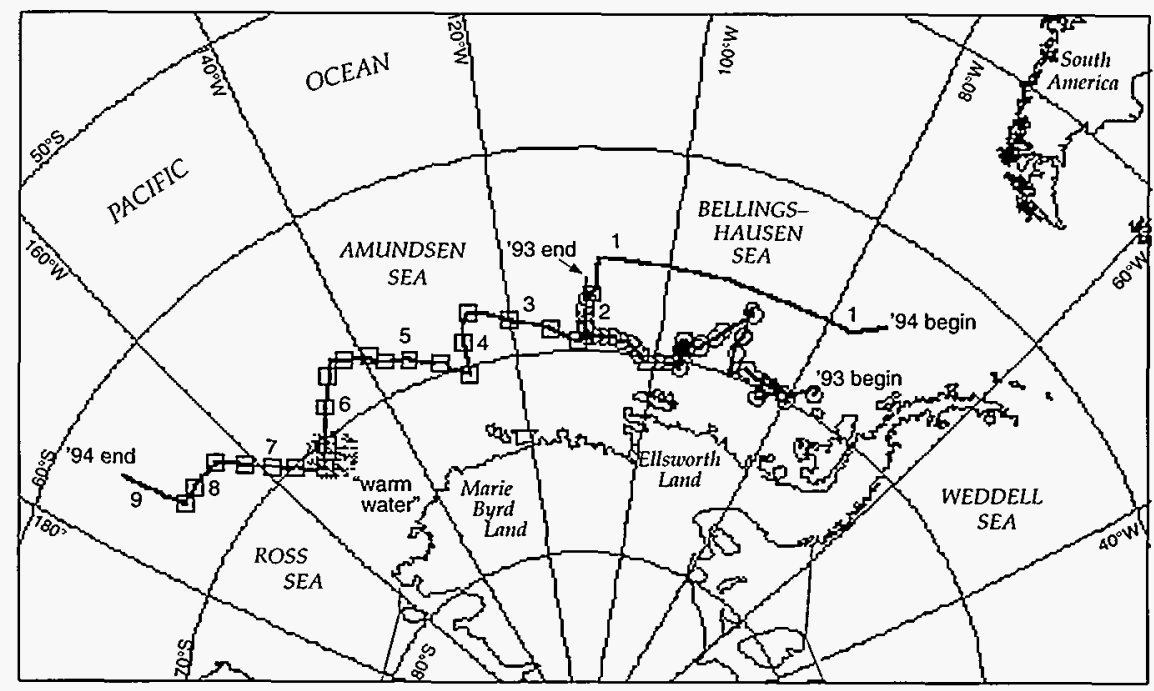

The map above traces the path of the R.V. Nathaniel B. Palmer in 1993 and 1994 when it operated in the Bellingshausen, Amundsen and Ross seas. 
thickness and freeboard distributions; 3) measurements of ice salinity and temperature; and 4) investigation of the structural and isotopic variability of the ice and identification of the processes responsible for ice growth. Further field research will be undertaken in the Ross, Amundsen, and Bellingshausen seas in 1995. Field observations and measurements will be used to develop and validate an antarctic sea ice growth model.

NSF Grant OPOP93-16767: Scientificpersonnel-M.O. Jeffries, $K$. Morris, S. Cushing, E. Maksym, $M$. Sturm (CRREL) and R. Massom (Australian Antarctic $(R C)$

Extinction of Near Ultraviolet and Visible Light in Summer Antarctic Sea Ice: High wavelength resolution measurements of downward-traveling light were taken from several depths in antarctic sea ice during early January. Spectral extinction was calculated in the ice layers where the measured light levels were proportional to downward flux in the ice. The wavelength range was 350 to $700 \mathrm{~nm}$ with a resolution of $1 \mathrm{~nm}$. The derived extinction values were considered as functions of the physical characteristics of the ice.

Extinction values in the near ultraviolet varied from 0.7 to $5 \mathrm{~m}^{-1}$ for different ice floes and layers in the ice. There was a minimum in extinction in the 450 to $500 \mathrm{~nm}$ band, where absorption by ice and water is small. It increased to values between 2 and $9 \mathrm{~m}^{-1}$ at $700 \mathrm{~nm}$ depending on the physical properties of the ice layers. Absorption by contaminants produced an absorption peak at $400 \mathrm{~nm}$ at all depths of one ice floe. Extinction in the 450 to $500 \mathrm{~nm}$ band was strongly dependent on contaminant concentration, and weakly dependent on ice depth and air content in the ice.

NSF Grant DPP 9070969: Scientific personnel-G. Wendler, $T$. Quakenbush, and C. Byers
The observed increase is, however, in agreement with Dumont d'Urville, the only ground station within the study area, which has displayed a similar temperature increase. Further, it is in agreement with the general temperature increase that has

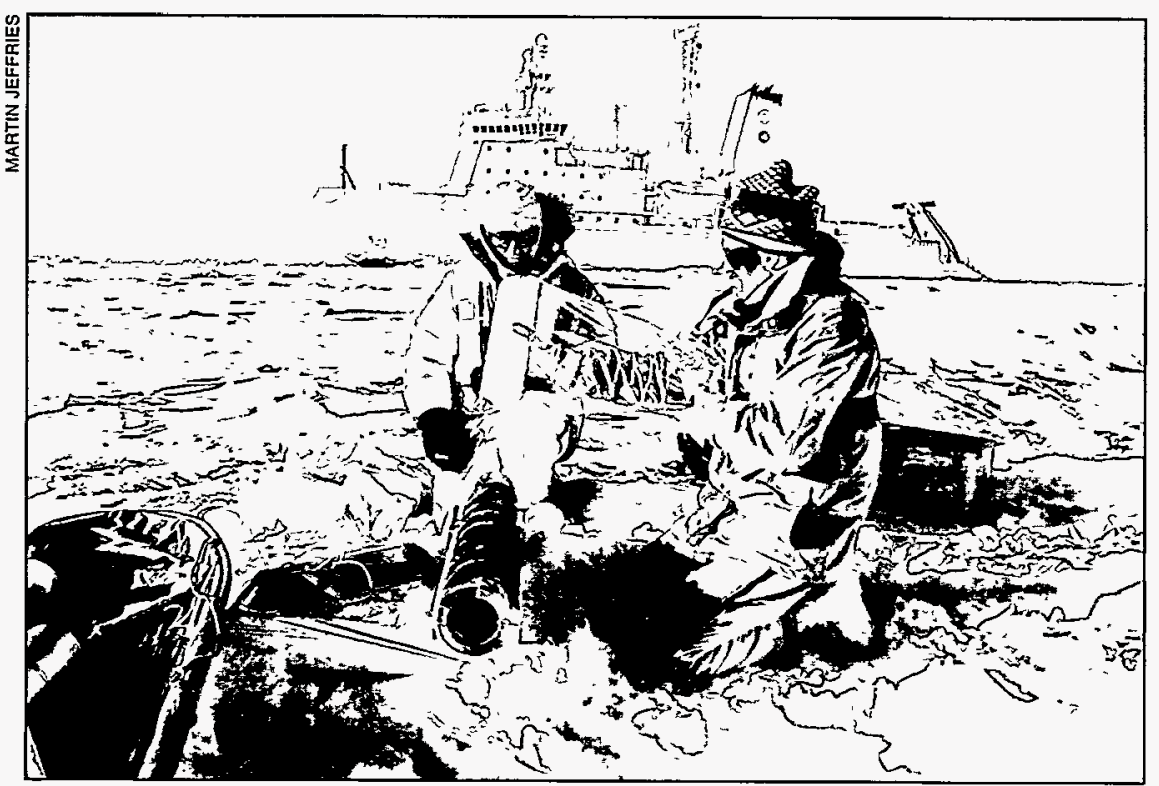

Research Associate Professor Martin Jeffries (right) and Marge Porter (Woodstock Academy, Connecticut) obtain ice cores from an antarctic sea ice floe. The research vessel Nathaniel B. Palmer is visible in the background.

Satellite-derived Outgoing Longwave Radiation, Surface Temperature and Sea Ice Concentration off the Coast of Adélie Land, Eastern Antarctica: Outgoing long-wave radiation (OLR) as seen from satellites (NOAA series), and sea ice concentration in Mer Dumont d'Urville, Eastern Antarctica were analyzed. For the period 1974 to 1990 , available radiative flux data showed a slight increase of $1.8 \mathrm{~W} / \mathrm{m}^{2}$ or 1 percent for the period. If this trend should continue (a trend over a 16-year period in any geophysical data is a rather questionable concept in isolation) a $4^{\circ} \mathrm{C}$ warming would be observed from space for this polar region over a century. been observed for the high southern latitudes. In addition, models of climatic change due to increased $\mathrm{CO}_{2}$ and other trace gases predict for polar regions values of similar size. Sea ice concentration showed a slight decrease for the period 1974 to 1989 , for which data are available.

However, a relationship existed between the radiative flux and the ice concentration, not only for the actual data, but also for the deviation series with the annual cycles removed.

A correlation factor of -0.74 was found; the sensitivity for an increase of 10 percent in ice concentration was $-2.9 \mathrm{~W} / \mathrm{m}^{2}$. This represents a temperature change as seen from 


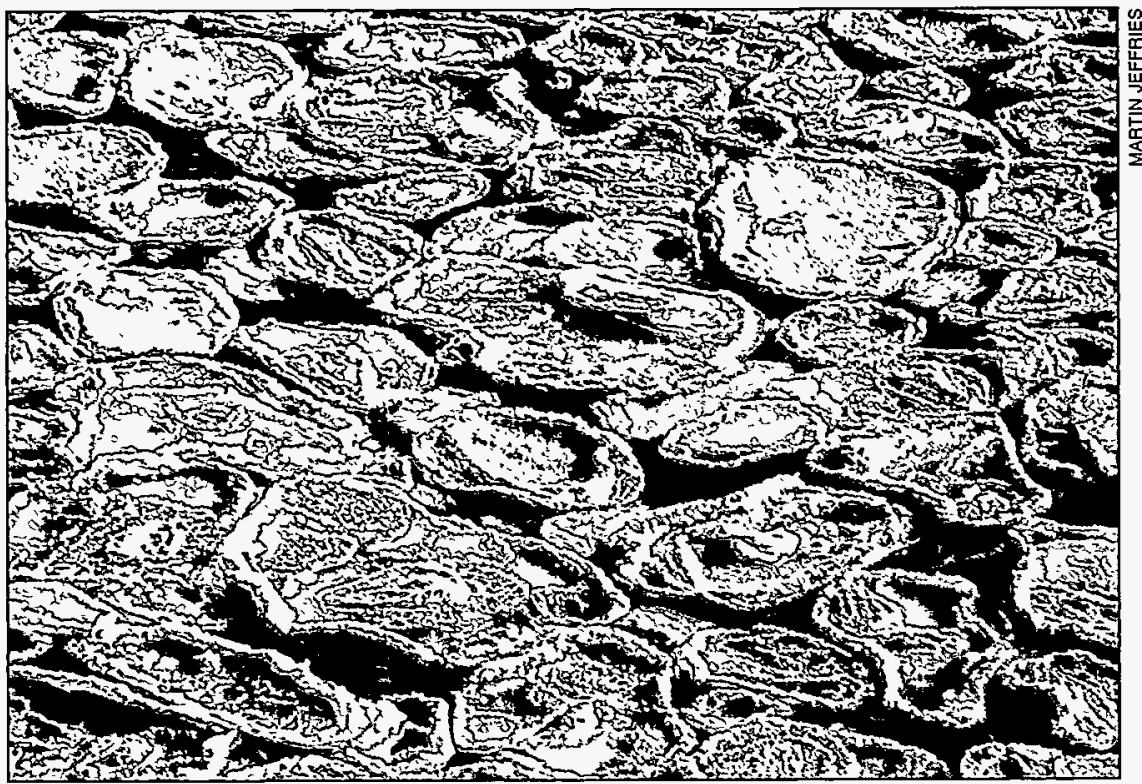

Pancake ice, comprising granular frazil ice crystals, makes a significant contribution to Antarctic pack ice floe development.

space of about $1^{\circ} \mathrm{C}$ for a 10 percent change in sea ice concentration.

NSF Grant DPP 9070969: Scientific personnel- $G$. Wendler and R. Meitner

East Siberian River Ice and Sea Ice Processes: The objective of this study is to obtain a basic understanding of river ice processes in the Kolyma, Indigirka and Anadyr rivers, and nearshore sea ice processes in the Gulf of Anadyr and in the East Siberian and Chukchi seas.

The aims of the study are to document: 1) river ice processes such as autumn freeze-up, grounding, overflows, spring breakup, ice jams and flooding; 2) estuarine processes related to winter runoff and freshwater input to the ocean; and 3) sea ice processes such as polynya and lead formation, ridge building, and ice motion.

An observational database of the study region will be developed from remotely sensed data. The primary data source will be synthetic aperture radar (SAR) images acquired at the Alaska SAR Facility. NOAA AVHRR data acquired at the Geophysical Institute also will be used.

Successful completion of the study will contribute to an improved understanding of the terrestrial and marine ice environments and their potential influence on the transport Siberian mainland to the Arctic Ocean.

Navy Grant N00014-94-I-0973: Scientific personnel-M.O. Jeffries, W.F. Weeks, and K. Morris

Interrelationships between Sea Ice Properties, Structure and Synthetic Aperture Radar Signatures During the Summer-to-Winter Transition in the Beaufort-Chukchi Sea: The objectives of this research in the late summer pack ice of the western Arctic are: 1) to document the salinity and temperature characteristics of floes; 2) to document the structural of hazardous materials from the east and isotopic variability of the ice and to identify the processes responsible for ice growth; and 3) to investigate the processes responsible for backscatter variability from the sea ice.

Field research was undertaken during August and September 1992 and 1993 aboard the U.S. Coast Guard icebreaker Polar Star. ERS-1 SAR data were acquired concurrently at the Alaska SAR Facility. M.S. thesis research by K. Schwartz investigated sea ice backscatter variability from late winter to early autumn and led to the development of a model to convert backscatter to a measure of the area of melt ponds and bare ice on floes and, thus, ice albedo.

NSF Grant OPP91-22948: Scientific personnel-M.O. Jeffries, S. Li, K. Schwartz, K. Morris, W.F. Weeks, S. Cushing, A.L. Veazey; H.R. Krouse (University of Calgary)

Deformation of Fast Ice Displayed in Satellite SAR Interferograms: With stable repeat orbits, the imaging synthetic aperture radar (SAR) aboard the first European Remote Sensing Satellite (ERS-1) provides a unique operational capability for the detection of subtle surface deformation on the order of millimeters to meters using satellite radar interferometry (SRI). A study of the deformation in fast ice near Prudhoe Bay was initiated using this new technique.

Fast ice is sea ice that forms fast and remains along the coast. Little knowledge has been gained on the detailed pattern of motion and deformation in fast ice because 1) conducting a field measurement of a motion field in sea ice is extremely expensive, if not impossible; and 
2) regular satellite remote sensing techniques have spatial resolution much coarser than the magnitude of motion in fast ice. On the other hand, such knowledge is critical to a better understanding of the mechanics interacting among sea ice, coast, and coastal constructions and of the momentum transfer among the atmosphere, ocean, sea ice and land. SRI will make possible a detailed quantitative study of the motion and deformation field in fast ice.

With SRI, the surface displacement is embedded in an intermediate fringe map that, for each pixel, depicts the phase difference of the SAR beam as determined from two repeat orbits. The phase difference is caused by path length difference viewed on a stereo pair of coherent complex SAR images and by the movement of surface and near-surface active scatterers during the time interval bracketed by the repeat images. After the effects of curvature of the earth ellipsoid are removed, the residual phase difference reflects the deformation pattern in fast ice.

NASA Grant NAGW-2716: Scientific personnel-S. Li, L.H. Shapiro, L. McNutt, and A. Jeffers
Quantitative Measurements of Deformation in Pack Ice Based on SAR Imagery: A grid-based automatic compurer algorithm has been developed for the extraction of quantitative measures of sea ice deformation from synthetic aperture radar (SAR) data sets.

The data sets utilized were the sequential fields of ice movement vectors produced by the Geophysical Processor System (GPS) of the Alaska SAR Facility (ASF). The algorithm utilizes the temporal changes in the size and the shape of each original 5 -by- $5 \mathrm{~km}$ grid element to calculate

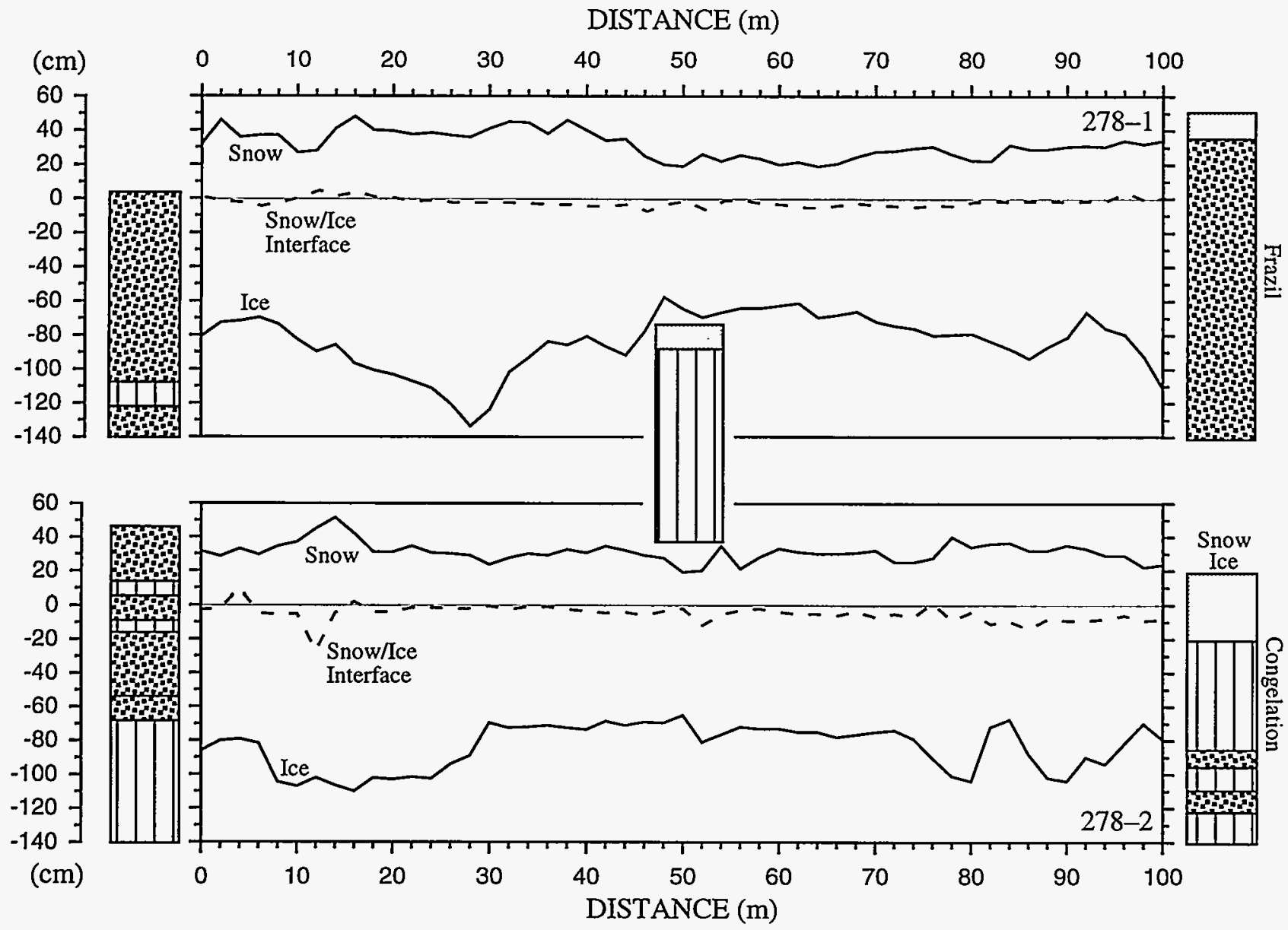

The snow and ice thickness profiles on an antarctic sea ice floe, pictured above, show there was extensive seawater flooding of the ice surface. The ice core structure diagrams reveal that floe development was dominated by frazil ice growth and rafting of ice layers. 
the convergence, the divergence, and the shear of the ice. Some isolated deformation cells were eliminated to minimize the effect of random positioning errors intrinsic in the determination of morion fields by a feature matching system, such as the GPS. Then, after threshold minimum deformation values were set, major deformation features were identified and the overall deformation values of those features were calculated by integrating the deformation values of the individual grid elements within the features. The algorithm made possible the rapid screening and extraction of significant ice deformation events without the necessity of looking at either the SAR images or at ice motion products on which no significant ice deformation occurred during the time interval bracketed by the image pairs. Image pairs showing severe deformation can be identified readily and selected for detailed study. To demonstrate the use of the algorithm, new intermediate-scale leads, pressure ridges and shear zones were identified, and their temporal variations and seasonal statistics were

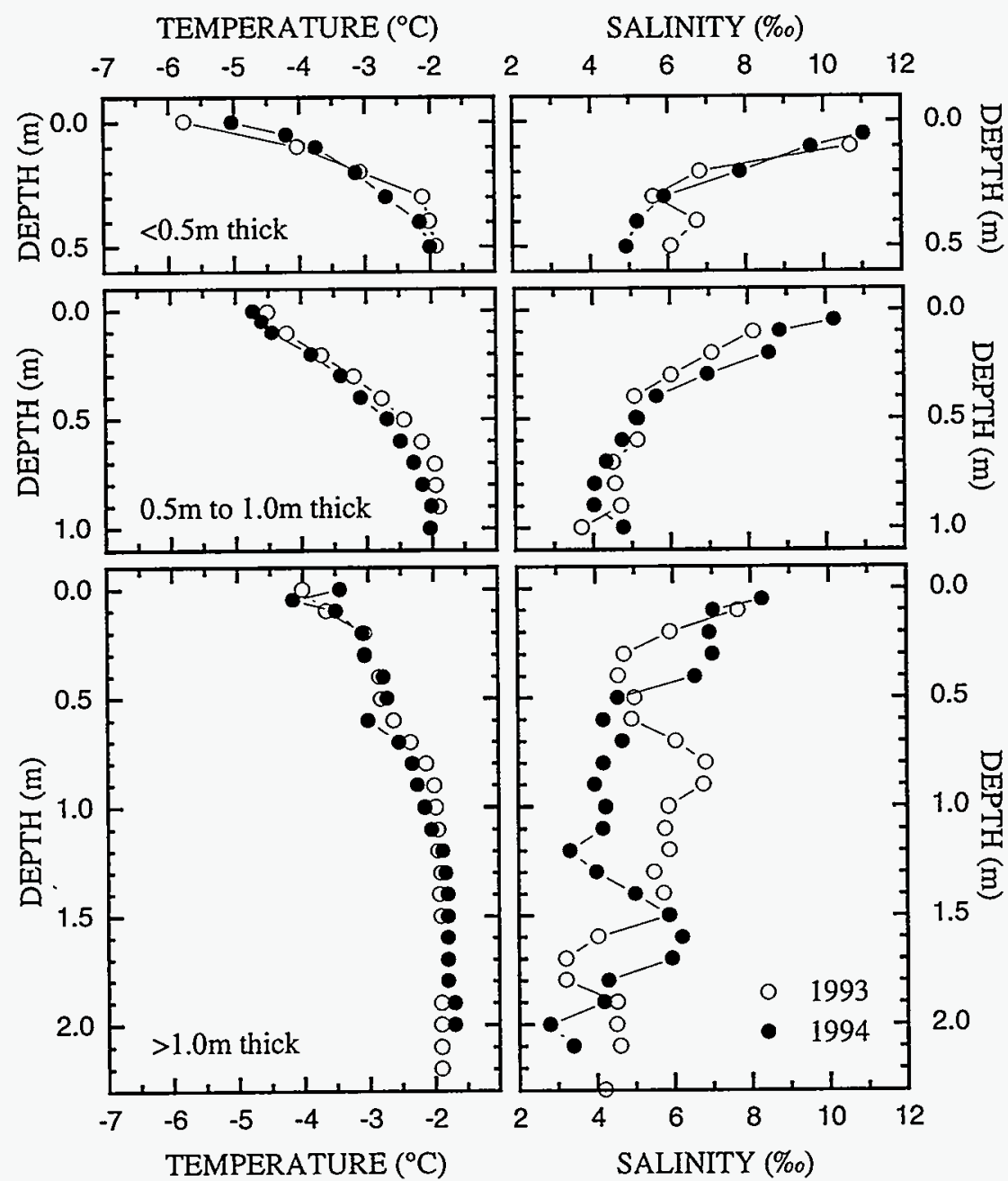

Average temperature and salinity profiles of three different Antarctic sea ice thickness categories in late winter show strong similarities (and a few differences) even though the data were obtained in different areas in different years. documented for a circular sampling area. That area covers a radius of 200 $\mathrm{km}$ centered at $81^{\circ} \mathrm{N}$ and $170^{\circ} \mathrm{W}$ in the Chukchi Sea portion of the Arctic Ocean for a six-month period during the winter of 1991-92.

NASA Grant NAGW-2716: Scientific personnel-S. Li, Z. Cheng, and W.F. Weeks

Northern Sea Route Reconnaissance Study: The political and economic changes in Russia since 1990 have led to the opening of the Northern Sea Route to transit shipping for ships from other nations, and the shorter distance from Europe to the Pacific Rim nations makes this appear initially attractive. However, the presence of sea ice for most of the year means that the shipping will probably be in convoys, using icereinforced vessels, with icebreaker support. The International Sea Route Program has been organized in Norway, with most of its scientific, engineering, legal, economic, environmental, and political science projects aimed at the assembly of all relevant information, for use by potential furure users of the Northern Sea Route. Alaska is a possible transshipment point for containerized cargo, and also is a possible exporter to Europe via the Northern Sea Route. This project was devised in response to a request from the Corps of Engineers, who are studying Alaskan uses of the Northern Sea Route, and was directed at summarizing the international activities that are taking place and making a linkage between the findings of the international programs and the needs of Alaska. Subjects examined include ice, oceanography, meteorology, 
communications, navigation, search and rescue, and system operational considerations.

U.S. Army District, Alaska Contract No. DACW85-94-P-0230: Scientificpersonnel-W.M. Sackinger

Use of an ROV for Studies of FirstYear Sea Ice Pressure Ridge Keels: In this program we deployed the Geophysical Institute's MitsuiRTV-1W remotely operated submersible vehicle (ROV) to make repeated observations of first-year sea

\section{Permafrost Studies}

Permafrost: A substantial fraction of the earth's surface, including its continental shelves, is underlain by permafrost, which is ground that has been frozen for two or more years. Some estimates put this fraction in excess of 80 percent of Alaska.

Permafrost is not merely of scientific interest, but because it is the only geologic hazard that is sensitive to the mean annual surface temperature (MAST), it is of vital interest to the geotechnical industry, which must assess the structural integrity of foundation soils. This assessment is primarily done by a relatively costly, site-specific, drilling and sampling program. However, some methods used in the geophysical exploration industry have proved effective in characterizing soils in a reconnaissance mode for further, more sitespecific investigation by drilling. ASTF has funded a project to improve a method used previously, namely the shallow-sounding terrain conductivity method, which is based on electromagnetic induction, as an indicator of the thermal ice pressure ridge keels near Barrow, Alaska. The purpose was to identify changes in the configuration and structure of the keels with time that might be due to processes related to consolidation, disintegration, or movement.

The deployments were made in March and May of 1992-93 and 1993-94 to study the keels of ridges that were mapped and profiled by drilling, and in which strings of thermistors had been installed to monitor temperature as a function of depth and time. The ROV provided information about the thermistor installations and the general arrangement of ice blocks in the keels that was important for interpretation of the temperature data and history of changes with time in the ridges. In addition, the ROV was used to recover several drill flights and a bit that had been lost through the ice.

Funded by a Grant from the West Coast National Undersea Research Center: Scientific personnel-L.H. Shapiro and W. Zito state of the soil (frozen soils are less conductive electrically than unfrozen soils). The modifications were to include the following:

- increase the sensitivity of sensors by considering in-phase output, coilcoil separation and/or frequency;

- improve boom design;

- convert all analog to digital data;

- develop a data acquisition system;

- develop software for testing, calibration, data storage/retrieval and data interpretation.

Development of a prototype system incorporating these modifications has proceeded with circuitry chips of unique design. The system is not functioning in its optimal state because there are severe noise/interference problems. The data acquisition system is working well and may be a commercially viable, stand-alone system. Calibration and rest sites have been established. An alternate design to the boom/coil-coil system is being considered.

ASTF Grant 89-1-155: Scientific personnel-G.G. Walker, $K$. Kawasaki, and J.G. Holt
Thawing of Warm Permafrost in Response to Climatic Change and Human Activities: Precise temperature measurements have been made in drill holes in discontinuous permafrost in Alaska since 1976. The holes are primarily in undisturbed permafrost on a north-south transect of Alaska along the trans-Alaska pipeline.

Most of the discontinuous permafrost south of the Yukon River is very warm, usually warmer than $-2^{\circ} \mathrm{C}$, with some of the marginal permafrost (e.g., at the toes of south-facing slopes) warmer than $-0.5^{\circ} \mathrm{C}$. Holes in undisturbed permafrost in this region have recently warmed.

The most warming occurred near Fairbanks at a cold site in the Chena River floodplain, where the mean temperature at the permafrost table $\left(-3.5^{\circ} \mathrm{C}\right)$ increased by about $1.5^{\circ} \mathrm{C}$ from 1990 to 1993 . Temperatures at this site were nearly stable prior to the warming (1983-1990). Permafrost near Birch Lake has warmed about $0.5^{\circ} \mathrm{C}$ from 1984 to 1993 . Direct evidence of talik formation 
and thawing at the permafrost table has been found at four sites in undisturbed permafrost. Measurements of the position of the permafrost table at a disturbed site (a field cleared for agricultural purposes), which have been made since 1976, show that the rate of thawing has increased since 1988. Temperature profiles in thawing permafrost change very slowly with time and, at some of these sites, there is an obvious freezing point depression at the base of the permafrost. These effects are interpreted to be a result of the presence of unfrozen water in the permafrost.

Collecrively, the above results show that much of the undisturbed discontinuous permafrost south of the Yukon River has warmed significantly and that some of it is thawing. Considering the magnitude and distribution of the warming indicated by these very sparse data, it is inferred that much of the discontinuous permafrost in Alaska south of the Yukon River and on the south side of the Seward Peninsula must be currently thawing. Additional measurements are needed to confirm this conclusion.

NSF Grant OPP92-24383: Scientific personnel-T.E. Osterkamp, T. Fei

Response of Gas Hydrates and Permafrost to Changes in Climate and Sea Level: A two-dimensional finite element model was used to investigate the thermal response of subsea permafrost and gas hydrates to changes in sea level and climare over a $121 \mathrm{~K}$ year period along a line offshore from Lonely, Alaska.

For subsea permafrost containing brines, the sparial distribution of the ice-bearing permafrost (IBP) is predicted to be wedge-shaped and to extend only $19 \mathrm{~km}$ offshore. There is significant lateral heat flow throughout the IBP section offshore and the depth to the IBP table increases almost linearly with distance offshore. For subsea permafrost with constant thermal parameters, IBP is predicted to extend $52 \mathrm{~km}$ offshore (water depth $-50 \mathrm{~m}$ ) and is nearly isothermal beyond $4 \mathrm{~km}$ offshore. Depth to the IBP table increases almost linearly with distance offshore and then becomes relatively shallow and nearly constant in depth.

Seabed temperatures and the assumed sea level history curve are especially important in determining the current distribution of subsea permafrost.

The full thickness of IBP onshore can be modeled better using constant thermal parameters. Depth to the IBP table offshore may be modeled better when it is assumed that the permafrost contains brines. The predicted depth zone for stability of methane gas hydrates is between 220 $\mathrm{m}$ and $650 \mathrm{~m}$ near shore.

For subsea permafrost containing brines, this zone extends $32 \mathrm{~km}$ offshore compared to $54 \mathrm{~km}$ (to the 55 $\mathrm{m}$ water depth) for constant thermal parameters. The time scale for producing methane gas from destabilized gas hydrates in the continental shelf near Lonely is on the order of $10^{4}$ years, much longer than previously predicted.

Funded by the Colorado School of Mines Contract P.O. 012330: Scientificpersonnel-T.E. Osterkamp

Influence of the Active Layer and Snow Cover on the Thermal Regime of Permafrost: Climatological data, active layer and permafrost measure- ments, and modeling are used to investigate the response of permafrost temperatures to changes in climate in Alaska north of the Brooks Range.

Mean annual air temperature (MAAT) from 1987 to 1991 within about $110 \mathrm{~km}$ from the Arctic Coast was $-12.4^{\circ}$ to $0.3^{\circ} \mathrm{C}$ along the coast to $-5.2^{\circ} \mathrm{C}$ inland.

Air temperature changes alone cannot explain the permafrost warming from the coast to inland. Measurements show that mean annual permafrost surface temperatures (MAPST) are about $3^{\circ} \mathrm{C}$ to $6^{\circ} \mathrm{C}$ warmer than MAAT in the region. The interaction of local microrelief and vegetation with snow appears to change the insulating effect of seasonal snow cover and may be the major factor that controls the permafrost temperature during the winter and, thus, the MAPST.

Sensitivity analyses show that for the same MAAT conditions, changes in seasonal snow cover parameters can increase or decrease the MAPST about $7^{\circ} \mathrm{C}$. Snowfall was greater during the cold years and less during the warm years and was poorly correlated between stations.

These results suggest that the effects of changes in air temperatures on permafrost temperatures historically also may have been modified by changes in snow cover. A numerical model was used to investigate the effect of changes in initial permafrost temperature conditions, MAAT, seasonal snow cover and thermal properties of soils on the permafrost temperatures.

Permafrost may have start warming about the same time as the atmosphere did in the late 1800 s, and the long-term mean surface temperature 
of the permafrost may have been established prior to this time. Variations in the penetration depth of the warming signal may be related to differences in thermal properties of permafrost. Variations in the magnitude of the permafrost surface warming may be due to the effect of local factors such as soil type, vegetation, microrelief, soil moisture, and seasonal snow cover. The effect of the interaction and snow cover may amplify temperature changes in the permafrost. A surprising result of this research is that permafrost temperatures may be changing in response to the solar cycle. Observations of permafrost temperatures in shallow drill holes in northern Alaska near the Beaufort Sea coast show that these temperatures have cycled during the decade from 1983 to 1993. The two sites nearest the coast indicate a period of about 10 years and an amplitude at the permafrost surface of about $2^{\circ} \mathrm{C}$. The two sites farthest from the coast have similar periods but reduced amplitudes of about $0.6^{\circ} \mathrm{C}$. Changes in air temperatures, snowfall, length of sea ice season, solar effects, and other factors could, in principle, produce the observed temperature changes. Barrow air temperatures have a periodicity of 10.1 years but currently lead the sunspot cycle. Snowfall also shows evidence for a periodicity near 10 years. Near Prudhoe Bay, the length of the sea ice season increased about one week from 1979 to 1986 , which could have caused a decrease in coastal air temperatures. The sunspot cycle (a 10to 11-year period) leads temperatures at the 30-m depth by two to three years, which is about the expected lead. During the descending part of the last solar cycle, satellite measurements of the solar total irradiance showed a decrease of abour $3 \mathrm{~W} \mathrm{~m} \mathrm{~m}^{-2}$, which is about four times the calculated mean energy flux at the permafrost table $(0.6$ to $0.7 \mathrm{~W}$ $\mathrm{m}^{-2}$ ) required to produce the observed temperature changes.

NSF Grant OPP91-22928: Scientificpersonnel-T.E. Osterkamp, V. Romanovski, and T. Zhang

\section{Glacier Studies}

The Measurement of Temperature in Ice Stream B, Antarctica: The western third of the Antarctic Ice Sheet is grounded below sea level. As a result, it may be vulnerable to rapid mechanical disintegration in response to minor climate or ocean perturbations; the present breakup of Columbia Glacier in Alaska may be a good analogue. A key role in the dynamics of the ice sheet is played by several great streams of fast-moving ice, 35 to $50 \mathrm{~km}$ wide, which drain into the Ross Ice Shelf. These mysterious streams seem to die, and new ones seem to be born in different locations, on the time scale of a few centuries. Our program is studying the role of the margins in the flow of Ice Stream B, at a location where the stream is $1 \mathrm{~km}$ thick and $35 \mathrm{~km}$ wide.

The first goal is to determine if the margins are significant in the force balance of the ice stream. In other words, are they exerting as much force on the ice stream (against the down-slope component of gravity) as the bed itself, despite the 35:2 ratio of bed-to-margin contribu- rions to the perimeter? The second goal is to determine if the positions of the margins are changing. Temperature data were obtained from sensors placed in holes across one of

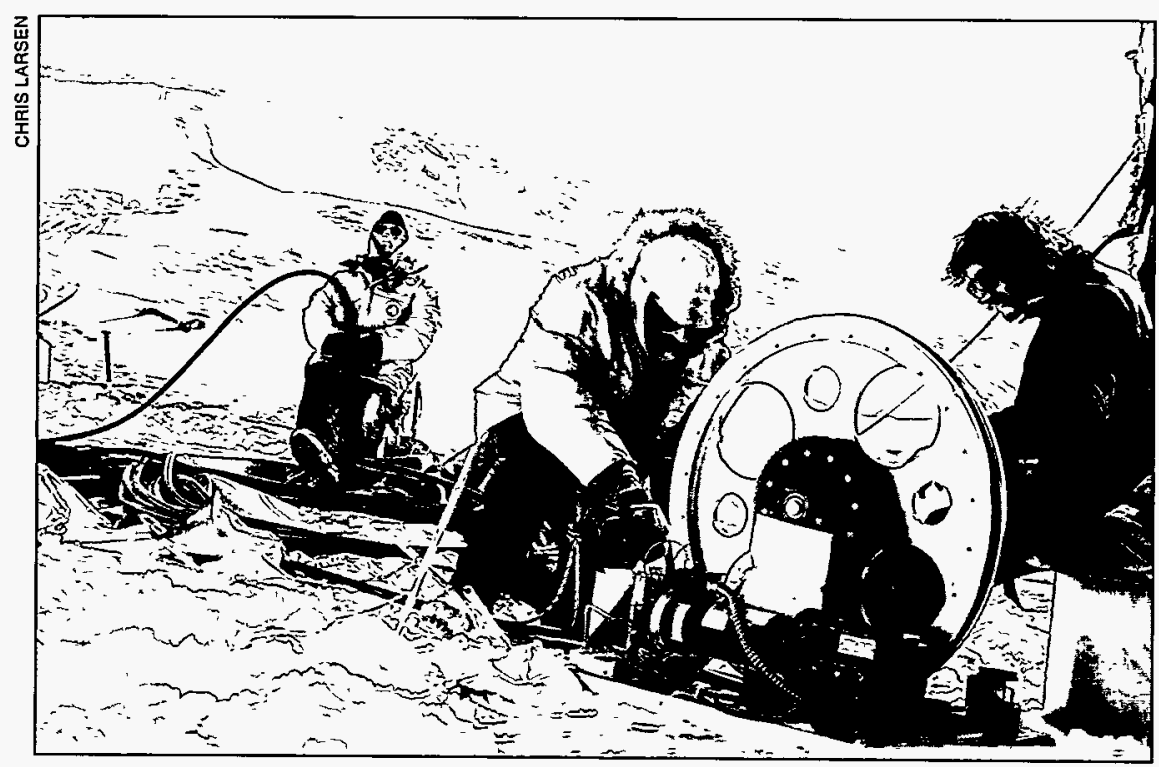

Hot-water drilling occurs in the margin of Ice Stream B. Hot water arrives by hose from the CalTech hot-water rig about $1.2 \mathrm{~km}$ distant; heat losses en route are made up by a heater just out of the field of view. 
the highly crevassed margins of the ice stream. The graph below illustrates what happens to the ice as it is fed into the side of the ice stream at a rate of one or two meters per year. "Stage" shows the temperature before it is fed into the ice stream. By the time the ice has progressed to "Lost Love," about $500 \mathrm{~m}$ into the ice stream, two things have occurred; it has warmed at the bottom and cooled from the top.

These processes continued at "Chaos," $1000 \mathrm{~m}$ in. The heating comes from the straining of the ice, and the implied side drag indicates that the margins are of about the same importance as the bed in the balance of forces. The cooling from the top is due to the ponding of cold air in the crevasses that permeate the margin. The depth of penetration of the cold into the ice below the crevasses implies that the ice at "Lost Love" and "Chaos" has been incorporated in the stream for about 50 and 100 years respectively. Given the one or two meters per year motion of ice into the ice stream, this can only be possible if the margin is unstable, moving outward at roughly 10 meters per year.

NSF Grant OPP91-17911: Scientificpersonnel-W.D. Harrison, K.A. Echelmeyer, and C. Larsen

Volume Change of Glaciers Measured from Airborne Laser Altimetry: Although mountain glaciers and small ice caps comprise

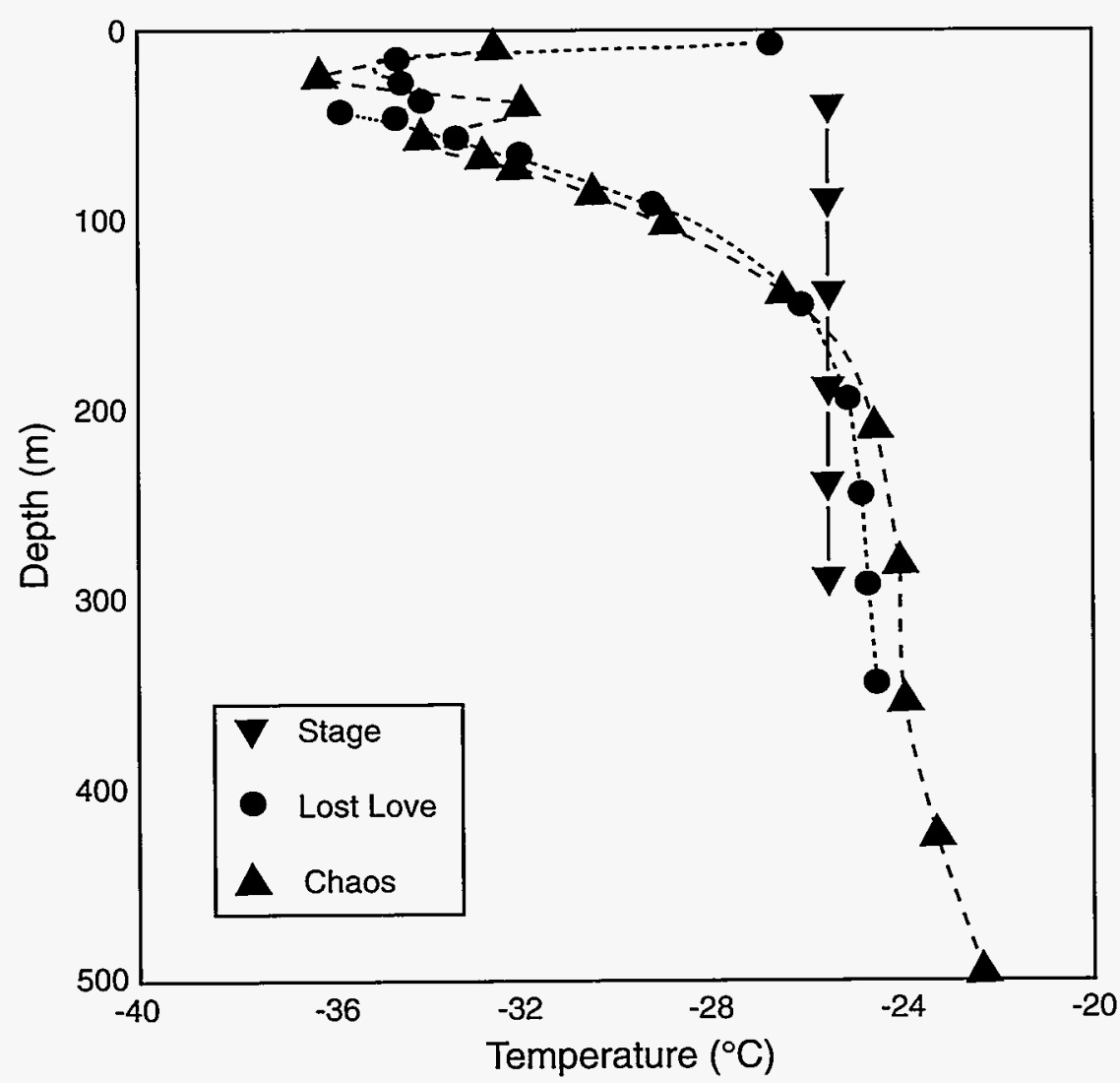

The graph shows temperature data from 3 holes. "Stage" is outside the ice stream. "Lost Love" and "Chaos" are about 500 and $1000 \mathrm{~m}$ into the margin, respectively. Anomalies at depths less than $50 \mathrm{~m}$ are mainly due to residual disturbances from hot-water drilling.

only about three percent of the glacierized area of the earth, they are particularly important in connection with changes in climate and sea level. An increase of $1 \mathrm{~K}$ in mean temperature over a long period is theoretically sufficient to cause up to a 20 percent decrease in North American glacier length.

A significant part of the ongoing 1 - to 2 -mm per year rise in sea level is believed to be due to the thinning of mountain glaciers and small ice caps. This is because of their high rates of mass exchange, with large accumulation in winter and large melting rates in summer.

Because it is difficult to measure glacier mass balance by conventional ground-based methods, balance is monitored on only a few glaciers in North America. Therefore, we have developed an airborne elevation profiling system suitable for rapid measurement of elevation, and, thus, volume changes of these glaciers.

The key components of the system are a laser altimeter, used for measuring the distance from a small aircraft to the glacier surface, a gyroscope and compass, used for determining the pointing direction of the laser beam, and continuous kinematic Global Positioning System (GPS) equipment, used for determining the exact position of the aircraft during the flight over a glacier.

The data is collected by an onboard computer and later processed to give an elevation profile along a specific track on the surface of the glacier. The resulting elevation profile is accurate to $0.3 \mathrm{~m}$ or better.

Several glaciers in Alaska have been profiled with this system, and many others will be. These recent profiles 
are being compared to topographic maps, most of which were made in the 1950s. Such comparisons will then be used to determine how the glaciers in North America have changed in the last few decades, and to infer what changes in the climate are leading to these changes in glacier volume. With the addition of real-time differential GPS navigation, we are also able to make repeat profiles on selected glaciers at one- to two-year intervals, and therefore measure volume changes on the time scale of a few years.

NASA Grant NAGW-3727 and NOAA Grant NA16RC0472-01: Scientificpersonnel-W.D. Harrison, K.A. Echelmeyer, C. Larsen, J. Sapiano, and J. Mitchell

Ice Thickness Measurements of Taku Glacier, Alaska: Taku Glacier, near Juneau, was a tidewater glacier until the 1940s, when it created and overrode a terminal moraine above sea level, ending calving. It advanced at higher rates than most tidewater glaciers until 1988, when its terminus stopped advancing. This advance is unusual in that most of the surrounding glaciers draining the same icefield have retreated.

Critical to the understanding of this tidewater glacier's dynamics is the knowledge of its thickness and depth below sea level (BSL). During the summer of 1993, we measured the depth along several transverse cross sections of Taku Glacier using seismic reflection techniques, in cooperation with the Alaska Department of Natural Resources (DNR-DGGS).

Seismic methods were needed because the expected depths were

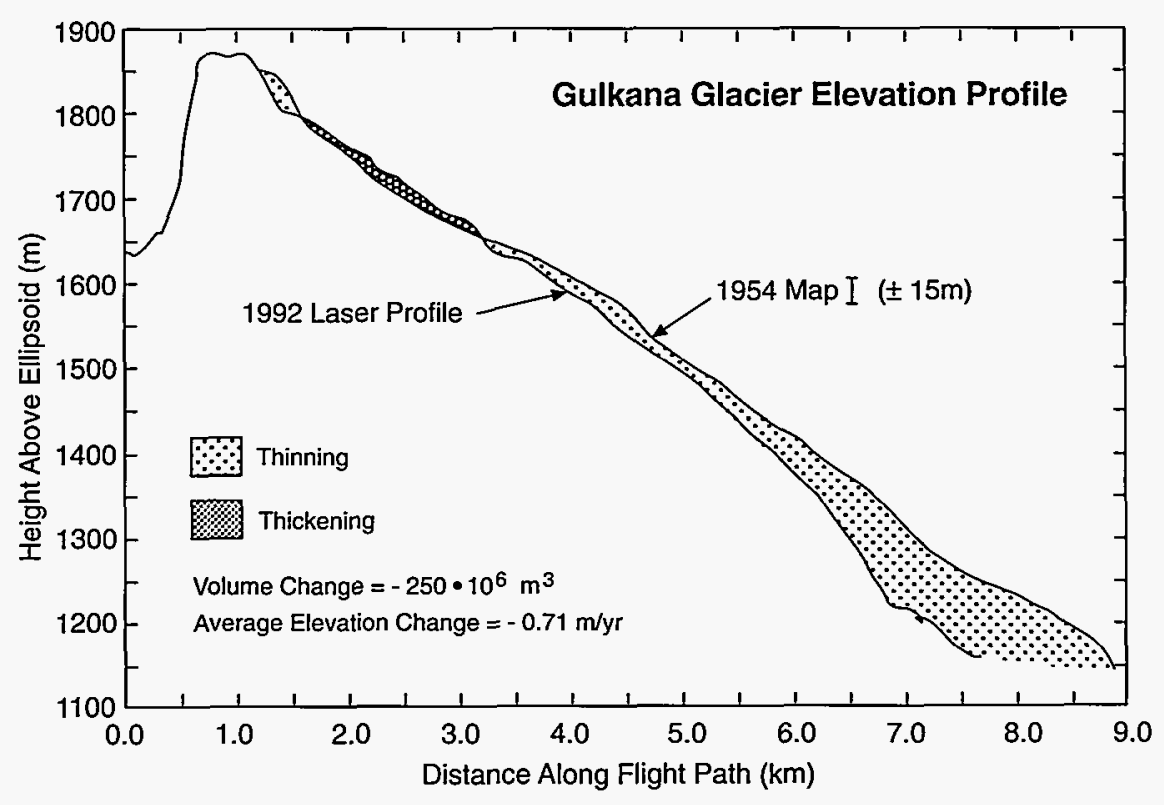

A 1992 elevation profile of Gulkana Glacier, obtained using airborne laser altimetry, is compared above with the elevation data from a 1954 map. beyond the range of ice radar equipment. The results show that Taku Glacier contains the thickest temperate ice yet measured. The maximum thickness is $1,477 \mathrm{~m}$, placing the base of the glacier over $600 \mathrm{~m}$ BSL. These data were combined with previous thickness measurements to create a longitudinal cross section running the entire length of the glacier. This cross section shows that the glacier fills a 40-km-long fjord. Perhaps the most significant finding about these depths is the fact that

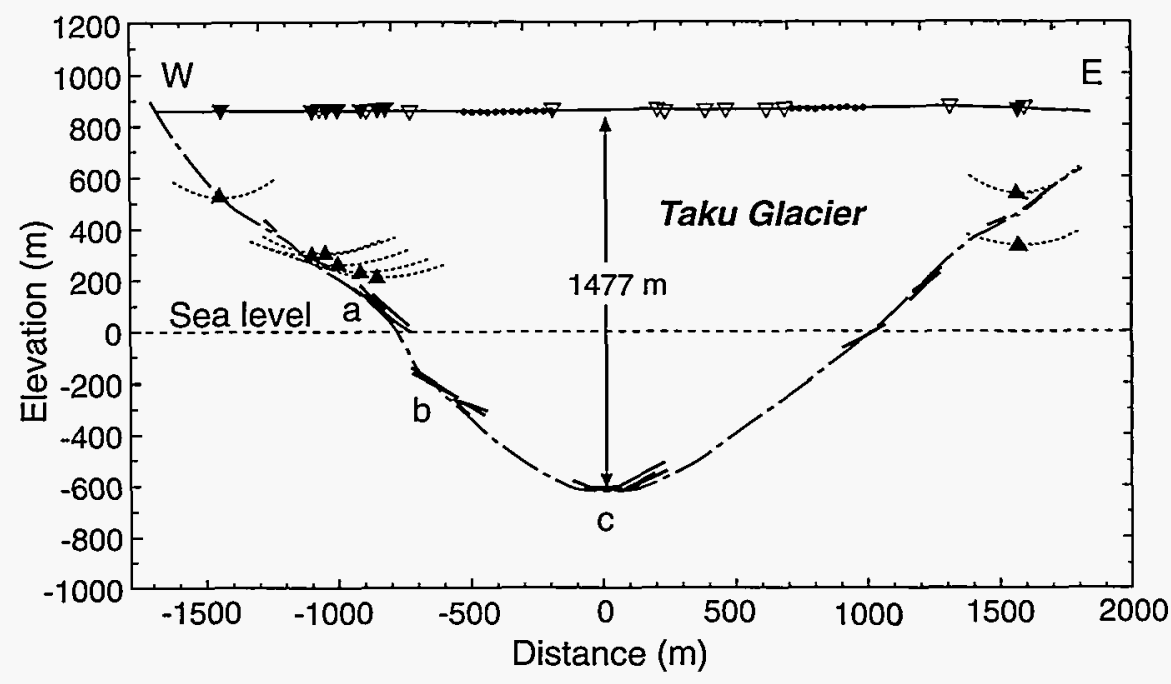

Shown above is a transverse cross section of the location known as Goat on Taku glacier. Measurements were taken using both seismic reflections (represented by thick line segments) and ice radar (represented by triangles). The maximum possible thickness of each ice radar measurement is represented by a triangle; the reflector itself could be anywhere on the ellipse segment that corresponds with each triangle. Inverted triangles and circles on the surface show equipment locations. This is the thickest ice measured on Taku and the thickest temperate ice yet measured anywhere. 
Taku Glacier's last retreat, which began about 1750 , ended over 25 $\mathrm{km}$ from the fjord's head, where the bed was over $300 \mathrm{~m}$ BSL.

This behavior is unusual for tidewater glaciers in Alaska, which typically retreat until reaching shallow water near the head of their fjords.

Asour seismic depth measurements are separated by many kilometers, it is possible that an undetected bedrock ridge exisrs berween our measurements which could have acted as a pinning point during the last retreat.

Internal funds: Scientific personnel-K.A. Echelmeyer, M. Nolan, C. Larsen; R. Motyka and D. Trabant (ADGGS)

SAR Investigations of Glaciers in Northwestern North America- the Surge of Bering Glacier: Synthetic aperture radar (SAR) images acquired by the European Space Agency satellite ERS-1 were employed to observe the 1993-94 surge of Bering Glacier, in the St. Elias and Chugach Mountains of Southcentral Alaska.

Bering Glacier-which is the largest glacier in North America and in the world, other than Antarctica and Greenland-descends $180 \mathrm{~km}$ from an ice divide north of Mt. St. Elias to the Gulf of Alaska coast, where it calves icebergs into proglacial Vitus Lake.

The total area of this glacier system, including tributaries, is 5200 $\mathrm{km}^{2}$. The width of the main trunk is about 8 to $12 \mathrm{~km}$. The mean thickness, estimated from the surface slopes (using USGS radar spot measurements of depth to bedrock on the lower glacier for control), appears to be approximately 600 to $700 \mathrm{~m}$. Near the coast the glacier expands laterally into a broad, 47-km-wide piedmont lobe.

The developing surge was confirmed in the field on 11 June 1993 by Austin Post, of the USGS, and observed by glaciologists from the State University of New York, the USGS, the Scripps Institution of Oceanography, and the Geophysical Institute during a June field program near the terminus.

Subsequent examination of SAR imagery acquired by ERS- 1 at the institute revealed the surge was in progress by April 30, and may have

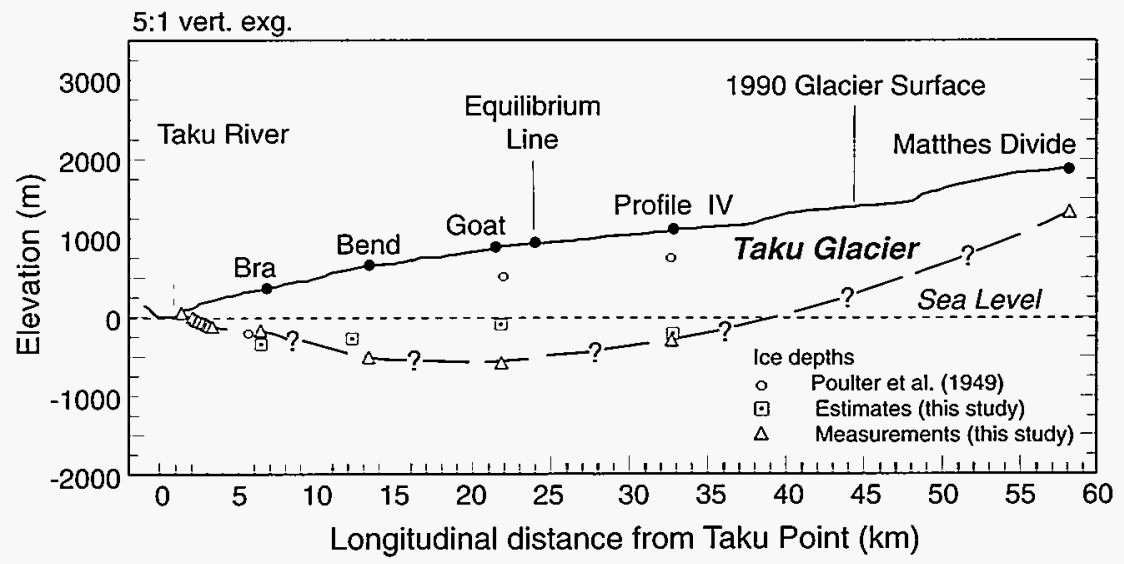

A longitudinal cross section of Taku Glacier is shown above. Using seismic and ice radar equipment, we made measurements that revealed the seismic measurements taken in 1949 were in error by as much as $1000 \mathrm{~m}$. been in its preliminary stages as early as March 26.

A time-lapse movie of the surge was constructed by J. Roush and R. Guritz, using subscenes of the lower glacier from sequential SAR images. The subscenes were terrain corrected using the USGS $90-\mathrm{m}$ digitalelevation model (DEM) of Alaska, which was interpolated onto a $30-\mathrm{m}$ grid to match the nominal resolution of the imagery.

The terrain-correction procedure resulted in geocoding (rotation to a standard orientation, i.e., such that north was up), and in referencing the pixels to geographic coordinates (latitude/longitude and universal transverse mercator).

The terrain-corrected subscenes were coregistered, as a result of being registered to the same underlying DEM. A slight additional adjustment was necessary for co-registration in some cases.

Measurements made with the sequential subscenes used to construct the time-lapse movie show the surge front propagating down-glacier at a mean velocity of $90 \mathrm{~m} /$ day between 19 May and 25 August 1993, reaching most of the broad perimeter of the terminus by and shortly after 25 August.

The calving terminus advanced rapidly into Vitus Lake, at a maximum rate in its central area of $19 \mathrm{~m} /$ day between 9 August and 18 October.

The mean rate of advance across the entire width of the terminus was $11 \mathrm{~m} /$ day during the same time period. The advancing surge front consisted of a distributed region of undulations and elongated bulges on the glacier having heights, estimated 
from the SAR data, of 40 to $110 \mathrm{~m}$ and widths (in the radar look direction, approximately transverse to the direction of glacier flow) varying from about 0.7 to $1.5 \mathrm{~km}$.

NASA Grant NAGW-2827: Scientific personnel-C.S. Lingle, W.D. Harrison, J. Roush and R. Güritz

SAR Investigations of Glaciers in Northwestern North America, Imaging and Photography of Late-Summer Snowline Position: Sequential photos were acquired showing the retreat of the transient snow line to its latesummer position on Black Rapids Glacier, which is a 45$\mathrm{km}$-long surge-type glacier located in the Alaska Range about $170 \mathrm{~km}$ southeast of Fairbanks.

The photos were acquired using an automatic camera, which was mounted on a rock outcrop overlooking the area of the equilibrium line during a spring 1993 program of field measurements. The camera and film were retrieved in spring 1994. Photos showing the maximum late-summer position of the snow line were acquired on 30 July and 7 August 1993. The 1993 ablation season ended when a snow storm occurred on 8 August.

The position of the late-summer snow line shown in the photos was subsequently reduced to geographic coordinates by Mikkel Tamstorf, an exchange student from Copenhagen, during summer 1994, and plotted on a map of the glacier made from recent survey data. The latesummer position of the snow line, identified in the photographs, was found to be approximately in agreement with the position of the late-summer snow line on Black Rapids Glacier identified in a winter SAR image acquired by the European Space Agency satellite ERS-1 on 5 December 1993.

Winter SAR images showing the positions of latesummer snow lines on a large number of glaciers in Alaska and the Yukon Territory were located by K. Ahlnäs in the archive of the Alaska SAR Facility.

NASA Grant NAGW-2827: Scientific personnel-W.D. Harrison, C.S. Lingle, K. Ablnäs and T. Heinrichs

Geophysical Investigation of Basal Processes on Black Rapids Glacier, Alaska: Black Rapids Glacier is a surgetype glacier in the central Alaska Range. During its last surge in 1937, the terminus rapidly advanced toward the Richardson Highway.

Large seasonal changes in surface speed have been observed each summer (up to 400 percent) during the

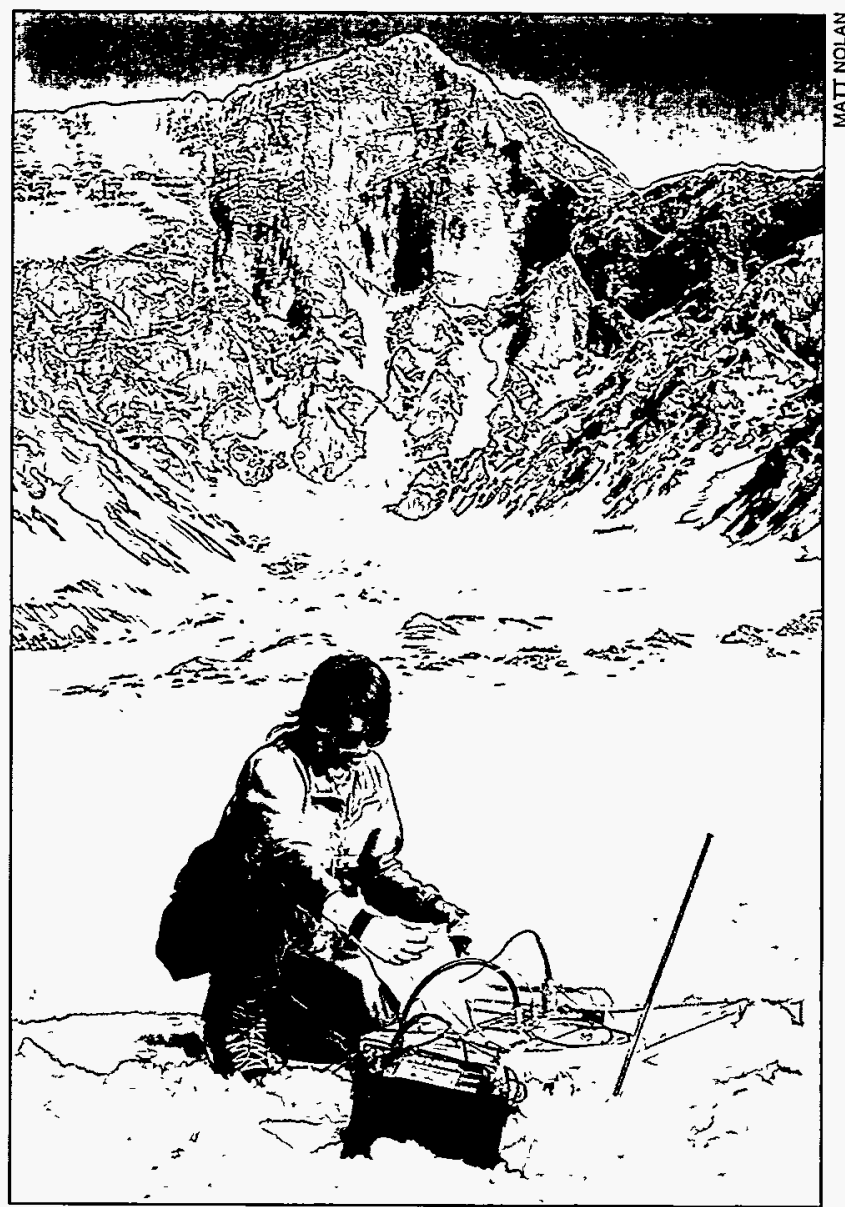

Graduate student Matt Nolan (above) records a seismogram of Black Rapids Glacier in front of $12660 \mathrm{ft}$ Mount Shand.

past twenty years. By studying the causes for these changes, which are due primarily to changes in basal motion, we hope to characterize the nature of ice/rock interface and describe the interrelations between this interface and seasonal surface water inputs. These studies may also help in understanding the surge mechanisms of this glacier during its next surge.

During the period of May to July, 1993, UAF and University of Washington researchers collected data related to the spring speed-up of the glacier. The velocity of several surface markers along the glacier was measured twice daily during this period.

This data set allows us to study the effects of changing basal conditions down a length of the glacier. Seismic reflection measurements of the bed were made every day, along with daily ice radar measurements by UW researchers. These data are used to measure changes at the bed directly that can then be correlared with changes in the surface speed. 


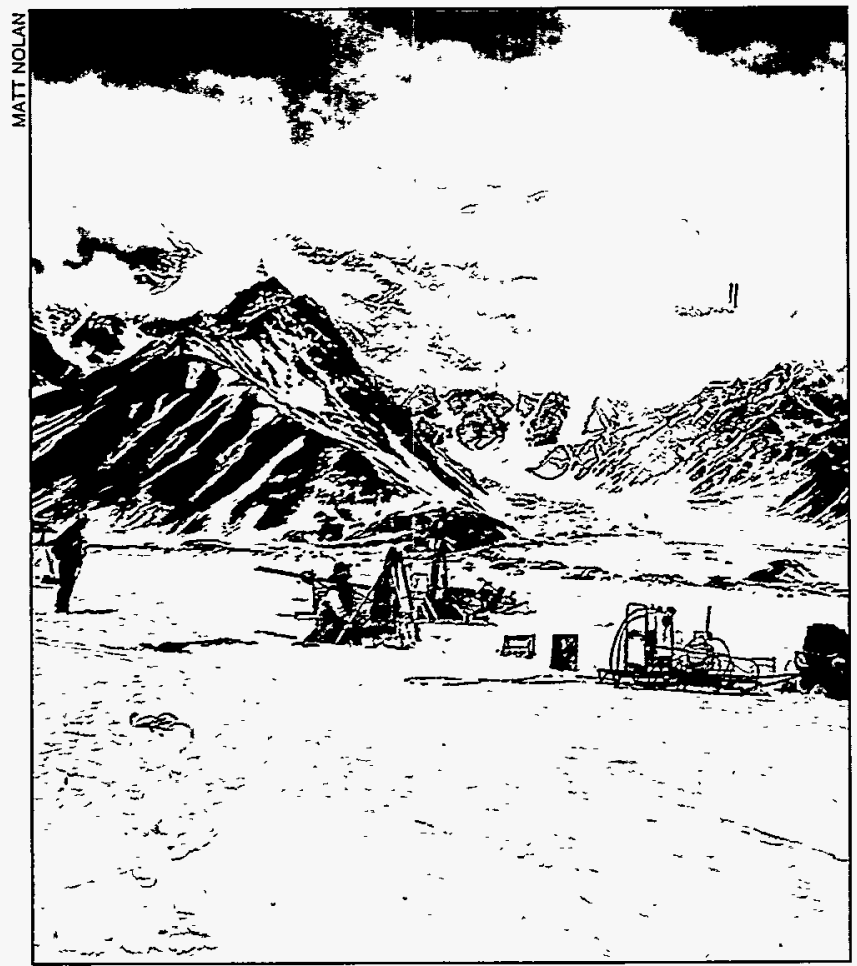

Geophysical Institute Research Technician Chris Larsen and Howard Conway (University of Washington) set off dynamite on Black Rapids glacier to generate seismic waves. The ice thickness at this location is approximately $600 \mathrm{~m}$.

In addition, the river emanating from the terminus was monitored continually throughout the summer. Stream measurements included stage and dye tracing, which provides us with an idea of how much water was stored at the bed and for how long. Electrical conductivity and turbidity provided important clues as to nature of the basal hydrology.

The analysis of this data is still ongoing. There is a wealth of data to analyze, each with its own unique thread of evidence, which we are trying to weave into a complete picture of basal hydrology. The analysis is further enhanced by measurements related to the draining of several ice marginal lakes during our summer field season.

These lakes drained subglacially, producing large increases in surface speed and changes in the stream record. By comparing the timing and discharge of these events with our measurements of glacier speed and changes at the bed and stream, we were able to test hypotheses regarding mechanisms of the spring speedup and basal motion in general.

NSF-OPP Grant OPP91-22783: Scientific personnel-K.A. Echelmeyer, W.D. Harrison, M. Nolan, and
O. Cochran; C. Raymond and T. Gades (University of Washington)

Volume Changes of McCall Glacier in Response to Climate Warming in the Arctic: McCall Glacier is located in the northeast Brooks Range of arctic Alaska. This glacier was studied during the IGY in 1957 and in the early 1970s. During 1993 and 1994, we returned to the glacier in order to investigate how it has changed over the last 36 years.

Measurements include surface elevation and velocity, mass balance, terminus position and local meteorological variables. Changes in volume will be interpreted in terms off climatic changed in actic Alaska.

The ice surface and the outline of the terminus were surveyed using optical and GPS methods, and ice thickness was determined by radio-echo sounding methods. Continuous measurements of temperature, precipitation, shortwave radiation and snow depth have been made since June 1993.

In addition, the surface elevation and termini of five other glaciers of different size and aspect within $20 \mathrm{~km}$ of McCall Glacier were surveyed. A contour map of the bed of McCall Glacier was made from the ice depth measurements. The present velocity field, the elevation and volume change from 1972 to 1993 , and the average mass balance during this period were determined from the surveys.

Annual velocities in 1993-94 were similar to those measured in the 1970s. However, in the ablation area, 1993 summer velocities were about 30 percent higher than the annual velocities there, indicating that McCall Glacier slides in summer. This may imply that McCall Glacier is not a true "cold," or healthy glacier in the strict sense.

Surveys show that the glacier surface has dropped everywhere, from a few meters in the accumulation zone to more than 40 meters near the terminus. A map of this elevation change (shown on the opposing page) illustrates that the total volume change was $-5.5 \times 10^{7} \mathrm{~m}^{3}$ of ice, and that the long-term mass balance was $-0.33 \mathrm{ma}^{-1}$.

These results indicate that $\mathrm{McC}$ all Glacier is not healthy, especially considering its low mass exchange rate (typical winter balance is only $0.2 \mathrm{ma}^{-1}$ ).

For the period of 1958 to 1971 , a mass balance of -0.13 $\mathrm{ma}^{-1}$ was found in an earlier study, indicating that the 
mass wastage has increased. Repeated surveys of detailed profiles across the lower ablation area from 1969 to 1994 show that a threefold increase in the rate of surface lowering occurred after 1975.

Studies are underway to relate these negative mass balances to changes in the climate of the Arctic.

NSF Grant DPP 92-14954: Scientific personnel-K.A. Echelmeyer, C.S. Benson, B. Rabus, and D. Trabant (USGS)

Analysis and Synthesis of Glaciological Data: This project has three parts-glacier facies, analysis of accumulation on the Greenland Ice Sheet, and snow pit and core data from the summir caldera of $\mathrm{Mt}$. Wrangell, Alaska.

Glacier facies: Glaciers, especially large ones such as the Greenland and Antarctic ice sheets, span several environments which give rise to different physical characteristics in the upper layers of snow and firn. A scheme of glacier facies was established in 1960 to define the differences in terms of physical measurements that penetrate several annual stratigraphic units of accumulation. The original definition of glacier facies was based on research in Greenland, and it included the full range of glacier facies. Some parts of the Antarctic Ice Sheet have only the upper end of the glacier facies spectrum, and many glaciers have only the lower end of the spectrum. For example, McCall Glacier in the Brooks Range of arctic Alaska does not extend to the upper limit of the wet snow facies. Modifications to the original definitions have been in the lower end of the spectrum, where com- plications due to melting are greatest. One goal of this project is to determine the extent to which glacier facies can be identified from satellite imagery. The facies boundaries we can expect to define on Landsat images are restricted to those that are visible at the surface. The potential of SAR imagery to see below the surface was discussed at the Fourth Workshop on Mass Balance and Related Topics, and Fahnestock and others have now shown that the drysnow and wet-snow lines may be mapped by SAR imagery (Science, Vol. 262, p. 1530-1534, 1993).

Analysis of accumulation on the Greenland Ice Sheet. We are redrawing a contour map of annual accumulation for the ice sheet by using the existing glaciological pit and core data from Greenland, combined with data from coastal meteorological sta-

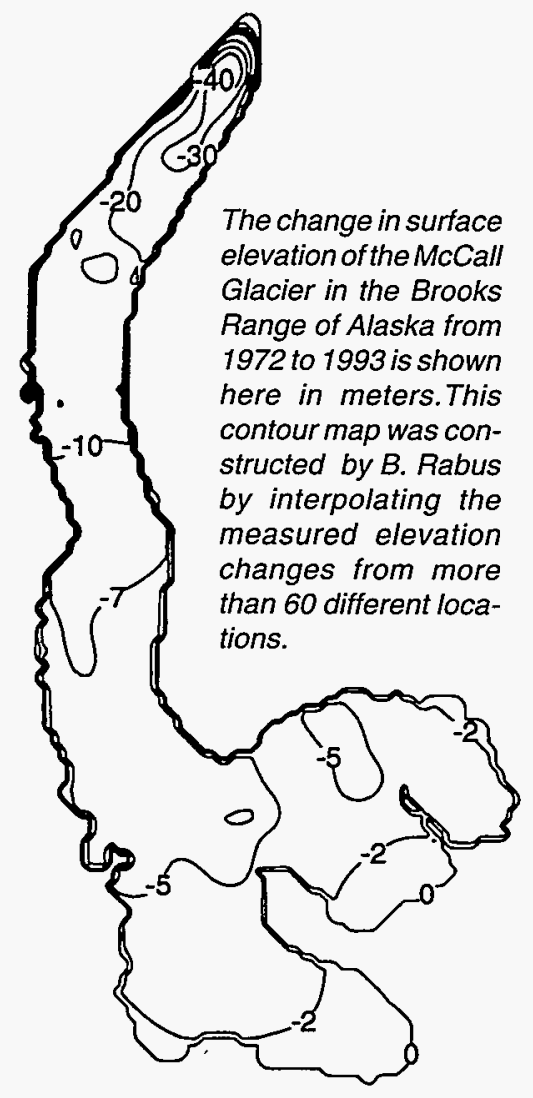

tions, and a model for orographic precipitation.

Snow pit and core data from the summit caldera of Mt. Wrangell, Alaska: Snow stratigraphy from the years 1950 to 1982 is being analyzed by using pit and core data that include detailed density determinations together with microparticle analysis and data on the stable isotopes of hydrogen and oxygen. The interpretation is made easier because the summit region lies in the dry-snow facies, so it experiences negligible melt.

NSF Grant DPP 90-02345: Scientific personnel-C.S. Benson

Satellite Radar Altimetry and Ice Sheet Dynamics: Altimeter data from the Geosat Exact Repeat Mission (ERM), which extended from fall 1986 through fall 1989, and Seasat, which extended from early July through early October 1978, were employed to measure mean changes in elevation on lower Lambert Glacier, the floating Amery Ice Shelf, and the adjacent East Antarctic Ice Sheet. This region, which is approximately south of India, is bounded by Latitudes $68^{\circ}$ to $72^{\circ} \mathrm{S}$ and Longitudes $66^{\circ}$ to $75^{\circ} \mathrm{E}$.

Lambert Glacier, which is the largest ice stream in East Antarctica, is about $90-\mathrm{km}$ wide in its lower reaches, by $400-\mathrm{km}$ long. It discharges ice from an accumulation area of about $900,000 \mathrm{~km}^{2}$.

Mean changes in elevation were measured on a same-season basis between the three-month Seasat time frame, austral late winter 1978, and the same time frame during each of the Geosat ERM years, to minimize the effects of seasonal changes 


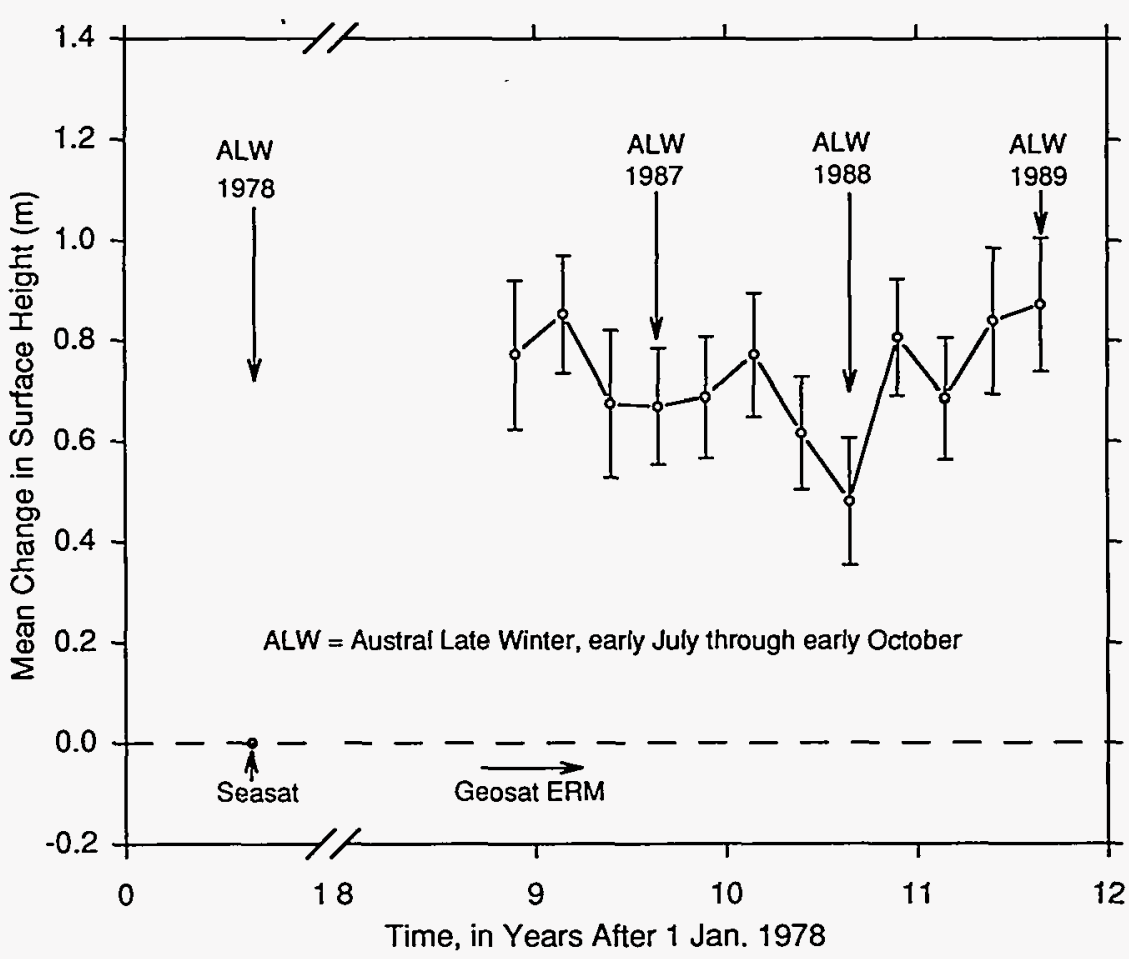

The graph above was computed from Seasat and Geosat ERM altimetry using orbit crossover analysis. It shows seasonal mean changes in the surface elevation of the Lambert Glacier-Amery Ice Shelf region, East Antarctica, from 1978 to 1987-89. The $137,200 \mathrm{~km}^{2}$ area is bounded by latitude $68^{\circ}$ to $72^{\circ} \mathrm{S}$ and longitude $66^{\circ}$ to $75^{\circ} \mathrm{E}$. The changes are computed with respect to the mean surface at the time of Seasat, which is the datum. Unfiltered altimeter data were employed. The results shown here have not been adjusted for apparent orbit bias (see text, section on satellite radar altimetry and ice sheet dynamics).

in penetration depth. The altimeterderived height measurements from both satellites, which were retracked at NASA Goddard Space Flight Center (GSFC), were referenced to Goddard Earth Model (GEM) T2 orbits. The elevation changes were measured via the method of orbit crossover analysis, using altimetry with random noise levels reduced by low-pass filtering, and also using unfiltered altimetry.

Apparent orbit bias between the two satellites, combined with systematic vertical error from other sources, was estimated using crossover differences from a region of austral late-winter sea ice seaward of the calving front of Amery Ice Shelf.
The apparent Seasat-Geosat ERM orbit bias was found to be negligible in the case of the low-pass filtered altimeter data $(-3 \pm 120 \mathrm{~mm})$, but more significant in the case of the unfiltered data $(80 \pm 130 \mathrm{~mm})$. Positive bias implies the Geosat surface was systematically higher.

The crossover results obtained using unfiltered altimetry were adjusted for this apparent orbit bias. The effects of crossover locations where the altimeters may have been measuring to two different points on the surface (perhaps the crests of two different undulations) were suppressed by using only crossover differences with absolute value $\leq 5 \mathrm{~m}$. Error ranges for the results were computed using variogram methods to estimate the spatially varying noise levels in the data, which were then propagated through the crossover computations.

On the lower Lambert Glacier, throughour an area including the grounding zone, extending from Latitude $72.1^{\circ}$ to $70.4^{\circ} \mathrm{S}$ (about $190 \mathrm{~km}$ in the along-flow direction), the mean rate of increase of the surface height was found to be $30 \pm 10 \mathrm{~mm} / \mathrm{yr}$ from 1978 to $1987-89$, using low-pass filtered altimetry. The mean rate of increase was found to be $40 \pm 50$ $\mathrm{mm} / \mathrm{yr}$ using unfiltered altimetry.

On the floating Amery Ice Shelf, the mean rate of increase of the surface height, measured using low-pass filtered altimetry, was found to be $30 \pm 5 \mathrm{~mm} / \mathrm{yr}$ during the same time period. The mean rate of increase was found to be $40 \pm 20 \mathrm{~mm} / \mathrm{yr}$ using unfiltered altimetry.

Throughout the entire $309 \mathrm{~km}$ east-west by $444 \mathrm{~km}$ north-south $\left(137,200 \mathrm{~km}^{2}\right)$ region, including the lower Lambert Glacier-Amery Ice Shelf system and the more steeplysloping grounded ice sheet on both sides, the surface elevations were found to be increasing at $40 \pm 3 \mathrm{~mm} / \mathrm{yr}$ during the same time period, using low-pass filtered altimetry. The mean rate of increase was found to be $60 \pm 20 \mathrm{~mm} / \mathrm{yr}$, using unfiltered altimetry. The results suggest that the East Antarctic Ice Sheet was characterized by a positive mass balance in this region from 1978 to $1987-89$.

NASA Grant NAGW-2614: Scientific personnel-C.S. Lingle, L.-H. Lee, V.A. Voronina; H.J.Zwally (NASA Goddard Space Flight Center); and T.C. Seiss (Hughes STX Corporation) 


\section{Snow Cover Studies}

Seasonal Snow on the Arctic Slope of Alaska: Our study is part of DOE's intensive ecological research at the R4D site near Toolik Lake in the northern foothills of the Brooks Range. (The acronym R4D stands for response, resistance, resilience, and recovery from disturbance in the arctic ecosystem.)

The seasonal snow studies were done in collaboration with research on the overall hydrology (D. Kane and L. Hinzman, UAF), stream chemistry (K. Everett, Byrd Polar Research Center, Ohio State University) and on the distribution of vegetarion types (D. Walker, INSTAAR, University of Colorado).

The research goals include measuring the amount of precipitation that comes as snow; the extent of wind transport of snow; the depositional pattern of drifted snow; the determination of the structure of the snowpack; the physical processes that operate within snowpack, and those that control snow melt; and the biological role of the arctic snowpack.

Our measurements of snow on the tundra indicate two major types, differing in depth, density, structure and thermal characteristics: the veneer facies, lying on and interacting with the tundra; and the drift facies, which forms in certain topographic settings. The thin veneer of depth hoar and windblown snow forms over most flat areas of the tundra.

The drifts consist of deeper, denser snow that forms in topographic depressions and in the lee of ridges and bluffs. The veneer facies and the drift facies can differ in depth by a factor of 10 and they are radically different in their stratigraphic structure, density and thermal character. The directions and magnitudes of windtransported snow from the primary winds have been measured at several locations on the Arctic Slope. We are attempting to identify the regions affected by specific wind transport directions. In the $\mathrm{R} 4 \mathrm{D}$ area, we measured and mapped the water equivalent of the maximum snow cover each year from 1985 to 1990 . During the years maps were made, 1988 was the minimum and 1989 was the maximum. These two maps are reproduced on the following pages.

The accompanying diagram (shown below) of snowpack ablation at this region has data from 1985 through 1994. In this nine-year data set, the maximum and minimum snowpacks occurred in 1992 and 1994 , respectively. It is clear that one can expect a very difficult time when trying to find a signal for long-term climate change from a data set like this (and this is by far the most detailed data set in existence for arctic Alaska).

The date at which the snowpack disappears has a variability of about one month. This noisy signal obscures efforts to find a smaller variation in the date of snow melt as a response to change in climate. The strongest correlation in the data set is between amount of snow and the date of disappearance of the snow. Two pairs of years, 1988-89 and 1991-92, are excellent cases of this point.

DOE Grant DE-FGO6 $84 E R$ 60245: Scientific personnel-C.S. Benson and S.A. Bowling; D.L. Kane and L.D. Hinzman (Water Research Center, UAF Institute of Northern Engineering); $M$. Sturm (CRREL). Continuation of this research, with the same personnel plus D.J. Goering, is beingdone through the Water Research Center of the School of Engineering under support from NSF Grant OPP-9214927 and OPP-9318535.

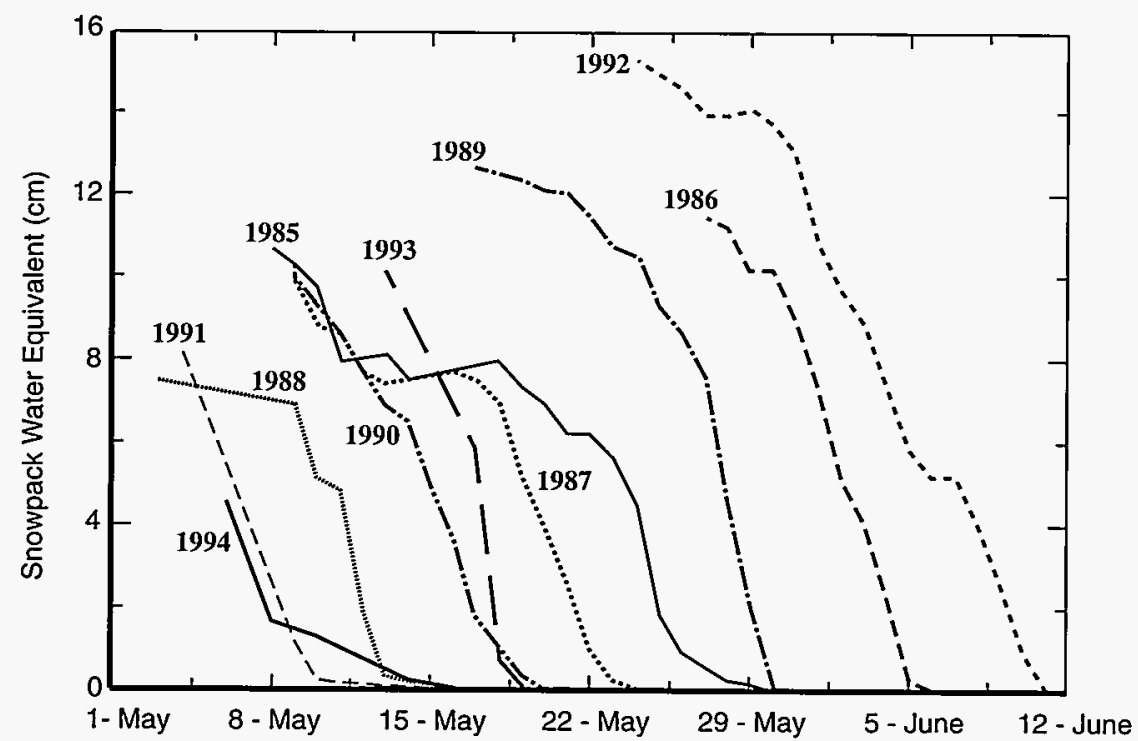

Snow ablation rates were measured on the east slope of Imnavait Creek near the runoff plots maintained by Hinzman and Kane. These values are a good index for the watershed. Onset of melt varies by a month, but once the process begins it is over in a week. 


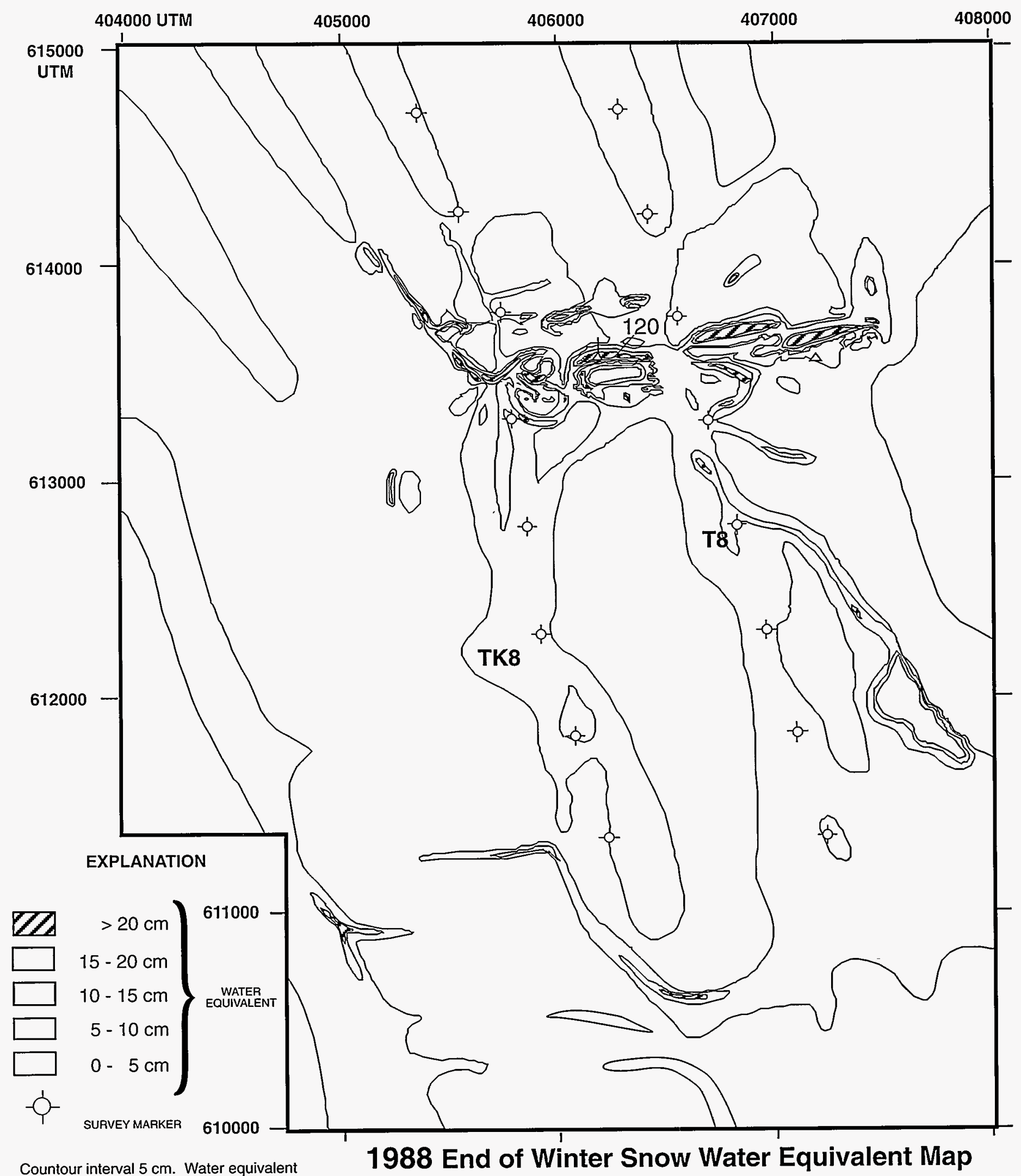

The above map shows the minimum snow distribution mapped during the six years (1985-90) that snow maps were made. 


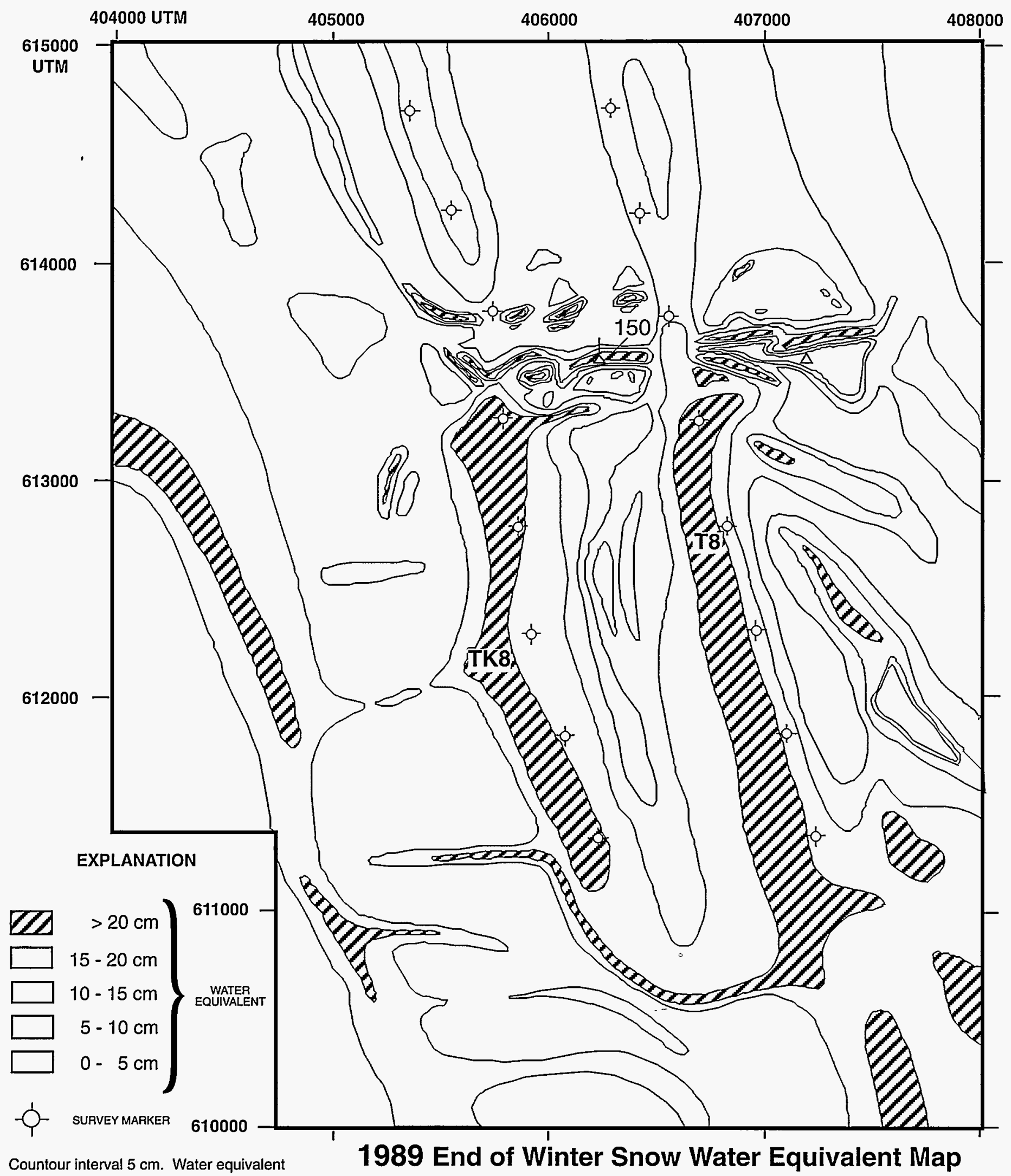

The above map shows the maximum snow distribution mapped during the six years (1985-90) that snow maps were made. 
Remote Sensing of Snow and Ice: This project has two parts-passive microwaves and photogrammetry, Landsat and SAR imagery.

In part one, passive microwave data were obtained from the Defense Meteorological Satellite Platform (DMSP) and the Special Sensor Microwave Imager (SSMI).

These data are being used to map the hemispheric extent of snow cover with a spatial resolution of $30 \mathrm{~km}$. Because the microwave radiation emanates from the ground beneath the snow as well as from the snow itself, the relationship between microwave brightness temperature and snow characteristics is complex.

We have focused on a traverse across Alaska from the Pacific Ocean at $58^{\circ} \mathrm{N}$, south of Alaska, to $73^{\circ} \mathrm{N}$ in the Arctic Ocean, north of Alaska. Our data set spans the all-time record low- temperature period in January 1989 , through the all-time high-temperature period in February 1989.

Variables include: the surface temperature; the moisture content and the general nature of the upper layers of rock and soil; the amount of snow cover and the extent of depth-hoar crystal development within the snow cover; the presence of ice lenses in the snow; and any liquid water present in the snow.

The hope still persists that microwave brightness temperature can be interpreted to obtain snow depth and liquid water content, but the complications are sobering, and in addressing them we expect to learn more about the overall soil-snow system and how it interacts.

During the past two years, we have learned that vegetation plays a major role in controlling the passive microwave signal received by the satellite. The vegetation map by A.W. Kuchler (USGS National Atlas, sheet No. 89) agrees well with the patterns of microwave brightness temperatures. Our paper, presented at the Fiftieth Eastern Snow Conference, deals with this subject.

A persistent anomaly, of unexpectedly low brightness temperature (approximately $45 \mathrm{~K}$ lower than surrounding values) is present in the data sets in the northern foothills of the Brooks Range during each year of the study. The exact location of the anomaly varies slightly from year to year. We have investigated several possible explanations for this anomaly, but so far without success. In the second part of the project, the 1993 and 1994 photogrammetry was successful over the summit of $\mathrm{Mt}$. Wrangell. It was paid for by the U.S. Geological Survey and the Alaska Division of Geological and Geophysical Surveys.

Analysis of ice volume changes in the North Crater ( $1 \mathrm{~km}$ in diameter) of Mt. Wrangell, based on photogrammetric, digital data of cross sections, $20 \mathrm{~m}$ apart, are still underway. In addition, we have made orthophoto maps of the glacier termini, paying special attention to parts that are advancing on the northeast flank of the mountain. The local nature of the advancing termini have been verified by examining Landsat imagery from 1973 and 1986.

The possibility of identifying facies boundaries on Mt. W rangell with SAR imagery, as mentioned in this report in connection with Greenland, has been successful. A publication on this subject is in preparation.

NASA Grant NA G5-887 and cooperative agreement NCC5-75: Scientific personnel-C. S. Benson; D. K. Hall (NASA Goddard Space Flight Center); and M. Sturm (CRREL) 


\section{Tectonics \& \\ Sedimentation}

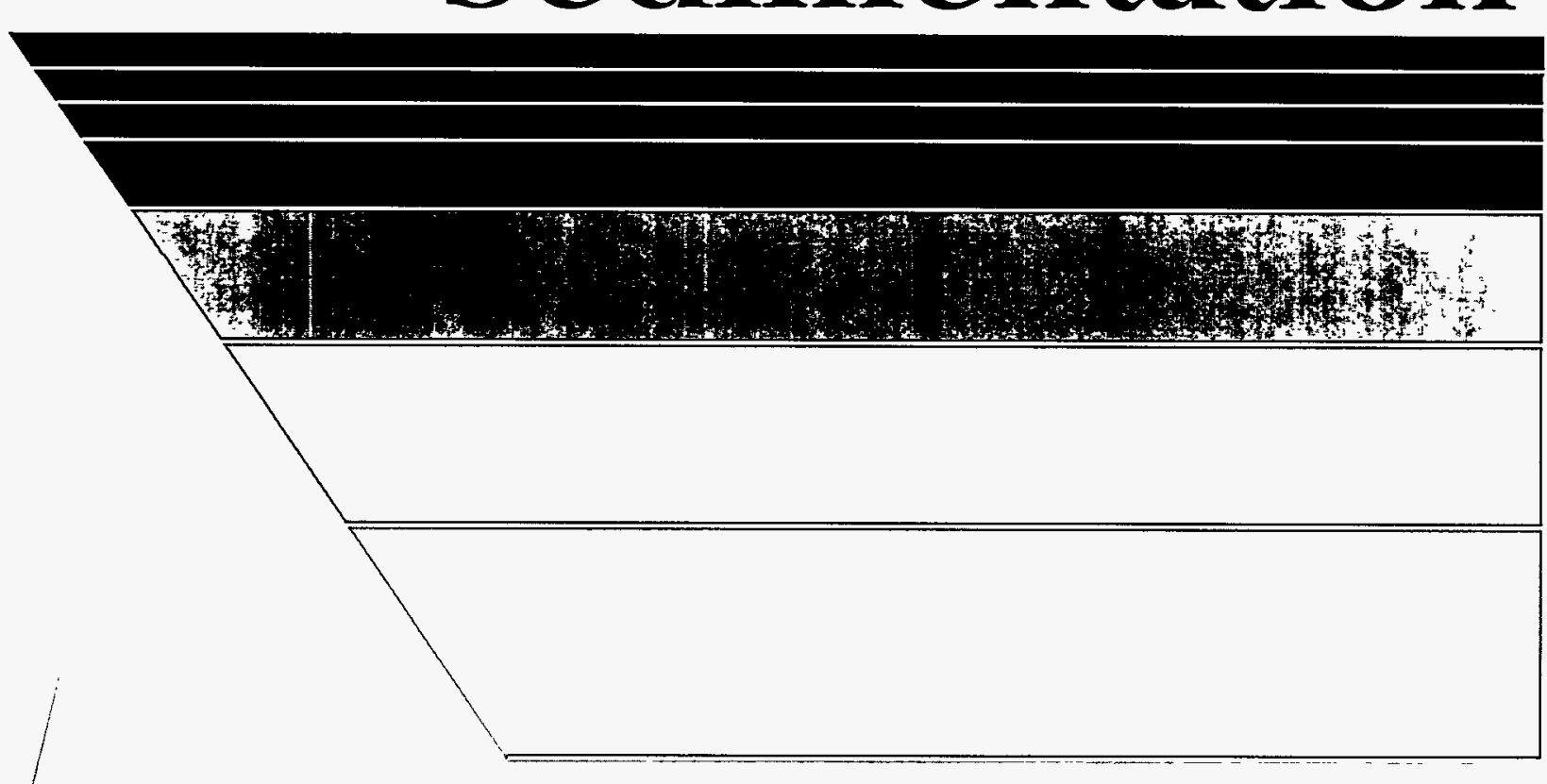




\section{TECTONICS and SEDIMENTATION}

The state of Alaska is located at the meeting point of the North American and Eurasian continents and the Pacific and Arctic Ocean basins. Because of this unique geographic position, Alaska is at the center of a region that has been geologically dynamic for many millions of years, and remains so today. The geologic history of this region is of great scientific interest, as are the processes of rock deposition and deformation recorded by that history. The Tectonics and Sedimentation Research Group consists of geologists and geophysicists whose studies aim to develop a better understanding of the history of this region and the processes that have shaped it.

The members of the group represent a diversity of disciplines and research interests, including tectonics, structural geology, sedimentary geology, geochronology, and paleomagnetism. The unifying goal of the group's work is to learn more about tectonics, or the processes and consequences of major movements of the earth's crust. The unusually diverse and complex tectonic activity of Alaska and the surrounding region are recorded in its rocks, providing insights into the history of the region and rectonic processes in general.

Alaska is a collage of "allochthonous terranes," or large pieces of crust that have moved great distances within ocean basins or along continental margins, and the process of assembling that collage continues today. The relative motion of lithospheric plates, or plate tectonics, has controlled where and how rocks have deformed, including the movements of allochthonous terranes. Where and how rocks at the earth's surface have been deformed offers clues to the plate tectonics of the past and to how different parts of the crust have moved and interacted. Seismic reflection and refraction data and gravity and magnetic field data help in determining subsurface crustal structure, and paleomagnetic data help in reconstructing past movements of crustal fragments, such as allochthonous terranes. Reconstruction of the deposition of ancient carbonate and terrigenous clastic rocks provides important insights into the climate, physiography, and depositional processes at various times in geological history, and these, in turn, reflect the tectonic setting at the time. Geochronology provides an essential means to date geologic events, thus calibrating the geological history reconstructed using other methods.

The group has worked mainly in Alaska, but is increasingly turning its attention to other parts of the northern Pacific margin and circum-Arctic region, including the Russian Far East. The group has been involved in numerous projects, large and small, and has worked in conjunction with various other researchers. A major focus has been a multi-year, multi-disciplinary study of the geol-ogy of the northeastern Brooks Range in the Arctic Na-tional Wildlife Refuge (ANWR). This project has been sponsored by the petroleum industry and the U.S. Department of Energy and has involved many graduate students and all of the faculty members of the group.

Several faculty members also have participated with the U.S. Geological Survey in the northern part of the Trans-Alaska Crustal Transect (TACT) project, across the central Brooks Range. Structural, paleomagnetic, geochron-ologic, and seismological studies in the Russian Far East are in progress in cooperation with Russian researchers. Numerous other projects have been conducted in cooperation with the Seismology and Volcanology groups of the Geophysical Institute, the Alaska Division of Geological and Geophysical Surveys, and other university and government researchers.

\section{Stratigraphy, Sedimentology, and Structural Geology}

The Geometry and Kinematics of Detachment Folds in the Northeastern Brooks Range: Detachment folds form as a result of the shortening and displacement of a strong layer, such as limestone or sandstone, above a weak layer, such as shale or salt. Graduate student Tom Homza is working with Associate Professor of Geology Wes Wallace on a docto- ral study to determine how detachment folds vary in shape, how they evolve, and what factors control their shape and evolution. Theirstudy consists of two parts. The first is to 
document the geometry and kinematics of detachment folds in the northeastern Brooks Range, which contains among the best exposed examples of detachment folds in the world.

The second part is to produce geometric models of detachment folds. These models are used to evaluate the geometric and kinematic implications of assumptions commonly made in constructing cross sections of detachment folds using the incomplete information that is typically available in real geologic situations.

The objective is to determine the model that best fits the observations of natural detachment folds. Results so far suggest that fold hinges remain fixed throughout the evolution of

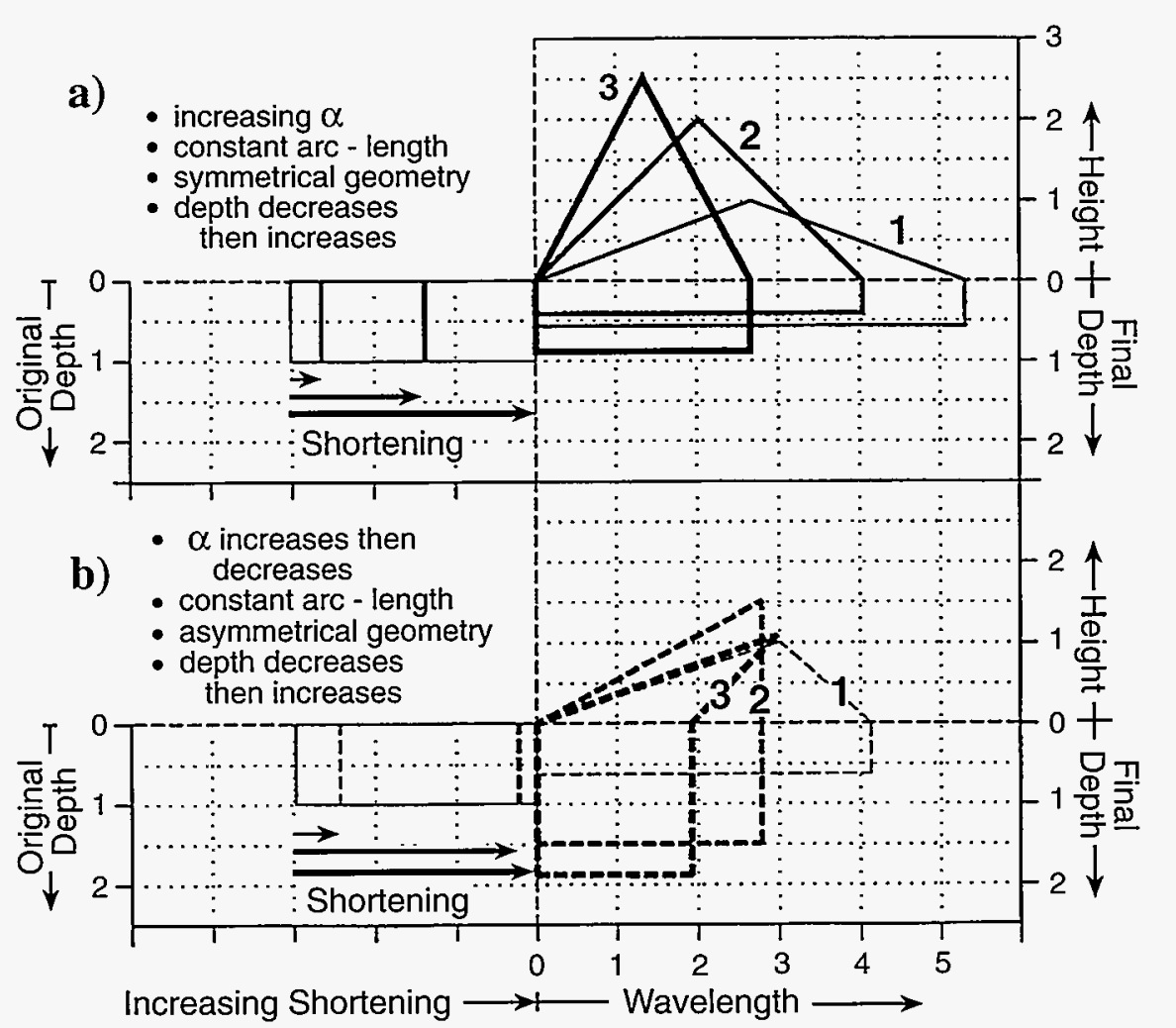

This geometric model shows the evolution of symmetrical (a) and asymmetrical (b)
detachment folds with increasing shortening (from 1 to 3). Hinges remain fixed with
respect to layering and the incompetent detachment unit beneath the fold changes
thickness during the evolution of each fold.

This geometric model shows the evolution of symmetrical (a) and asymmetrical (b)
detachment folds with increasing shortening (from 1 to 3). Hinges remain fixed with
respect to layering and the incompetent detachment unit beneath the fold changes
thickness during the evolution of each fold.

This geometric model shows the evolution of symmetrical (a) and asymmetrical (b)
detachment folds with increasing shortening (from 1 to 3). Hinges remain fixed with
respect to layering and the incompetent detachment unit beneath the fold changes
thickness during the evolution of each fold.

This geometric model shows the evolution of symmetrical (a) and asymmetrical (b)
detachment folds with increasing shortening (from 1 to 3). Hinges remain fixed with
respect to layering and the incompetent detachment unit beneath the fold changes
thickness during the evolution of each fold.

detachment folds in the northeastern Brooks Range, and that the weak layer that underlies these folds varies in thickness as the folds evolve. Both of these results differ from the assumptions used in published models for detachment folds. This study will be of value in defining criteria for the recognition of detachment folds, which commonly are misinterpreted as other types of folds, and in providing new models for the construction of cross sections of detachment folds from incomplete information.

These results will be useful for the assessment of subsurface structures for petroleum exploration and production and for earthquake hazard.

NSF Grant EAR 93-04482: Scientific personnel-W.K. Wallace and T. Homza
Geological Evolution of the Northeastern Brooks Range in the Arctic National Wildlife Refuge: The Tectonics and Sedimentation Research Group has been studying the geological evolution of the northeastern Brooks Range since 1986. These studies address various aspects of the geology of the northeastern Brooks Range, a rugged and inaccessible part of northern Alaska. The goal of these studies is to develop a comprehensive understanding of the character and evolution of the region while establishing the geological framework of the coastal plain of the Arctic National Wildlife Refuge. Because of the high potential for petroleum discoveries on the coastal plain, this work has been funded primarily by the petroleum industry and the U.S. Department of Energy.

The group's efforts have focused on the sedimentary rocks of the region, their environments of deposition, and their subsequent deformation. Most of the rocks were deposited on the rifted edge of an ancient continent, similar to the continental margins now bounding the Atlantic Ocean. Unlike present geography, the continent lay to the north and the ocean lay to the south at the time of deposition.

Associate Professor of Geology Wes Wallace, Research Assistant Professor of Geology Cathy Hanks, and their graduate students studied how rocks folded and faulted to form the northeastern Brooks Range, providing clues to the origin of mountain belts in general. As in the RockyMountains, this deformation occurred within a continent, but was related to relative plate motions at a distant convergent continental margin. 
Associate Professor of Geology Keith Crowder and his graduate students have studied a variety of ancient terrigenous clastic rocks to interpret their environments of deposition and erosional gaps in the sedimentary record. These deposits were derived from erosion of a continent to the north and were deposited in ancient rivers, deltas, and continental shelves.

Associate Professor of Geology Keith Watts and his graduate students have studied carbonate rocks to reconstruct the paleogeography of the ancient continental margin and to understand depositional cycles that reflect changes in relative sea level. These rocks are mainly limestones and dolostones derived from marine organisms, comparable to those forming today in the Bahamas and in the Persian Gulf.

Associate Professor of Geophysics Paul Layer has begun ${ }^{40} \mathrm{Ar} /{ }^{39} \mathrm{Ar}$ dating of granitic rocks to constrain the age of tectonically significant thermal events. These dates will be combined with the results of structural studies and fission-track dating by Paul O'Sullivan (LaTrobe University, Australia) to determine when and where deformation and uplift occurred in the northeastern Brooks Range, and how those events affected the coastal plain to the north.

ARCO Alaska, BP Exploration (Alaska), Chevron USA, Exxon Company USA, Japan National Oil Corp., Mobil Exploration and Producing U.S., Phillips Petroleum, Unocal, and U.S. Department of Energy funds: Scientific personnelW.K. Wallace, C.L. Hanks, R.K. Crowder, K.F. Watts, P.W. Layer, D.B. Stone, A. Anderson, W. Camber, J. Clough, M. Eckstein, N. Harun, T. Homza, A. Krumbardt, D. LePain, S. Morgan, M. Myers, E. Pavia, P. Peapples, and C. Petersen (joint participation between the UAF Geophysical Institute and the Department of Geology and Geophysics)

Structure of the North-central Brooks Range Along the TransAlaska Crustal Transect: The U.S. Geological Survey (USGS) has conducted the Trans-Alaska Crustal Transect (TACT), a combined geo- physical and geological study along the trans-Alaska pipeline route from Valdez to the North Slope. The final phase of this project addressed the northern part of the Brooks Range from Atigun Pass to the mountain front. Associate Professor of Geology Wes Wallace worked with Tom Moore and other USGS researchers in the geological studies along this northernmost part of the transect.

This work documented a wellexposed cross section across the external part of a mountain belt, which can serve as a basis for comparison with other mountain belts throughout the world.

Among the most striking and significant features of the region are the many well-exposed examples of fold-and-thrust structures. The exposures allow three-dimensional reconstruction of the geometry of thrust-truncated detachment folds, thrust faults, and duplexes, thereby providing new insights into the geometry and evolution of these important types of structures. The transect crosses the boundary between greatly deformed rocks that

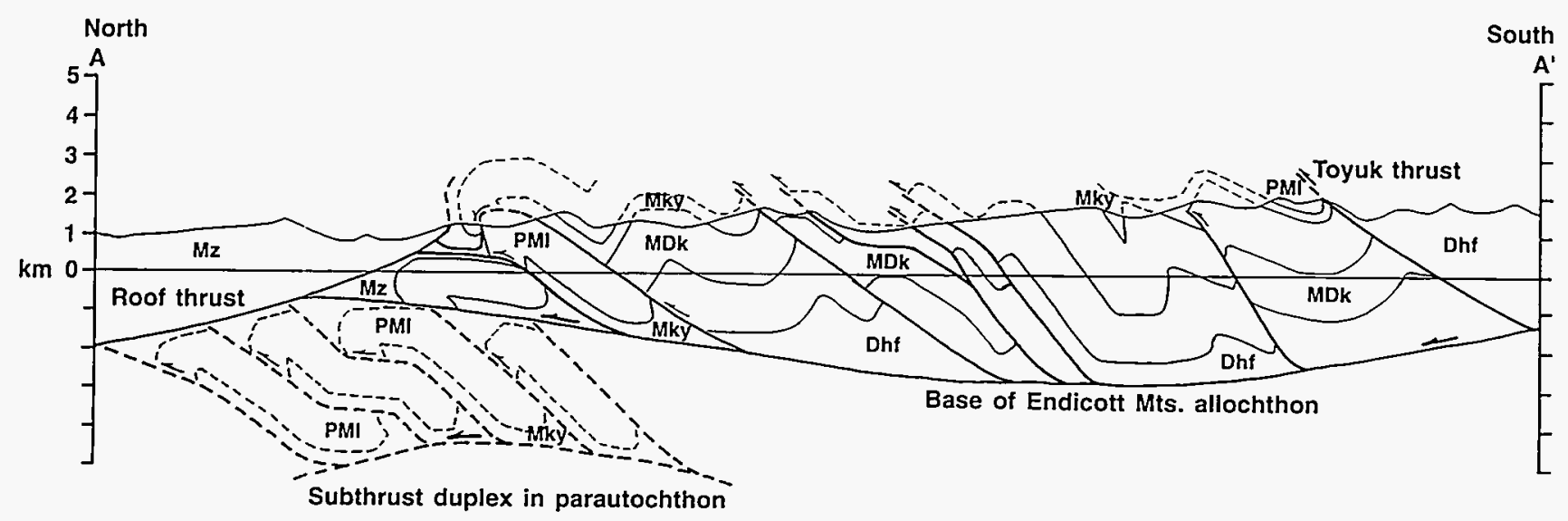

Shown is the schematic cross section south of the Brooks Range mountain front and east of the pipeline. The structure is characterized by a series of asymmetrical folds that have been truncated by northward-directed thrust faults.

Rock units are as follows: $M z=$ foredeep deposits; $P M I=$ Lisburne Limestone; $M k y=$ Kayak Shale; MDk = Kanayut Conglomerate; Dhf $=$ Hunt Fork Shale. 


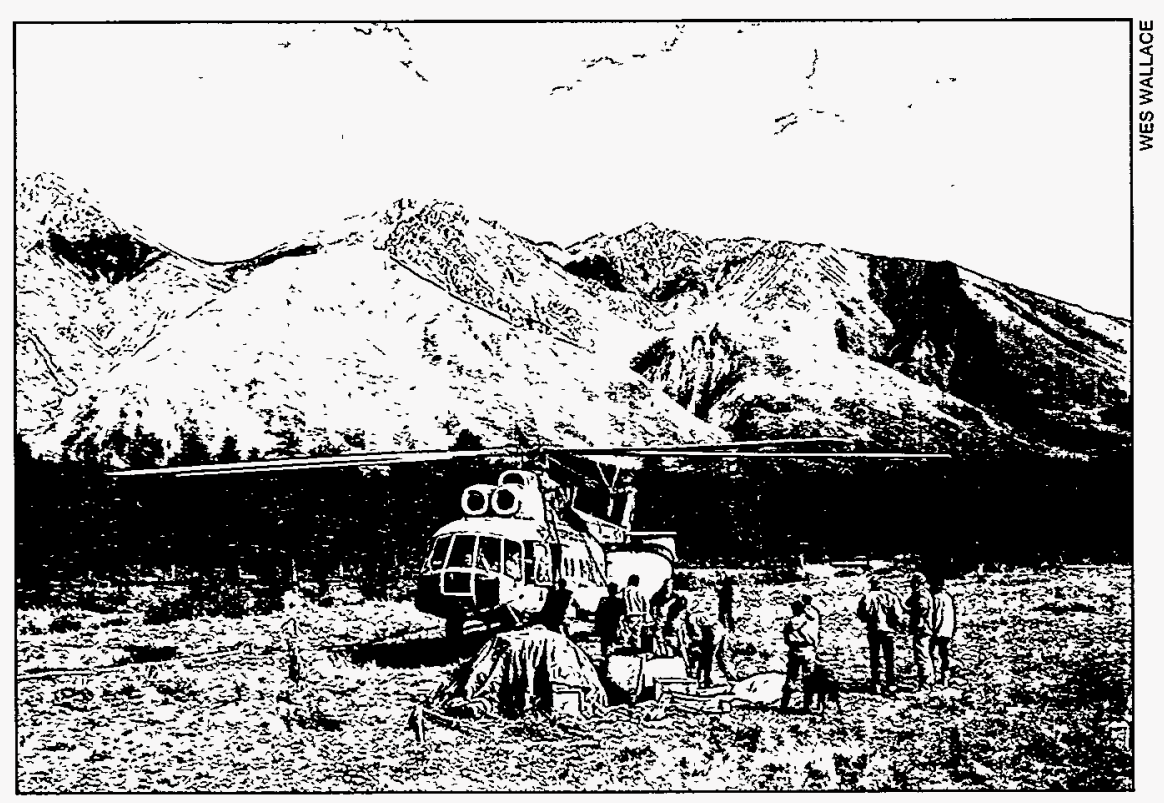

Researchers at the Chersky Range site prepare for a camp move. The baking of sedimentary rocks during the intrusion of light-colored granite creates reddish hues on the mountain slopes in the high peaks (shown as darker hues above).

have been displaced great distances and other rocks that have been displaced much less, including some that were derived by erosion from the ancestral Brooks Range. Such boundaries are an important element of all mountain belts; this study offers clues to the complex relationships among age, displacement, and the geologic structures associated with such boundaries.

This study is of regional significance because it documented structures typical of those found in the northcentral Brooks Range, and it documented the boundary berween these structures and the very different structures typical of the northeastern Brooks Range. The geologic maps and orher field observations from the project will be used to construct a series of restorable cross sections across the north-central Brooks Range.

These will be combined with the results of the TACT geophysical studies and fission-track dating to de- velop an interpretation of the subsurface structure of the northcentral Brooks Range and foothills, and to determine how that structure evolved.

In addition to the insights gained into the evolution of mountain belts, this research will have direct implications for the subsurface structure of the Alaskan North Slope petroleum province, and implications for the timing of sediment deposition and hydrocarbon generation, migration, and trap formation.

ARCO Alaska, BP Exploration (Alaska), Chevron USA, Exxon Company USA, Japan National Oil Corp., Mobil Exploration and Producing U.S., Phillips Petroleum, Unocal, and USGS funds: Scientific personnel-W.K. Wallace

Distribution and Character of Fractures in the Lisburne Group, Northeastern Brooks Range, Alaska: The Carboniferous Lisburne Group is a thick, heterogeneous, and highly fractured sequence of carbonate rocks that acts as the reservoir horizon for the Lisburne Oil Field of the North Slope. The rock group also is widely exposed throughout northern Alaska and is an important element of the Brooks Range fold-and-thrust belt.

The Lisburne Group was recognized as a potential reservoir horizon very early during petroleum exploration of northern Alaska and was considered by many to be the primary exploration target during the early phases of drilling on the North Slope.

As in many carbonate reservoirs, naturally-occurring fractures in the Lisburne Group play a major role in hydrocarbon production by providing both porosity and permeability. However, the distribution, density and character of fractures within the Lisburne Group are variable and difficult to predict, and may depend on the lithology of the host rock, the structural setting of the reservoir and other unidentified factors.

In the Lisburne Oil Field, less than 10 percent of the 2 billion barrels in place is recoverable at the present time. A clearer understanding of the distribution, character and origin of fractures has the potential to aid in the development of secondary and tertiary recovery programs for this large, but difficult to produce, reservoir.

This study uses examples of the Lisburne Group exposed in the northeastern Brooks Range to determine the relarionship between lithology, structural position, and fracture patterns within this carbonate sequence. This information will help predict the distribution and effect of fractures on hydrocarbon production within the Lisburne Group of the North Slope. 
Funded by DOE and Sandia National Labs Grant AG-4303: Scientific personnel-C.L. Hanks; $A$. Krumbardt and J. Lorenz (Sandia National Labs); and L. Teufel (New Mexico Institute of Technology and Sandia National Labs)

Origin of Arcuate Structural Trends in the Chersky Range, Northeastern Siberia: Associate Professor of Geology Wes Wallace has worked for two summers in northeastern Siberia with structural geologists of the Yakutian Institute of Geological Sciences, Russian Academy of Sciences.

His research is one part of a cooperative project that also includes studies of paleomagnetism (Stone), geochronology (Layer), and seismicity (Fujita and Stone), which are described elsewhere in this biennial report.

The Chersky Range of northeastern Siberia displays a sharp bend that is convex westward, toward the interior of the continent. This bend probably is partly a reflection of the shape of an originally irregular ancient continental margin against which later structures have conformed.

A major objective of this study is to determine whether the arcuate trend of these later structures was formed by oroclinal bending of originally straighter structures, or whether they formed in place, perhaps during multiple events with different displacement directions.

Field observations in the two limbs of the bend indicate that at least two generations of structures formed during convergence in different directions. Early northwestward convergence may reflect oblique collision of displaced continental terranes with the Siberian continental margin.

Later southwestward convergence may reflect collision of arctic Alaska with the Siberian continental margin and the terranes accreted to it. This second event resulted in the Eurasian and North American continents being sutured together in northeastern Siberia, thus allowing the separation of the Arctic and Pacific ocean basins.

These significant observations will help reconstruct the tectonic evolution of northeastern Siberia and its relationship to the tectonic evolution of Alaska, and they will offer a possible analog for other arcuate structural trends elsewhere in the world.

ARCO Alaska, BP Exploration (Alaska), Chevron USA, Exxon Company USA, Japan National Oil Corp., Mobil Exploration and Producing US, Phillips Petroleum, Unocal, and Russian Academy of Sciences funds: Scientific personnel-W.K. Wallace
Synthetic Aperture Radar (SAR) and Geologic Reconnaissance in Arctic Regions: Regional and detailed geologic studies of northern Alaska and other parts of the Arctic are hampered by the high cost of doing field work in this remote and generally inhospitable region. Satellite-based syntheric aperture radar (SAR) could provide a relatively inexpensive remote sensing alternative for regional geologic studies in arctic regions.

SAR systems have the advantage of being able to "see" the earth's surface regardless of cloud and light conditions. While SAR imagery has been utilized in geologic studies of tropical regions, its urility in arctic regions, where ground-cover is minimal, is still being tested.

The northeastern Brooks Range was chosen to test the usefulness of SAR in geologic studies in arctic regions because of the relatively large amount of ground data available from the area. ERS-1 data was terraincorrected and compiled into a mosaic of the entire range.

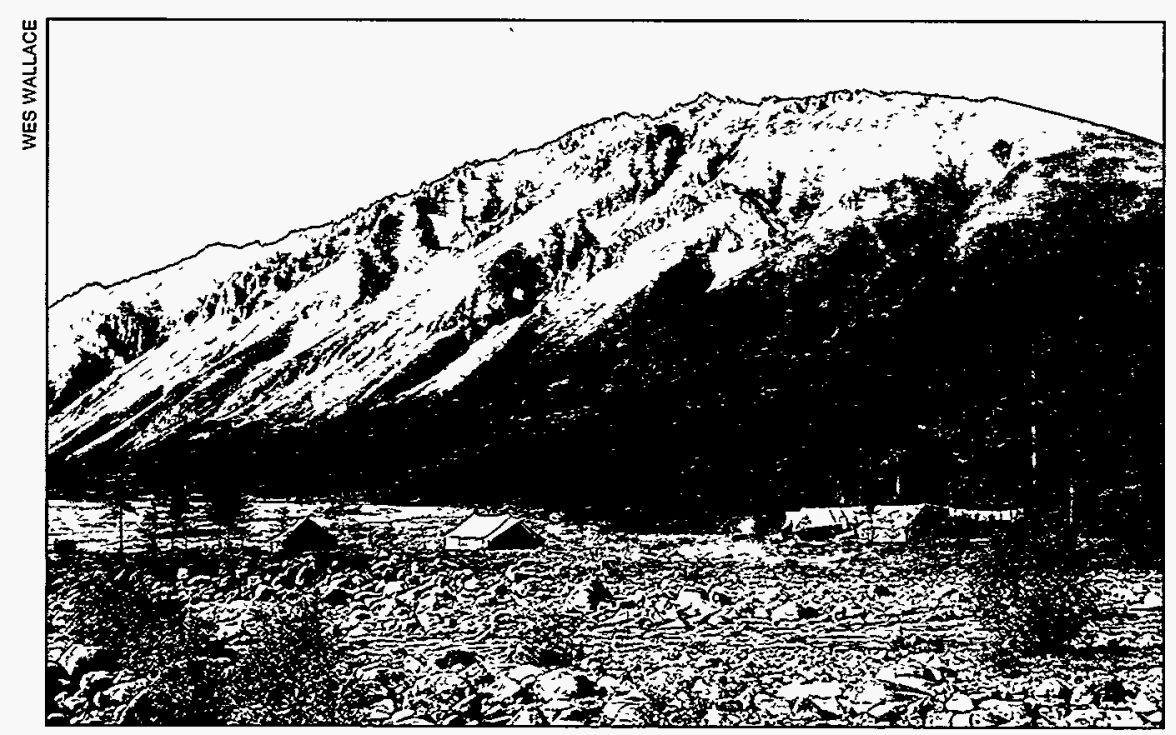

The background of this campsite in the Chersky Range of northeastern Siberia includes the view of a major fault that separates the light-colored rocks in the peaks from the dark rocks in the lower slopes. 
Preliminary analysis suggests that structural features and gross lithologic differences are readily discernible on individual images and on the mosaic. Carbonate and clastic rocks involved in regional-scale folding give different radar response and can be distinguished on the imagery. Faults can be distinguished as linear features or juxtaposition of different rock types. The effect of surface roughness and water content, and lithology, can be enhanced by removing the effect of the terrain on the radar response. The resulting image shows no topography but highlights lithologic variations. Future studies aim at using ERS-1 ERS-2, and RadarSat radar data, and ADEOS optical data in geologic studies of the central and western Brooks Range, northwestern Canada, and the Russian arctic.

ARCO Alaska, BP Exploration (Alaska), Chevron USA, Exxon Company USA, Japan National Oil Corp., Mobil Exploration and Producing U.S., Phillips Petroleum, Unocal, and USGS funds: Scientific personnel-C.L. Hanks and R. Guritz

\section{Geochronology}

The Geochronology Laboratory: The UAF Geochronology Laboratory conducts research to determine the ages of rocks and minerals by using the ${ }^{40} \mathrm{Ar} /{ }^{39} \mathrm{Ar}$ step-heating dating method. Major areas of research emphasis include determining the tectonic history of Alaska and adjacent Russia, the relationship between plutonism and ore mineralization, and the eruptive history of Alaska's volcanoes. The laboratory

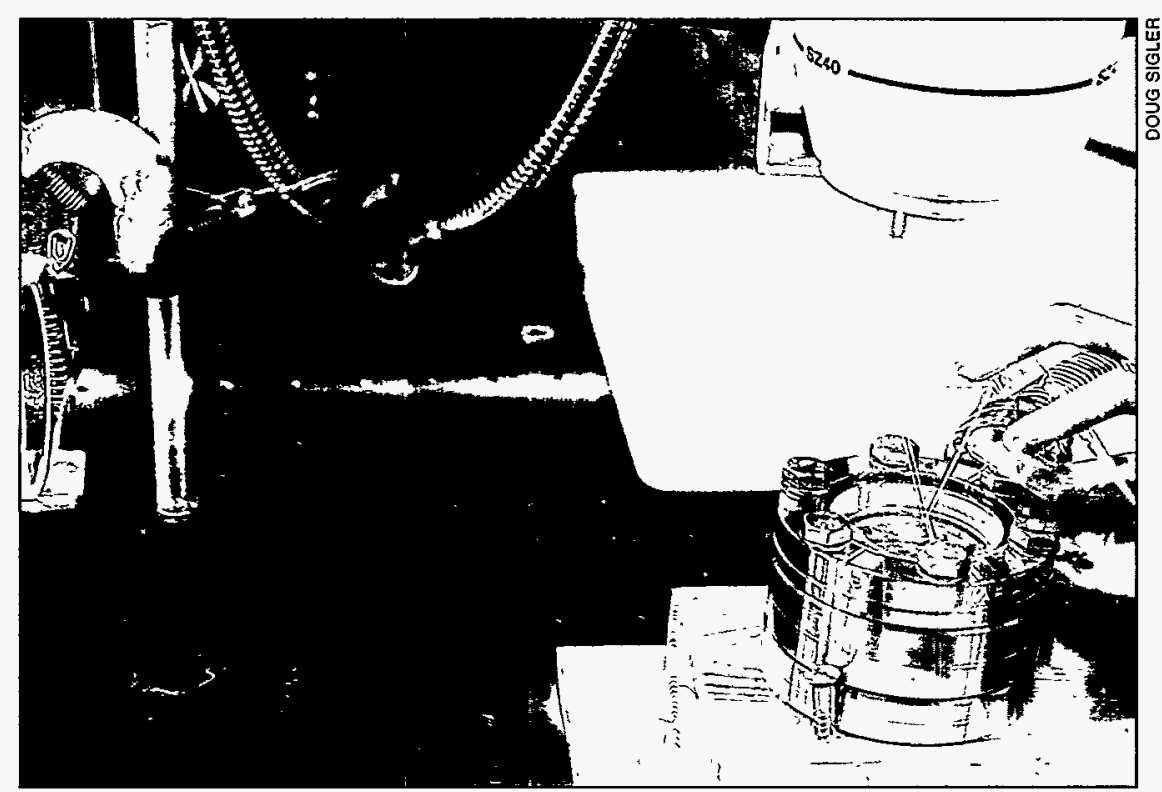

Shown above is the single crystal laser fusion device housed in the Geochronology Lab. The laser beams heat up rock crystals until they turn into small glass beads, about the size of grains of sand. The meltdown forces the release of argon gas, which is funneled into a mass spectrometer, where it is analyzed to determine the rock's age. has two mass spectrometer systemsa Nuclide system installed in 1970 with an on-line extraction furnace and a VG3600 laser system installed in 1994 . We completed over 2000 mass spectrometry analyses on 143 samples in 1993, and 2500 mass spectrometry analyses on 158 samples in 1994 on the Nuclide mass spectrometer system. In 1994, we completed over 1200 mass spectrometry analyses on the newly installed laser

system. Many of these analyses were done for research grants and for internally supported projects discussed below, while other analyses were done for externally supported contracts and in support of student theses.

Corporations which supplied support for the geochronology laboratory in exchange for age analyses in 1993 and 1994 include: Placer Dome Inc.; ASA Inc.; Ryan Lode Mines Inc.; Fairbanks Gold Mining Inc.; Arco; and Chevron Minerals Division. Universities that provided contract support or collaboration include Michigan State University, Beloit College, Stanford University, University of Pittsburgh, University of Witwatersrand in South Africa, and Lulea University in Sweden. Supporting government agencies include the Alaska Division of Geological and Geophysical Surveys, the U.S. Geological Survey, the U.S. Bureau of Land Management and the U.S. Bureau of Mines.

For theses, students investigated the plutons of the northeastern Brooks Range, the mineralization in the Fairbanks district, the history of the Southwest Nevada Volcanic Field, the igneous and metamorphic rocks in Russia, mineralization in 
southern Africa and Australia, and the thermal history of the McKinley pluton.

Supported by internal funds through variousagencies: ScientificpersonnelP.W. Layer and J. Drake

${ }^{40} \mathrm{Ar} /{ }^{39} \mathrm{Ar}$ Dating of the McKinley Pluton, Denali National Park, Alaska: The McKinley pluton is one of the northernmost plutons of the Alaska-Aleutian Range batholith. This biotite granite intrusion composes most of the 6194-meter-high Mt. McKinley massif. Previous K-Ar and apatite fission-track studies suggest that the pluton has had a protracted cooling/uplift history since its emplacement at $-56 \mathrm{Ma}$. The great local relief available for sampling makes the McKinley pluton an ideal intrusion for a thermochronometric study through ${ }^{40} \mathrm{Ar} /{ }^{39} \mathrm{Ar}$ dating of $\mathrm{K}$-feldspars and biotites. The samples analyzed were collected from a range in elevation of 2100 to 5990 meters. The K-feldspars can be modeled as having multiple domains; thus, cooling histories spanning $-35 \mathrm{Ma}$ can be estimated for each elevation.

Six biotites and one primary white mica have relatively flat age spectra with plateau ages between 55.1 and $55.8 \mathrm{Ma}$. They show a very slight age/elevation dependence; the lower biotites are younger. These consistent mica ages most likely reflect uniform cooling of the pluton.

The K-feldspars exhibit saddleshaped age spectra that vary significantly from each other. Cycled heating experiments carried out on the K-feldspars resulted in arrhenius plots indicating that the samples may contain three or four separate diffusional domains. The arrhenius plots and age spectra were fitted using the programs of Lovera (1992).

The results show the pluton cooled very slowly with rates of $10^{\circ} \mathrm{C} / \mathrm{Ma}$ to $4^{\circ} \mathrm{C} / \mathrm{Ma}$, with the lower samples cooling at the slower rates. The pluton came into equilibrium with the geothermal gradient at $-30 \mathrm{Ma}$ and at a temperature of $-250^{\circ} \mathrm{C}$. The $\mathrm{K}$-feldspars record uplift rates of $0.13 \mathrm{~mm} / \mathrm{yr}$, from $43 \mathrm{Ma}$ to $25 \mathrm{Ma}$. The small domains of the K-feldspars do not record the rapid uplift of Mt. McKinley that began at $5 \mathrm{Ma}$.

Internal funds: Scientific personnel-P.W. Layer and A. West

Acquisition of a High Sensitivity ${ }^{40} \mathrm{Ar} /{ }^{39} \mathrm{Ar}$ Dating System for the University of Alaska: In 1992, we received initial funding to upgrade the analytical facilities in the geochronology laboratory. In 1993, construction began on an automated laser ${ }^{40} \mathrm{Ar} /{ }^{39} \mathrm{Ar}$ dating system. This system was to bring state-of-the-art capability to UAF with applications to hazard assessment, mineral resource evaluation, and basic scientific research. In February 1993, the final funds were secured from the Alaska Science and Technology Foundation and the University of Alaska Natural Resources Fund. In April 1993, the VG3600 mass spectrometer was ordered from England from Fisons Instruments. Construction of the argon extraction system was completed in 1993. In December 1993, the mass spectrometer was delivered and in February 1994, it was installed, tested and calibrated.

A coherent 6-watt argon-ion laser was delivered in April 1994, and the first samples were measured in May. Preliminary results from these first analyses were displayed at the International Conference of Geochronology, Cosmochronology, and Isotope Geochemistry held in Berkeley, California in June 1994. The average turnaround from the time of delivery of the spectrometer to presentation of first results is typically about two years. Our less than sixmonth turnaround is the fastest on record for a Fisons Instruments spectrometer. By the end of 1994, we were in the process automating the system with the LabWindows software package.

Preliminary results: As a first application of the laser dating system, we initiated a collaborative investigation with Tom Vogel and Kris Huysken from Michigan State University to determine the age of a tephra sequence from the Southwest Nevada Volcanic Field by dating single sanidine crystals. The single grain laser approach is critical in such studies because material from older eruptions is incorporated into younger tephra flows. By dating single crystals, older, inherited crystals can be distinguished from younger, primary ones. The results are shown in the figure on the following page.

Crystals from three of the four units dated show unimodal age distributions indicating that the mean age is the age of eruption and that the sequence was emplaced over a time span from 12.7 to $11.6 \mathrm{Ma}$. The fourth unit, 12R8-4a, shows a distribution of ages indicating that this eruption entrained a significant portion of older material. The high precision of the laser dating system allows distinction of units that differ by as little as 0.5 percent of the age. 
Research applications include dating young volcanic units from Alaska's volcanoes, dating obsidian from archeological sites, and dating single minerals from ore deposits.

Funded by NSF Grant EAR9214375 and ASTF Grant 89-1-187: Scientific personnel-P.W. Layer

${ }^{40} \mathrm{Ar} /{ }^{39} \mathrm{Ar}$ Dating of Eocene Obsidian from the BatzaTena Archeological Site, West-Central Alaska: This investigation explored the potential of using ${ }^{40} \mathrm{Ar} /{ }^{39} \mathrm{Ar}$ dating to help fingerprint the source of stone archeological artifacts. As a preliminary investigation, the age of obsidian was determined from the BatzaTena archeological site, a principal source of obsidian in Alaska. The BatzaTena (Indian River) obsidian site in westcentral Alaska is thought to be the source for many obsidian artifacts found in northern Alaska.The BatzaTena obsidian occurs as subround pelletal fragments up to $10-\mathrm{cm}$ long thought to have formed as residual inclusions (marekanites or Apache

\section{Single Sanidine Ages}

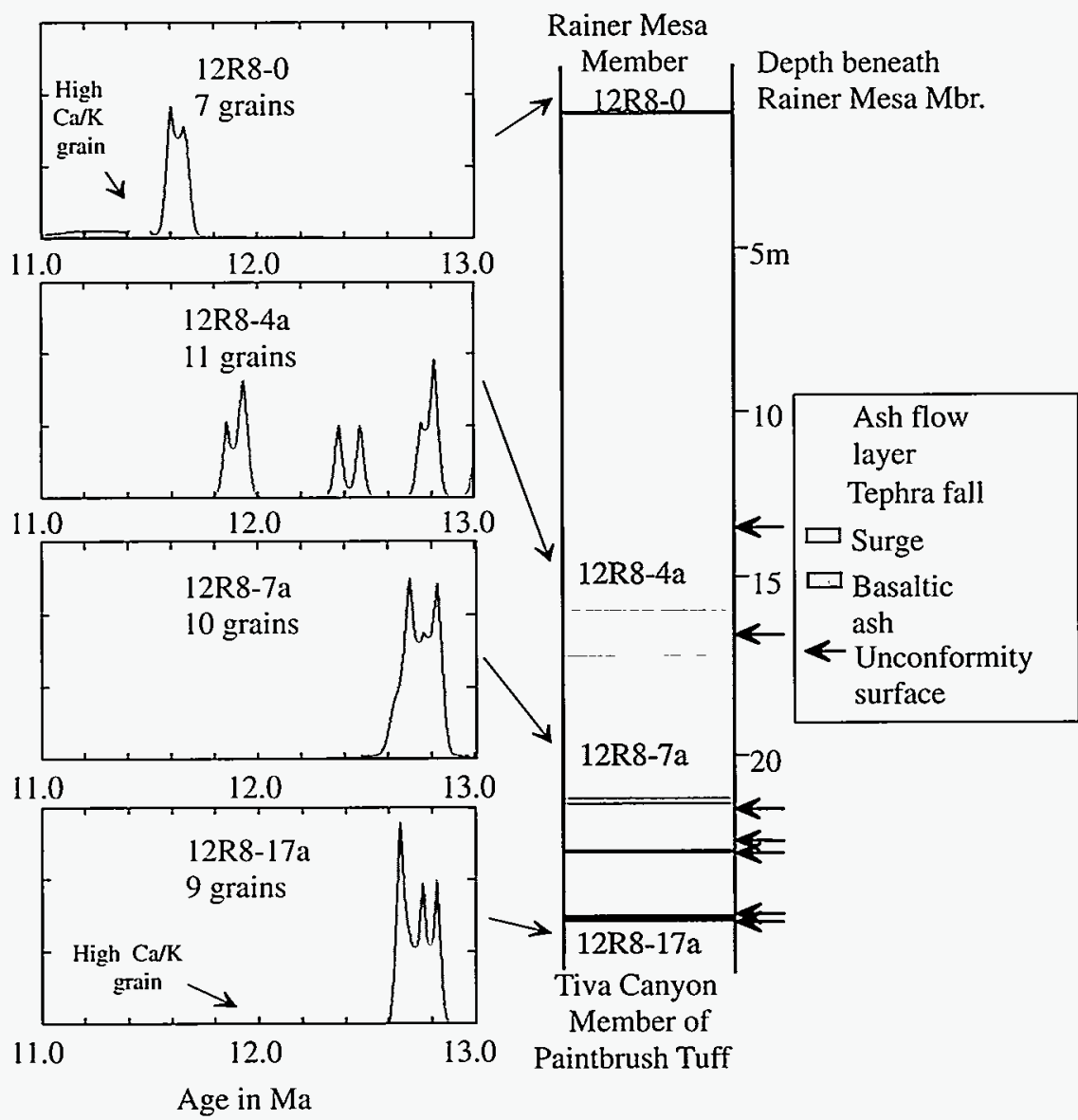

Results of single grain sanidine dating of four units from the tephra sequence beneath the Rainer Mesa Member of the Timber Mountain Tuff, Southwest Nevada Volcanic Field. The age and age uncertainty for each grain is shown by the 'bell curve' on the age versus probability plot. For units $12 R 8-0$ and 12R8-17a one grain showed anomalous ages and high $\mathrm{Ca} / \mathrm{K}$ ratios indicative of a plagioclase rather than a sanidine grain. The scatter in ages seen in unit 12R8-4a is probably due to entrainment of older sanidine in an $11.9 \mathrm{Ma}$ unit.

tears) in hydrated perlitic volcanic glass. Several fresh obsidian fragments analyzed by ${ }^{40} \mathrm{Ar} /{ }^{39} \mathrm{Ar}$ step heating show no evidence of alteration. Age spectra from the samples are flat with an average age of $41 \mathrm{Ma}$, an age consistent with stratigraphic controls that may reflect the eruptive age of the obsidian.

If $41 \mathrm{Ma}$ obsidian is unique to this site, then these results may be used with instrumental neutron activation analysis, $\mathrm{x}$-ray fluorescence microprobeand hydration measurements (compiled by John Cook at the Bureau of Land Management) to provide a distinctive fingerprint to identify artifacts from BatzaTena. Laser ${ }^{40} \mathrm{Ar} /{ }^{39} \mathrm{Ar}$ dating techniques allow for small $(<1 \mathrm{~mm})$ fragments from artifacts to be dated without destroying the sample. There are few accounts of reliable ${ }^{40} \mathrm{Ar} /{ }^{39} \mathrm{Ar}$ ages from terrestrial glass samples older than $-10 \mathrm{Ma}$. The BatzaTena study that indicates reliable ages may be obtainable from old obsidian elsewhere.

Internal funds: Scientific personnel-P.W. Layer and J. Cook (U.S. Bureau of Land Management)

Cooling History of the Okpilak Batholith Determined from ${ }^{40} \mathrm{Ar} /{ }^{39} \mathrm{Ar}$ K-Feldspar Thermochronometry: This study was done under the Research Experience for Undergraduates program at the Geophysical Institute. The thermal history of the Okpilak batholith of the northeastern Brooks Range was investigated using ${ }^{40} \mathrm{Ar} /{ }^{39} \mathrm{Ar}$ thermochronology of potassium feldspars. This Okpilak batholith presents an unusual case of intimate involvement of crystalline rocks in thrusting near the foreland 
basin of a fold-and-thrust belt. Various dating techniques provide an outline of the batholith's uncommon history: U-Pb zircon dating yielded an initial crystallization age of $380 \pm 10 \mathrm{Ma}$; K-Ar and ${ }^{40} \mathrm{Ar} /{ }^{39} \mathrm{Ar}$ dating of recrystallized biotite indicates metamorphism and cooling at -59 ; and apatite fission track analyses produced ages of $31-43 \mathrm{Ma}$ for subsequent structural uplift and unroofing. In this study, the multidomain theory of argon retention in $\mathrm{K}$-feldspar was used to provide additional information about the history of the batholith.

Samples from four locations in the batholith yielded age spectra indicative of the multidomain behavior of feldspar. We modeled the cooling history of the batholith in a temperature range from $-450^{\circ} \mathrm{C}$ to $220^{\circ} \mathrm{C}$ and in a time from $-130 \mathrm{Ma}$ to $-30 \mathrm{Ma}$. Coupled with apatite and biotite age data, a consistent history emerges.

The batholith underwent slow cooling (or uplift) from about $130 \mathrm{Ma}$ to about $45 \mathrm{Ma}$. The pluton underwent rapid uplift and cooling from a temperature of about $250^{\circ} \mathrm{C}$ to less than $100^{\circ} \mathrm{C}$ in about $10 \mathrm{Ma}$. The rocks uplifted from a depth $>10 \mathrm{~km}$ (assuming a $25^{\circ} \mathrm{C} / \mathrm{km}$ geothermal gradient) in a relatively short period. These data rule out a model of gradual uplift from $>10 \mathrm{~km}$ depth between 59 and $43 \mathrm{Ma}$, as had been proposed.

Funded by NSF Grant EAR9322471: Scientificpersonnel-P.W. Layer and J. Paegle (University of Utah summer intern)

Geochronologic Studies of Yakutia (Northeast Russia): The geochronologic studies in Yakutia are part of a multidisciplinary collaborative in- vestigation which includes paleomagnetism, seismology and structural geology, conducted to gain an understanding of the tectonic history of the Sakha Republic (Yakutia).

Most geochronologic investigations focus on determining the timing of events affecting the Chersky Range in eastern Yakutia. We collected over 300 samples for geochronologic and geochemical analysis. Two areas of focus in 1993 and 1994 were the description and the chronology of ophiolites, and the timing of granitic intrusion in the region.

Ophiolites in the Chersky Range. Mafic and ultramafic rocks are found in isolated complexes along the Chersky Range. Five of the six exposures are located on the eastern side of a Devonian-Ordovician carbonate platform and appear to be thrust over it; the sixth exposure, the Debin fragment, is located to the west.

On the basis of lithology, geochemical composition and structural position, the ultramafic rocks and their associated mafic volcanics and deep-marine sedimentary rocks are interpreted as ophiolites or ophiolite fragments derived from back-arc or oceanic crust that was located to the east of the Chersky Range.

Faunal and radiometric dating indicate that the oceanic crust has an age of about 370-430 Ma, Early to Middle Devonian. The ages of metamorphic minerals suggest that the ophiolites were obducted and metamorphosed during the amalgamation of the Kolyma-Omolon superterrane, about 170-174 Ma (Callovian, late Middle Jurassic), at which time olistostromes containing fragments of the ophiolite were formed. This age predates the formation of the Uyandina-Yasachnaya volcanic arc, believed to represent the final closure of the ocean basin between the Kolyma-Omolon superterrane and the North Asian craton. It is possible that the southernmost ophiolite, the Debin fragment, has a different history from the other Chersky Range ophiolites and was emplaced as a result of the accretion of the Kolyma-Omolon superrerrane.

Granitic Rocks in the Chersky Range. Our geochronologic investigations of plutonic rocks from the Kolymian Structural Loop (KSL) are intended to investigate whether KSL formed in response to a single tectonic event or to discrete events. Precise ages were obtained from ${ }^{40} \mathrm{Ar} /{ }^{39} \mathrm{Ar}$ step-heating of biotite and, in some cases, hornblende, muscovite or feldspar. Plutons from the main northwest-southeast trending axis of the Chersky Range show no significant age variations along strike; however, there is a decrease in age across the belt from $142 \mathrm{Ma}$ in the east to $135 \mathrm{Ma}$ in the west. These ages are significantly older than ages from granites of the east-west trending Polousney Range north of the Chersky Range. These plutons show a decrease in age across the belt from $127 \mathrm{Ma}$ in the south to $120 \mathrm{Ma}$ along the northern edge of the belt. Our preliminary data suggest that discrete tectonic events are responsible for the formation of the Chersky (western KSL) and Polousney (northern KSL) ranges. Linear granitic belts which radiate away from the main KSL tend to be significantly younger than the KSL granites. A $106 \mathrm{Ma}$ north-trending transverse belt extending from the Polousney Range north to Bolshoi Lyakhov Island 
shows no age progression. A southwest trending belt into the Verkhoyansk foldbelt shows a much clearer age progression.

The ages range from $132 \mathrm{Ma}$ near the Adycha-Taryn fault and then decrease westward to $124 \mathrm{Ma}$. These results indicate that various parts of the KSL as well as other structures in eastern Yakuria formed over a pro- tracted time in the early Cretaceous. In addition to these Cretaceous intrusions, we confirmed the presence of at least one Carboniferous intrusive complex to the east of the Chersky Range, the Tommor Massif, which is composed of alkaline-ultrabasic rocks, alkaline and subalkaline gabbroids, and alkaline and quartz syenites. It appears this complex region has escaped regional Cretaceous thermal overprinting events which seem to have reset the paleomagnetic signature and radiometric ages in volcanics and other rocks.

Funded by NSF Grant OPP9224029: Scientific personnel-P.W. Layer, D.B. Stone, and D. Hackett; $K$. Fujita (Michigan State University); $L$. Parfenov and V. Oxman (Yakutsk)

\section{Paleomagnetism and Solid-Earth Geophysics}

Magnetic Records in Sediment Cores: A lake-sediment coring project was set up to search for possible records of great earthquakes in the magnetic signatures of the sediments. Skilak Lake on the Kenai Peninsula, Alaska, was chosen as a target because of its close proximity (within about $100 \mathrm{~km}$ ) to the epicenter of the Great Alaska Earthquake of 1964, because it has a very uniform sediment source, and because it does not suffer from bioturbation.

A clear record of volcanic activity, indicated by the high magnetic susceptibility associated with glassy tephra layers, was produced in the process of searching for the earthquake signature. Our original cores penetrated about 1 meter, but our coring capability improved; we now have several $3+$ meter cores. These have not yer been fully analyzed but show clear, high-amplitude magnetic susceptibility peaks at about $1.5-\mathrm{m}$ and 2.2-m depths, presumably indicating major eruptive events. A linear extrapolation of the sedimentation rate from the shallow cores put these eruptions at 1250 and 1850 years BP. A number of interior Alaska lakes were sampled with the large Mack- ereth corer. From these cores, magnetic secular variation reference curves are being built for the last $9000+$ years to help correlate paleoenviromental data from lake to lake.

In addition to the lake cores, the paleomagnetic laboratory is measuring the magnetization of cores from ODP leg 152 to the Arctic Ocean north of Spitzbergen. The preliminary results of these studies show a well defined magnetostratigraphy.

U.S. Science Support PALE Grant ATM92-00600, NSF pass-through funds from the U.S. Science Support Program account 151-20801b, and State of Alaska funds: Scientific personnel-D.B. Stone, W.K. Witte, and B. Finney

Tectonics of Eastern Siberia: The aim of this project is to understand the present-day tectonic activity and the tectonic history of the Sakha Republic (previously known as Yakutia). The project began in 1990 with a reconnaissance field program to see what cooperative studies could be conducted with scientists from the Yakutsk Science Center. It has developed into a major program of paleomagnetic, geochronologic and structural geology studies combined with investigations of present-day seismicity. The main focus of the program is to investigate the geologic origin of the Kolyma Structural Loop (KSL). The KSL stands out as a horseshoe-shaped pattern on geologic maps of the area east of the Verkhoyansk mountain ranges. This pattern is formed by exposures of rock types including Paleozoic shelf sediments, island arc volcanic rocks, slices of oceanic crust (ophiolites) and major belts of intrusive rocks.

The principal findings of the geochronologic studies involve obtaining more precise control over the ages of emplacement of the major intrusive rocks that clearly outline the KSL. Work also involves obtaining better data relating to the age and history of the belt of associated ophiolitic rocks and the volcanic rocks representing the ancient volcanic arc.

The main thrust of the paleomagnetic work done so far has been to investigate whether the KSL was originally formed with the horseshoe shape seen today, whether it is an example of oroclinal bending, or whether it is made up of disparate fragments which have more or less 
accidentally gathered in this pattern. So far, the studies have been plagued with discoveries that most of the rocks have had their original magnetizations overprinted by some later event. This magnetic overprint is so pervasive that it has now turned into a major focus of our work. The principle reason for this shift in focus is the huge area over which the re- markably consistent near-vertical overprinting of magnetization is found. Since this remagnetization affects rocks of all lithologies, it has to represent the effects of a major
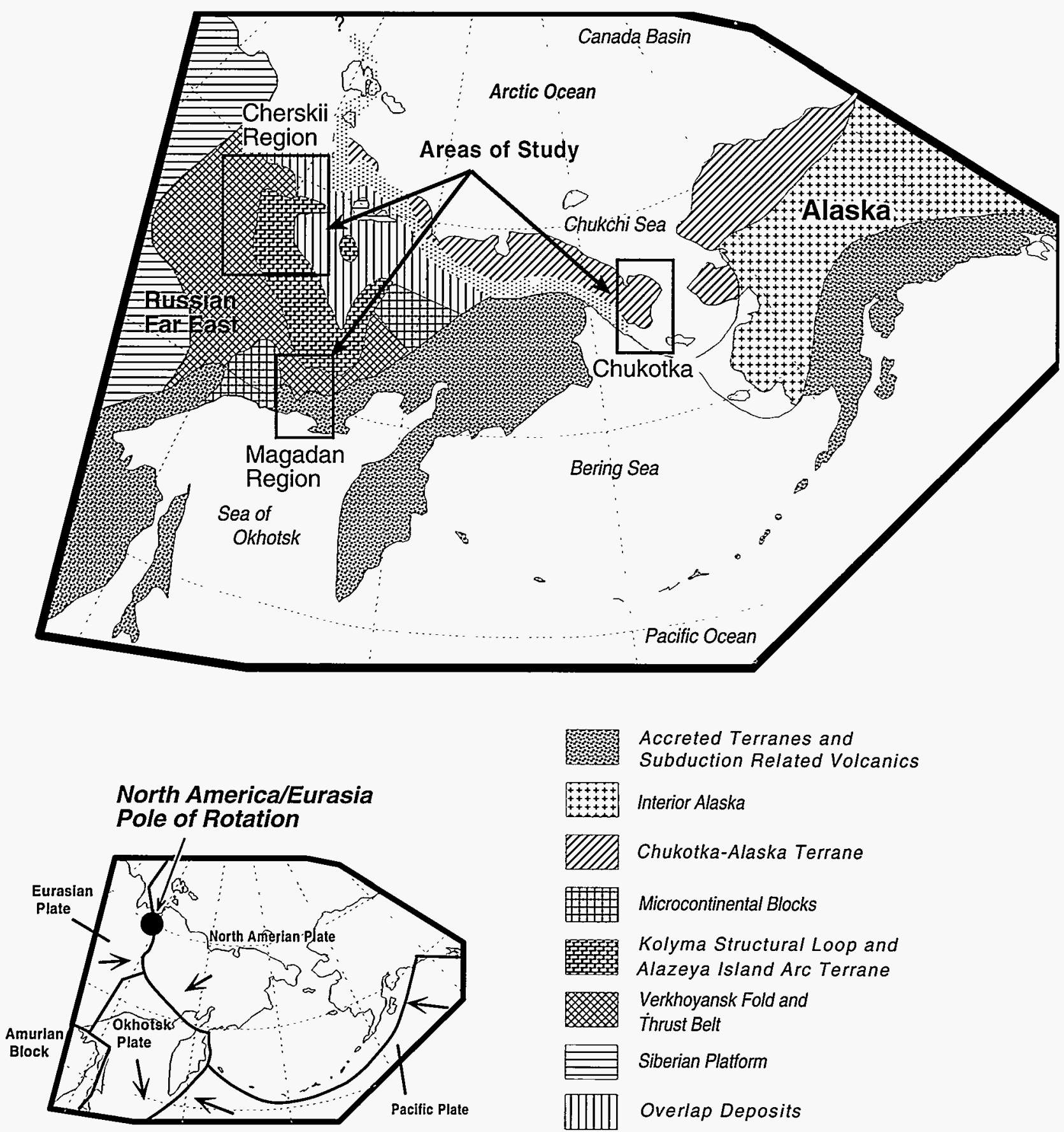

Accreted Terranes and

Subduction Related Volcanics

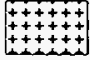

Elle

曲

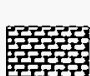

m

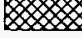

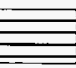

\|\|

Overlap Deposits

Interior Alaska

Microcontinental Blocks

Verkhoyansk Fold and

Thrust Belt

Siberian Platform

Chukotka-Alaska Terrane

Kolyma Structural Loop and

Alazeya Island Arc Terrane

South Anyui Suture System

This map shows major tectonic provinces mentioned in the text. The inset map shows the modern simplified plate configuration. Note that the Pacific Plate is subducting beneath the North American and Eurasian plates. Arrows indicate relative motion between the plates. 
geologic event; its timing and origin are important to understanding the history of the area.

The overprint seems to have occurred at roughly the same time as the emplacement of intrusive rocks, but preliminary conclusions point to a chemical, rather than a thermal, cause for the remagnetization. Our hypothesis is that the remagnetization resulted from the migration of hot fluids associated with the compressional events related to the formation of the major fold and thrust belts of the area.

The results of two seasons of studying the structural geology of the area suggest that the northwest corner of the KSL formed during two different deformational events with different directions of displacement.

The studies of present-day seismic activity involve both the operation of digitally recording seismometer stations and the restudy of records from older earthquakes as recorded by the Yakursk analog seismic network. The results of this work show that the most probable locations for the active North American-Eurasian-Pacific plate boundaries now involve a separate Okhorsk plate.

NSF Grant OPP92-24029, Incorporated Research Institutes for Seismology Grant IRIS-0159, and funds from ARCO Alaska Inc. and Exxon Exploration Co.: Scientific personnel-D.B. Stone, P.W. Layer, W.K. Wallace, S. Crumley and D. Hackett; $K$. Fujita and K. Mackey (Michigan State University)

Chukotka Seismic Experiment: The North Atlantic Treaty Organization (NATO) awarded funds for this experiment under its Linkage Grant program. This experiment is designed to encourage collaboration and communication among scientists of the former Soviet Union and scientists in western countries in the hopes that it will lead to ongoing (fundable) research projects.

Two collaborative experiments were set up through the Northeast Interdisciplinary Research Institute (NEISRI) in Magadan. One experiment involved paleomagnetic sampling of the Okhotsk-Chukotka volcanic belt where it is exposed on the Chukotka Peninsula, and the other involved establishing a chain of temporary seismic stations along the coast of Chukotka near the planned track of the R.V. Ewing.

The R.V. Ewingcarried out a Multi-Channel Seismic (MCS) experiment between the Bering Sea and the Arctic Ocean during August 1994. This experiment offered a unique opportunity to record the large $\left(8000 \mathrm{in}^{3}\right)$ airgun blasts onshore and to obtain information relating to the deep crustal structure. Stations were set up at Provideniya, New Chaplino, and Lavrentia, and records were collected from stations at Gambell (St. Lawrence Island) and at Tin City (northwest of Nome). We hope the records will permit the modeling of the lower crust in the area when combined with the MCS data collected by the R.V. Ewingand the onshore data from Alaska collected by the USGS.

The combined data should allow us to make much better interpretations of the geologic connections across the Bering Straight region, and it should allow us to tie the geology of this region of Alaska with Russia. Although the experiment was set up as part of the NATO Linkage Grant project, it was too expensive for this grant alone, and additional funds were obtained from other sources listed below.

The paleomagnetic studies included joint sampling of the Okhotsk-Chukotka volcanic belt of the Late Cretaceous age, and involved the sharing of samples collected earlier by our Russian collaborators. The bulk of these latter samples came from exposures of the OkhotskChukotka volcanic belt near Lake Elgygytgyn, an ancient impact structure thought to be about 4 million years old.

The remainder of the samples are from the Omolon district; we are currently working on them in Fairbanks and Magadan paleomagnetic laboratories. These samples are of Paleozoic age, an age critical to the understanding of the tectonic history of the area.

As part of the Linkage Grant package, we brought the deputy director of NEISRI (L. Izmailov) and the head of their paleomagnetic laboratory (T. Linkova) to Fairbanks to show them our facilities, to discuss our research, and to establish new memoranda of understanding.

Funded by NSF Grant OPP9224029, NATO Grant 930919 and Incorporated Research Institutes for Seismology Grant IRIS-0159; and by ARCO Alaska Inc., Exxon Exploration Co. and Royal Dutch Shell: Scientific personnel-D.B. Stone, S. Crumley and B. Kennedy; K. Fujita and K. Mackey (Michigan State University) 


\section{Seismology}
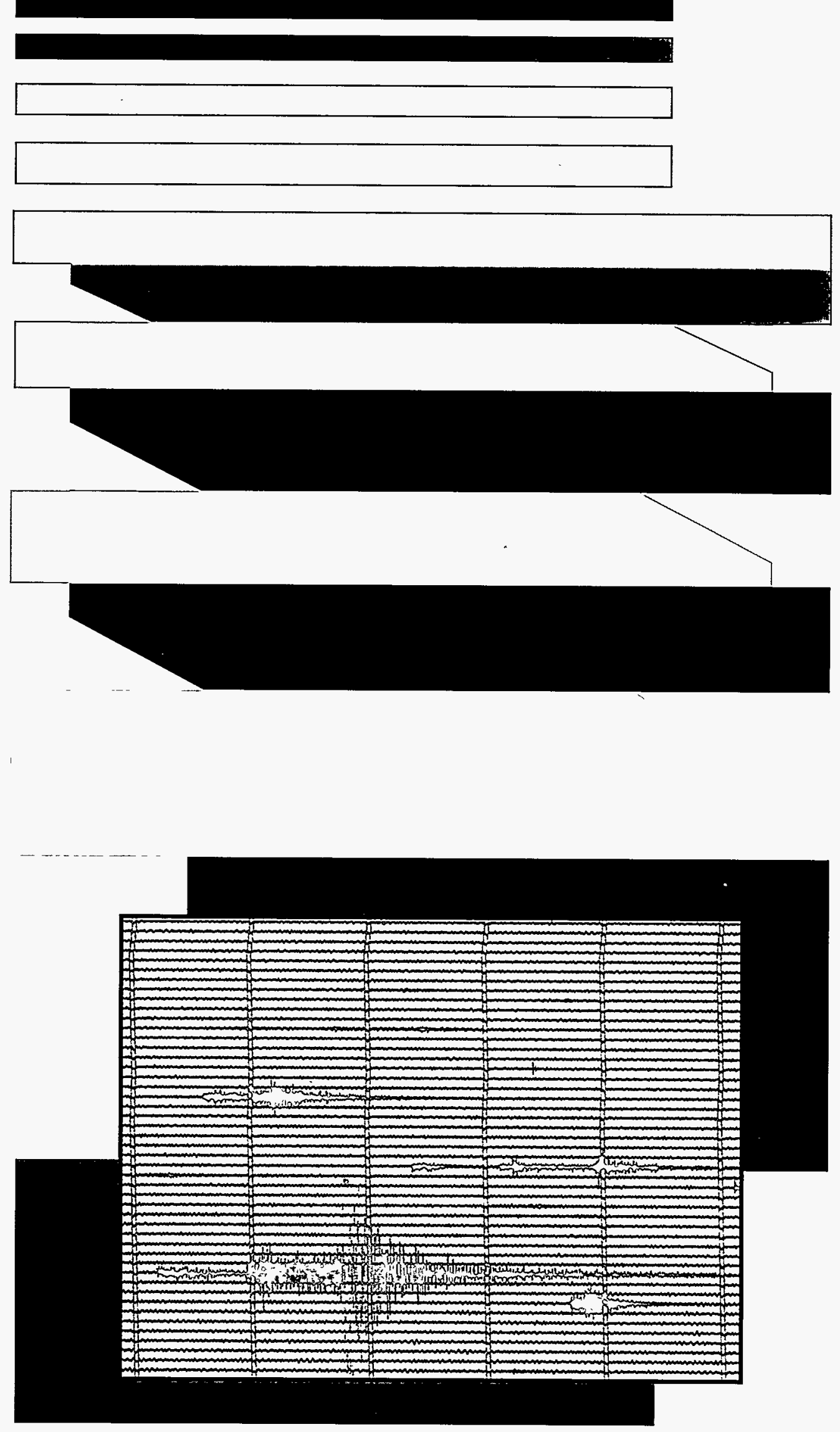


\section{SEISMOLOGY}

The seismology group consists of about 20 professors, researchers, and staff members of the Geophysical Institute (GI) and the U.S. Geological Survey (USGS), and approximately a dozen students. To monitor the seismicity of Alaska, we operate a seismograph network with about 150 short-period stations, of which 20 have three components. Currently, 11 high-dynamic-range, broadband seismographs are operating in Alaska. Clusters of seismographs monitor the four active volcanoes nearest Anchorage. Close collaboration between GI and USGS nersonnel in earthquake and volcano monitoring and research is the foundation of our vigorous program. The Alaska Earthquake Information Center (AEIC) analyzes seismic data and distributes earthquake catalogs, phase data and information on earthquake hazard. Approximately 5000 earthquakes are located, and a terabite of data is archived each year. Roger Hansen has joined the group as Alaska's State Seismologist.

The seismology group is involved in a number of active research projects, which include: monitoring earthquakes in the Shumagin seismic gap, where an increase in seismic moment release rate can be interpreted as the prelude to a larger rupture; analyzing the relationship of seismicity to volcanic eruptions; mapping the properties of the crust, the upper mantle of the earth, and the stress field; refining the technique of predicting earthquakes based on seismic quiescence; mitigating earthquake hazards by microzonation of the expected strong ground motions; cataloging and understanding the characteristics of seismicity in Alaska; compiling information on historic earthquake intensities and constructing an authoritative seismic zoning map; improving instrumentation and processing strategies that will record and perform near-real-time analyses of volcanic seismicity; creating models for the geometry and moment release of earthquakes in Alaska, and constructing tectonic models to explain the distribution of seismicity, the mapped faults, the directions of slip, the orientation of stress, the moment distribution in great earthquakes, the surface deformation and the velocity structure.

The seismology group uses a variety of techniques including: array processing to aid seismic monitoring; GPS techniques to monitor volcanoes and plate motions, to model slip along faults and to study mountain building; and SAR interferometry to apply to measuring surface deformations associated with earthquakes, volcanic activity, thickness of lava flows in inaccessible areas, glacier flow and ocean ice movements. Jeff Freymueller will join the institute as a research assistant professor of geophysics to lead the study of crustal deformation.

\begin{abstract}
Estimating Stress Directions: In addition to inverting fault plane solutions from Alaska for stress directions, we have analyzed data sets from California, Iran, Italy and Hawaii. In all data sets, we are able to achieve very small mean misfits (angles of a few degrees) by subdividing spatially, even in sets of greatly varied fault plane solutions. The volumes of uniform stress directions we thus identify have dimensions of approximately ten times the dimensions of the earthquakes used
\end{abstract}

(in Hawaii and Iran these volumes have radii of a few $\mathrm{km}$ and a few hundred km, respectively). Departing from these observations, we designed a method to define segments of plate boundaries quantitatively based on mapping the misfit of individual fault plane solutions along the plate boundary. In a test of the method used for the southern San Andreas fault, we found close agreement with an independent estimate of the segmentation. In the Aleutians, we found that the segment- ation, based on stress directions, seems to correlate with intersections of fracture zones on the Pacific plate, with the presence of major asperities as estimated from great ruptures, and to a lesser degree with ends of ruptures, but not at all with morphological divisions of the island arc. We are refining our method for defining segmentation of plate boundaries. After analyzing several plate boundaries we hope to identify the cause of segmentation, and to define its influence on great ruptures. 


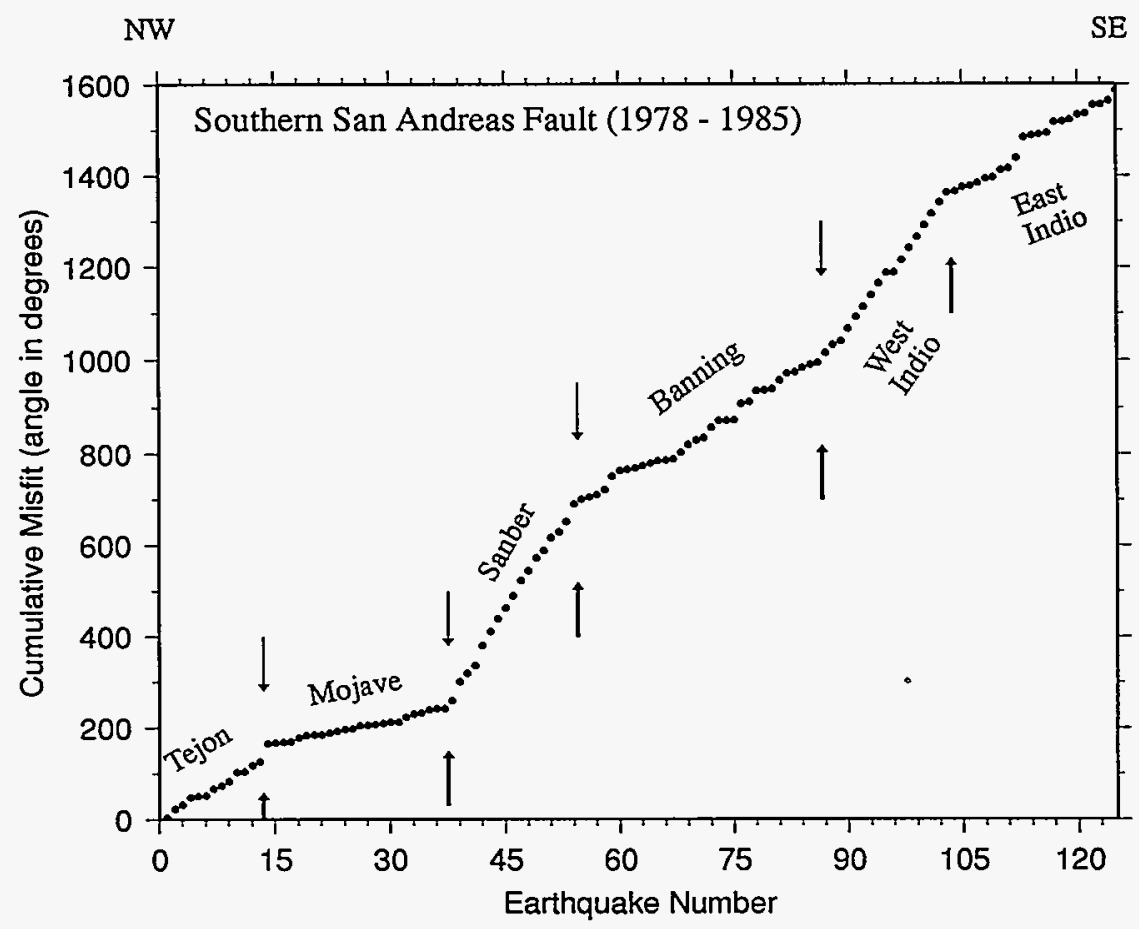

The segmentation of the southern San Andreas fault, from the Carizo planes to its terminus near Indio, can be defined based on changes in the mean misfit to an assumed stress tensor. The misfit is the angle between the shear stress (calculated from the assumed stress tensor) resolved into the earthquake fault plane, and the observed slip direction in that plane. The misfit is estimated for each earthquake with a known fault plane solution. The cumulative curve shows segments of constant slope that differ significantly from the slopes in other segments. This means that within segments the stress directions are uniform, but different from that in other segments. The abscissa shows distance along the fault in a variable scale because the units are earthquake sequential numbers. Our quantitative segmentation (arrows up) compares favorably with the subjectively chosen subdivisions based on geomorphologic evidence (arrows down).

We believe that in Hawaii we detected a rotation of the stress tensor directions associated with magma intrusions in the crust. Strain directions also change with time. A comparison of stress and strain directions (strain released by the earthquakes) showed that the two are often not parallel. The greatest principal stress is usually oriented at a high angle to the major fault planes, which means that these planes are oriented unfavorably for failure, i.e. they are weak. The estimate of the strain directions depends strongly on the largest earthquake in the data set, which can only occur on the weak major fault. Therefore, strong variations in strain direc- ticle motion in the NNE-SSW direction, which is different from that expected based on the fault plane orientations. The earthquake sources producing the signals were located at approximately $80 \mathrm{~km}$ depth. This source-station geometry and the results are similar to those in an experiment in South America, where the anisotropy was attributed to mantle flow in the wedge above the subduction zone. We plan to analyze signals from more sources at different depths and recorded at different stations before we construct a model for the observed anisotropy in Alaska.

\section{Scientific personnel-M. Wyss and} G. Tytgat

The Use of SAR Interferometry for Volcano Monitoring: The remote sensing and seismology groups are conducting a pilot program to measure crustal deformations and changes at Alaska volcanoes.

Substantial differences in elevation brought about by lava flows or explosive eruptions can clearly be measured by SAR-image interferometry. The question is can elevation changes with amplitudes of several $\mathrm{cm}$ be resolved.

These deformations can precede and accompany eruptions. The targets in this pilot study are Katmai, where some GPS ground truth data exist, and Westdahl, where a lava flow was extruded.

Scientificpersonnel-K.G. Dean, R. Fatland, S. Li, Z. Lu, and M. Wyss

Seismic Quiescence as an Earthquake Prediction Tool: We improved the tools for analyzing seismicity patterns and the reporting history of earthquake catalogs. The 
program Z-MAP will be part of the IASPEI software package. With it, seismicity rate changes, as well as focal depth changes, can be mapped as a function of space and time.

In addition, earthquake catalogs can be declustered, and detection threshold changes and magnitude shifts can be identified and modeled. The very significant seismic quiescences before the Landers (M7.3) and Big Bear (M6.5) earthquakes of 1992 have now been mapped in detail in time and space. The Irpinia, Italy (M6.9), earthquake also was preceded by a seismic quiescence, which we documented together with one false alarm.

Currently, we are developing an algorithm with which we will test the feasibility of predicting earthquakes based on seismic quiescence.

U.S. Department of Interior Grant 1434-92-G-2234: Scientific personnel- $M$. Wyss and $S$. Wiemer

\section{Relationship Between JHD Station} Corrections and Velocity Variations in the Cook Inlet Area, Southcentral Alaska: The pattern of JHD (Joint Hypocenter Determination method) station corrections computed from clustered events can be used to make inferences about the presence of velociry variations under local seismic networks. We used the onser times of $\mathrm{P}$-waves and $\mathrm{S}$-waves from 1,931 events with epicenters between 146 degrees and 157 degrees and 58 degrees and 62 degrees $\mathrm{N}$, recorded from June 1988 to December 1993.

The events satisfy the following criteria: focal depths greater than 40 $\mathrm{km}$; number of $\mathrm{P}$-wave arrivals 10 and greater; number of $S$-wave arrivals 5 and greater; azimuthal gap between stations less than 170 degrees; magnitude 2.5 and greater; and rms residual less than $0.5 \mathrm{sec}$.

The events were subdivided into subsets depending on the focal depth and geographic location of the hypocenters and relocated with the JHD method. As a result, P-wave and $S$-wave station corrections for 75 stations located in and around the Cook Inlet area were computed for the different subsets of events. The station corrections are highly dependent on the subset of events used. The station corrections span a very wide range ( $-2.2 \mathrm{sec}$ to $2.6 \mathrm{sec}$ ), which indicates the presence of large lateral velocity variations.

Regardless of the subset of events used, the large positive corrections are associated with the Cook Inlet sedimentary basin, while the nega-

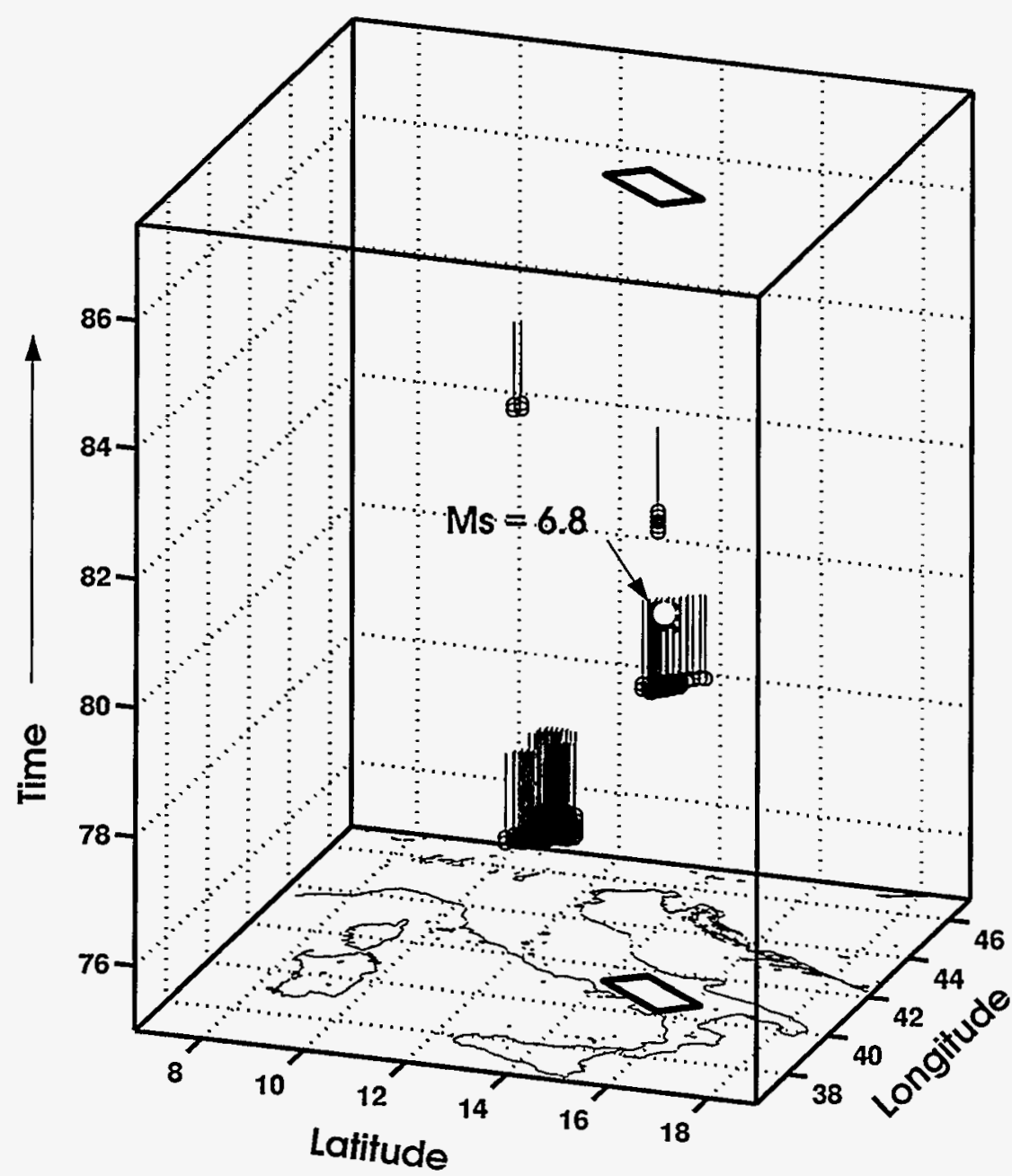

In this crystal cube for earthquake prediction, we show the map of ltaly at the bottom ( $x$ and $y$ are longitude and latitude, respectively), with the source area of the 1980 Irpinia, M6.9, earthquake outlined by a polygon. Time progresses from the bottom to the top vertically ( $z$-axis is in calendaryears). Alarms for possible earthquake occurrence, based on the detection of seismic quiescence, are indicated by vertical lines with a circle at their beginning. The alarm status is evaluated by algorithm for every month and every 0.1 degree latitude and longitude. The occurrence of the Irpinia earthquake is marked by the arrow. The Irpinia source area contains one of the two alarms, which is interpreted as a precursor. The earlier alarm, further north in Italy, was a false alarm; it was not followed by a major earthquake. 
tive station corrections tend to correlate with ray paths through the high-velocity Pacific Plate subducting beneath southcentral Alaska.

Alaska Energy Authority Grant 0850006: Scientific personnel-N.N. Biswas and N. Ratchkovsky; J. Pujol (University of Memphis)

\section{Investigation of Strong Ground} Motion in the Anchorage Area: A systematic study of strong motion in the Anchorage area has been initiated. The scope of the study includes: (a) the compilation of the available geotechnical information; (b) determination of the ground amplification factor in the frequency range of 0.1 to $10 \mathrm{~Hz}$ for geologically typical sites; and (c) characteristics of strong ground motions for the maximum magnitude interplate and intraplate earthquakes. The results of the study will be used in developing seismic zonation for the study area.

In order to achieve the above goals, a digital strong motion network comprising 15 stations has been installed in the Anchorage area. The network trigger level is set for magnitudes equal to and greater than 3 . For the determination of the site amplification factor, onsite measurement has been divided into two sets: the first one consisted of microtremor measurement at about 80 sites to cover the frequency range from 0.1 to 1 $\mathrm{Hz}$; the second one consisted of weak ground motion measurement for local sources to cover the frequency range from 1 to $10 \mathrm{~Hz}$. For this part, we operated 19 IRIS-PASSCAL stations for two months in the Anchorage area. We are now processing the data obtained from these measurements.
ASTF Grant 91-2-125: Scientific personnel-N.N.Biswas, M. Succarieh and Y. Chen; A. Phuakan (University of Alaska Anchorage); $M$. Dravinsky (University of Southern California, LA); A. Papageorgieu (Rensselaer Polytechnic Institute, Troy); C. Saikia (Woodward Clyde Consultants, Pasadena)

Determination of Site Amplification Factor by Coda Method in the Cook Inlet Area: Seismograms of $S$-wave coda recorded by the seismic stations of the Alaska regional network in the Cook Inlet area in southcentral Alaska were analyzed to determine site factor in the frequency range of 2 to $16 \mathrm{~Hz}$. The network covers an area between latitudes 58 degrees $N$ and 62 degrees $N$ and longitudes 146 degrees $W$ and 156 degrees W. The local earthquakes selected are in the magnitude range of 3.0 and 4.5. In order to eliminate the source and path effecrs, the coda amplitudes as a function of frequency were normalized by the network average amplitude values.

The resultant values of the site factor obtained for a given site were then compared with those obtained for other sites. The four active volcanoes (Augustine, Spurr, Iliamna, and Redoubt) on the west side of Cook Inlet show the following changes in the values of site factors: for sites on Augustine and Iliamna volcanoes, the values increase uniformly from 1.9 and 0.5 at $2 \mathrm{~Hz}$ to 4.2 and 0.9 , respectively, as $16 \mathrm{~Hz}$; for Spurr, the values decrease from 0.53 at $2 \mathrm{~Hz}$ to 0.19 at $16 \mathrm{~Hz}$; for Redoubt a peak value of 1.14 occurs at $8 \mathrm{~Hz}$ and it decreases to 0.85 and 0.63 at $2 \mathrm{~Hz}$ and $16 \mathrm{~Hz}$, respectively. For hill sites which are mostly composed of hard rocks, a peak value of 0.38 occurs at $10 \mathrm{~Hz}$, which decreases to 0.31 and 0.26 at $2 \mathrm{~Hz}$ and $16 \mathrm{~Hz}$, respectively. Sites underlain by thick sedimentary formations of the Cook Inlet basin show large variations in the average value of the site factor. The values vary from 5.03 at $2 \mathrm{~Hz}$ to 1.69 at 16 $\mathrm{Hz}$. The average values for the sires located along the edge of the Cook Inlet basin show a low of 0.49 at 12 $\mathrm{Hz}$, which increases to 0.85 and 0.54 at $2 \mathrm{~Hz}$ and $16 \mathrm{~Hz}$, respectively.

We interpret the above results as highly dependent on the site lithology-the hard rock sites show relatively smaller variation of the site factor compared to those located on softer formations.

ASTF Grant 91-2-125: Scientific personnel-N.N. Biswas and Y. Chen

The Rupture Process of Large Earthquakes in the Alaska/Aleutian Subduction Zone: The interplate coupled zone is made up of weak regions that tend to slip aseismically, and strong regions (asperities) that store the majority of stress accumulating from plate motions. By using teleseismic body waves to study the rupture process of earthquakes, it is possible to map the moment release that occurs during an event and to relate this moment history to the distribution of asperities in the faulted region. Asperities are important because it is precisely the breaking of these strongly coupled regions that produces large destructive earthquakes.

Many of the world's largest earthquakes have occurred in the Alaskal Aleutian subduction zone. Research is under way to determine the asper- 
ity distributions of some of these events. The long-term goal is to develop an asperity distribution map of the North Pacific subduction zones. Such regions tend to accumulate stress and may be the source of future large earthquakes in the region.

Internal funds: Scientific personnel-D.H. Christensen

\section{Source Characteristics of Historic} Alaska Earthquakes: Although occurring infrequently, large earthquakes produce the most damage and pose the greatest risk to life and property. Because they occur so infrequently, an important part of earthquake research has been to study major and large historic events in as much detail as possible. Examples of historic earthquake studies that led to predictions occurred in the Parkfield, California, and Kaoiki, Hawaii. Most recently, the Loma Prieta, California, earthquake has renewed interest in the detailed analysis of the earlier 1906 San Francisco earthquake. Through understanding historic seismicity, we are better able to devise physical models of the rupture process and to determine the most likely occurrence of future events.

Alaska has a rich history of large earthquakes, which only now are being studied fully. In addition to many large and great earthquakes located in the interplate regions of Alaska, large intraplate earthquakes in Alaska occur at fairly regular intervals. At least 10 large intraplate events with $M>7$, and a total of at least 21 events with $M>6.2$, occurred during this century. Many of these events occurred in the vicinity of Fairbanks and Anchorage (the two largest cities in Alaska) and are important for the earthquake risk assessment of Alaska. The significance of historic seismicity is obvious when one considers the overall infrequent occurrence of large earchquakes in any one region. At best, we can expect only one or two cycles to occur in any one century. Given the rather short history of seismic observation throughout the world, it is important that we make the best possible use of the resource.

Efforts are currently under way to study the historic earthquake record of Alaska thoroughly to determine long-term seismicity patterns and the detailed rupture dynamics of individual large earthquakes.

Department of Interior Grant 1434 92-G-2221: Scientific personnelD.H. Christensen and H. Fletcher

Crustal and Upper Mantle Velocity Structure in Alaska: Knowing the velocity structure of the crust and upper mantle provides constraints on geologic interpretation, which has strong implications for the tectonic evolution of a region. Determining the velocity structure can be accomplished through a variety of means. In addition to the classical methods of refraction and reflection surveys, the crustal and upper mantle velocity structure also can be determined through passive methods. These techniques range from the simple interpretation of local earthquake travel times at network stations to the multistation inversion of teleseismic and local arrivals for three-dimensional variations in velocity structure (tomography). The technique of receiver function inversion requires as little as one high-quality three-component seismic station to determine the crustal thickness and the velocity structure of the crust and upper mantle beneath the station (although the resolution is grearly increased by summing waveform information for many events). S-wave splitting studies to determine anisotropy in the crust and upper mantle also can be performed using the same data set. Stations can be deployed for a short period (one to six months) depending on the availability of teleseismic activity, and thus provide a relatively inexpensive way to determine local crustal and upper mantle structure. Because this method uses seismic waves from earthquake sources, explosions are not required and information can be gathered anywhere that an appropriate station can be deployed for the necessary period.

We also are involved in a project to determine the crustal and upper mantle velocity structure at several points along the Trans-Alaska Crustal Transect (TACT) profile across Alaska using the method of teleseismic receiver function inversion. In this way, we hope to further constrain the deeper structure and to contrast the results from this study with the results from the reflection/ refraction profile. Principle stress directions which may be related to anisotropy also can be determined from this data using results from $S$ wave splitting studies.

We have gathered intermediate and broad-band data from eight sites along the TACT profile across Alaska. Four temporary stations were deployed in the Brooks Range during the summer of 1990 .

These stations were deployed in southcentral Alaska, near Glennallen, during the fall of 1990 , then 
removed in the fall of 1991 . The data are being analyzed.

NSF Grant EAR 90-04112 Scientificpersonnel-D.H. Christensen and C. Searcy; G. Zandt (Lawrence Livermore National Laboratory); and M. Savage (University of Nevada Reno)

Tomographic Studies of Seismic Velocity Structure in Alaska: During the next two years, we plan to use information available from the Alaska Earthquake Information Center (AEIC) to produce a series of tomographic images of the velocity strucrure under Alaska at several scales.

Our first goal will be to determine the gross features of the seismically acrive and most densely instrumented area of Alaska using the existing digital data set. As the project unfolds, we also will be able to increase coverage and quality of the data set in important areas either by accessing older predigital data or by deploying temporary arrays into such regions.

Following an initial study, additional efforts will be aimed at detailed studies of smaller regions with tighter station coverage (of individual volcanoes, the Cook Inlet region, etc.) and at larger scale teleseismic studies aimed at refining the deeper structure under Alaska.

A two-year NSF grant and a grant from the Alaska Volcano Observatory (AVO; Department of the Interior) awarded to D.H. Christensen and D. Zhao will support the initial steps of this project.
The inversion should allow us to distinguish details of the subducting slab and those present in the upper mantle, and to observe crustal velocity contrasts. Teleseismic arrival times will be used for large-scale inversions that will emphasize the deeper structure beneath much of Alaska, including the aseismic extension of the subducting plate. Smaller scale studies will be conducted in regions more densely covered by stations and seismicity, such as individual volcanoes in Cook Inlet, to determine a more detailed picture of the plumbing system within these active volcanoes.

USGS Grant 14-08-0001-A0573 and NSF Grant EAR 91-18090: Scientific personnel-D.H. Christensen, H.Pulpan, C. Searcy, J. Clippard, R. Lorentzen; and D. Zhao (California Institute of Technology)

Northeast Russia Seismic Studies: The main purpose of these studies is to better define the tectonic boundarybetween the North American and Eurasian plates, and between them and the Pacific plate. The active plate boundary north of Siberia in the Arctic Ocean is a well-defined oceanic spreading center. Its tectonic style and location is less well known where it comes on-shore and passes the Euler pole representing the opening of the Arctic and Atlantic oceans.

Under the sponsorship of the Incorporated Research Institutes for Seismology (IRIS), we have been operating digitally recording seismometers in northeastern Russia since 1991 and reanalyzing analog records from the Russian networks.

Under this program, we installed digital recorders on Russian seismometers in the Sakha Republic (Yakutsk and Batagai, 1991, Tiksi, 1992-93) and in 1993 expanded into the Magadan region (Stekol'nyi). In 1994, we installed four temporary stations along the Chukotka Peninsula to record local events and the air-gun being used for a marine crustal profile by the R. V. Ewing.

Using a combination of older analog records from northeastern Russia, the new digital data and evidence of recent ground motion from studies of the surficial geology, we have put together a new plate boundary map/model connecting the North America, Eurasia and Pacific plates.

This model requires that the Sea of Okhotsk region be a separate plate, currently being pushed our over the Pacific Plate along the KurileKamchatka subduction zone. Using the analog records, we also have constructed a first-order crustal thickness map, which generally agrees with our current models of the tectonic situation.

Funded by NSF Grant OPP9224029, NATO Grant 930919 and Incorporated Research Institutes for Seismology Grant IRIS-0159; and by ARCO Alaska Inc., Exxon Exploration Co. and Royal Dutch Shell: Scientific personnel-D.B. Stone, S. Crumley and B. Kennedy; K. Fujita and K. Mackey (Michigan State University) 


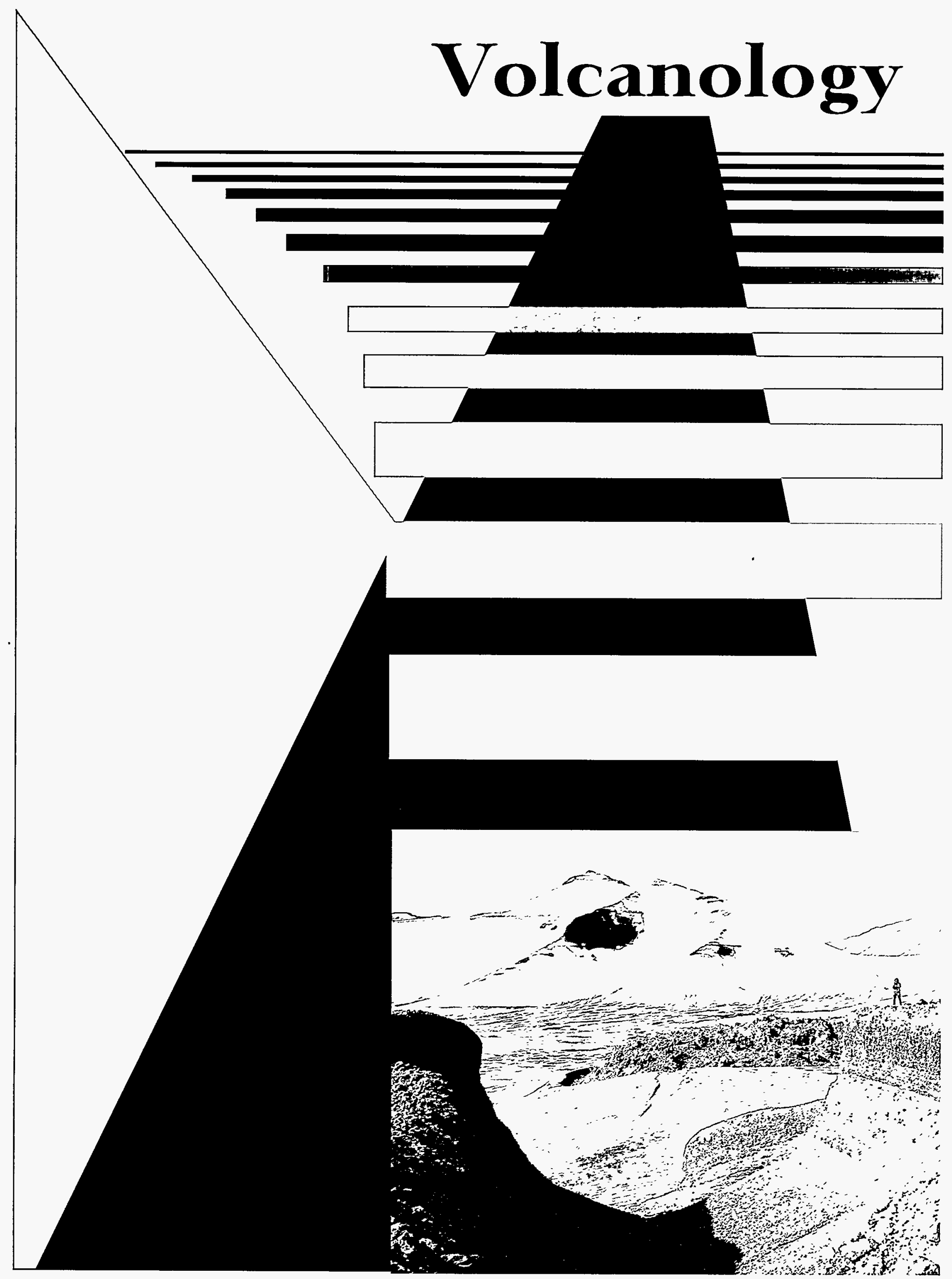




\section{VOLCANOLOGY}

The Volcanology Group seeks to understand the physical and chemical processes that comprise active volcanism. Although our interest begins with the origin of magmas at mantle depths, our focus is to use the dynamic experiments provided by the frequent eruptions in Alaska's Aleutian Arc to elucidate the behavior of magmas as they approach and erupt at Earth's surface.

Volcanology is a strongly multidisciplinary scientific inquiry, encompassing areas of geophysics, geochemistry, and geology. The Volcanology Group was recently formalized as a separate entity, but has long existed as part of the Solid Earth Group at the institute. The relationship of Volcanology to the Seismology Group and the Tectonics and Sedimentation Group remains close. We also have important ties with the Atmospheric Sciences, Remote Sensing, and the Snow, Ice, and Permafrost groups.

Volcanism is among the most spectacular and devastating natural phenomena to occur on the planet. The conditions that exist in erupting systems are the most extreme in Earth's accessible environment. Volcanism is important because it disrupts human activity, sometimes lethally, because volcanic systems contain a vast potential source of energy, and because such magmatism represents the continued chemical evolution of the planet. A part of this evolution is the upward migration and emplacement of low-melting material (new or recycled additions) to the crust. In explosive events, some of this material is fragmented to form ash and associated gases, and is widely dispersed. Very large explosive eruptions, such as the recent Pinatubo event (Philippines, 1991) or the even larger Katmai event (Alaska, 1912), may actually alter worldwide weather patterns. Therefore, explosive volcanism has an environmental impact that goes beyond the immediate disaster and is a key to the character and history of Earth and its surface environment. In the U.S., explosive volcanism is first an Alaskan problem.

Despite its importance, volcanism is poorly understood. Generally, volcanoes erupt because magma is buoyant within the crust and contains dissolved gases which are rapidly released as magma reaches shallow depth. In detail, however, there are major areas of ignorance. These areas include: the mechanisms of melt generation and subsequent fractionation; the regions and conditions of melt storage in the crust; the trigger for final rise to the surface and the cause of cessation of that rise; and the controls that lead to explosive or effusive (nonexplosive) behavior.

The core of our work in volcanology at the institute is the Alaska Volcano Observatory. This effort-collaborative with the U.S. Geological Survey and the Alaska Division of Geological and Geophysical Surveys-is an exciting and rewarding, monitoring-intensive activity, which calls on researchers to apply their knowledge in practical ways and introduces students to science in direct service to society. Around this core are many other efforts, including basic volcano seismology research, drilling exploration of active volcanic systems, reconstruction of the history of volcanism in Alaska, and investigation of processes of magmatic evolution and eruption. At the institute, we are privileged to live in an active part of our planet, where volcanology presents intense physical and intellectual challenges. Our primary intellectual challenge is to combine the seismological observation of active volcanoes with the petrologic investigation of their eruption products to provide new and valuable insights into the magmatic environment.

\section{Monitoring and Hazard Mitigation}

Alaska Volcano Observatory: The Alaska Volcano Observatory (AVO) is one of three observatories operated under the U.S. Geological Survey's (USGS) Volcano Hazard Program. AVO is responsible for monitoring and alerting businesses and commu- nities about eruptions in the Aleutian Arc, home of the most active explosive volcanoes in the nation. Although the primary hazard of an eruption is to aircraft over the heavily traveled North Pacific airspace, there is the continued potential for dam- age to transportation and energy facilities and to the interruption of everyday life in Alaska communities.

AVO is a cooperative effort of the USGS, the UAF Geophysical Institute, and the Alaska Division of Geological and Geophysical Surveys 
(ADGGS). The primary facilities of AVO are at the USGS in Anchorage, at the Geophysical Institute in Fairbanks, and at the USGS in Menlo Park, California.

Seismology is the largest component of AVO work at the Geophysical Institute, but significant investigations are under way in igneous and hydrothermal geochemistry, eruption mechanisms, volcano hazards (rephrachronology and tsunami modeling) and remote sensing.

Since its formation in 1988 following the eruption of St. Augustine Volcano, AVO has responded to a seismic crisis at Mount Dutton, to the Redoubt eruptions of 1989-90, and to the violent multiple eruptions of Mount Spurr in 1992.

Although efforts are now mostly restricted to the Cook Inlet region, AVO intends to extend its coverage to active volcanoes on the Alaska Peninsula and Aleutian Islands in the interest of air safety. Elements of the AVO effort are reported separately in this section of the biennial report.

USGS Grant 14-08-0001A0574: Scientific personnel-J.C. Eichelberger, D.H. Christensen, K.G. Dean, J. Kienle, S.R. McNutt, C.J. Nye, S.E. Swanson, and J.E. Begét

AVO Coordination: The coordinating scientist for AVO provides leadership for the observatory's efforts in Fairbanks, and he reports to the scientist-in-charge at the USGS in Anchorage. The coordinating scientist is also responsible for producing AVO's bimonthly report.

USGS Grant 14-08-0001-A0574: Scientificpersonnel-J.C. Eichelberger

\section{Volcano Seismology} Following the successful response to the 1992 eruptions of Mount Spurr, research efforts in 1993 and 1994 focused on gaining physical insight into the eruptive processes and comparing Mount Spurr's activity to that of other volcanoes worldwide.

We have compiled an electronic database of over 600 earthquake swarms at volcanoes from around the world. The database reveals that the average volcanic swarm lasts
Volcano Seismology of Mount Spurr: about a week. The ten-month swarm of Mount Spurr, thus, was much longer than average, and the swarm may reflect slow movement of magma or a source at greater depths.

We have revised the velocity model used to locate earthquakes at Mount Spurr, and we have revised the models used for Mount Redoubt, Mount St. Augustine, and Katmai volcanoes.

Based on the new model, Mount Spurr appears to have more deep

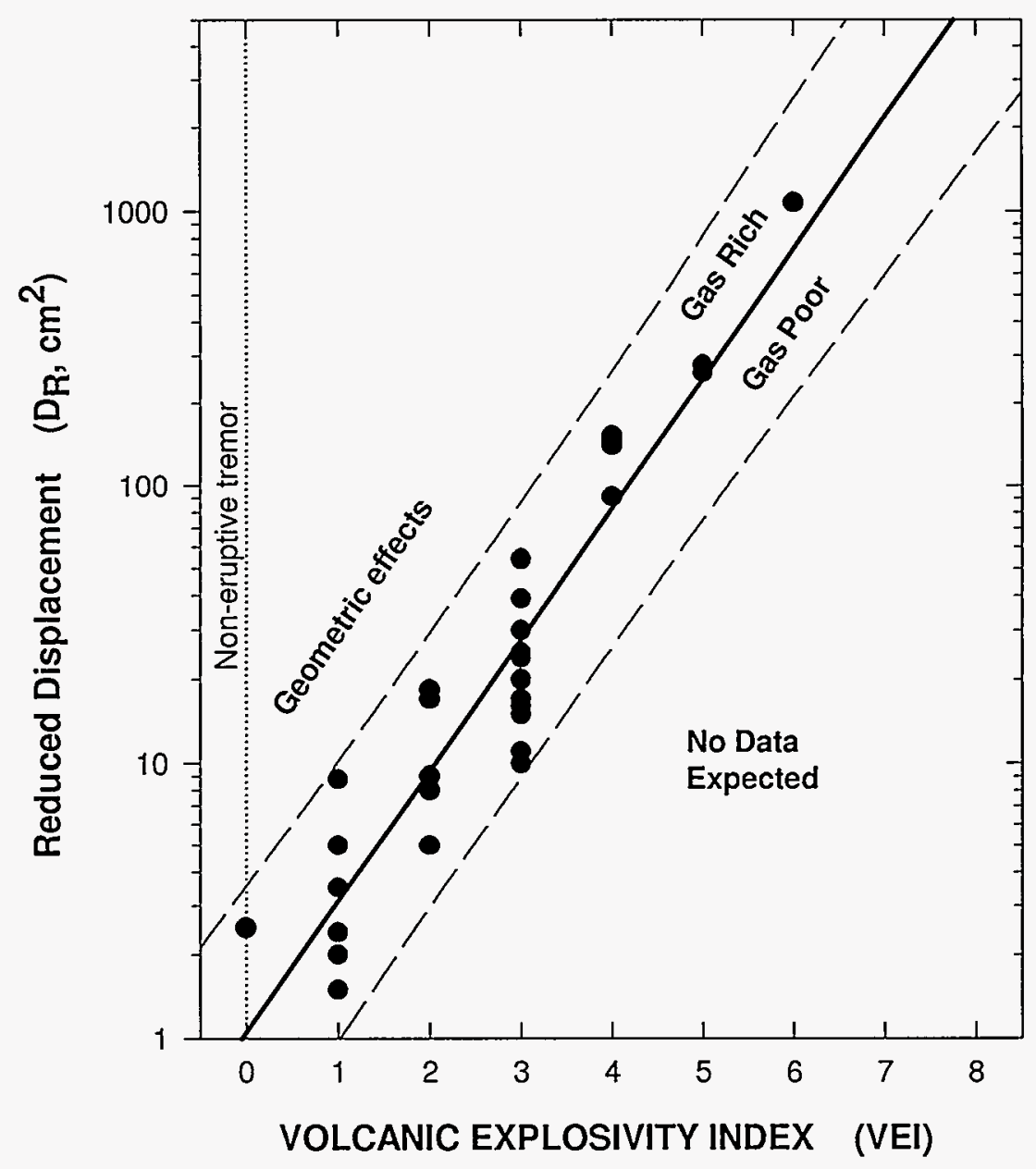

Shown is volcanic tremor reduced displacement versus the Volcanic Explosivity Index of Newhall and Self (1982). The plots show that bigger eruptions have stronger tremor. The plotted line is a linear regression fit to the data. The correlation coefficient, $r$, is 0.93 . 
earthquakes ( 20 to $40 \mathrm{~km}$ ) than the other volcanoes, which may well reflect different properties of the crust or differences in the magma plumbing system.

We compared the amplitude of Mount Spurr's volcanic tremor, a continuous signal produced during eruptions, with that of several other volcanoes.

Mount Spurr's tremor is comparable to that observed elsewhere for eruptions of the same size (Volcano Explosivity Index) after normalizing the amplitude to reduced displacement $\left(D_{R}\right)$, which is the root-meansquare amplitude multiplied by the distance.

The normalized amplitude is roughly proportional to the kinetic energy of the eruptions as shown in the figure on the previous page.

In general, we have found eruptions that are more gas rich produce stronger tremor than those that are gas poor, and geometric effects, such as the shape of the conduit, also can change the tremor amplitude for a given eruption size.

Thus, using seismic data, we are able to measure the size of an eruption at the time it is occurring. Such information is very useful to mitigate volcanic hazards, particularly those pertaining to aviation.

USGS Grant 14-08-0001-A0574: Scientific personnel-S.R. McNutt, G. Tytgat, J. Benoit, A. Jolly, S. Estes and L. Rao

Seismic Tomography of Redoubt Volcano: Mount Redoubt is one of several active volcanoes located along the western margin of Cook Inlet. Less than $200 \mathrm{~km}$ from Anchorage, it last erupted in 1989-90.
The associated rapidly rising ash plume caused the engines of a large jet aircraft to shut down temporarily, nearly resulting in a major disaster. This near accident indicates the need for careful monitoring of these active volcanoes.

The space-time behavior of seismic activity inside a volcanic edifice, probably associated with magma movement or pressure-induced fracturing, can be useful in assessing whether or not a major eruption is imminent.

In this project, we set out to investigate the three-dimensional seismic velocity structure of the volcano to obtain more accurate hypocenter locations of seismic events and to obtain some information on the interior geological features.

Travel time residuals of 1480 local earthquakes and three man-made explosions were inverted to derive three-dimensional velocity adjustments of an initially horizontally layered model.

The derived velocity model, with block sizes of $1.6 \times 1.6 \times .5 \mathrm{~km}^{3}$, shows a core of high $\mathrm{P}$-wave velocity and low $S$-wave velocity anomalies.

This core extends from two kilometers above sea level $(1 \mathrm{~km}$ below the peak) to one kilometer below sea level. The high vp/vs ratio indicates the presence of partial-melt or water-saturated rocks there.

USGS Grant 14-08-0001-A0574 and State of Alaska Funds: Scientific personnel-D.H. Christensen, $H$. Pulpan, and R. Lorentzen

Seismic Tomography of Mount St. Augustine Volcano: The present central dome complex of Mount St. Augustine volcano was largely formed by repeated silicic dome intrusions following a major edifice collapse in 1883 .

After the collapse, four eruptions ending with dome formation regenerated the former edifice to more than its original height. These eruptions occurred in 1933, 1963-1964, 1976, and most recently, in 1986.

Understanding the internal structure of the dome complex is important when assessing its stability. A future large-scale collapse, such as the one that occurred in 1883 , could spawn a major landslide and a tsunami, which could have a potentially catastrophic impact on several lower Cook Inlet coastal communities.

We propose to conduct an active seismic experiment in the summer of 1996 to study the detailed internal velocity structure of the volcano.

The deeper parts of the dome complex most likely are still molten and above the Curne isotherm, meaning they are probably not magnetized.

Based on seismological research on Showa-shinzan in Hokkaido, Japan, we also expect to find large velocity contrasts between the fast, molten elements of the Augustine dome complex and the slower elements that have developed significant fracture porosity by forming columnar joints.

The goal of this research is to resolve the fine-scale velocity and the magnetic structure of the dome complex.

NSF Grant EAR94-05471 with logistic support from USGS Grant 14-08-0001-A0574: Scientific personnel-J. Kienle, D.H. Christensen, J. Clippard, and S. Crumley 


\section{Geochemistry and Petrology}

Petrology of Some Recent Eruptions in the Aleutian Arc, Alaska: In the past three years, confirmed eruptive activity occurred at eight volcanoes along the Aleutian arc. Most of the eruptions occurred in remote areas making sampling of eruptive material difficult. We describe products from three recently active volcanoes, Mount Spurr, Bogoslof, and Kanaga, from which we were able to obtain samples.

Mount Spurr (in the eastern Aleutian arc) and Kanaga (in the western arc) both lie directly on the volcanic front while Bogoslof (in the central arc) lies about $50 \mathrm{~km}$ behind the main volcanic front. As with most andesitic arcs, the eruption products are crystalrich and eruptive style varies greatly and seemingly independently of magma chemistry. The 1992 Bogosloferuption was basaltic, about $51 \mathrm{wt}$ percent $\mathrm{SiO}_{2}$; it produced a silicic-lava-like dome at sea level. Trace element chemistry shows that although basaltic, Bogoslof lava is evolved and enriched in some elements, e.g. Rb, consistent with its back-arc nature. The 1992 Mount Spurr and 1994 Kanaga eruptions were both andesitic, about 57 and 60 wt percent $\mathrm{SiO}_{2}$, respectively.

Despite their bulk-chemical similarity, groundmass glass compositions for Mount Spurr and Kanaga are significantly different, -60 wt percent $\mathrm{SiO}_{2}$ at Mount Spurr and - 76 wt percent $\mathrm{SiO}_{2}$ at Kanaga.

The Mount Spurr eruption produced three vulcanian to subplinian tephra columns 14 - to $15-\mathrm{km}$ high accompanied by minor pyroclastic and debris flows; the Kanaga eruption was of intermittent low-level tephra emission and block lava flows.

The mineral assemblage for all three eruptions is plagioclase, clinopyroxene, and FeTi oxides; hornblende is present in Mount Spurr

Juvenile Tephra and Crustal Xenoliths from the 1992 Mount Spurr Eruptions: During the 1992 eruptions of Mount Spurr, both juvenile tephra and partially melted metamorphic rocks (buchites) were ejected. Mineral assemblages in the buchites can be used to constrain the temperature and pressure of metamorphism and melting.

The possible role of crustal melting, as represented by the buchites, in petrogenesis of Spurr magma is also being evaluated. Compositions $\stackrel{2}{\text { CHRIS NYE }}$ of both igneous and

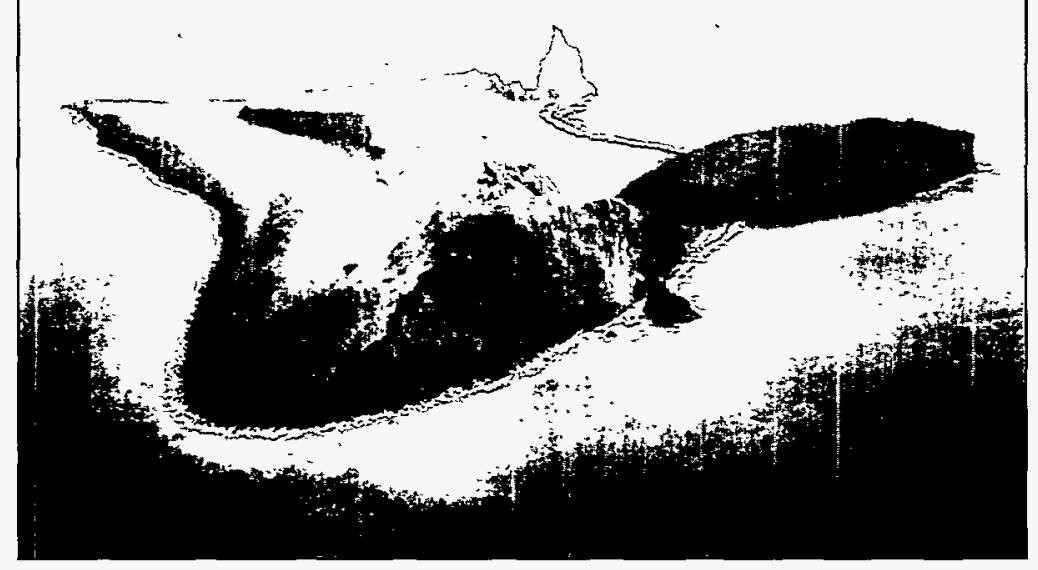

The 1992 eruption of Bogoslof Volcano, located in the Aleutian chain, produced the dome in the foreground above. Kenyon Dome (center) was extruded in 1927, and the spine (rear), Castle Rock, is a remnant of the dome extruded in 1796. were determined through electron microprobe analysis. Plagioclase, hornblende, and pyroxene analyses from the igneous rocks have provided clues into the nature of the 1992 magma. Detailed zoning profiles of the plagioclase indicate that the magma from all three eruptions was essentially and Bogoslof lava, and orthopyroxene is found in Mount Spurr and Kanaga samples. Mount Spurr proximal deposits also contain partially melted, highly inflated, felsic granulites.

Project supported by the USGS Volcano Hazards and Geothermal Studies Programs and State of Alaska funds: Scientific personnel-M.L. Harbin, C.J. Nye, J.C. Eichelberger, and S.E. Swanson identical, implying a single magmatic source. Also, the plagioclase zoning records a single episode of disequilibrium prior to the June eruption and no recharge of the system after each eruption.

The presence of hornblende phenocrysts requires that the magma contained at least $3 \mathrm{wt}$ percentage of water. Additionally, lack of reaction coronas on the hornblende indicate that the magma rose quickly. Pyrox- 
ene compositions were used to constrain magmatic temperatures, yielding an estimated range from about $850^{\circ}$ to $1150^{\circ} \mathrm{C}$.

Compositions of garnet and biotite in the buchites were used to roughly set temperatures of metamorphism, while the phase assemblage allowed tentative pressures to be established. Temperatures of $650^{\circ}$ to $700^{\circ} \mathrm{C}$ were calculated and pressures were estimated at 4 to $7 \mathrm{kbar}$ ( -12 to $20 \mathrm{~km}$ ) during metamorphism.

USGS Grant 14-08-0001A0574: Scientific personnel-S.E. Swanson, J.C. Eichelberger, and M.L. Harbin

Degassing and Crystallization Behavior of Redoubt Magma: Dense clasts in proximal 1989-90 pyroclastic deposits on the Piedmont Lobe of the Drift River Glacier represent quenched samples of extruding dome lava. As such, they provide a record of the response of magma to shallow emplacement and effusion. Crystallinity and retained volatile content $\left(\mathrm{H}_{2} \mathrm{O}, \mathrm{F}, \mathrm{Cl}\right)$ are being measured in relation to repose time since the last dome-forming event and to elapsed time since the onset of eruption.

The initial major eruptive event (15 December 1989) involved variations not simply correlative in bulk and melt composition; perhaps they arose from seismically suggested sidewall tapping of a boundary zone in the reservoir. A dacitic melt-bearing component was microlite-rich and contained reaction-rimmed hornblende. A rhyolitic melt-bearing component was microlite-free with pristine hornblende. Chemical heterogeneity declined after the second large event (2 January 1990), which was the first to destroy an observed juvenile lava dome. Crystallinity increased with time through the episode. Magmatic water in quenched coarse proximal clasts of pyroclastic flows ( $\geq 2$ January 1990) was uniformly low. If these are representative of flow material, the source domes were completely degassed when they failed 9 to 30 days after onset of growth, and little or no magmatic water was available to propel flows and loft plumes.

Chlorine concentration was variable but may have decreased somewhat with time. Samples with low $\mathrm{Cl}$ exhibit thick reaction rims on hornblende. Both characteristics may be evidence of shallow residence, and their distribution within samples indicates that each major pulse, even that comprising the final dome, involved both old conduit and new

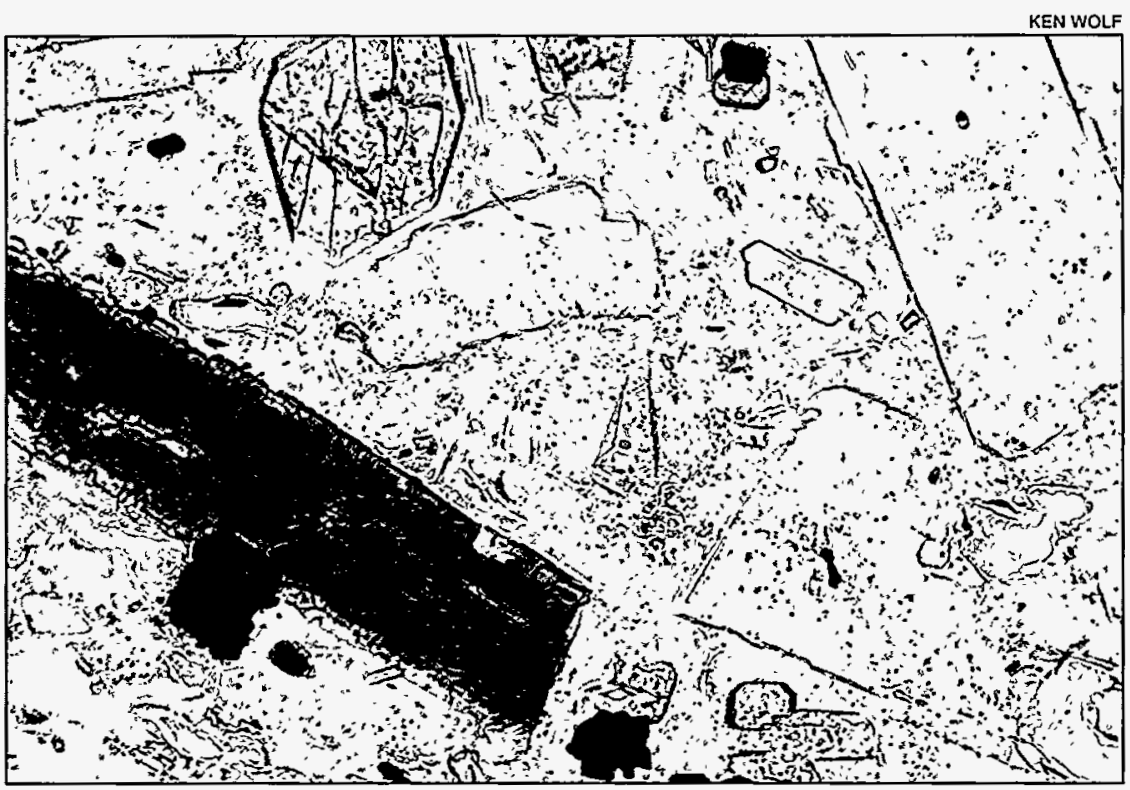

Pictured above is a photomicrograph of a typical dacite clast that contains rhyolite glass from the 1989-90 eruption of Redoubt Volcano. This sample is microlite-free, contains unrimmed hornblende, and has elevated chlorine concentrations, which are all apparent indicators of rapid ascent from the magma reservoir to the surface. Many samples throughout the eruption contain these characteristics, which indicates that the eruption consisted of a series of magma pulses that ascended prior to each of the eruptive events.

reservoir magma. Taking the simple view that conduit volume must be less than erupted volume for new magma to appear, conduit radius was less than about $10 \mathrm{~m}$. Debris shed from the vent hours before the 15 December 1989 tephra eruption included abundant dense (but with relict vesicle texture) glassy prismatically jointed blocks with reaction rim-free hornblende.

These blocks were fully degassed to $0.11 \pm 0.04$ we percent $\mathrm{H}_{2} \mathrm{O}$, a degassing perhaps recorded in intense shallow 12/13-14 seismicity. We interpret this as indicating that a magma column was rapidly established and extended essentially to the surface just prior to the onset of major tephra production.

The Redoubt eruption repeatedly crossed the boundary between explosive and effusive behavior.

To further highlight the problem of effusive eruption and controls on 
explosivity, a special session was held on the topic at the Spring AGU meeting (May 1994). The session was comprised of theoretical papers on bubble growth, degassing and magma flow, and of field-oriented

\section{Modeling}

Tsunamis from Mount St. Augustine Volcano: On 6 October 1883, a rsunami in lower Cook Inlet nearly destroyed the settlement of English Bay on the Kenai Peninsula of what is now Alaska. The source of this tsunami was St. Augustine volcano, located $85 \mathrm{~km}$ east of English Bay.

A large section of the north flank of the volcano collapsed, creating a landslide-technically, a debris avalanche-that surged into the sea and generated the tsunami.

Numerical modeling of the propagation of this tsunami illustrates that it took just less than one hour for the waves to travel across Cook Inler in to English Bay where local wave runup reached up to 6 meters in places. Fortunately, it was low tide when the tsunami occured, otherwise the settlement may have been lost.

We now have evidence from bathymetric data and geologic mapping indicating that edifice collapse of sufficient volume to reach the sea has occurred fairly frequently on Augustine Island.

We have carbon-14 dated organic material from 11 debris avalanches that traveled up to $5 \mathrm{~km}$ offshore from the island. Many, if not all, of those past debris avalanches could have generated a tsunami. presentations concerning the eruptive behavior of the volcanoes of the Cascades and the Aleutian Range.

USGS Grant 14-08-0001-A0574: Scientificpersonnel-J.C. Eichelberger, S.E. Swanson, and K. Wolf
The 11 rock avalanches analyzed are all younger than 2000 years old, suggesting that summit failure occurs at an average rate of as high as 150 to 200 years per cycle.

At present, the edifice of Mt. St. Augustine has been rebuilt by four dome-building eruptions since the last edifice collapse in 1883 . The most recent of those eruptions occurred in 1986, but no tsunami ensued at that time. Today, lower Cook Inlet is much more populated than it was in 1883.

Another tsunami generated by Mt. St. Augustine, especially a tsunami that occured at mid or high tide, could pose a serious hazard to Cook Inlet coastal communities.

A better model of tsunami generation by landslides is being developed.

Wave propagation models needed to solve the difficult problem of wave runup and wave inundation on shoaling shores are being developed also. These models use detailed bathy-

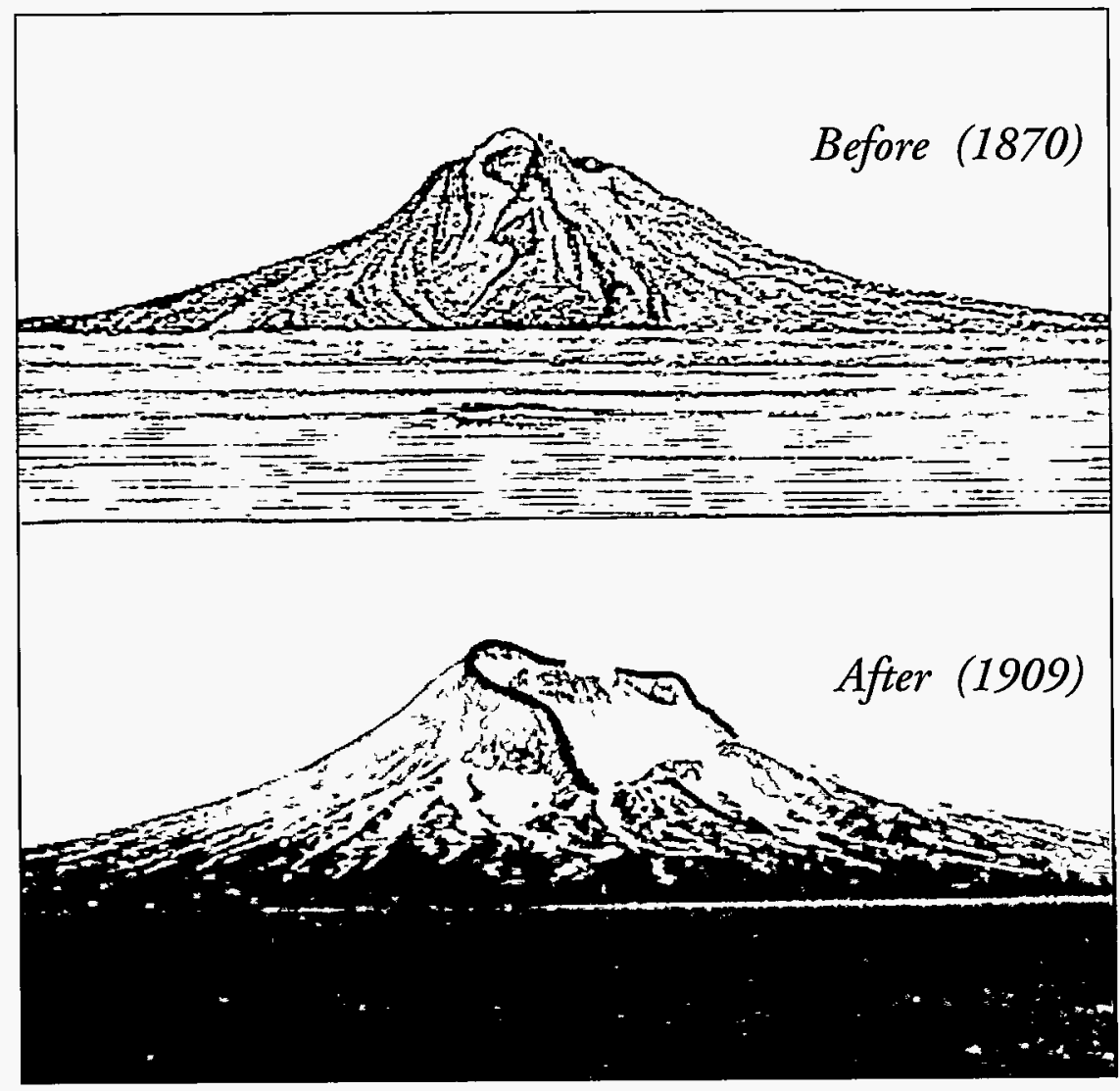

The massive slope failure at Mt. St. Augustine is evident when comparing the 1870 sketch at the top against the 1909 photo at the bottom. A dome and spine that existed before 1883 slid into the sea generating the 6 October tsunami. The resulting horseshoe-shaped crater was $0.7 \mathrm{~km}$ wide and open to the north. 

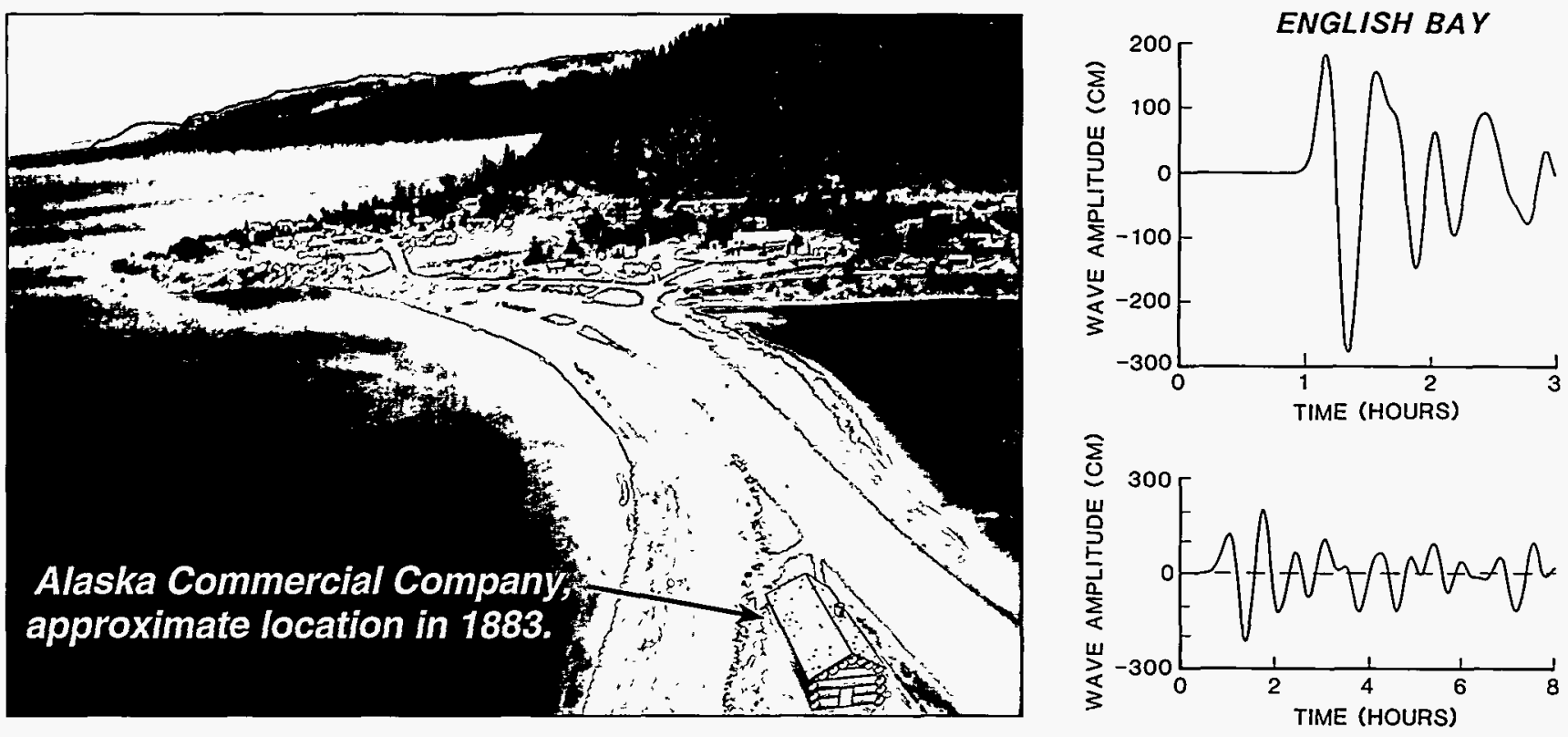

Seen from the south in the image above is Nanwalek (formerly English Bay or, in the days of the Russian traders, Alexandrovsky) and the sand spit on which the Alaska Commercial Company trading post was once located. The building was inundated by several meters of water on the morning of 6 October 1883 from a tsunami generated by the massive slope failure of Mt. St. Augustine. The graphs next to the image illustrate calculated wave amplitudes on two different time scales, modeling the effect of the tsunami. Similar models have been constructed for other Cook Inlet communities, such as Homer and Anchorage.

metry from the vicinities of several Cook Inlet coastal towns.

The first target for calculating inundation levels, runup and current velocities for Augustine-generated tsunamis is the fishing port of Homer, a major tourism center on the Kenai Peninsula.

Alaska Sea Grant College Program Grant 39147-239525 and USGS Grant 14-08-0001-A0574:Scientific personnel-J. Kienle, J.E. Begét; $Z$. Kowalik and E. Troshina (UAF Institute of Marine Science)

Cook Inlet Volcano Monitoring Eruption Processes: During the last eruptive cycle of Mt. Redoubt Volcano from December 1989 to April 1990, we had the rare opportunity to video record the rise and dispersal of several so-called coignimbrite thermals with a remote radio-telemetered camera. These thermals are distinct from eruption plumes; they develop over hot ashcovered ground, often far from the actual vent.

On Redoubt Volcano, two coignimbrite thermals rose from hot ash flow deposits that were spawned by dome collapse at the vent on April 15 and 21 . The deposits spread out 3 to $5 \mathrm{~km}$ north of the main crater over an ice piedmont at the base of an ice canyon. The thermals developed into large plumes rising $12 \mathrm{~km}$ into the stratosphere where they spread out rapidly to form a large umbrella cloud that was later dispersed by wind.

A new model of the dynamics describing the ascent of such thermals suggests that erupted material travelled as a pyroclastic flow down the ice canyon. After a few minutes, the upper parts of the pyroclastic flow became buoyant, and a large, hot ash cloud ascended off the flow. The cloud initially ascended rather sluggishly, since it was only buoyant on rising from the pyroclastic flow. However, as it ascended, it entrained and heated up more air, generating more buoyancy. It continued to accelerate upward. Only after it got much higher did the velocity decrease again as the thermal energy of the ash cloud became exhausted. The pyroclastic flow took about $200 \mathrm{sec}$ onds to become buoyant. Plume rise into the stratosphere to a height of $12 \mathrm{~km}$ took another 250 seconds.

The model shows that the height of rise of such thermals depends mainly upon the initial thermal energy of the cloud and on the atmospheric stratification.

We have modelled the lateral spreading of the umbrella cloud as a gravity current and we have shown that the theory is in good agreement with the observation.

USGS Grant 14-08-0001-A0574: Scientific personnel-J. Kienle and A.W. Woods (Cambridge University) 


\section{Subsurface Investigations}

Katmai Scientific Drilling Project: The Katmai Scientific Drilling Project sought to understand shallow intrusion and eruption of silicic magma through a three-dimensional investigation of an exemplary igneous system that formed by the great eruption of 1912 near Mount Katmai on the Alaska Peninsula. Specific objectives being pursued at the Geophysical Institute were to coor-

Thousand Smokes and led to the establishment of Katmai National Monument (now a national park and preserve) as a place to "study...the causes of catastrophe...for generations to come," (President Woodrow Wilson, 1918). Following the acceptance of the Katmai Scientific Drilling Science Plan by the Interagency Coordinating Group (ICG) for Continental Scientific Drilling

JOHN EICHELBERGER

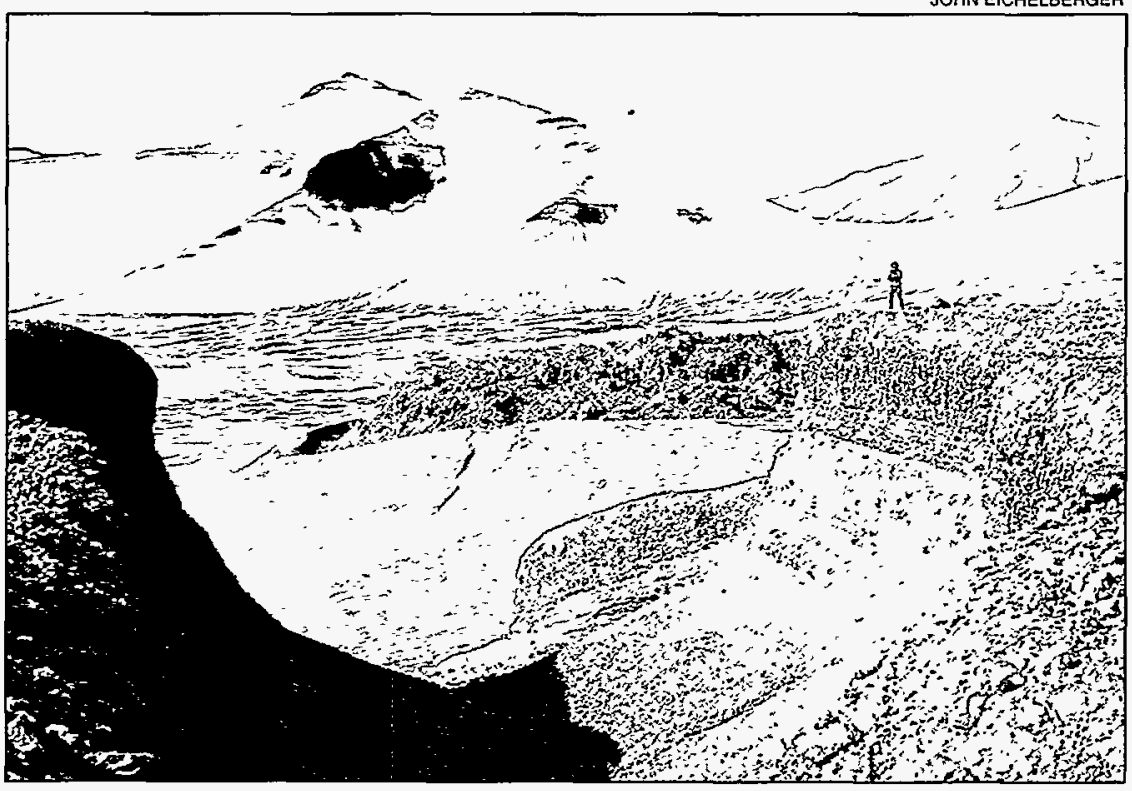

Pictured above is the northward view across the vent for the great eruption of 1912 in what is now Katmai National Park. Craggy Novarupta Dome marks the center of the 2- to 3-km diameter crater.

dinate the project during the $\mathrm{Na}$ tional Environmental Policy Act (NEPA) Process and to undertake an investigation of vent lithologies and processes as a prelude to drilling.

On 6-8 June 1912, $12 \mathrm{~km}^{3}$ of magma reached the surface through a new vent that breached a geologically simple basement in a cataclysmic outburst, which is by far the largest eruption of this century. The eruption formed the Valley of Ten in 1989 , a panel appointed by the National Academy of Sciences affirmed the site selected as uniquely suited to a drilling investigation of explosive volcanism.

The plan received favorable peer and panel reviews by the three agenit was authored by a consortium of some 40 scientists, and was led by the present Principal Investigator. Extensive surface geophysical, geocies of the ICG (DOE, NSF, USGS); logical, and geochemical investigations were undertaken simultaneously to better define the eruptive vent and its products in helicoptersupported operations characterized by scrupulous compliance with environmental regulations.

The final version of the plan involved three core holes penetrating a total of $2.4 \mathrm{~km}$ into the still-cooling system. The ICG formally requested permission to drill from the National Park Service (NPS) in 1990 and the NPS published a Notice of Intent to consider the application in the Federal Register early in 1991, officially beginning the NEPA Process. A principle effort of this process was the preparation of an Environmental Impact Statement (EIS) by Dames and Moore under contract to NPS with funding from ICG agencies.

The PI participated in the intensive review of the EIS in its various stages of development, and of supporting documents, including NEPA-required revisions and amendments to the OperationsPlan.

Although the Preliminary Draft EIS found the project to be benign in terms of long-term environmental effects, NPS determined that the project exceeded the scope of what was deemed desirable for research activities in the park, that it was beyond the permitting authority of NPS, and that it would require direct congressional action. As a consequence, the request for permission to drill was withdrawn, and the EIS was not published.

At this writing, it appears that this example of a failed evaluation pro- 
cess may help motivate a National Academy of Sciences study of the broader issue of research in protected public lands.

The remarkable variety of 1912 eruption products and the time constraints on their emplacement provide an opportunity to gain insight into the important processes of magmatic degassing and mixing, even without drilling.

Because the 1912 eruption involved three magmas (rhyolite, dacite, and andesite), our first step was to identify those components in the complex vent-fill and dome-lava lithologies. We then evaluated the response of these magmatic components to the different pressure/temperature paths represented by the eruption products. Among the results to date are:

- contact between the chemically distinct magmas did nor begin until the onset of eruption;

- observations are consistent with the vent having a nested funnel structure, as proposed by Hildreth (1983);

- degassing and welding of intravent material occurred within hours of emplacement, perhaps instantaneously;

- emplacement of Novarupta Dome began immediately after cessation of explosive activity and was heralded by emplacement of pyroclastic dikes at shallow depth;

- water is effectively outgassed during decompression, whereas halogens are lost primarily through subsequent crystallization (second boiling);

- crystallization rapidly followed emplacement of Novarupta Dome, beginning before the flow of magma had entirely ceased;

- crystallization of lava was caused by decompression rather than cooling; rapid spherulite growth forced the melt toward the low-pressure liquidus minimum and was followed by granophyric crystallization.

Evidence for shallow emplacement of pyroclastic dikes and for decompression-driven crystallization have important implications for how eruptions start and stop, respectively.

DOE Grant DE-FG06-92ER 14234: Scientific personnel-J.C. Eichelberger, S.E. Swanson, T. Bates, and D. Wiesneth

The Long Valley Exploratory Well: Caldera systems provide the best opportunity to understand where and how large volumes of silicic magma are stored in continental crust. The Long Valley Exploratory Well (LVEW) reached intact prevolcanic floor in the center of Long Valley Caldera in eastern California, the most active caldera in the contiguous United States.

Geologic analysis of LVEW samples at the Geophysical Institute support the classic view of epicontinental calderas, with the basement floor forming an ill-fitting piston which sank into the subjacent chamber as magma sprayed catastrophically from a bounding ring fault. The LVEW reveals intracaldera tuff that was thickened by syneruptive collapse and invaded by rising early post-caldera magmas.

Our current work is concerned with determining the character and history of hydrothermal activity in the central part of the caldera. The present intracaldera regime is relatively cool, given the geologically and geophysically supported expectation of shallow magma. Geochemical evidence suggests that higher temperatures prevailed only very soon after caldera formation, either because the magma was deeper than has been thought or because deep circulation of cool groundwater was established early. Grain-scale oxygen isotopic data from components of the intracaldera tuff reveal substantial disequilibrium, limiting the time that the hydrothermal system could have persisted. Evidence of both normal and inverted paleoremperature gradients were found within the caldera's resurgent dome, indicating convective overturn of the hydrothermal system. Strontium isotopic data show that calcite, a major precipitate of the hydrothermal fluids, was derived predominantly from volcanic sources, with only a small proportion from fluid interaction with the basement floor.

Sandia National Laboratory (U.S. DOE funds) Contracts 87-0400 and AA-9228: Scientific personnelJ.C. Eichelberger, P.W. Layer and $V$. Mc Connell; and M. Keskinen (UAF Department of Geology and Geophysics) 


\section{Remote Sensing}
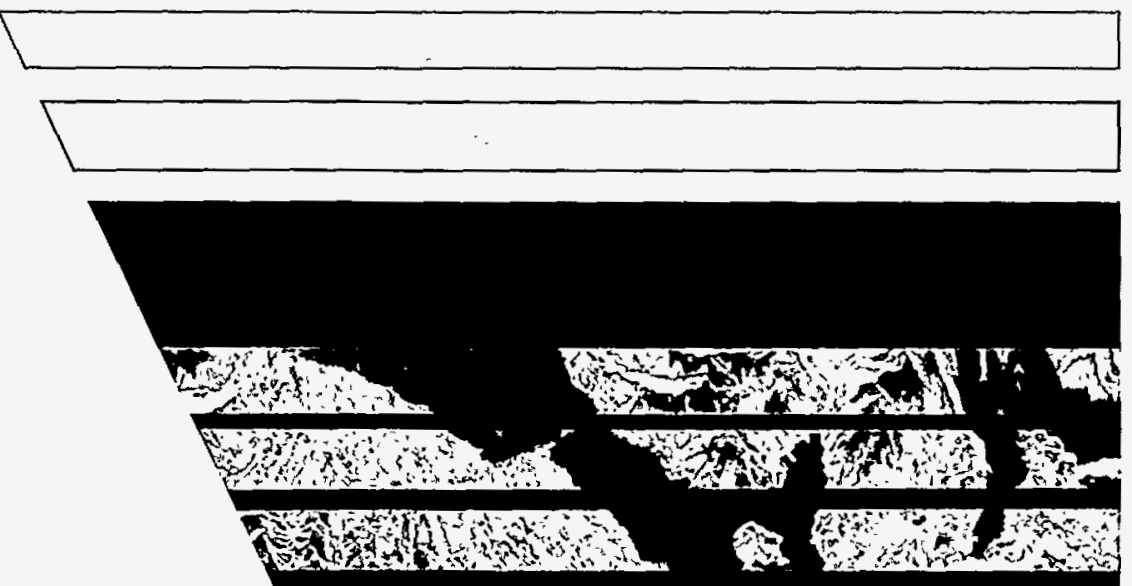

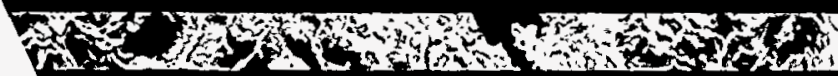

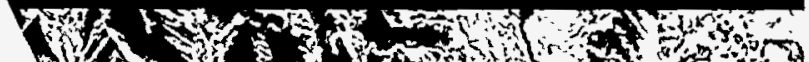

(ver)

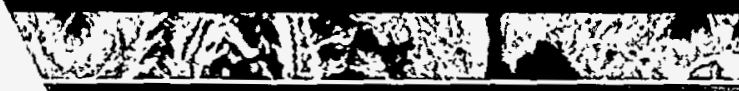
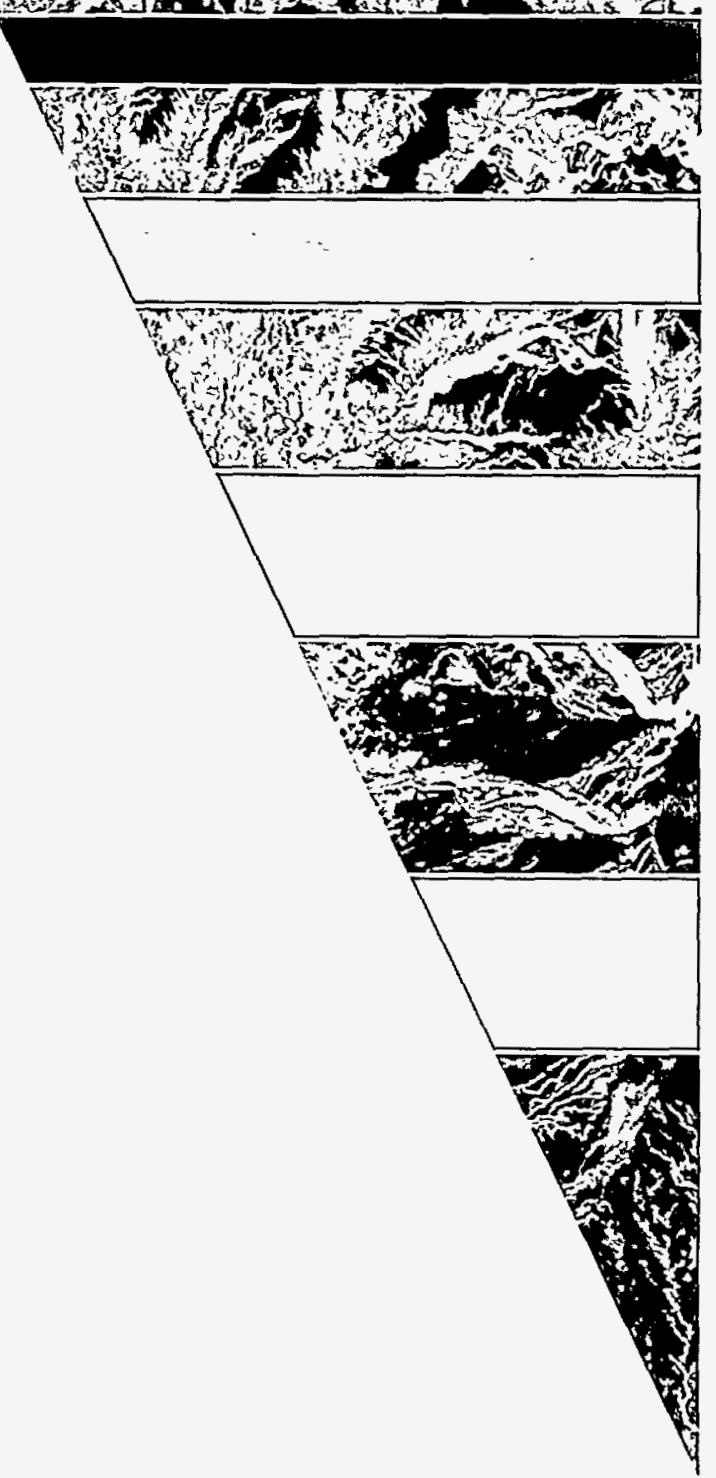


\section{REMOTE SENSING}

Remote sensing-based research transcends traditional scientific boundaries to take an interdisciplinary view of earth science problems. It provides a unique opportunity to observe phenomena over time, measure attributes and behavior of various media on regional to global scales, and to provide these data and observations to researchers for initialization and validation of their models.

Monitoring the ozone layer and developing daily UV indexes are examples of remote sensing research. Remote sensing has contributed to the development of a new discipline, Earth System Science, which emphasizes modeling, especially in climatology and on physical interactions across boundaries. Remote sensing is important not only to the research community, but also to policy makers and resource managers at the state and local level, and to private industry.

The Remote Sensing Group at the Geophysical Institute (GI) draws together researchers from several earth science disciplines at the University of Alaska Fairbanks (UAF). The Remore Sensing Group emphasis at GI is processes in polar regions. GI and UAF are uniquely positioned on the globe to contribute research and analysis of remote sensing data in support of these studies. There also are other areas where UAF and GI researchers have the resources needed to be at the leading edge of development. One example is the use of UAF's Cray supercomputer system for data analysis and visualization.

Future satellite data sources offer the GI and UAF a unique science opportunity; data reception and processing will occur on site at the Alaska Synthetic Aperture Radar Facility in the institute, and access to other research data sources will be available through the Alaska SAR Distributed Active Archive Center (DAAC) on the
EOSDIS System. The following research areas are included in current and future remote sensing studies of polar processes at GI/UAF: climate change and climate modeling (mesoscale studies); regional studies (areal restricted phenomena); applied studies; and advanced methods.

Climate studies include the role of polar processes in climate change, mesoscale modeling, and research into sources and sinks of carbon and methane, atmospheric chemistry, cloud properties, atmospheric circulation, radiation and albedo, ice sheets and glaciers, and polar storms. It also includes land-cover characterization (e.g., boreal forest and wetlands) and high-latitude oceanatmosphere modeling.

Regional studies include research into basin-wide hydrology/water resources, regional geology, geomorphology and volcanology, oceanic productivity, glaciology, and atmospheric chemistry and pollution. It also includes forestry and ecosystem classification/characterization, landscape characterization and classification, lightning detection and areal distribution, and the study of surface landforms and deformation.

Applied studies include hazard monitoring (e.g., volcanic eruptions, oil spills, flooding), applications (e.g., ship detection, landscape disturbance), environmental assessment (offshore and land-based), and educational outreach. Advanced methods include mapping and data integration, parallel processing and supercomputer applications, spatial statistics for regional-scale phenomena, vector and raster data integration and visualization, data extraction, development of geophysical processing systems, SAR interferometry, stereo mapping and storage and manipulation of large data sets.

\section{Scientific Activities}

Measurement of Surface Velocity and Elevations with Synthetic Aperture Radar Imagery-Application to Glaciers and the Solid Earth: Full-resolution and complex synthetic aperture radar (SAR) images are suitable for measuring surface velocities on large Alaska glaciers and polar ice streams and for measuring changes in the positions of glacier termini. Terrain correction is required, however, to rectify the distortion of the topography caused by the side-looking imaging geometry, particularly if the sequential images employed for measurement of displacements are not acquired from exact repeat orbits.

SAR interferometry can be employed for measurement of displacements of the earth's surface using 
sequential images from exact-repeat orbits, and can also be employed for synthesis of digital elevation models (DEMs)—given image pairs satisfying suitable criteria-without need for terrain correction.

For terrain correction, a DEM of the imaged area is required a priori. SAR images are necessarily transformed during terrain correction, such that the resolution of the terrain-corrected image is equal to the resolution of the underlying DEM.

The highest resolution DEM available for most of Alaska is $90 \mathrm{~m}$. Full-resolution SAR images, however, have a pixel size of $12.5 \mathrm{~m}$ and a nominal resolution of $30 \mathrm{~m}$. Therefore, software has been developed to enhance the resolution of DEMs to match full-resolution imagery using the optimal interpolation method of kriging.

Kriging throughout areas of this size is computationally intensive, so this software was developed to run on the Cray system of the UAF Arctic Region Supercomputing Center (ARSC). The resulting terrain-corrected SAR images retain the visual information that would otherwise be lost due to the dropout of in-between pixels. This software also has been generalized for use with spatially distributed data sers not defined on regular grids.

Preliminary work on glacier velocity measurement has been performed using software developed by $\mathrm{R}$. Bindschadler and T. Scambos (of the NASA Goddard Space Flight Center) for measuring velocities on large antarctic ice streams using cross-correlation of sequential Landsat Themaric Mapper (TM) images. This software was adapted for use with full-resolution SAR imagery processed at the Alaska SAR Facility.

Cross-correlation of SAR (or Landsat TM) imagery is computationally intensive, so this software also has been converted to run on the ARSC Cray system.

Malaspina Glacier in the St. Elias Mountains of southcentral Alaska was selected as a test case because of its large surface area, which is characterized by folded medial moraines that are suitable for correlation between sequential images.

Cross-correlation of images acquired 9 January 1993 (during Phase C of ERS-1, which had a 35-day orbit repeat cycle) and 26 April 1994 (during Phase E, which had a 168day orbit repeat cycle) yielded large numbers of displacement vectors on the areas of the glacier having folded medial moraines, and in general, throughout debris-covered areas of the glacier surface.

The preliminary results suggest, however, that this method must be used with SAR images that have either been terrain corrected or acquired from exact repeat orbits.

Sponsored by Cray Research, Inc., 1993 University Research and Development Grant Program; administered by the UAF Arctic Region Supercomputing Center; research performed at the Geophysical Institute: Scientific personnel-C.S. Lingle, V.A. Voronina, and D. R. Fatland

The Surface Velocity Field on Bagley Icefield, Alaska, Before and During the 1993-94 Surge of Bering Glacier, from ERS-1 SAR Interferometry: Bagley Icefield, which is the primary accumulation area for Bering Glacier in the St.
Elias and Chugach Mountains of Southcentral Alaska, flows west for $90 \mathrm{~km}$ from an elevation of about $2100 \mathrm{~m}$ at its ice divide below Mt. St. Elias to an elevation of about $1200 \mathrm{~m}$, where it turns southwest and descends to the Gulf of Alaska coast as the main trunk of Bering Glacier. The entire system-which is about $180 \mathrm{~km}$ long from Bagley ice divide to the Bering terminus-forms the largest glacier in North America, and in the world outside Antarctica and Greenland.

Bagley Icefield was imaged sequentially by the synthetic aperture radar (SAR) onboard the First European Remote Sensing Satellite (ERS-1) during both of the mission ice phases, which occurred during the winters of 1991-92 and 1993-94. Both the first and second ice phases were characterized by three-day repeat orbits. Digital image processing techniques were employed to synthesize interferograms from suitable sequential image pairs acquired during the ice phases. The first ice phase was prior to the recent surge of Bering Glacier, which began during spring 1993. The second ice phase, which was well after surge onset, occurred when the surge had propagated up-glacier into Bagley Icefield as well as downglacier to the terminus.

The SAR images used to synthesize interferograms are analogous to stereo-optical pairs, and the interferometric baselines created by the cross-track separation between repeat orbits are analogous to optical baselines. The transverse separation of repeat orbits for ERS-1 can vary from a few meters to a few kilometers, and is typically of the order of several hundred meters. 
If the transverse separation is small (a few meters to tens of meters), the interference fringes primarily represent glacier motion parallel to the radar look direction. If the transverse separation is relatively large (about 50 to a few hundred meters), the interference fringes primarily represent elevation differences in the area.

An image pair that included central Bagley Icefield-acquired by ERS-1 on 4 and 7 February 1994 while the surge of the Bering Glacier was in progress-proved suitable for the synthesis of an interferogram, which showed the component of ice motion parallel to the radar look direction on the ice field and its tributaries. An analogous interferogram was synthesized from an image pair acquired prior to the surge onset.

A third interferogram, synthesized from an image pair acquired 4 and 7 February 1994 over the unglaciated Chitina River Valley-a relatively short distance northwest of Bagley Icefield - showed that the fringes of the Bagley interferograms represent primarily ice motion (not elevation differences). From the changes in the interference fringes, it was possible to estimate how far upglacier into Bagley Icefield the surge propagated.

The velocity on Bagley Icefield during the surge, estimated from the interference fringes, was found to be similar to the velocity that had been measured independently using the Global Positioning System (GPS) during field work on the ice field in late July 1994.

NSF Grant OPP-9319873: Scientific personnel-C.S. Lingle and D. R. Fatland
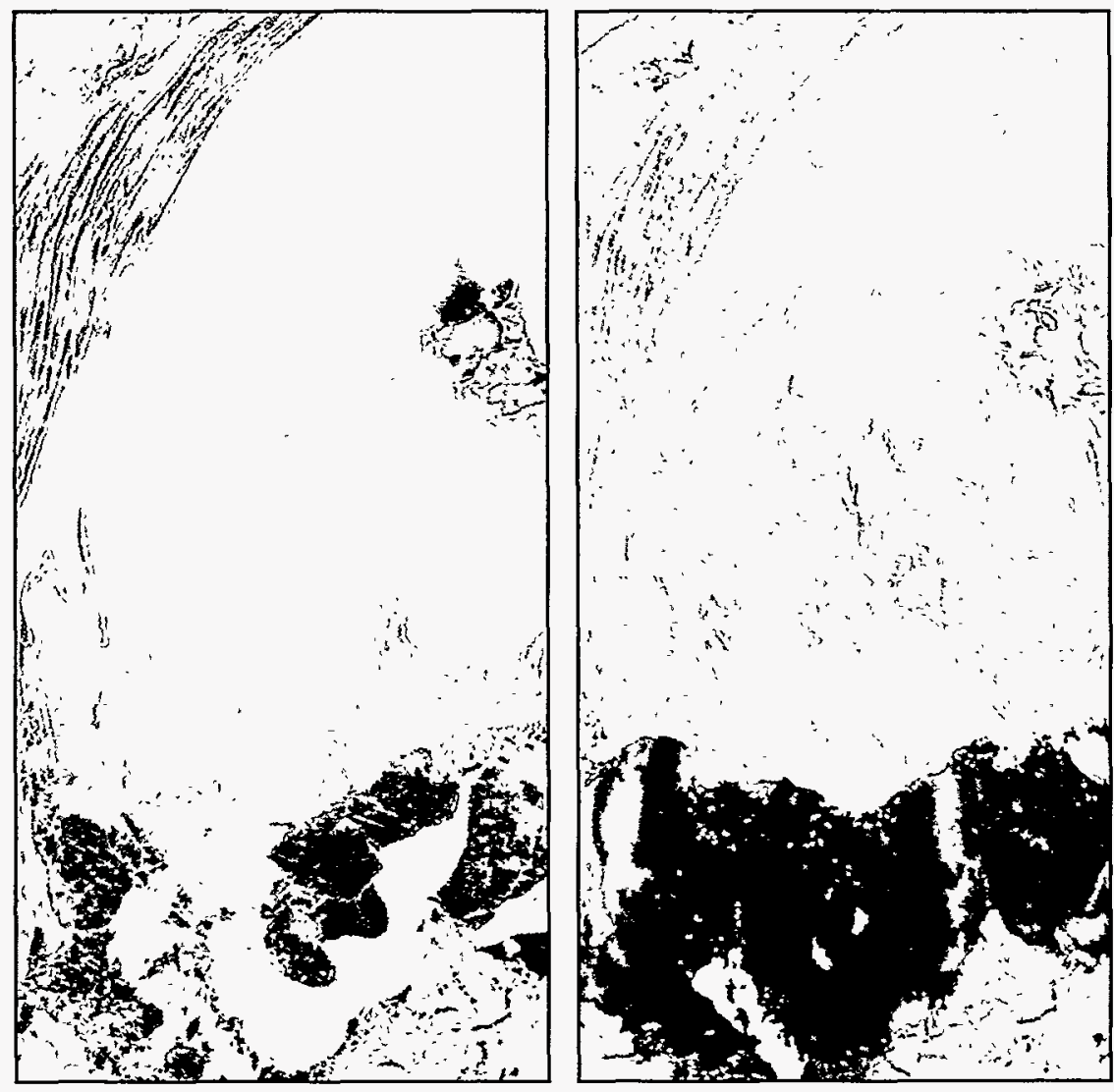

The two panels above (scene IDs 59540 and 72703) are ERS-1 SAR images showing the rapid surge of the Bering Glacier. A comparison between the first panel, an image taken on 22 November 1992 before the surge, and the second panel, taken on 9 August 1993, shows the effects of the surge transforming the glacier surface from smooth to undulating, which is characteristic of fast-flowing ice. The images were created by J. Roush, C. Lingle, and R. Guritz, and submitted to the Journal of Glaciology in 1994.

Monitoring and Assessing Volcanic Eruptions Using Remote Sensing Data: Advanced Very High Resolution Radiometer (AVHRR) satellite images are used to monitor and analyze volcanic eruptions in the North Pacific region (Alaska and Russia, Kamchatka Peninsula). The one kilometer spatial resolution, $2500-\mathrm{km}$ swath width and almost hourly coverage (at high latitudes) in visible and thermal infrared data bands are well suited for monitoring eruptions.

The AVHRR data are received and processed in real time to detect eruption clouds and hot spots. The spatial extent, direction of movement, composition, and spectral characteristics of eruption clouds are derived from these data within minutes of a satellite pass.

Analysis techniques include band ratio, subtraction and level slicing. Cloud temperatures, derived from the thermal infrared bands, are compared to the atmospheric temperature profile to estimate the height of the cloud. These images are also used to tune an eruption cloud-tracking model. The surface terrain of volcanoes prior to, during, and after an eruption are analyzed on synthetic aperture radar (SAR) data acquired from the Alaska SAR Facility (ASF). 


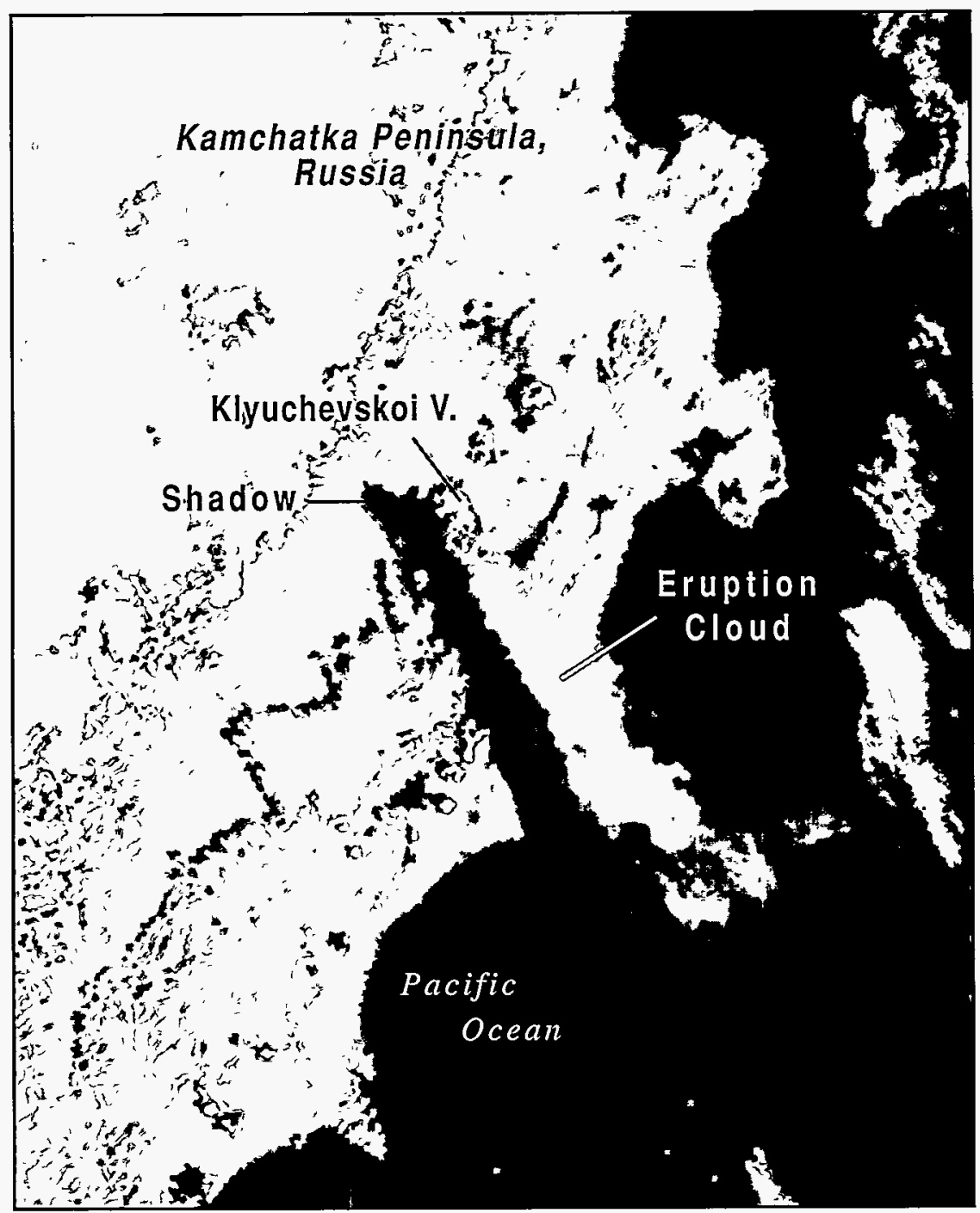

Satellite images like the one above are used by AVO to detect and monitor volcanic eruptions. This image shows Klyuchevskoi Volcano on 30 September 1994. An eruption cloud $>250 \mathrm{~km}$ long is drifting to the southeast into air traffic routes in the North Pacific.

The high spatial resolution and the cloud-penetrating capabilities of the SAR data are used to assess and to map recent changes in land surface resulting from an eruption. Newly formed lava flows, landslides, avalanches, ice pits and cones have been detected.

The SAR data are especially valuable in remote areas where it is expensive and logistically complicated to assess changes using more conventional techniques. Information derived from these data are provided to
USGS AVO Grant 1434-93-A1127: Scientific personnel-K.G. Dean, S. George, C. Wyatt and K. Engle

The Use of SAR-Interferometry for Volcano Monitoring: This project is described in the Seismology section on page 136 of this report.

Puff-A Tracking and Prediction Model of Volcanic Eruption Clouds: The Puff model was developed to track the movement of young, volcanic eruption clouds as they drift across Alaska. Young eruption clouds are defined as those less than 48 hours old and within a few hundred kilomerers of the source volcano.

Initially, the model was used as an emergency response tool by the Alaska Volcano Observatory (AVO) to forecast the position and direction of movement of blowing ash and its dispersal near populated areas or aircraft routes. Soon after implementation, Puff began providing information when satellite images were not available, thus becoming intricately related to remote sensing systems used to monitor eruptions.

The model, which originally was developed by H.L. Tanaka, is based on the three-dimensional Lagrangian form of the diffusion equation. It assumes a uniformly distributed, vertical column of particles above the source volcano that extends to a designated height in the armosphere.

The individual ash particles are set in motion using forecasted windfield data. Fallout and diffusion are also included in their motion. The diffusion uses an approximation of pollutant turbulent mixing described by a stochastic process. In a Lagrangian framework, this is realized using 
a random walk with a Gaussian distribution. Gravitational fallout is approximated by Stoke's law.

The model currently uses Unidata for the wind fields. Unidata is updated and transmitted twice daily to include the measured wind, as well as the forecast for up to 60 hours.

Software has been developed for executing the model in a real-time emergency situation . It is currently running under the Silicon Graphics workstations in the GI/ARSC visualization lab. A graphical user interface for easier operation is also under development.

When initiated, Puff provides a continuous prediction of the position of an eruption cloud. Satellite images, acquired approximately every hour, are compared to the model to check the accuracy of the prediction. When the prediction does not completely agree with the satellite images, input parameters are tuned to improve accuracy.

UCAR Grant S94-45514: Scientificpersonnel-K.G. Dean and C. Searcy

The Influence of River Discharge on the Thawing of Sea Ice in the Mackenzie River Delta: Multitemporal satellite images, field observations, and field measurements were used to investigate the mechanisms by which sea ice melts offshore from the Mackenzie River delta, of the Arctic Ocean. Advanced Very High Resolution Radiometer (AVHRR) satellite data

\section{AVHRR Thermal Image: $\quad$ Model Output:}

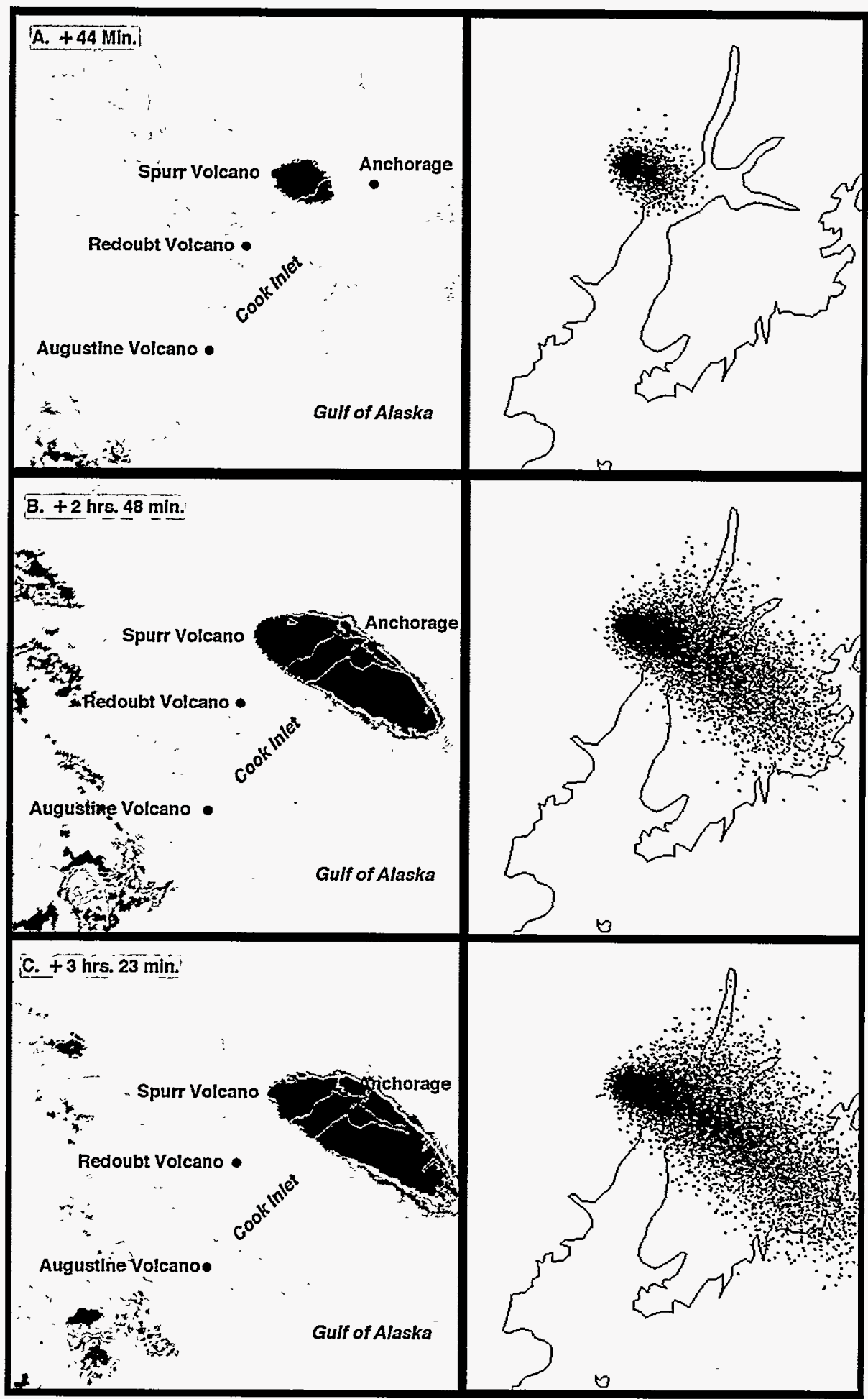

The eruption of Mount Spurr on 18 August 1992 resulted in an eruption cloud drifting to the east over Anchorage. The cloud's position was recorded on a thermal infrared satellite image (shown above left); the prediction made by the Puff model (shown above right) closely agreed with the observed position. 
recorded between April and August 1986 were corrected to a map projection and calibrated such that albedo and temperature values could be compared, and physical relationships investigated.

Three stages in the thawing of sea ice were identified: flooding (overflows); melting of the top surface by incoming solar radiation (insolation effects); and melting of the bottom surface by warm river water.

Overflows are the first features observed in the melt process. The overflows contribute heat to the melt process due to their warmer temperatures in part caused by their low albedo values, which can be as low as $1 / 7$ that of ice values.

Approximately two weeks after the overflows develop, sea surface temperatures rise as the river discharge peaks and becomes the dominant source of energy.
As the summer progresses, the albedo of ice decreases by $1 / 3$, thus increasing absorption of solar energy due to insolation. By this process, ice removal in the delta regime is initiated two months earlier than adjacent coasts with minimal runoff. However, the net result is only a twoweek acceleration of ice removal in the delta region.

A one-dimensional, thermodynamic model was generated for the spring melt season based on satellite image observations and field measurements. The model simulated the vertical melting of the nearshore ice as a function of the surface heat fluxes and the sensible heat contributed by the river to the bottom of the ice cover. Model parameters were tuned by comparing the results with the satellite imagery until a best-fit occurred. The simulations indicate that nearly half of the heat used to melt the nearshore ice came from river discharge. Once calibrated, the model was used to investigate the sensitiv-ity of the region to changes in the river parameters, such as the initial date of river breakup far upstream, or warmer river discharge. In the first case, a one-week change in the initial river breakup responded with about a four-day change in the date of ice removal. The same response occurred for a doubling of the river temperature.

Three papers have been written based on this investigation. The first is published in the Polar Research Journal, and the other two have been submitted to the Journal of Geophysical Research.

NASA Grant NAGW 1835: Scientific personnel-K.G. Dean, W.J. Stringer, K. Ablnäs, C. Searcy; $T$. Weingartner (UAF Institute of $M a-$ rine Science)
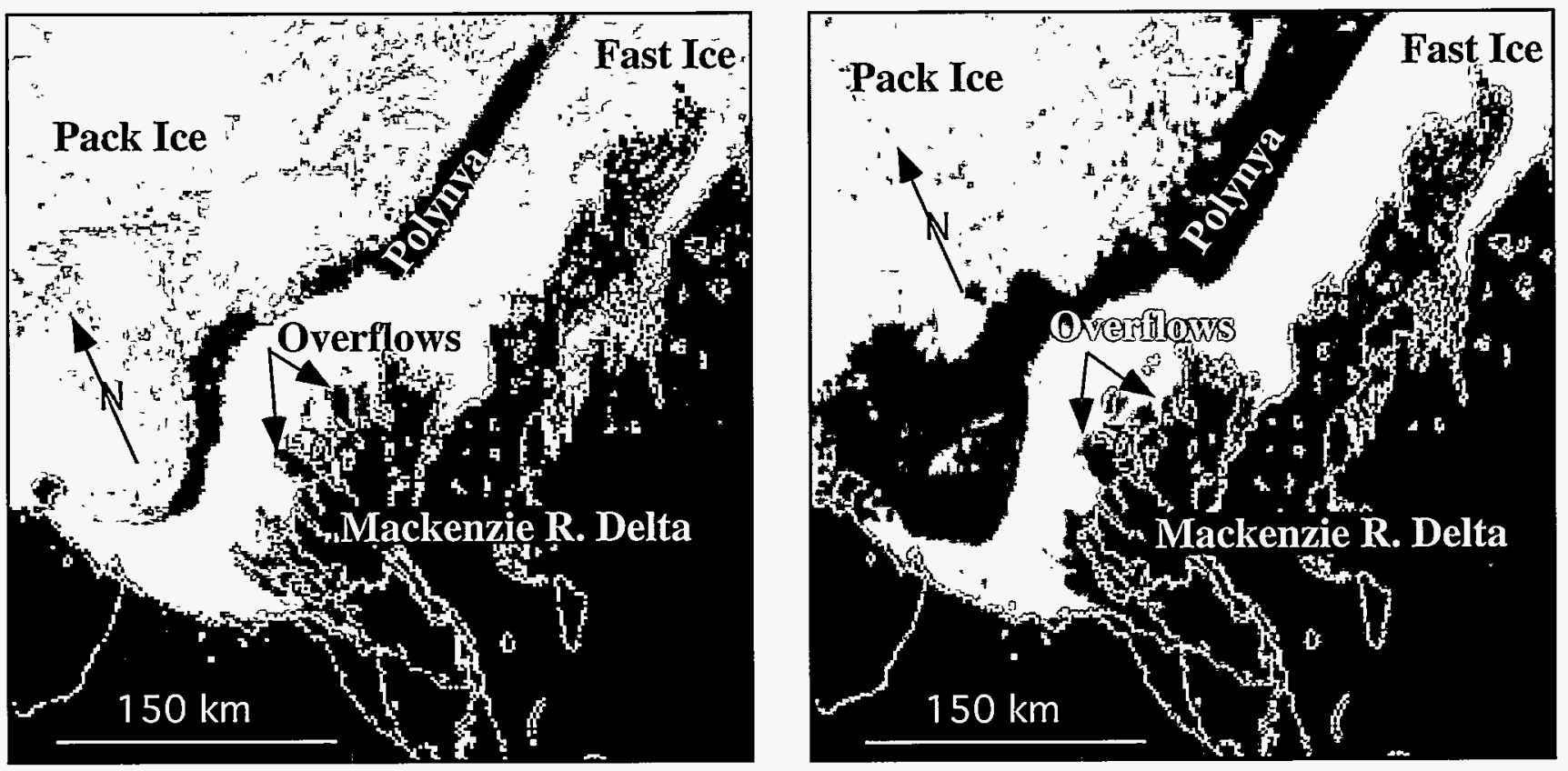

Shown above is a comparison between two near-infrared (Band2), AVHRR satelite images taken ten days apart (4 and 14 June 1986). The two images show flooding (overflows) of the fast ice offshore from the western Mackenzie River channels, pack ice, and the Cape Bathyhurst Polynya. The 14 June images show the formation of a bight in the pack ice edge which is attributed to melting by warm river water discharged by the western Mackenzie River channels. The bight developed after the 4 June image was recorded. 
Beaufort and Arctic Storms Experiment Sea Ice Motion Project: The Beaufort and Arctic Storms Experiment (BASE) will be conducted over the southern Beaufort Sea and Mackenzie River Delta in the fall of 1994. Its purpose is two-fold: to better predict the weather that affects this region; and to better understand the climate implications of these weather systems.

The original funding for BASE was provided by the Federal Panel on Energy, Research, and Development (PERD) because improved weather forecasting potentially can affect the safety and efficiency of offshore operations. Because BASE represents one of the first experiments designed to improve understanding of the weather in Canada's Arctic, it has also attracted considerable interest from the climate community.

The Canadian Global Energy and Water Cycle Experiment (GEWEX), which is focusing its activities on the Mackenzie River Basin, will be a major beneficiary of the information to be obtained from BASE. For example, BASE will provide unique information on the distribution of precipitation, the nature and impact of high-latitude cloud systems, and the atmospheric water budget in this region. Furthermore, BASE should allow for an examination of the largescale impact caused by the freezeover of the Beaufort Sea.

A range of weather systems affects the northern Mackenzie Basin and southern Beaufort Sea during the autumn. Extra-tropical cyclones pass over the area about twice per week; these may rapidly deepen, undergo secondary cyclogenesis in the lee of the nearby mountains, or bring only weak precipitation. In the rear of these systems, cold air outbreaks with associated snow squalls commonly occur. Mesoscale vortices can develop and, if sufficiently intense, form a polar low. None of these systems have been studied with detailed measurements before, and it will be important to compare them with their southern counterparts not subjected to such a high Coriolis parameter or low tropopause.

A major alteration of surface characteristics also occurs during this period. The Beaufort Sea, initially characterized by a few hundred kilometer wide band of open water warmed by the inflow of the Mackenzie River, is completely icecovered by mid to late October.

BASE therefore represents a crucial step in terms of acquiring detailed measurements of arctic weather systems. The benefits will be an improved understanding of cold region, high-latitude meteorological processes and the role they play in the global climate system.

Supported through NASA and Atmospheric Environmental Service, Canada: Scientific personnel-L. McNutt; T. Agnew, R. Stewart, K. Moore, and D. Hudak (Atmospheric Environment Service, Canada)

Response of Sea Ice to ArcticStorms: The response of arctic sea ice to atmospheric forcing has been a topic of research throughout this century. Although storms may not be responsible for the long-term behavior of ice, they very likely represent the most dynamic ice events and times when local atmospheric forcing is the most dominant factor in ice behavior. At other times, other factors, such as sea slope, long-range internal forces and currents play major, and in some cases dominant, roles in ice dynamics.

As his masters dissertation research, C. Byers undertook a project to determine if relationships can be found between ice behavior and atmospheric forcing during arctic storms.

Atmospheric pressure and floe location for a one million square kilometer area located in the Canada Basin were obtained from arctic marine buoys during winter months for a seven-year period. Statistical analysis of derived wind data led to a definition of storms as winds above 16 meters per second (two standard deviations of the data set).

For statistical analysis purposes, the wind data were divided into three subsets, with divisions at 8 and 16 meters per second. Threshold vorticity values were determined to divide the data into cyclonic, anticyclonic and zonal flow conditions. Thus, a matrix of nine subsets of the data was defined.

After deriving a set of atmospheric and ice parameters from the buoy data, descriptive statistics and correlations were calculated for the entire data set and for the matrix of nine subsets as described. Strong correlations were found between wind and ice speed, between ice speed and shear within the ice and between atmospheric vorticity and ice vorticity. However, significant correlations were absent berween all wind parameters and both ice divergence and turning angle. Coupling between atmosphere and ice appeared more effective during episodes of high winds and cyclonic circulation. 
Supported by the Minerals Management Service Cooperative Agreement 14-35-0001-30560 and by state of Alaska funds: Scientific personnelW.J. Stringer and C. Byers

Multitemporal and Multisensor Data to Initialize and Validate Mesoscale Air-Sea-Ice Interaction Models for the Beaufort and Chukchi Seas: This study will build on on-going research to evaluate the use of remote sensing data to derive geophysical measurements of sea ice for use in validating and initializing mesoscale air-sea-ice interactions in the Beaufort and Chukchi seas.

Current work concentrates on using AVHRR data with SAR images and National Meteorological Center (NMC) information. In the ADEOS time frame, RADARSAT SAR data will be used with AVNIR, OCTS, and NSCAT to evaluate the potential of these instruments to provide high-resolution visual and tempera-

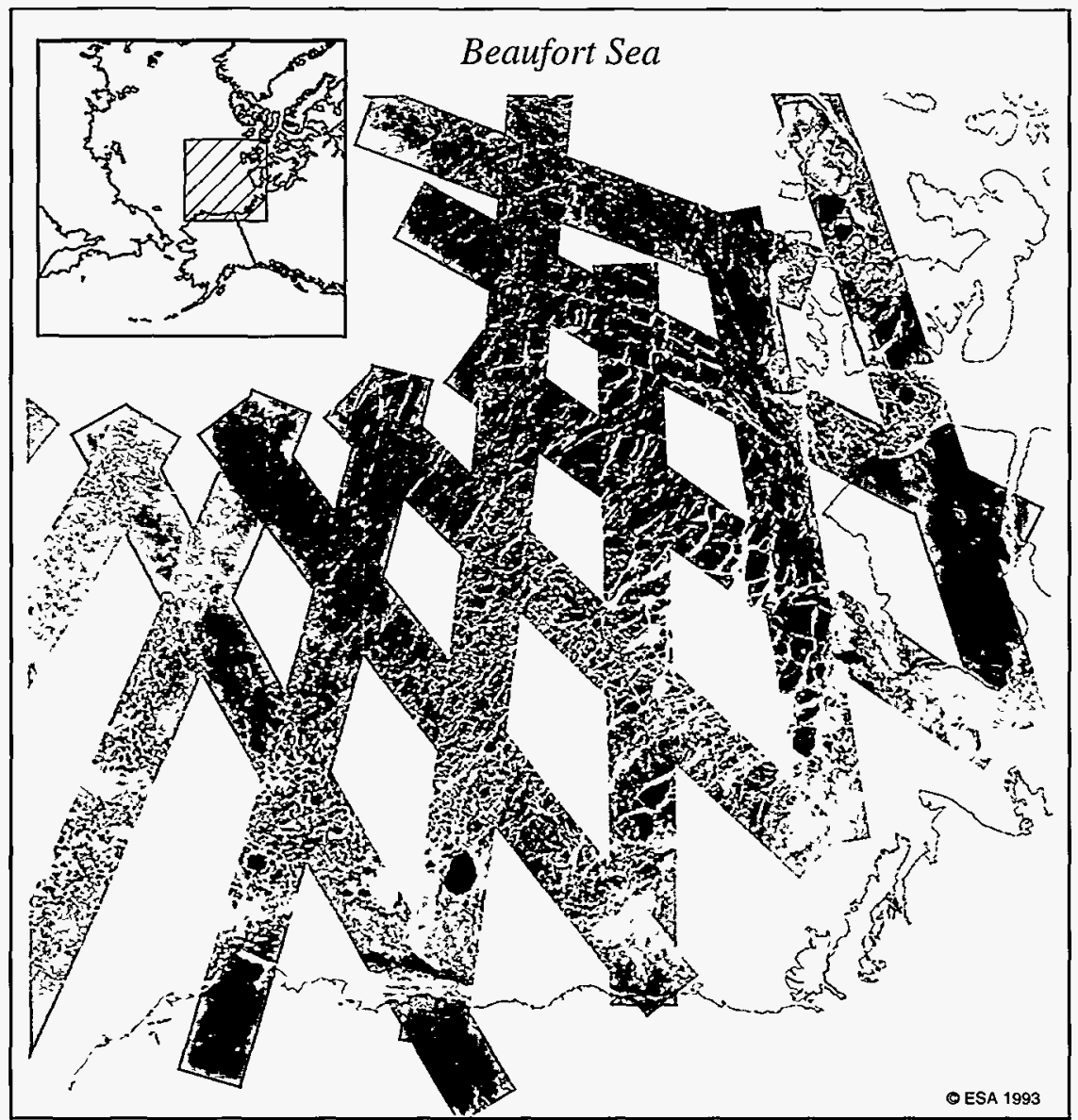

The mosaic above combines more than 350 SAR images from the ERS-1 satellite with coincident, wide-swath, thermal infrared AVHRR imagery. The 2100-by-2100 km region includes the Beaufort Sea north of Alaska from Point Barrow (west) to Banks Island, Canada (east). The long strips represent SAR data obtained over a three-day period in March 1994. The AVHRR data provide both imagery data and temperatures of the sea surface in cloud-free areas while SAR can also image the sea ice through clouds and in darkness. The two sources, when mosaicked together, provide complementary information on sea ice conditions. Patterns evident on this mosaic, created by the Science Computing Program on the ASFInteractive Image Analysis System, are consistent with ice dynamics and theology for the region. ture data. In situ measurements are taken from buoy arrays used to measure floe-floe interactions, and to form associated field observations obtained when the buoys were placed on the sea ice.

The objective of the larger study is to find a way to provide routine geophysical measurements on $5 \times 5$ $\mathrm{km}$ (or smaller) grids. The complete suite of measurements is still under discussion. AVNIR and OCTS, with RADARSAT and, later, MODIS and MERIS data, are expected to contribute to the evaluation of ice compression and divergence, and, indirectly, to estimates of heat flux.

Scientific personnel-L. McNutt, J. Overland, and S. Li

Regional Deformation of Sea Ice in the Beaufort and Chucki Sea Related to Storm Track Climatology, Ice Rheology and Basin Orientation: The objective of the investigation is to provide the information necessary to understand the relationship between small-scale ice features and the basin-wide modeling of ice dynamics, especially for mesoscale ice climatology models in the Beaufort and Chukchi Seas.

Most models used in this region continue to rely on the same internal stress and forcing equations developed for the Central Arctic Ocean pack ice. These basin-scale models use the ellipse yield curve and ice rheology similar to that developed by Hibler (1979). This type of approach does not take into account the scale effects, especially for areas $<200 \mathrm{~km}$. Some attempts have been made to apply an anistropic constitutive model to the sea ice. For regional models, including mesoscale 
models, an examination of the state variables is necessary.

Supported through NASA and NOAA: Scientific personnel-L. McNutt, S. Li, J. Overland, and R. Wade

A Study of Physical and Structural Factors Affecting SAR Signatures of Ice-covered Arctic Lakes: The objecrive of this research is to improve our ability to use SAR for studies of lake ice variability in relation to environmental change by identifying those factors and processes responsible for backscatter variability from lake ice.

Field investigations of ice cores and ice thickness were undertaken at shallow lakes near Barrow, NW Alaska, in spring 1992 and 1993. ERS-1 SAR data were acquired for shallow and deep subarctic and arctic lakes in Alaska and Canada.

Backscatter time series were compiled from the time of initial ice formation in autumn until the onset of melt in spring and during the complete removal of the ice cover in early summer. A lake-ice backscatter model has been developed. A numerical ice growth model, initially developed for alpine lakes in Montana, has been used in conjunction with the SAR detection of the complete freezing of shallow lakes on the North Slope to determine the depth distribution of those lakes. The depth distribution model is useful for water resources management.

NASA Grant NAG5-1731: Scientific personnel-M.O. Jeffries, W.F. Weeks, K. Morris; H. Wakabayashi (NASDA); D.K. Hall (NASAI GSFC); G.E. Liston (Colorado State University)
Investigation of the 1994 Delta Junction, Alaska, Forest Fire Using SAR Imagery: Synthetic Aperture Radar (SAR) can be used to identify the location, size and severity of boreal forest fires and to estimate the amount of carbon that they release into the atmosphere. We are developing techniques to do this by studying the fire that occurred in the summer of 1994 near Delta Junction. These techniques can be used to study fires in remote locations in Alaska and other northern regions.

The study of this particular fire is also important because the area that burned was used by the U. S. Army to test biological and chemical, as well as conventional, weapons between 1953-1967. Some unexploded ammunition still remains within the burn area. We hope to use SAR and other data to evaluate potential environmental hazards in the area.

To determine a radar signature of a burned area, we will compare data acquired by SAR, which records information in the microwave region, with data acquired by instruments that record visible light- - aerial photographs and Landsat images. The aerial photos were taken after the fire was extinguished, but the Landsat images were acquired while it was still burning. To correlate the radar signature with burn intensity, we will compare the images to depth of burn data, acquired through field studies. We will also make timeseries analyses of the change in radar signatures of the burned area by comparing over 50 SAR images acquired from 1993 to 1995.

Natural Resources Fund: Scientific personnel-L. McNutt; C. Williams (Institute of Northern Forestry)
Remote Sensing of Oil Spills: Working under a reimbursable services agreement with the Alaska Department of Environmental Conservation, we have performed a comprehensive review of the stateof-the-art remote sensing of oil spills.

There have been two main objectives of this study. The first was to examine the capabilities of all possible providers of remote sensing data in the case of a future Alaska spill and to summarize their capabilities and limitations, while the second was to examine the capability of the Geophysical Institute to provide oil spill data from remote sensing satellites in the case of a future spill. The second objective also included an examination of the capability of synthetic aperture radar satellites to provide data for search and rescue operations involving vessels in Alaska waters.

Although this work was largely of a service nature, several areas of possible research were identified relating to the interpretation of SAR imagery. There are research needs related to the positive identification of oil versus other phenomena, such as internal waves. The wakes of large and small vessels as seen on SAR imagery require interpretation in terms of vessel speed and displacement. Very often part of a ship's wake appears similar to an oil slick. It is not clear how this occurs but it is clearly important to be able to differentiate between this phenomenon and spilled oil if we wish to use SAR to look for ships dumping oily ballast water.

Finally, the identification of a vessel against an ocean background on SAR imagery may depend on ocean state as well as vessel size, configura- 
tion, composition and orientation. However, very little is known about this topic.

State of Alaska funds: Scientific personnel-W.J. Stringer

Identification of Potential Sites of Radionuclide Pollution in the Former Soviet Union: The opening of the former Soviet Union in recent years has revealed the problem of radionuclide pollution resulting from weapons production and testing, reactor waste collection, treatment, storage and disposal, and other acrivities relared to both the civil and military nuclear programs that may have worldwide consequences. Some sites are well known and have been identified for some time while others are still being discovered.

Many of these sites are located on land in close proximity to waterways that lead into the Arctic Ocean and in some cases the entry of radioactive materials into these pathways has already been documented. It is possible some of these sites have not been fully documented or that other sites exist but have not yet been discovered due to secrecy at earlier times.

As part of an overall assessment of this problem, we are conducting research to determine if remote sensing methods can be employed to examine the known sites of radioactive pollution. We hope to map their full extent, to estimate their poten- tial contaminant release and to develop a signature to be employed in a search for additional sites which may not be known to us at the present. High resolution, multispectral satellite imagery obtained by the Landsat Multispectral Scanner has been obtained of the Mayak nuclear facility located north of the city of Chelyabinsk, and just east of the Ural Mountains.

This facility is currently the best documented site of terrestrial radionuclide pollution and will be used as the starting point for this investigation.

Supported by US Navy funds: Scientific personnel-W.J. Stringer and S. George

\section{Data Collection and Ancillary Services}

The Alaska Synthetic Aperture Radar Facility (ASF) Science Division: The ASF Science Division is responsible for the Science Program of the ASF. Currently, the program focuses on the use of SAR and other remote sensing data to monitor polar processes related to global climate change. The emphasis is on sea ice, oceans, glaciology, hydrology, land cover studies, volcanology, and hazard analysis. The ASF Science Division includes the Science, Techno logy, and Education Program (STEP), a science computing program which develops SAR processing algorithms and various user tools for SAR data manipulation. The ASF Science Division supports visiting scientists as well as local and remote users, and has an active graduate student education program. The ASF Science Division works with the GI and other groups at UAF, such as the Institute of Marine Science (IMS), the Institute of Northern Forestry (INF), and the Cooperative Extension Program. The STEP program works closely with the Arctic Region Supercomputing Center (ARSC) and its visualization laboratory on joint research and development projects, including a graduate student program.

NASA Contract NAS5-32393: Scientific personnel-L. McNutt, M.O. Jeffries, S. Li, C.S. Lingle, C. Olmsted, K. Dean, L. Shapiro, $R$. Wade, J. Groves, R. Fatland, F. Hao, J. Roush, K. Schwartz, M. Shindle and T. Logan

Terrain Correction of the Alaska SAR Facility SAR Imagery Using the T3D Massively Parallel Processor at the Arctic Region Supercomputing Center: Terrain correction of satellite synthetic aperture radar (SAR) images is essential be- cause the range mapping under the side-looking configurations of the SAR instrument causes severe geometric and radiometric distortions on the acquired image in the sense of a normal orthographic map projection.

Computer codes exisring for correction of the geometric distortions on the SAR images were slow. The goals of the project have been to convert the existing codes available to us into codes suitable for parallel processing under the CRAY T3D environment at the Arctic Region Supercomputing Center (ARSC) at the University of Alaska Fairbanks and thus to meet the urgent needs of the user community for fast correction of satellite SAR images acquired at the Alaska SAR Facility (ASF).

In 1994, conversion of the serial computer code for geometric correction to parallel computer code on the 
CRAY T3D system at ARSC was completed. As a result, the turnaround time for the terrain correction of a single satellite SAR image has been reduced from the original four and one-half hours on a Sun Sparc2 workstation to only six and one-half minutes on the T3D system. This development will facilitate land applications of satellite SAR images acquired at ASF. Further investigation on radiometric correction of the SAR signatures on rugged surfaces using T3D at ARSC will be the goal for the next year.

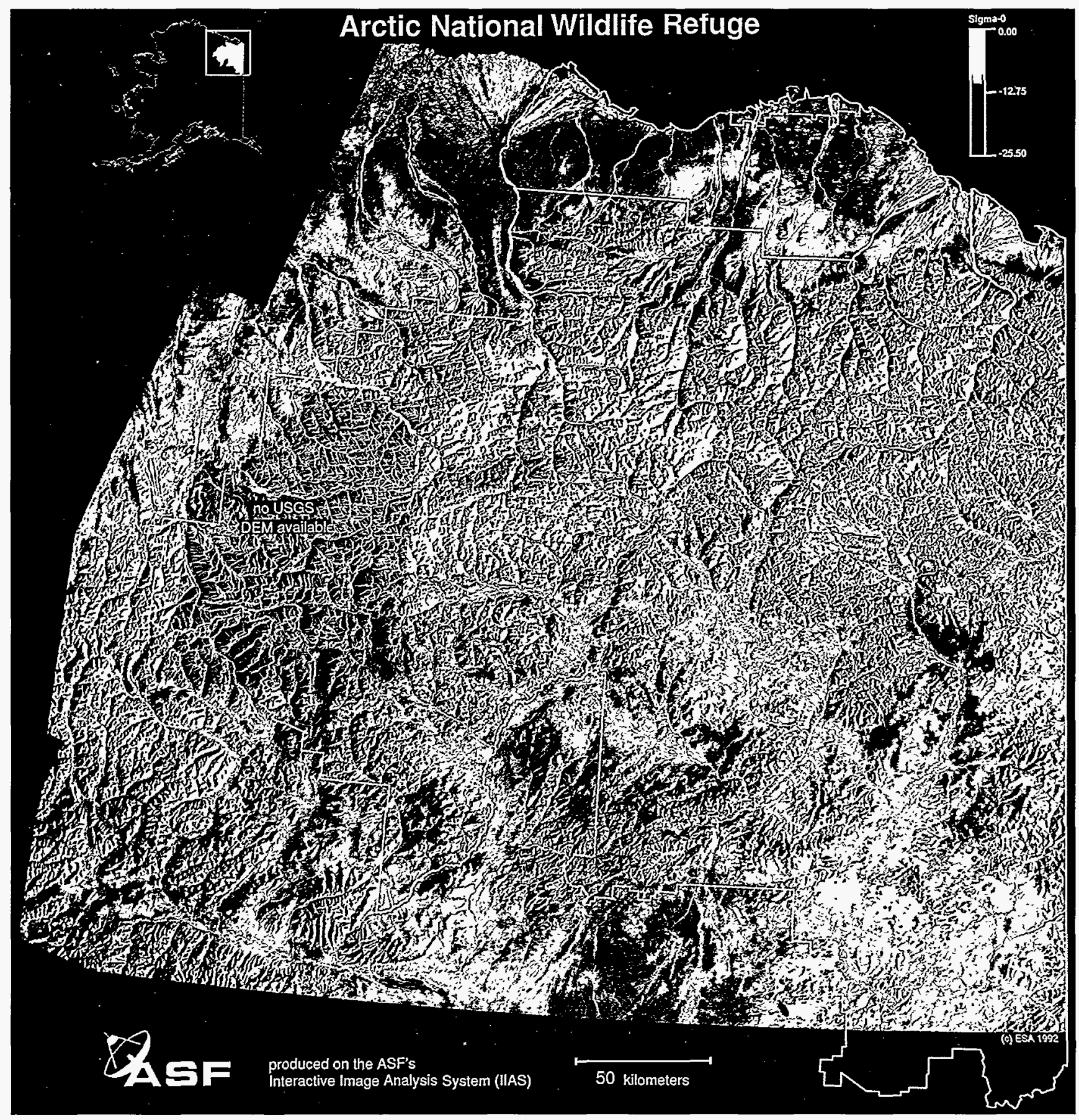

To test the parallel terrain correction code, a mosaic of the Arctic National Wildlife Refuge was created and successfully compared with this ANWR mosaic, which was made during 1993 using the ASF STEP lab facilities. 
Funded by Cray Research, Inc.: Scientific personnel-T. Logan, S. Li, R. Guritz, and C. Olmsted

AVHRR Satellite Data Receiving Station: This project operates a satellite receiving station that receives, processes, archives, and distributes image data from satellites that operate medium-resolution earth sensors in polar orbits.

Thestation provides real-time digital images for analysis by researchers and students to address dynamically changing natural events, including ongoing research and monitoring key Alaska resources.

The satellite receiving station is the automated High Resolution Picture Transmission (HRPT) type; it receives visible and infrared images from Advanced Very High Resolution Radiometer (AVHRR) sensors on two NOAA weather satellites. HRPT refers to the particular kind of L-band telemetry downlink and modulation schemes used by the NOAA weather satellites to transmit images to inexpensive ground starions. An AVHRR sensor covers very large regions with high spatial resolution relative to the area covered.

The directly acquired digital data coming into this station are mosaicked into synoptic images showing regional coverage from the Russian Far East well into western Canada. The displays are updated hourly, using unique techniques developed here. Individual image swaths as well as the mosaicked regional coverage are available on-line and can be downloaded by desktop computers anywhere within the university system, as well as by the World Wide Web on Internet.
Sponsored by the Natural Resources Fund, University of Alaska and Geophysical Institute: Scientific personnel-J.M. Miller, K.G. Dean, K. Engle, and S. George

Cryospheric System for Monitoring Global Change in Canada: There are three objectives for EOS proposed in this work which follow from Canadian work in preparation for ERS- 1 and RADARSAT.

The first is to continue to develop and implement the algorithms necessary to extract geophysical information related to the cryosphere. The second is to access information from other related data bases, especially in Canada, so that a complete record of information on cryospheric processes can be obtained. This includes historical data bases, operational information, and future products. The third objective is to use the information from these sources in models to examine the effects of global change as manifested by cryospheric processes. This includes the dissemination of the pertinent data to investigators involved in modeling global change, and access to the archive catalog of data produced by the information systems in the $\mathrm{Ca}$ nadian system.

Supported through NASA and Atmospheric Environment Service, Canada: Scientific personnel-L. McNutt; B. Goodison (Atmospheric Environment Service, Canada); J. Falkingham (Ice Centre, Environment Canada); J. Chilar (Canada Centre for Remote Sensing); R. Keeley (Canada Department of Fisheries and Oceans); H. French (University of Ottawa); and R. Koerner (Geological Survey of Canada)
A Two-way Transformation Between the Geographical Coordinates and the ASF SAR Image Coordinates: A two-way algorithm has been developed to implement a fast, yetaccurate, conversion between geographical and ASF SAR image coordinates, given the geographical locations of the corner points and the center of the image.

The concerned area is mapped under a local orthographic projection onto an intermediate plane tangential to the image center. The mapping between the geographic and intermediate coordinates involves a rotation of coordinate systems followed by a separation of the horizontal coordinates from the vertical one.

The mapping between the intermediate and image planes is approximated by a set of simple quadratic equations. Linkages between the geographical and intermediate coordinates and between the intermediate and image coordinates are thus established for the control points, and conversion coefficients and functions derived. Then a twostep mapping allows an easy twoway conversion between the two-end coordinate systems.

The algorithm is used at ASF for determining the locations of features of concern to users on the ASF SAR images and in the fields to improve field data sampling and data analysis procedure.

Also, this algorithm is included into other algorithms to create image mosaic strips along track, and to coregister image or image strips to form a time series for image animation.

NASA Contract NAS5-32393: Scientific personnel-S. Li 


\section{Other \\ Projects}
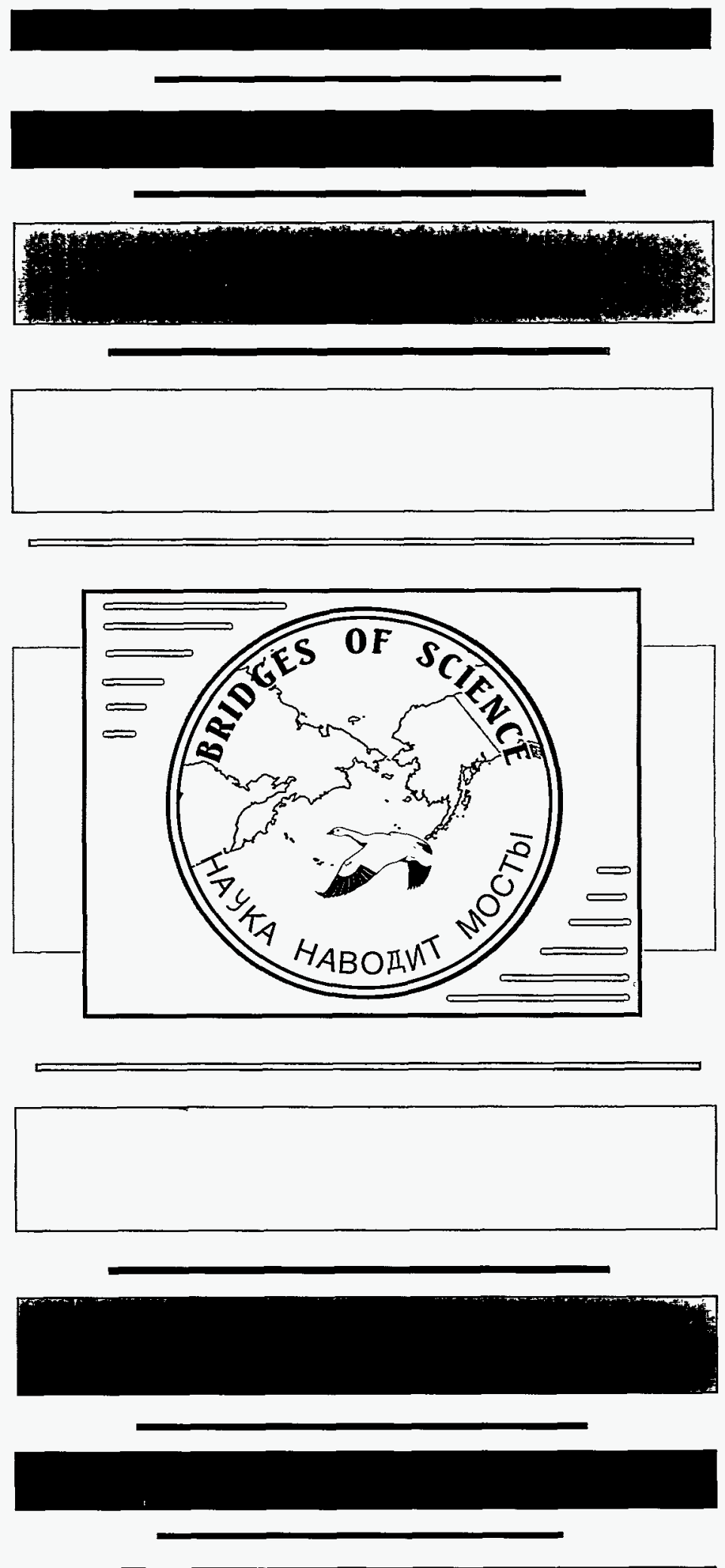


\section{OTHER PROJECTS}

Collaboration with Japanese Scientists on Studying the Arctic Environment: Several researchers at the institute are collaborating with Japanese scientists on issues relating to the arctic environment.

In November 1994, R. Collins and G. Shaw actended an International Symposium on the Arctic Environment at Nagoya University and a workshop on the Arctic Environment at Fukuoka University. Visits also were made to the College of Science at Tohoku University in Sendai and to the Communication Research Laboratory in Tokyo.

Geophysical Institute and Japanese scientists are collaborating on several research projects. The projects include investigations of the lower atmosphere and stratosphere with a laser radar (lidar) system from $\mathrm{Na}$ goya Universiry, snow and air contamination studies with Nagoya and Fukuoka universities and investigations of the middle atmosphere with researchers from the Communication Research Laboratory.

Scientific personnel: G.E. Shaw and R. Collins

The Center for Global Change and ArcticSystem Research: The Center for Global Change and Arctic System Research at UAF formed in 1990 to provide a campus-wide framework for developing, coordinating, and implementing interdisciplinary research initiatives that address the role of the Arctic in the earth system.

The center's scientific focus is to understand the chain of physical, biological, and social processes in the arctic system that interact with the rotal earth system and global change. Its aim is to improve predictions of environmental changes on decade to century time scales, and to help formulate policy options and engineering solutions in response to anticipated impacts.

These efforts are guided by a ScienceSteering Committee, composed of faculty with expertise in areas such as agriculture, forestry, ecology, political science, engineering, geophysics, marine science, anthropology, archaeology, climatology, and economics.

Several working groups have been established to address a variety of research themes and topics in education. Interdisciplinary proposals have been submitted to several agencies.

The center now serves as the Science Management Office for the Land-Atmosphere-Ice Interaction (LAII) studies of NSF's Arctic System Science Program. The center also houses and staffs the Cooperative Institute for Arctic Research, established in 1994.

State of Alaska funds: Scientific personnel-G. E. Weller (scientific director), P. Anderson (associate director), and $K$. Van Cleve (chairman of scientific steering committee, UAFForestry Sciences Department). The LAII$S M O$ is funded by NSF Grant OPP-9403647.

Cooperative Institute for Arctic Research (CIFAR): The Cooperative Institute for Arctic Research, or CIFAR, was established through a Memorandum of Understanding signed by NOAA Administrator Jim Baker and University of Alaska President Jerome Komisar in April 1994.

CIFAR is the latest of eight national NOAA-University joint institutes; the oldest is CIRES, the Cooperative Institute for Research in Environmental Sciences in Boulder, Colorado, formed in 1967. The institutes promote closer cooperation between researchers from NOAA laboratories and universities. CIFAR is the only cooperative institute exclusively concerned with arctic studies; it works closely with NOAA's Pacific Marine Environmental Laboratories (PMEL) in Seatrle.

Research topics include atmospheric studies, climate dynamics and variability, hydrographic studies and sea-ice dynamics, tsunami research and prediction, fisheries oceanography, and environmental assessment, monitoring and numerical modeling. Base funding from NOAA supports the studies; an Office of Naval Research grant funds monitoring and assessing arctic Alaska contaminants.

An executive board with members from NOAA and the university sets policy for CIFAR. Proposals can be submited through CIFAR; a Council of Fellows makes recommendations on proposed and future research. CIFAR is collocated with and staffed by UAF's Center for Global Change and Arctic System Research.

NOAA Grant NA57RJ0046: Scientific personnel-G.E. Weller (director), and P. Anderson (associate director); ONR Grant NO001494-1-1028: R. Benner, S. George, and G. Shaw; T. DeLaca (UAF Office 
of Arctic Research); J. Kelley (UAF

Institute of Marine Science)

International Bridges of Science Conference, Vladivostok, Russia: The annual Arctic Science Conference organized by the Arctic Division of the American Association for the Advancement ofScience occurred in two cities in 1994. The introductory sessions in Anchorage were followed by the main meeting in Vladivostok. The Far East Branch of the Russian Academy of Sciences was the host in Vladivostok. More than 500 abstracts of papers were submitted; the attendance list included 269 Russians and 108 North Americans.

The conference covered five major scientific areas: biological sciences (resources, biodiversity, ecosystems, flora and fauna, fisheries), physical sciences (climate change, contaminants, technology), Beringian Heritage International Park (geology, biogeography and paleogeography), peoples and cultures (archaeology, ethnology, folklore, socioeconomic development, and human migrations) communications, and information exchanges.

Russians and North Americans made many individual contacts during the technical sessions, poster presentations, discussion groups, and excursions, which was the primary goal of this conference, and others that may follow.

State of Alaska funds, UA President's Natural Resources Fund, and U.S. Park Service Travel Grant, plus grants from NSF, U.S. Department of State and EPA to AAAS headquarters in Washington D.C.: Conference organizers-P. Anderson, G.E. Weller; R. Meehan (USFWS)
International Conference on Arctic Margins, ICAM94: The Solid Earth Sciences Group of the Geophysical Institute and the U.S. Minerals Management Service (MMS) cohosted the ICAM94 meeting in Magadan, Russia, remorely. The North East Science Center of the Russian Academy of Sciences served as the on-site host.

Travel money was scarce, but the meeting attracted 64 foreign attendees out of 140 registered participants. Russian attendees came from many parts of the country, including Moscow, St. Petersburg and Yakutsk.

One hundred fifty papers were presented (out of 270 abstracts submitted) on a wide range of geologic topics related to the arcric margins. These included presentations in workshops, oral and poster sessions, and a roundtable discussion about a proposed coordinated oil and gas lease sale in the Chukchi Sea to be conducted by the North East Petroleum Operating (NEPO) Agency in Magadan, the Russian Committee on Geology, and the MMS. Milestone dates for the proposed salel tender are to be determined.

The proceedings volume for the ICAM meeting is expected to be published in 1996 by the Russian Academy of Sciences. Abstract volumes are available from the North East Science Center in Magadan.

Funded by the Alaska Geological Society, the U.S. Minerals Management Service, Exxon Production Research Company, the North East Petroleum Operating Agency, Union Texas Petroleum Company, and the International Science Foundation: Conference organizers-D.K. Thurston (USMMS); D.B. Stone (Geophysical
Institute); and K. Fujita (Michigan State University)

The 3rd Circumpolar Symposium on Remote Sensing of Arctic Environments (University of Alaska Fairbanks, 16-20 May 1994): More than 100 scientists from the international community met at the University of Alaska Fairbanks on 16-20 May 1994 to attend the symposium, which was designed to promote interdisciplinary discussion and understanding among physical, biological, social and applied scientists working on arcric and antarctic problems using remote sensing data.

Both environmental monitoring techniques and results from recent accomplishments and activities were presented, discussed and debated. The symposium had a workshop atmosphere conducive to interactions among researchers, governmental agencies and the local community.

Remote sensing systems discussed included using SAR, ground-based radar, passive microwave, AVHRR, Landsat and GIS techniques. Topics discussed included geologic mapping in ANWR, diamonds in Canada, interferometry of the Bering Glacier, analysis of volcanic hazards, modeling using remote sensing data, sea ice morphology and dynamics, snow studies, reindeer habitats and lichen land cover, analysis of boreal forests and tundra, oceanographic studies in the Bering, Beaufort and Greenland seas, detection of wind shear and turbulence, measurements of gas fluxes, meteorites in Antarctica, and northern sea routes.

After meetings, participants took diverse tours and field trips showing remote sensing systems and Alaska 
lifestyles and cultures. Tours included a satellite-data receiving station and image processing systems at the Alaska SAR Facility (ASF), the University of Alaska Museum, and a tunnel into permafrost providing an in situ glimpse of ice lenses, wedges and frozen ground.

The fourth symposium, to be held in Copenhagen in 1996, will be hosted by Preben Goodmansen. Due to the increasing Antarctic research presented at the symposiums, the title will change to the Circumpolar Symposium of Remote Sensing of Polar Environments.

The symposium wassponsored by the Geophysical Institute, NSF, NASAI ASF, NOAA, NWS, and CRRELL: Conference chair-K.G. Dean

International Coordination of the Implementation Phase of the SolarTerrestrial Energy Program-A Community Initiative: The purpose of this project is to help the PI carry out his duties as the international coordinator for the Solar-Terrestrial Energy Program (STEP).

STEP has been conceived as an ambitious and complex program of international cooperation under the guidance of the Scientific Committee on Solar Terrestrial Physics (SCOSTEP). Research councils in Japan, Sweden and Germany have agreed to provide support for the coordinator's office operations, publications and travel, which at present amounts to approximately $\$ 36,500$ per year. The Geophysical Institute is the fiscal agent for this support.

The following are the most important tasks for the International STEP Coordinator: to sell STEP to government agencies, to the public and to the scientists themselves in participating countries; to learn firsthand about the principal STEP-related research programs of the different groups in participating countries and to propose ways to integrate such programs into the international effort; to brief government agencies in participating countries on the progress of STEP and on the significance of the scientists' contributions to this program; to identify problems in participating countries specifically related to international coordination and to advise their scientists on possible solutions; to propose specific actions and recommendations to SCOSTEP in support of those national plans for STEP that would benefit from such official action; to keep informed about the development of other international programs such as the International Geosphere-Biosphere Program (IGBP) and the World Climate Research Program (WCRP) and to propose some means to foster working relationships with STEP.

During 1993 and 1994, the project PI visited Australia, Argentina, Brazil, Chile, Mexico, Cuba, Taiwan, Austria and Russia to accomplish the above coordination tasks. Numerous recommendations were proposed to research groups and government agencies in these countries.

NSF Grants ATM 12638 and ATM 04200: Scientific personnel-J.G. Roederer

Research Experience for Undergraduates (Summer Intern Program): These projects provide support for undergraduate students to participate in scientific research during a ten-week intern program at the
Geophysical Institute. Students work under the supervision of faculty members and are given hands-on research experience in a graduate school setting. The program is open to undergraduates majoring in physics, geophysics, geology, or related physical science programs. The goal is to encourage students to go to graduate school and to pursue research careers.

Two intern programs run at the Geophysical Institute. The original program, which focuses on atmospheric and space sciences, started in 1987. A second project, which focuses on solid earth geophysics, began in 1994. During the summers of 1993 and 1994, 17 and 24 interns, respectively, were accommodated.

The interns come from colleges across the country. The program relies primarily on mentoring by faculty members who assign projects, provide guidance and are responsible for providing necessary facilities. Most faculty members outline a single self-contained project, enabling the intern to finish the program with a sense of accomplishment.

A series of weekly lectures composes the more formal part of the program. During these lectures, faculty and support staff members representing various subfields and activities present overviews of their research.

The lectures serve to expose the interns to the broad range of research conducted and to the infrastructure supporting research at the Geophysical Institute. There are usually eight formal presentations, along with visits to shops and facilities. Field trips are conducted to various sites and natural attractions, including Poker 
Flat Research Range and the permafrost tunnel. The interns are encouraged to attend seminars and thesis defenses and to participate in various social functions.

At the end of the program each intern presents a detailed report; some deliver it orally. Exit interviews conducted by senior administrative staff have revealed that interns find their time in Alaska educationally and recreationally worthwhile.

NSF Grant ATM92-00570: Administrative personnel-D.W. Swift, R.W. Smith and G.E. Shaw; and NSF Grant EAR93-22471: Administrative personnel-D.H. Christensen, P.W. Layer, and D.B. Stone

The Private Industry Council: The Geophysical Institute Keith B. Mather Library continues to participate in the Private Industry Council summer program. Students are selected to assist the library in shelving, circulation, binding, filing, and new book processing.

The program is intended to develop participants' skills and to enhance their job opportunities as library technicians. Selected participants in 1993 were Loretta Linus and Amber Thomas; selected participants in 1994 were Loretta Linus and Sandra Laflin.

Fairbanks Private Industry Council funds: Library personnelJ. Triplehorn

Geophysical Exploration for a Buried Ivory Cache on Campus: During the 1930s, the Fairbanks Exploration Company removed a tremendous amount of overburden for placer gold mining dredges in the
Fairbanks district. The loess was removed by intense streams of water directed into the material. As the silt was removed, often the remains of Pleistocene animals were revealed, which included many mammoth and mastodon tusks.

The University of Alaska collected these specimens during the summer, employing students to drive the "bone wagon." Neil Davis, in his book The College Hill Chronicles, relates: "The bone wagon was finding so many mammoth tusks that the world's museums were refusing to accept any more of them, and so the tusks were piling up on the campus."

Locals also took the tusks to use as yard decorations or to cut up into ivory.

In the summer of 1938 , to stop the losses, Leo Rhode, a former University of Alaska Regent, participated in the digging of an approximately sixfoot deep pit in which about 30 of the best tusks were buried for safe storage. As the years went by, the location of the hole was forgotten.

To an exploration geophysicist, this presented an interesting problem: What known or new techniques can be used to locate the cache?

Rhode helped narrow the area of search by indicating on aerial photos and maps where he remembers the location of the cache to be.

While digging the pit, he remembers looking across a narrow road into a small warehouse containing parts of Ben Eielson's aircraft. That warehouse no longer exists, but the site where it used to stand appears to be at the intersection of Taku Drive and Tanana Loop, near the U.S. Forest Service building . In addtion to the roadway, postburial construc- tion has added at least five feet of fill over the site.

The existing method most likely to produce results in searching for the cache is ground penetrating radar (GPR). GPR systems operate using pulses of electromagnetic waves for taking profiles of near surface materials (soils, rocks, pavement, hazardous waste dumps, etc.).

A transmitting antenna directs a pulse into the ground, and the signal is reflected off subsurface discontinuities in the density and electrical conductivity. The reflected waves received by a second antenna are recorded on a computer for display and processing.

In the summer of 1994, radar surveys were run using equipment borrowed from the U.S. Army Corps of Engineers Cold Regions Research and Environmental Laboratory. An anomaly that might be the old pit containing the tusks was found under the intersection of Taku Drive and Tanana Loop.

Before test drilling, however, more historical searching needs to be done to narrow the area of investigation; primarily we want to identify the prior location of the warehouse Rhode remembers as the harborer of Ben Eielson's aircraft.

Photos of two paleontology labs with an unknown person sitring on the dock of one thought to be the aircraft warehouse will be published in the University of Alaska alumni magazine to encourage possible recognition and identification of the site.

University of Alaska President's Special Project Fund, Project 1302: Scientific personnel-E.M. Wescott, K. Kawasaki and T.N. Davis 


\section{Staff}

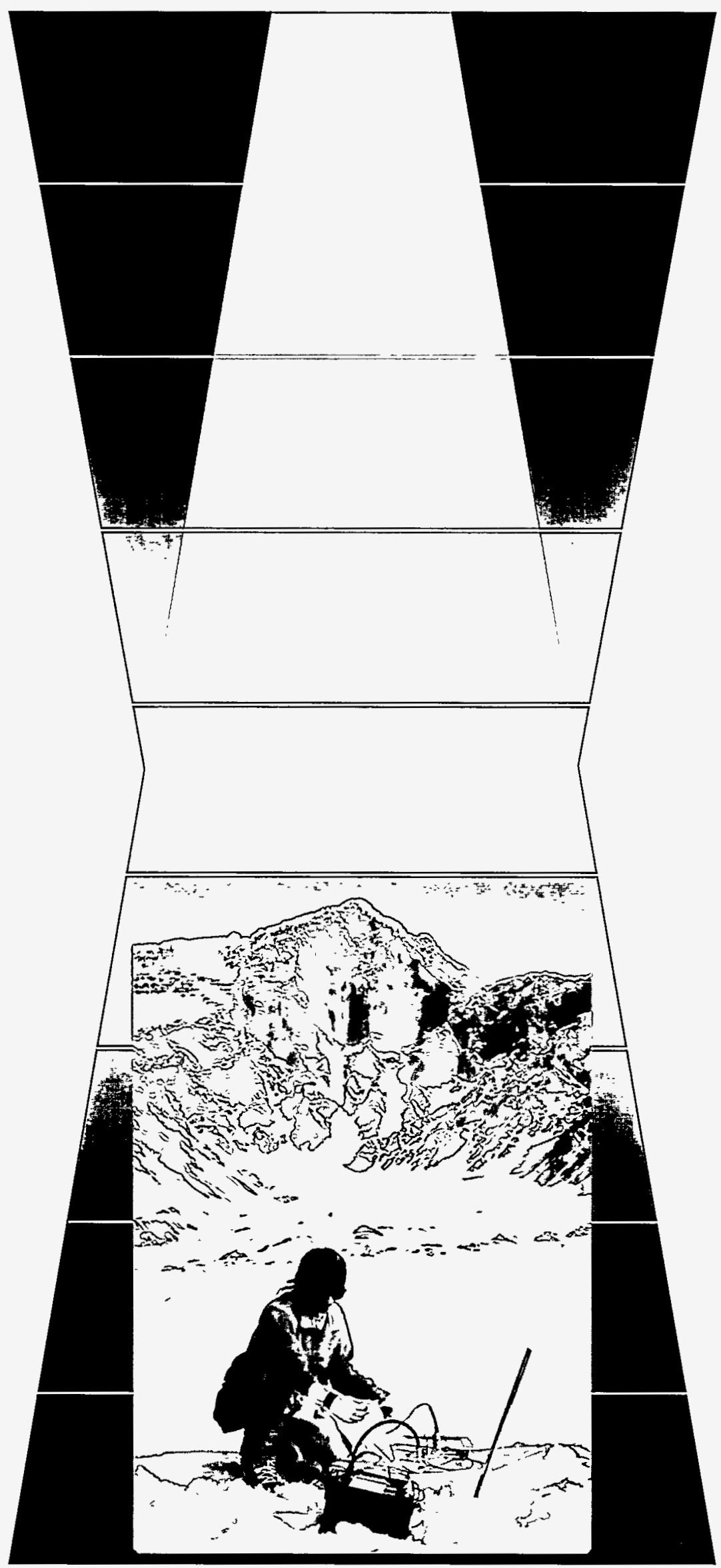




\section{Faculty and Scientific Staff Listing by Group}

\section{Space Physics}

Syun-Ichi Akasofu, Professor of Geophysics and Geophysical Institute Director

Neal Brown, Research Assistant Professor of Geophysics

Richard Collins, Research Assistant Professor of Space Physics and Aeronomy

John Craven, Professor of Physics

Charles Deehr, Professor Emeritus of Geophysics and Science Director of Poker Flat Research Range

Thomas Hallinan, Professor of Geophysics

Joseph Hawkins, Associate Professor of Electrical Engineering and Alaska Space Grant Program Director

Robert Hunsucker, Professor Emeritus of Physics and Electrical Engineering

Joseph Kan, Professor of Physics

Lou-Chuang Lee, Professor of Physics

Dirk Lummerzheim, Research Associate Professor of Aeronomy

John Olson, Professor of Physics

Antonius Otto, Research Associate Professor of Space Physics

Manfred Rees, Professor Emeritus of Geophysics

Juan Roederer, Professor Emeritus of Physics

Davis Sentman, Associate Professor of Physics (also see Atmospheric Sciences)

Roger Smith, Professor of Physics

Hans Stenbaek-Nielsen, Professor of Geophysics

Daniel Swift, Professor Emeritus of Physics

Brenton Watkins, Professor of Physics (also see Atmospheric Sciences)

Daniel Weimer, Research Associate Professor of Space Physics

Eugene Wescott, Professor of Geophysics

\section{Atmospheric Sciences}

Richard Benner, Assistant Professor of Chemistry

Sue Ann Bowling, Assistant Professor of Geophysics

Victor Filyushkin, Research Associate of Atmospheric Sciences

Daniel Jaffe, Associate Professor of Chemistry

Elena Leontieva, Research Associate of Atmospheric Sciences

Amanda Lynch, Assistant Professor of Atmospheric Sciences

Takeshi Ohtake, Professor Emeritus of Geophysics

Davis Sentman, Associate Professor of Physics (also see Space Physics)

Glenn Shaw, Professor of Physics

Knut Stamnes, Professor of Physics

John Walsh, Visiting Wadati Research Professor

Brenton Watkins, Professor of Physics (also see Space Physics)

Gunter Weller, Professor of Geophysics, Geophysical Institute Deputy Director and Center for Global

Change and Arctic System Research Director (also see Snow, Ice and Permafrost)

Gerd Wendler, Professor of Geophysics (also see Snow, Ice and Permafrost)

Tingjun Zhang, Post-Doctoral Fellow (also see Snow, Ice and Permafrost)

Snow, Ice and Permafrost

Kristina Ahlnäs, Remote Sensing Specialist (also see Remote Sensing)

Carl Benson, Professor Emeritus of Geophysics and Senior Research Consultant

Keith Echelmeyer, Associate Professor of Geophysics

William Harrison, Professor of Physics

Martin Jeffries, Research Associate Professor of Geophysics (also see Remote Sensing)

Koji Kawasaki, Associate Professor of Geophysics

Craig Lingle, Research Associate Professor of Geophysics (also see Remote Sensing)

Thomas Osterkamp, Professor of Physics 
William Sackinger, Associate Professor of Geophysics and Electrical Engineering

Tatsumi Sasaki, Visiting Scientist (on sabbatical from University of Hokkaido)

Lewis Shapiro, Senior Research Consultant (also see Remote Sensing)

Vera Voronina, Geophysicist

Wilford Weeks, Professor of Geophysics (also see Remote Sensing)

Gunter Weller, Professor of Geophysics, Geophysical Institute Deputy Director and Center for Global

Change and Arctic System Research Director (also see Atmospheric Sciences)

Gerd Wendler, Professor of Geophysics (also see Atmospheric Sciences)

Tingjun Zhang, Post-Doctoral Fellow (also see Atmospheric Sciences)

\section{Seismology}

Niren Biswas, Professor of Geophysics

Douglas Christensen, Associate Professor of Geophysics

Kazuya Fujita, Adjunct Professor of Geophysics (Michigan State University)

Bob Hammond, USGS Geophysicist

Roger Hansen, State Seismologist, Research Professor of Geophysics

John Lahr, Affiliate Professor of Seismology (USGS, Menlo Park)

Stephen McNutt, Research Professor and Volcano Seismologist (also see Volcanology)

Hans Pulpan, Associate Professor of Geophysics

Charlotte Rowe, Deputy State Seismologist

Max Wyss, Wadati Professor of Seismology

Tectonics and Sedimentation

Keith Crowder, Associate Professor of Geology

Catherine Hanks, Research Assistant Professor of Geology

Paul Layer, Associate Professor of Geophysics

David Stone, Professor of Geophysics

Wesley Wallace, Associate Professor of Geology

Keith Watts, Associate Professor of Geology

\section{Volcanology}

Kenneson Dean, Research Assistant Professor of Geology (also see Remote Sensing)

John Eichelberger, Professor of Volcanology

Juergen Kienle, Professor of Geophysics

Stephen McNutt, Research Professor and Volcano Seismologist (also see Seismology)

Christopher Nye, Research Assistant Professor of Geology, ADGGS

\section{Remote Sensing}

Kristina Ahlnäs, Remote Sensing Specialist (also see Snow, Ice and Permafrost)

Kenneson Dean, Research Assistant Professor of Geology (also see Volcanology)

Martin Jeffries, Research Associate Professor of Geophysics (also see Snow, Ice and Permafrost)

Shusun Li, Research Assistant Professor of Geophysics

Craig Lingle, Research Associate Professor of Geophysics (also see Snow, Ice and Permafrost)

Lyn McNutt, Research Associate Professor, ASF Chief Scientist

John Miller, ASF Senior Adjunct Applications Engineer Emeritus

Lewis Shapiro, Senior Research Consultant (also see Snow, Ice and Permafrost)

William Stringer, Associate Professor of Geophysics

Carl Wales, ASF Project Director

Wilford Weeks, Professor of Geophysics (also see Snow, Ice and Permafrost)

Poker Flat Research Range

Charles Deehr, Science Director 


\section{Faculty and Scientific Staff}

KRISTINAAHLNÄS, Remote Sensing Specialist; University of Helsinki, '60, B.S., '61, M.S. Ahlnäs' past research activities include physical oceanography and marine geology. Ahlnäs joined the Geophysical Institute in 1973 to work with geochronology, paleomagnetism, glaciology, meteorology and volcanology. Ahlnäs investigated the use of satellite data for Alaska and for sea ice studies in Antarctica when, respectively, the NOAA-VHRR satellite became operational and a NOAA-station was established in McMurdo, Antarctica. Analyzing Landsat TM-data, she discovered multiple dipole eddies in the Gulf of Alaska. Since the launch of the ERS-1 SAR satellite in 1991, Ahlnäs has been operated ASF's geophysical processor system (GPS). Ahlnäs' current research interests include the analysis and color enhancement of SAR data for glaciers in Alaska and dipole eddies around St. Matthew Island in the Bering Sea.

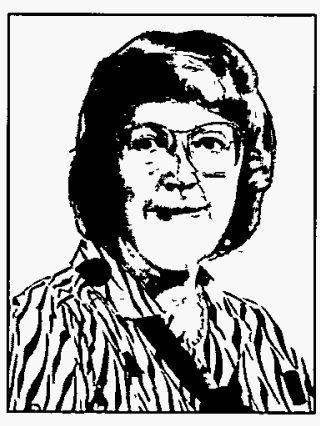

Ahlnäs, K. 1994. Nanoq-The Arctic Museum of Finland. Information North, Arctic Institute of North America, v. 20(1), pp. 4-5.

Ahlnäs, K. 1994. Tidally Generated Dipole Eddies Around St. Matthew Island, Bering Sea. Report Series in Geophysics of the University of Helsinki, v. 28, pp. 11-17.

Ahlnäs, K., and C.S. Lingle. 1993. Computer-Enhanced Synthetic Aperture Radar (SAR) Images of the Malaspina Glacier, the Bagley Icefield and the Logan-Walsh Glacier System in the St. Elias Mountains of Alaska and the Yukon Territory. Cover of Geophysical Institute Biennial Report, 1991-1992.

Dean, K.G., W.J. Stringer, K. Ahlnäs, C. Searcy and T. Weingartner. 1994. The Influence of River Discharge on the Thawing of Sea Ice, Mackenzie River Delta: Albedo and Temperature Analyses. Polar Research, Norsk Polarinstitutt, Oslo, v. 13(1), pp. 83-94.

SYUN-ICHI AKASOFU, Geophysical Institute Director since 1 September 1986, and Professor of Geophysics; Tohoku University '53, B.S., '57, M.S.; University of Alaska '61, Ph.D. Dr. Akasofu, who specializes in space physics, joined the Geophysical Institute in 1958 as a graduate student. He continues to be involved in studies of the aurora and the magnetosphere. His study of global auroral activities in 1964 has become the basis of the discipline of magnetospheric substorms. As Director, he has been instrumental in establishing a number of new research activities. He was named among the 1,000 most-cited contemporary scientists in 1981, and he received the Chapman Medal from the Royal Astronomical Society of London in 1976. He also received the Japan Academy Award in 1977, the John Fleming Medal from the American Geophysical Union in 1979, the University of Alaska Distinguished Alumnus Award in 1980, and the first Chapman Chair Professorship of the University of Alaska in 1985.

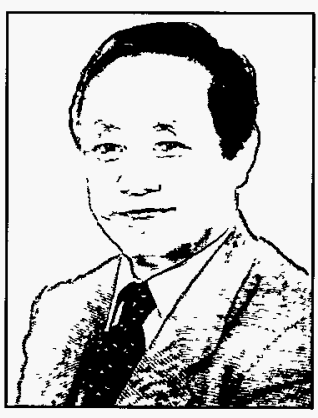

In addition, he was selected as the Alaska Centennial Alumnus by the National Association of State Universities and Land Grant Colleges in 1987, and he received the Japan Foreign Minister Award in 1993.

Akasofu, S.-I. 1994. Assessing the Magnetic Reconnection Paradigm. EOS, v. 75(22), pp. 249-252.

Akasofu, S.-I. 1994. Geospace Environment Data Display System (GEDDS): A New Way to Integrate Ground-based and Satellite-based Observations and Theoretical Modelings. Proceedings of COSPAR Colloquium No. 5, 1992 STEP Symposium.

Akasofu, S.-I. 1994. Past, Present and Future of Substorm Research. Proceedings of the International Conference on Substorms (ICS-2), 7-11 March, Fairbanks, Alaska, pp. 13-26.

Akasofu, S.-I. 1994. The Arctic Circle: A Treasure House of Earth Science. Springer Science, v. 9(4), pp. 2-6.

Akasofu, S.-I. 1994. The Shape of the Solar Corona. Sky and Telescope, v. 88, pp. 24-27.

Akasofu, S.-I. 1993. Linking Ground- and Space-based Observations in Space Physics. EOS, v. 74(23), pp. 259-260.

Akasofu, S.-I. 1993. Space Research From the Ground. NSF Directions, Spring Issue, v. 6(1), pp. 8-9.

Akasofu, S.-I., and J.B. Townshend. 1993. 50 Years of Service. EOS, v. 74(29), pp. 235.

Akasofu, S.-I., and T. Saito. 1993. Reversals of the Solar Source Surface Magnetic Field and of the Planers. Flow and Creep in the Solar System: Observations, Modeling and Theory, D.B. Stone and S.K. Runcorn (Eds.), pp. 45-57. 
Dean, K., S.-I. Akasofu and H. Tanaka. 1993. Volcanic Hazards and Aviation Safery: Developing Techniques in Alaska. Aviation Safety Journal, v. 3(1), pp. 11-15.

Garrido, D.E., R.W. Smith, D.W. Swift, S.-I. Akasofu, R.M. Robinson and Y.T. Chiu. 1994. Imaging the Plasmasphere and Trough Regions in the Extreme Ultraviolet Region. Optical Engineering, v. 33(2), p. 371.

Iijima, T., M. Watanabe, T.A. Potemra, L.J. Zanetti, J.R. Kan and S.-I. Akasofu. 1993. Substorm Currents in the Equatorial Magnetotail. Journal of Geophysical Research, v. 98(A10), pp. 17283-17298.

Lee, L.C., L. Zhang, M. Yan, H.J. Cai, G.S. Choe, A. Otto and S.-I. Akasofu. 1994. Formation of Very Thin Current Sheet During Substorm Growth Phase and Plasma Dynamics in the Recovery Phase. Proceedings of the International Conference on Substorms (ICS-2), 7-11 March, Fairbanks, Alaska, p. 143-148.

Sun, W., Y. Kamide, J.R. Kan and S.-I. Akasofu. 1993. Inversion of the Auroral Electrojets from Magnetometer Chain Data Based on the Flexible Tolerance Method. Journal of Geomagnetism and Geoelectricity, v. 45, pp. 1151 1157.

Saito, T., S.-I. Akasofu, Y. Kozuka, T. Takahashi and S. Numazawa. 1993. The Solar Coronal Sheet During the Period of Sunspot Maximum. Journal of Geophysical Research, v. 98(A4), pp. 5639- 5644.

Tanaka, H.L., K.G. Dean and S.-I. Akasofu. 1993. Predicting the Movement of Volcanic Ash Clouds. EOS, Transactions of the American Geophysical Union, v. 74(20), p. 231.

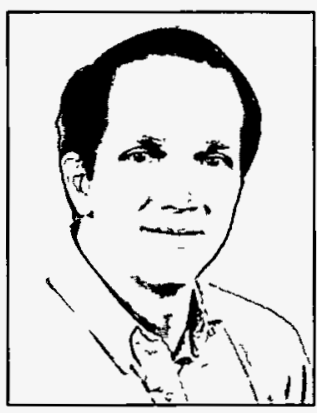

RICHARD L. BENNER, Assistant Professor of Chemistry; State University of New York at Oswego'81, B.S.; Washington State University'85, M.S.; University of Denver'91, Ph.D. Dr. Benner came to the institute in 1991 as a joint appointment with the Department of Chemistry. His research interests include the biogeochemical cycling of sulfur species, parcicularly in the Arctic. These investigations require the development of new analytical instrumentation and measurement techniques to allow direct determination of surface flux of naturally occurring trace sulfur gases. Another area that he is currently active in is measurement of the chemical composition of atmospheric aerosols and the role of composition on heterogeneous chemical reactions and cloud nucleating properties. These investigations also involve development of new instrumental techniques for the purpose of making low level measurements of species important to gaseous and aerosol chemistry in the atmosphere. Adaptation and optimization of chemical instrumentation for the purpose of making meaningful chemical measurements from aircraft platforms.

Benner, R.L., and D.H. Stedman. 1994. Chemical Mechanism and Efficiency of the Sulfur Chemiluminescence Detecror. Applied Spectroscopy, v. 48(7), pp. 848-851.

Benner, R.L., and D.A. Veazey. 1994. Automobile Emissions and Control Strategies for Cold Temperature Communities. Association of Finnish Civil Engineers, Fourth International Symposium on Cold Region Development Proceedings, June 13, 1994, p. 327.

Schorren, D.E., C. Fought, D.F. Miller, W.G. Coulombe, R.L. Benner and D.H. Stedman. 1994. A Quasi-real-time Detection System for Low Part-per-trillion Levels of Sulfur Dioxide in the Atmosphere. Environmental Science and Technology, v. 28, pp. 1307-1311.

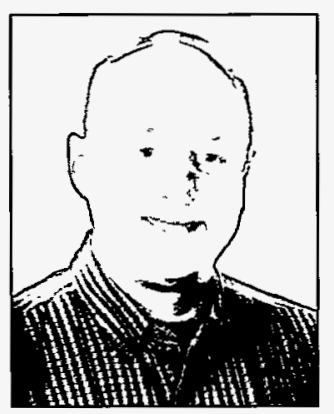

CARL S. BENSON, Professor of Geophysics and Geology Emeritus; University of Minnesota '50, B.S., '56, M.S.; California Institute of Technology '60, Ph.D. Dr. Benson's research background is in glaciology. His past activities include glaciological research on the ice sheets of Greenland and Antarctica. After joining the Geophysical Institute in 1960, he has investigared the seasonal snow cover of interior and arctic Alaska, low temperature air pollution problems (including ice fog), the freezing of small turbulent streams, and glacier mass balance problems on the McCall Glacier of the Brooks Range. Dr. Benson also has researched glacier-volcano interactions in the Wrangell Mountains and on the west side of Cook Inlet. Professor Benson taught in the Department of Geology and Geophysics and served as Department Chairman from 1969 to 1973. 
Benson, C.S. 1994. Glacier Facies: With Special Reference to Greenland. In: Mass Balance and Related Topics of the Greenland Ice Sheet; Report of the 4th Workshop held in Amsterdam, 22-24 November 1993. Open File Series 94/13 Geological Survey of Greenland, June 1994, pp. 11-14.

Benson, C.S., G.A. Bender and S.A. Bowling. 1994. Accumulation on the Greenland Ice Sheet. In: Mass Balance and Related Topics of the Greenland Ice Sheet; Report of the 4th Workshop held in Amsterdam, 22-24 November 1993. Open File Series 94/13 Geological Survey of Greenland, June 1994, pp. 15-17.

Benson, C.S. 1993. An Overview of Research on Snow in Alaska. In: Snow Watch '92, Detection Strategies for Snow and Ice; International Workshop on Snow and Lake Ice Cover and the Climate System, held in Ontario, Canada, March 1992. Proceedings edited by R.G. Barry, B.E. Goodison, and E.F. LeDrew, p. 135-143. See also: Glaciological Data Report GD-25, World Data Center A for Glaciology.

Benson, C.S., and M. Sturm. 1993. Structure and Wind Transport of Seasonal Snow on the Arctic Slope of Alaska. Proceedings of the International Symposium on Snow and Snow-Related Problems held in Nagaoka, Japan, September 1992. Annals of Glaciology, v. 18, p. 261-267.

Lingle, C.S., C.S. Benson and T.K. Ahlnäs. 1994. Glacier Facies on Nabesna Glacier and Mt. Wrangell, Alaska, from Synthetic Aperture Radar (SAR) Data. Third Circumpolar Symposium on Remote Sensing of Arctic Environments held in Fairbanks, Alaska, 16-20 May 1994, abstract only, p. 34.

Hall, D.K., C.S. Benson and J.Y.L. Chein. 1993. Analysis of Visible and Microwave Satellite Data for Snow Mapping in Alaska. Proceedings of the 50th Annual Eastern Snow Conference, held in Quebec City, Quebec, Canada, 8-10 June 1993, pp. 129-136.

NIRENDRAN. BISWAS, Professor of Geophysics; Indian Institute of Technology, Khangpur, India '57, M. Tech.; University of California, Los Angeles'71, Ph.D. Dr. Biswas has a research background in solid earth geophysics. His past activities include basic and applied interpretation of geophysical data. After joining the Geophysical Institute in 1971, he has studied the basic and applied aspects of Alaska earthquakes and the earth's structure. Professor Biswas teaches courses in seismology.

SUE A. BOWLING, Assistant Professor of Geophysics; Radcliffe College '63, A.B.; University of Alaska '67, M.S., '70, Ph.D. Dr. Bowling's research background is in air pollution meteorology, in human influences on climate and in climatic change. After joining the Geophysical Institute in 1970, she has been involved in the study of circulation, climate, and paleoclimate in Alaska, and in the study of human influences on climate, solar-weather relationships, and applied research in air pollution. Dr. Bowling has taught dynamic meteorology and has developed and taught geophysics for nonscientists.

Bowling, S.A. 1993. Climate Change as Seen by Trees and by Climate Modelers. Forest Development in Cold Climates, J. Alden (Ed.), pp. 189-202.

NEAL B. BROWN, Assistant Professor of Physics; Washington State University '61, B.S.; University of Alaska, '66, M.S. Mr. Brown has been a participant in various upper atmospheric studies involving noctilucent clouds, and near-infrared spectroscopy of aurora and airglow. He served as Director of Poker Flat Research Range from 1971 through 1989. He has a strong interest in teaching and communicating science at the undergraduate level and to the public. He developed and ran the Alaska Space Academy for middle school students.
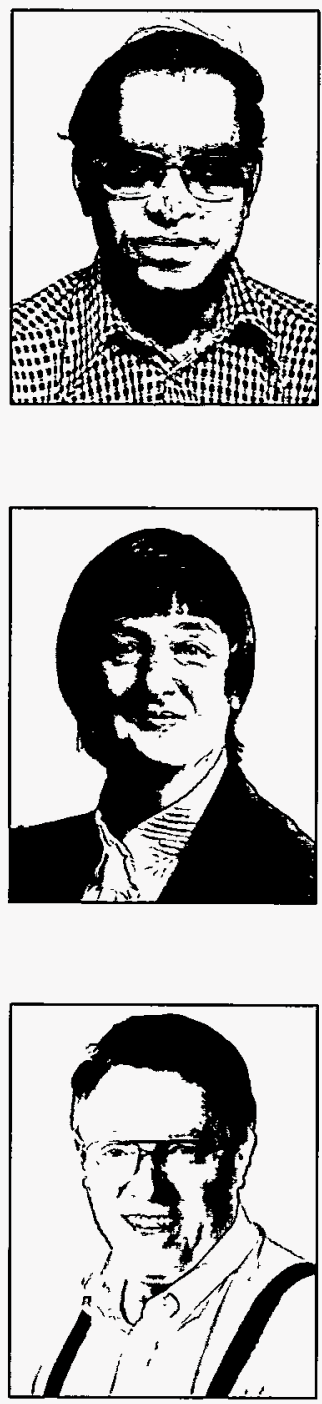


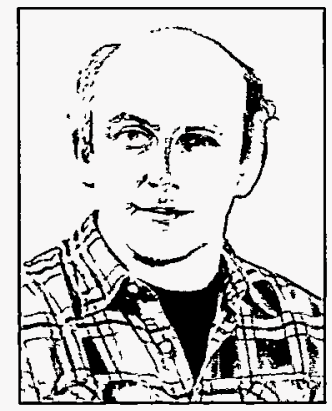

DOUGLAS H. CHRISTENSEN, Associate Professor of Geophysics; University of Utah '77, B.S.; University of Michigan '79, M.S., '87, Ph.D. Dr. Christensen joined the Geophysical Institute in 1987. His primary interests include earthquake seismology and earth structure studies. His research activities in earthquake seismology include detailed studies of earthquake sources using waveform analysis; rupture process of large earthquakes; determination and resolution of hypocentral parameters; spatial and temporal variations of interplate and intraplate seismicity; and relationship to subduction zone dynamics. Earth structure studies include an active program in tomographic imaging at both regional and local scales and the interpretation of local and teleseismic waveforms for structure and anisotropy using broadband data.

Christensen, A.B., R.L. Waterscheild, M.N. Ross, C.-I. Meng, L.J. Paxton, D.E. Anderson, G. Crowly, S. Avery, J.D. Craven, R.R. Meier and D.J. Strickland. 1944. The Global Ultraviolet Imager (GUVI) for the NASA TIMED Mission. Optical Spectroscopic Techniques and Instrumentation for Atmospheric and Space Research, SPIE, v. 2266, pp. 451-466.

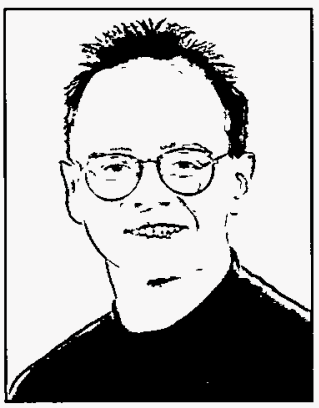

RICHARD L. COLLINS, Assistant Research Professor; University College Dublin '86, B.S.; Case Western Reserve University'88, M.S.; University of Illinois'94, Ph.D. Dr. Collins joined the institute in 1994 as a member of the space physics group. His interests include the aeronomy of the middle atmosphere, and the development of electro optic systems for remote sensing. In particular he has developed and deployed laser radar (lidar) systems to study the structure and dynamics of the atmosphere at high latitudes.

Collins, R.L., A. Nomura and C.S. Gardner. 1994. Gravity Waves in the Upper Mesosphere Over Antarctica: Lidar Observations at the South Pole and Syowa. Journal of Geophysical Research, v. 99(D3), pp. 5475-5485.

Collins, R.L., K.P. Bowman and C.S. Gardner. 1993. Polar Stratospheric Clouds at the South Pole in 1990: Lidar Observations and Analysis. Journal of Geophysical Research. v. 98(D1), pp. 1001-1010.

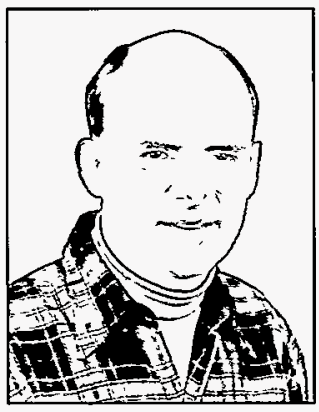

JOHN D. CRAVEN, Professor of Physics; University of Iowa '63, B.A.; '64, M.S.; '69, Ph.D. Before joining the University of Alaska in July of 1991, Dr. Craven was involved in the design, fabrication, calibration and flight operations for spacecraft-based scientific instruments, and in the analysis of observations obtained during those missions. Research included studies of the geomagnetically trapped energetic radiation, low-energy magnetospheric plasmas, and faintlight imaging and photometry of the aurora and other emission sources at visible and/or ultraviolet wavelengths. This research included P/Comet Halley and the diffuse galactic UV background. His present research work concentrates on large-scale variations in thermospheric composition in the dayside hemisphere driven by magnetospheric energy deposition at auroral latitudes, large-scale auroral morphology and dynamics and their dependence on the orientation of the interplanetary magnetic field, and spatial mappings between the aurora and magnetotail. Academic duties in the physics department include teaching undergraduate general physics and the advanced laboratory for physics majors, and the graduate course in space physics. He is a member of the AGU, EGS, AAAS, Sigma Xi, the UAF Faculty Senate, the AGU/SPA Education and Public Information Committee, and the Management Operations Working Group for NASA's Ionosphere, Thermosphere and Mesosphere Branch.

Craven, J.D., L.A. Frank and C.T. Russel. 1994. Motion of the Auroral Bulge's Poleward Boundary in the Substorm Recovery Phase. Proceedings of the Second International Conference on Substorms, held at the University of Alaska Fairbanks, 7-11 March 1994, J. R. Kan, J. D. Craven, S.-I Akasofu, (Eds.), pp. 305-311. 
Craven, J.D., A.C. Nicholas, L.A. Frank, D.J. Strickland and T.J. Immel. 1994. Variations in FUV Dayglow after Intense Auroral Activity. Geophysical Research Letters, v. 21, pp. 2793-2796.

Christensen, A.B., R.L. Waterscheild, M.N. Ross, C.-I. Meng, L.J. Paxton, D.E. Anderson, G. Crowly, S. Avery, J.D. Craven, R.R. Meier and D.J. Strickland. 1994. The Global Ultraviolet Imager (GUVI) for the NASA TIMED Mission. Optical Spectroscopic Techniques and Instrumentation for Atmospheric and Space Research, SPIE, v. 2266, pp. 451-466.

Fujii, R. ., A. Hoffman, P.C. Anderson, J.D. Craven, M. Sugiura, L.A. Frank and N.C. Maynard. 1994. Electrodynamic Parameters in the Nighttime Sector During Auroral Substorms. Journal of Geophysical Research, v. 99, pp. 6093-6112.

Weimer, D.R., J.D. Craven, L.A. Frank, W.B. Hanson, N.C. Maynard, R.A. Hoffman and J.A. Slavin. 1994. Satellite Measurements Through the Center of a Substorm Surge. Journal of Geophysical Research, v. 99, pp. 2363923649.

Weimer, D.R., J.D. Craven, L.A. Frank, W.B. Hanson and J.A. Slavin. 1994. Electric Fields and Currents Associated with a Substorm Surge. Proceedings of the Second International Conference on Substorms, held at the University of Alaska Fairbanks, 7-11 March 1994, J. R. Kan, J. D. Craven, S.-I Akasofu, (Eds.), pp. 455-460.

Yahnin, A., M.V. Malkov, V.A. Sergeev, R.J. Pellinen, O.A. Aulamo, S. Vennerström, E. Friis-Christensen, K. Lassen, C. Danielsen, J.D. Craven, C. Deehr and L.A. Frank. 1994. Features of Steady Magnetospheric Convection. Journal of Geophysical Research, v. 99, pp. 4039-4051.

Anderson, P.C., W.B. Hansen, R.A. Heelis, J.D. Craven, D.N. Baker and L.A. Frank. 1993. A Proposed Production Model of Rapid Subauroral Ion Drifts and Their Relationship to Substorm Evolution. Journal of Geophysical Research, v. 98, pp. 6069-6078.

Baker, D.N., T.I. Pulkkinen, R.L. McPherron, J.D. Craven, L.A. Frank, R.D. Elphinstone, J.S. Murphree, J.F. Fennell, R.E. Lopez and T. Nagai. 1993. CDAW 9 Analysis of Magnetospheric Events on 3 May 1986: Event C. Journal of Geophysical Research, v. 98, pp. 3815-3834.

Frank, L.A., D.J. Williams, E.C. Roelof, D.L. Carpenter, Y.-T. Chiu, J.D. Craven, H.O. Funsten, R.E. Gold, D.J. Gorney, B.K. Hultqvist, E.P. Keath, R.N. Lundin, B.H. Mauk, D.J. McComas, R.R. Meier, C.-I. Meng, D.G. Mitchell, K.R. Moore, J.F. Seely, M. Schulz, J.B. Sigwarth, G.L. Siscoe, B.W. Smith and R.A. Wolf. 1993. Imagers for the Magnetosphere, Aurora, and Plasmasphere (IMAP). Instrumentation for Magnetospheric Imagery II, SPIE v. 2008, pp. 11-34.

Reiff, P.H., G. Lu, J.L. Burch, J.D. Winningham, L.A. Frank, J.D. Craven, W.K. Peterson and R.A. Heelis. 1993. On the High- and Low-Altitude Limits of the Auroral Electric Field Region. Auroral Plasma Dynamics, Geophysical Monograph Series, American Geophysical Union, Washington, D.C., v. 80, pp. 143-154.

Burch, J.L., N.A. Saflekos, D.A. Gurnett, J.D. Craven and L.A. Frank. 1992. The Quiet-time Polar Cap: DE 1 Observation and Conceptual Model. Journal of Geophysical Research, v. 97, pp. 19403-19412.

Laakso, H., A. Pedersen, J.D. Craven and L.A. Frank. 1992. Magnetospheric Electric Fields and Auroral Oval. Proc. of the First International Conference on Substorms (ICS-1), ESA publication SP-335, 499-504, 1992.

Sharber, J.R., E.W. Hones, Jr., R.A. Heelis, J.D. Craven, L.A. Frank, N.C. Maynard, J.A. Slavin and J. Birn. 1992. Dynamics Explorer Measurements of Particles, Fields, and Plasma Drifts over a Horse-collar Auroral Pattern. Journal of Geomagnetism and Geoelectricity, v. 44, pp. 1225-1237.

R. KEITH CROWDER, Associate Professor of Geology; Radford University '78, B.S.; University of Arkansas' 80 , M.S.; University of Iowa '83, Ph.D. Dr. Crowder is a field-oriented sedimentologist, stratigrapher, and sedimentary petrologist. His research focuses mainly on paleogeographic reconstructions of ancient terrigenous and marine depositional systems, and the relationship between tectonics and sedimentation. He is directing research projects on the evolution of Paleozoic and Mesozoic depositional environments in the Brooks Range and Arctic Slope, and is involved in paleogeographic reconstructions of Cretaceous strata of the Alaska Peninsula and Wrangell Mountains. His particular interest is the development of the Cretaceous margin of northern Alaska. He and his students are relaring depositional environments and sediment dispersal patterns to the occurrence of hydrocarbons in Alaska.

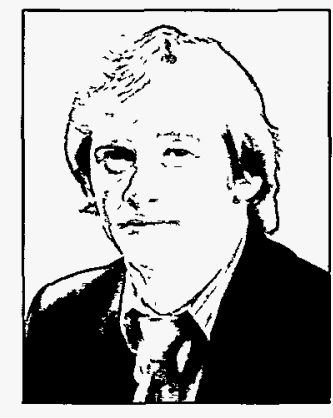




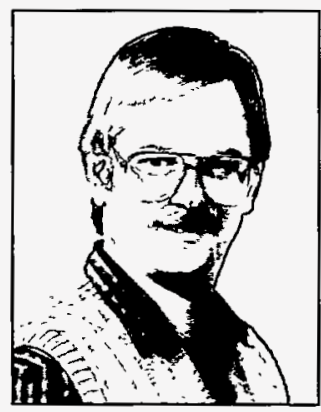

KENNESON G. DEAN, Research Assistant Professor; Northern Arizona University '72, B.S.; University of Alaska Fairbanks '79, M.S. Dean's current research interests focus on monitoring and analysis of environmental hazards using remote sensing data. His ongoing studies include monitoring of volcanic eruptions and associated hazards, and development of an eruption cloud tracking model. Investigations include pollution in the Arctic, surficial geology, quaternary geology, hydrology, and coastal oceanography. Past projects include the eruption of Redoubt Volcano, the influence of river discharge on the distribution of sea ice, potential effects of global warming, and the Exxon Valdez oil spill. He was president of the Arctic Division of the American Association for the Advancement of Science (AAAS) in 1993, and was Chairman of the 3rd Circumpolar Symposium on Remote Sensing of Arctic Environments, 1994.

Dean, K. 1994. Satellite Analyses of Movement and Characteristics of the Redoubt Volcano Plume, 8 January 1990. Journal of Volcano Geothermal Research, v. 62(1-4), pp. 339-352.

Dean, K.G., W.J. Stringer, K.Ahlnäs, C. Searcy and T. Weingartner. 1994. The Influence of River Discharge on the Thawing of Sea Ice, Mackenzie River Delta: Albedo and Temperature Analyses. Polar Research, v. 13(1), pp. 82-94.

Dean, K.G., L. Whiting and H. Jiao. 1994. An Aircraft Encounter with a Redoubt Ash Cloud (A Satellite View). T.J. Casadevall (Ed.). Volcanic Ash and Aviation Safety; Proceedings of the First International Symposium on Volcanic Ash and Aviation Safety held in Seattle, Washington, 8 July 1991, pp. 333-339.

Dean, K.G., S.-I. Akasofu and H. Tanaka. 1993. Volcanic Hazards and Aviation Safety: Developing Techniques in Alaska. Aviation Safety Journal, v. 3(1), pp. 11-15.

Alaska Volcano Observatory. 1993. Mr. Spurr's 1992 Eruption. EOS Transactions, American Geophysical Union, v. 74(19), pp. 217-222.

Tanaka, H.L., K.G. Dean and S.-I. Akasofu. 1993. Predicting the Movement of Volcanic Ash Clouds. EOS, Transactions of the American Geophysical Union, v. 74(20), p. 231.

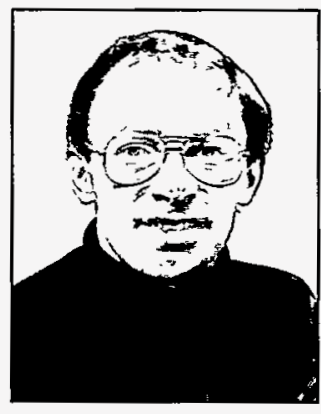

CHARLES S. DEEHR, Scientific Director of Poker Flat Research Range and Professor Emeritus of Physics, Reed College, '58, AB; University of Alaska '61, MS; '68, Ph.D. Deehr has been involved with the long-range planning of the Poker Flar Research Range Improvement and Modernization Program and is the chairman of the Poker Flat Science Working Group. He is charged with the coordination of research at Poker Flat, and is involved with the development of cooperative efforts in the circumpolar area, including a station for dayside auroral observations in Svalbard and the middle atmosphere research program of the Communications Research Laboratory of the Japanese Ministry of Post and Telecommunication. His main research interests involve spectrophotometric studies of atmospheric emissions, mainly associated with observations of the dayside and nightside aurora and the polar airglow as signatures of the coupling between the solar wind and the ionosphere.

Deehr, C.S. 1994. Ground-based Optical Observations of Hydrogen Emissions in the Auroral Aubstorm. Proceedings 2nd International Conference on Substorms, University of Alaska Fairbanks.

Deehr, C.S. 1993. Ionospheric Signatures of Ion Precipitation in the Dayside Aurora. The 1993 Magnetopause Workshop, Geophysical Institure University of Alaska Fairbanks, September 20-23.

Egeland, W.J. Burke, N.C. Maynard, E.M. Basinska, J.D. Winningham, and C.S. Deehr. 1994. Ground and Satellite Observations of Postdawn Aurorae Near the Time of a Sudden Storm Commencement. Journal of Geophysical Research, v. 99, (A2) 2095-2108.

Fasel, G.J., J. . Minow, L.C. Lee, R.W. Smith and C.S. Deehr. 1994. Poleward-Moving Auroral Forms: What Do We Really Know About Them? Physical Signatures of Magnetospheric Boundary Layer Processes, pp. 211-226.

Fasel, G.J., J.I. Minow, R.W. Smith, C.S. Deehr, and L.C. Lee. 1994. Multiple Brightenings of Poleward-Moving Dayside Auroral Forms. Solar Wind Sources of Magnetospheric ULF Waves, Geophys. Monogr. Ser., 81, M. Engebretson, K. Takahashi, and M. Scholer, 201-211, American Geophysical Union, Washington, D.C.

Sigernes, F., D.A. Lorentzen, C.S. Deehr and K Henriksen. 1994. Calculation of Auroral Balmer Volume Emission Height Profiles in the Upper Atmosphere. Journal of Atmospheric \& Terrestrial Physics, v. 56, 503. 
Yahnin, A., M.V. Malkov, V.A. Sergeen, R.J. Pellinen, O. Aulamo, S. Vennerstrom, E. Fris-Christensen, K. Lassen, C. Danielsen, J.D. Craven, C.S. Deehr and L.A. Frank. 1994. Features of Steady Magnetospheric Convection. Journal of Geophysical Research, v. 99, no. A3, 4039-4051.

Fasel, G.J., J.I. Minow, R.W. Smith, C.S. Deehr and L.C. Lee. 1993. Ionospheric Signatures of Solar-Wind Magnetosphere Interaction in Dayside Aurora. Airglow and Aurora, 2050, S. Leontiev, (Ed.), 20-26, SPIE, Bellingham, WA.

Sigernes, D.A. Lorentzen, C.S. Deehr and K. Henriksen. 1993. Modulation of the Auroral Proton Spectrum in the Upper Atmosphere. Journal of Atmospheric \& Terrestrial Physics, v. 55, (9) 1289-1294.

BRETT DELANA, Senior Project Engineer; Oregon State University '71, B.S.E.E.; University of Alaska '73, M.S. Delana joined the Geophysical Institute as a project engineer in 1975 to work with various field programs involving ionospheric and magnetospheric measurements. He joined the Alaska SAR Facility project in 1986 and presently serves as Chief Engineer for the program.

Wales, C., M. Jeffries, T. George, C. Wilson, B. Delana, G. Reynolds, L. Sweet and D. Sandberg. 1994. Alaska Synthetic Aperture Radar, Union Radio-Scientifique Internationale, URSI Bulletin, September, p. 270.

KEITH A. ECHELMEYER, Associate Professor of Geophysics; University of Colorado '76, B.S.; California Institute of Technology '78, M.S., '83 Ph.D. Dr. Echelmeyer has a research background in glacier mechanics, including studies of glacier surges and their response to climatic change; problems in geophysical continuum mechanics and the high pressure phases of ice; finite element modeling of nonlinear fluids; and uses of GPS in geophysics. With Dr. William Harrison, he has studied Jakobshavns Glacier in Greenland, the world's fastest glacier, and its relation to ice sheet dynamics. He also is involved in studies of ice stream dynamics in West Antarctica, and the geophysical investigation of the thickness and bed of glaciers. $\mathrm{He}$ is also involved in studies of the role of glaciers in sea level change and climate, including airborne Laser/GPS altimetry of glaciers.

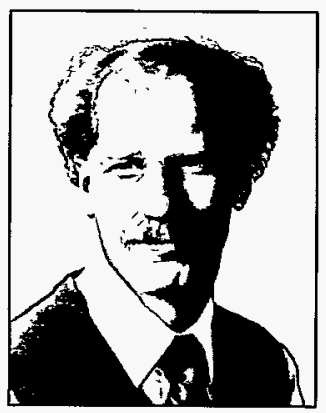

Echelmeyer, K., and W. Harrison. 1994. Temperature Measurements in the Margin of Ice Stream B, 1993-1994. Antarctic Journal of the United States, 1994 Review, pp. 4.

Echelmeyer, D.A., W.D. Harrison, C. Larson and J.E. Mitchell. 1994. The Role of the Margins in the Dynamics of an Active Ice Stream. Journal of Glaciology, v. 40(136), pp. 527-538.

Echelmeyer, K., and W. Harrison. 1993. Temperature Measurements in the Margin of Ice Stream B, West Antarctica. Antarctic Journal of the United States, 1993 Review, pp. 4.

Echelmeyer, K., and W. Harrison. 1993. The Role of Ice Stream Margins in Ice Stream Dynamics. NASA Conference Publication, R. Bindschadler (Ed.), (3222), p. 33.

Funk, M., K. Echelmeyer and A. Iken. 1994. Temperature Modeling and the Mechanisms of Flow in Jakobshavns Isbrae, Greenland. Journal of Glaciology, v. 40(136), pp. 569-586.

Harrison, W.D., K.A. Echelmeyer, E.F. Chacho, C.F. Raymond and R.J. Benedict. 1994. The 1987-88 Surge of West Fork Glacier, Susitna Basin, Alaska, U.S.A. Journal of Glaciology, v. 40(135), pp. 241-254.

Scambos, T.A., K.A. Echelmeyer, M.A. Fahnestock and R.A. Bindschadler. 1994. Development of Enhanced Ice Flow at the Southern Margin of Ice Stream D, Antarctica. Annals of Glaciology, v. 20, pp. 313-318.

Harrison, W.D., K.A. Echelmeyer and H. Engelhardt. 1993. Short-Period Observations of Speed, Strain and Seismicity on Ice Stream B, Antarctica. Journal of Glaciology, v. 39(133), pp. 463-470.

Iken, A., K. Echelmeyer, W. Harrison and M. Funk. 1993. Borehole Measurements of Ice Temperature and Water Pressure, Jakobshavns Isbrae, Greenland. Journal of Glaciology, v. 38 (131), pp. 15-25.

Iken, A., K. Echelmeyer, W. Harrison and M. Funk. 1993. Mechanisms of Fast Flow in Jakobshavns Isbrae, West Greenland: Part I. Measurements of Temperature and Water Level in Deep Boreholes. Journal of Glaciology, v. 39(131), pp. 15-25. 


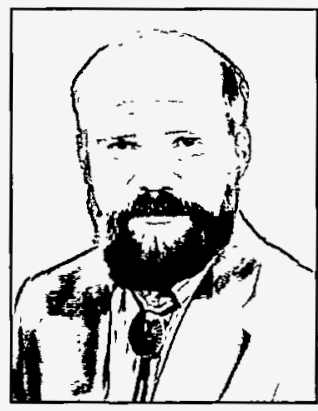

JOHN C. EICHELBERGER, Professor of Volcanology; Massachusetts Institute of Technology '71, B.S. and M.S.; Stanford University '74, Ph.D. Dr. Eichelberger has researched magmatic processes and eruption mechanisms. His current research emphasis is on understanding the behavior of volatiles in magmas. He serves as Coordinating Scientist of the Alaska Volcano Observatory, which monitors Alaska's active Cook Inlet volcanoes. He also serves as chief scientist of the Katmai Scientific Drilling Project, in which scientists seek to drill into the vent of the greatest eruption on Earth in this century. He also is active in research drilling in Long Valley Caldera, California, and is chairman of the Organizing Committee of the Continental Scientific Drilling Forum, a group established by DOE, NSF, and USGS to aid in the development of research drilling projects. He joined the Geophysical Institute in 1991. Dr. Eichelberger is a fellow of the Geological Society of America and teaches classes in geology, petrology, and volcanology.

Alaska Volcano Observatory (compiled and edited by C.J. Nye and J.C. Eichelberger). 1993. Spurr Erupts. EOS, v. 74(19), pp. 217 and 221-222.

Eichelberger, J.C., and A. Sattler. 1994. Conflict of Values Necessitates Public Lands Research Policy. EOS, Transactions, American Geophysical Union, v. 75(43), pp. 505-508.

Vogel, 'T.A., T.B. Woodburne, J.C. Eichelberger and P.W. Layer. 1994. Chemical Evolution and Periodic Eruption of Mafic Lava Flows in the West Moat of Long Valley Caldera, California. Journal of Geophysical Research, v. 99, pp. 19829-19842.

Westrich, H.R. and J.C. Eichelberger. 1994. Gas Transport and Bubble Collapse in Rhyolitic Magma: An Experimental Approach. Bulletin of Volcanology, v. 56, pp.447-458.

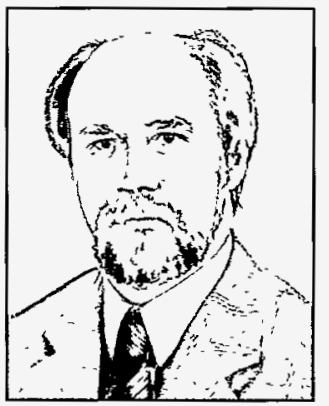

VICTOR V. FILYUSHKIN, Research Associate; Moscow Institute of Physics and Technology, Moscow, Russia '82, Candidate of Sciences (Physics and Mathematics), dissertation in plasma-chemistry. Filyushkin joined the Geophysical Institute in 1993 to participate in studies of cloud/radiation interaction and ozone chemistry in the Arctic. Most recently he has enhanced his background in atmospheric photochemical and cloud modeling during his visiting appointments at Atmospheric Chemistry Division, NCAR (1990-1991) and Cooperative Institute for Mesoscale Meteorological Studies, NOAA and University of Oklahoma (1992-1993).

Filyushkin, V.V., S. Madronich, G.P. Brasseur and I.V. Petropavlovshikh. 1994. Fast Two-Stream Method for Computing Actinic Flux in Vertically Inhomogeneous Atmospheres. Journal of the Armospheric Sciences, v. 51(8), pp. 1077-1088.

Filyushkin, V.V., and D.K. Lilly. 1993. The Application of a 3D Delta-Eddington Radiative Transfer Model to Calculation of Solar Heating and Photolysis Rates in a Stratocumulus Cloud Layer. Proceedings, SPIE 2049, pp. 56-57.

Kogan, Y.L., K.K. Lilly, Z.N. Kogan and V.V. Filyushkin. 1994. The Effect of CCN Regeneration on the Evolution of Stratocumulus Cloud Layers. Atmospheric Research, pp. 137-150.

Stamnes, K., V.V. Filyushkin and B.D. Zak. 1994. Use of Advanced Earth Observing Satellite to Study Radiation/ Cloud/Climate Interactions and Ozone Chemistry in the Arctic. Dynamics of the Arctic Climate System: Proceedings, Goteborg, Sweden, November, 1994.

Lilly, D.K., Y.L. Kogan, Z.N. Kogan and V.V. Filyushkin. 1993. Simulation of Stratocumulus Clouds with Emphasisn on Particle Physics and Aerosol Distributions. Proceedings of the 4th Symposium on Global Change Studies at the 73rd AMS Annual Meeting, January, 1993, pp. 203-207. 
KAZUYA FUJITA, Visiting Research Professor of Geophysics; Northwestern University, '73, B.A., '76, M.S., '79, Ph.D. Dr. Fujita has taught for the Department of Geological Sciences at Michigan State since 1979 and has been collaborating in tectonics research in Siberia with scientists from the Geophysical Institute since 1990. His research interests include the tectonics of plate margins, especially in Northeast Asia and the Arctic. Dr. Fujita received an Excellence in Teaching Award sponsored by the Amoco Foundation in 1992. He is currently an editorial board member for Tectonophysics and Journal of Physics of the Earth, and a member of the IRIS Standing Committee for the Global Seismograph Network.

Fujita, K., and B.M. Koz'min. 1994. Seismicity of the Amerasian Arctic Shelf and Its

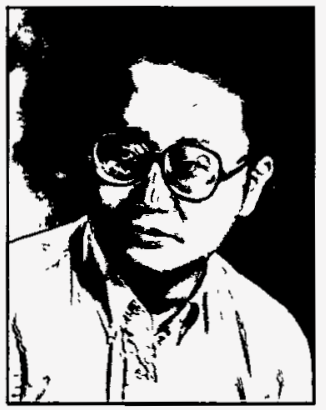
Relationship to Tectonic Features, In: D. Thurston and K. Fujita, Eds., 1992 Proceedings of the International Conference on Arctic Margins. U.S. Minerals Management Service, OCS Study, MMS 94-0040, p. 307-312.

Heiphetz, A., W. Harbert and P.W. Layer. 1994. Preliminary Reconnaissance Paleomagnetism of Some Late Mesozoic Ophiolites, Kuyul Region, Koryak Superterrane, Russia. D.K. Thurston and K. Fujita. 1992 Proceedings International Conference on Arctic Margins. U.S. Minerals Management Service, OCS Study 1994, MMS 94-0040: 229-234.

Imaev, V.S., L.P. Imaeva, B.M. Koz'min and K. Fujita. 1994. Active Faults and Contemporary Geodynamics of the Seismic Belts of Yakutia. Geotektonika, v. 2, pp. 57-71 (in Russian).

Riegel, S.A., K. Fujita, B.M. Koz'min, V.S. Imaev and D.B. Cook. 1993. Extrusion Tectonics of the Okhotsk Plate, Northeast Asia. Geophysical Research Letters, v. 20, pp. 607-610.

THOMAS H. GEORGE, Planning and Development Manager, Alaska SAR Facility and DAAC Manager; Oregon State University '73, B.S.; University of Alaska Fairbanks '85 M.S. Specialist in remote sensing technology for earth science applications. He is active in acquisition of remote sensing data from satellite and aircraft platforms, and data systems development. His current projects include planning for the Alaska SAR Facility; participation in development of the NASA's EOS Data and Information System network and; acquisition of airborne remote sensing data. He also teaches courses in remote sensing, aerial photography data acquisition, and map reading for pilots.

Wales C., M. Jeffries, T. George, C. Wilson, B. Delana, G. Reynolds, L. Sweet and D.

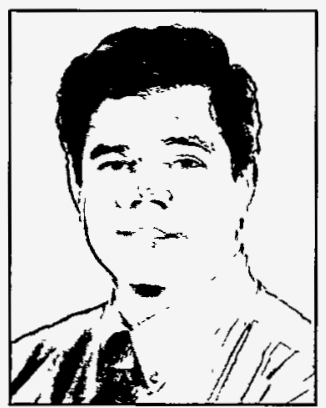
Sandberg. 1994. Alaska Synthetic Aperture Radar, Union Radio-Scientifique Internationale. URSI Bulletin, September, p. 270.

Stow, D., A. Hope and T. George. 1993. Reflectance Characteristics of Arctic Tundra Vegetation from Aerial Radiometry and Videography. Photogrammetric Engineering and Remote Sensing, v. 14, pp. 1239-1244.

THOMAS J. HALLINAN, Professor of Geophysics; Cornell University '64, B.S.E.E.; University of Alaska '69, M.S., '76, Ph.D. Dr. Hallinan has been interested primarily in the mechanisms responsible for the characteristic shapes of auroral forms. To study these shapes, he has developed systems for auroral imaging using low-light-level television cameras. His other interests include rocket-borne electron accelerators, electron beam experiments in large vacuum chambers ( $>20 \mathrm{~m}$ path lengths) and barium shaped-charge injections. He has conducted auroral imaging experiments from rockets and from the Space Shuttle.

Hallinan, T.J. 1993. Video Imaging of Charge IIB Auroral Streaks. Rome Laboratory, Air Force Material Command, Griffiss Air Force Base, New York, RL-TR-93-242 Final

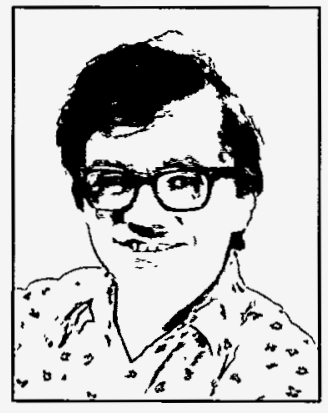
Technical Report, 16 pp. 
Hoch, E.L., T.J. Hallinan and H.C. Stenbaek-Nielsen. 1994. Quantitative Measurements of Vaporization, Burst Ionization and Emission Characteristics of Shaped Charge Barium Releases. Journal of Geophysical Research, v. 99, pp. 13263-13271.

Jack, T.M., and T.J. Hallinan. 1994. Measurement of Auroral Rays From the Space Shuttle. Journal of Geophysical Research, v. 99(A5), pp. 8865-8872.

Hoch, E.L., and T.J. Hallinan. 1993. Measurements of the Time Constant for Steady Ionization in Shaped Charge Barium Releases. Journal of Geophysical Research, v. 98, pp. 7765-7773.

Sivjee, G.G., R.M. Hamwey and T.J. Hallinan. 1993. Optical Observations of the Beam Plasma Discharge Phenomenon. Planetary Space Science, v. 41, pp. 633-639.

Stenbaek-Nielsen, H.C., E.M. Wescott and T.J. Hallinan. 1993. Observed Barium Emission Rates. Journal of Geophysical Research, v. 98(A10), pp. 17,491-17,500.

Wescotr, E.M., T.J. Hallinan, H.C. Stenbaek-Nielsen, D.W. Swift and D.D. Wallis. 1993. Rapid Ray Motions in Barium Plasma Clouds and Auroras. Journal of Geophysical Research, v. 98(A3), pp. 3711- 3724.

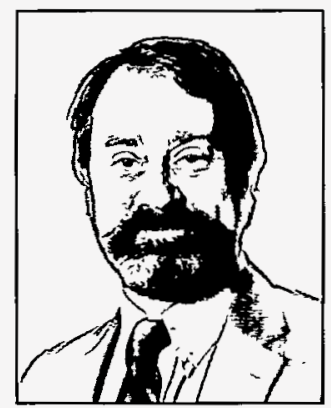

WILLIAM R. HAMMOND, Geophysicist, U.S. Geological Survey; University of Montana '81, B.A., University of Idaho '91, M.S. Hammond is the liaison between the Alaska Earthquake Information Center (AEIC) and the USGS National Earthquake Information Center, Golden, Colorado. At AEIC, he works with Geophysical Institute personnel on earthquake data acquisition, analysis and dissemination. He is involved in rapid response to earthquakes in Alaska, production of Alaska earthquake catalogs, public education about earthquake hazards in Alaska, the study of the Salcha Seismic Zone near Fairbanks, and compilation of intensity data for earthquakes near Anchorage. His background includes analysis of tectonic and mining-induced seismicity in northern Idaho, design of a rockburst monitoring system for an active silver mine in Idaho, installation and operation of a digitally recorded seismograph network in northern Idaho, and radar detection and delineation of sulfide mineralization beneath glaciers in Alaska. In 1989, he was director of the North Idaho Seismic Network. He was a geophysicist at the Alaska Tsunami Warning Center in Palmer, Alaska, during 1990. He has eight years experience in applied geophysics for mineral exploration and geotechnical engineering. His other interests include the design and use of ice-penetrating radar systems in glacier studies and operational aspects of air- and water-borne vehicles. He is a member of AGU, Seismological Society of America, Sigma Xi, and the International Cessna 120/140 Association.

Hammond, W.R., J.C. Lahr, C.D. Rowe, J.A. Power and R.A. Page. 1993. The Salcha Seismic Zone Near Fairbanks, Alaska (abs.). EOS, Transactions of the American Geophysical Union, v. 74(43), p. 417.

Fogleman, K., C. Rowe, C. Stephens and B. Hammond. 1994. Earthquakes in Alaska. January - August. 1991, Alaska State Seismologist's Report 93-01-01 through 91-01-08, Geophysical Institute, University of Alaska Fairbanks.

Fogleman, K., C. Rowe, C. Stephens and B. Hammond. 1994. Earthquakes in Alaska. January 1993, Alaska State Seismology Report 93-01-01, Geophysical Institute, University of Alaska Fairbanks.

Nye, C.J., W.R. Hammond and G.C. Tytgat. 1993. Kidazgeni Glacier Outburst Flood of June 29, 1993. Alaska Volcano Observatory Bi-monthly Report, May 1 - June 30, 1993, v. 5(3).

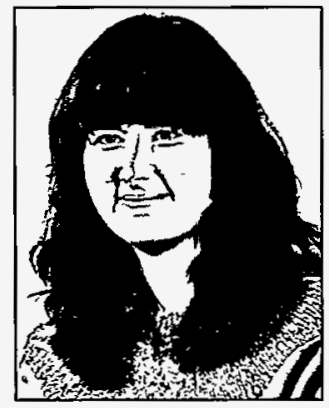

CATHERINE L. HANKS, Research Assistant Professor, with the Tectonics and Sedimentation Research Group; Rice University '78, B.A.; University of Washington '81, M.S.; University of Alaska Fairbanks '91, Ph.D. Dr. Hanks works as a structural geologist with an emphasis in research on the structural evolution of fold-and-thrust belts. Dr. Hanks is specifically interested in researching the map-scale and mesoscopic-scale structural behavior of the wide range of lithologies that can be found in a fold-and-thrust belt. These lithologies include both crystalline and sedimentary rocks. In addition, Dr. Hanks is involved in investigating the viability of using satellite imagery to determine the structural analysis of foldand-thrust belts in arctic regions. Her current research focuses on the northeastern Brooks Range of Alaska. 
Hanks, C.L., and R.M. Guritz. 1994. Geologic Analysis of an Arctic Fold-and-Thrust Belt Using Syntheric Aperture Radar (SAR): A Pilot Project in the Arctic National Wildlife Refuge (ANWR), Northeastern Brooks Range, Alaska. Third Circumpolar Symposium on Remote Sensing of Arctic Environments, p. 28.

Hanks, C.L., and R.M. Guritz. 1994. Synthetic Aperture Radar (SAR) and Geologic Reconnaissance in Arctic Regions: A Pilot Study in the Northeastern Brooks Range of Alaska. Geological Society of America Abstracts with Programs, v. 26(7), p. A266.

Hanks, C.L., J.C. Lorenz and A.P. Krumhardt. 1994. Mechanical Stratigraphy of the Lisburne Group, Eastern Sadlerochit Mountains: A Preliminary Report of Field Results. Alaska Division of Geological and Geophysical Surveys Public Data File 94-19: 29.

Hanks, C.L., W.K. Wallace and P. O'Sullivan. 1994. The Cenozoic Structural Evolution of the Northeastern Brooks Range, Alaska. 1992 Proceedings, International Conference on Arctic Margins, Anchorage, Alaska. U.S. Department of the Interior Minerals Management Service OCS Study, MMS, 94-0040, pp. 263-268.

Hanks, C.L. 1993. Rapid Evaluation of Regional Geometry and Shortening of a Fold-and-Thrust Belt: An Example from the Northeastern Brooks Range, Alaska. The American Association of Petroleum Geologists Bulletin. v. 77(1), pp. 19-28.

Hanks, C.L.. 1993. The Cenozoic Structural Evolution of a Fold-and-Thrust Belt, Northeastern Brooks Range, Alaska. Geological Society of America Bulletin, v. 105, pp. 306-322.

ROGER A. HANSEN, State Seismologist and Professor of Geophysics; University of California, Berkeley, '75 B.A., '77 M.A., '81 Ph.D. Dr. Hansen has a broad background of seismology ranging from very long period phenomenon to regional seismic array processing and earthquake hazards assessment. He has made considerable contributions to real-time monitoring of earthquakes and explosions for the purpose of discrimination of seismic sources and the verification of compliance to nuclear weapons treaties. Much of this work has been carried out while employed at NORSAR (NORwegian Seismic ARray), the University of Colorado, the U.S. Bureau of Reclamation, and the Air Force Technical Applications Center. Key research interests include earth structure studies, earthquake source process studies, wave propagation characteristics, seismic array processing, earthquake hazards assessment, and near

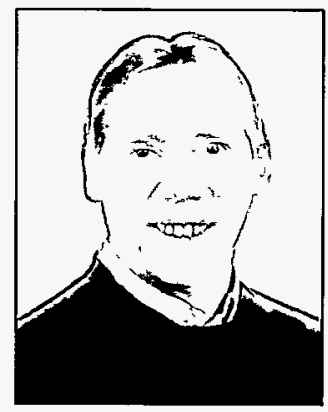
real-time broad-band seismology.

Hansen, R. 1994. Central Asian Bulletin and Catalog. IRIS Data Management Center, Technical Reference Manual. v. 44. Hansen, R. 1994. Central Asian Waveform Product. IRIS Data Management Center, Technical Reference Manual. v. 21. Hansen, R.A., D.J. Harvey and F.L. Vernon. 1994. Preliminary Analysis of the IRIS/Kyrgyz Network Data: Phase I of a Central Asian Bulletin and Catalog (abstract). 27th General Assembly of the International Association of Seismology and Physics of the Earth's Interior; Wellington, New Zealand.

Harvey, D. and R. Hansen. 1994. Contributions of IRIS Data to Nuclear Monitoring. IRIS Newsletter, v. 13(2), p. 1-6.

WILLIAM D. HARRISON, Professor of Physics; Mount Allison University '58, B.Sc.; University of London '60, B.Sc. (Special); California Institute of Technology '66, Ph.D. A nuclear physicist by training, Dr. Harrison transferred his interest to snow and ice problems and studied glaciers while at California Institute of Technology and the University of Washington before coming to Alaska. His present research is focused on the dynamics of glacier motion and on marine permafrost and heat and mass transport processes in it.

Harrison, W.D. , K.A. Echelmeyer, E.F. Chacho, C.F. Raymond and R.J. Benedict. 1994. The 1987-88 Surge of West Fork Glacier, Susitna Basin, Alaska, U.S.A. Journal of Glaciology, v. 40(135), pp. 241-254.

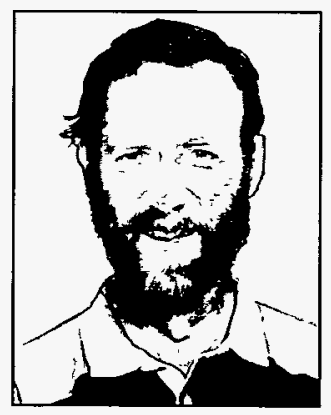

Harrison, W.D., K.A. Echelmeyer and H. Engelhardt. 1993. Short-Period Observations of Speed, Strain and Seismicity on Ice Stream B, Antarctica. Journal of Glaciology, v. 39(133), pp. 463- 470.

Echelmeyer, D.A., W.D. Harrison, C. Larson and J.E. Mitchell. 1994. The Role of the Margins in the Dynamics of an Active Ice Stream. Journal of Glaciology, v. 40(136), pp. 527-538. 
Iken, A., K. Echelmeyer, W. Harrison and M. Funk. 1993. Mechanisms of Fast Flow in Jakobshavns Isbrae, West Greenland: Part I. Measurements of Temperature and Water Level in Deep Boreholes. Journal of Glaciology, v. 39(131), pp. 15-25.

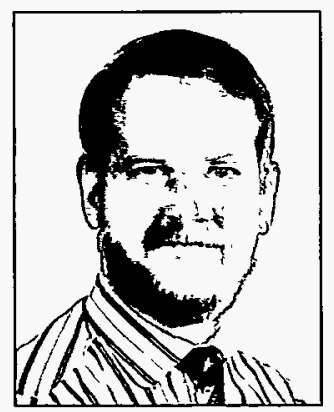

JOSEPH G. HAWKINS, Associate Professor of Electrical Engineering and Director of the Alaska Space Grant Program; University of Alaska Fairbanks '82, B.S., Stanford University '84, M.S., '88, Ph.D. Dr. Hawkins' research interests include space plasma physics and space systems engineering. His past research efforts include experimental studies of spacecraft charging and electron beam emission processes. He currently is studying the interaction between the solar wind and the magnetosphere using numerical simulation techniques. In 1990, he established the Alaska Student Rocket Program at UAF in which students are involved in the design, construction, and flight testing of sounding rocket payloads from the nearby Poker Flat Research Range.

Hawkins, J.G., L.C. Lee, M. Yan, Y. Lin, F.W. Perkins and M. Yamada. 1994. A Mechanism to Produce a Dawn-Dusk Component of Plasma Flow During Magnetic Reconnection to the Magnetotail. Journal of Geophysical Research, v. 99, pp. 5869.

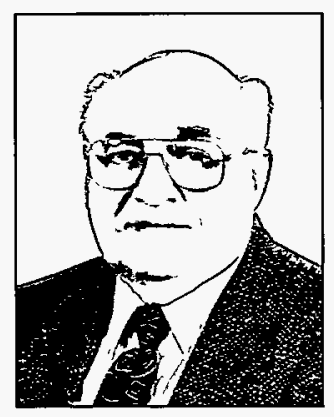

ROBERT D. HUNSUCKER, Senior Consultant and Professor Emeritus of Physics and Electrical Engineering; Oregon State University '54, B.S., '58, M.S.; University of Colorado '69, Ph.D. Dr. Hunsucker has a background of research in radio physics and propagation in the mid-latitude and high-latitude ionosphere. His past activities include use of several different radio techniques for investigating the irregular ionosphere. He joined the Geophysical Institute in 1958 and participated in high-latitude ionospheric propagation studies until 1964, when he joined CRPL/NBS of Boulder, Colorado. He returned to the institute in 1971 and has been engaged in aeronomy and propagation studies using incoherent scatter radar and other radio techniques. He is especially interested in gravity wave sources and propagation in the high-latitude ionosphere and in radio techniques for probing the ionosphere. He is a Fellow of the American Association for the Advancement of Science, a life fellow of the Institute of Electrical and Electronic Engineers (IEEE), an associate editor of the URSI Radio Scientist and Bulletin and Senior Editor of Radio Science.

Hunsucker, R.D. 1993. A Review of Ionospheric Radio Techniques: Present Status and Recent Innovations. Reviews of Radio Science (1990-1993), Oxford University Press, Ch. 22, pp. 429-490.

Hunsucker, R.D., R.B. Rose, R.A. Adler and G.K. Lott. 1993. An Auroral-E HF Propagation Experiment in Alaska: Results from the First Year Data Analysis. National Radio Science Meeting, January 5, 1993 Boulder, CO.

LaBelle, J., A.T. Weatherwax, M.L. Trimpe, R. Brittain, R.D. Hunsucker and J.V. Olson. 1994. The Spectrum of LF/ MF/HF Radio Noise at Ground Level During Storms. Geophysical Research Letters, v. 21, n. 24, pp. 27492752.

Jing, N., and R.D. Hunsucker. 1993. A Theoretical Investigation of Sources of Large and Medium Scale Atmospheric Gravity Waves in the Auroral Oval. Journal of Atmospheric and Terrestrial Physics, v. 55(13), pp. 1667-1679.

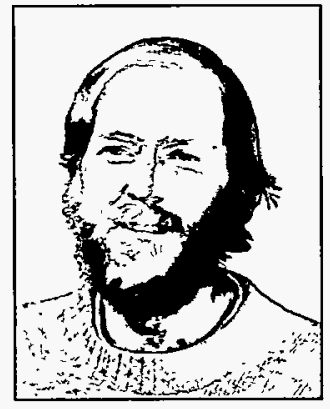

DANIEL A. JAFFE, Assistant Professor of Chemistry; Massachusetts Institute of Technology '79, B.S.; University of Washington '83, M.S., '87 Ph.D. Dr. Jaffe's background is in spectroscopic measurements of air pollutants. Under his direction, several high-sensitivity instruments for the measurement of nitrogen oxides, ozone and carbon monoxide have been built. Currently, his research group is collecting dara on gaseous, aerosol, and precipitation indicators of global- and arctic-scale pollution. He is particularly interested in tropospheric ozone and other greenhouse gases, and he has proposed several new theories related to the behavior of these gases in the Arctic. He currently has active field projects going in Alaska, East Asia, the North Pacific region and Russia. Dr. Jaffe has a joint appointment at UAF with the Geophysical Institute and the Department of Chemistry. 
Jaffe, D.A., B.M. Cerundolo and J. Kelley. 1994. The Influence of Mt. Redoubt Emissions on Snow Chemistry. Journal of Volcanology and Geophysical Research, v, 62, pp. 359-367.

Jaffe, D.A., E. Leighton and M.A. Tumeo. 1994. Environmental Impact on the Polar Regions. Forum for Applied Research and Policy, v. 9, pp. 65-70.

Jaffe, D.A.. 1993. Relationship between Anthropogenic Nitrogen Oxides and Ozone Trends in the Arctic Troposphere, In: eds H. Niki and K.H. Becker, The Tropospheric Chemistry of Ozone in the Polar Regions. NATO ASI Series, Springer-Verlag, N.Y., pp. 105-115.

Jaffe, D.A. and M.D. Zukowski. 1993. Nitrate Deposition to the Alaskan Snowpack. Armospheric Environment, v. 27A(17/18), pp. 2935-2941.

Baklanov, A., D.A. Jaffe and B.M. Cerundolo. 1993. Deposition of Air Pollutants on the Kola Peninsula During 1990-1991. In: ed, M. Kozlov, Aerial Pollution in the Kola Peninsula, University of Turku Press, Turku, Finland, pp. 106-111.

MARTIN O. JEFFRIES, Associate Research Professor; University of Sheffield '79, B.A.; Victoria University of Manchester '81, M.Sc.; University of Calgary'85, Ph.D. Dr. Jeffries has been with the Geophysical Institute since 1985. His research interests focus on field investigations of the growth, structure and properties of sea ice and lake ice, remote sensing studies of sea and lake ice processes, and their relationship to environmental variability in the Arcric and Antarctic. Since 1990 he has made five sea ice research cruises in the Arctic Ocean and the Southern Ocean. In 1993 and 1994 he was Chief Scientist aboard the Antarctic research vessel Nathaniel B. Palmer during sea ice and oceanographic investigations in the Bellingshauen, Amundsen and Ross seas. He is a member of the U.S. Antarctic Program Research Vessel User Panel, and of the NASA Polar DAAC Advisory Group. He is also an editorial advisor to Polar Record, the journal of the Scott Polar Research Institute.

Jeffries, M.O. 1994. Book Review. The Crystal Desert: Summers in Antarctica. Arctic, v. 47(3), pp. 313-315.

Jeffries, M.O. 1994. R/V Nathaniel B. Palmer Cruise NBP 93-5: Sea Ice Physics and Biology in the Bellingshausen and Amundsen Seas, August and September 1993. Antarctic Journal of the United States, v. 29(1), pp. 7-8.

Jeffries, M.O., and H. Johnson (Eds.). 1994. Alaska SAR Facility, ERS-1 SAR Image Sampler. CD-ROM, Alaska SAR Facility, Geophysical Institure, University of Alaska Fairbanks.

Jeffries, M.O., K. Morris, W.F. Weeks and H. Wakabayashi. 1994. Structural and Stratigraphic Features and ERS1 SAR Backscatter Characteristics of Ice Growing on Shallow Lakes in N.W. Alaska, Winter 1991-92. Journal of Geophysical Research, v. 99(C11), pp. 22459-22471.

Jeffries, M.O., K. Morris, A.L. Veazey and H.R. Krouse. 1994. Depositional Environment of the Snowcover on West Antarctic Pack Ice Floes. Annals of Glaciology, v. 20, pp. 33-38.

Jeffries, M.O., K. Morris, A.P. Worby and W.F. Weeks. 1994. Late Winter Characteristics of the Seasonal Snow Cover on Sea Ice Floes in the Bellingshausen and Amundsen Seas. Antarctic Journal of the United States, v. 29(1), pp. 9-10.

Jeffries, M.O., K. Morris, A.P. Worby and W.F. Weeks. 1994. Late Winter Sea Ice Properties and Growth Processes in the Bellingshausen and Amundsen Seas. Antarctic Journal of the United States, v. 29(1), pp. 11-13.

Jeffries, M.O., R.A. Shaw, K. Morris, A.L. Veazey and H.R. Krouse. 1994. Crystal Structure, Stable Isotopes $\left(\delta^{18} \mathrm{O}\right)$ and Development of Sea Ice in the Ross, Amundsen and Bellingshausen Seas, Antarctica. Journal of Geophysical Research, v. 99(C1), pp. 985-995.

Jeffries, M.O., K. Schwartz and S. Li. 1993. Preliminary Results of A Study of Sea Ice Backscatter Variation During Freeze-up in the Western Beaufort Sea. ASF SAR User Meeting, 1993 July 27, Battelle Conference Center, Seattle, Washington, S-17-19.

Jeffries, M.O., and M. . Shaw. 1993. The Drift of Ice Islands from the Arctic Ocean into the Channels of the Canadian Arctic Archipelago; The History of Hobson's Choice Ice Island. Polar Record, v. 29(171), pp. 305-312.

Jeffries, M.O., H. Wakabayashi and W.F. Weeks. 1993. ERS-1 SAR Backscatter Changes Associated with Ice Growing on Shallow Lakes in Arctic Alaska. Proceedings of the 1994 International Geoscience and Remote Sensing Symposium (IGARSS '93), Tokyo, Japan, August 1993, v. IV, pp. 2001-2004.

Jeffries, M.O., W.F. Weeks, R.A. Shaw and K. Morris. 1993. Structural Characteristics of Congelation and Platelet Ice and Their Role in the Development of Antarctic Landfast Sea Ice. Journal of Glaciology, v. 39 (132), pp. 223-238. 
Jeffries, M.O., and W.F. Weeks. 1993. Structural Characteristics and Development of Sea Ice in the Western Ross Sea. Antarctic Science, v. 5 (1), pp. 63-75.

Schwartz, K., M.O. Jeffries and S. Li. 1994. Using ERS-1 SAR Data to Monitor the State of the Arctic Ocean Sea Ice Surface Between Spring and Autumn, 1992. Proceedings of the International Geoscience and Remote Sensing Symposium (IGARSS '94), Pasadena, California, 8-12 August 1994, pp. 1759-1762.

Veazey, A.L., M.O. Jeffries and K. Morris. 1994. Small Scale Variability of Physical Properties and Structural Characteristics of Antarctic Landfast Sea Ice. Annals of Glaciology, v. 20, pp. 61-66.

Wakabayashi, H., M.O. Jeffries and W.F. Weeks. 1994. C-band Backscatter Variation and Modeling for Lake Ice in Northern Alaska. Journal of the Japanese Remote Sensing Society, v. 14(3), pp. 18-27.

Wales C., M.O. Jeffries, T. George, C. Wilson, B. Delana, G. Reynolds, L. Sweet and D. Sandberg. 1994. The Alaska SAR Facility: What it is. What it does. Union Radio-Scientifique Internationale. URSI Bulletin. September, n. 270, pp. 127-131.

Worby, A.P., W.F. Weeks, M.O. Jeffries, K. Morris and R. Jaña. 1994. Late Winter Sea Ice and Snow Thickness Distribution in the Bellingshausen and Amundsen Seas. Antarctic Journal of the United States, v. 29(1), pp. 13-15.

Danielson, A. and M.O. Jeffries. 1993. Small Scale Variability of Physical Properties and Structural Characteristics of a Single Ice Floe. Antarctic Journal of the United States, 1992 Review, XXVII:85-87.

Morris, K., and M.O. Jeffries. 1993. Ice Thickness Variability of the McMurdo Sound Fast Ice Runway. Antarctic Journal of the United States, 1992 Review, XXVII:83-85.

Wakabayashi, H., M.O. Jeffries and W.F. Weeks. 1993. C-band SAR Backscatter From Ice on Shallow Tundra Lakes: Observations and Modeling. Proceedings of the First ERS-1 Symposium-Space at the Service of our Environment, Cannes, France, 4-6 November 1992, European Space Agency Publication, ESA SP-359, 333-337.

Wakabayashi, H., W.F. Weeks and M.O. Jeffries. 1993. A C-band Backscatter Model for Lake Ice in Alaska. Proceedings of the 1994 International Geoscience and Remote Sensing Symposium (IGARSS '93), Tokyo, Japan, August 1993, Vol. III, pp. 1264-1266.

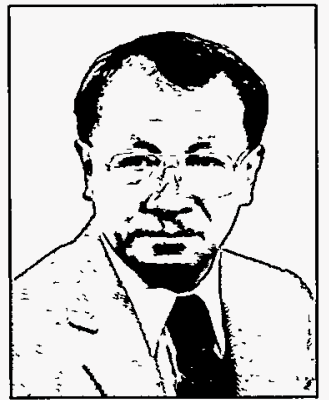

JOSEPH R. KAN, Professor of Physics; National Cheng Kung University, Taiwan '61, B.S.; Washington State University '66, M.S.; University of California, San Diego '69, Ph.D. Dr. Kan does basic research in plasma physics and space physics. His past activities include studying waves and instabilities in space plasmas. After joining the Geophysical Institute in 1972, he has been involved in theoretical studies of auroral arc formation, magnetopause and boundary layer structures, collisionless shock waves in space, and magnetospheric substorms. Dr. Kan teaches general physics, electrodynamics, statistical physics, plasma physics, and space physics.

Kan, J.R., L.H. Lyu and C.F. Kennel. 1994. Unloading Instability Triggered by Alfvén Waves Disrupting the CrossTail Current in the Near-Earth Plasma Sheet. Proceedings of the Second International Conference on Substorms, held at the University of Alaska Fairbanks, 7-11 March 1994, J.R. Kan, J.D. Craven, S.-I. Akasofu, (Eds.), p. 183.

Kan, J.R. 1993. A Global Magnetosphere-Ionosphere Coupling Model of Substorms. Journal of Geophysical Research, v. 98, p. 17263.

Kan, J.R. 1993. On the Cause of Substorm Expansion Phase Onset and the Processes Driving the Substorm Expansion Phase. Journal of Atmospheric and Terrestrial Physics, v. 55, p. 979.

Iijima, T., M. Watanabe, T.A. Potemra, L.J. Zanetti, J.R. Kan and S.-I. Akasofu. 1993. Substorm Currents in the Equatorial Magnetotail. Journal of Geophysical Research, v. 98, p. 17283.

Lyu, L.H., and J.R. Kan. 1993. Ion Dynamics in High-Mach-Number Quasi-Parallel Shocks. Journal of Geophysical Research, v. 98, p. 18985.

Sun, W., Y. Kamide, J.R. Kan and S.-I. Akasofu. 1993. Inversion of the Auroral Electrojets from Magnetometer Chain Data Based on the Flexible Tolerance Method. Journal of Geomagnetism and Geoelectricity, v. 45, p. 1151.

Voronina, V., and J.R. Kan. 1993. A Kinetic Model of the Plasma Sheet: Isotropic Nonuniform Temperature. Journal of Geophysical Research, v. 98, p. 13395. 
KOJI KAWASAKI, Associate Professor of Geophysics; University of California, Berkeley ' 60 , B.A.; University of Alaska '67, M.S., '71, Ph.D. Dr. Kawasaki has a background of research in geomagneric disturbance phenomena, the main field of the earth, aurora and magnetospheric physics, and teaching experience in general undergraduate physics and graduate and undergraduate exploration geophysics. He also has a strong interest in science education. Dr. Kawasaki rejoined the institute in 1980 after several years of study and research at NOAA, the University of Tokyo, the Universiry of Alberta, and the U.S. Geological Survey. He is presently engaged in research on permafrost, snow, and ice, especially in the application of geophysical exploration techniques and in geomagnetism.

JUERGEN KIENLE, Professor Geophysics; Swiss Federal Institute of Technology (ETH) '64, Diploma; University of Alaska '69, Ph.D. Dr. Kienle does research in volcanology and has conducted various land and marine gravity studies in Alaska and Antarctica. He rejoined the Geophysical Institute in 1970 after working for two years in marine geophysics at the LamontDoherty Geologic Observatory of Columbia University. Currently he is engaged in volcanic studies of the eastern Aleutian arc, with focus on Mt. St. Augustine and other Cook Inlet volcanoes; he also has carried out volcanologic and tectonic studies on Mr. Erebus and in Northern Victoria Land, Antarctica. He teaches undergraduate geology and graduate courses in volcanology. His volcanologic research primarily concerns the volcanism of subduction zones and includes volcano structure, seismicity, deformation, microgravity, physical volca-

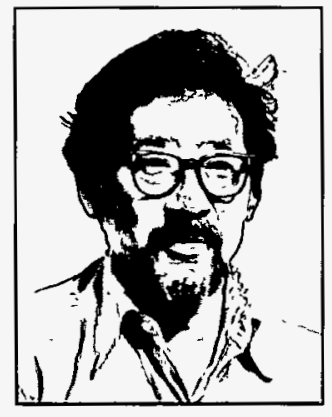
nology and volcanic hazard evaluation.

Kienle, J. 1994. Volcanic Ash - Aircraft Incidents in Alaska Prior to the Redoubt Eruption on 15 December 1989. T. J. Casadevall. Volcanic Ash and Aviation Safety, Proceedings of the First International Symposium on Volcanic Ash and Aviation Safety held in Seattle, Washington, 8 July 1991, pp. 119-123.

Kienle, J., T.F. Redfield and A.M. Goodliffe. 1993. Gravity Study of the Mt. Melbourne Quadrangle and the Lower Rennick Glacier are in North Victoria Land, Antarctica, and the Relation of the Rennick Graben Structure to Rifting Processes in the Ross Sea. In: Recent Progress in Antarctic Earth Science, TERRAPUB, pp. 523534.

Woods, A.W., and J. Kienle. 1994. The Injection of Volcanic Ash into the Atmosphere. T.J. Casadevall. Volcanic Ash and Aviation Safety, Proceedings of the First International Symposium on Volcanic Ash and Aviation Safety held in Seatrle, Washington, 8 July 1991, pp. 101-106.

Alaska Volcano Observatory, including J. Kienle. 1993. Mt. Spurr's 1992 Eruption. EOS Transactions, American Geophysical Union, v. 74(19), pp. 217, 221-222.

Redfield, T.F., J. Kienle and F. Heimberg. 1993. Complete Bouguer Gravity Map of North Victoria Land Between the Rennick and the Matusevich. Geologishes Jahrbuch, E47, pp. 185-195.

Redfield, T.F., J. Kienle and F. Heimberg. 1993. Complete Bouguer Gravity Map of the Mr. Melbourne Quadrangle, North Victoria Land. Geologisches Jahrbuch, E47, pp. 169-184.

Woods, A.W. and J. Kienle. 1993. The Dynamics and Thermodynamics of Volcanic Clouds: Theory and Observations From the April 15 and April 21, 1990 Eruptions of Redoubt Volcano, Alaska. Journal of Volcanology and Geothermal Research, v. 62, pp. 273-299.

JOHN C. LAHR, Adjunct Professor of Seismology; Rensselaer Polytechnic Institute '66 B.S., Columbia University'75 Ph.D. Dr. Lahr began working for the USGS Alaska Seismic Studies Project in Menlo Park, California, in 1971 and has been the chief of this project since 1975. In 1993 he joined the Geophysical Institute as senior USGS seismologist and acts as liaison between the USGS and the Geophysical Institute for two cooperative efforts: the Alaska Earthquake Information Center and the Alaska Volcano Observatory. He has authored and co-authored many papers on Alaskan seismicity and tectonics and is experienced in all aspects of seismic investigation, from field deployment and instrumentation to computer algorithms for earthquake analysis and location. His current work is focused on improving the speed and reliability of the computer systems and techniques used to monitor Alaska for earthquake

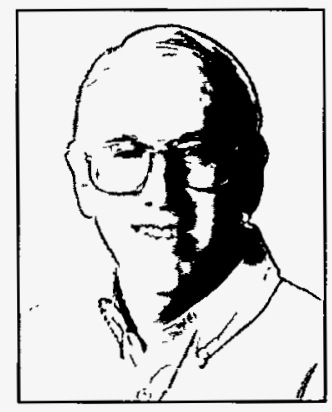


occurrences and volcanic unrest and to rapidly convey this information to federal, state, local, and business officials; increasing public understanding of Alaska's tectonic regime and public awareness of the hazard posed by Alaska's earthquakes and volcanoes; using seismology to investigate volcanic processes; and building a seismological and tectonic foundation for improved estimates of the long-term and intermediate-term potential for hazardous earthquakes.

Lahr, J.C., B.A. Chouet, C.D. Stephens, J.A. Power and R.A. Page. 1994. Earthquake Classification, Location, and Error Analysis in a Volcanic Environment: Implications for the Magmatic System of the 1989-1990 Eruptions at Redoubt Volcano, Alaska. Journal of Volcanology and Geothermal Research, v. 62, pp. 137-151.

Lahr, J.C., C.D. Stephens, R.A. Page and K.A. Fogleman. 1994. Characteristics of the Aleutian Wadati-Benioff Zone Seismicity beneath Southern Alaska (extended abs.). SUBCON, An Interdisciplinary Conference on the Subduction Process held in Avalon, California, 12- 17 June 1994, pp. 301-303.

Chouet, B.A., R.A. Page, CD. Stephens, J.C. Lahr and J.A. Power. 1994. Precursory Swarms of Long-period Events at Redoubt Volcano (1989-1990), Alaska: Their Origin and Use as a Forecasting Tool. Journal of Volcanology and Geothermal Research, v. 62, pp. 95-125.

Page, R.A., J.C. Lahr, B.A. Chouet, J.A. Power and C.D. Stephens. 1994. Statistical Forecasting of Repetitive Dome Failures During the Waning Eruption of Redoubt Volcano, Alaska, February-April 1990. Journal of Volcanology and Geothermal Research, v. 62, pp. 183-196.

Plafker, G., L.M. Gilpin and J.C. Lahr. 1994. Neotectonic Map of Alaska, in Plafker, G., and H.C. Berg, Eds., The Geology of Alaska: Boulder, Colorado, Geological Society of America. The Geology of North America. G1(plate 12) scale 1:2,500,000.

Power, J.A., J.C. Lahr, R.A. Page, B.A. Chouet, C.D. Stephens, D.H. Harlow, T.L. Murray and T.N. Davies. 1994. Seismic Evolution of the 1989-90 Eruption Sequence of Redoubt Volcano, Alaska. Journal of Volcanology and Geothermal Research, v. 62, pp. 69-94.

Stephens, C.D., B.A. Chouet, R.A. Page, J.C. Lahr and J.A. Power. 1994. Seismological Aspects of the 1989-1990 Eruptions at Redoubr Volcano, Alaska: The SSAM Perspective. Journal of Volcanology and Geothermal Research, v. 62, pp. 153-182.

Fogleman, K.A., J.C. Lahr, C.D. Stephens and R.A. Page. 1993. Earthquake Locations Determined by the Southern Alaska Seismograph Network for October 1971 through May 1989. U.S. Geological Survey. Open-file Report, 93-309, 54 p.

Hammond, W.R., J.C. Lahr, C.D. Rowe and J.P. Benoit. 1993. The Salcha Seismic Zone Near Fairbanks, Alaska (abs.). EOS, Transactions of the American Geophysical Union. v. 74(43), p. 417.

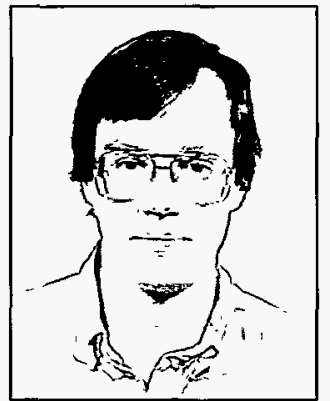

PAUL W. LAYER, Associate Professor of Geophysics; Michigan State University '81, B.S.; Stanford University '84, M.S., '86, Ph.D. Dr. Layer is head of the Geochronology Laboratory. His research focuses on use of the 40-argon/39-argon dating technique to investigate geological and geophysical problems in Alaska and elsewhere. Applications include determining the age of plutonism and tectonic events, dating of volcanoes in Alaska, constraining the time of formation of mineral deposits and determination of the sources of archaeological artifacts. Dr. Layer is involved in international collaborative projects with scientists from Russia, Germany, Canada, Mexico, Sweden and South Africa. Dr. Layer teaches courses on general and advanced geochronologic techniques and on the use of statistical methods in the geosciences.

Layer [Leier], P.W., L.M. Parfenov, A A. Surnin and V.F. Timofeev. 1993. First 40Ar/39Ar age Determinations of Magmatic and Metamorphic Rocks of the Verkhoyana-Kolyma Mesozoides'(in Russian). Daklady Adademii Nauk Rossiya, 329, pp. 621-624.

Heipherz, A., W. Harbert, P.W. Layer. 1994. Preliminary Reconnaissance Paleomagnetism of Some Late Mesozoic Ophiolites, Kuyul Region, Koryak Superterrane, Russia. D. K. Thurston and K. Fujita. 1992 Proceedings International Conference on Arctic Margins. U.S. Minerals Management Service, OCS Study, MMS 940040, pp. 229-234.

Huysken, D.T., T.A. Vogel, P.W. Layer. 1994. Incremental Growth of a Large Volume, Chemically Zoned Magma Body: A Study of the Tephra Sequence Beneath the Rainer Mesa Ash Flow Sheet of the Timber Mountain Tuff. Bulletin of Volcanology, 56, pp. 377-386.

Oxman [Oksman], V.S., L.M. Parfenov, A.V. Prokopiev, V.F. Timoveev, F.F. Tretyakov, V.D. Nedosekin and P.W. Layer [Leier]. 1994. The Chersky Range Ophiolites (in Russian). Geologiya i Geofizika, 36(6): 3-20. 
Smith, P.E., D. York, R.M. Easton, Ö. Özdemir and P.W. Layer. 1994. A Laser 40Ar/39Ar Study of Minerals Across the Grenville Front: Investigation of Reproducible Excess Ar patterns. Canadian Journal of Earth Sciences , 31, pp. 808-817.

Vogel, T.A., T.B. Woodburne, J.C. Eichelberger and P.W. Layer. 1994. Chemical Evolution and Periodic Eruption of Mafic Lava Flows in the West Moat of Long Valley Caldera, California. Journal of Geophysical Research, 99, pp. 19829-19842.

Solie, D.N., and P.W. Layer. 1993. The Hayes Glacier Fault Southern Alaskan Range: Evidence for Post-paleocene Movement. Alaska Division of Geology and Geophysics, Professional Report 113, pp. 71-80.

LOU-CHUANG LEE, Professor of Physics; National Taiwan University'69, B.S.; California Institute of Technology '72, M.S.; '75, Ph.D. Dr. Lee has a background of research in plasma physics, space physics, and astrophysics. His past activities include studies of wave propagation in random media, plasma turbulence in interstellar space and in the cometary tail, synchrotron radiation in Tokamaks, and auroral kilometric radiation. In 1978, he joined the Geophysical Institute, where he is involved in theoretical and simulation studies of the magnetopauseboundary layer, magnetic reconnection, substorm growth phase and onset, auroral arc formation, auroral kilometric radiation, solar flare, and the formation of solar prominences. Dr. Lee teaches classical mechanics, electromagnetic theory, statistical mechanics, atmospheric fluid dynamics, plasma physics and space physics.

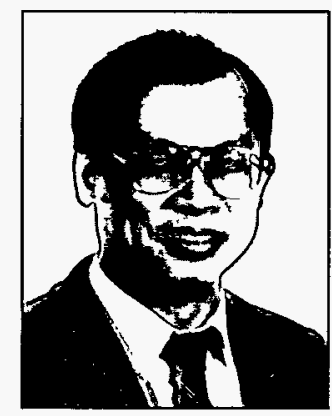

Lee, L.C., G.S. Choe and S.-I. Akasofu. 1994. A Simulation Study of the Formation of Solar Prominences. Micro/Meso Scale Phenomena in Space Plasma, AGU Monograph, M. Ashour-Abdalla, T. Chang and P. Dusenbary (Eds.), pp. 29-42.

Lee, L.C., and G.J. Fasel. 1994. Patchy Multiple X -Line Reconnection and Poleward-Moving Auroral Forms. Proceedings of NATO Workshop on "Physical Signatures of Magnetospheric Boundary Layer Processes," pp. 291-306.

Lee, L.C., J.R. Johnson and Z.W. Ma. 1994. Kinetic Alfvén Waves as a Source of Plasma Transport at the Dayside Magnetosphere. Journal of Geophysical Research, v. 99, pp. 17405.

Lee, L.C. and Y. Lin. 1994. A Hybrid Simulation of Magnetopause. Proceedings of the Lanczos Centenary Conference, SIAM, p. 461.

Lee, L.C., L. Zhang, M. Yan, H.J. Cai, G.S. Choe, A. Otto and S.-I. Akasofu. 1994. Formation of Very Thin Current Sheet During Substorm Growth Phase and Plasma Dynamics in the Recovery Phase. Proceedings of the International Conference on Substorms (ICS-2), p. 143.

Lee, L.C., and Y. Lin. 1993. Chaotic Ion Motion in Slow Shocks. Nonlinear Space Plasma Physics, R. Z. Sagdeev (Ed.), American Institute of Physics, pp. 104-139.

Lee, L.C., Z.W. Ma, Z.F. Fu and A. Otto. 1993. Topology of Magnetic Flux Ropes and Formation of Fossil Flux Transfer Events and Boundary Layer Plasmas. Journal of Geophysical Research, v. 98(A3), pp. 3943-3951.

Lee, L.C., and C.Q. Wei. 1993. Interaction of Solar Wind with the Magnetopause-Boundary Layer and Generation of Magnetic Impulse Events. Journal of Atmospheric and Terrestrial Physics, v. 55(7), pp. 967-978.

Cai, H.J., D.Q. Ding and L.C. Lee. 1994. Momentum Transport Near a Magnetic X Line in Collisionless Reconnection. Journal of Geophysical Research, v. 99, pp. 35.

Cai, H.J., Y. Lin and L.C. Lee. 1994. Equilibrium Structure and Disruption of Thin Current Sheet in the Magnetotail. Proceedings of the Second International Conference on Substorms, held at the University of Alaska Fairbanks, 7-11 March 1994, J.R. Kan, J.D. Craven, S.-I Akasofu, (Eds.), p. 481.

Cao, F., and L.C. Lee. 1994. Plasma Pressure and Anisotrophy Inferred from the Tsyganenko Magnetic Field Model. Annales Geophysicae, v. 12, pp. 286.

Fasel, G.J., J.I. Minow, L.C. Lee, R.W. Smith and C.S. Deehr. 1994. Poleward-Moving Auroral Forms: What Do We Really Know About Them? Proceedings of NATO Workshop on "Physical Signatures of Magnetospheric Boundary Layer Processes," p. 211-226.

Fasel, G.J., J. Minow, R.W. Smith, C.S. Deehr and L.C. Lee. 1994. Multiple Brightenings of Poleward Moving Dayside Auroral Forms. In: Solar Wind Sources of Magnetospheric ULF Waves, Geophysical Monograph Serial, Eds. M. Engebretson, K. Takahashi, M. Scholer, AGU Washington, D.C., pp. 201-212. 
Hawkins, J.G., L.C. Lee, M. Yan, Y. Lin, F.W. Perkins and M. Yamada. 1994. A Mechanism to Produce a Dawn-Dusk Component of Plasma Flow During Magnetic Reconnection to the Magnetotail. Journal of Geophysical Research, v. 99, pp. 5869.

La Belle-Hamer, A.L., A. Otto and LC. Lee. 1994. Magnetic Reconnection in the Presence of Sheared Plasma Flow: Intermediate Shock Formation. Physics of Plasmas, v. 1(3), pp. 706-713.

Lin, Y., and L. C. Lee. 1994. Generation of Region 1 and Mantle Field-Aligned Currents by the Secondary Rotational Discontinuity. Solar Wind Sources of Magnetospheric Ultra-Low-Frequency Waves, M.J. Engelbretson and K. Takahashi (Eds.). AGU Monograph 81, pp. 213-221.

Lin, Y., and L.C. Lee. 1994. Reconnection Layer at the Flank Magnetopause in the Presence of Shear Flow. Geophysical Research Letters, v. 21, p. 855.

Lin, Y., and L.C. Lee. 1994. Structure of Reconnection Layers in the Magnetosphere. Space Science Reviews, v. 65, pp. 59-179.

Ma, Z.W., A. Otto and L.C. Lee. 1994. Core Magnetic Field Enhancement in SingleX line, Multiple Xline and Patchy Reconnection. Journal of Geophysical Research, v. 99(A4), pp. 6125-6136.

Wu, B.H., J.K. Chao, W.H. Tsai, Y. Lin and L.C. Lee. 1994. A Hybrid Simulation of Contact Discontinuity. Geophysical Research Letters, v. 21, p. 2059.

Yan, M., and L.C. Lee. 1994. Generation of Slow-Mode Waves in Front of the Dayside Magnetopause. Geophysical Research Letters, v. 21(7), pp. 629-632.

Yan, M., A. Otto, D. Muzzell and L. C. Lee. 1994. Tearing Mode Instability in a Multiple Current Sheet System. Journal of Geophysical Research, v. 99(A5), pp. 8657-8669.

Chao, J.K., H.H. Chen, A.J. Chen and L.C. Lee. 1993. A Quantitative Model of the 22-year Variation of Geomagnetic Acrivity. Journal of Atmospheric and Terrestrial Physics, v. 55, pp. 959.

Choe, G.S. and L.C. Lee. 1993. Formation of Solar Prominences with Normal and Inverse Polarities. Proceedings of the IAU Colloguim 141 on the Magnetic Field of Solar Active Regions, p. 98.

Ding, D.Q., L.C. Lee and H.J. Cai. 1993. Collisionless Magnetic Field Reconnection at the Dayside Magnetopause. Physics of Space Plasmas (1992), SPI Conference Proceedings and Reprint Series, v. 12, pp. 379-392.

Fasel, G.J., L.C. Lee and R.W. Smith. 1993. A Mechanism for the Multiple Brightenings of Dayside Poleward-Moving Auroral Forms. Geophysical Research Letters, v. 20(20), pp. 2247-2250.

Lin, Y., and L.C. Lee. 1993. Structure of the Dayside Reconnection Layer in Resistive MHD and Hybrid Models. Journal of Geophysical Research, v. 98(A3), pp. 3919-3934.

Ma, Z.W., L.C. Lee, Y. Shi and A. Otto. 1993. By Enhancements in Magnetospheric Current Sheets and Magnetic Flux Tubes. Physics of Space Plasmas (1992), SPI Conference Proceedings and Reprint Series, v. 12, pp. 575582.

Wei, C.Q., and L.C. Lee. 1993. The Coupling of Magnetopause - Boundary Layer to the Polar Ionosphere. Journal of Geophysical Research v. 98(A4), pp. 5707-5725.

Wu, B.H., M.E. Mandt, L.C. Lee and J.K. Chao. 1993. Magnetospheric Response to Solar Wind Dynamic Pressure Variations: Interaction of Interplanetary Tangential Discontinuities with the Bow Shock. Journal of Geophysical Research, v. 98, pp. 21297-21311.

Yan, M., L.C. Lee and E.R. Priest. 1993. Magnetic Reconnection with Large Separatrix Angles. Journal of Geophysical Research, v. 98, pp. 7593.

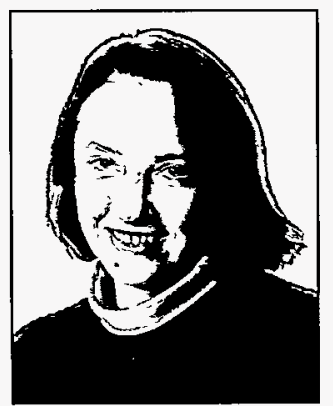

ELENA N. LEONTIEVA, Research Associate, Moscow Power Engineering Institute '78, M.S. With a background in radiophysics, she has been engaged in research on clouds and radiation in the atmosphere with the Institute of Atmospheric Physics Russian Academy of Sciences, Moscow, Russia. She joined the Geophysical Institure in August 1991 as a Research Scholar. Her present studies are focused on the Arctic radiate energy budget with the emphasis on the retrievals of cloud optical properties from ground-based and satellite-borne radiation measurements. 
Leontieva, E., and K. Stamnes. 1994. Remotely Sensed Measurements of Cloud Optical Depth Using a Ground-Based Multi-Filter Rotating Shadow Band Radiometer in the Arctic. Third Circumpolar Symposium on Remote Sensing of Arctic Environments, Programs and Abstracts, p. 33.

Leontieva, E., K. Stamnes and J.A. Olseth. 1994. Cloud Optical Properties at Bergen (Norway) Based on the Analysis of Long-Term Solar Irradiance Records. Theoretical and Applied Climatology, v. 50, pp. 73-82.

Leontyeva, E., and K. Stamnes. 1994. Estimations of Cloud Optical Thickness From Ground-Based Measurements of Incoming Solar Radiation in the Arctic. Journal of Climate, v. 7(4), pp. 566-578.

Leontyeva, E.N., and K.H. Stamnes. 1993. Cloud/Albedo Effects on the Radiation Budget in the Arctic. Atmospheric Radiation, SPIE Proceedings, v. 2049, p. 111-118.

SHUSUN LI, Research Assistant Professor; Peking University '66, B.A.; University of California, Santa Barbara '82, M.A., '85, Ph.D. Dr. Li has background in geography and remote sensing. He joined the Geophysical Institute in 1990 . His present research activities include SAR interferometry, general SAR and optical sensor remote sensing applications in quantifying and modeling interactions among the atmosphere, sea ice and ocean.

Li, S. 1994. A DEM-Free Satellite Radar Interferometric Technique for the Detection of Surface Elevation Changes. Proceedings of Third Circumpolar Symposium on Remote Sensing of Arctic Environments, Fairbanks, Alaska, 16 May 1994, p. 33.

Li, S. 1994. Deformation of Fast Ice Near Prudhoe Bay Revealed by Satellite Radar

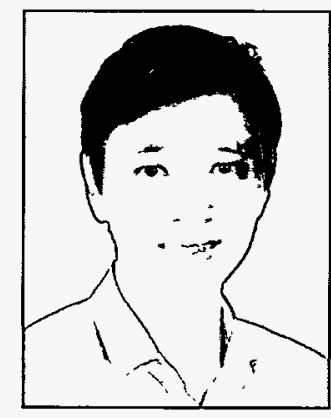

Interferomotery. Global Glimpses, Center for Global Change and Arctic Research, v. 2, pp. 2-3.

Li, S. 1993. Development and Applications of an Algorithm for a Two-Way Transformation Between the Geographical Coordinates and the ASF SAR Image Coordinates. ASF SAR User Meeting, 27 July 1993, Battelle Conference Center, Seattle, Washington, S1-21.

Li, S., R. Wade, Z. Cheng and W. Weeks. 1993. Identification of the Formation of Intermediate Scale Leads, Shear Zones, and Pressure Ridges Using ERS-1 SAR Ice Motion Products. ASF SAR User Meeting, 27 July 1993, Battelle Conference Center, Seattle, Washington, SI-14.

Cheng, Z., S. Li and W.F. Weeks. 1994. A Multi-Level Analysis of the Motion, Deformation, and Heat Exchange of Pack Ice in the Central Arctic Using a Grid Oriented Algorithm for the Extraction of Quantitative Measures of Intermediate-Scale Deformation from SAR Ice Motion Products. Proceedings of Third Circumpolar Symposium on Remote Sensing of Arctic Environments, Fairbanks, Alaska, 16 May 1994, p. 16.

McNutt, L., S. Li and R.M. Guritz. 1994. The Role of the Interactive Image Analysis System (IIAS) at the ASF DAAC. Geophysical Institute, University of Alaska Fairbanks: Alaska SAR Facility, 6 June 1994, ASF-SD94-001.

Schwartz, K., M.O. Jeffries and S. Li. 1994. Using ERS-1 SAR Data to Monitor the State of the Arctic Ocean Sea Ice Surface Between Spring and Autumn 1992. Proceedings of the International Geoscience and Remote Sensing Symposium (IGARSS '94), California Institute of Technology, Pasadena, California, 8 August 1994, pp. $1759-1762$.

Jeffries, M.O., K. Schwartz and S. Li. 1993. Preliminary Results of A Study of Sea Ice Backscatter Variation During Freeze-Up in the Western Beaufort Sea. ASF SAR User Meeting, 27 July 1993, Battelle Conference Center, Seattle, Washington, S-17-19.

CRAIG S. LINGLE, Research Associate Professor; University of Washington '67, B.S.; University of Maine-Orono '78, M.S.; University of Wisconsin-Madison '83, Ph.D. Dr. Lingle joined the Geophysical Institute in 1990. His research interests include measurement of elevation changes and high-resolution mapping of surface topography on the Antarctic ice sheet with satellite radar altimetry, and interpretation of the results in terms of ice-sheet dynamics. Other interests are the use of satellite synthetic aperture radar (SAR) imagery to study the glaciers and icefields of Alaska and the Yukon Territory (including the 1993-'94 surge of Bering Glacier in the Chugach Mountains), and numerical modeling of Antarctic ice shelves. Dr. Lingle is a member of the International Glaciological Society, the American Geophysical Union, and the American Association for the Advancement of Science.

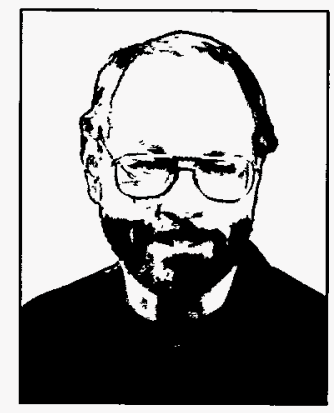


Lingle, C.S., L.-H. Lee, H.J. Zwally and T.C. Seiss. 1994. Recent Elevation Increase on Lambert Glacier, Antarctica, From Orbit Crossover Analysis of Satellite Radar Altimetry. Annals of Glaciology, v. 20, pp. 26-32.

Lingle, C.S., J.J. Roush and D.R. Fatland. 1994. Time of Onset of the 1993-94 Surge of Bering Glacier, Alaska, and Effect on Iceberg Calving of Surge Arrival at the Terminus. EOS, v. 75 (1994 Fall Meeting Supplement, November 1) p. 64.

Lingle, C.S., A. Post, U.C. Herzfeld, B.F. Molnia, R.M. Krimmel and J.J. Roush. 1993. Bering Glacier Surge and Iceberg-Calving Mechanism at Vitus Lake, Alaska, U.S.A. (correspondence). Journal of Glaciology, v. 39(133), pp. 722-727.

Fatland, D.R., and C.S. Lingle. 1994. The Surface Velocity Field on Bagley Icefield, Alaska, Before and During the 1993-94 Surge of Bering Glacier, From ERS-1 SAR Interferometry. EOS, v. 75 (1994 Fall Meeting Supplement, November 1) p. 62.

Herzfeld, U.C., C.S. Lingle and L.-H. Lee. 1994. Recent Advance of the Grounding Line of Lambert Glacier, Antarctica, Deduced From Satellite Radar Altimetry. Annals of Glaciology, v. 20, pp. 43-47.

Roush, J.J., C.S. Lingle and R.M. Guritz. 1994. The Surge of Bering Glacier, Alaska, Observed with Sequential ERSI Synthetic Aperture Radar Imagery. EOS, v. 75 (1994 Fall Meeting Supplement, November 1) p. 63.

Voronina, V.A., and C.S. Lingle. 1994. The Velocity of Seward and Malaspina Glaciers, Alaska, from ERS-1 Synthetic Aperture Radar Imagery. EOS, v. 75 (1994 Fall Meeting Supplement, November 1), p. 224.

Herzfeld, U.C., C.S. Lingle and L.-H. Lee. 1993. Geostatistical Evaluation of Satellite Radar Altimetry for HighResolution Mapping of Lambert Glacier, Antarctica. Annals of Glaciology, v. 17, pp. 77-85.

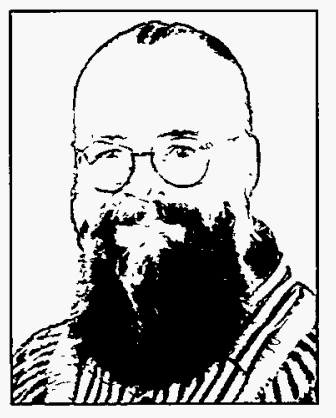

DIRK LUMMERZHEIM, Associate Research Professor; Georg August Universität, Göttingen, Federal Republic of Germany '81, Diplom Geophysik; University of Alaska Fairbanks '87, Ph.D. His research interest lies in the study of auroral processes, auroral arc formation, the penetration of auroral electrons into the atmosphere, and the subsequent optical emissions. He was at the High Altitude Observatory/National Center for Atmospheric Research, Boulder, Colorado, as a visiting scientist in 1990-93.

Lummerzheim, D., and J. Lilensten. 1994. Electron Transport and Energy Degradation in the Ionosphere: Evaluation of the Numerical Solution, Comparison with Laboratory Experiments and Auroral Observations. Annales Geophysics, v. 12, p. 1039.

Lanchester, B.S., J.R. Palmer, M.H. Rees, D. Lummerzheim, K. Kaila and T. Turenen. 1994. Energy Flux and Characteristic Energy of an Elemental Auroral Structure. Geophysical Research Letters, v. 21, p. 2789.

Watermann, J., O. de la Beaujardière, D. Lummerzheim, J. Woch, P.T. Newell, T.A. Potemra, F.J. Rich and M. Shapshak. 1994. The Dynamic Cusp at Low Latitude: a Case Study Comining Viking, DMSP and Sondrestrom Radar Observations. Annales Geophysics, v. 12, p. 1144.

Watermann, J., D. Lummerzheim, O. de La Beaujardière, P.T. Newell and F.J. Rich. 1994. The Ionospheric Footprint of Magnetosheath-like Particle Precipitation Observed by an Incoherent Scatter Radar. Journal of Geophysical Research, v. 99, p. 3855.

Min, Q.L., D. Lummerzheim, M.H. Rees and K. Stamnes. 1993. Effects of a Parallel Electric Field and the Geomagnetic Field in the Topside Ionosphere on Auroral and Photoelectron Energy Distributions. Journal of Geophysical Research, v. 98(A11), pp. 19223-19234.

Sharber, J.R., R.A. Frahm, J.D. Winningham, J.C. Biard, D. Lummerzheim, M.H. Rees, D.L. Chenette, E.E. Gaines, R.W. Nightingale and W.I. Imhof. 1993. Observations of the UARS Particle Environment Monitor and Computation of Ionization Rates in the Middle and Upper Atmosphere During a Geomagnetic Storm. Geophysical Research Letters, v. 20, p. 1319.

Winningham, J.D., J.R. Sharber, R.A. Frahm, J.L. Burch, N. Eaker, R.K. Black, V.A. Belvins, J.P. Andrews, J. Rudzki, D.L. Chenette, D.W. Datlowe, E.E. Gaines, W.I. Imhof, R.W. Nightingale, S.S. Prasad, R.M. Robinsonn, T.L. Schumaker, R.R. Vondrak, P.F. Bythrow, B.J. Anderson, T.A. Potemra, L.J. Zanetti, D.B. Holland, M.H. Rees, D. Lummerzheim, G.C. Reid, R.G. Roble, C.R. Clauer and P.M. Banks. 1993. The UARS Particle Environment Monitor. Journal of Geophysical Research, v. 98, p. 10649. 
AMANDA LYNCH, Assistant Professor of Atmospheric Sciences, Geophysical Institute, University of Alaska Fairbanks; Monash University '86 B.Sc.(Hons); University of Melbourne ' 92 Ph.D. Dr. Lynch was previously involved in many aspects of numerical modeling of the atmosphere at the Australian Bureau of Meteorology Research Centre, as part of the Mesoscale Modeling Group, and the University of Melbourne. These studies ranged from mesoscale weather modeling and real-time forecasting of the tropics and mid-latitudes to long-term global and Antarctic climate studies, concentrating mainly on long-term variability, large-scale momentum budgets, and surface effects. She joined the Geophysical Institute in August 1992 as a post-doctoral fellow, and has been developing a regional Arctic model of ocean-iceatmosphere and land-atmosphere interactions (ARCSyM), which will be used to study the impact of polar processes upon both the global climate and local weather.

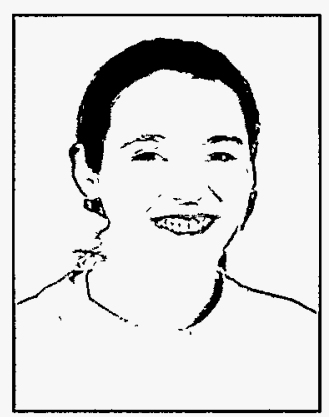

Lynch, A.H., M.F. Glueck, W.L. Chapman, D.A. Bailey and J.E. Walsh. 1994. The Use of Remote Sensing Data (SAR, AVHRR, SSM/I) for Verification of a Coupled Atmosphere-Sea Ice Regional Model. Journal of Climatology, v. 51, pp. 182-193.

Walsh, J.E., A.H. Lynch, W.L. Chapman and D. Musgrave. 1993. A regional model for Studies of Atmosphere-IceOcean Interaction in the Western Arctic. Meteorology Atmosphere Physics, v. 51, pp. 179-194.

S. LYN MCNUTT, Research Associate Professor and Alaska SAR Facility Chief Scientist; University of California Los Angeles '72, BA, '78 MA; University of Washington '82, Ph.D. Dr. McNutt began serving 1 September 1993 as the Chief Scientist for the Alaska Synthetic Aperture Radar (SAR) Facility within the Geophysical Institute, where she is a Research Associate Professor in the Remote Sensing Group. Dr. McNutt has worked for the National Oceanic and Atmospheric Administration (NOAA), for the Pacific Marine Environmental Laboratory (PMEL), for the Canada Centre for Remote Sensing (CCRS), and for the Canadian Space Agency (CSA). Dr. McNutt has experience in all aspects of planning and implementing complete Earth observation from space programs. Her experience ranges from the technical specification and the building of sensors and satellites, to the management and the organiza-

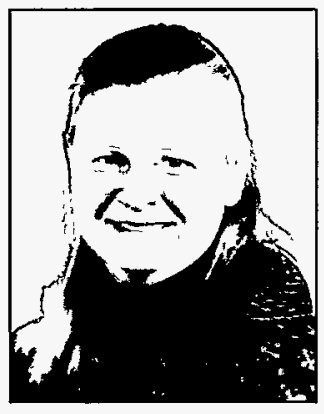
tion of field programs and scientific projects for instrument and geophysical validation. Dr. McNutt has almost twenty years of experience in air-sea-ice research, beginning with the SEASAT pre-launch validation studies. She also has been involved in data processing for airborne and satellite sensors, and in ground segment design as part of the SEASAT, RADARSAT, ERS-1 and 2, and ENVISAT Programs. Before coming to the Alaska Synthetic Aperture Radar Facility, McNutt was the manager of the Earth Observation Program Office for the Canadian Space Agency. She also was selected by NASA as a principal investigator for the Cryospheric System for Monitoring Global Change in Canada (CRYSYS) Program within the EOS Program. Dr. McNutt was a founding member, and currently serves as the co-chair, of the Program for International Polar Ocean Research (PIPOR). She also heads the Science Steering Committee for the CRYSYS Program. Dr. McNutt has previously served as a coordinating investigator for ERS-1. She is currently group leader for the Remote Sensing Group at the Geophysical Institute. Dr. McNutt also serves on the following science advisory groups: the Earth Observing System (EOS); the Interdisciplinary Working Group (IWG); and the National Research Council (NRC) SAR Discipline Working Group (oceanography).

McNutt, L., and F. Carsey. 1994. Description of the Alaska SAR Facility Science Capabilities for the RADARSAT/ ERS-2 Era. Version 1.0.3, November 1994, ASF SD94-001, 49 p.

McNutt, L., S. Li and R.M. Guritz. 1994. The Role of the Interactive Image Analysis System (IIAS) at the ASF DAAC. Geophysical Institute, University of Alaska Fairbanks: Alaska SAR Facility, June 6, 1994, ASF-SD94-001.

McNutt, L., and D. Sandberg. 1994. ASF SAR Research Results Bibliography: Version 1.1 Draft. October 1994, ASF SD94-003, $126 \mathrm{p}$. 


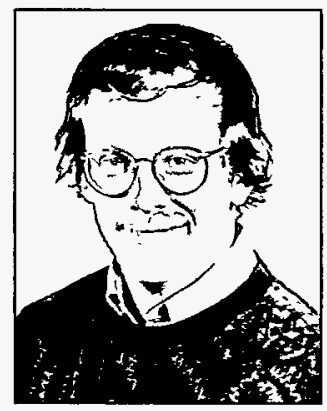

STEPHEN R. McNUTT, Research Professor, Volcano Seismology, Alaska Volcano Observatory; Wesleyan University '77, B.A.; Columbia University '82, M.A., '84, M. Phil., '85, Ph.D. Dr. McNutt joined the institute in November 1991. Prior to his arrival, he was a senior seismologist with the California Division of Mines and Geology in Sacramento, California. His research interests include volcano seismology (especially studies of source and propagation effects for volcanic tremor) low-frequency events; explosion earthquakes; volcanic hazard assessments in Alaska, California, and Central America; and mechanical behavior of volcanoes, including periodicity of eruptions, and the effects of earth tides, sea level variations, and tectonic stresses on eruptive activity. He is a member of the American Geophysical Union, the International Association of Volcanology and Chemistry of the Earth's Interior, the International Society for the Prevention and Mitigation of Natural Hazards, the Seismological Society of America, and Sigma Xi. He also is an active participant in the Alaska Volcano Observatory.

McNutt, S.R. 1994. Volcanic Tremor Amplitude Correlated with Eruption Explosivity and its Potential Use in Determining Ash Hazards to Aviation. U.S. Geological Survey Bulletin 2047, pp. 377-385.

Aramaki, S., F. Barberi, T. Casadevall and S. McNutt. 1994. Safety for Volcanologists. Bulletin of Volcanology, v. 56, pp. 151-154.

Hill, D., P. Gasparini, S. McNutt and H. Rymer. 1994. Preface, Special Issue, Internal Structure of Volcanoes and Geophysical Precursors of Eruptions. Journal of Volcanology and Geothermal Research, v. 61, p. 3-4.

Lu, Z., M. Wyss, G. Tytgat, S. McNutt and S. Stihler. 1994. Aftershocks of the 13 May 1993 Shumagin Alaska Earthquake. Geophysical Research Letters, 21(6): 497-500.

Alaska Volcano Observatory. 1993. Spurr Erupts. EOS Transactions American Geophysical Union, v. 74, pp. 217, 221-222.

Reeder, J., S. McNutt and G. Abers. 1993. Pavlof Volcano Eruption Report. Bulletin of Volcanic Eruptions, No. 30, Volcanological Society of Japan and IUGG, pp. 64-65.

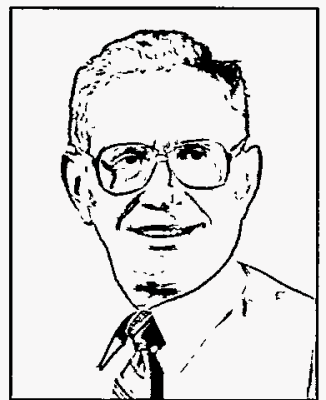

JOHN M. MILLER, Adjunct Applications Engineer, Emeritus; University of Alaska '60 B.S.E.E.; University of Alaska '68 M.S.E.M; Registered Professional Engineer, State of Alaska '61; University of Alaska'94 Adjunct Applications Engineer, Emeritus. Mr. Miller's experience embraces engineering aspects of research particularly relating to instrumentation, logistics, and systems operations. Since 1971, his work has focused upon aerospace applications of remotesensing technology to resource management and assessments of geophysical hazards in the Arctic and subarctic.

Delana, B., T. George, M. Jeffries, J. Miller, W. Weeks, G. Weller and C. Wilson. 1993. The Alaska SAR Facility. Polar Triennial Report on Science, NASA.

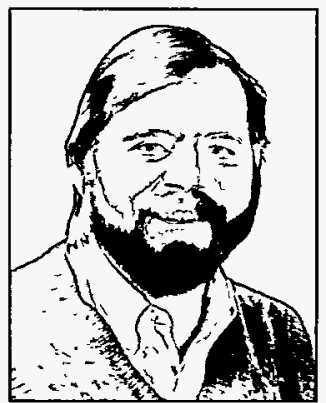

CHRISTOPHER J. NYE, Research Assistant Professor of Geology; University of Alaska Fairbanks '76, B.S., '78, M.A.; University of California at Santa Cruz '83, Ph.D. Dr. Nye's research focuses on the geochemistry, petrology and geology of convergent margin volcanoes, most notably those in Alaska. Applications include petrogenesis of volcanic rocks, recycling of crustal material in subduction zones, and description of temporal variations in magmatic plumbing systems. The last is used to further basic understanding of volcanoes and the magmatic systems that produce geothermal reservoirs. Dr. Nye is an active participant in the Alaska Volcano Observatory.

Nye, C.J., S.E. Swanson, V.F. Avery and T.P. Miller. 1994. Geochemistry of the 1989-1990 Eruption of Redoubt Volcano: Part I, Whole-Rock Major- and Trace Element Chemistry. Journal of Volcanology and Geothermic Research, v. 62, pp. 427-450. 
Nye, C.J., C.A. Neal and R.G. McGimsey. 1993. Extreme and Abrupt Transition From Tholeiitic to Calcalkaline Volcanism at Aniakchak Volcano, Eastern Aleutian Arc. EOS, v. $74(43$ supplement), pp. 674.

Alaska Volcano Observatory (compiled and edited by C.J. Nye and J.C. Eichelberger). 1993. Spurr Erupts. EOS, 74(19): 217 and 221-222.

Beget, J.E., and C.J. Nye. 1993. Postglacial Eruption History, Long-Term Eruptive Patterns, and Future Hazards at Mt. Redoubt, Alaska. Journal of Volcanology and Geothermal Research, v. 62, pp. 31-54.

Motyka, R.J., and C.J. Nye. 1993. Thermal Water and Fumarole Gas Chemistry, Crater Peak, Mt. Spurr, Alaska. In: D.N. Solie, F. Tannian, (Eds.), Short Notes on Alaskan Geology, Alaska Division of Geological and Geophysical Surveys. Professional Report PR-113: pp. 31-40.

Motyka, R.J., C.J. Nye, D.L. Turner and S.A. Liss. 1993. The Geyser Bight Geothermal Area, Umnak Island, Alaska. Geothermics, v. 22, pp. 301-327.

Motyka, R.J., S.A. Liss, C.J. Nye and M.A. Moorman. 1993. Geothermal Resources of the Aleutian Arc. Alaska Division of Geological and Geophysical Surveys, Professional Report PR-114 Ed.

Solie, D.N., M.B. Werdon, C.J. Nye and R.A. Combellick. 1993. Land Selection Unit 6 (Tyonek Quadrangle): References, DGGS Sample Locations, Geochemical and Major Oxide Data. Alaska Division of Geological and Geophysical Surveys, Public Data File 93-6 Ed.

Solie, D.N., M.B. Werdon, C.J. Nye and R.A. Combellick. 1993. Land Selection Unit 7 (Tyonek and Kenai Quadrangles): References, DGGS Sample Locations, Geochemical and Major Oxide Data. Alaska Division of Geological and Geophysical Surveys, Public Data File 93-7 Ed.

Swanson, S.E., C.J. Nye, P. Miller and V.F. Avery. 1993. Geochemistry of the 1989-1990 Eruption of Redoubt Volcano: Part II, Magma Mixing Evidence Form Mineral and Glass Chemistry. Journal of Volcanology and Geothermal Research, v. 62, pp. 453-468.

TAKESHI OHTAKE, Professor Emeritus of Geophysics; Tohoku University '52, B.Sc., '61, D. Sc. Dr. Ohtake has carried out meteorological investigations on the size distribution of snowflakes and raindrops, and their relationship to radiosonde data and radar reflectivity, as well as investigated nucleation processes in the formation of ice fog crystals and atmospheric ice crystals from the polar cloudless skies. He has considerable experience in applying the electron microscope to the study of aerosols and condensation, and ice nuclei contained in individual cloud droplets and ice crystals. He also is presently interested in the nucleation of polar stratospheric cloud particles. Dr. Ohtake retired from the institute in December 1987. In recognition of his distinguished career in experimental meteorology, he was elected a Fellow of the American Meteorological Society in 1990.

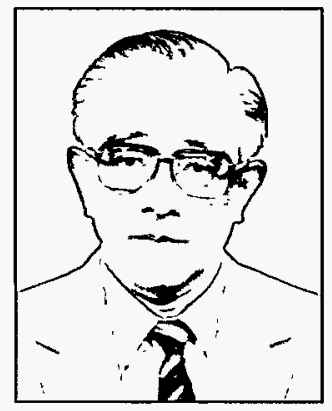

Ohtake, T., and K. Okada. 1994. 1993 Disappearing Sulfuric Acid Particles From South Pole in Winter. Meteorological Society of Japan, Tokyo, Spring Conference Proceedings, v. 65(14).

Ohtake, T. 1993. Freezing Points of $\mathrm{H}_{2} \mathrm{SO}_{4}$ Aqueous Solutions and Formation of Stratospheric Ice Clouds. Tellus, v. 45B, pp. 138-144.

Ohtake, T., and K. Okada. 1993. Winter Aerosol Particles at the South Pole. EOS AGU Fall Meeting, v. 175.

COERT OLMSTED, Senior Programmer Analyst with the Alaska Synthetic Aperture Radar Facility (ASF), and Scientific Consultant with the Arctic Region Supercomputing Center; Harvard University '64, A.B. Mathematics; University of Alaska, Fairbanks '88, Ph.D. Mathematics. Dr. Olmsted has a background in data analysis, numerical algorithms, geophysics, oceanography, and computer systems. His specialties include scientific computing, data management, applied mathematics, simulation, computer graphics/cartography, solar windmagnetosphere interactions, image processing for remote sensing, and synthetic aperture radar (SAR). He has been working at the ASF in algorithm development for SAR processing, particularly in SAR image terrain correction and in SAR signal processing on parallel computers. He has written a general information document for scientific SAR users that has been well received by investigators and students in the field of SAR remote sensing. At ARSC

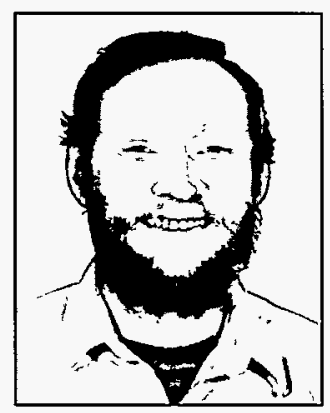
he has been awarded a Cray University Research and Development Grant to develop a massively parallel version of Antonius Otto's fluid and MHD supercomputer codes. These projects have applications in magnetospheric simulation. 
Olmsted, C. 1993. Alaska SAR Facility Scientific SAR User's Guide. Alaska SAR Facility Document ASF-SD-003, Geophysical Institute, University of Alaska Fairbanks.

Wivell, C., C. Olmsted, D. Steinwand and C. Taylor. 1993. An Earth Remote Sensing Satellite-1 Synthetic Aperture Radar Mosaic of the Tanana River Basin in Alaska. Photogrammetric Engineering and Remote Sensing, v. 59, p. 527 (and cover illustration).

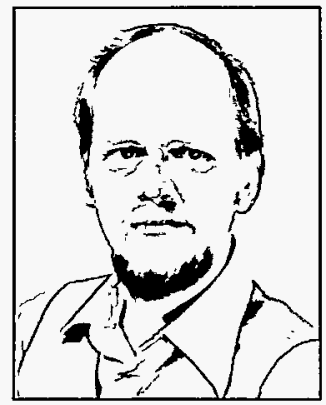

JOHN V. OLSON, Professor of Physics; University of California at Los Angeles'63, B.A., '64, M.S. '70, Ph.D. Dr. Olson joined the Geophysical Institute in 1979. His research interests have included the study of geomagnetic pulsations at high latitudes associated with the nightside aurorae and with the dayside magnetospheric boundaries. He also has carried out programs of ULF and VLF diagnostics for ionospheric heating programs and has a strong interest in digital signal analysis. He is a member of the American Geophysical Union and the American Association for the Advancement of Science.

Olson, J.V., and B.J. Fraser. 1994. Pc3 Pulsations in the Cusp. Solar Wind Sources of Magnetospheric ULF Waves, AGU Monograph, v. 81, pp. 325-334.

Olson, J.V., and B.J. Fraser. 1993. Conjugate ULF Pulsation in the Cusp. COSPAR/STEP Proceedings.

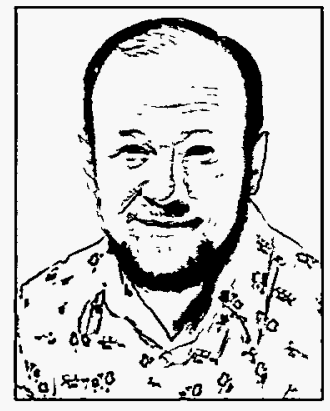

THOMAS E. OSTERKAMP, Professor of Physics; Southern Illinois University '62, B.A.; Saint Louis University '64, M.S., '68, Ph.D. in Physics; Department of Physics 1968-1973. Dr. Osterkamp joined the Geophysical Institute in 1973. His past research has been on the physics of snow, ice, and permafrost, particularly the scientific aspects of environmental, engineering, and scientific problems. This has included applications of the principles of soil physics, thermodynamics, heat and mass flow, and ice growth and decay theories to natural systems where ice is a component of the system (e.g., lake ice, river ice, sea ice, permafrost, subsea permafrost, seasonally frozen ground, and the seasonal snow cover). Dr. Osterkamp has taught undergraduate and graduate physics courses and specialty sources on the physics of ice, snow, sea ice, and permafrost.

Osterkamp, T.E. 1994. Evidence for Warming and Thawing of Discontinuous Permafrost in Alaska. EOS Transactions of the American Geophysical Union, v. 75(44), p. 85.

Osterkamp, T. E., T. Zhang and V. Romanovsky. 1994. Evidence for a Cyclic Variation of Permafrost Temperatures in Northern Alaska. Permafrost and Periglacial Processes, v. 5(3), pp. 137-144.

Osterkamp, T.E., and T. Fei. 1993. Potential Occurrence of Gas Hydrates in the Continental Shelf Near Lonely, Alaska. Proceedings of the 6th International Conference on Permafrost, July 1993, Beijing, China.

Romanovsky, V.E., and T.E. Osterkamp. 1994. Subsea Permafrost and Gas Hydrate Dynamics Since the Late Pleistocene Near Cape Thompson and Barrow, Alaska. Proceedings of the AAAS 45th Arctic Science Conference, August 1994, Anchorage, Alaska, pp. 208-209.

Romanovsky, V.E., and T.E. Osterkamp. 1994. Temporal and Spatial Behavior of the Active Layer in Northern Alaska: 1986-1993. EOS Transactions of the American Geophysical Union, v. 75(44), p. 86.

Nelson, F.E., A.H. Lachenbruch, M.K. Woo, E.A. Koster, T.E. Osterkamp, M.K. Gavrilova and G.O. Chang. 1993. Permafrost and Changing Climate. Proceedings of the 6th International Conference on Permafrost, July 1993, Beijing, China, v. 2.

Zhang, T., and T.E. Osterkamp. 1993. Changing Climate and Permafrost Temperatures in the Alaskan Arctic. Proceedings of the 6th International Conference on Permafrost, Beijing, China. 
ANTONIUS OTTO, Research Associate Professor; Ruhr-Universität Bochum '83, Diplome, '87, Ph.D. Dr. Otto's major research interests are in space plasma theory and simulation. His activities address the linear and nonlinear dynamics of macroscopic current-carrying plasma systems with applications in the field of solar wind-magnetosphere interaction, magnetotail dynamics, and the formation and evolution of discrete auroral structures. In 1992, Dr. Otto joined the Geophysical Institute where his current work addresses the three-dimensional evolution of the plasma and magnetic flux transport at magnetospheric boundaries, the corresponding magnetosphere-ionosphere coupling, and the formation of current filaments and thin auroral arc structures.

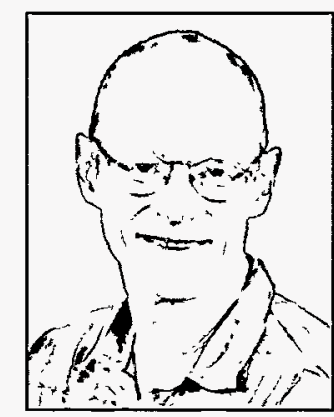

Otto, A., and G.T. Birk. 1994. Formation of Thin Discrete Auroral Arcs. Substorms 2, Proceedings of the Second International Conference on Substorms, Ed. by J.R. Kan, J.D. Craven, and S.-I. Akasofu, University of Alaska Fairbanks, pp. 127-131.

Otto, A. 1993. Simulation of Dynamic Processes at the Dayside Magnetopause. In: The Solar Wind - Magnetosphere System, Ed. by H.K. Biernat, S.J. Bauer and M. Heindler, Austrian Academy of Sciences, p. 179.

Otto, A., and G.T. Birk. 1993. Formation of Thin Auroral Arcs by Current Striation. Geophysical Research Letters, v. 20(24), pp. 2833-2836.

Otto, A., H. Ziegler and G.T. Birk. 1993. Plasma and Magnetic Field Signatures Generated by Three-Dimensional MHD Simulations, In: Spatio-Temporal Analysis for Resolving Plasma Turbulence(START), ESA WPP-47, European Space Agency, p. 337.

La Belle-Hamer, A.L., A. Otto and L.C. Lee. 1994. Magnetic Reconnection in the Presence of Sheared Plasma Flow: Intermediate Shock Formation. Physics of Plasmas, v. 1(3), pp. 706-713.

Ma, Z.W., L.C. Lee, Y. Shi and A. Otto. 1994. By Enhancements in Magnetospheric Current Sheets and Magnetic Flux Tubes. In: Physics of Space Plasmas (1992), SPI Conference Proceedings and Reprint Series, T. Chang, G.B. Crew and J.P. Jasperse, Eds., Scientific Publishers Inc., Cambridge, MA, Vol. 12, p. 575.

Ma, Z.W., A. Otto and L.C. Lee. 1994. Core Magnetic Field Enhancement in Single X Line, Multiple X Line and Patchy Reconnection. Journal of Geophysical Research, v. 99(A4), pp. 6125-6136.

Voge, A., K. Schindler and A. Otto. 1994. Nonlinear Current-Sheet Formation in Ideal Plasmas. Journal of Geophysical Research, v. 99, p. 21241.

Yan, M., A. Otto, D. Muzzel and L.C. Lee. 1994. Tearing Mode Instability in a Multiple Current Sheet System. Journal of Geophysical Research, v. 99(A5): 8657-8669.

Birk, G.T., A. Otto and H. Ziegler. 1993. A 3-D Three-Fluid Code to Study the Magnetosphere-IonosphereThermosphere System, In: Spatio-Temporal Analysis for Resolving Plasma Turbulence (START), ESA SPP47, European Space Agency, p. 357.

HANS PULPAN, Associate Professor of Geophysics; Montanistische Hochschule, Leoben, Austria '61, Dipl. Eng.; University of Illinois '64, M.Sc., '68, Ph.D. Dr. Pulpan has a background of research in tectonophysics and the physics of flow in porous media. His past activities include the deduction of the tectonic stress field from hydraulic well-fracturing data. After joining the Geophysical Institute in 1968, he has been engaged in seismotectonic studies of the eastern portion of the Aleutian-Alaska arc system, wave propagation in heterogeneous media, and volcano seismology. His applied research includes seismic risk and soil response studies in various parts of Alaska.

Petersen, H., M. Wyss, and H. Pulpan. 1993. Stress Tensor Inversion in the Alaska Subduction

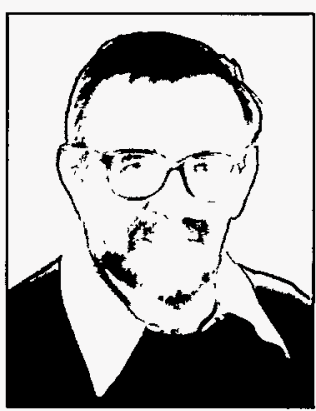
Zone. EOS, 74(3), p. 95. 


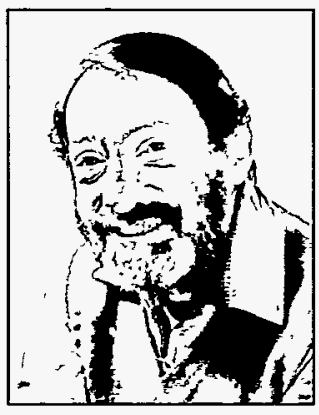

MANFRED H. REES, Professor Emeritus of Geophysics; West Virginia University '48, B.S.E.E.; University of Colorado'56, M.Sc., '58, Ph.D. A former member of the staff, Dr. Rees rejoined the institute in 1975 after several years at the University of Colorado, where he was engaged in research and in teaching physics and chemistry of planetary atmospheres. He worked on a variety of upper-atmosphere physics problems, including auroral spectroscopy, energetic particle interactions, ionospheric electric fields, thermospheric dynamics and chemistry, and ionospheric-magnetospheric interactions. His publications are principally in auroral physics.

Lanchester, B.S., J.R. Palmer, M.H. Rees, D. Lummerzheim, K. Kaila, and T. Turunen. 1994. Energy Flux and Characteristic Energy of an Elemental Auroral Structure. Geophysical Research Letters, v. 21, p. 2789.

Min, Q.L., D. Lummerzheim. M.H. Rees and K. Stamnes. 1993. Effects of a Parallel Electric Field and the Geomagnetic Field in the Topside Iososphere on Auroral and Photoelectron Energy Distributions. Journal of Geophysical Research, v. 98(A1 1), pp. 19223-19234.

Sharber, J.R., R.A. Frahm, J.D. Winningham, J.C. Biard, D. Lummerzheim, M.H. Rees, D.L. Chenette, E.E. Gaines, R.W. Nightingale and W.I. Imhof. 1993. Observations of the UARS Particle Environment Monitor and Computation of Ionization Rates in the Middle and Upper Armosphere During a Geomagnetic Storm. Geophysical Research Letters, v. 99, p. 3855.

Winningham, J.D., J.R. Sharber, R.A. Frahm, J.L. Burch, N. Eaker, R.K. Black, V.A. Belvins, J.P. Andrews, J. Rudzki, D.L. Chenette, D.W. Datlowe, E.E. Gaines, W.I. Imhof, R.W. Nightingale, S.S. Prasad, R.M. Robinsonn, T.L. Schumaker, R.R. Vondrak, P.F. Bythrow, B.J. Anderson, T.A. Potemra, L.J. Zanetti, D.B. Holland, M.H. Rees, D. Lummerzheim, G.C. Reid, R.G. Roble, C.R. Clauer and P.M. Banks. 1993. The UARS Particle Environment Monitor. Journal of Geophysical Research, v. 98, p. 10,649.

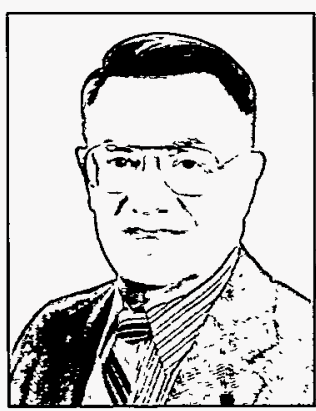

JUAN G. ROEDERER, Professor Emeritus of Physics and Senior Consultant; University of Buenos Aires '52, Ph.D. Dr. Roederer has a background in high-energy physics, cosmic rays and magnetospheric physics. His work on numerical modeling led to a quantitative understanding of the earth's radiation belts. He also has conducted research, taught courses, and written a book on psychoacoustics and neuropsychology. Dr. Roederer was Director of the Geophysical Institute from 1977 to 1986, and Dean of the College of Environmental Sciences from 1979 to 1982 . His research interest centers on magnetospheric plasma physics of Earth and Jupiter. He is a fellow of the American Association for the Advancement of Science and the American Geophysical Union, corresponding member of the Academies of Science of Argentina and Austria, Member of the Third World Academy of Sciences, Past president of the ICSU Committee on Solar-Terrestrial Research, and an accomplished organist. He served as Chairman of the United States Arctic Research Commission from 1987 to 1991.

Roederer, J.G. 1994. Our Forgotten Colleagues in Cuba. EOS, Transactions, American Geophysical Union, v. 75(20), pp. 228-229.

Roederer, J.G. 1994. STEP in Today's World. STEP International, v. 4(2), pp. 8-9.

Roederer, J.G. 1994. STEP: Needs for International Scientific Coordination. In: COSPAR Colloquium No. 5: 1992 STEP Symposium, Pergamon Press, Oxford, pp. 15-16.

Roederer, J.G. 1993. Arctic Research: Asking the Right Questions. MAB Northern Sciences Network Newsletter, v. 13, pp. 18-21.

Roederer, J.G. 1993. STEP and Global Change. STEP International, v. 3(4), pP. 1-4.

Lester, M., A.J. Coates, R.A. Harrison, D. Rees, J.G. Roederer, M.J. Rycroft and M.A. Saunders. 1993. International Solar Terrestrial Energy Programme and the UK Participation. Surveys in Geophysics, v. 14, pp. 555-583. 
CHARLOTTE A. ROWE, Deputy State Seismologist; New Mexico Institute of Mining and Technology '81, B.S., University of Alaska Fairbanks '88, M.S. Ms. Rowe joined the Geophysical Institute in 1988. Her research background includes eruption monitoring at Mount St. Helens; seismic studies on Mount Erebus volcano, Antarctica; seismic hazard evaluation ar Makushin Volcano on Unalaska; and seismic velocity studies in Yukon Flats. She is currently investigating reservoir-type induced sources for earthquake swarms in Southeast Alaska, and is developing regular earthquake information features with the news media. As cooperative PI with the Princeton Earth Physics Project, she is working on a program to incorporate seismology into the school science curricula statewide. Ms. Rowe works cooperatively with USGS investigators to supervise data analysis, archiving and distribution for the

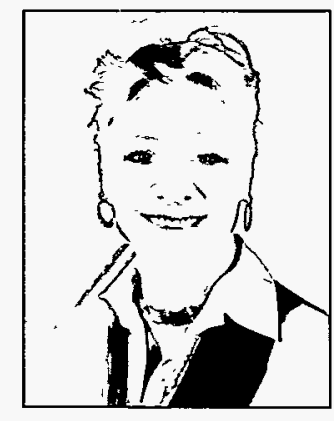
Alaska Earthquake Information Center.

Rowe, C. 1994. Historic Seismicity in Alaska. Proceedings from Conference on the Status of Knowledge and Preparedness for Earthquake Hazards in Alaska. Earthquake Alaska: Are We Prepared? Eds. R. Combellick, R. Head, and R. Updike, U. S. Geological Survey Open-File Report, 94-218, pp. 3-8.

Rowe, C. 1994. Seismology in Alaska Schools. Princeton Earth Physics Project (PEPP) Newsletter, V. 2. Princeton University, New Jersey.

Dibble, R.R., B. O'Brien, and C.A. Rowe. 1994. The Velocity Structure of Mount Erebus, Antarctica, and its Lava Lake. Volcanological and Environmental Studies of Mount Erebus, Antarctica, P.R. Kyle (Ed.), Antarctic Research Series, American Geophysical Union, v. 66, pp. 1-16.

Fogleman, K., C. Rowe, C. Stephens and B. Hammond. 1994. Earthquakes in Alaska: January-August 1991. Alaska State Seismologist's Reports, numbers 91-01-01 through 91-01-08, Geophysical Institute, University of Alaska Fairbanks.

Fogleman, K., C. Rowe, C. Stephens and B. Hammond. 1994. Earthquakes in Alaska: January 1993. Alaska State Seismologist's Report, number 93- 01-01, Geophysical Institute, University of Alaska Fairbanks.

Hammond, W.R., J.C. Lahr, C.A. Rowe and J.P. Benoit. 1993. The Salcha Seismic Zone Near Fairbanks, Alaska (abs.). EOS, Transactions of the American Geophysical Union, v. 74(43), p. 417.

WILLIAM M. SACKINGER, Associate Professor of Geophysics and Electrical Engineering; University of Notre Dame'59, B.S.; Cornell University '61, M.S., '69, Ph.D. After an 11 -year career in industry, Dr. Sackinger joined the University of Alaska in 1970 and became involved in research on electrical properties of ice and snow, dynamics of sea ice motion, and stresses in sea ice. He also has studied adfreeze bonding of sea ice to structures; corrosion of pipelines and arctic offshore structures; ice island generation and movement; spray sea ice; and environmental assessment and regulation for arctic development. He is co-editor of the three-volume book, Port and Ocean Engineering under Arctic Conditions, and of the books Ice Technology for Polar Operations and Advances in Ice Technology. His recent interests are in the conversion of methane to higher hydrocarbons using ion impacts on surfaces. He serves on several international committees and corporate boards, and is Director of Intellectual Property and Licensing for the University of Alaska.

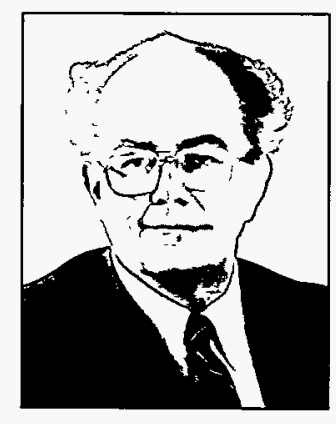

Sackinger, W.M., and V.A. Kamath. 1994. Direct Conversion of Northern Natural Gas to Higher Hydrocarbon Liquids Near the Wellhead. POLARTECH '94, 22 March 1994; Lulea, Sweden, pp. 445-450.

Sackinger, W.M. and O.P. Smith. 1994. Delay of Arctic Shoreline Erosion Through the Use of Arrificial Spray Ice/ Sediment Massifs. POLARTECH '94, 22 March 1994; Lulea, Sweden, pp. 347-354.

Sackinger, W.M., V.A. Kamath, B.L. Morgan and R.W. Airey. 1993. Natural Gas Conversion to Higher Hydrocarbons Using Plasma Interactions with Surfaces. Final Report Ed.; Fairbanks, Alaska, Geophysical Institute, Universiry of Alaska Fairbanks, December, 1993, Grant No. DE-FG21-90MC27420.

Lou, M. and W.M. Sackinger. 1993. Analysis of a Fracture of a Multi-Year Sea Ice Flow Acting Upon a Compliant Offshore Structure. Proceedings of the 12th International Conference on Port and Ocean Engineering Under Arctic Conditions, 17-20 August 1993, Hamburg Germany, v. 2, pp. 547-558. 

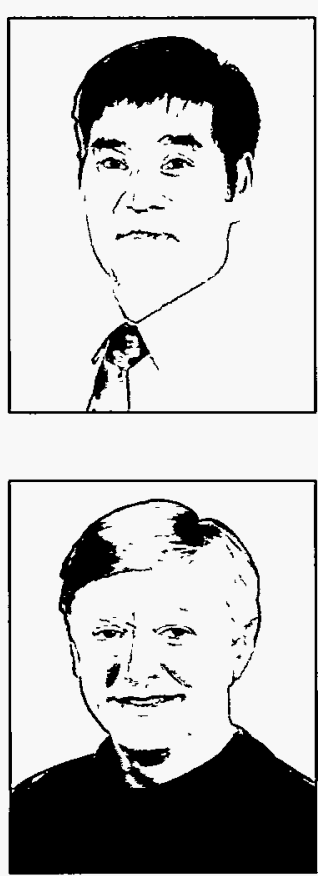

TATSUMI SASAKI, Exchange Visitor from Hokkaido University of Education; Tokyo University of Education '74, B.S., '76, M.S.; University of Tsukuba '83, D.Sc., Dr. Sasaki's research has been in the field of coastal geomorphology, especially in the study of topographic changes in the foreshore zones of sandy beaches. He is presently engaged in research on ice-push phenomena which occur along the shorelines of inland lakes. During his stay at the Geophysical Institute, he has conducted field investigations of land forms along the shores of several lakes and is monitoring ice movement in Harding Lake through field survey methods.

Sentman, D.D. 1994. A Critical Comparison of Schumann Resonance Theory and Observations. Fifth Symposium on Global Change Studies and Symposium on Global Change and the Meteorological Applications of Lightning Information. American Meteorological Society, Boston, pp. 311-314.

Sentman, D.D. 1994. Schumann Resonances. CRC Handbook of Atmospheric Electrodynamics, (Hans Volland, editor), CRC Press, Boca Raton, pp. 267-295.

Sentman, D.D., and D.A. Ehring. 1994. Midlatitude Detection of ELF Whistlers. Journal of Geophysical Research, v. 99 , p. 2183-2190.

Sentman, D.D., and E.M. Wescott. 1994. Preliminary Observations From the Sprites 94 Aircraft Campaign: 1. Red Sprites. Geophysical Research Letters, pp. 1205-1208.

Sentman, D.D., and E.M. Wescott. 1994. Recent Observations of a Variety of Optical Emissions Extending Upward From the Tops of Thunderstorms to the Stratosphere, Mesosphere, and Ionosphere. Physics of Plasmas, pp. 2514-2522.

Senrman, D.D., and E.M. Wescott. 1994. Red Sprites and Blue Jets. Geophysical Institute Video Production, University of Alaska, 9 July.

Sentman, D.D., and E.M. Wescott. 1993. Observations of Upper Atmospheric Optical Flashes Recorded From an Aircraft. Geophysical Research Letters, v. 20, pp. 2857-2860.

Wescott, E.M., and D.D. Sentman. 1994. Preliminary Observations From the Sprites 94 Aircraft Campaign: 2. Blue Jets. Geophysical Research Letters, pp. 1209-1212.

Williams, E., K. Blasch, D. Sentman and B. Boldi. 1994. Extraction of Information on Global Lightning Activity From Single-Station Measurements in the Schumann Band. Fifth Symposium on Global Change Studies and Symposium on Global Change and the Meteorological Applications of Lightning Information, American Meteorological Society, pp. 307-310.

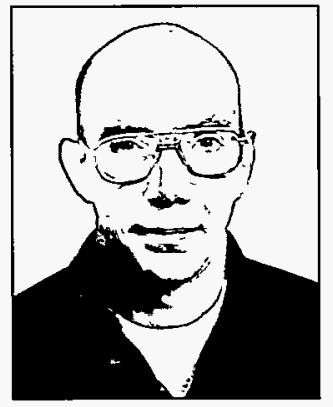

LEWIS H. SHAPIRO, Senior Research Consultant; South Dakota School of Mines and Technology'62 B. S.; University of Minnesota '71, Ph.D. Dr. Shapiro joined the staff in 1971 after previous work on the origin of gold deposits in the Black Hills and the regional structural geology of the Middle Rocky Mountain Province. He has since studied the relationship between earthquakes and structural geology in interior Alaska and remote sensing applications to geologic problems. For several years he has been involved principally in sea ice studies, particularly the determination of mechanical properties, and the deformation and movement in near-shore areas. He retired from the institute in February 1990. 
Shapiro, L.H., and W.F. Weeks. 1993. The influence of Crystallographic and Structural Properties on the Flexural Strength of Small Sea Ice Beams. Ice Mechanics - 1993; 1st Joint Mechanics Meeting of ASME, ACSE and SES, Charlottesville, VA, Dempsey, J.P., Z.P. Bazant, Y.D.S. Rajopakse, and S.S. Sunder, (eds.); AMD v. 163, pp. 177-188.

GLENN E. SHAW, Professor of Geophysics; Montana State University'63, B.S.; University of Southern California '65, M.S.; University of Arizona '71, Ph.D. Dr. Shaw joined the Geophysical Institute in 1971 and has worked on a wide variety of problems in the atmospheric sciences, including experimental studies of solar variability, modeling and experimental investigation of the effects of clouds and aerosols on climate, and theoretical work in radiation transfer through planetary atmospheres. Dr. Shaw was an early investigator of arctic haze and has continued to have a deep involvement with studies involving the chemistry and physical chemistry of aerosols in the arctic regions. He is a Fellow of the Royal Meteorological Society, a member of the Polar Research Board at the National Academy of Sciences and recipient of the Terris Moore Prize.

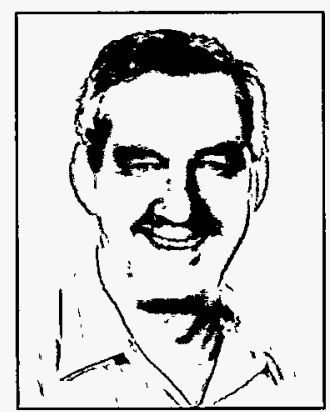

Shaw, G.E., J.A. Shaw and R.A. Shaw. 1993. The Snows of Interior Alaska. Atmospheric Environment, v. 27A(13), pp. 2091- 2096.

Shaw, G.E., K. Stamnes and Y.X. Hu. 1993. Arctic Haze: Perturbation to the Radiation Field. Meteorology and Atmospheric Physics, v. 51, pp. 227-235.

Kienle, J., Q. Ji and G.E. Shaw. 1993. Physics and Microphysics of Volcanic Plumes. Alaska Volcano Observatory Publication, v. 5(2), p. 12.

Sturges, W.T., and G.E. Shaw. 1993. Halogens in Central Alaska. Atmospheric Environment, v. 27A(17/18), pp. 2969-2977.

ROGER W. SMITH, Professor of Physics, University of Exeter '63, B.S., '67, Ph.D. Dr. Smith joined the Geophysical Institute in July, 1984. His research is specialized in the study of upper-atmospheric dynamics and auroral dynamics at high latitudes by optical methods. Wind and temperature studies of the thermosphere have been conducted in both the Arctic and Antarctic. His interests have extended to optical investigations of the mesosphere using the same techniques. Studies of auroral transients have been conducted at Svalbard. Some new projects are being developed in the optical imaging of ionospheric convection and magnetospheric dynamics from space. He teaches in both graduate and undergraduate programs in the department of physics.

Smith, R.W. 1994. Dayside Aurora and Magnetospheric Processes. In: Physical Signatures of Magnetospheric Boundary Layer Processes, A. Egeland, J. Holtet and P.E. Sandholt (Eds.), Kluwer Academic Publishers, pp. 141-155.

Smith, R.W., G. Hernandez, K. Price, G. Fraser, K.C. Clark, W.J. Schulz, S. Smith and M. Clark. 1994. The June 1991 Thermospheric Storm Observed in the Southern Hemisphere. Journal of Geophysical Research, v. 99(A9), pp. 17,609-17,615.

Fasel, G.J., J.I. Minow, R.W. Smith and C.S. Deehr. 1994. Poleward-Moving Auroral Forms: What Do We Really Know About Them? Physical Signatures of Magnetospheric Boundary Layer Processes, pp. 21 1-226.

Fasel, G.J., J. Minow, R.W. Smith, C.S. Deehr and L.C. Lee. 1994. Multiple Brightenings of Poleward Moving Dayside Auroral Forms. In: Solar Wind Sources of Magnerospheric ULF Waves, Geophysical Monograph Serial, Eds. M. Engebretson, K. Takahashi, M. Scholer, AGU Washington, D.C., pp. 201-212.

Garrido, D., R.M. Robinson, Y.T. Chiu, H.L. Collin, R.W. Smith and D.W. Swift. 1994. Magnetospheric Imaging of High Latitude Outflows. Annales Geophysicae, v. 12, pp. 169-182.

Garrido, D.E., R.W. Smith, D.W. Swift, S.-I. Akasofu, R.M. Robinson and Y.T. Chiu. 1994. Imaging the Plasmosphere and Trough Regions in the Extreme Ultraviolet Region. Optical Engineering, v. 33, p. 371. 
Minow, J.I., R.W. Smith, W.F. Denig and P.T. Newel. 1994. Dayside Auroral Dynamics During Reconfiguration of the Auroral Oval. In: Physical Signatures of Magnetospheric Boundary Layer Processes, A. Egeland, J. Holtet and P.E. Sandholt (Eds.), Kluwer Academic Publishers, pp. 201-210.

Conner, J.F., R.W. Smith and G. Hernandez. 1993. Techniques for Deriving Doppler Temperatures from MultipleLine Fabry-Perot Profiles: An Analysis. Applied Optics, v. 32(23), pp. 4437- 4444.

Fraser, G.J., G. Hernandez and R.W. Smith. 1993. Eastward-Moving 2-4 Day Waves in the Winter Antarctic Mesosphere. Geophysical Research Letters, v. 20, pp. 1547-1550.

Fasel, G.J., L.C. Lee and R.W. Smith. 1993. A Mechanism for the Multiple Brightenings of Dayside Poleward-Moving Auroral Forms. Geophysical Research Letters, v. 20(20), pp. 2247-2250.

Garrido, D.E., R.W. Smith, C.A. Marsh, A.B. Christensen and S. Chakrabarti. 1993. Inversion of Photometric $\mathrm{He}^{+}$ $(30.4 \mathrm{~nm})$ Intensities to Obtain $\mathrm{He}^{+}$Distributions, SPIE, v. 2008, p. 121.

Minow, J.I., and R.W. Smith. 1993. Optical Remote Sensing of the Ion Convection Pattern in the High-Latitude Ionosphere from a Polar Orbiting Satellite. Geophysical Research Letters, v. 20(7), pp. 559-562.

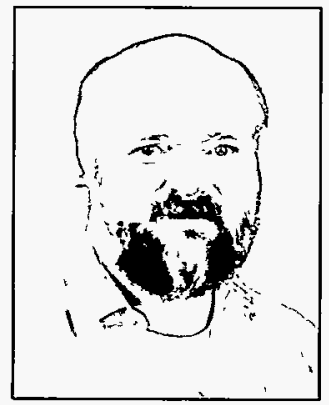

KNUT H. STAMNES, Professor of Physics, University of Oslo '69, B.S., '72, M.S.; University of Colorado '78, Ph.D. A former member of the staff, Dr. Stamnes rejoined the institute in 1988 after several years at the University of Tromso where he was engaged in teaching and research. His past work includes radio wave propagation in the ionosphere, energetic electron interaction with the atmosphere, and modeling and interpretation of auroral observations. His present research interests include radiation transport in planetary atmospheres, atmospheric response to solar terrestrial radiation and climatic implications (energy balance), satellite remote sensing, and kinetic theory of planetary atmospheres. He has taught undergraduate courses in physics and numerical methods, and developed and taught a graduate course in radiative transfer.

Stamnes, K. 1993. Modeling of UV Penetration Through the Atmosphere and Ocean. In: The Role of the Stratosphere in Global Change, M.L. Chanin (Ed.), NATO ASI Series, Springer-Verlag, Berlin, v. 18.

Stamnes, K. 1993. The Stratosphere as a Modulator of Ultraviolet Radiation into the Biosphere. Surveys in Geophysics, v. 14, pp. 167-186.

Stamnes, K., and B.D. Zak. 1993. The Atmospheric Radiation Measurements (ARM) Program's North Slope of Alaska Climate Research Site: ARM's Window on the Arctic. Atmospheric Radiation, June 30, Tromsö, Norway, SPIE, v. 2049, pp. 107-110.

Jin, Z., and K. Stamnes 1994. Radiative Transfer in Nonuniformly Refracting Layered Media: Atmosphere-Ocean System. Applied Oprics, v. 33(3), pp. 431-442.

Jin, Z., K. Stamnes, W.F. Weeks and S.C. Tsay. 1994. The Effect of Sea Ice on the Solar Energy Budget in the Atmosphere-Sea Ice-Ocean System: A Model Study. Journal of Geophysical Research, v. 99(C12), pp. 25,281-25,294.

Leontyeva, E., and K. Stamnes. 1994. Estimations of Cloud Optical Thickness from Ground-based Measurements of Incoming Solar Radiation in the Arctic. Journal of Climate, v. 7(4), pp. 566- 578.

Leontieva, E., and K. Stamnes. 1994. Remotely Sensed Measurements ofCloud Optical Depth Using a Ground-Based Multi-Filter Rotating Shadow Band Radiometer in the Arctic (Methodology). Proceedings of the Third Circumpolar Symposium on Remote Sensing of Arctic Environments, May 16 - 20, Fairbanks, Alaska, p. 33.

Leontieva, E., K. Stamnes and J.A. Olserh. 1994. Cloud Optical Properties at Bergen (Norway) Based on the Analysis of Long-Term Solar Irradiance Records. Theoretical and Applied Climatology, v. 50, pp. 73-82.

Zeng, J., R. McKenzie, K. Stamnes M. Wineland and J. Rosen. 1994. Measured UV Spectra Compared with Discrete Ordinate Method Simulations. Journal of Geophysical Research, v. 99(D11), pp. 23,019-23,030.

Hu, Y.-X., and K. Stamnes. 1993. An Accurate Paramererization of the Radiative Properties of Water Clouds Suitable for Use in Climate Models. Journal of Climate. v. 6, n. 4, pp. 728-742.

Kylling, K., K. Stamnes, R.R. Meier and D.E. Anderson. 1993. The 200- to 300-nm Radiation Field in the Stratosphere: Comparison Between Theory and Experiment. Journal of Geophysical Research, v. 98(D2), pp. 2741-2745. 
Leontieva, E.N., and K.H. Stamnes. 1993. Cloud/Albedo Effects on the Radiation Budget in the Arctic. Atmospheric Radiation, June 30, Tromso, Norway, SPIE, v. 2049, p. 111-118.

Min, Q.L., D. Lummerzheim, M.H. Rees and K. Stamnes. 1993. Effects of a Parallel Electric Field and the Geomagnetic Field in the Topside Ionosphere on Auroral and Photoelectron Energy Distributions. Journal of Geophysical Research, v. 98(A11), pp. 19223-19234.

Mobley, C.D., B. Gentili, H.R. Gordon, Z. Jin, G.W. Kattawar, A. Morel, P. Reinersman, K. Stamnes and R.H. Stavn. 1993. Comparison of Numerical Models for Computing Underwater Light Fields. Applied Optics, v. 32, n. 36, pp. 7484-7504.

Shaw, G.E., K. Stamnes and Y.X. Hu. 1993. Arctic Haze: Perturbation to the Radiation Field. Meteorology and Atmospheric Physics, v. 51, pp. 227-235.

Zeng, J., Z. Jin and K. Stamnes. 1993. Impact of Stratospheric Ozone Depletion on UV Penetration into the Ocean at High Latitudes. Underwater Light Measurements; 29 June 1993, Tromso, Norway, H. Chr. Eilertsen (Ed.), SPIE, v. 2048, pp. 56-63.

HANS C. STENBAEK-NIELSEN, Professor of Geophysics; Royal Technical University of Denmark '65, M.Sc. Professor Nielsen has a research background in auroral and magnetospheric physics. At the institute, he has worked mainly in auroral research. During the last 10 years, he has been engaged in chemical release experiments, notably the barium shaped-charge program, and in auroral work. Professor Nielsen also is teaching graduate and undergraduate courses within the Department of Physics.

Stenbaek-Nielsen, H.C. and E.M. Wescotr. 1994. Observing Magnetospheric Topology. Proceedings of the Second International Conference on Substorms, held at the University of Alaska Fairbanks, 7-11 March 1994, J.R. Kan, J.D. Craven, S.-I Akasofu, (Eds.), p. 275.

Stenbaek-Nielsen, H.C., E.M. Wescott and T.J. Hallinan. 1993. Observed Barium Emission Rates. Journal of Geophysical Research, v. 98(A10), pp. 17,491-17,500.

Hoch, E.L., T.J. Hallinan and H.C. Stenbaek-Nielsen. 1994. Quantitative Measurements of Vaporization, Burst Ionization and Emission Characteristics of Shaped Charge Barium Releases. Journal of Geophysical Research, v. 99, pp. 13263-13271.

Wescott, E.M., H.C. Stenbaek-Nielsen, D.L. Hampton and P.A. Delamere. 1994. Results of Critical Velocity Experiments with Barium, Strontium, and Calcium Releases from CRRES Satellite. Journal of Geophysical Research, v. 99(A2), pp. 2145-2158.

Milinevsky, G.P., A.I. Kashirin, Yu, A. Romanovsky, H.C. Stenbaek-Nielsen and M.C. Kelley. 1993. Long-Lived Artificial Ion Clouds in the Earth's Ionosphere. Geophysical Research Letters, v. 20, p. 1019.

Wescott, E.M., T.J. Hallinan, H.C. Stenbaek-Nielsen, D.W. Swift and D.D. Wallis. 1993. Rapid Ray Motions in Barium Plasma Clouds and Auroras. Journal of Geophysical Research, v. 98(A3), pp. 3711- 3724.

DAVID B. STONE, Professor of Geophysics; University of Keele North Staffordshire '56, B.A.; University of Newcastle upon Tyne '63, Ph.D. Dr. 's current research interests are in paleomagnetism and tectonics. His ongoing paleomagnetic studies include work on tectonostratigraphic terranes in Alaska and Siberia and their paleogeographies, the application of paleomagnetic techniques to paleoclimate studies, and investigations as to whether great earthquakes leave a paleomagnetic record in lake sediments. His other ongoing research includes the use of seismic, gravity and magnetic methods to study the structures of accreted terranes. Dr. teaches geophysics and tectonics and related geospeculation.

Stone, D. G., S. G. Crumley, K. Fujita, A. Neustroev and L. Parfenov. 1994. Crystalline

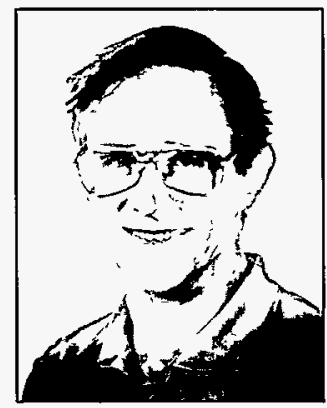
Terrane, Northeast Russia. Supplement to EOS, April 1994, p. 124.

Stone, D. G., S. G. Crumley, A. Neustroev and L. M. Parfenov. 1994. Paleomagnetic Constraints on the Paleogeography of Northeast Russia. International Conference on Arctic Margins, held in Magadan, September 1994. 
Stone, D. B., S. G. Crumley and L. M. Parfenov. 1994. Paleomagnetism and the Koyma Structural Loop. Proceedings of the International Conference on Arctic Margins, held in Anchorage, Alaska, D. K. Thurston, K. Fujita (Eds.), pp. 189-194.

Stone, D. B., and S. K. Runcorn (Eds.). 1993. Flow and Creep in the Solar System: Observations, Modeling and Theory. NATO ASI Series C, pp. 506.

Beget, J. E., S.D. Stihler and D. B. . 1994. A 500-Year Long Record of Tephra Falls From Mt. Redoubt and Other Volcanoes in Upper Cook Inlet, Alaska. Journal of Volcanology and Geothermal Research, v. 62, pp. 55-67.

Raikevitch, M., A. Harathunian and D. . 1994. Paleomagnetism and Chronology of the Okhotsk-Chukotka Volcanic Belt of Northeast Russia. Supplement to EOS, November 1994, p. 202.

Crumley, S. G. and D. B. . 1993. Paleomagnetic Overprint Shows No Post-Accretion Deformation Across the Western Kolyma-Verkhoyansk Collision Zone. Supplement to EOS, Fall 1993, p. 214.

Stihler, S. D., D. B. and J. E. Beget. 1993. "Varve" Counting Versus Tephrochronology and ${ }^{137} \mathrm{Cs}$ and ${ }^{210} \mathrm{~Pb}$ Dating: A Comparative Test at Skilak Lake, Alaska: Reply to Comment. Geology, v. 21, p. 763.

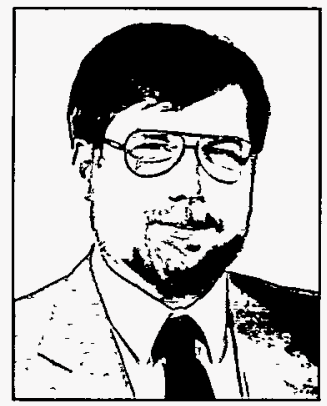

WILLIAM J. STRINGER, Associate Professor of Geophysics; New Mexico State University '62, B.S.; University of Alaska'66, M.S., '71, Ph.D. Dr. Stringer's past activities include studies of auroral morphology and the role of atomic hydrogen emissions in auroral displays. His recent activities relate to applications of satellite imagery to Alaska problems, including archaeology, flood hazards, soil surveys, vegetation mapping, and oceanic ice. He has conducted a comprehensive characterization of Alaska nearshore ice conditions for the Outer Continental Shelf Environmental Assessment Program. Dr. Stringer also has produced movies and exhibits and conducted community college courses designed to increase public understanding of science. His community activities include six years as a member of the Fairbanks North Star Borough Assembly. Currently he is involved in a remote sensing investigation of sources of radionuclide pollution in the former Soviet Union.

Stringer, W., and K. G. Dean. 1993. Remote Sensing Data Acquisition. Analysis and Archival Final Report, OCSEAP Research Unit 716, available from the Minerals Management Service, 949 E. 36th Avenue, Anchorage, Alaska 99508-4302, 209 pp.

Dean, K. G., W. J. Stringer, K. Ahlnäs, C. Searcy and T. Weingartner. 1994. The Influence of River Discharge on the Thawing of Sea Ice, Mackenzie River Delta: Albedo and Temperature Analyses. Polar Research, v. 13, pp. $83-94$.

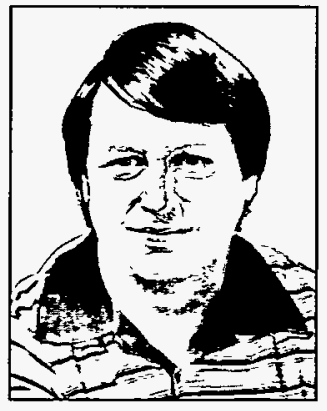

SAMUELE. SWANSON, Associate Professor of Geology; University of California, Davis' 68 , B.S., '70, M.S.; Stanford University'74, Ph.D. Dr. Swanson held a joint appointment between the Department of Geology and Geophysics and the Geophysical Institute through August 1994. His specialty is in igneous petrology. His past activities included crystal growth from silicate liquids, phase equilibria in granitic systems, mineralogy and petrology of granitic and ultramafic rocks and timing of plutonism and volcanism in western North America. He also conducted studies involving magmatism in the eastern Aleutian arc, petrogenesis of granites associated with ore deposits, and textural development in igneous rocks.

Swanson, S. E., and J. E. Beget. 1994. Melting Properties of Volcanic Ash. T. J. Casadevall, (Ed.), Volcanic Ash and Aviation Safery, Proceedings of the First International Symposium on Volcanic Ash and Aviation Safery held in Seattle, Washington, 8 July 1991, pp. 87-92.

Swanson, S. E., C. J. Nye, T. P. Miller and V. F. Avery. 1993. Geochemistry of the 1989-1990 Eruption of Redoubt Volcano: Part II. Magma Mixing Evidence Form Mineral and Glass Chemistry. Journal of Volcanology and Geothermal Research, v. 62, pp. 453-468.

Nye, C. J., S. E. Swanson, V. F. Avery and T. P. Miller. 1994. Geochemistry of the 1989-1990 Eruption of Redoubt Volcano: Part I. Whole-Rock Major- and Trace-Element Chemistry. Journal of Volcanology and Geothermic Research, v. 62, pp. 427-450. 
DANIEL W. SWIFT, Professor of Physics Emeritus; Haverford College'57, B.A.; Massachusetts Institute of Technology '59, M.S. Professor Swift joined the Geophysical Institute in 1963. His background is in theoretical research and numerical simulation of fluids and plasmas. His past work is in ionospheric physics, radio wave propagation, and simulation of convective flow in porous media. He was the coordinator of the summer intern program for undergraduates from 1987 to 1993 . His recent research efforts have focused on using methods of numerical simulation to understand magnetospheric processes responsible for auroral and geomagnetic storm phenomena. He has contributed to the analysis of barium injection experiments in space. He is engaged in a collaborative effort to develop a global-scale numerical model of the earth's magnetosphere using a hybrid code and is developing numerical techniques to simulate performance of high-powered microwave devices. Professor Swift has

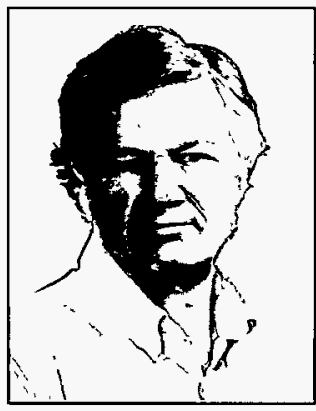
taught graduate courses in electricity and magnetism, mathematical physics, classical mechanics, and has developed a course in methods of numerical simulation.

Garrido, D. E., R. W. Smith, D. W. Swift, S.-I. Akasofu, R. M. Robinson and Y. T. Chiu. 1994. Imaging the Plasmosphere and Trough Regions in the Extreme Ultraviolet Region. Optical Engineering, v. 33, p. 371.

Wescott, E. M., T. J. Hallinan, H. C. Stenbaek-Nielsen, D.W. Swift and D.D. Wallis. 1993. Rapid Ray Motions in Barium Plasma Clouds and Auroras. Journal of Geophysical Research, v. 98(A3), pp. 3711- 3724.

HIROSHI L. TANAKA, Affiliate Assistant Professor of Physics (University of Tsukuba Institute of Geoscience, Tsukuba 305, Japan); University of Tsukuba, Japan '80, B.S.; University of Missouri-Columbia '88, Ph.D. Dr. Tanaka has a background of research in global energetics of atmosphere circulation, climatology, and numerical modeling of largescale motions. His past research includes spectral energetics analyses of the Global Weather Experiment during 1979, the development of three-dimensional normal mode energetics of the general circulation, and theoretical stability analyses of atmospheric baroclinic waves. Dr. Tanaka is analyzing nonlinear energy interactions of large-scale atmospheric motions over the horizontal and vertical spectral domains. He also is working on a particle-tracking Lagrangian model for the volcanic plume prediction. Dr. Tanaka received the Yamamoto-Shono Award from the Meteorological Society of Japan in 1992. He left the Geophysical Institute in August 1991 but maintains a working association with researchers here.

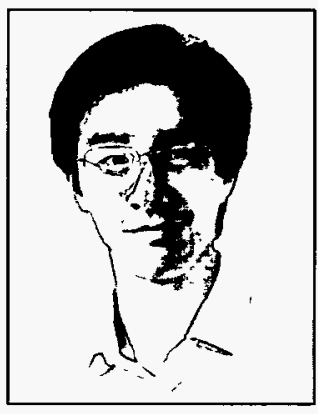

Tanaka, H. L. 1994. Development of a Prediction Scheme for Volcanic Ash Fall from Redoubt Volcano, Alaska. T. J. Casadevall, (Ed.). Volcanic Ash and Aviation Safety, Proceedings of the First International Symposium on Volcanic Ash and Aviation Safety held in Seattle, Washington, 8 July 1991, pp. 283-291.

Tanaka, H. L., K. G. Dean and S.-I. Akasofu. 1993. Predicting the Movement of Volcanic Ash Clouds. EOS, Transactions of the American Geophysical Union. v. 74(20), p. 231.

Akasofu S.-I. and H. L. Tanaka. 1994. Hurry up to develop volcanic plume tracking model. Kagaku Asahi, 5, 121124 (in Japanese).

Dean, K., S.-I. Akasofu and H. Tanaka. 1993. Volcanic Hazards and Aviation Safety: Developing Techniques in Alaska. Aviation Safety Journal, v. 3(1), pp. 11-15.

JULIA H. TRIPLEHORN, Associate Professor of Library Science; Ohio Wesleyan '57, B.A.; University of Illinois '59, M.S.L.S. Ms. Triplehorn is a scientific reference librarian whose major responsibility is the administration of the Keith B. Mather Library in the Geophysical Institute. Her research interests are in arctic science information and the compilation of scientific bibliographies.

Triplehorn, J. 1993. Tracking Information Sources in Gold Mining. Geoscience Information Proceedings, v. 24, pp. 125-128.

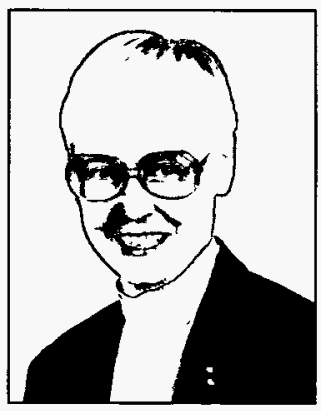




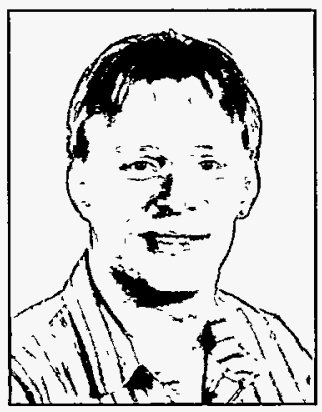

GUY TYTGAT, Geophysicist; University of Alaska Fairbanks '86, B.S. Tytgat is currently working toward an M.S. in Geological Engineering at UAF. He was a student assistant at the institute from August 1981 through December 1985, gathering and processing seismic data and doing field work. From January 1988 until October 1990, he performed data analysis and research on seismic source parameters of subduction earthquakes, and on intraplate seismicity as a research assistant. He is a geophysicist for the Alaska Volcano Observatory, performing signal analysis of tremor and earthquakes related to volcanic activity for eruptions of Redoubt Volcano in 1989-90 and Mt. Spurr Volcano in 1992.

Lu, Z., M. Wyss, G. Tytgat and S. McNutt. 1994. Aftershocks of the 13 May 1993 Shumagin Alaska Earthquake. Geophysical Research Letters, v. 21(6), pp. 497-500.

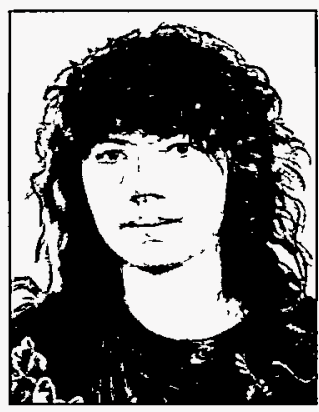

VERA A. VORONINA, Geophysicist; Moscow State University, Russia '89, M.S., Moscow State University, Russia '90, Ph.D. Dr. Voronina joined the Geophysical Institute in 1991. For two years she has studied space plasma physics. Current research interests include geostatistical data analysis, determining glacier velocities using SAR imagery, and measurement of ice sheet elevations by satellite radar altimetry. Dr. Voronina taught a graduate class in Stochastic Processes in 1994.

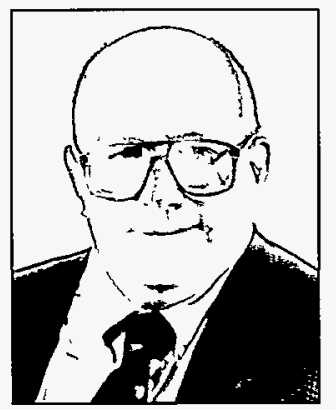

CARL A. WALES, Project Director, Alaska Synthetic Aperture Radar Facility, Massachusetts Institure of Technology, S.B. 1976, S. M. 1982, Ocean Engineer 1982. Wales joined the Geophysical Institute in the fall of 1993. He came to the Geophysical Institute after a 27-year naval career. The last ten years of his active duty career was devoted to directing research and development projects related to the arctic and submarine operations in the Arctic. His undergraduate and graduate training was primarily in the areas of mechanical engineering, naval architecture, and ocean engineering. His undergraduate research was in the areas of acoustics and cavitation, while his graduate research was devoted to systems engineering and development for systems to do acoustic research in arctic ice camps. Since graduate school, his primary interest is in technical program and project management along with assisting in research programs related to sea ice.

Wales C., M. O. Jeffries, T. George, C. Wilson, B. Delana, G. Reynolds, L. Sweet and D. Sandberg. 1994. Alaska Synthetic Aperture Radar, Union Radio-Scientifique Internationale. URSI Bulletin, September, p. 270. 
WESLEY K. WALLACE, Associate Professor of Geology; Rice University'72, B.A.; Fulbright scholar University of Helsinki, '72-'73; University of Washington, '76, M.S., '81, Ph.D. Dr. Wallace has a joint appointment with the Department of Geology and Geophysics and the Geophysical Institute. His research interests include the tectonic evolution of Alaska and the structure of mountain belts, in particular the geometry and evolution of fold-and-thrust belts. His major current research focus is on the geometry and kinematics of detachment folds and duplexes in the Brooks Range of the Arctic National Wildlife Refuge and near the Trans-Alaska Pipeline. He also has recently conducted research in the Chersky Range of northeastern Siberia on the origin of arcuate mountain belts and the interference of folds and faults of different age and orientation.

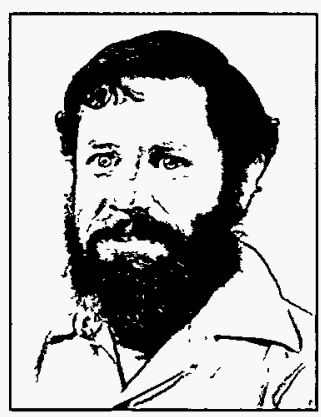

Wallace, W. K. 1993. Detachment Folds and a Passive-Roof Duplex: Examples from the Northeastern Brooks Range, Alaska. In: D. N. Solie and F. Tannian, Eds., Short Notes on Alaskan Geology 1993. Alaska Division of Geological and Geophysical Surveys Geologic Report, v. 113, pp. 81-99.

Anderson, A. V., W. K. Wallace and C. G. Mull. 1994. Depositional Record of a Major Tectonic Transition in Northern Alaska: Middle Devonian to Mississippian Rift-Basin Margin Deposits, Upper Kongakut River Region, Eastern Brooks Range, Alaska. In: D. Thurston and K. Fujita, Eds., 1992 Proceedings International Conference on Arctic Margins, U.S. Minerals Management Service Outer Continental Shelf Study 94-0040, pp. 71-76.

Decker, J., S. C. Bergman, R. B. Blodgett, S. E. Box, T. K. Bundtzen, J. G. Clough, W. L. Coonrad, W. G. Gilbert, M. L. Miller, J. M. Murphy, M. S. Robinson and W. K. Wallace. 1994. Chapter 9: Geology of Southwestern Alaska. In: G. Plafker and H.C. Berg, Eds., The Geology of Alaska: The Geology of North America, Geological Society of America, Boulder, Colorado, v. G1, pp. 285-310.

Hanks, C. L., W. K. Wallace and P. O'Sullivan. 1994. The Cenozoic Structural Evolution of the Northeastern Brooks Range, Alaska. In: D. Thurston and K. Fujita, Eds., 1992 Proceedings, International Conference on Arctic Margins. U.S. Minerals Management Service Outer Continental Shelf Study 94-0040, pp. 263-268.

LePain, D. L., R. K. Crowder and W. K. Wallace. 1994. Early Carboniferous Transgression on a Passive Continental Margin: Deposition of the Kekiktuk Conglomerate, Northeastern Brooks Range, Alaska. American Association of Petroleum Geologists Bulletin, v. 78(5), p. 679-699.

Moore, T.E., W. K. Wallace, K. J. Bird, S. M. Karl, C. G. Mull and J. T. Dillion. 1994. Chapter 3: Geology of Northern Alaska. In: Plafker, G. and H. C. Berg, Eds., The Geology of Alaska: The Geology of North America, Geological Society of America, Boulder, Colorado, v. G1, p. 49-140.

Moore, T. E., W. K. Wallace, C. G. Mull, S. M. Karl and K. J. Bird. 1994. Generalized Geologic Map and Secrions for Northern Alaska. In: G. Plafker and H. C. Berg, Eds., The Geology of Alaska: The Geology of North America, Geological Society of America, Boulder, Colorado, v. G1, Plate 6.

JOHN E. WALSH, Visiting Wadati Research Professor; Dartmouth College '70, B.A.; Massachusetts Institute of Technology '74, Ph.D. Dr. Walsh is an atmospheric scientist who has studied recent climatic change in the polar regions, particularly as it is affected by interactions between sea ice and the atmosphere. He has synthesized several decades of sea ice data to evaluate trends of ice coverage. More recently, he has become involved in the use of the Arctic System Regional Model to obtain projections of climate change in the Alaska region. Other interests include the hydrologic cycle in polar regions, large-scale snow variability, and the arctic performance of global climate models.

Walsh, J. E., X. Zhou, D. Portis and M. Serreze. 1994. Atmospheric Contribution to

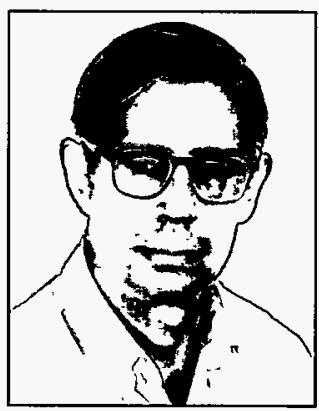
Hydrologic Variations in the Arctic. Atmosphere-Ocean, v. 32, pp. 733-755.

Walsh, J. E. 1993. Continental Snow Cover and Climate Variability. Climate Variability on Decade-to-Century Timescales. National Academy Press, National Research Council, Washington, D.C., pp. 181-195.

Walsh, J. E. 1993. The Elusive Arctic Warming. Nature, v. 361, pp. 300-301. 
Walsh, J. E. 1993. Observational and Modeling Studies of the Influence of Sea Ice Anomalies on the Atmospheric Circulation. Prediction of Interannual Climate Variations (J. Shukla, Ed.), NATO ARW Series I, No. 6, Springer-Verlag, Heidelberg, pp. 71-88.

Walsh, J. E., A. H. Lynch, W. L. Chapman and D. Musgrave. 1993. A Regional Model for Studies of ArmosphereIce-Ocean Interaction in the Western Arctic. Meteorology and Atmospheric Physics, v. 51, pp. 182-193.

Chapman, W. L., W. J. Welch, K. P. Bowman, J. Sacks and J. E. Walsh. 1994. Arcric Sea Ice Variability: Model Sensitivities and a Multidecadal Simulation. Journal of Geophysical Research, v. 99, pp. 919-935.

Chapman, W. L. and J. E. Walsh. 1993. Recent Variations of Sea Ice and Air Temperatures in High Latitudes. Bulletin of the American Meteorological Society, v. 74, pp. 33-47.

Serreze, M. D., J. E. Box, R. G. Barry and J. E. Walsh. 1993. Characteristics of Arctic Synoptic Activity. Meteorology and Atmospheric Physics, v. 51, pp. 182-193.

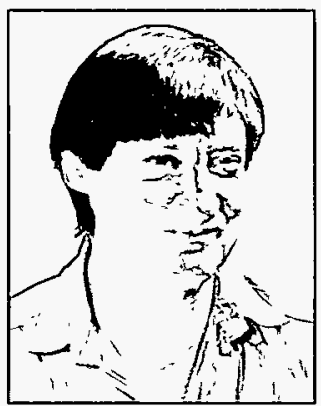

BRENTON J. WATKINS, Professor of Physics; University of Adelaide '69, B.S.; La Trobe University '72, M.S.; University of Alaska '76, Ph.D. Dr. Warkins was previously with the Australian Antarctic Research Program, MIT Lincoln Lab and Haystack Observatory, working on various upper atmosphere research problems. His primary interests are ionosphere research using incoherent scatter radar troposphere/stratosphere studies using turbulence scatter radar and numerical modeling.

Bristow, W. A., and B. J. Watkins. 1994. Effect of Large-Scale Convection Electric Field Structure on the Formation of Thin Ionization Layers at High Latitudes. Journal of Atmospheric and Terrestrial Physics, v. 56, p. 401.

Bristow, W. A., and B. J. Watkins. 1993. Incoherent-Scatter Radar Observations of Thin Ionization Layers at Sondrestrom. Journal of Atmospheric and Terrestrial Physics, v. 55, p. 873.

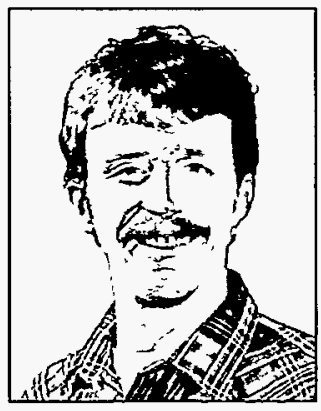

KEITH F. WATTS, Associate Professor of Geology; Utah State University '78, B.S.; University of California, Santa Cruz '85, Ph.D. Dr. Watts is a field-oriented carbonate sedimentologist specializing in the study of ancient limestones and dolostones in deformed mountain belts. His present research program focuses on the Carboniferous Lisburne Group of the Brooks Range in northern Alaska. His research is aimed at determining the paleogeography, depositional history, and tectonic implications of this widespread sequence of limestones. Forming one of the world's largest relatively unstudied carbonate provinces, the Lisburne Group also holds many clues to the origin and history of the Brooks Range and other circumarctic regions, a major unresolved problem. Watts' previous research focused on the evolution of Mesozoic slope-facies carbonates in the Oman Mountains (Middle East, Arabian carbonate platform margin), a mountain belt remarkably similar to the Brooks Range. In 1990, Watts was a sedimentologist on Leg 133 of the Ocean Drilling Program, analyzing the northeastern Australia margin including the Great Barrier Reef and associated carbonate platforms. Watts left the institute in July 1994.

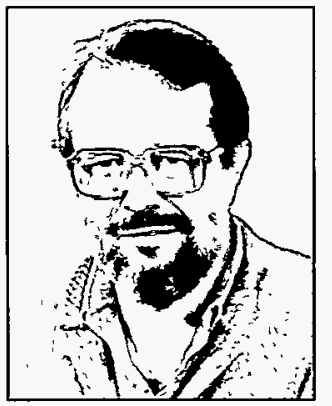

WILFORD F. WEEKS, Professor of Geophysics; University of Illinois '51, B.S., '53, M.S.; University of Chicago '56, Ph.D. Dr. Weeks is primarily interested in the geophysics of sea ice, with particular emphasis on interactions among growth conditions, ice structure, and ice properties. His current activities include applying remote sensing techniques to studies of ice dynamics and thermodynamics; numerical simulations of aspects of ice gouging and ice property profiles; statistical aspects of pressure ridges; and the internal structure and composition of sea ice masses. Dr. Weeks is a member of the U.S. National Academy of Engineering and a Fellow of the American Geophysical Union. He has been president of the International 
Glaciological Society and in 1989 was the recipient of their highest award, the Seligman Crystal. From 1986 to 1993 , he also was the Chief Scientist of the Alaska Synthetic Aperture Radar Facility.

Weeks, W. F. 1994. Possible Roles of Sea Ice in the Transport of Hazardous Material in the Arctic Ocean. Workshop on Arctic Contamination, Arctic Research of the United States, U.S. Interagency Arctic Research Policy Committee 8, pp. 34-52.

Weeks, W. F. 1993. Report on the National Academy of Sciences-Academy of Sciences of the USSR Workshop on the Mechanics of Ice and its Applications. National Academy Press, $84 \mathrm{pp}$.

Groat, C. G., W. F. Weeks and 15 others. 1994. Environmental Information for Outer Continental Shelf Oil and Gas Decisions in Alaska. National Research Council, Washington, D.C., 274 pp.

Jeffries, M. O., K. Morris and W. F. Weeks. 1994. Structural and Stratigraphic Features and ERS 1 Synthetic Aperture Radar Backscatter Characteristics of Ice Growing on Shallow Lakes in NW Alaska, Winter 1991-1992. Journal of Geophysical Research, v. 99(C11), pp. 22,459-22,471.

Jeffries, M. O., K. Morris, A. P. Worby and W. F. Weeks. 1994. Late Winter Sea-Ice Properties and Growth Processes in the Bellingshausen and Amundsen Seas. Antarctic Journal of the United States, v. 29(1), pp. 11-13.

Jeffries, M. O., K. Morris, A. P. Worby and W. F. Weeks. 1994. Late Winter Characteristics of the Seasonal Snow Cover on Sea-Ice Floes in the Bellingshausen and Amundsen Seas. Antarctic Journal of the United States, v. 29(1), pp. 9-11.

Jin, Z., K. Stamnes and W. F. Weeks. 1994. Transport of Photosythetically Active Radiation in Sea Ice and the Ocean. SPIE Proceedings Series, Ocean Optics XII, v. 2258, pp. 954-964.

Jin, Z., K. Stamnes, W. F. Weeks, and S. -C. Tsay. 1994. The Effect of Sea Ice on the Solar Energy Budget in the Atmosphere-Sea Ice-Ocean System: A Model Study. Journal of Geophysical Research, v. 99(C12), pp. 25,281-25,294.

Wade, R. H., and W. F. Weeks. 1994. Radar Backscatter Estimates From a Surface Scattering Model of First Year Sea Ice. EARSeL (European Association of Remote Sensing Laboratories), Advances in Remote Sensing, 3(2-XII), pp. 24-30.

Wakabayashi, H., M. O. Jeffries and W. F. Weeks. 1994. C-Band Backscatter Variation and Modeling for Lake Ice in Northern Alaska. Journal of the Japanese Remote Sensing Society, v. 14(3), pp. 18-27.

Worby, A. P., W. F. Weeks, M. O. Jeffries, K. Morris and R. Jana. 1994. Late Winter Sea-Ice-Thickness and Snow Thickness Distributions in the Bellingshausen and Amundsen Seas. Antarctic Journal of the United States, v. 29(1), pp. 13-15.

Jeffries, M. O., H. Wakabayashi and W. F. Weeks. 1993. ERS-1 SAR Backscatter Changes Associated with Ice Growing on Shallow Lakes in Arctic Alaska. Proceedings of the 1994 International Geoscience and Remote Sensing Symposium (IGARSS '93), 1993 August, Tokyo, Japan, v. 4, pp. 2001-2004.

Jeffries, M. O., and W. F. Weeks. 1993. Structural Characteristics and Development of Sea Ice in the Western Ross Sea. Antarctic Science, v. 5(1), pp. 63-75.

Jeffries, M. O., W. F. Weeks, R. Shaw and K. Morris. 1993. Structural Characteristics of Congelation and Platelet Ice and Their Role in the Development of Antarctic Land-Fast Sea Ice. Journal of Glaciology, v. 39(132), pp. 223238.

Shapiro, L. H., and Weeks, W. F. 1993. Influence of Crystallographic and Structural Properties on the Flexural Strength of Small Sea Ice Beams. Ice Mechanics-93, First Joint Mechanics Meeting of the American Society for Civil Engineering-Engineering Mechanics Division/American Society of Mechanical Engineers-Applied Mechanics Division/Society of Engineering Science (June 1993, Charlottesville, VA), AMD - v. 163, pp. $173-188$.

Wakabayashi, H., W. F. Weeks and M.O. Jeffries. 1993. A C-band Backscatter Model for Lake Ice in Alaska. Proceedings of the 1994 International Geoscience and Remote Sensing Symposium (IGARSS '93), 1993 August, Tokyo, Japan, v. 3, pp. 1264-1266.

Wakabayashi, H., M. O. Jeffries and W. F. Weeks. 1993. C-Band SAR Backscatter from Ice on Shallow Tundra Lakes: Observations and Modeling. First ERS-1 Symposium - Space at the Service of our Environment, 4 November 1992, Cannes, France, ESA, 1993 March, ESA SP-359, pp. 333-337. 


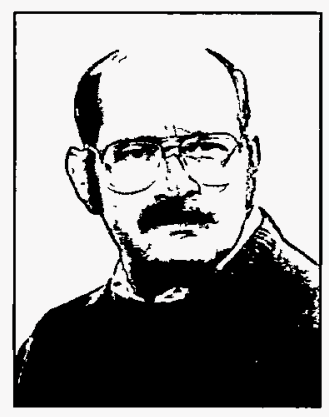

DANIEL R. WEIMER, Research Associate Professor; University of Michigan '77, B.S., B.S.E.; University of Iowa '83, M.S., '84, Ph.D. Dr. Weimer joined the Geophysical Institute in 1989. His primary interests are satellite measurements of auroral electric fields and solar wind-magnetosphere-ionosphere coupling processes. Dr. Weimer is Scientist-in-Charge of the Space Physics/Aeronomy Data Analysis Center.

Weimer, D. R. 1994. Substorm Time Constants. Journal of Geophysical Research, v. 99(A6), pp. 11005-11015.

Weimer, D. R., J. D. Craven, L. A. Frank, W. B. Hanson, N. C. Maynard, R. A. Hoffman and J. A. Slavin. 1994. Satellite Measurements Through the Center of a Substorm Surge. Journal of Geophysical Research, v. 99, pp. 23639-23649.

Weimer, D. R., J. D. Craven, L. A. Frank, W. B. Hanson and J. A. Slavin. 1994. Electric Fields and Currents Associated with a Substorm Surge. Proceedings of the Second International Conference on Substorms, which was held at the University of Alaska Fairbanks, 7-11 March 1994, J. R. Kan, J. D. Craven, S.-I Akasofu, (Eds.), pp. 455-460.

Weimer, D. R. and D.A. Gurnett. 1993. Large-Amplitude Auroral Electric Fields Measured with DE 1. Journal of Geophysical Research, v. 98(A8), pp. 13557-13564.

Menietti, J. D., D. R. Weimer, M. Andre and L. Eliasson. 1994. DE 1 and Viking Observations Associated with Electron Conical Distributions. Journal of Geophysical Research, v. 99, pp. 23673- 23684.

Shue, J. H., and D. R. Weimer. 1994. The Relationship Between Ionospheric Convection and Magnetic Acrivity. Journal of Geophysical Research, v. 99(A1), pp. 401-415.

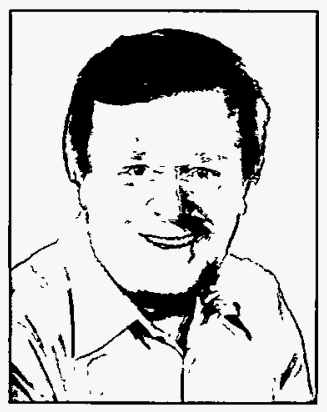

GUNTER E. WELLER, Professor of Geophysics and Deputy Director; University of Melbourne, Australia '62, B.S., '64, M.S., '68, Ph.D. Dr. Weller has a background of research in arctic and antarctic meteorology and glaciology. He joined the Geophysical Institute in 1968 to study the heat and mass balance of glaciers, arctic sea ice, the microclimates of the tundra, and air pollution. Dr. Weller was program manager of the National Science Foundation's polar programs in meteorology from 1972 to 1974; Project Manager of NOAA-BLM's Outer Continental Shelf Environmental Assessment Program in the Arctic from 1975 to 1982; Project Director of the NASA-UAF Alaska SAR Facility from 1986 to 1993; and is now the Director of UAF's Center for Global Change and Arctic System Research and the NOAA-UA Cooperative Institute for Arctic Research (CIFAR). He was the Chairman of the National Academy's Polar Research Board from 1985 to 1990.

Weller, G. 1994. Global Climate Warming-Real, Not Imagined. Earth Space Review v. 3, pp. 20-25.

Weller, G 1994. Global Pollution and its Effect on the Climate of the Arctic. Proceedings of the International Symposium on the Ecological Effects of Arctic Airborne Contaminants, Reykjavik, Iceland, The Science of the Total Environment, v. 160, 161, pp. 19-24.

Weller, G. 1994. The Polar Environment. The Illustrated Library of the Earth, Polar Regions, pp. 14-25.

Weller, G. 1993. Global Change and Its Implication for Alaska. Proceedings of Symposium on the Impacts of Climate Change on Resource Management of the North; 1993; Whitehorse, YT, University of Waterloo, pp. 17-22.

Weller, G. 1993. Polar Meteorology. Meteorology and Atmospheric Physics, v. 51, pp. 141-143.

Weller, G. and P. Anderson. 1993. The Polar Regions and Global Change. Center for Global Change and Arctic System Research, University of Alaska Fairbanks, 20 p. 
GERD D. WENDLER, Professor of Geophysics; Ieopold Franzens Universitat, Innsbruck, Austria '64, Ph.D. Meteorology, glaciometeorology, and radiation were his background when he joined the Geophysical Institute in 1966. Here, he initiated micrometeorological studies, starting with a project on McCall Glacier. Presently, he works in the Arctic and Antarctic on various programs in the field of meteorology and climatology.

Wendler, G., and T. Quakenbush. 1994. Ultraviolet Radiation and its Extinction in Arctic Sea Ice. Antarctic Journal of the United States, Annual Review, pp. 84-85.

Wendler, G., J. C. André, P. Pettré, J. Gosink and Thomas Parish. 1993. Katabatic Winds in Adélie Land. Antarctic Meteorology and Climatology, Studies Based on Automatic Weather Stations, AGU, Antarctic Research Series, v. 61, pp. 23-45.

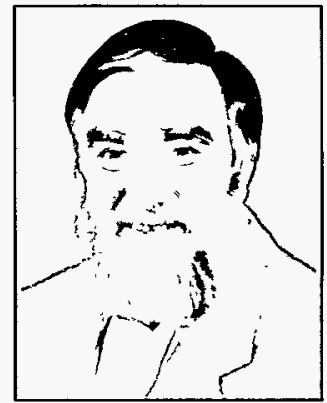

Wendler, G., and Y. Kodama. 1993. The Kernlose Winter in Adélie Land. Antarctic Meteorology and Climatology, Studies Based on Automatic Weather Station, Antarctic Research Series, v. 61, pp. 139-147.

Wendler, G., and R. Meitner. 1993. Satellite-Derived Outgoing Longwave Radiation, Surface Temperature and Sea Ice Concentration off the Coast of Adélie Land, Eastern Antarctica. Meteorology and Atmospheric Physics, v. 51, pp. 219-226.

Wendler, G., and M. Pook. 1993. On the Half-Yearly Pressure Oscillation in Eastern Antarctica. Antarctica Journal of the United States, 1992 Annual Review, pp. 284-285.

Allison, I., G. Wendler and U. Radok. 1993. A Climatology of the East Antarctic Ice Sheer $\left(100^{\circ} \mathrm{E}\right.$ to $\left.140^{\circ} \mathrm{E}\right)$ Derived from Automatic Weather Stations. Journal of Geophysical Research, v. 98(D5), pp. 8815-8823.

André, J. C., P. Pettré; G. Wendler and M. Zephoris. 1993. Vertical Structure and Downslope Evolution of Antarctic Katabatic Flows. Waves and Turbulence in Stably Stratified Flows, Oxford, Clarendon Press, pp. 92-104.

Parish, T., P. Pettré and G. Wendler. 1993. A Numerical Study of the Diurnal Variation of the Adélie Land Katabatic Wind Regime. Journal of Geophysical Research. v. 98(D7), pp. 12933-12947.

Parish, T., P. Pettré and G. Wendler. 1993. The Influence of Large-Scale Forcing on the Katabatic Wind Regime at Adélie Land, Antarctica. Meteorology and Atmospheric Physics, v. 51, pp. 165-176.

EUGENE M. WESCOTT, Professor of Geophysics; University of California at Los Angeles '55, A.B.; University of Alaska' 60, M.S., '64, Ph.D. Dr. Wescott has a background of research in solid earth geophysics and space physics. His past activities include research in telluric currents, auroral audibility, magnetoconjugate phenomena, electrodynamics of the ionosphere and magnetosphere, auroral induced corrosion in arctic pipelines, earthquake prediction, and geothermal resource exploration. After joining the Geophysical Institute in 1958, he investigated a wide range of magnetically conjugate geophysical phenomena. He is currently involved in auroral and magnetospheric electric field studies and plasma physics experiments using barium and calcium plasma rocket injections. The most exciting current project, a joint collaboration with Davis Sentman, is investigating optical flashes in the upper atmosphere to $90 \mathrm{~km}$ above mesoscale thunderstorms; red-colored "Sprites" and "Blue Jets." Dr. Wescott teaches courses in geophysical methods and field geophysical prospecting.

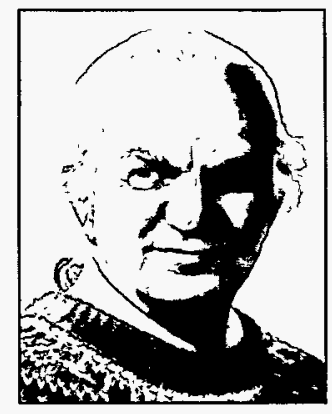

Wescott, E. M., H. C. Stenbaek-Nielsen, D. L. Hampton and P. A. Delamere. 1994. Results of Critical Velocity Experiments with Barium, Strontium, and Calcium Releases from CRRES Satellite. Journal of Geophysical Research, v. 99(A2), pp. 2145-2158.

Wescott, E. M., T. J. Hallinan, H.C. Stenbaek-Nielsen, D. W. Swift and D. D. Wallis. 1993. Rapid Ray Motions in Barium Plasma Clouds and Auroras. Journal of Geophysical Research, v. 98(A3), pp. 3711-3724.

Sentman, D. D., and E. M. Wescott. 1993. Observations of Upper Atmospheric Optical Flashes Recorded from an Aircraft. Geophysical Research Letters, v. 20(24), pp. 2857-2860.

Stenbaek-Nielsen, H. C., E. M. Wescott and T. J. Hallinan. 1993. Observed Barium Emission Rates. Journal of Geophysical Research, v. 98(A10), pp. 17501-17516.

Weiss, L. A., E. J. Webber, P. H. Reiff, J. R. Sharber, J. D. Winningham, F. Primdahl, I. S. Mikkelsen, C. Seifring and E. M. Wescott. 1993. Convection and Electrodynamic Signatures in the Vicinity of a Sun-Aligned Arc: Results from the Polar Acceleration Regions and Convection Study (Polar ARCS). Auroral Plasma Dynamics, Geophysical Monograph 80, published by the American Geophysical Union, p. 69-80. 


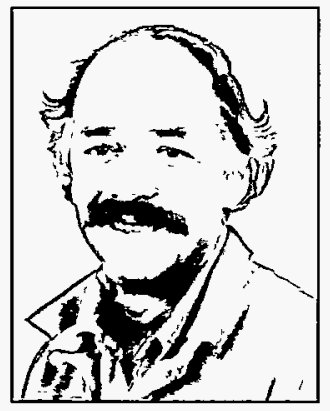

MAX WYSS, Wadati Professor of Seismology; Federal Institute of Technology, Zuerich, Switzerland '64, M.S.; California Institute of Technology, Pasadena '70, Ph.D. Dr. Wyss has worked at the University of Colorado, Boulder, on problems regarding earthquake source processes, tectonics, earthquake hazards and prediction. He joined the Geophysical Institute in 1991 to study Alaska earthquakes and the hazards they pose. He edited a number of volumes on earthquake prediction, including a 1991 evaluation of prediction methods while he was the chairman of the IASPEI Subcommission on Earthquake Prediction. In 1985, he won a Senior U.S. Scientist Award of the Alexander von Humboldt Foundation. He is estimating the directions of the stress tensor in the seismically active areas of Alaska and along plate boundaries and is testing the hypothesis that major earthquakes may be predicted based on seismic quiescence.

Wyss, M. 1993. How Much Sense Does Earthquake Prediction Make? Continental Earthquakes, D. Guoyu and C. Zhangli (Eds.), IASPEI Publication Series for the IDNDR, v. 3, pp. 85-90.

Beisser, M., D. Gillard and M. Wyss. 1994. Inversion for Source Parameters from Sparse Data Sets: Test of the Method and Application to the $1951(\mathrm{M}=6.9)$ Kona, Hawaii, Earthquake. Journal of Geophysical Research, v. 99(B10), pp. 19,661-19,678.

Weimer, S., and M. Wyss. 1994. Seismic Quiescence Before the Landers $(M=7.5)$ and Big Bear $(M=6.5) 1992$ Earthquakes. Bulletin of the Seismological Society of America, v. 84(3), pp. 900-916.

Lu, Z., M. Wyss, G. Tytgat and S. McNutt. 1993. Aftershocks of the 13 May 1993 Shumagin Alaska Earthquake. Geophysical Research Letters, v. 21(6), pp. 497-500.

Neri, G., and M. Wyss. 1993. Preliminary Results from Stress Tensor Inversion of Earthquake Fault-Plane Solutions in the Southern Tyrrhenian Region. Bollettino di Geofisica Teorica ed Applicata, v. 35(139), pp. 349-362.

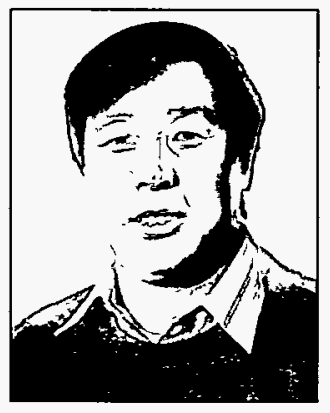

TINGJUN ZHANG, Postdoctoral Fellow; Lanzhou University, China '79, B.S.; Lanzhou Institute of Glaciology and Geocryology, China '85, M.S.; University of Alaska Fairbanks'93, Ph.D. Dr. Zhang has a background of research in climate, heat and mass transfer in porous media, snow, ice and permafrost, and geomorphological phenomena related with freezing and thawing. Dr. Zhang joined the Geophysical Institute in 1993. His past activities include land classification and slope development on the Tebit Plateau. He also has conducted research on the investigation and the modeling of the interaction between the atmosphere and surface, seasonal snow cover. He also has researched the physical and thermal processes of the active layer and permafrost at high latitudes. His present research includes the investigation and modeling of snowmelt, the development of a land surface model suitable for the arctic environments, and the potential response of the active layer and permafrost to elevated carbon dioxide-induced climate warming at high latitudes.

Zhang, T., and T.E. Osterkamp. 1994. Considerations in Determining Thermal Diffusivity from Temperature Time Series Using Numerical Methods. Geophysical Institute Report, UAG-R-324, 18 pp.

Zhang, T., K. Stamnes and S.A. Bowling. 1994. Effect of Clouds on Snowmelt at High Latitudes. Proceedings of the Conference on the Dynamics of the Arctic Climate System, Goteborg, Sweden, pp. 103-107.

Zhang, T., K. Stamnes and S.A. Bowling. 1994. Impact of the Atmosphere and Clouds on Snowmelt at High Latitudes: Data Analysis and Application. EOS, Transactions of the American Geophysical Union, v. 75(44), p. 126.

Zhang, T., K. Stamnes and S.A. Bowling. 1994. Impact of the Atmosphere and Clouds on Snowmelt at High Latitudes: Sensitivity Analysis. EOS, Transactions of the American Geophysical Union, v. 75(44), p. 105.

Zhang, T. and T.E. Osterkamp. 1993. Changing Climate and Permafrost Temperatures in Alaskan Arctic. Proceedings of the Gth International Conference on Permafrost, Beijing, China, Science Press, pp. 783-788.

Zhang, T. and T.E. Osterkamp. 1993. Climate and Permafrost Temperatures in Alaska North of the Brooks Range. EOS, Transactions of the American Geophysical Union, v. 74(16), p. 89.

Zhang, T. and T.E. Osterkamp. 1993. Response of Permafrost Temperatures to Changes in Climate in Alaska North of the Brooks Range. EOS, Transactions of the American Geophysical Union, v. 74(43), p. 142.

Osterkamp, T. E., T. Zhang and V. Romanovsky. 1994. Evidence for a Cyclic Variation of Permafrost Temperatures in Northern Alaska. Permafrost and Periglacial Processes, v. 5(3), p. 137-144. 


\section{Administrative Staff by Department}

(as of December 1994)

\section{ADMINISTRATION}

Director

907-474-7282

Syun-Ichi Akasofu, Director

Gunter Weller, Deputy Director

Helga Wilm, Executive Officer for Administration

Catherine Barr, Human Resource Manager

Business Office

907-474-7644

Neta Stilkey, Business Manager

Johni Meshell, General Ledger Accountant

Operations Office

907-474-7291

Robert Grove, Operations Manager

\section{SUPPORT SERVICES}

\section{Computer Resource Center}

907-474-7489

Robert Grove, Acting Supervisor

Celia Rohwer, Vax System Manager

Scort Chesney, Network Manager

\section{Electronics Shop}

907-474-7283

John Benevento, Supervisor

Engineering

907-474-7291

Robert Grove, Supervisor

James Desrochers, Project Support Engineer

Ed Hoch, Design Engineer

Daniel Osborne, Project Engineer

Donald Rice, Project Engineer

\section{Geo-Data Center}

907-474-7487

Carl Wales, ASF Project Director

Rose Watabe, Data Center Specialist

High-Latitude Monitoring Station

907-552-4531

Robert Grove, Supervisor

Ed Luteran, Station Manager 
Information/Publications Office

907-474-7558

Kathryn Berry, Science Editor-in-Chief/Information Officer

Machine Shop

907-474-7506

Lawrence Kozycki, Supervisor

Photo-Graphics Service Center

907-474-7572

Evelyn Trabant, Supervisor

Seismology Lab

907-474-5533

Roger Hansen, State Seismologist

Charlotte Rowe, Deputy State Seismologist

Steve Estes, Project Engineer

Guy Tytgat, Geophysicist

Word Processing Service Center

907-474-7562

Sheila Finch, Supervisor

KEITH B. MATHER LIBRARY

907-474-7503

Julia Triplehorn, Librarian, Associate Professor of Library Science

ALASKA SAR FACILITY

907-474-6714

Carl Wales, Project Director

Cindy Wilson, Assistant Project Director

Brett Delana, Senior Project Engineer

Lyn Mcnutt, Chief Scientist

Larry Sweet, Systems Engineer

Paul Bundschuh, Systems Manager

\section{POKER FLAT RESEARCH RANGE}

907-474-7015

Charles Deehr, Science Director

Stanley Schwafel, Assistant Director

Ron Pierce, Range Manager

Ray Martinez, Launch Officer

Ralph Robertson, Range Safety Officer

James Strandberg, External Projects Coordinator 


\section{Geophysical Institute Staff Listing}

(as of December 1994)

Kevin C. Abnett, Software Engineer

T. Kristina Ahlnäs, Remote Sensing Specialist

Syun-Ichi Akasofu, Director, Professor of Geophysics

R. Donald Ames, Expediter II

Mark P. Anderson, Database Programmer

Daniel Armstrong, Maintenance Mechanic

Jesse Atencio, Microcomputer Specialist

Patricia Babcock, Technical Secretary

Michele M. Bach, Clerk Specialist III

David Bailey, Research Programmer

Dolores J. Baker, Drafter I

James N. Baldridge, Senior Programmer Analyst

(-3 March 1994)

Matthew Barkdull, Microcomputer Specialist

Joanne M. Ballew, Technical Secretary (-14 July 1994)

Carherine M. Barr, Human Resource Manager

Michelle R. Barr, ASF User Services Specialist

Amy Barrow, Technical Secretary

John Benevento, Electronics Shop Supervisor

Cynthia L. Benner, Post-Doctoral Fellow

(-26 February 1993)

Richard L. Benner, Assistant Professor of Chemistry

Kathryn A. Berry, Science Editor-in-Chief

Niren N. Biswas, Professor of Geophysics

Duwayne J. Bostow, Professional Instrument

Technician

Sue Ann Bowling, Assistant Professor of Geophysics

Neal Brown, Research Assistant Professor of Geophysics

Paul A. Bundschuh, Systems Manager

James R. Burton, CAD Specialist

H. Scott Chesney, Network Manager

Douglas H. Christensen, Associate Professor of Geophysics

Deborah C. Coccia, Drafter III

Michael J. Cogan, Senior Electronics Technician

Saberina S. Coleman, Electronics Technician

(-27 March 1993)

Patricia A. Collins, Electronics Technician III

Richard Collins, Research Assistant Professor of Space

Physics and Aeronomy

Mark Conde, Post-Doctoral Fellow

James Conner, User Services Consultant

David N. Covey, Programmer Analyst III

John D. Craven, Professor of Physics

Norman Cushing, Software Design Engineer

Janet A. Dalrymple, Administrative Assistant

Rick A. Danielson, Property Control Officer

Jacqueline L. Dashiell, Accounts Clerk IV

Kenneson Dean, Research Assistant Professor of

Geology
Charles S. Deehr, Science Director for Poker Flat

Research Range

Brett S. Delana, Senior Project Engineer

Paul J. Delys, Programmer Analyst II

Jeannette L. DeMallie, Research Technician

Jim T. Desrochers, Project Support Engineer

Craig R. Dowell, Maintenance Mechanic II

(-6 August 1993)

Jeffry Drake, Senior Lab Technician

Ruth E. Duerr, Science Data Manager

Julie Duhrsen, Administrative Assistant II

(-31 August 1994)

Keith A. Echelmeyer, Associate Professor of

Geophysics

John C. Eichelberger, Professor of Volcanology

Steven A. Estes, Project Engineer

Mary R. Farrell, Administrative Assistant II

Ronald I. Faust, Research Technician

Victor Filyushkin, Research Associate of Atmospheric Sciences

Sheila G. Finch, Word Processing Center Supervisor

Marcus Forbes, Research Technician/Analyst

Beverly L. Frey, Data Control Clerk I

Thomas H. George, Applications Specialist

Carol S. Gering, Technical Secretary (-29 July 1994)

Crystal A. Goula, Data Control Clerk

O. Dean Gramling, Jr., Research Technician

Roberta L. Greenlee, Accounts Clerk IV

Robert A. Grove, Operations Manager

Joanne E. Groves, Technician III

Richard M. Guritz, Senior Systems Analyst

Cheryl L. Haase, Database Administrator

Henry B. Hahn III, Research Technician/Analyst

Thomas J. Hallinan, Professor of Geophysics

Catherine L. Hanks, Research Assistant Professor of Geology

Roger Hansen, State Seismologist, Research Professor of Geophysics

William Harrison, Professor of Physics

Edwin E. Heath, Electronic Technician II

Merritt R. Helfferich, Elvey Project Director

Douglas Henderson, Software Engineer

Jack Herring, Post-Doctoral Research Associate

Susan Hetrick, Mail Clerk, Clerk Specialist

Edward L. Hoch, Design Engineer

Jeanne H. Hume, Administrative Assistant III

(-31 December 1993)

Deborah J. Ice, Adminstrative Assistant

Daniel A. Jaffe, Associate Professor of Chemistry 
Martin O. Jeffries, Research Associate Professor of Geophysics

Jay Johnson, Post-Doctoral Fellow (-9 September 1993)

Henry Johnson, CD-ROM Designer

Joseph R. Kan, Professor of Physics

Cheryl Katje, Publications Assistant

Koji Kawasaki, Associate Professor of Geophysics

Larry R. Kent, Communications Engineer

Johnny M. Kerr, Mechanic/Operator

Sharon Kessey, Clerk Specialist

Juergen Kienle, Professor of Geophysics

Lawrence V. Kozycki, Jr., Machine Shop Supervisor

Chris Lace, Administrative Assistant

Christopher F. Larsen, Research Technician

Daniel K. LaSota, Microcomputer Specialist

Kathleen M. Lawson, Electronic Technician II

Paul W. Layer, Associate Professor of Geophysics

Chi-Hsaung Lee, Test Engineer

Li-Her C. Lee, Programmer Analyst II

Lou-Chuang Lee, Professor of Physics

Zuh-Yao Lee, Systems Engineer

Elena Leontieva, Research Associate of Atmospheric Sciences

Shusun S. Li, Research Assistant Professor of Geophysics

$\varnothing y s t e i n$ Lie-Svendsen, Post-Doctoral Fellow (-19 November 1993)

Craig S. Lingle, Research Associate Professor of Geophysics

Thomas Logan, Software Engineer

Dirk Lummerzheim, Research Associate Professor of Aeronomy

Amanda H. Lynch, Assistant Professor of Atmospheric Sciences

John F. Mahoney, Maintenance Technician

(-1 December 1994)

Arthur E. Manning, Machinist

Tim A. Manning, Machinist

Richard Marlin, ASF Development Coordinator

Raymond L. Martinez, Poker Flat Research Range Launch Officer

Judith A. Martsolf, Data Technician

Joan L. McInerney, Electronic Technician I

Lyn McNutt, Research Associate Professor, ASF Chief Scientist

Stephen R. McNutt, Research Professor of Volcanology

Morna W. Mellor, Computer Resource Center Supervisor/ Sr. Programmer Analyst (-27 October 1994)

Johni M. Meshell, General Ledger Accountant

John M. Miller, Senior Applications Engineer (-1 July 1994)
Rebecca Miller, Clerk Specialist

Richard P. Million, Senior Systems Analyst

Clifton S. Moore, Research Technician

Kim Morris, Research Technician

Jane Nanto, Technical Secretary

Jeffrey D. Nelson, Machinist

Penelope Noecker, Operations Assistant

Coert D. Olmsted, Senior Programming Analyst

John V. Olson, Professor of Physics

Daniel L. Osborne, Project Engineer

Thomas E. Osterkamp, Professor of Physics

Antonius H. Otto, Research Associate Professor of Space Physics

June Pelehowski, Project Engineer

Ronald M. Pierce, Poker Flat Research Range Manager

Ronald G. Pigg, Research Technician

(-19 August 1993)

Gregory W. Pippin, Research Technician

Hans Pulpan, Associare Professor of Geophysics

Lalitha N. Rao, Data Control Clerk

Manfred H. Rees, Professor of Geophysics (-1 July 1993)

Thomas A. Reimers, Electronic Technician III

Al J. Renfroe, Research Technician

Greta J. Reynolds, User Services Manager

Donald D. Rice, Project Engineer

Kathe S. Rich, Operations Controller

Ralph E. Robertson, Jr., Poker Flat Research Range Safety Officer

Mitchell R. Robinson, Systems Analyst

Juan G. Roederer, Professor of Physics (-1 July 1993)

Celia M. Rohwer, VAX System Manager

Michael Ross, Database Administrator

Charlotte A. Rowe, Deputy State Seismologist

Susan L. Royston, Payroll Accountant

Ned Rozell, Science Writer/Grant Developer

Walter L. Rutherford, System Manager

William M. Sackinger, Associate Professor of Geophysics and Electrical Engineering

Donna E. Sandberg, Technical Secretary

Stanley R. Schwafel, Poker Flat Research Range Assistant Director

Valerie J. Scullion, Administrative Assitant III

Davis D. Sentman, Associate Professor of Physics

Glenn E. Shaw, Professor of Physics

Debra A. Shrider, Drafter II

Douglas L. Sigler, Photographics Lab Technician (-10 June 1994)

Roger W. Smith, Professor of Physics

Jean A. Sobolik, Technical Secretary 
G. H. Cole Sonafrank, System Manager/Senior Systems Analyst

Knut H. Stamnes, Professor of Physics

Hans Stenbaek-Nielsen, Professor of Geophysics

Neta J. Stilkey, Business Manager

David Stone, Professor of Geophysics

James S. Strandberg, Poker Flat Research Range

External Projects Coordinator

William Stringer, Associate Professor of Geophysics

Mary K. Stuart, Research Technician

Wei Sun, Research Associate of Space Physics

Larry R. Sweet, Systems Engineer

Daniel Swift, Professor of Physics (-1 July 1994)

Hal R. Tippens, Programmer Analyst II

Heidi A. Titchenal, Software Engineer

Evelyn I. Trabant, Photo-Graphics Service Center Supervisor

Ann M. Trent, Accounts Clerk IV

Julia H. Triplehorn, Associate Professor of Library Science

Guy C. Tytgat, Geophysicist

Jesse A. Venable, Research Technician
Vera Veronina, Geophysicist

Carl Wales, ASF Project Director

Raymond E. Ward, Maintenance Worker

Rosemary A. Watabe, Data Center Specialist

Brenton J. Watkins, Professor of Physics

Cynthia E. Weatherby, Programmer Analyst II

Wilford F. Weeks, Professor of Geophysics

Daniel R. Weimer, Research Associate Professor of Space Physics

Gunter Weller, Deputy Director, CGCASR Director, Professor of Geophysics

Gerd Wendler, Professor of Geophysics

Eugene M. Wescott, Professor of Geophysics

Debi-Lee Wilkinson, Research Technician

Jason D. Williams, Calibration Engineer

Helga Wilm, Executive Officer for Adminstration

Cynthia L. Wilson, ASF Project Coordinator

Max Wyss, Wadati Professor of Seismology

Tracy L. Zeiler, Systems Engineer

Tingjun Zhang, Post-Doctoral Fellow

William J. Zito, Electronic Technician IIB

\section{Personalia}

\section{Professors Honored}

Professor of Physics Lou-Chuang Lee received the Emil Usibelli Distinguished Research Award in 1994. The prestigious award is presented each year to individuals who display extraordinary excellence in teaching, research and public service.

Professor of Physics Glenn Shaw was elected a member of the Polar Research Board, established by the National Academy of Sciences in 1958 to provide independent advice to the federal government on matters of science and technology affecting public policy on environmental quality, natural resources, and other issues in polar regions. The board assists federal agencies in the development and maintenance of strong programs of polar research that promote national interests and international opportunities in the Arctic and the Antarctic.

Robert Hunsucker, a senior consultant and professor emeritus, was selected from a nationwide pool of candidates to be the new editor of Radio Science, an international bimonthly journal that publishes papers on all aspects of radio communication. The American Geophysical Union appointed Hunsucker to the three-year position. Hunsucker also became a Life Member of the Institute of Electrical and Electronics Engineers, Inc., a status reserved for those who have had a long association with IEEE, and who have contributed knowledge through years of professional experience.

Professors Davis Sentman, Eugene Wescott, Engineer Daniel Osborne and the team of NASA/UAF scientists, pilots, and technical specialists that captured the first color video footage of Sprites and Jets, those spectacular flashes of light reaching up to 60 miles above thunderstorms, received the Laurel Award from Aviation Week and Space Technology magazine. 
After a lengthy selection process, Assistant Professor of Atmospheric Sciences Amanda Lynch was chosen as one of five female scientists in Alaska to be featured on a five-part series on the Alaska Public Radio Network. APRN prepared fiveminute segments on each scientist which were aired on the Alaska News Nightly broadcast in August 1995. The scientists also were featured on five fifteen-minute segments that were aired on public radio stations throughout the state and then bundled together on cassette and distributed along with a curriculum guide to school districts in Alaska by the Department of Education.

The Japanese government has presented Institute Director Syun-Ichi Akasofu with an award in 1993 for promoting academic exchanges between Japan and Alaska. The award was presented in Tokyo by Japan's Foreign Minister Muto. Akasofu was also honored for his auroral research, which spans more than three decades.

Professor of Geophysics Gunter Weller received an Edith R. Bullock Prize for Excellence in 1993. The Trustees of the University of Alaska Foundation give out two such $\$ 15,000$ awards each year in recognition of outstanding work on behalf of the University of Alaska. Weller also was elected to the rank of Fellow by the American Association for the Advancement of Science, the largest federation of scientists in the world. Each year the council elects members whose efforts on behalf of the advancement of science or its applications are scientifically distinguished. Weller was selected the first director of the Cooperative Institute For Arctic Research. Formed in 1994 to promote closer cooperation between university scientists and federal and state agencies, the new institute is collocated with the UAF Center for Global Change and Arctic System Research, which Weller also directs.

Professor Emeritus Carl Benson was elected to Fellowship in the American Geophysical Union in 1993.

Research Associate Professor Martin Jeffries was elected a Fellow of the Arctic Institute of North America in 1993.

Professor of Physics Juan Roederer was appointed in 1993 to the five-member Consultative Committee of the International Center for Theoretical Physics based in Trieste, Italy. ICTP is a research institute operated under the joint sponsorship of the international Atomic Energy Agency of the United Nations, UNESCO, and the Italian government.

Professor Emeritus of Geophysics Takeshi Ohtake has been elected a Fellow of the American Meteorological Society in recognition of his distinguished career in experimental meteorology.

\section{Terris and Katrina Moore Prize}

Associate Professor of Geophysics Keith Echelmeyer received the 1994 Terris and Katrina Moore prize, an award established by former Geophysical Institute Director Terris Moore to recognize the contributions of deserving researchers in their field of science. Echelmeyer's research focuses on glacier flow and mass balance. Professor of Physics Glenn Shaw received the award in 1993. Shaw earned the honor for his research on arctic haze and global climate modulation by clouds.

\section{Graduate Students Honored}

Nettie LaBelle-Hamer won an Outstanding Student Paper award at the 1994 spring American Geophysical Union meeting in Baltimore. Her paper on space plasma was titled "Magnetic Reconnection in the Presence of Sheared Flow."

Post-Doctoral Fellow Yu Lin won the 1993 Ed Hones Space Physics Award for her exceptional work in plasma physics and numerical simulation. The $\$ 1,200$ award is given to recognize outstanding research by a space physics graduate student.

Alice Danielson won third place for Best Student Poster in the physical sciences category of the national AAAS meeting in Boston in 1993. A graduate student in physical oceanography, her poster was titled "The Role of Snow in Antarctic 
Sea Ice Growth." Her poster and presentation also won the Best Student Paper award at the 1992 meeting of AAAS in Valdez. Carl Byers, a graduate student in applied geoscience, shared the Valdez award for his presentation, which was titled "Ground-truthing SMMR Brightness Temperatures of Arctic Multiyear Ice."

\section{Other Honors}

A three-dimensional video animation produced by Geophysical Institute Senior Systems Analyst Rick Guritz and UAF Associate Professor Mitch Roth was chosen from an international field of applicants to become part of a video produced annually by SIGGRAPH, a leading professional organization in the computer graphics industry.

The Geophysical Institute Quarterlynewsletter won the Bronze Award for outstanding work in the 1994 CASE (Council for Advancement and Support of Education) Circle of Excellence Awards. This is a prestigious nationwide competition, entered by large institutions such as Vassar University and the University of Michigan. The newsletter was produced by Science Editor Kathy Berry, Graphic Artist Deb Coccia and Publications Assistant Jan Dalrymple.

Elvey Project Director Merritt Helfferich received the 1992 UAF Alumni Achievement Award for Professional Excellence in 1993. Helfferich graduated from UAF with a BA in English in 1966, the year he began working at the Geophysical Institute as an engineering aid. He later advanced into a variety of positions.

\section{Science Operations Center Dedicated}

The Science Operations Center, one of the buildings to be constructed as part of the upgrade and modernization of Poker Flat Research Range, was dedicated to T. Neal Davis in September, 1993. Davis, the first director of the range, served the Geophysical Institute in many capacities from 1962 through 1982, including professor of geophysics, assistant director, deputy director, and acting director. A professor emeritus, Davis currently serves the institute as a research consultant. Davis also was named by the UAF Alumni Association as UAF's 1994 Distinguished Alumnus.

\section{Employee Recognition Program}

The University of Alaska Fairbanks Employee Recognition Program recognized Professor of Physics Daniel Swift for 30 years of service. Research Assistant Professor Neal Brown, Associate Professor of Physics Hans Pulpan, Professor of Geophysics Gunter Weller and Executive Officer for Administration Helga Wilm were recognized for 25 years. Recognized for 20 years of service were CAD Specialist James Burton and Computer Resource Center Supervisor Morna Mellor. Senior Electronics Technician Michael Cogan, Administrative Assistant Janet Dalrymple, and Professor of Physics Lou-Chuang Lee were recognized for 15 years of service. Those recognized for 5 years of service were Drafter Dolores Baker, Associate Professor of Geophysics Doug Christensen, Accounts Clerk Roberta Greenlee, System Manager Walter Rutherford, Maintenance Worker Ray Ward, and Data Center Specialist Rose Watabe.

\section{Employee Recognition Program}

The University of Alaska Fairbanks Employee Recognition Program recognized Remote Sensing Specialist Kristina Ahlnäs and Professor of Physics Tom Osterkamp for 25 years of service. Human Resource Manager Kate Barr and Programmer Analyst Cynthia Weatherby were recognized for 20 years service. Recognized for 15 years of service were Supervisor of the Electrical Service Shop John Benevento, Electronics Technician Patricia Collins, Systems Analyst Richard Guritz, Electronics Technician Joan McInerney, Ledger Supervisor Johni Meshell, and Professor of Physics John Olson. Those recognized for 10 years of service were Associate Professor of Geophysics Keith Echelmeyer, ASF Mission Planner Greta Reynolds and Professor of Physics Roger Smith. Those recognized for 5 years of service were 
Systems Manager Paul Bundschuh, Property Control Officer Rick Danielson, Project Support Engineer Jim Desrochers, Operations Manager Bob Grove, Research Technician Chris Larsen, Associate Professor of Geophysics Paul Layer, Data Technician Judy Martsolf, Project Engineer Don Rice, Systems Analyst Mitch Robinson, Deputy State Seismologist Charlotte Rowe, Technical Secretary Donna Sandberg, Research Associate in Space Physics Wei Sun, and Research Associate Professor of Space Physics Dan Weimer.

\section{Promotions}

In 1993, Professor of Physics John Craven and Professor of Volcanology John Eichelberger were awarded tenure. Dan Jaffe and Doug Christensen also were awarded tenure and promoted to associate professors, Jaffe in chemistry and Christensen in geophysics. Paul Layer was promoted to Assistant Professor of Geophysics and received tenure in 1994; Cathy Hanks was promoted to research assistant professor of geology.

\section{Commencement}

Advanced degrees were earned in 1993 and 1994 as follows:

\section{Master of Science Degrees}

1993

Terri Bates, geology

Matthew Bierer, space physics

Carl Byers, applied geoscience

Bianca Cerundolo, chemistry

Stephen Crumley, geology

Carrie Decker, geology

Andrew Goodliffe, geophysics

Ming-Shuai $\mathrm{Hu}$, geophysics

Zhijian Li, geophysics

Collette Marsh, physics

Andrew Nicholas, space physics

1994

Wendy Camber, geology

Ze Cheng, geophysics

Mary Eckstein, geology

Nina Harun, geology

North Larsen, atmospheric sciences

Robert Lorenzen, geophysics

Paige Peapples, geology

Kenneth Schwartz, physics

Alice Veazey, oceanography

Dave Veazey, chemistry

Andrew West, geology

\section{Doctor of Philosophy Degrees}

1993

Dominique Gillard, geophysics

Yu Lin, space physics

Wentong $\mathrm{Lu}$, atmospheric sciences

M. Geoff McHarg, physics

Qilong Min, physics

De-Li Shen, physics

Jih-Hong Shue, space physics

Robert Hampton Wade, geophysics

Tingjun Zhang, geophysics

1994

Arlene Anderson, geology

James F. Conner, physics

Dante Espino Garrido, space physics

Yong-Xiang $\mathrm{Hu}$, atmospheric sciences

Annette Labelle-Hamer, space physics

David L. LePain, geology

Zhiwei Ma, space physics

Mark D. Myers, geology

James Robert Slusser, atmospheric sciences

Timothy Quakenbush, atmospheric sciences 


\section{Finances}

The Geophysical Institute and the State of Alaska operate on a fiscal year starting each July 1. Although this report reviews scientific activity for the calendar years 1993 and 1994, institute funding is reported below by fiscal year.

\begin{tabular}{|l|r|r|r|r|}
\hline \multicolumn{2}{|c|}{ SUMMARY OF EXPENDITURES BY FUNDING SOURCE FY91-FY94 } \\
\hline NSF & \multicolumn{1}{c|}{ FY 91 } & \multicolumn{1}{c|}{ FY 92} & FY 93 & \multicolumn{1}{c|}{ FY 94} \\
DOE & $1,822,863$ & $1,955,149$ & $1,779,619$ & $2,182,258$ \\
DOD & 424,345 & 560,763 & 424,314 & 297,309 \\
NOAA & 454,818 & $2,341,400$ & $1,028,770$ & 812,298 \\
NASA & 178,887 & 254,212 & 90,075 & 315,501 \\
MISC. FEDERAL & $7,240,336$ & $7,141,701$ & $8,398,957$ & $13,447,889$ \\
MISC. OTHER & $1,306,576$ & $2,748,352$ & $1,770,841$ & $1,082,813$ \\
STATE OF ALASKA & 582,566 & 480,554 & 873,209 & $1,168,023$ \\
TOTAL RESTRICTED & $12,328,969$ & $15,616,028$ & $14,693,541$ & $19,675,359$ \\
MISCELLANEOUS & 129,590 & 126,362 & 96,237 & 113,273 \\
STATE APPROPRIATION & $4,038,339$ & $3,932,735$ & $4,417,584$ & $3,965,756$ \\
TOTAL & $16,494,899$ & $19,675,126$ & $19,207,362$ & $23,754,389$ \\
\hline
\end{tabular}


9 


\section{GEOPHYSICAL INSTITUTE}

Biennial Report

1993-1994

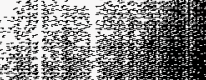

$\because$
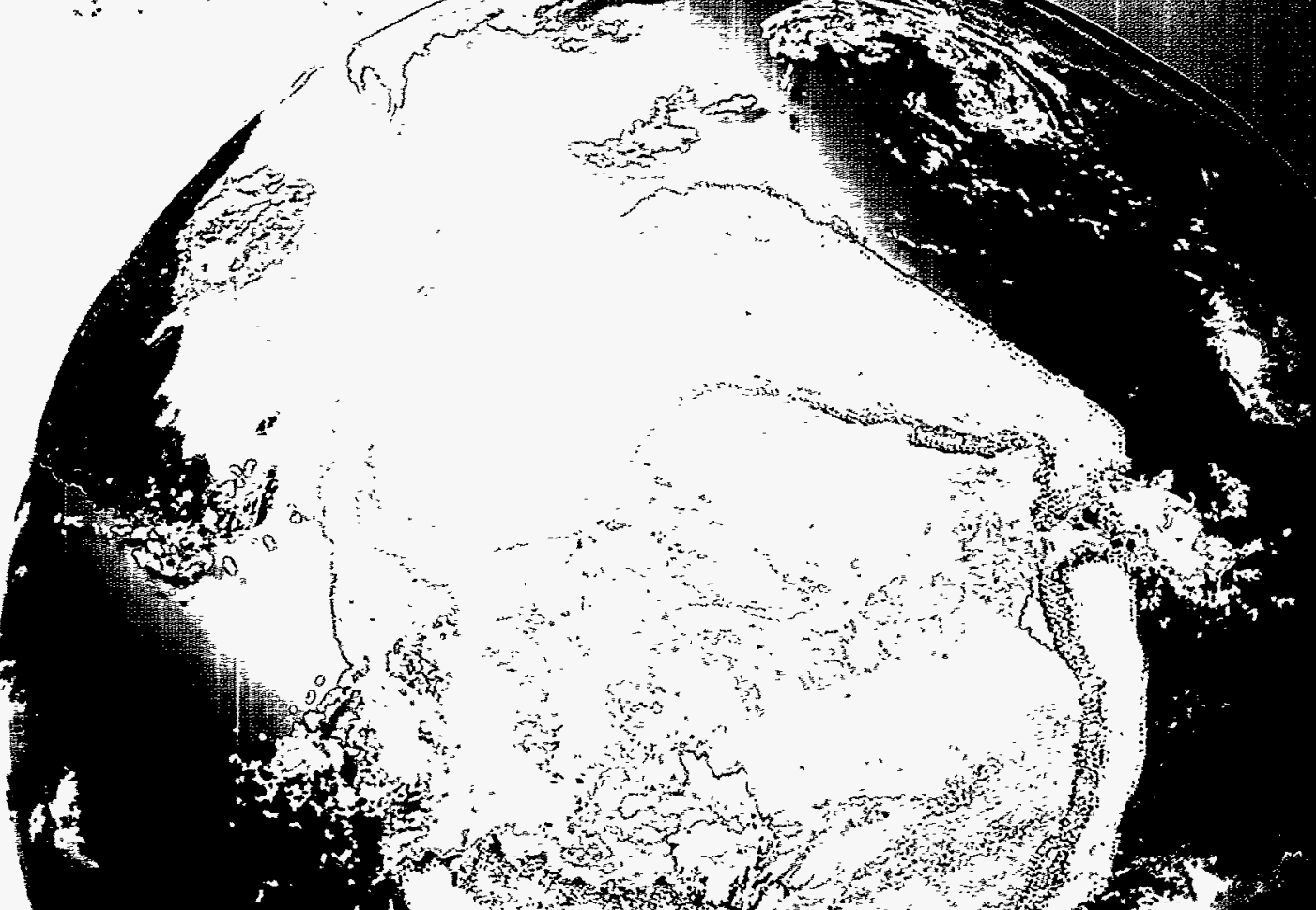

soth (t) N. 